Decoding genetic messages in extracellular vesicles released by immune cells lessons for biomarker applications 


\section{Colofon}

Cover design: Tom Driedonks

Layout and printing: proefschrift-aio.nl

ISBN: 978-90-393-7204-3

(c) Tom Driedonks. All rights reserved. Published papers were reprinted in this publication with permissions from the publisher. No parts of this publication may be reproduced or transmitted in any form or by any means, electronical or mechanical, without permission of the author.

This work was performed at the Department of Biochemistry \& Cell Biology, Faculty of Veterinary Medicine, Utrecht University, Utrecht, The Netherlands, and was funded by the European Research Council under the European Union's Seventh Framework Programme.

Printing of this thesis was financially supported by Infection \& Immunity Utrecht and by the Department of Biochemistry \& Cell Biology, Faculty of Veterinary Medicine, Utrecht University. 


\title{
Decoding genetic messages in extracellular vesicles released by immune cells - lessons for biomarker applications
}

Ontcijferen van genetische boodschappen in membraanblaasjes uitgescheiden door immuuncellen - lessen voor toepassing als biomarker

(met een samenvatting in het Nederlands)

Proefschrift

ter verkrijging van de graad van doctor aan de Universiteit Utrecht op gezag van de rector magnificus, prof.dr. H.R.B.M. Kummeling, ingevolge het besluit van het college voor promoties in het openbaar te verdedigen op dinsdag 5 november 2019 des middags te 12.45 uur

door

\section{Tom Adrianus Paulus Driedonks}

\author{
geboren op 1 november 1986 \\ te Boxtel
}




\section{Promotor:}

Prof. dr. M.H.M. Wauben

\section{Copromotor:}

Dr. E.N.M. Nolte-t Hoen 
"Let's think the unthinkable, let's do the undoable.

Let us prepare to grapple with the ineffable itself, and see if we may not eff it after all"

Douglas Adams 



\section{Table of Contents}

\section{Chapter 1}

General introduction

\section{Chapter 2}

Comprehensive multi-center assessment of small RNA-seq methods

for quantitative miRNA profiling

\section{Chapter 3}

Technical approaches to reduce interference of Fetal Calf Serum derived RNA in the analysis of extracellular vesicle RNA from cultured cells

\section{Chapter 4}

Immune stimuli shape the small non-coding transcriptome of extracellular vesicles released by dendritic cells

\section{Chapter 5}

Circulating Y-RNAs in extracellular vesicles and ribonucleoprotein complexes; implications for the immune system

\section{Chapter 6}

Y-RNA subtype ratios in plasma extracellular vesicles are cell type- specific and are candidate biomarkers for inflammatory diseases

\section{Chapter 7}

The potential role of Y-RNA binding proteins in TLR-induced sorting of Y-RNAs into extracellular vesicles

\section{Chapter 8}

General Discussion

\section{Appendix}

Nederlandse samenvatting

Curriculum Vitae

Dankwoord 
Chapter 1

General introduction 
1. General introduction 


\section{Introduction}

Extracellular vesicles (EV) are $50-1000 \mathrm{~nm}$ sized nanoparticles, composed of lipid membranes, proteins and RNA, which are released by virtually all cells in the body $(1,2)$. EV are also released by 'lower' organisms including bacteria and fungi, although we will focus on EV from mammalian cells in this thesis. EV are abundantly present in a wide range of body fluids, such as milk, urine, seminal fluid, and blood plasma $(3,4)$. The intercellular exchange of EV constitutes a recently discovered route of cell-cell communication that exists next to receptor-ligand interactions and communication via cytokines and chemokines $(3,5)$. The collective term 'EV' refers to both exosomes and microvesicles, which are formed via distinct pathways at different locations in the cell. Exosomes are formed as intraluminal vesicles by inward budding of the multi-vesicular endosomes (MVE), and are released upon fusion of the MVE with the plasma membrane. Microvesicles are generated by direct outward budding of the plasma membrane (2). Several sorting machineries are involved in the recruitment of molecular cargo (e.g. proteins and RNA) into exosomes and microvesicles. Exosome biogenesis is mediated by clustering of molecular cargo by components of the endosomal sorting complex required for transport (ESCRT)(6), or through ESCRT-independent pathways involving tetraspanins (7) or the formation of ceramide by neutral sphingomyelinase (nSMase) (8). Although a number of proteins involved in exosome biogenesis are additionally involved in the formation of microvesicles, such as ESCRT proteins and nSMase, the exact mechanisms underlying microvesicle biogenesis need to be elucidated further $(2,9)$. What determines which proteins and RNAs are selected for incorporation into EV is a major question in the field.

EV can influence the function of target cells via different routes: EV can directly stimulate target cells by binding to receptors, they can transfer receptors to target cells, and they can furthermore deliver intraluminal proteins and RNA molecules into recipient cells (10). It is known that the molecular composition of EV depends on the (patho)physiological status of the EV-producing cell. The resultant alterations in the composition of EV can lead to changes in the functional effect that these EV have on recipient cells. Furthermore, because EV contain molecular information about the (patho)physiological status of the EV-producing cell, and are abundantly detected in body fluids, EV are intensively investigated for their potential application as noninvasive biomarkers for the diagnosis and monitoring of diseases.

The RNA cargo of EV has received a great deal of attention in the last few years (11-13). First of all, the EV-mediated transfer of RNA may play an important role in intercellular communication. Furthermore, there is accumulating evidence that EVassociated RNA in biofluids has great biomarker potential for a wide range of diseases. Important questions in the EV-RNA research field include how RNA cargo is selected for 
incorporation into $\mathrm{EV}$, how these processes are controlled by the status of the cell, and to what extent the EV-transcriptome reflects the cellular transcriptome. Answering these questions is not only important to further elucidate the role of EV-RNA in intercellular communication, but is also an important prerequisite to correctly interpret changes in the RNA content of EV present in body fluids for biomarker purposes. In this thesis, we will predominantly focus on the small non-coding RNAs present in EV. Of these noncoding RNA species, microRNAs (miRNAs) are most intensively studied because their role in gene regulation is well understood. Numerous studies have addressed changes in miRNA profiles in EV due to changes in cell signaling, oncogenic transformation, or other disease-related processes (14-20). Besides miRNAs, EV contain a wide range of other small non-coding RNA species, such as tRNAs, snRNAs, snoRNAs, Y-RNAs, Vault RNAs, and 7SL, many of which are more abundantly present in EV than miRNAs $(16,21)$. Whether the abundance of these other RNA species in EV can change as a result of changes in the (patho)physiological status of EV-producing cells is largely unexplored. In this thesis, we critically evaluated and optimized technologies to characterize and quantify non-coding RNA species in EV released in in vitro cell cultures and in EV present in blood plasma. In addition, we explored how the incorporation of a broad range of non-coding RNA species into EV is modulated by environmental stimuli.

Below, we will introduce the different types of RNA that have been detected in EV. Next, we will explain how commonly used methods to purify EV from culture medium and biofluids can influence EV-RNA analysis. In addition, we summarize possibilities and limitations of RT-qPCR and RNA sequencing, techniques that were extensively used in our studies, for characterization and quantification of EV-RNA. Finally, we give an overview of how EV-RNA can play a role in intercellular communication and how EV-RNA in body fluids may be used as biomarkers of disease.

\section{Non-coding RNAs in EV}

Since it was discovered in 2007 that EV contain miRNA and mRNA, the concept emerged that EV mediate horizontal transfer of genetic messages between mammalian cells (22-24). Later, it was discovered that the EV-transcriptome was much more diverse than initially thought. EV contain various types of non-coding RNA, such as miRNAs, tRNAs, snRNAs, snoRNAs, Y-RNAs, Vault RNAs, and 7SL $(16,21)$. Many of these RNAs are RNA polymerase III transcripts which are found in RNP complexes involved in housekeeping functions in cells $(25,26)$. Furthermore they have been implicated in various diseases, either in full-length or fragmented form $(27,28)$. The presence of these non-coding RNAs in EV suggests that they also serve functions in EV-mediated communication 
between cells. Per RNA type (Figure 1), we will discuss their cellular functions, the proteins with which they interact, their potential involvement in diseases, and what is known about these RNAs in relation to EV.

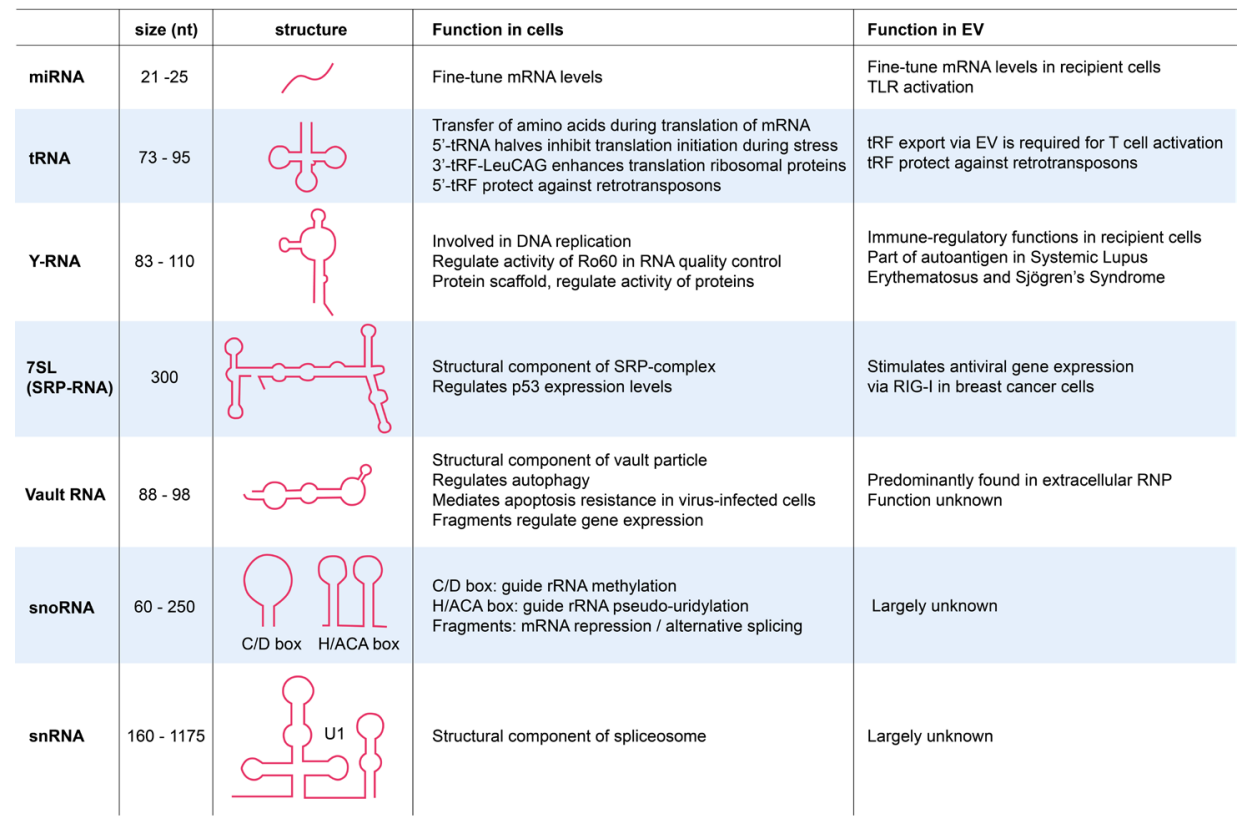

Figure 1 - Overview of the structure, size and functions of various small non-coding RNAs in EV and cells

\section{microRNA}

MicroRNAs (miRNAs) are a family of 21 - 25 nucleotide sized RNAs that negatively regulate gene expression at the transcriptional and translational level (29). The first discovered miRNA, which was called lin-4, was shown to cause changes in the embryonic development of C.elegans (30). A decade later, miRNAs were additionally discovered in other species such as mice and humans $(31,32)$. All miRNAs are transcribed by polymerase II as long pri-miRNA transcripts that are processed by Drosha into a pre-miRNA hairpin. After nuclear export by Exportin-5, the pre-miRNAs are processed by Dicer into miRNA duplexes, after which one of the two strands is assembled into Argonaute (AGO), an essential component of the RNA-induced silencing complex (RISC). The maturation of miRNAs is tightly regulated, as pre-miRNA precursors were found to interact with 180 different RNA binding proteins (RBP) that are involved in their maturation (33). MiRNAs function by basepairing with complementary regions on mRNAs, which may either lead to translational repression or to mRNA cleavage, thereby downregulating gene expression (29). Because the function of miRNAs relies 
on base pairing with target mRNAs, binding sites of miRNAs can be computationally predicted (34). Furthermore, this allows the design of luciferase reporter constructs that contain miRNA target sites in their 3'-untranslated regions (UTR). These constructs are a commonly used tool to validate the miRNA-mediated repression of gene expression. Almost two decades of research have demonstrated the importance of miRNAs in fine-tuning gene expression in a wide range of physiological and pathophysiological processes (27,35-39). Accumulating evidence has shown that many miRNAs are selectively expressed in specific cells and tissues $(40,41)$. Furthermore, aberrant expression of miRNAs is correlated with and/or functionally involved in a wide range of diseases, such as Alzheimer's disease $(17,39)$, cancer $(42)$, autoimmune disorders (43), and cardiovascular diseases (44).

The mechanisms by which miRNAs exert their functions inside cells have been intensively studied. This knowledge provided a basis to investigate the functions of miRNAs in EV-mediated communication. The presence of miRNAs in EV was first discovered in EV from mast cells (22). These EV also contained mRNAs, which could be delivered to recipient cells and subsequently be translated into proteins, proving that RNAs could be functionally transferred via EV (22). Subsequent studies have shown that EV can also mediate functional transfer of miRNAs between cells. For example, $T$ cells which overexpressed miR-335 could transfer this miRNA via EV into B cells which normally do not express miR-335 (45). Similarly, EV released by primary dendritic cells (DC) were shown to transfer miR-148a into a DC cell line which did not express this miRNA (15). To demonstrate functionality of miRNA transfer, both of these studies showed that luciferase reporter constructs fused to miRNA target sites expressed in the recipient cells could be suppressed by the miRNAs transferred via the EV $(15,45)$. Besides luciferase repression, repression of endogenous miRNA targets was shown (46). Addition of EV from wildtype DC, which contained miR-155 and miR-146a, into cells deficient for these miRNAs, resulted in repression of their respective target genes (46). Apart from these 'classical' functions of EV-miRNAs in repression of gene expression, triggering of endosomal RNA sensors in recipient cells has been described as alternative function of EV-associated miRNAs. It was shown that miR-21 and miR-29a in EV released by lung cancer cell lines could stimulate Toll-like receptors (TLR) 7 and 8, which triggered the expression of inflammatory cytokines TNFa and IL-6 (47). It is unclear whether this TLR-activating function is specific within the context of cancer, as these miRNAs have also been abundantly detected in EV from DC and T cell co-cultures (21). Besides their presence in EV, miRNAs have additionally been detected in ribonucleoprotein complexes (RNP) and associated with lipoprotein particles (LPP) in plasma (48-50).

Interestingly, the miRNA composition of EV can change in response to environmental cues imposed on the EV-producing cells. This has been shown for a wide range of conditions, such as prion infection, oncogenic mutations, metabolic stress, virus 
infection, and immune-related stimuli $(4,11,15,16,18,20,51-53)$. Longstanding questions within the field of EV research are which molecular mechanisms underly sorting of RNAs into EV and how this process is influenced by the activation- or differentiation status of the EV producing cell. Cell stimulation-induced changes in EV-associated miRNA levels are potentially controlled by the total levels of miRNA inside cells (54). It is known that cellular miRNA levels robustly and dynamically respond to external stimuli (55). Furthermore, overexpression of miRNAs inside cells was shown to increase the levels of these miRNAs in EV due to an increased availability of these miRNAs for packaging (54). Although this suggests that the incorporation of miRNAs is a passive process that is mainly driven by the sheer abundance of a miRNA in the cytoplasm, several other reports have shown the involvement of RNA binding proteins in the recruitment of cytoplasmic miRNAs into EV (11). For instance, human ribonucleoprotein $\mathrm{A} 2 \mathrm{~B} 1$ ( $\mathrm{hnRNP} A 2 \mathrm{~B} 1$ ) was found to shuttle miRNAs containing specific nucleotide motifs into T cell-derived EV (56). In liver cells, the related protein hnRNP Q (SYNCRIP) was implicated in miRNA shuttling into EV through recognition of a different nucleotide motif than the motif recognized by hnRNP A2B1 (57). Additionally, Y-box protein 1 (YBX1) was found to mediate the packaging of miR-223 in EV released by HEK293T cells (58). In liver cells, the protein embryonic-lethal abnormal vision like (ELAVL, also known as HuR) was shown to mediate the displacement of miR-122 from mRNA/AGO complexes and drive its subsequent export via EV (51). Furthermore, AGO2 has been implicated in miRNA shuttling $(59,60)$, although this point is controversial since other studies have reported that $\mathrm{AGO} 2$ is not associated to EV in culture medium or plasma $(48,49,61)$. It is possible that the incorporation efficiency of AGO2 may differ extensively between cell types $(59,61)$ and has been shown to depend on the mutational status of cells (60). Based on the current literature, the incorporation of miRNAs into EV seems to depend on the cellular context, with specific miRNA-binding proteins being active in specific cell types. It is likely that more cell-specific RNA shuttling proteins will be discovered in the future. Furthermore, it is largely unknown how cellular activation signals modulate the activity of these proteins and how this affects RNA-shuttling.

\section{Transfer RNA}

Transfer RNAs (tRNAs) are historically known for delivering amino acids to ribosomes during the translation of mRNA into proteins. However, various studies have revealed that tRNAs are involved in various other functions (62). tRNAs are approximately 7395 nucleotides long and fold into a typical cloverleaf structure, which is stabilized through posttranscriptional modifications at multiple bases (63-65). tRNA is transcribed by RNA polymerase III as a precursor (pre-tRNA) containing a 5 '-leader and $3^{\prime}$-trailer sequences. During tRNA maturation, the $5^{\prime}$-leader is cleaved off by RNase $P$, 
and the 3 '-trailer is removed by RNase $Z$, after which the bases 'CCA' are added to the 3 '-end (66). Ten percent of the human tRNA genes contain introns which are spliced out by a tRNA-splicing endonuclease complex to form mature tRNA (66). Conjugation of a cognate amino acid to the $3^{\prime}$ end of a tRNA is achieved by an amino acid-specific aminoacyl tRNA synthetase, of which 20 exist, each specific for one amino acid/ tRNA pair (67). Accumulating evidence suggests that the relative abundance of tRNA subtypes in cells influences protein expression. As a result, expression levels of human tRNA subtypes are tissue specific and mirror the codon bias of tissue-specific proteins (68). Aberrant expression levels of tRNAs have been detected in human breast cancers and it was shown that this alters the expression of specific proteins (69). Together, this suggests that the relative abundance of specific tRNAs shapes protein translation (70).

Besides full-length tRNA, various fragmented forms of tRNA have been discovered, such as tRNA halves and tRNA-fragments (tRFs). During cell stress, for instance induced by UV-irradiation, arsenite or heat shock, mature tRNAs are cleaved at specific sites in the anticodon loop by the ribonuclease angiogenin, which leads to the formation of 5'- and 3'-tRNA halves. Specifically the 5'-tRNA-halves inhibit protein translation by displacement of initiation factors from the mRNA m7G cap (71,72). In addition, 5'tRNA halves promote the assembly of stress granules, in which untranslated mRNAs are sequestered (73). This implicates 5'-tRNA halves and angiogenin as effectors of stress-induced translational repression. It is currently unknown whether 3 '-tRNA halves have any function. Interestingly, angiogenin-mediated cleavage is controlled by tRNA methylation (74). Loss of the cytosine- 5 methyltransferase NSun2, which methylates tRNA at specific nucleotides, leads to accumulation of 5'-tRNA halves, because angiogenin has a higher affinity for unmethylated tRNAs (74). Consequently, NSun2 knockout cells were more sensitive toward oxidative stress (74) and exhibited reduced protein synthesis (75). Furthermore, certain neurological and developmental abnormalities in mice and humans are caused by mutations in NSun2 $(74,76)$. Together, this exemplifies that posttranscriptional modifications are essential for tRNA folding and stability $(65,77)$. Consequently, tRNA instability and the ensuing tRNA fragmentation makes cells more sensitive to environmental stress. Nevertheless, as 5'-tRNA halves have been detected in hematopoietic and lymphoid mouse tissues in homeostatic conditions, 5'-tRNA halves may have additional physiological functions that are yet to be uncovered (78).

Besides the generation of tRNA halves, tRNA can also be cleaved into $12-30 \mathrm{nt}$ tRNA fragments (tRFs) (66). 5'-tRFs can be generated through Dicer-dependent (79) and Dicer-independent mechanisms (80-82). The formation of Dicer-dependent tRFs is controlled by the Lupus autoantigen La (83), which is required for correct folding of tRNAs into cloverleaf structures by binding to their $3^{\prime}$ oligo-uridine motifs $(84,85)$. Knockdown of La increased mis-folding of tRNAs into hairpin structures. These mis- 
folded tRNAs were translocated by Exportin-5 from the nucleus into the cytoplasm, where they were processed into tRFs by Dicer (83). Whether the methylation status of tRNAs influences its processing into tRFs, similar to angiogenin-mediated tRNA processing into tRNA-halves, is not known. Because tRFs are comparable in size to miRNAs, and have been found in association to AGO, tRFs were initially thought to mediate gene silencing (86-88). However, there is no experimental evidence that AGO-associated tRNA mediates mRNA repression (83). Instead, tRFs may serve distinct functions. For example, tRFs may regulate the translation of $m R N A$ s encoding ribosomal proteins. It was recently found that a specific fragment of tRNA-LeuCAG (LeuCAG3'tsRNA) binds mRNAs that encode two ribosomal proteins (RPS28 and RPS15), thereby enhancing their translation efficiency (89). Other specific 3'-tRFs were shown to protect against retrotransposition of endogenous retroviruses (ERV) in developing stem cells (90). Like retroviruses, retrotransposon RNA transcripts need a tRNA fragment to prime their reverse transcription into CDNA, which is then re-integrated into the host genome. An 18nt 3'-tRF was shown to compete for tRNA-primer binding sites on the retrotransposon RNA, thereby prohibiting the formation of CDNA products and subsequent retrotransposition (90). These examples show that tRNA fragments are emerging as elements of translational control and may additionally confer protection against endogenous retroviruses. Notably, the mechanisms underlying these functions are markedly different from what is known from the well-studied class of miRNAs.

tRNAs and various types of tRNA fragments have been found in EV from several biological sources $(16,21,52,91-97)$. Not all extracellular tRNA has been found in association with EV. Instead, tRNAs have been abundantly detected in plasma LPPs $(78,98)$ and occur as RNase-resistant RNA:RNA duplexes (99). Interestingly, the abundance of extracellular tRNAs differs greatly between biofluids $(100,101)$, with high amounts of (fragmented) tRNA being present in urine, bile, seminal plasma and amniotic fluid $(93,101,102)$. The functions of EV-associated tRNA are largely unexplored, but a few studies have suggested potential effects of tRNA shuttling via EV. First, the disposal of tRNA fragments via EV may be required for activation of T-cells (97). In EV from anti-CD3-activated T-cells, increased amounts of tRNA fragments 5'-tRNALeu-TAA and -TAG were found relative to unstimulated conditions. The shuttling of 3'-fragments was unaffected. To test whether increased tRF release influenced cellular 5 '-tRF levels, EV release was blocked by chemical inhibition of the nSMase protein, which is important for the formation of EV. This indeed reduced the release of EV and EV-associated 5'-tRFs, which led to the accumulation of this tRF in cells. To address the function of cellular $5^{\prime}$-tRF, their function was inhibited with antisense oligonucleotides. This resulted in higher T-cell activation and consequent release of the cytokine IL-2. This suggested that activated T-cells release tRF-containing EV to dispose of tRFs that can inhibit their activation and cytokine release (97). Second, tRNA enclosed within EV in 
sperm were shown to repress endogenous retroelements in embryos, thereby affecting embryonic development (103). EV in sperm from mice consuming a low-protein diet contained EV with an increased level of 5'-tRF-Gly. Microinjection of 5'-tRF-Gly into early embryos resulted in repression of the endogenous retroelement MERVL. This effect was recapitulated in embryos generated with sperm containing EV with high amounts of 5'-tRF-Gly versus low amounts of 5'-tRF-Gly. Besides repressing MERVL, 5'-tRF-Gly was shown to repress ribosomal protein expression. As a consequence, embryos generated with sperm from low-protein diet mice showed slower cell division during early embryonic development (103). Although the long term effects were not described, this indicates that the paternal diet may influence the tRNA content of EV in sperm, which may affect the phenotype of offspring (103). Summarizing, although tRNAs have been intensively studied in cells, relatively little is known about the functions of tRNAs and tRNA fragments in EV. The recent discoveries of multiple different functions of (fragmented) tRNAs in cellular processes may guide studies on the functional effects of tRNAs in EV.

\section{Y-RNA}

Y-RNA was discovered because it is associated to the Ro60 autoantigen, which is a clinically important target of autoantibodies in Systemic Lupus Erythematosus (SLE) and Sjögren's Syndrome (SS) patients (104). Y-RNAs are 83 - 110 nucleotide long RNAs which fold into stem-loop structures and are evolutionary conserved in vertebrates $(105,106)$, nematodes (107), and bacteria (108). Four different Y-RNA subtypes (Y1, Y3, Y4 and Y5) exist in the human genome, while only two ( $Y 1$ and $Y 3$ ) exist in mice (109). $Y$-RNA interacts with several proteins. The conserved stem region is bound by Ro60 protein and the 3'-oligouridine tail is bound by La. Ro60 is required for stabilization of Y-RNA, since Ro60 knockout leads to an almost complete loss of Y-RNA (110). The loop regions differ between Y-RNA subtypes and are known to interact with various other RNA-binding proteins $(111,112)$. Y-RNA is implicated as chaperone for interacting proteins, thereby influencing the localization and the activity of these proteins $(113,114)$. In this way, intracellular Y-RNA is involved in a number of housekeeping functions, such as DNA replication (115) and quality control of misfolded non-coding RNA $(116,117)$. For example, Ro60 is known to bind misfolded non-coding RNAs in its central cavity, which can be sterically blocked by Y-RNA $(116,118)$. Furthermore, Y3-RNA in neuronal cells regulates the localization of enhancer protein $\mathrm{HuD}$, which enhances the translation of mRNAs encoding proteins involved in neuronal development (119). Y3-RNA binding counteracts the translation enhancer activity of HuD by dissociating it from bound mRNAs (119). The functional consequences of the interactions between Y-RNA and other proteins remain to be further investigated.

Y-RNA has been detected in EV from various biological sources, and multiple studies have shown a strong enrichment of Y-RNA in EV relative to cellular levels $(16,21,52,91-$ $93,95,120,121)$. In line with this, Y-RNA is among the most abundant non-coding RNAs 
in human plasma $(100,101)$. In addition to full-length Y-RNA, Y-RNA fragments derived from the stem regions have been detected in EV (reviewed in (112)). Currently, there is inconsistency in the EV-field over the actual abundance of full-length versus fragmented Y-RNA in EV. We further address this in Chapter 4, 5, 6 and in the General Discussion (Chapter 8). Y-RNA has also been found complexed to RNP in plasma of SLE and SS patients (104), and in culture supernatant of glioblastoma cells $(61,122)$. Importantly, we discovered that Y-RNA levels in EV are not static, but can be regulated by external signals imposed on cells ((52), Chapter 4, 6 and 7). This suggested that Y-RNAs, in addition to miRNAs, contribute to the unique molecular identity of EV. The function of Y-RNA in EV is largely unknown, although a number of reports suggested that Y-RNAs in EV may have immune regulatory functions $((94,112,121,123)$ Chapter 5$)$. An extensive review of the literature on the presence of Y-RNAs in EV, their function in intercellular communication, and biomarker potential can be found in Chapter 6 (112).

\section{$7 S L$ (SRP-RNA)}

7SL is the RNA component of the signal-recognition particle (SRP), which consists of six signal-recognition proteins (SRPs). This RNP complex recognizes signal sequences on nascent polypeptides and mediates translocation of nascent secretory proteins across the ER membrane (124). Besides this essential function in the production of secretory proteins, 7SL is mainly known for its involvement in the development of cancer. 7SL is highly expressed in cancer tissues, and its repression resulted in a significant reduction of cell proliferation (125). It was shown that 7SL dissociates the enhancer protein HuR from the 3 '-untranslated region of tumor suppressor gene p53 (125). This leads to downregulation of p53 expression, thereby favoring tumor growth (125).

$7 S L$ has been abundantly detected in $E V$ released by various cell types $(16,21,52,95,126)$. Nevertheless, its function in intercellular communication is largely unexplored. Interestingly, recent data indicate that $7 \mathrm{SL}$ triggers the production of the antiviral cytokine interferon in breast cancer cells (126). Indeed, interferon production is a characteristic of certain types of breast cancer that are resistant to radiation therapy (127). Interferon production can be induced by the activation of the intracellular RNA sensor RIG-I, which specifically detects (viral) 5'-triphosphorylated RNA (128). In the case of breast cancer, EV from activated stromal cells were shown to contain non-protein bound, 5 '-triphosphorylated 7SL, which triggered RIG-I in breast cancer cells resulting in interferon production which stimulated the outgrowth of radiation therapy resistant tumor cells $(126,129)$. This illustrates the important role that RNA-binding proteins have in EV, which prevent the activation of RNA sensors by the transferred EV-RNA. Whether EV-associated 7SL has other functions remains to be investigated. 


\section{Vault RNA}

Vault RNA (vtRNA) is the RNA component of the largest known RNP complex, i.e. the 13 MDa Vault particle (130). This large, barrel-shaped RNP consists of three proteins; the major vault protein (MVP), telomerase-associated protein (TEP1), and the vault poly(ADP)ribose polymerase (VPARP) (131). The majority of vtRNA is found in the cytoplasm and is associated with the vault particle through interaction with TEP1 (132). What kind of physiological functions the vault particles fulfill is a longstanding question in the field. Upregulation of vault particles has been reported in different disease-related conditions, such as in drug-resistant cancer cell lines (132), in cancer cells responding to IFNy (133), and in DC activated with LPS (134), although the exact consequence of this upregulation has not been resolved. Functions of vtRNA are even less well understood, although it was recently discovered that vtRNA has a role in the regulation of autophagy (135). A key step in the initiation of autophagy is the oligomerization of p62, which was shown to be controlled by the interaction between vtRNA and p62 (135). Furthermore, it has been shown that vtRNA can be upregulated by viruses such as Epstein Barr virus (EBV) (136) and Influenza A (137). In the case of influenza, vtRNA was shown to bind and suppress the viral dsRNA sensor PKR, thereby promoting viral replication (137).

Like many other small non-coding RNAs, vtRNA can be cleaved into multiple fragments, which are referred to as small vtRNAs (svRNAs) $(28,138)$. Cleavage of vtRNA is mediated by Dicer (139), and may additionally depend on RNA methylation, as NSun2 knockout cells contained strongly reduced levels of svRNAs (140). Similar to miRNAs, svRNAs can interact with AGO and functionally repress mRNA translation. One of these fragments, named svRNA-b, downregulates expression of cytochrome P450 3A4 (CYP3A4), which has an important role in metabolizing various drugs (138). A fragment that was strongly reduced in Nsun2 knockout cells, svRNA-4, was shown to repress levels of voltage-gated calcium channels (140). This may to some extent explain neurological defects which were reported in NSun2-deficient mice (74).

VtRNA has been detected in EV from cultured cells and in serum $(21,52,95,120,141-$ 143). Lässer et al. have reported that vtRNA is predominantly found in high-density particles in culture medium of mast cells (92). This is supported by a recent report that predominantly detected vtRNA in high-density particles (61). Moreover, intact vault particles were observed by electron microscopy in high-density fractions (61). In our own experiments, we predominantly detected vtRNA in high-density particles, although a minor amount of vtRNA could additionally be detected in high-grade purified EV (Driedonks, unpublished data). The amount of EV-associated vtRNA may depend on the used cell type, as Jeppesen et al. detected vtRNA in glioblastoma-EV, but not in breast cancer-EV (61). Functions of EV-mediated transfer of vtRNA or vtRNA fragments have not yet been addressed. 


\section{snoRNA}

SnoRNAs (small nucleolar RNAs) reside in the nucleolus and aid in processing and modification of ribosomal RNAs. There are two families of snoRNA, the 'C/D box' and ' $\mathrm{H} / \mathrm{ACA}^{\prime}$ ' snoRNAs. Both groups of snoRNAs use antisense complementarity to ribosomal RNAs to guide methyltransferases or pseudo-U-synthetases to specific sites on the rRNAs that need to be methylated and pseudouridylated, respectively $(144,145)$. These base modifications are essential for normal ribosomal function, as loss of rRNA methylation causes slower rates and lower accuracy of mRNA translation (146). Most snoRNAs are encoded in introns of ribosome-related genes, indicating that snoRNA expression and ribosomal gene expression are tightly co-regulated (147). Thus, snoRNAs have an important role in the modification of rRNA which is required for normal ribosomal function. In addition, aberrant snoRNA expression levels have been associated with carcinogenesis (reviewed in (148)). Additionally, a defect in a genetic locus that encodes SNORD116 and SNORD115 is implicated in Prader-Willi syndrome (149). The mechanisms by which aberrant snoRNA expression results in disease are not understood, and may be unrelated to the functions of snoRNA in rRNA modification. Similar to other small non-coding RNAs, snoRNAs can be processed into small RNAs by Dicer-dependent and -independent mechanisms $(139,150)$. Some of these snoRNA fragments have been shown to influence gene expression. The snoRNA fragment ACA45, for example, was shown to repress expression of CDC2L6, which is part of a protein complex involved in the regulation of polymerase II transcription (151).

SnoRNAs are predominantly retained in the nucleus of cells, and therefore the detected snoRNA levels in EV are low $(21,52,91,142)$. It is not clear whether EVassociated snoRNAs are fragmented or full length. Nevertheless, it was reported that detection of snoRNAs in plasma was feasible, which highlighted their potential in minimally invasive liquid biopsies (152). It remains to be investigated whether snoRNAs in plasma are EV-associated or complexed to different macromolecular structures, such as LPPs.

\section{snRNA}

snRNAs (small nuclear RNAs) are an important component of the spliceosome, which is a complex macromolecular machine that catalyzes mRNA splicing $(153,154)$. This complex is composed of multiple RNP subunits, each of which consists of a snRNA molecule (U1, U2, U4, U5, U6, U11 and U12) complexed with multiple spliceosomal proteins named snRNPs. The snRNAs carry out various essential functions during mRNA splicing. This multi-step process starts with the recognition of $5^{\prime}$ and $3^{\prime}$ splice sites by $U 1$ and $U 2$ snRNPs, respectively. The $U 1$ and $U 2$ snRNPs then interact with each other to fold the intron into a loop. Subsequently, the U4-U6-U5 snRNP is recruited, which catalyzes the reaction that excises the intron and mediates ligation of the exons 
(reviewed in (154)). Appropriate processing and folding of snRNAs is important for cell survival (155). Defects in snRNA function may underlie various diseases such as spinal muscular atrophy (156) and growth defects (157). Therefore cells are employed with various surveillance mechanisms that ensure snRNA quality by mediating degradation of aberrant snRNAs (155-158).

Various studies have reported the presence of snRNAs in EV $(18,52,91,92,95)$. Not all snRNA subtypes are equally abundant in EV, as we have found that U2, U5, U1 and U6 are the most abundant snRNA in EV, based on counts in our RNA sequencing datasets (52). Besides its association with EV, a considerable amount of snRNA may additionally be associated with high density protein complexes (92). Furthermore, snRNA have been found in plasma, and U2 was proposed as biomarker potential for pancreatic and colorectal adenocarcinoma (159). The relatively abundant presence of snRNA in EV and/or RNP seems to contradict their predominant nuclear localization in cells. However, snRNA maturation involves a number of processing steps that take place in the cytoplasm (154). Additionally, snRNAs have been shown to localize to cytoplasmic RBP granules, called 'U-bodies' (160). It was suggested that these U-bodies have a role in the assembly of snRNPs, or serve in storage of snRNPs before their re-import into the nucleus. Cytoplasmic localization of snRNAs was further confirmed by results of the GENCODE project, which reported that snRNAs were equally abundant in the cytosol as in the nucleus of 15 different cell lines (161). Functions of EV-mediated snRNA transfer have not yet been addressed.

Together, this illustrates that extracellular RNA consists of a highly heterogeneous group of non-coding RNA classes. These RNA species serve different roles in the cell and aberrant expression of these RNAs may lead to development of disease. A limited number of studies has indicated that not only miRNAs but also other non-coding RNA types present in EV may serve a role in cellular communication. There is emerging evidence that many of the non-coding RNAs in the extracellular space can be associated to both EV, RNP and LPP particles. Because these different RNA carriers have distinct physical characteristics, the methods that are applied to concentrate extracellular RNA may greatly impact the outcomes of an experiment.

\section{Commonly used methods for the separation of EV from other RNA- containing structures}

One of the challenges in EV-research is the isolation and concentration of EVs from culture supernatant or biofluids. Various EV purification methods are being used, all of which aim to separate EV from other bioactive structures (such as protein complexes and lipoprotein particles) and/or concentrate EVs from a large volume of liquid $(162,163)$ (summarized in Table 1). These methods differ in the purity and yield of recovered EV (sub) populations, and in the type of contaminating structures remaining in the EV preparation. 
Because these contaminating structures may contain extracellular RNA, the purity of EV will greatly affect the composition of the isolated RNA. Indeed, EV preparations isolated by different methods show differences in protein and RNA composition (164-167). Great efforts have recently been made to identify experimental and reporting requirements for the isolation and characterization of EV (168) and to improve accurate reporting of methodological details of EV-purification and characterization in publications (163).

Table 1 - Overview of the processing time and pros/cons of commonly used EVpurification methods

\begin{tabular}{|c|c|c|c|c|}
\hline Method & $\begin{array}{l}\text { Typical starting } \\
\text { volume }\end{array}$ & $\begin{array}{l}\text { Typical } \\
\text { processing time }\end{array}$ & Pro & Con \\
\hline $\begin{array}{l}\text { Differential } \\
\text { ultracentrifugation }\end{array}$ & $10 \mathrm{~mL}-250 \mathrm{~mL}$ & $\begin{array}{l}0.5 \text { days hands-on } \\
\text { time }\end{array}$ & Straightforward & $\begin{array}{l}\text { - Contamination with RNP } \\
\text { - Advanced equipment required }\end{array}$ \\
\hline $\begin{array}{l}\text { Density gradient } \\
\text { centrifugation }\end{array}$ & $10 \mathrm{~mL}-250 \mathrm{~mL}$ & $\begin{array}{l}1.5 \text { day hands- } \\
\text { on time plus } \\
\text { overnight } \\
\text { centrifugation }\end{array}$ & High purity & $\begin{array}{l}\text { - Long processing time } \\
\text { - Advanced equipment required } \\
\text { - Loss of EV by sticking to } \\
\text { centrifugation tubes }\end{array}$ \\
\hline SEC & $<0.5 \mathrm{~mL}$ & $\begin{array}{l}60 \text { min hands-on } \\
\text { time }\end{array}$ & $\begin{array}{l}\text { Quick, no } \\
\text { advanced } \\
\text { equipment } \\
\text { required }\end{array}$ & $\begin{array}{l}\text { - Contamination with large LPP } \\
\text { - Requires concentrated sample } \\
\text { - Loss of EV by sticking to } \\
\text { column }\end{array}$ \\
\hline $\begin{array}{l}\text { Precipitation-based } \\
\text { methods }\end{array}$ & $10-250 \mathrm{~mL}$ & $\begin{array}{l}<60 \text { min } \\
\text { hands-on time, } \\
\text { plus overnight } \\
\text { precipitation }\end{array}$ & Cheap, scalable & $\begin{array}{l}\text { - Co-precipitation of } \\
\text { contaminant proteins and LPP }\end{array}$ \\
\hline
\end{tabular}

\section{Differential ultracentrifugation}

Differential ultracentrifugation is the most commonly used EV-isolation technique for isolation of EV from culture supernatant $(162,163)$. First, low-speed centrifugation steps are carried out to deplete cells, apoptotic bodies and cell debris from culture supernatant, after which EV are pelleted at very high g-forces, typically 100,000 $200,000 \mathrm{~g}$ (Figure 2A). Although this is a straightforward technique to concentrate $\mathrm{EV}$, one must be aware that other high-density structures may be co-isolated, such as ribonucleoprotein complexes (RNPs) and high-density lipoprotein particles (HDL) $(11,52,165)$. In contrast, low-density lipoprotein particles (LDL) are not pelleted by ultracentrifugation (169). Furthermore, particles are pelleted less efficiently from viscous fluids $(170,171)$. 


\section{Size exclusion chromatography}

Size-exclusion chromatography (SEC) is used to separate macromolecular structures based on size. This method is quickly gaining popularity in the EV research field (172-176). A SEC column consists of a porous resin through which a buffer is run. A concentrated EV-containing sample is loaded onto the column, and differently sized structures are collected in different eluate fractions (Figure 2B). Smaller particles can access the pores in the resin and thus have a long retention time, eluting in the later fractions, while large particles do not enter the pores and elute in the early fractions. SEC can be performed in any lab since it does not require any specialized equipment such as ultracentrifuges. SEC columns can be manually prepared (172) or purchased commercially (173). Furthermore, processing times are shorter than with ultracentrifugation, and there is some indication that the function of EV purified by SEC may be better preserved than after using ultracentrifugation (177). Disadvantages of SEC are that only small sample volumes can be loaded, and that EV will become considerably diluted in the eluted fractions. Therefore, concentration before SEC and/ or after SEC may be required, depending on the downstream application. Notably, SEC is a crude separation method that only separates structures based on size, which is not sufficient to completely separate EV from other RNA-containing structures in complex biofluids. For example, EV-containing fractions will additionally contain large lipoprotein particles (such as chylomicrons, VLDL and LDL) because of the overlap in size. Additional techniques, such as density gradient centrifugation, are required to further separate EV from contaminant structures ((176) and Chapter 6).

\section{Density gradient centrifugation}

Density gradient centrifugation can be used to separate particles based on their buoyant density (Figure 2C). Concentrated particle samples are either loaded on top of a density gradient (top-down), or are added to the bottom of a tube and are subsequently overlaid with a gradient (bottom-up). These density gradients may be prepared from highly concentrated sucrose solutions or iodixanol (Optiprep), which each have their advantages and disadvantages. lodixanol is capable of forming self-generating gradients, is iso-osmotic at all densities, has a low viscosity, and is non-toxic when added to cells. However, efficient pelleting of EV from iodixanol fractions requires at least 15fold dilution of the fractions (Driedonks, unpublished data). In contrast, sucrose is more viscous, has a high osmolarity, and is toxic to cells. Lower dilution factors are required to pellet EV from sucrose fractions (4-fold dilution is sufficient). Generally speaking, the choice for a density medium depends on the intended downstream application; sucrose is optimal for characterization of the protein / RNA content of EV because of the lower dilution factors required for EV-pelleting from sucrose, whereas iodixanol is preferred when studying the function of EV because of its low toxicity on cells. Density gradients 
are centrifuged at high speeds $(100,000-200,000 \mathrm{~g})$ for long periods of time (15-18 h) to allow the particles to migrate to their equilibrium density. The density of EV is typically $1.11-1.18 \mathrm{~g} / \mathrm{mL}$. Since most LDL have densities of $0.93-1.06 \mathrm{~g} / \mathrm{mL}$, and RNP $>1.25 \mathrm{~g} / \mathrm{mL}$, they can be separated from EV using a gradient. In contrast, the density of HDL $(1.06-1.21 \mathrm{~g} / \mathrm{ml})$ overlaps with the density of EV, and HDL particles may therefore contaminate EV-containing fractions $(169,176)$. It was shown that a combination of sizebased and density-based separation methods can to a large extent separate EV from LPP and RNP ((176) and Chapter 6).

A

Ultracentrifugation

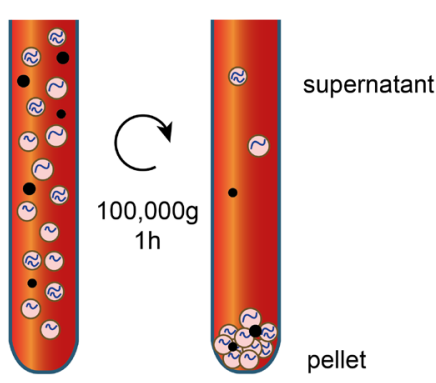

C

$$
\begin{aligned}
& \text { Density gradient } \\
& \text { centrifugation }
\end{aligned}
$$

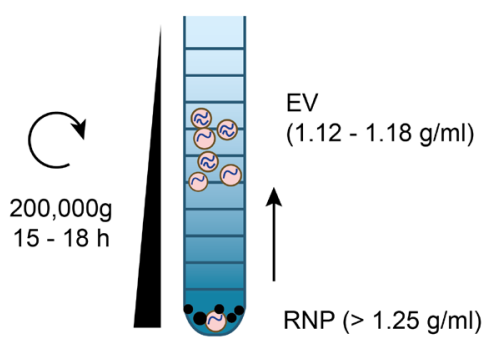

B

Size-exclusion chromatography

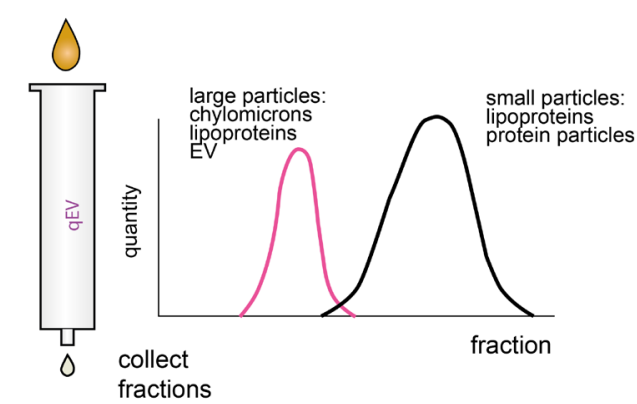

D

\section{Precipitation}

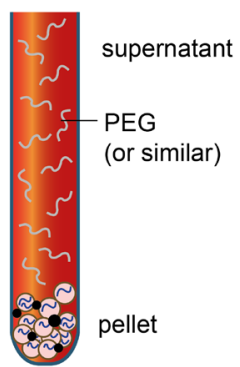

Figure 2 - Schematic representation of commonly used methods for EV enrichment or purification

A) EV (pink) and other dense particles (black) are pelleted by applying high g-forces during ultracentrifugation

B) In size-exclusion chromatography, a concentrated EV sample (brown drop) is applied onto a size-exclusion column through which a buffer is run. Particles with a large diameter elute in early fractions (pink line) while particles with a small diameter elute in later fractions (black line)

C) Differential density gradient centrifugation separates $\mathrm{EV}$ from other structures by differences in buoyant density

D) In precipitation, water soluble polymers such as PEG are added to the EV-containing liquid to reduce the solubility of $\mathrm{EV}$, which allows pelleting at low g-forces. 


\section{Precipitation-based methods}

Precipitation-based methods have frequently been used to concentrate EV from large volumes of fluid (Figure 2D) $(165,178)$. Most precipitation methods use soluble polymers and low temperatures to reduce the solubility of proteins (and EV), which can subsequently be pelleted at low centrifugation speed. Several commercial EV isolation kits are available, such as the miRCURY Exosome Isolation Kit, ExoQuick and Invitrogen Total Exosome Isolation Reagent (179). In-house prepared poly-ethyleneglycol (PEG) solution have also been used as precipitation agent (178). This method is relatively inexpensive, does not require advanced equipment, and can be used in large scale applications. However, co-precipitation of contaminant proteins and LPP is an important caveat $(165,178,179)$. Despite the high the protein and RNA yields obtained by precipitation, the purity is low. Furthermore, co-precipitated proteins and LPP greatly affect the protein and RNA composition of samples (165), which biases the outcomes of EV-RNA characterization or studies on the functional effects of EV.

\section{Contaminant RNAs derived from cell culture medium supplements}

It has been known for a long time that bovine serum that is used to supplement cell culture medium contains EV (170), and that these EV contain bovine RNAs (180). Depletion of bovine EV from fetal calf serum (FCS) is commonly employed, for instance by prolonged ultracentrifugation, before using this medium to culture cells for EV-production. Depletion of FCS-EV is important in studies aiming to characterize the molecular content of EV. This is particularly important in studies addressing the RNA content of cell culture EV because of the sequence similarity of non-coding RNAs in various mammalian genomes. In 2016, it was reported that even prolonged ultracentrifugation does not remove all bovine RNA from culture medium (181). Since then, several studies by us and others have determined the extent to which bovine RNAs contaminate RNA preparations of cell culture EV (180-186). Most of these studies agree on the point that ultracentrifugation does not deplete all RNA-containing carriers from FCS $(180,181,183,185,186)$. Nevertheless, an important unanswered question is to what extent residual FCS-RNA in EV-depleted medium affects analysis of the RNA content of cell culture-derived EV. In Chapter 3 (185) we addressed the efficiency with which different types of non-coding RNAs can be depleted from FCS and how the influence of these bovine RNAs on the characterization of RNA in cell culture EV can be minimized.

\section{Technical aspects of EV-RNA quantification and characterization}

Research interest in characterization and quantification of EV-RNA has strongly increased, because EV-RNA potentially plays an important role in intercellular communication, and can be exploited as biomarker for disease. The most commonly used methods for characterization and quantification of cellular RNA and EV-RNA are 
RNA-sequencing (RNA-seq) and reverse-transcriptase quantitative polymerase chain reaction (RT-qPCR). RT-qPCR relies on a priori selection of genes of interest and does not allow the discovery of novel transcripts. In contrast, RNA-seq is a high-end technique which determines the nucleotide sequences of thousands of unique RNA molecules in parallel, thereby providing a comprehensive overview of the transcriptome of a cell or EV. Nevertheless, each method comes with its own difficulties. Biases in RNA sequencing are a common challenge for both cellular and EV-RNA characterization. In addition, a specific challenge in EV-RNA analysis is the lack of known reference genes that can be used to normalize RT-qPCR data. In this paragraph, we will elaborate on these technical challenges and propose strategies to deal with them.

\section{Analysis of EV-RNA by small non-coding RNA sequencing and sources of sequencing bias}

Analysis of the highly complex and dynamic eukaryotic transcriptome has been revolutionized by the RNA-seq methodology (187). In RNA-seq, all transcripts in a sample are converted to cDNA in a series of processing steps (sequencing library preparation), after which the nucleotide sequence of all transcripts is determined in a next-generation DNA sequencer (NGS). The sequencing data are then computationally processed, in which the abundance of each transcript is counted. Sequencing libraries may vary in the absolute number of read counts per library, therefore the abundance of individual transcripts is usually expressed relative to the total read counts of the library, so-called counts per million (cpm). Various specialized protocols exist to convert the input RNA transcripts into cDNA. The studies in this thesis employed small RNA-seq analysis of non-coding RNAs. Most small RNA libraries are prepared via ligation of oligonucleotide adapters to the $5^{\prime}$ - and 3 '-ends of the RNA transcripts. These adapters allow annealing of a reverse-transcription primer, which allows the reverse transcriptase to generate cDNA from the RNA templates. The next steps include PCR amplification, library cleanup (and optional size-selection) and nextgeneration sequencing. Although small RNA sequencing library preparation methods are generally reproducible among laboratories $(188,189)$, different commercially available library preparation kits differ in the exact types of enzymes, adapters and other reagents that are employed. Due to differences in the specificity of RNA ligases from different kits, different transcripts may be captured with variable efficiency (190). Other biases may arise from 5 ' and 3 '-end modifications, differences in G/C content, and secondary structure of transcripts $(191,192)$. Although, RNA sequencing data obtained using the same library preparation kit are generally reproducible $(188,189)$, not much was known about the comparability of results obtained using different kits, about whether these kits allow accurate quantification of all different RNA molecules in a sample, and on how well quantitative differences in RNA transcripts are reflected 
in the final RNA-seq dataset. To investigate this, we took part in a multicenter study in which an equimolar pool of 1152 synthetic miRNAs was analyzed by RNAseq using a number of different protocols by various labs (Chapter 2). Identification of methodological limitations of RNA sequencing is a step into the right direction and may guide experimental design to circumvent biases, or may help in setting up computational approaches to reduce bias (189).

\section{Analysis of EV-RNA by RT-qPCR and normalization of data}

While RNA sequencing is a powerful technology to measure the expression of a wide range of RNAs in parallel, reverse transcription quantitative polymerase chain reaction (RT-qPCR) allows for relatively simple, quantitative measurement of selected target genes of interest. For the analysis of small RNA by RT-qPCR, small RNA molecules are poly-adenylated and reverse-transcribed into cDNA. Alternatively, stem-loop primers can be used for reverse transcription, which circumvents poly-adenylation (193). Subsequently, qPCR is performed to quantify the transcripts of interest. In this reaction, target CDNA products are exponentially amplified in a PCR reaction using oligonucleotide primers and DNA polymerase. Each PCR cycle typically results in a duplication of the gene product, and this amplification can be measured in real-time using a fluorescent dye that intercalates into the double-stranded DNA products (194). The fluorescent signal is low during the first amplification signals and does not exceed the background, but as the amount of PCR product accumulates, the fluorescent signal increases exponentially. The more copies of a specific transcript were initially present, the fewer cycles are required to reach the quantification threshold, which is termed the $\mathrm{Cq}$ value (quantification cycle). Therefore, differences in $\mathrm{Cq}$ value $(\mathrm{dCq})$ between samples reflect differences in transcript abundance. This illustrates that RT-qPCR data are not absolute measures of the abundance of a transcript. Nevertheless, RT-qPCR is a useful method to compare the relative abundance of transcripts in different samples over a wide dynamic range.

Reliable comparison of gene expression in different samples requires normalization to compensate for differences in the amount of biological material in the tested samples, and for technical variability in RT-qPCR. For quantification of cellular gene expression, 'reference genes' are generally used as internal controls to normalize for differences in RNA input and technical variation between samples. It should be noted that there is no universal reference gene that exhibits invariable expression levels under all conditions (195). This also holds true for non-coding RNAs (196)). Therefore, the stability of expression of an internal reference gene under the given experimental conditions should be validated. Various software tools have been developed to assess the suitability of reference genes (geNorm (197), NormFinder (198), BestKeeper (199), RefGenes (200)). Finding suitable reference transcripts to normalize RT-qPCR data of 
RNAs in EV or body fluids is even more complicated (201). In contrast to cells, there are no known housekeeping processes in EV that can guide the search for stable reference genes. Even RNAs that are stably expressed in cells may be differentially sorted into EV and are therefore not necessarily reliable for EV-RNA normalization (11). The current consensus on strategies to normalize EV-RNA data is that the stability of putative reference genes should be extensively validated, for instance by making use of publicly available data (202) or by testing the stability of transcripts over multiple biological replicates of isolated EV (203). Furthermore, normalization to the mean of multiple reference genes may improve the normalization accuracy, by reducing biases from inherent technical variability in the assessment of single reference genes (197). In addition, synthetics spike-in miRNAs have been used to normalize for RNA isolation efficiency between samples $(11,49,204)$. In contrast to internal reference genes, spikeins cannot correct for technical variability in the efficiency of EV purification before RNA isolation. Furthermore, a workaround to circumvent the reliance on EV reference transcripts would be to quantify relative differences in RNA transcripts released by equal numbers of cells in equal volumes of supernatant. This may be useful approach when working with limited numbers of cells. Of course, the MIQE guidelines for transparent reporting of qPCR values should be followed to ensure reproducibility of results (205). The increasing availability of EV-RNA-seq data may be utilized for the identification of universal EV-reference transcripts to be used in the normalization of RT-qPCR data obtained from EV-RNA $(12,206)$.

\section{Utilization of knowledge of EV-RNA characterization to study intercellular communication and biomarker potential}

The fundamental knowledge of the strengths and limitations of EV-purification techniques and EV-RNA characterization is instrumental to study the role of EV-RNA in intercellular communication, and to correctly utilize EV-RNA as disease biomarker. We will discuss the involvement of EV-RNA in immune-regulation to illustrate the role of EV-RNA in intercellular communication. Furthermore we will discuss how the profiling of EV-RNA in biofluids can be employed to diagnose and monitor diseases

\section{The role of EV-enclosed RNA in immune cell communication}

Intercellular communication is essential to coordinate effective immune responses to invading pathogens (5). Well-known means of cellular communication include direct cell-cell contact via membrane bound receptors and their ligands, paracrine signaling via soluble molecules (cytokines and chemokines) which act on nearby cells, and endocrine signaling via hormones acting on distant cells. Over the last decade, EV have emerged as an additional route of immune-cell communication (4,207-210). EV contain a subset of lipids, proteins and RNA from their parental cells that can be 
functionally transferred to recipient cells. EV from immune cells have been implicated in antigen presentation, immune activation and immune suppression. For example, dendritic cell-derived and B cell derived EV carry functional MHC complexes which display antigenic peptides to $T$ cells, thereby inducing antigen-specific $T$ cell responses $(211,212)$. EV have also been implicated in 'cross-presentation', in which peptide-MHC complex-bearing EVs bind to the surface of non-professional APCs, thereby presenting their peptides to T cells (213). Such a mechanism may increase the number of cells that display particular peptides, thereby amplifying the initiation of adaptive immune responses. Immune-activating and immune-suppressive effects of EV are highly specific and depend on the type of EV-producing immune cell, the type of stimulus / environmental conditions and the type of recipient cell. For instance, EV from M1 polarized macrophages induced the release of proinflammatory cytokines in DC and macrophages (214). In contrast, EV from DC treated with the anti-inflammatory cytokine IL-10 were shown to suppress inflammation in a collagen-induced arthritis model (215). Some immune-related effects of EV are clearly mediated by EV-associated proteins, for example antigen presentation via EV. Other reported immune-regulatory effects of EV may be mediated by EV-associated lipids, RNAs, or a combination of EV-cargo.

The roles that miRNAs play in fine-tuning of immune-activation in cells have been intensively investigated. Pro- and anti-inflammatory stimuli greatly influence the cellular miRNA transcriptome, which fine-tunes immune responses $(55,216,217)$ and steers immune cell development and function via complex regulatory mechanisms (218). Furthermore, ongoing research has highlighted the functional involvement of EV-associated miRNAs in EV in communication between immune cells. Various studies have shown that immune-related stimuli cause changes in the RNA composition of EV $(15,52,219,220)$. As noted previously, the functional transfer of miRNAs has been shown by various studies $(45,46,219)$. For example, overexpressed miR-335 in Jurkat T cells was shown to be transferred via EV to Raji B cells which do not endogenously express this miRNA (45). Functional transfer of miR-155 and miR-146a was also demonstrated by Alexander et al., who used miR-155-/- and miR-146a-/- knockout DCs as recipient cells to assess the transfer of these miRNAs via EV released by wildtype DC (46). The data in this study indicated that incubation of knockout DC with wildtype EV resulted in repression of target genes for miR-155 and miR-146 in the recipient cells. In miR-155/- and miR-146a-/- mice injected with EV from wildtype or knockout DC, these miR-155 and miR-146 were taken up by the spleen and the liver. Furthermore, injection of WT EV in miR-155-/- mice resulted in an elevated inflammatory response, whereas injection of these EV in miR-146a-/- mice resulted in repression of inflammation induced by LPS injection. The authors concluded that the transfer of these two miRNAs can alter the ability of recipient cells to respond to inflammatory stimuli (46). It remains to be investigated to what extent the transfer of these miRNAs influence inflammatory 
processes in a wildtype recipient cell. Other EV-enclosed miRNAs have also been implicated in the induction of tolerance to inflammatory stimuli. For instance, miR10a-5p containing endothelial-cell derived EV suppressed LPS-activation in THP1 cells through repression of IRAK4, which is an effector molecule that activates NF-kB (220). Furthermore, EV-mediated transfer of miRNAs let-7b and let-7d by regulatory $\mathrm{T}$ cells was shown to suppress helper $\mathrm{T}$ cells and reduce systemic inflammation in mice (221). Besides effects on inflammation and immune cell activation, EV-miRNA can also affect cell differentiation. For example, the differentiation of naïve monocytes to macrophages was induced by miR-223-containing EV from macrophages (222). Furthermore, EV-associated miRNAs can also influence cell migration. EV from LPSstimulated macrophages, for example, were shown to contain elevated levels of miR150. Uptake of these EV by endothelial cells enhanced their migratory capacity via suppression of c-Myb (219). Together, these examples highlight that EV-associated RNAs should be considered as important players in immune cell communication and immune regulation.

While the studies described above strongly suggest that miRNA can be functionally transferred between cells via EV, they largely relied on artificial systems (knockout/ overexpressing cell lines) to enable discrimination between miRNAs transferred by EV from miRNAs that were endogenously expressed in the recipient cell. However, knockdown of miRNAs may lead to changes in the phenotype and/or function of recipient cells, because miRNA knockdown leads to increased expression of the mRNAs it normally suppresses. However, testing the effects of miRNA transfer between non-modified cells is a lot more difficult. For example, the miRNA-of-interest may be endogenously expressed by the recipient cells, which decreases the detection window in which the transfer of the miRNA-of-interest can be measured. Furthermore, treatment with EVs may induce the expression of the miRNA-of-interest in the recipient cells, instead of transferring this miRNA via EV. Hence, the currently available data have not yet provided undisputed evidence that individual miRNAs transferred via EV can induce changes in target cell function. Additionally, there is an ongoing debate on how many copies of individual miRNAs are present per EV, and how many EV should be transferred to target cells to allow for miRNA-mediated effects on the function of these cells (223-225). It has been proposed that single cells may take up hundreds of EV over a period of time, which may be sufficient to induce a regulatory effect (46). In addition, multiple different miRNAs that affect the same signaling pathway may be co-transferred via EV. Proteins and lipids in EV may further enhance the targeting efficiency and act synergistically with the RNA components. Future studies addressing the function of EV-associated RNAs should consider this multi-component nature of $E V$, e.g. by applying integrated omics approaches for parallel assessment of proteins, lipids and RNAs in EV. In addition, there should be increased awareness of alternative 
functions that RNAs transferred via EV may perform in target cells, e.g. TLR stimulation by miRNAs. Finally, the function of EV-associated RNA types other than miRNAs should be addressed in more detail. Summarizing, EV from various types of immune cells have been implicated in immune-regulatory effects, which exemplify the biological roles of EV in intercellular communication.

\section{Biomarker potential of EV-associated RNAs in biological fluids}

EV are abundantly present in biofluids and contain molecular information about the (disease) status of their parental cells. This has put EV and EV-RNA in the limelight as biomarkers for a wide range of diseases, including Alzheimer's disease, rheumatoid arthritis, various types of cancer, and (auto)inflammatory diseases (17,226-232). Especially the RNA content of EV is interesting for biomarker purposes, since RNA can be characterized and quantified via highly sensitive methods such as RNA sequencing and RT-qPCR. The basis for using EV-RNA as biomarkers for disease is that changes in the transcriptional profile of diseased cells are reflected in the RNA content of EV. It is expected that monitoring extracellular RNA in samples of biological fluids, the socalled 'liquid biopsies', will provide significant benefits over current disease monitoring strategies. Taking cancer as an example, the (geno)type of the tumor can be deduced from regular tissue biopsies. For tumors that are difficult to access, such as brain tumors, taking tissue biopsies imposes significant risks on patients. Less invasive techniques to monitor cancer progression in patients, such as MRI, have a limited resolution and will thus not detect small tumors (233). Ideally, tumor-specific mRNAs or miRNAs detected in plasma may be used for diagnosis and classification of the type of cancer, and to monitor the response of the tumor to treatment. Various candidate RNA biomarkers for diagnosis and monitoring of cancer have been identified (14,234-236). Furthermore, a number of miRNAs are frequently reported by multiple studies on cancer biomarkers (237). Besides applications in cancer, liquid biopsies may prove useful for the diagnosis of neurodegenerative diseases such as Alzheimer's and Parkinson's disease. These diseases are characterized by a long preclinical phase before the onset of dementia, which presents a challenge for early diagnosis. RNA profiling in plasma may provide a cost-effective alternative to current detection methods, such as detection of Alzheimerassociated $A \beta$ in cerebrospinal fluid or PET-scans. Furthermore extracellular RNA-based patient stratification will benefit drug development allowing the inclusion of more truepositive AD cases in patient cohorts (17). Candidate biomarkers have been identified for diagnosis of Alzheimer's disease $((17,238)$ and reviewed in $(239))$. Nevertheless, there is inconsistency between different studies in the types of miRNAs that are identified and their differential expression (239). Thus, although RNA biomarkers provide a significant advantage for diagnosis of neurodegenerative diseases over currently available methods, much more work is required to increase the consistency and reliability of 
results for clinical application. Analysis of circulating RNA may also provide opportunities for detection of inflammatory conditions, such as autoimmune- and infectious diseases $(231,240-242)$. For example in sepsis, inflammation caused by bacterial infections is currently diagnosed by overnight microbial culture $(231,242)$, while circulating RNA may allow for a more rapid diagnosis. Furthermore, EV are thought to be both mediators and markers of sepsis, which enables their use as diagnostic, therapeutic or both (242). A number of miRNAs have been identified which are related to sepsis $(166,243-247)$. Of these markers, miR-150 has been identified in two studies and appears to correlate well with levels of inflammatory cytokines $(243,247)$. Nevertheless, there is striking variability in dysregulated miRNAs reported by different studies $(248,249)$. Of note, the etiology of sepsis is very heterogeneous with regard to the site of infection, causative organism and health status of the patient (250), which may in part explain the interstudy variability.

Although these examples illustrate the great opportunities that RNA profiling in biofluids provides for diagnosis and monitoring of various diseases, many fundamental questions remain to be answered before assessment of EV-RNA can be accurately and reliably applied in a clinical setting. Importantly, biofluids are highly complex and contain various other macromolecular structures that may co-purify with EV and contain additional pools of non-coding RNA (48-50). There is strong evidence that the methods selected for EV-enrichment and RNA purification method heavily influence the composition of the isolated pool of extracellular RNA $(166,167)$. Furthermore, standardization of biofluid collection and accurate reporting of technical details is required to improve data comparability between studies and between laboratories $(168,251)$. In addition to these technical points, we still lack fundamental understanding on how disease-related changes in cells cause changes in the RNA content of EV. It must be noted that most plasma RNA biomarker studies to date compared the extracellular transcriptome of healthy versus diseased individuals. While this approach may yield sufficient information for diagnosis of individual patients, it does not provide information on whether the altered RNA profiles represent 'cellular snapshots' derived from the diseased cells, or whether the alterations are caused by the collective response of multiple cell types. Conversely, analysis of the transcriptome of EV released by in vitro cultured cells may not be representative for RNA profiles observed in biofluids, which additionally contain other RNA-containing structures and EV that are derived from other tissues. Although much work remains to be done to bridge the gap between in vitro studies and patient cohort studies, increasing our understanding of how circulating RNA profiles in biofluids are altered during disease will lead to significant improvements in disease monitoring, and may provide cost-effective means to screen the population for early disease detection. 


\section{Outline of this thesis}

The research in this thesis explores whether the incorporation of various small non-coding RNA species into EV is regulated by external stimulation imposed on immune cells in in vitro and in vivo systems. While the field of research on EV-RNA has centered its attention on the miRNA content of EV, we have mainly focused on other non-coding RNA species present in EV. We analyzed which non-coding RNA types were differentially incorporated into EV upon cell stimulation as a first step in uncovering their function in EV-mediated signaling processes and to address their biomarker potential. To technically support this research, we evaluated and optimized technologies to characterize and quantify non-coding RNA species in EV released in in vitro cell cultures and in EV present in blood plasma. We first performed a systematic assessment of the reliability and reproducibility of various small non-coding RNA sequencing protocols on pools of synthetic RNA with a defined composition (Chapter 2). Furthermore, we compared various protocols to deplete EV from fetal calf serum (Chapter 3), as residual bovine EV-RNA may cause biases in RNA-seq datasets of EVRNA from cultured cells. This resulted in an optimized protocol to deplete FCS-EV from culture medium and a method to assess the levels of background RNA levels in purified EV samples, which we employed in our further studies on EV from cultured cells. In these studies, we made use of the capacity of dendritic cells and macrophages to strongly respond to exogenous stimuli, which leads to changes in the molecular cargo and function of EV released by these cells. First, we investigated how immuneactivating (LPS) or -suppressing (VitaminD3) stimuli imposed on primary dendritic cells affected the RNA composition of EV (Chapter 4). We discovered that besides miRNA, the levels of Y-RNA in EV can be regulated by these exogenous stimuli. This suggested that $Y$-RNA may be involved in communication between immune cells and may also be explored as biomarker in immune-related conditions. Interestingly, Y-RNA is abundantly detected in EV in various studies and has been functionally implicated in immune-regulatory processes, which we have reviewed in Chapter 5. In Chapter 6 we investigate whether the different Y-RNA subtypes in plasma are associated to EV or other RNA carriers. Moreover, we provide proof-of-concept that Y-RNA levels in the pool of plasma EV are indicative of neutrophil responses during endotoxemia, and may therefore be candidate biomarkers for inflammatory conditions, such as sepsis. Finally, we designed an experimental approach to investigate which Y-RNA binding proteins drive enhanced shuttling of Y-RNA into EV released by TLR-activated macrophages. In this approach, we performed parallel analysis of the colocalization of Y-RNA and various Y-RNA binding proteins in various cellular organelles and in EV (Chapter 7). Based on the acquired data, we speculate on the potential role of EVmediated removal of Y-RNA in balancing the activity of mRNA stability enhancing 
proteins in EV-producing cells. Our work provides support for a role of non-miRNA species in immune-cell communication, and highlights advantages of these RNA species for biomarker purposes. 


\section{References}

1. Raposo G, Stoorvogel W. Extracellular vesicles: Exosomes, microvesicles, and friends. J Cell Biol (2013) 200:373-383. doi:10.1083/jcb.201211138

2. van Niel G, D’Angelo G, Raposo G. Shedding light on the cell biology of Extracellular Vesicles. Nat Rev Mol Cell Biol (2018) 19:213-228. doi:10.1038/nrm.2017.125

3. Colombo M, Raposo G, Théry C. Biogenesis, Secretion, and Intercellular Interactions of Exosomes and Other Extracellular Vesicles. Annu Rev Cell Dev Biol (2014) 30:255-289. doi:10.1146/annurev-cellbio-101512-122326

4. Yáñez-Mó M, Siljander PRM, Andreu Z, Zavec AB, Borràs FE, Buzas El, Buzas K, Casal E, Cappello F, Carvalho J, et al. Biological properties of extracellular vesicles and their physiological functions. J Extracell Vesicles (2015) 4:1-60. doi:10.3402/jev.v4.27066

5. van der Grein SG, Nolte-'t Hoen ENM. "Small Talk" in the Innate Immune System via RNA-Containing Extracellular Vesicles. Front Immunol (2014) 5:1-8. doi:10.3389/fimmu.2014.00542

6. Colombo M, Moita C, van Niel G, Kowal J, Vigneron J, Benaroch P, Manel N, Moita LF, Théry C, Raposo G. Analysis of ESCRT functions in exosome biogenesis, composition and secretion highlights the heterogeneity of extracellular vesicles. J Cell Sci (2013) 126:5553-65. doi:10.1242/jcs.128868

7. van Niel G, Charrin S, Simoes S, Romao M, Rochin L, Saftig P, Marks MS, Rubinstein E, Raposo G. The Tetraspanin CD63 Regulates ESCRT-Independent and -Dependent Endosomal Sorting during Melanogenesis. Dev Cell (2011) 21:708-721. doi:10.1016/j.devcel.2011.08.019

8. Trajkovic K, Hsu C, Chiantia S, Rajendran L, Wenzel D, Wieland F, Schwille P, Brügger B, Simons M. Ceramide Triggers Budding of Exosome Vesicles into Multivesicular Endosomes. Science (80-) (2008) 319:1244-1248.

9. Mathieu M, Martin-Jaular L, Lavieu G, Théry C. Specificities of secretion and uptake of exosomes and other extracellular vesicles for cell-to-cell communication. Nat Cell Biol (2019) 21:9-17. doi:10.1038/s41556-0180250-9

10. Camussi G, Deregibus MC, Bruno S, Cantaluppi V, Biancone L. Exosomes/microvesicles as a mechanism of cell-to-cell communication. Kidney Int (2010) 78:838-848. doi:10.1038/ki.2010.278

11. Mateescu B, Kowal EJK, van Balkom BWM, Bartel S, Bhattacharyya SN, Buzás El, Buck AH, de Candia P, Chow FWN, Das $S$, et al. Obstacles and opportunities in the functional analysis of extracellular vesicle RNA - an ISEV position paper. J Extracell Vesicles (2017) 6:1286095. doi:10.1080/20013078.2017.1286095

12. Murillo OD, Thistlethwaite W, Rozowsky J, Subramanian SL, Lucero R, Shah N, Jackson AR, Srinivasan S, Chung A, Laurent CD, et al. exRNA Atlas Analysis Reveals Distinct Extracellular RNA Cargo Types and Their Carriers Present across Human Biofluids. Cell (2019) 177:463-477.e15. doi:10.1016/j.cell.2019.02.018

13. Das S, Ansel KM, Bitzer M, Breakefield XO, Charest A, Galas DJ, Gerstein MB, Gupta M, Milosavljevic A, McManus MT, et al. The Extracellular RNA Communication Consortium: Establishing Foundational Knowledge and Technologies for Extracellular RNA Research. Cell (2019) 177:231-242. doi:10.1016/j. cell.2019.03.023

14. Skog J, Würdinger T, van Rijn S, Meijer DH, Gainche L, Curry WT, Carter BS, Krichevsky AM, Breakefield XO. Glioblastoma microvesicles transport RNA and proteins that promote tumour growth and provide diagnostic biomarkers. Nat Cell Biol (2008) 10:1470-1476. doi:10.1038/ncb1800

15. Montecalvo A, Larregina AT, Shufesky WJ, Stolz DB, Sullivan MLG, Karlsson JM, Baty CJ, Gibson GA, Erdos G, Wang Z, et al. Mechanism of transfer of functional microRNAs between mouse dendritic cells via exosomes. Blood (2012) 119:756-66. doi:10.1182/blood-2011-02-338004

16. Bellingham SA, Coleman BM, Hill AF. Small RNA deep sequencing reveals a distinct miRNA signature released in exosomes from prion-infected neuronal cells. Nucleic Acids Res (2012) 40:10937-10949. doi:10.1093/nar/gks832

17. Cheng L, Doecke JD, Sharples RA, Villemagne VL, Fowler CJ, Rembach A, Martins RN, Rowe CC, Macaulay $\mathrm{SL}$, Masters CL, et al. Prognostic serum miRNA biomarkers associated with Alzheimer's disease shows concordance with neuropsychological and neuroimaging assessment. Mol Psychiatry (2014)1-9. doi:10.1038/mp.2014.127 
18. Cha DJ, Franklin JL, Dou Y, Liu Q, Higginbotham JN, Beckler MD, Weaver AM, Vickers K, Prasad N, Levy S, et al. KRAS-dependent sorting of miRNA to exosomes. Elife (2015) 4:1-22. doi:10.7554/eLife.07197

19. Thompson AG, Gray E, Heman-Ackah SM, Mäger I, Talbot K, El Andaloussi S, Wood MJ, Turner MR. Extracellular vesicles in neurodegenerative disease-pathogenesis to biomarkers. Nat Rev Neurol (2016) 12:346-357. doi:10.1038/nrneurol.2016.68

20. Datta Chaudhuri A, Dastgheyb RM, Yoo SW, Trout A, Talbot CC, Hao H, Witwer KW, Haughey NJ. TNFa and $I L-1 \beta$ modify the miRNA cargo of astrocyte shed extracellular vesicles to regulate neurotrophic signaling in neurons. Cell Death Dis (2018) 9: doi:10.1038/s41419-018-0369-4

21. Nolte-'t Hoen ENM, Buermans HPJ, Waasdorp M, Stoorvogel W, Wauben MHM, 't Hoen PAC. Deep sequencing of RNA from immune cell-derived vesicles uncovers the selective incorporation of small non-coding RNA biotypes with potential regulatory functions. Nucleic Acids Res (2012) 40:9272-85. doi:10.1093/nar/gks658

22. Valadi H, Ekström K, Bossios A, Sjöstrand M, Lee JJ, Lötvall JO. Exosome-mediated transfer of mRNAs and microRNAs is a novel mechanism of genetic exchange between cells. Nat Cell Biol (2007) 9:654-659. doi:10.1038/ncb1596

23. Ratajczak J, Miekus K, Kucia M, Zhang J, Reca R, Dvorak P, Ratajczak MZ. Embryonic stem cell-derived microvesicles reprogram hematopoietic progenitors: Evidence for horizontal transfer of mRNA and protein delivery. Leukemia (2006) 20:847-856. doi:10.1038/sj.leu.2404132

24. Baj-Krzyworzeka M, Szatanek R, Węglarczyk K, Baran J, Urbanowicz B, Brański P, Ratajczak MZ, Zembala M. Tumour-derived microvesicles carry several surface determinants and mRNA of tumour cells and transfer some of these determinants to monocytes. Cancer Immunol Immunother (2006) 55:808-818. doi:10.1007/s00262-005-0075-9

25. Mattick JS, Makunin I V. Non-coding RNA. Hum Mol Genet (2006) 15 Spec No:R17-29. doi:10.1093/ $\mathrm{hmg} / \mathrm{ddl} 046$

26. White RJ. Transcription by RNA polymerase III: more complex than we thought. Nat Rev Genet (2011) 12:459-463. doi:10.1038/nrg3001

27. Esteller M. Non-coding RNAs in human disease. Nat Rev Genet (2011) 12:861-74. doi:10.1038/nrg3074

28. Tuck AC, Tollervey D. RNA in pieces. Trends Genet (2011) 27:422-432. doi:10.1016/j.tig.2011.06.001

29. He L, Hannon GJ. MicroRNAs: small RNAs with a big role in gene regulation. Nat Rev Genet (2004) 5:522-31. doi:10.1038/nrg1379

30. Lee RC, Feinbaum RL, Ambros V. The C. Elegans Heterochronic Gene Lin-4 Encodes Small RNAs With Antisense Complementarity To Lin-14. Cell (1993) 75:843-854.

31. Lagos-Quintana M, Rauhut R, Yalcin A, Meyer J, Lendeckel W, Tuschl T. Identification of tissue-specific microRNAs from mouse. Curr Biol (2002) 12:735-739.

32. Sempere LF, Freemantle S, Pitha-Rowe I, Moss E, Dmitrovsky E, Ambros V. Expression profiling of mammalian microRNAs uncovers a subset of brain-expressed microRNAs with possible roles in murine and human neuronal differentiation. Genome Biol (2004) 5:R13. doi:10.1186/gb-2004-5-3-r13

33. Treiber T, Treiber N, Plessmann U, Harlander S, Daiß J-L, Eichner N, Lehmann G, Schall K, Urlaub H, Meister G. A Compendium of RNA-Binding Proteins that Regulate MicroRNA Biogenesis. Mol Cell (2017) 66:270-284.e13. doi:10.1016/j.molcel.2017.03.014

34. Agarwal V, Bell GW, Nam J-W, Bartel DP. Predicting effective microRNA target sites in mammalian mRNAs. Elife (2015) 4:1-38. doi:10.7554/elife.05005

35. Turner ML, Schnorfeil FM, Brocker T. MicroRNAs regulate dendritic cell differentiation and function. $J$ Immunol (2011) 187:3911-7. doi:10.4049/jimmunol.1101137

36. O'Connell RM, Zhao JL, Rao DS. MicroRNA function in myeloid biology. Blood (2011) 118:2960-2969 doi:10.1182/blood-2011-03-291971

37. O'Connell RM, Rao DS, Chaudhuri A a, Baltimore D. Physiological and pathological roles for microRNAs in the immune system. Nat Rev Immunol (2010) 10:111-122. doi:10.1038/nri2708 
38. Baltimore D, Boldin MP, O'Connell RM, Rao DS, Taganov KD. MicroRNAs: new regulators of immune cell development and function. Nat Immunol (2008) 9:839-45. doi:10.1038/ni.f.209

39. Lau P, Bossers K, Janky R, Salta E, Frigerio CS, Barbash S, Rothman R, Sierksma ASR, Thathiah A, Greenberg D, et al. Alteration of the microRNA network during the progression of Alzheimer's disease. EMBO Mol Med (2013) 5:1613-1634. doi:10.1002/emmm.201201974

40. Kent OA, McCall MN, Cornish TC, Halushka MK. Lessons from miR-143/145: The importance of cell-type localization of miRNAs. Nucleic Acids Res (2014) 42:7528-7538. doi:10.1093/nar/gku461

41. Juzenas S, Venkatesh G, Hübenthal M, Hoeppner MP, Du ZG, Paulsen M, Rosenstiel P, Senger P, Hofmann-Apitius M, Keller A, et al. A comprehensive, cell specific microRNA catalogue of human peripheral blood. Nucleic Acids Res (2017) 45:9290-9301. doi:10.1093/nar/gkx706

42. Taylor DD, Gercel-Taylor C. MicroRNA signatures of tumor-derived exosomes as diagnostic biomarkers of ovarian cancer. Gynecol Oncol (2008) 110:13-21. doi:10.1016/j.ygyno.2008.04.033

43. Zhu S, Pan W, Song X, Liu Y, Shao X, Tang Y, Liang D, He D, Wang H, Liu W, et al. The microRNA miR-23b suppresses IL-17-associated autoimmune inflammation by targeting TAB2, TAB3 and IKK-a. Nat Med

(2012) 18:1077-1086. doi:10.1038/nm.2815

44. Small EM, Frost RJA, Olson EN. MicroRNAs Add a New Dimension to Cardiovascular Disease. Circulation (2010) 121:1022-1032. doi:10.1161/CIRCULATIONAHA.109.889048

45. Mittelbrunn M, Gutiérrez-Vázquez C, Villarroya-Beltri C, González S, Sánchez-Cabo F, González MÁ, Bernad A, Sánchez-Madrid F. Unidirectional transfer of microRNA-loaded exosomes from T cells to antigen-presenting cells. Nat Commun (2011) 2:282. doi:10.1038/ncomms1285

46. Alexander M, Hu R, Runtsch MC, Kagele DA, Mosbruger TL, Tolmachova T, Seabra MC, Round JL, Ward DM, O'Connell RM. Exosome-delivered microRNAs modulate the inflammatory response to endotoxin. Nat Commun (2015) 6:7321. doi:10.1038/ncomms8321

47. Fabbri M, Paone A, Calore F, Galli R, Gaudio E, Santhanam R, Lovat F, Fadda P, Mao C, Nuovo GJ, et al. MicroRNAs bind to Toll-like receptors to induce prometastatic inflammatory response. Proc Natl Acad Sci (2012) 109:E2110-E2116. doi:10.1073/pnas.1209414109

48. Arroyo JD, Chevillet JR, Kroh EM, Ruf IK, Pritchard CC, Gibson DF, Mitchell PS, Bennett CF, PogosovaAgadjanyan EL, Stirewalt DL, et al. Argonaute2 complexes carry a population of circulating microRNAs independent of vesicles in human plasma. Proc Natl Acad Sci U S A (2011) 108:5003-8. doi:10.1073/ pnas.1019055108

49. Turchinovich A, Weiz L, Langheinz A, Burwinkel B. Characterization of extracellular circulating microRNA. Nucleic Acids Res (2011) 39:7223-33. doi:10.1093/nar/gkr254

50. Vickers KC, Palmisano BT, Shoucri BM, Shamburek RD, Remaley AT. MicroRNAs are transported in plasma and delivered to recipient cells by high-density lipoproteins. Nat Cell Biol (2011) 13:423-33. doi:10.1038/ncb2210

51. Mukherjee K, Ghoshal B, Ghosh S, Chakrabarty Y, Shwetha S, Das S, Bhattacharyya SN. Reversible HuRmicroRNA binding controls extracellular export of miR122 and augments stress response. EMBO Rep (2016) 17:11841203. doi:10.15252/embr

52. Driedonks TAP, van der Grein SG, AriyurekY, Buermans HPJ, Jekel H, Chow FWN, Wauben MHM, Buck AH, Hoen PAC, Nolte-'t Hoen ENM. Immune stimuli shape the small non-coding transcriptome of extracellular vesicles released by dendritic cells. Cell Mol Life Sci (2018)3857-3875. doi:10.1007/s00018018-2842-8

53. Ressel S, Rosca A, Gordon K, Buck AH. Extracellular RNA in viral-host interactions: Thinking outside the cell. Wiley Interdiscip Rev RNA (2019)1-23. doi:10.1002/wrna.1535

54. Squadrito ML, Baer C, Burdet F, Maderna C, Gilfillan GD, Lyle R, Ibberson M, De Palma M. Endogenous RNAs Modulate MicroRNA Sorting to Exosomes and Transfer to Acceptor Cells. Cell Rep (2014)

8:1432-1446. doi:10.1016/j.celrep.2014.07.035

55. Mann M, Mehta A, Zhao JL, Lee K, Marinov GK, Garcia-Flores Y, Baltimore D. An NF-kB-microRNA regulatory network tunes macrophage inflammatory responses. Nat Commun (2017) 8: doi:10.1038/ s41467-017-00972-z 
56. Villarroya-Beltri C, Gutiérrez-Vázquez C, Sánchez-Cabo F, Pérez-Hernández D, Vázquez J, MartinCofreces N, Martinez-Herrera DJ, Pascual-Montano A, Mittelbrunn M, Sánchez-Madrid F. Sumoylated hnRNPA2B1 controls the sorting of miRNAs into exosomes through binding to specific motifs. Nat Commun (2013) 4:2980. doi:10.1038/ncomms3980

57. Santangelo L, Giurato G, Cicchini C, Montaldo C, Mancone C, Tarallo R, Battistelli C, Alonzi T, Weisz A, Tripodi M. The RNA-Binding Protein SYNCRIP Is a Component of the Hepatocyte Exosomal Machinery Controlling MicroRNA Sorting. Cell Rep (2016) 17:799-808. doi:10.1016/j.celrep.2016.09.031

58. Shurtleff MJ, Temoche-Diaz MM, Karfilis KV., Ri S, Schekman R. Y-box protein 1 is required to sort microRNAs into exosomes in cells and in a cell-free reaction. Elife (2016) 5:1-23. doi:10.7554/eLife.19276

59. Melo SA, Sugimoto H, O'Connell JT, Kato N, Villanueva A, Vidal A, Qiu L, Vitkin E, Perelman LT, Melo CA, et al. Cancer Exosomes Perform Cell-Independent MicroRNA Biogenesis and Promote Tumorigenesis. Cancer Cell (2014) 26:707-721. doi:10.1016/j.ccell.2014.09.005

60. Mckenzie AJ, Hoshino D, Hong NH, Coffey RJ, Patton JG, Weaver AM, Mckenzie AJ, Hoshino D, Hong $\mathrm{NH}$, Cha DJ, et al. KRAS-MEK Signaling Controls Ago2 Sorting into Report KRAS-MEK Signaling Controls Ago2 Sorting into Exosomes. Cell Rep (2016) 15:1-10. doi:10.1016/j.celrep.2016.03.085

61. Jeppesen DK, Fenix AM, Franklin JL, Higginbotham JN, Zhang Q, Zimmerman LJ, Liebler DC, Ping J, Liu Q, Evans R, et al. Reassessment of Exosome Composition. Cell (2019) 177:428-445.e18. doi:10.1016/j. cell.2019.02.029

62. Kirchner S, Ignatova Z. Emerging roles of tRNA in adaptive translation, signalling dynamics and disease. Nat Rev Genet (2015) 16:98-112. doi:10.1038/nrg3861

63. Phizicky EM, Alfonzo JD. Do all modifications benefit all tRNAs? FEBS Lett (2010) 584:265-271. doi:10.1016/j.febslet.2009.11.049

64. Hori H. Methylated nucleosides in tRNA and tRNA methyltransferases. Front Genet (2014) 5:1-26. doi:10.3389/fgene.2014.00144

65. Delaunay S, Frye M. RNA modifications regulating cell fate in cancer. Nat Cell Biol (2019) 21:552-559. doi:10.1038/s41556-019-0319-0

66. Anderson P, Ivanov P. TRNA fragments in human health and disease. FEBS Lett (2014) 588:4297-4304. doi:10.1016/j.febslet.2014.09.001

67. Ibba M, Söll D. Aminoacyl-tRNA synthesis. Annu Rev Biochem (2000) 69:617-650. doi:10.1146/annurev. biochem.69.1.617

68. Plotkin JB, Robins H, Levine AJ. Tissue-specific codon usage and the expression of human genes. Proc Natl Acad Sci (2004) 101:12588-12591. doi:10.1073/pnas.0404957101

69. Goodarzi H, Nguyen HCB, Zhang S, Dill BD, Molina H, Tavazoie SF. Modulated expression of specific tRNAs drives gene expression and cancer progression. Cell (2016) 165:1416-1427. doi:10.1016/j. cell.2016.05.046

70. Pavon-Eternod M, Gomes S, Geslain R, Dai Q, Rosner MR, Pan T. tRNA over-expression in breast cancer and functional consequences. Nucleic Acids Res (2009) 37:7268-7280. doi:10.1093/nar/gkp787

71. Yamasaki S, Ivanov P, Hu GF, Anderson P. Angiogenin cleaves tRNA and promotes stress-induced translational repression. J Cell Biol (2009) 185:35-42. doi:10.1083/jcb.200811106

72. Ivanov P, Emara MM, Villen J, Gygi SP, Anderson P. Angiogenin-Induced tRNA Fragments Inhibit Translation Initiation. Mol Cell (2011) 43:613-623. doi:10.1016/j.molcel.2011.06.022

73. Emara MM, Ivanov P, Hickman T, Dawra N, Tisdale S, Kedersha N, Hu G, Anderson P. Angiogenininduced tRNA-derived Stress-induced RNAs Promote Stress-induced Stress Granule Assembly. J Biol Chem (2010) 285:10959-10968. doi:10.1074/jbc.M109.077560

74. Blanco S, Dietmann S, Flores J V, Hussain S, Kutter C, Humphreys P, Lukk M, Lombard P, Treps L, Popis $M$, et al. Aberrant methylation of tRNAs links cellular stress to neuro-developmental disorders. EMBO J (2014) 33:1-20. doi:10.15252/embj.201489282

75. Tuorto F, Liebers R, Musch T, Schaefer M, Hofmann S, Kellner S, Frye M, Helm M, Stoecklin G, Lyko F. RNA cytosine methylation by Dnmt2 and NSun2 promotes tRNA stability and protein synthesis. Nat Struct Mol Biol (2012) 19:900-905. doi:10.1038/nsmb.2357 
76. Blanco S, Kurowski A, Nichols J, Watt FM, Benitah SA, Frye M. The RNA-Methyltransferase Misu (NSun2) Poises Epidermal Stem Cells to Differentiate. PLoS Genet (2011) 7:e1002403. doi:10.1371/journal. pgen.1002403

77. Motorin Y, Helm M. TRNA stabilization by modified nucleotides. Biochemistry (2010) 49:4934-4944. doi:10.1021/bi100408z

78. Dhahbi JM, Spindler SR, Atamna H, Yamakawa A, Boffelli D, Mote P, Martin DIK. 5' tRNA halves are present as abundant complexes in serum, concentrated in blood cells, and modulated by aging and calorie restriction. BMC Genomics (2013) 14:298. doi:10.1186/1471-2164-14-298

79. Cole C, Sobala A, Lu C, Thatcher S, Bowman A, Brown J, Green P, Barton G, Hutvagner G. Filtering of deep sequencing data reveals the existence of abundant Dicer- dependent small RNAs derived from tRNAs. RNA (New York, NY) (2009)2147-2160. doi:10.1261/rna.1738409.2008b

80. Babiarz JE, Ruby JG, Wang Y, Bartel DP, Blelloch R. Mouse ES cells express endogenous shRNAs, siRNAs, and other microprocessor-independent, dicer-dependent small RNAs. Genes Dev (2008) 22:27732785. doi:10.1101/gad.1705308

81. Haussecker D, Huang Y, Lau A, Parameswaran P, Fire AZ, Kay MA. Human tRNA-derived small RNAs in the global regulation of RNA silencing. RNA (2010) 16:673-695. doi:10.1261/rna.2000810.also

82. Li Z, Ender C, Meister G, Moore PS, Chang Y, John B. Extensive terminal and asymmetric processing of small RNAs from rRNAs, snoRNAs, snRNAs, and tRNAs. Nucleic Acids Res (2012) 40:6787-6799. doi:10.1093/nar/gks307

83. Hasler D, Lehmann G, Murakawa Y, Klironomos F, Jakob L, Gr??sser FA, Rajewsky N, Landthaler M, Meister G. The Lupus Autoantigen La Prevents Mis-channeling of tRNA Fragments into the Human MicroRNA Pathway. Mol Cell (2016) 63:110-124. doi:10.1016/j.molcel.2016.05.026

84. Chakshusmathi G, Kim S Do, Rubinson DA, Wolin SL. A La protein requirement for efficient pre-tRNA folding. EMBO J (2003) 22:6562-6572. doi:10.1093/emboj/cdg625

85. Hasler D, Meister G. From tRNA to miRNA: RNA-folding contributes to correct entry into noncoding RNA pathways. FEBS Lett (2016) 590:2354-2363. doi:10.1002/1873-3468.12294

86. Burroughs AM, Ando Y, de Hoon MJL, Tomaru Y, Suzuki H, Hayashizaki Y, Daub CO. Deep-sequencing of human Argonaute-associated small RNAs provides insight into miRNA sorting and reveals Argonaute association with RNA fragments of diverse origin. RNA Biol (2011) 8:158-177. doi:10.4161/rna.8.1.14300

87. Kumar P, Anaya J, Mudunuri SB, Dutta A. Meta-analysis of tRNA derived RNA fragments reveals that they are evolutionarily conserved and associate with AGO proteins to recognize specific RNA targets. BMC Biol (2014) 12:78. doi:10.1186/s12915-014-0078-0

88. Thomson DW, Pillman KA, Anderson ML, Lawrence DM, Toubia J, Goodall GJ, Bracken CP. Assessing the gene regulatory properties of Argonaute-bound small RNAs of diverse genomic origin. Nucleic Acids Res (2015) 43:470-481. doi:10.1093/nar/gku1242

89. Kim HK, Fuchs G, Wang S, Wei W, Zhang Y, Park H, Roy-Chaudhuri B, Li P, Xu J, Chu K, et al. A transferRNA-derived small RNA regulates ribosome biogenesis. Nature (2017) doi:10.1038/nature25005

90. Schorn AJ, Gutbrod MJ, Leblanc C, Martienssen R. LTR-Retrotransposon Control by tRNA-Derived Small RNAs. Cell (2017) 170:61-71.e11. doi:10.1016/j.cell.2017.06.013

91. Tosar JP, Gambaro F, Sanguinetti J, Bonilla B, Witwer KW, Cayota A. Assessment of small RNA sorting into different extracellular fractions revealed by high-throughput sequencing of breast cell lines. Nucleic Acids Res (2015)1-16. doi:10.1093/nar/gkv432

92. Lässer C, Shelke GV, Yeri A, Kim D-K, Crescitelli R, Raimondo S, Sjöstrand M, Gho YS, Van Keuren Jensen K, Lötvall J. Two distinct extracellular RNA signatures released by a single cell type identified by microarray and next-generation sequencing. RNA Biol (2017) 14:58-72. doi:10.1080/15476286.2016.12 49092

93. Vojtech L, Woo S, Hughes S, Levy C, Ballweber L, Sauteraud RP, Strobl J, Westerberg K, Gottardo R, Tewari $\mathrm{M}$, et al. Exosomes in human semen carry a distinctive repertoire of small non-coding RNAs with potential regulatory functions. Nucleic Acids Res (2014) 42:7290-7304. doi:10.1093/nar/gku347 
94. Cambier L, de Couto G, Ibrahim A, Echavez AK, Valle J, Liu W, Kreke M, Smith RR, Marbán L, Marbán E. Y RNA fragment in extracellular vesicles confers cardioprotection via modulation of IL-10 expression and secretion. EMBO Mol Med (2017)e201606924. doi:10.15252/emmm.201606924

95. Shurtleff MJ, Yao J, Qin Y, Nottingham RM, Temoche-Diaz MM, Schekman R, Lambowitz AM. Broad role for YBX1 in defining the small noncoding RNA composition of exosomes. Proc Natl Acad Sci (2017)

114:E8987-E8995. doi:10.1073/pnas.1712108114

96. Lambertz U, Oviedo Ovando ME, Vasconcelos EJR, Unrau PJ, Myler PJ, Reiner NE. Small RNAs derived from tRNAs and rRNAs are highly enriched in exosomes from both old and new world Leishmania providing evidence for conserved exosomal RNA Packaging. BMC Genomics (2015) 16:151. doi:10.1186/ s12864-015-1260-7

97. Chiou NT, Kageyama R, Ansel KM. Selective Export into Extracellular Vesicles and Function of tRNA Fragments during T Cell Activation. Cell Rep (2018) 25:3356-3370.e4. doi:10.1016/j.celrep.2018.11.073

98. Allen RM, Zhao S, Solano MAR, Michell DL, Shyr Y, Sethupathy P, Linton MF, Graf GA, Sheng Q. Bioinformatic analysis of endogenous and exogenous small RNAs on lipoproteins. J Extracell Vesicles (2018) 7: doi:10.108 0/20013078.2018.1506198

99. Tosar JP, Gámbaro F, Darré L, Pantano S, Westhof E, Cayota A. Dimerization confers increased stability to nucleases in 5 halves from glycine and glutamic acid tRNAs. Nucleic Acids Res (2018) 1:1-13. doi:10.1093/ nar/gky495

100. Yeri A, Courtright A, Reiman R, Carlson E, Beecroft T, Janss A, Siniard A, Richholt R, Balak C, Rozowsky J, et al. Total Extracellular Small RNA Profiles from Plasma, Saliva, and Urine of Healthy Subjects. Sci Rep (2017) 7:44061. doi:10.1038/srep44061

101. Godoy PM, Bhakta NR, Barczak AJ, Cakmak H, Fisher S, Mackenzie TC, Patel T, Price RW, Smith J, Woodruff PG, et al. Large Differences in Small RNA Composition Between Human Biofluids. Cell Rep (2018) 25:13461358. doi:10.2139/ssrn.3155656

102. Peng H, Shi J, Zhang Y, Zhang H, Liao S, Li W, Lei L, Han C, Ning L, Cao Y, et al. A novel class of tRNA-derived small RNAs extremely enriched in mature mouse sperm. Cell Res (2012) 22:1609-1612. doi:10.1038/ cr.2012.141

103. Sharma U, Conine CC, Shea JM, Boskovic A, Derr AG, Bing XY, Belleannee C, Kucukural A, Serra RW, Sun F, et al. Biogenesis and function of tRNA fragments during sperm maturation and fertilization in mammals. Science (80-) (2016) 351:391-397. doi:10.1126/science.aad6780

104. Hendrick JP, Wolin SL, Rinke J, Lerner MR, Steitz JA. Ro small cytoplasmic ribonucleoproteins are a subclass of La ribonucleoproteins: further characterization of the Ro and La small ribonucleoproteins from uninfected mammalian cells. Mol Cell Biol (1981) 1:1138-1149. doi:10.1128/MCB.1.12.1138.Updated

105. Pruijn GJM, Wingens PAETM, Peters SLM, Thijssen JPH, Van Venrooij WJ. Ro RNP associated Y RNAs are highly conserved among mammals. Biochim Biophys Acta-Gene Struct Expr (1993) 1216:395-401. doi:10.1016/0167-4781(93)90006-Y

106. Mosig A, Guofeng M, Stadler BMR, Stadler PF. Evolution of the vertebrate Y RNA cluster. Theory Biosci (2007) 126:9-14. doi:10.1007/s12064-007-0003-y

107. van Horn DJ, Eisenberg D, O'Brien CA, Wolin SL. Caenorhabditis elegans embryos contain only one major species of Ro RNP. RNA (1995) 1:293-303.

108. Sim S, Wolin SL. Bacterial Y RNAs: Gates, Tethers, and tRNA Mimics. Microbio/ Spectr (2018) 6:1-14. doi:10.1128/microbiolspec.RWR-0023-2018

109. Perreault J, Noël JF, Brière F, Cousineau B, Lucier JF, Perreault JP, Boire G. Retropseudogenes derived from the human Ro/SS-A autoantigen-associated hY RNAs. Nucleic Acids Res (2005) 33:2032-2041. doi:10.1093/nar/gki504

110. Xue D, Shi H, Smith JD, Chen X, Noe DA, Cedervall T, Yang DD, Eynon E, Brash DE, Kashgarian M, et al. A lupus-like syndrome develops in mice lacking the Ro 60-kDa protein, a major lupus autoantigen. Proc Natl Acad Sci U S A (2003) 100:7503-8. doi:10.1073/pnas.0832411100

111. Köhn M, Ihling C, Sinz A, Krohn K, Hüttelmaier S. The $Y 3^{* *}$ ncRNA promotes the $3^{\prime}$ end processing of histone mRNAs. Genes Dev (2015) 29:1998-2003. doi:10.1101/gad.266486.115 


\section{General introduction}

112. Driedonks TAP, Nolte-'t Hoen ENM. Circulating Y-RNAs in Extracellular Vesicles and Ribonucleoprotein Complexes; Implications for the Immune System. Front Immunol (2019) 9:1-15. doi:10.3389/ fimmu.2018.03164

113. Belisova A, Semrad K, Mayer O, Kocian G, Waigmann E, Schroeder R, Steiner G. RNA chaperone activity of protein components of human Ro RNPs. RNA (2005) 11:1084-1094. doi:10.1261/rna.7263905

114. Boccitto M, Wolin SL. Ro60 and Y RNAs: structure, functions, and roles in autoimmunity. Crit Rev Biochem Mol Biol (2019) 0:1-20. doi:10.1080/10409238.2019.1608902

115. Christov CP, Gardiner TJ, Szüts D, Krude T. Functional requirement of noncoding Y RNAs for human chromosomal DNA replication. Mol Cell Biol (2006) 26:6993-7004. doi:10.1128/MCB.01060-06

116. Stein AJ, Fuchs G, Fu C, Wolin SL, Reinisch KM. Structural insights into RNA quality control: The Ro autoantigen binds misfolded RNAs via its central cavity. Cell (2005) 121:529-539. doi:10.1016/j. cell.2005.03.009

117. Chen X, Taylor DW, Fowler CC, Galan JE, Wang HW, Wolin SL. An RNA degradation machine sculpted by Ro autoantigen and noncoding RNA. Cell (2013) 153:166-177. doi:10.1016/j.cell.2013.02.037

118. Fuchs G, Stein AJ, Fu C, Reinisch KM, Wolin SL. Structural and biochemical basis for misfolded RNA recognition by the Ro autoantigen. Nat Struct Mol Biol (2006) 13:1002-1009. doi:10.1038/nsmb1156

119. Tebaldi T, Zuccotti P, Peroni D, Köhn M, Gasperini L, Potrich V, Bonazza V, Dudnakova T, Rossi A, Sanguinetti G, et al. HuD Is a Neural Translation Enhancer Acting on mTORC1-Responsive Genes and Counteracted by the Y3 Small Non-coding RNA. Mol Cell (2018) 71:256-270. doi:10.1016/j.molcel.2018.06.032

120. van Balkom BWM, Eisele AS, Pegtel DM, Bervoets S, Verhaar MC. Quantitative and qualitative analysis of small RNAs in human endothelial cells and exosomes provides insights into localized RNA processing, degradation and sorting. J Extracell vesicles (2015) 4:26760. doi:10.3402/jev.v4.26760

121. Haderk F, Schulz R, Iskar M, Cid LL, Worst T, Willmund KV, Schulz A, Warnken U, Seiler J, Benner A, et al. Tumor-derived exosomes modulate PD-L1 expression in monocytes. Sci Immunol (2017) 2:eaah5509. doi:10.1126/sciimmunol.aah5509

122. Wei Z, Batagov AO, Schinelli S, Wang J, Wang Y, El Fatimy R, Rabinovsky R, Balaj L, Chen CC, Hochberg F, et al. Coding and noncoding landscape of extracellular RNA released by human glioma stem cells. Nat Commun (2017) 8:1145. doi:10.1038/s41467-017-01196-X

123. Hizir Z, Bottini S, Grandjean V, Trabucchi M, Repetto E. RNY (YRNA)-derived small RNAs regulate cell death and inflammation in monocytes/macrophages. Cell Death Dis (2017) 8:e2530. doi:10.1038/cddis.2016.429

124. Akopian D, Shen K, Zhang X, Shan S. Signal Recognition Particle: an essential protein targeting machine. Annu Rev Biochem (2013) 30:311-328. doi:10.1016/j.cll.2010.01.003.Lyme

125. Abdelmohsen K, Panda AC, Kang M-J, Guo R, Kim J, Grammatikakis I, Yoon J-H, Dudekula DB, Noh JH, Yang $X$, et al. 7SL RNA represses p53 translation by competing with HuR. Nucleic Acids Res (2014) 42:10099-111. doi:10.1093/nar/gku686

126. Nabet BY, Qiu Y, Shabason JE, Wu TJ, Yoon T, Kim BC, Benci JL, DeMichele AM, Tchou J, Marcotrigiano J, et al. Exosome RNA Unshielding Couples Stromal Activation to Pattern Recognition Receptor Signaling in Cancer. Cell (2017) 170:352-366.e13. doi:10.1016/j.cell.2017.06.031

127. Weichselbaum RR, Ishwaran H, Yoon T, Nuyten DSA, Baker SW, Khodarev N, Su AW, Shaikh AY, Roach P, Kreike $\mathrm{B}$, et al. An interferon-related gene signature for DNA damage resistance is a predictive marker for chemotherapy and radiation for breast cancer. Proc Natl Acad Sci (2008) 105 :

128. Hornung V, Ellegast J, Kim S, Brzózka K, Jung A, Kato H, Poeck H, Akira S, Conzelmann K-K, Schlee M, et al. 5'-Triphosphate RNA is the Ligand for RIG-I. Science (80-) (2006) 314:994-997. doi:10.1126/science.1132505

129. Boelens MC, Wu TJ, Nabet BY, Xu B, Qiu Y, Yoon T, Azzam DJ, Twyman-Saint Victor C, Wiemann BZ, Ishwaran $\mathrm{H}$, et al. Exosome transfer from stromal to breast cancer cells regulates therapy resistance pathways. Cell (2014) 159:499-513. doi:10.1016/j.cell.2014.09.051

130. Kong LB, Siva AC, Kickhoefer VA, Rome LH, Stewart PL. RNA location and modeling of a WD40 repeat domain within the vault. RNA (2000) 6:890-900. doi:10.1017/S1355838200000157 
131. Berger W, Steiner E, Grusch M, Elbling L, Micksche M. Vaults and the major vault protein: Novel roles in signal pathway regulation and immunity. Cell Mol Life Sci (2009) 66:43-61. doi:10.1007/s00018-0088364-z

132. Kickhoefer VA, Rajavel KS, Scheffer GL, Dalton WS, Scheper RJ, Rome LH. Vaults are up-regulated in multidrug-resistant cancer cell lines. J Biol Chem (1998) 273:8971-8974. doi:10.1074/jbc.273.15.8971

133. Steiner E, Holzmann K, Pirker C, Elbling L, Micksche M, Sutterluty H, Berger W. The major vault protein is responsive to and interferes with interferon-gamma-mediated STAT1 signals. J Cell Sci (2006) 119:459-469. doi:10.1242/jcs.02773

134. Schroeijers AB, Reurs AW, Scheffer GL, Stam AGM, de Jong MC, Rustemeyer T, Wiemer EAC, de Gruijl TD, Scheper RJ. Up-Regulation of Drug Resistance-Related Vaults During Dendritic Cell Development. J Immunol (2002) 168:1572-1578. doi:10.4049/jimmunol.168.4.1572

135. Horos R, Alleaume A-M, Kleinendorst R, Tarafder AK, Schwarzl T, Zielonka EM, Adak A, Castello A, Huber W, Sachse C, et al. The small non-coding vault RNA1-1 acts as a riboregulator of autophagy. Cell (2019) 176:1-14. doi:10.1101/177949

136. Mrázek J, Kreutmayer SB, Gräser FA, Polacek N, Huettenhofer A. Subtractive hybridization identifies novel differentially expressed ncRNA species in EBV-infected human B cells. Nucleic Acids Res (2007) 35:1-9. doi:10.1093/nar/gkm244

137. Li F, Chen Y, Zhang Z, Ouyang J, Wang Y, Yan R, Huang S, Gao GF, Guo G, Chen J-L. Robust expression of vault RNAs induced by influenza A virus plays a critical role in suppression of PKR-mediated innate immunity. Nucleic Acids Res (2015) 43:gkv1078. doi:10.1093/nar/gkv1078

138. Persson H, Kvist A, Vallon-Christersson J, Medstrand P, Borg A, Rovira C. The non-coding RNA of the multidrug resistance-linked vault particle encodes multiple regulatory small RNAs. Nat Cell Biol (2009) 11:1268-71. doi:10.1038/ncb1972

139. Langenberger D, Çakir MV, Hoffmann S, Stadler PF. Dicer-Processed Small RNAs: Rules and Exceptions. J Exp Zool Part B Mol Dev Evol (2013) 320:35-46. doi:10.1002/jez.b.22481

140. Hussain S, Sajini A a, Blanco S, Dietmann S, Lombard P, Sugimoto Y, Paramor M, Gleeson JG, Odom DT, Ule $J$, et al. NSun2-mediated cytosine-5 methylation of vault noncoding RNA determines its processing into regulatory small RNAs. Cell Rep (2013) 4:255-61. doi:10.1016/j.celrep.2013.06.029

141. Koppers-Lalic D, Hackenberg M, Bijnsdorp I V., van Eijndhoven MAJ, Sadek P, Sie D, Zini N, Middeldorp JM, Ylstra B, de Menezes RX, et al. Nontemplated nucleotide additions distinguish the small RNA composition in cells from exosomes. Cell Rep (2014) 8:1649-1658. doi:10.1016/j.celrep.2014.08.027

142. Lunavat TR, Cheng L, Kim D-K, Bhadury J, Jang SC, Lässer C, Sharples RA, López MD, Nilsson J, Gho YS, et al. Small RNA deep sequencing discriminates subsets of extracellular vesicles released by melanoma cells evidence of unique microRNA cargos. RNA Biol (2015) 12:810-823. doi:10.1080/15476286.2015.1056975

143. Umu SU, Langseth H, Bucher-Jonannessen C, Fromm B, Keller A, Meese E, Lauritzen M, Leithaug M, Lyle R, Rounge T. A comprehensive profile of circulating RNAs in human serum. RNA Biol (2017) 0:1-9. doi:10.1080/1 5476286.2017.1403003

144. Eddy SR. Non-coding RNA genes and the modern RNA world. Nat Rev Genet (2001) 2:919-929.

145. Sergiev PV., Aleksashin NA, Chugunova AA, Polikanov YS, Dontsova OA. Structural and evolutionary insights into ribosomal RNA methylation. Nat Chem Biol (2018) 14:226-235. doi:10.1038/nchembio.2569

146. Baudin-baillieu A, Fabret C, Liang XH, Piekna-Przybylska D, Fournier MJ, Rousset JP. Nucleotide modifications in three functionally important regions of the Saccharomyces cerevisiae ribosome affect translation accuracy. Nucleic Acids Res (2009) 37:7665-7677. doi:10.1093/nar/gkp816

147. Dieci G, Preti M, Montanini B. Eukaryotic snoRNAs: A paradigm for gene expression flexibility. Genomics (2009) 94:83-88. doi:10.1016/j.ygeno.2009.05.002

148. Martens-Uzunova ES, Olvedy M, Jenster G. Beyond microRNA - Novel RNAs derived from small non-coding RNA and their implication in cancer. Cancer Lett (2013) 340:201-211. doi:10.1016/j.canlet.2012.11.058

149. Duker AL, Ballif BC, Bawle EV., Person RE, Mahadevan S, Alliman S, Thompson R, Traylor R, Bejjani BA, Shaffer $L G$, et al. Paternally inherited microdeletion at 15q11.2 confirms a significant role for the SNORD 116 C/D box snoRNA cluster in Prader-Willi syndrome. Eur J Hum Genet (2010) 18:1196-1201. doi:10.1038/ejhg.2010.102 
150. Scott MS, Ono M. From snoRNA to miRNA: Dual function regulatory non-coding RNAs. Biochimie (2011) 93:1987-1992. doi:10.1016/j.biochi.2011.05.026

151. Ender C, Krek A, Friedländer MR, Beitzinger M, Weinmann L, Chen W, Pfeffer S, Rajewsky N, Meister G. A Human snoRNA with MicroRNA-Like Functions. Mol Cell (2008) 32:519-528. doi:10.1016/j.molcel.2008.10.017

152. Liao J, Yu L, Mei Y, Guarnera M, Shen J, Li R, Liu Z, Jiang F. Small nucleolar RNA signatures as biomarkers for non-small-cell lung cancer. Mol Cancer (2010) 9:1-10. doi:10.1186/1476-4598-9-198

153. Will CL, Lührmann R. Spliceosomal UsnRNP biogenesis, structure and function. Curr Opin Cell Biol (2001) 13:290-301. doi:10.1016/S0955-0674(00)00211-8

154. Matera AG, Wang Z. A day in the life of the spliceosome. Nat Rev Mol Cell Biol (2014) 15:108-21. doi:10.1038/ nrm3742

155. Chen X, Smith JD, Shi H, Yang DD, Flavell RA, Wolin SL. The Ro Autoantigen Binds Misfolded U2 Small Nuclear RNAs and Assists Mammalian Cell Survival after UV Irradiation. Curr Biol (2003) 13:2206-2211. doi:10.1016/j. cub.2003.11.028

156. Zhang Z, Lotti F, Dittmar K, Younis I, Wan L, Kasim M, Dreyfuss G. SMN Deficiency Causes Tissue-Specific Perturbations in the Repertoire of snRNAs and Widespread Defects in Splicing. Cell (2008) 133:585-600. doi:10.1016/j.cell.2008.03.031

157. Shukla S, Parker R. Quality control of assembly-defective U1 snRNAs by decapping and 5'-to-3' exonucleolytic digestion. Proc Natl Acad Sci (2014) 111:E3277-E3286. doi:10.1073/pnas.1412614111

158. Ustianenko D, Pasulka J, Feketova Z, Bednarik L, Zigackova D, Fortova A, Zavolan M, Vanacova S. TUT-DIS3L2 is a mammalian surveillance pathway for aberrant structured non-coding RNAs. EMBO J (2016) 35:2179-2191. doi:10.15252/embj.201694857

159. Baraniskin A, Nöpel-Dünnebacke S, Ahrens M, Jensen SG, Zöllner H, Maghnouj A, Wos A, Mayerle J, Munding J, Kost D, et al. Circulating U2 small nuclear RNA fragments as a novel diagnostic biomarker for pancreatic and colorectal adenocarcinoma. Int J Cancer (2013) 132:48-57. doi:10.1002/ijc.27791

160. Liu J-L, Gall JG. U bodies are cytoplasmic structures that contain uridine-rich small nuclear ribonucleoproteins and associate with P bodies. Proc Natl Acad Sci (2007) 104:11655-11659. doi:10.1073/pnas.0704977104

161. Djebali S, Davis CA, Merkel A, Dobin A, Lassmann T, Mortazavi A, Tanzer A, Lagarde J, Lin W, Schlesinger F, et al. Landscape of transcription in human cells. Nature (2012) 489:101-8. doi:10.1038/nature11233

162. Gardiner C, Vizio D Di, Sahoo S, Théry C, Witwer KW, Wauben M, Hill AF. Techniques used for the isolation and characterization of extracellular vesicles: Results of a worldwide survey. J Extracell Vesicles (2016) 5 doi:10.3402/jev.v5.32945

163. Van Deun J, Mestdagh P, Agostinis P, Akay Ö, Anand S, Anckaert J, Martinez ZA, Baetens T, Beghein E, Bertier $L$, et al. EV-TRACK: transparent reporting and centralizing knowledge in extracellular vesicle research. Nat Methods (2017) 14:228-232. doi:10.1038/nmeth.4185

164. Crescitelli R, Lässer C, Szabó TG, Kittel A, Eldh M, Dianzani I, Buzás El, Lötvall J. Distinct RNA profiles in subpopulations of extracellular vesicles: Apoptotic bodies, microvesicles and exosomes. J Extracell Vesicles (2013) 2:1-10. doi:10.3402/jev.v2i0.20677

165. Van Deun J, Mestdagh P, Sormunen R, Cocquyt V, Vermaelen K, Vandesompele J, Bracke M, De Wever O, Hendrix A. The impact of disparate isolation methods for extracellular vesicles on downstream RNA profiling. J Extracell Vesicles (2014) 3:1-14. doi:10.3402/jev.v3.24858

166. Buschmann D, Kirchner B, Hermann S, Märte M, Wurmser C, Brandes F, Kotschote S, Bonin M, Steinlein OK, Pfaffl MW, et al. Evaluation of serum extracellular vesicle isolation methods for profiling miRNAs by nextgeneration sequencing. J Extracell Vesicles (2018) 7: doi:10.1080/20013078.2018.1481321

167. Srinivasan S, Yeri A, Cheah PS, Chung A, Danielson K, De Hoff P, Filant J, Laurent CD, Laurent LD, Magee R, et al. Small RNA Sequencing across Diverse Biofluids Identifies Optimal Methods for exRNA Isolation. Cell (2019) 177:446-462.e16. doi:10.1016/j.cell.2019.03.024

168. Théry C, Witwer KW, Aikawa E, Alcaraz MJ, Anderson JD, Andriantsitohaina R, Antoniou A, Arab T, Archer F, Atkin-Smith GK, et al. Minimal information for studies of extracellular vesicles 2018 (MISEV2018): a position statement of the International Society for Extracellular Vesicles and update of the MISEV2014 guidelines. J Extracell Vesicles (2018) 8:1535750. doi:10.1080/20013078.2018.1535750 
169. Feingold K, Grunfeld C. "Introduction to Lipids and Lipoproteins," in Endotext, eds. K. Feingold, B. Anawalt, A. Boyce (MDtext.com). Available at: https://europepmc.org/books/ NBK305896;jsessionid=FC86076A4DE6F06B7F048A4DFF4C7F05\%0A

170. Théry C, Amigorena S, Raposo G, Clayton A. Isolation and Characterization of Exosomes from Cell Culture Supernatants. Curr Protoc cell Biol (2006) Chapter 3:1-29. doi:10.1002/0471143030.cb0322s30

171. Momen-Heravi F, Balaj L, Alian S, Trachtenberg AJ, Hochberg FH, Skog J, Kuo WP. Impact of biofluid viscosity on size and sedimentation efficiency of the isolated microvesicles. Front Physiol (2012) 3

MAY:1-6. doi:10.3389/fphys.2012.00162

172. Böing AN, Pol E Van Der, Grootemaat AE, Coumans F a., Sturk A, Nieuwland R. Single-step isolation of extracellular vesicles from plasma by size-exclusion chromatography. J Extracell Vesicles (2014) 3:118. doi:10.3402/jev.v3.23430

173. Welton JL, Webber JP, Botos LA, Jones M, Clayton A. Ready-made chromatography columns for extracellular vesicle isolation from plasma. J Extracell Vesicles (2015) 4:1-9. doi:10.3402/jev.v4.27269

174. Baranyai T, Herczeg K, Onódi Z, Voszka I, Módos K, Marton N, Nagy G, Mäger I, Wood MJ, El Andaloussi S, et al. Isolation of exosomes from blood plasma: Qualitative and quantitative comparison of ultracentrifugation and size exclusion chromatography methods. PLoS One (2015) 10:1-13. doi:10.1371/ journal.pone.0145686

175. Willms E, Johansson HJ, Mäger I, Lee Y, Blomberg KEM, Sadik M, Alaarg A, Smith CIE, Lehtiö J, El Andaloussi S, et al. Cells release subpopulations of exosomes with distinct molecular and biological properties. SciRep (2016) 6:1-12. doi:10.1038/srep22519

176. Karimi N, Cvjetkovic A, Jang SC, Crescitelli R, Hosseinpour Feizi MA, Nieuwland R, Lötvall J, Lässer C. Detailed analysis of the plasma extracellular vesicle proteome after separation from lipoproteins. Cell Mol Life Sci (2018)1-14. doi:10.1007/s00018-018-2773-4

177. Mol EA, Goumans MJ, Doevendans PA, Sluijter JPG, Vader P. Higher functionality of extracellular vesicles isolated using size-exclusion chromatography compared to ultracentrifugation. Nanomedicine (2017) 13:2061-2065. doi:10.1016/j.nano.2017.03.011

178. Ludwig AK, De Miroschedji K, Doeppner TR, Börger V, Ruesing J, Rebmann V, Durst S, Jansen S, Bremer M, Behrmann E, et al. Precipitation with polyethylene glycol followed by washing and pelleting by ultracentrifugation enriches extracellular vesicles from tissue culture supernatants in small and large scales. J Extracell Vesicles (2018) 7: doi:10.1080/20013078.2018.1528109

179. Karttunen J, Heiskanen M, Navarro-Ferrandis V, Das Gupta S, Lipponen A, Puhakka N, Rilla K, Koistinen A, Pitkänen A. Precipitation-based extracellular vesicle isolation from rat plasma co-precipitate vesicle-free microRNAs. J Extracell Vesicles (2018) 8: doi:10.1080/20013078.2018.1555410

180. Shelke GV, Lässer C, Gho YS, Lötvall J. Importance of exosome depletion protocols to eliminate functional and RNA-containing extracellular vesicles from fetal bovine serum. J Extracell Vesicles (2014) 3:1-8. doi:10.3402/jev.v3.24783

181. Wei Z, Batagov AO, Carter DRF, Krichevsky AM. Fetal Bovine Serum RNA Interferes with the Cell Culture derived Extracellular RNA. Sci Rep (2016) 6:31175. doi:10.1038/srep31175

182. Tosar JP, Cayota A, Eitan E, Halushka MK, Witwer KW. Ribonucleic artefacts: are some extracellular RNA discoveries driven by cell culture medium components? J Extracell Vesicles (2017) 6:1-10. doi:10.1080/20 013078.2016.1272832

183. Kornilov R, Puhka M, Mannerström B, Hiidenmaa H, Peltoniemi H, Siljander P, Seppänen-Kaijansinkko R, Kaur S. Efficient ultrafiltration-based protocol to deplete extracellular vesicles from fetal bovine serum. $J$ Extracell Vesicles (2018) 7:1422674. doi:10.1080/20013078.2017.1422674

184. Lehrich BM, Liang Y, Khosravi P, Federoff HJ, Fiandaca MS. Fetal Bovine Serum-Derived Extracellular Vesicles Persist within Vesicle-Depleted Culture Media. Int J Mol Sci (2018) 19: doi:10.3390/ijms19113538

185. Driedonks TAP, Nijen-Twilhaar MK, Nolte-'t Hoen ENM. Technical approaches to reduce interference of Fetal calf serum derived RNA in the analysis of extracellular vesicle RNA from cultured cells. J Extracell Vesicles (2018) 8: doi:10.1080/20013078.2018.1552059 


\section{General introduction}

186. Mannerström B, Paananen RO, Abu-Shahba AG, Moilanen J, Seppänen-Kaijansinkko R, Kaur S.

Extracellular small non-coding RNA contaminants in fetal bovine serum and serum-free media. Sci Rep (2019) 9:5538. doi:10.1038/s41598-019-41772-3

187. Wang Z, Gerstein M, Snyder M. RNA-Seq: a revolutionary tool for transcriptomics. Nat Rev Genet (2009) 10:57-63. doi:10.1038/nrg2484

188. 't Hoen PAC, Friedländer MR, Almlöf J, Sammeth M, Pulyakhina I, Anvar SY, Laros JFJ, Buermans HPJ, Karlberg O, Brännvall M, et al. Reproducibility of high-throughput mRNA and small RNA sequencing across laboratories. Nat Biotechnol (2013) 31:1015-22. doi:10.1038/nbt.2702

189. Giraldez MD, Spengler RM, Etheridge A, Godoy PM, Barczak AJ, Srinivasan S, De Hoff PL, Tanriverdi K, Courtright A, Lu S, et al. Comprehensive multi-center assessment of small RNA-seq methods for quantitative miRNA profiling. Nat Biotechnol (2018) doi:10.1038/nbt.4183

190. Linsen SEV, de Wit E, Janssens G, Heater S, Chapman L, Parkin RK, Fritz B, Wyman SK, de Bruijn E, Voest EE, et al. Limitations and possibilities of small RNA digital gene expression profiling. Nat Methods (2009) 6:474-6. doi:10.1038/nmeth0709-474

191. Giraldez MD, Spengler RM, Etheridge A, Goicochea AJ, Tuck M, Choi SW, Galas DJ, Tewari M. Phospho-RNAseq: a modified small RNA-seq method that reveals circulating mRNA and IncRNA fragments as potential biomarkers in human plasma. EMBO J (2019)e101695. doi:10.15252/embj.2019101695

192. Raabe CA, Tang T-H, Brosius J, Rozhdestvensky TS. Biases in small RNA deep sequencing data. Nucleic Acids Res (2014) 42:1414-1426. doi:10.1093/nar/gkt1021

193. Chen C, Ridzon DA, Broomer AJ, Zhou Z, Lee DH, Nguyen JT, Barbisin M, Xu NL, Mahuvakar VR, Andersen MR, et al. Real-time quantification of microRNAs by stem-loop RT-PCR. Nucleic Acids Res (2005) 33:1-9. doi:10.1093/nar/gni178

194. Kubista M, Andrade JM, Bengtsson M, Forootan A, Jonák J, Lind K, Sindelka R, Sjöback R, Sjögreen B, Strömbom L, et al. The real-time polymerase chain reaction. Mol Aspects Med (2006) 27:95-125. doi:10.1016/j. mam.2005.12.007

195. Bustin SA. Absolute quantification of mRNA using real-time reverse transcription polymerase chain reaction assays. J Mol Endocrinol (2000) 25:169-193. doi:10.1677/jme.0.0250169

196. Galiveti CR, Rozhdestvensky TS, Brosius J, Lehrach H, Konthur Z. Application of housekeeping npcRNAs for quantitative expression analysis of human transcriptome by real-time PCR. RNA (2010) 16:450-461. doi:10.1261/rna.1755810

197. Vandesompele J, De Preter K, Pattyn F, Poppe B, Van Roy N, De Paepe A, Speleman F. Accurate normalization of real-time quantitative RT-PCR data by geometric averaging of multiple internal control genes. Genome Biol (2002) 3: doi:10.1186/gb-2002-3-7-research0034

198. Andersen CL, Jensen JL, Ørntoft TF. Normalization of Real-Time Quantitative Reverse Transcription-PCR Data: A Model-Based Variance Estimation Approach to Identify Genes Suited for Normalization, Applied to Bladder and Colon Cancer Data Sets. Cancer Res (2004) 64:5245-5250. doi:10.1158/0008-5472.CAN-04-0496

199. Pfaffl MW, Tichopad A, Prgomet C, Neuvians T. Determination of most stable housekeeping genes, differentially regulated target genes and sample integrity : BestKeeper. Biotechnol Lett (2004) 26:509-515. doi:JCMM395 [pii]\r10.1111/j.1582-4934.2008.00395.x

200. HruzT, Wyss M, Docquier M, Pfaffl MW, Masanetz S, Borghi L, Verbrugghe P, Kalaydjieva L, Bleuler S, Laule O, et al. RefGenes: identification of reliable and condition specific reference genes for RT-qPCR data normalization. BMC Genomics (2011) 12:156. doi:10.1186/1471-2164-12-156

201. Marabita F, de Candia P, Torri A, Tegnér J, Abrignani S, Rossi RL. Normalization of circulating microRNA expression data obtained by quantitative real-time RT-PCR. BriefBioinform (2015)bbv056. doi:10.1093/bib/ bbv056

202. Popovici V, Goldstein DR, Antonov J, Jaggi R, Delorenzi M, Wirapati P. Selecting control genes for RT-QPCR using public microarray data. BMC Bioinformatics (2009) 10:1-10. doi:10.1186/1471-2105-10-42

203. Gouin K, Peck K, Antes T, Johnson JL, Li C, Vaturi SD, Middleton R, de Couto G, Walravens A-S, RodriguezBorlado $L$, et al. A comprehensive method for identification of suitable reference genes in extracellular vesicles. J Extracell Vesicles (2017) 6:1347019. doi:10.1080/20013078.2017.1347019 
204. Locati MD, Terpstra I, de Leeuw WC, Kuzak M, Rauwerda H, EnsinkWA, van Leeuwen S, Nehrdich U, Spaink $\mathrm{HP}$, Jonker MJ, et al. Improving small RNA-seq by using a synthetic spike-in set for size-range quality control together with a set for data normalization. Nucleic Acids Res (2015)1-10. doi:10.1093/nar/gkv303

205. Bustin S a, Benes V, Garson J a, Hellemans J, Huggett J, Kubista M, Mueller R, Nolan T, Pfaff MW, Shipley GL, et al. The MIQE guidelines: minimum information for publication of quantitative real-time PCR experiments. Clin Chem (2009) 55:611-22. doi:10.1373/clinchem.2008.112797

206. Liu T, Zhang Q, Zhang J, Li C, Miao Y-R, Lei Q, Li Q, Guo A-Y. EVmiRNA: a database of miRNA profiling in extracellular vesicles. Nucleic Acids Res (2018)3-7. doi:10.1093/nar/gky985

207. Robbins PD, Morelli AE. Regulation of immune responses by extracellular vesicles. Nat Rev /mmunol (2014) 14:195-208. doi:10.1038/nri3622

208. Lindenbergh MFS, Stoorvogel W. Antigen Presentation by Extracellular Vesicles from Professional AntigenPresenting Cells. Annu Rev Immunol (2018) 36:annurev-immunol-041015-055700. doi:10.1146/annurevimmunol-041015-055700

209. Groot Kormelink T, Mol S, de Jong EC, Wauben MHM. The role of extracellular vesicles when innate meets adaptive. Semin Immunopathol (2018)1-14. doi:10.1007/s00281-018-0681-1

210. Veerman RE, Güçlüler Akpinar G, Eldh M, Gabrielsson S. Immune Cell-Derived Extracellular Vesicles - Functions and Therapeutic Applications. Trends Mol Med (2019) 25:382-394. doi:10.1016/j. molmed.2019.02.003

211. Raposo G, Nijman HW, Stoorvogel W, Leijendekker R, Harding C V., Melief CJM, Geuze HJ. B Lymphocytes Secrete Antigen-presenting Vesicles. J Exp Med (1996) 183:1161-1172.

212. Zitvogel L, Regnault A, Lozier A, Wolfers J, Flament C, Tenza D, Ricciardi-Castagnoli P, Raposo G, Amigorena S. Eradication of established murine tumors using a novel cell-free vaccine: Dendritic cell-derived exosomes. Nat Med (1998) 4:594-600. doi:10.1038/nm0598-594

213. Théry C, Duban L, Segura E, Væron P, Lantz O, Amigorena S. Indirect activation of naïve CD4+ T cells by dendritic cell-derived exosomes. Nat Immunol (2002) 3:1156-1162. doi:10.1038/ni854

214. Cheng L, Wang Y, Huang L. Exosomes from M1-Polarized Macrophages Potentiate the Cancer Vaccine by Creating a Pro-inflammatory Microenvironment in the Lymph Node. Mol Ther (2017) 25:1665-1675. doi:10.1016/j.ymthe.2017.02.007

215. Kim S-H, Lechman ER, Bianco N, Menon R, Keravala A, Nash J, Mi Z, Watkins SC, Gambotto A, Robbins PD Exosomes Derived from IL-10-Treated Dendritic Cells Can Suppress Inflammation and Collagen-Induced Arthritis. J Immunol (2014) 174:6440-6448. doi:10.4049/jimmunol.174.10.6440

216. Pedersen IM, Cheng G, Wieland S, Volinia S, Croce CM, Chisari FV, David M. Interferon modulation of cellular microRNAs as an antiviral mechanism. Nature (2007) 449:919-22. doi:10.1038/nature06205

217. Pedersen AW, Holmstrøm K, Jensen SS, Fuchs D, Rasmussen S, Kvistborg P, Claesson MH, Zocca M-B. Phenotypic and functional markers for 1alpha,25-dihydroxyvitamin D(3)-modified regulatory dendritic cells. Clin Exp Immunol (2009) 157:48-59. doi:10.1111/j.1365-2249.2009.03961.x

218. Mehta A, Baltimore D. MicroRNAs as regulatory elements in immune system logic. Nat Rev Immunol (2016) 16:279-294. doi:10.1038/nri.2016.40

219. Zhang Y, Liu D, Chen X, Li J, Li L, Bian Z, Sun F, Lu J, Yin Y, Cai X, et al. Secreted Monocytic miR-150 Enhances Targeted Endothelial Cell Migration. Mol Cell (2010) 39:133-144. doi:10.1016/j.molcel.2010.06.010

220. Njock M-S, Cheng HS, Dang LT, Nazari-Jahantigh M, Lau AC, Boudreau E, Roufaiel M, Cybulsky MI, Schober A, Fish JE. Endothelial cells suppress monocyte activation through secretion of extracellular vesicles containing anti-inflammatory microRNAs. Blood (2015) doi:10.1182/blood-2014-11-611046

221. Okoye IS, Coomes SM, Pelly VS, Czieso S, Papayannopoulos V, Tolmachova T, Seabra MC, Wilson MS. Exosomes Suppress Pathogenic T Helper 1 Cells. Immunity (2014) 41:89-103. doi:10.1016/j.immuni.2014.05.019

222. Ismail N, Wang Y, Dakhlallah D, Moldovan L, Agarwal K, Batte K, Shah P, Wisler J, Eubank TD, Tridandapani S, et al. Macrophage microvesicles induce macrophage differentiation and miR-223 transfer. Blood (2013) 121:984995. doi:10.1182/blood-2011-08-374793 
223. Chevillet JR, Kang Q, Ruf IK, Briggs Ha, Vojtech LN, Hughes SM, Cheng HH, Arroyo JD, Meredith EK, Gallichotte EN, et al. Quantitative and stoichiometric analysis of the microRNA content of exosomes. Proc Natl Acad Sci US A (2014) 111: doi:10.1073/pnas.1408301111

224. Turchinovich A, Tonevitsky AG, Cho WC, Burwinkel B. Check and mate to exosomal extracellular miRNA: New lesson from a new approach. Front Mol Biosci (2015) 2:10-12. doi:10.3389/fmolb.2015.00011

225. Sverdlov ED. Amedeo Avogadro's cry: What is $1 \mu \mathrm{g}$ of exosomes? BioEssays (2012) 34:873-875. doi:10.1002/ bies.201200045

226. Sellam J, Proulle V, Jüngel A, Ittah M, Miceli Richard C, Gottenberg J-E, Toti F, Benessiano J, Gay S, Freyssinet $J-M$, et al. Increased levels of circulating microparticles in primary Sjögren's syndrome, systemic lupus erythematosus and rheumatoid arthritis and relation with disease activity. Arthritis Res Ther (2009) 11:R156. doi:10.1186/ar2833

227. Buzas El, György B, Nagy G, Falus A, Gay S. Emerging role of extracellular vesicles in inflammatory diseases. Nat Rev Rheumatol (2014) 10:356-64. doi:10.1038/nrrheum.2014.19

228. Redzic JS, Balaj L, van der Vos KE, Breakefield XO. Extracellular RNA mediates and marks cancer progression. Semin Cancer Biol (2014) 28:14-23. doi:10.1016/j.semcancer.2014.04.010

229. Zijlstra C, Stoorvogel W. Prostasomes as a source of diagnostic biomarkers for prostate cancer. J Clin Invest (2016) 126:1144-1151. doi:10.1172/JCl81128

230. Repetto E, Lichtenstein L, Hizir Z, Tekaya N, Benahmed M, Ruidavets J-B, Zaragosi L-E, Perret B, Bouchareychas L, Genoux A, et al. RNY-derived small RNAs as a signature of coronary artery disease. BMCMed (2015) 13:259. doi:10.1186/s12916-015-0489-y

231. Correia CN, Nalpas NC, McLoughlin KE, Browne JA, Gordon SV., MacHugh DE, Shaughnessy RG. Circulating microRNAs as potential biomarkers of infectious disease. Front Immunol (2017) 8:1-17. doi:10.3389/ fimmu.2017.00118

232. Mustapic M, Eitan E, Werner JK, Berkowitz ST, Lazaropoulos MP, Tran J, Goetzl EJ, Kapogiannis D. Plasma extracellular vesicles enriched for neuronal origin: A potential window into brain pathologic processes. Front Neurosci (2017) 11:1-12. doi:10.3389/fnins.2017.00278

233. Santiago-Dieppa DR, Steinberg J, Gonda D, Cheung VJ, Carter BS, Chen CC. Extracellular vesicles as a platform for "liquid biopsy" in glioblastoma patients. Expert Rev Mol Diagn (2014) 14:819-25. doi:10.1586/14737159.201 4.943193

234. Dhahbi JM, Spindler SR, Atamna H, Boffelli D, Martin DIK. Deep Sequencing of Serum Small RNAs Identifies Patterns of 5'tRNA Half and YRNA Fragment Expression Associated with Breast Cancer. Biomark Cancer (2014) 6:37-47. doi:10.4137/BIC.S20764.RECEIVED

235. Martinez BV, Dhahbi JM, Nunez Lopez YO, Lamperska K, Golusinski P, Luczewski L, Kolenda T, Atamna H, Spindler SR, Golusinski W, et al. Circulating small non coding RNA signature in head and neck squamous cell carcinoma. Oncotarget (2015) 6:19246-19263. doi:10.18632/oncotarget.4266

236. van Eijndhoven MAJ, Zijlstra JM, Groenewegen NJ, Drees EEE, van Niele S, Baglio SR, Koppers-Lalic D, van der Voorn H, Libregts SFWM, Wauben MHM, et al. Plasma vesicle miRNAs for therapy response monitoring in Hodgkin lymphoma patients. JCI Insight (2016) 1:1-16. doi:10.1172/jci.insight.89631

237. Matsuzaki J, Ochiya T. Circulating or tissue micrornas and extracellular vesicles as potential lung cancer biomarkers: A systematic revie. Int J Clin Onc (2017) 22:413-420. doi:10.5301/ijbm.5000307

238. Guo R, Fan G, Zhang J, Wu C, Du Y, Ye H, Li Z, Wang L, Zhang Z, Zhang L, et al. A 9-microRNA Signature in Serum Serves as a Noninvasive Biomarker in Early Diagnosis of Alzheimer's Disease. J Alzheimer's Dis (2017) 60:1365-1377. doi:10.3233/JAD-170343

239. Swarbrick S, Wragg N, Ghosh S, Stolzing A. Systematic Review of miRNA as Biomarkers in Alzheimer's Disease. Mol Neurobiol (2019) doi:10.1007/s12035-019-1500-y

240. Furer $\mathrm{V}$, Greenberg JD, Attur M, Abramson SB, Pillinger MH. The role of microRNA in rheumatoid arthritis and other autoimmune diseases. Clin Immunol (2010) 136:1-15. doi:10.1016/j.clim.2010.02.005

241. Zeng L, Cui J, Wu H, Lu Q. The emerging role of circulating microRNAs as biomarkers in autoimmune diseases. Autoimmunity (2014) 47:419-29. doi:10.3109/08916934.2014.929667 
242. Raeven P, Zipperle J, Drechsler S. Extracellular vesicles as markers and mediators in sepsis. Theranostics (2018) 8:3348-3365. doi:10.7150/thno.23453

243. Vasilescu C, Rossi S, Shimizu M, Tudor S, Veronese A, Ferracin M, Nicoloso MS, Barbarotto E, Popa M, Stanciulea O, et al. MicroRNA fingerprints identify miR-150 as a plasma prognostic marker in patients with sepsis. PLoS One (2009) 4: doi:10.1371/journal.pone.0007405

244. Wang HJ, Zhang PJ, Chen WJ, Feng D, Jia YH, Xie LX. Four serum microRNAs identified as diagnostic biomarkers of sepsis. J Trauma Acute Care Surg (2012) 73:850-854. doi:10.1097/TA.0b013e31825a7560

245. Goodwin AJ, Guo C, Cook JA, Wolf B, Halushka P V., Fan H. Plasma levels of microRNA are altered with the development of shock in human sepsis: An observational study. Crit Care (2015) 19:1-10. doi:10.1186/ s13054-015-1162-8

246. Caserta S, Kern F, Cohen J, Drage S, Newbury SF, Llewelyn MJ. Circulating Plasma microRNAs can differentiate Human Sepsis and Systemic Inflammatory Response Syndrome (SIRS). Sci Rep (2016) 6:1-13. doi:10.1038/ srep28006

247. Ma Y, Liu Y, Hou H, Yao Y, Meng H. MiR-150 predicts survival in patients with sepsis and inhibits LPS-induced inflammatory factors and apoptosis by targeting NF-KB1 in human umbilical vein endothelial cells. Biochem Biophys Res Commun (2018) 500:828-837. doi:10.1016/j.bbrc.2018.04.168

248. Benz F, Roy S, Trautwein C, Roderburg C, Luedde T. Circulating MicroRNAs as biomarkers for sepsis. Int J Mol Sci (2016) 17: doi:10.3390/ijms17010078

249. Ho J, Chan H, Wong SH, Wang MHT, Yu J, Xiao Z, Liu X, Choi G, Leung CCH, Wong WT, et al. The involvement of regulatory non-coding RNAs in sepsis: A systematic review. Crit Care (2016) 20:1-12. doi:10.1186/s13054016-1555-3

250. Angus DC, van der Poll T. Severe sepsis and septic shock. N Eng/ J Med (2013) 369:703-707. doi:10.1007/97881-322-0535-7_88

251. Witwer KW, Buzás El, Bemis LT, Bora A, Lässer C, Lötvall J, Nolte-'t Hoen EN, Piper MG, Sivaraman S, Skog J, et al. Standardization of sample collection, isolation and analysis methods in extracellular vesicle research. $J$ Extracell vesicles (2013) 2:1-25. doi:10.3402/jev.v2i0.20360 


\title{
Chapter 2
}

\section{Comprehensive multi-center assessment of small RNA-seq methods for quantitative miRNA profiling}

\author{
MD Giraldez ${ }^{\star}, 1$, RM Spengler ${ }^{*}$, A Etheridge ${ }^{5}$, PM Godoy $^{6}$, AJ Barczak ${ }^{6}$, S Srinivasan ${ }^{7}$,

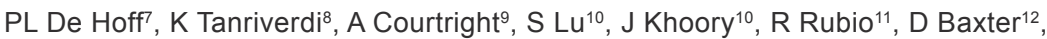 \\ TAP Driedonks $^{13}$, HPJ Buermans ${ }^{14}$, ENM Nolte-'t Hoen ${ }^{13}, \mathrm{H}$ Jiang ${ }^{3,15}, \mathrm{~K}$ Wang $^{12}$, I Ghiran ${ }^{10}$, \\ Y Wang ${ }^{11}$, K Van Keuren-Jensen ${ }^{9}$, JE Freedman ${ }^{8}$, PG Woodruff ${ }^{16}$, LC Laurent ${ }^{7}$, DJ Erle ${ }^{6}$, \\ DJ Galas $\$, 5$, and M Tewari $\$, 1,2,3,4$
}

1 Department of Internal Medicine, University of Michigan

2 Department of Biomedical Engineering, University of Michigan

3 Center for Computational Medicine and Bioinformatics, University of Michigan

4 Biointerfaces Institute, University of Michigan

5 Pacific Northwest Research Institute

6 Lung Biology Center, Department of Medicine, University of California San Francisco

7 University of California, San Diego, Department of Reproductive Medicine and Sanford Consortium for Regenerative Medicine, 2880 Torrey Pines Scenic Drive, La Jolla, CA. 92037

8 University of Massachusetts Medical School, Department of Medicine, Division of Cardiovascular Medicine, Worcester, Massachusetts 01605, USA

9 Neurogenomics, The Translational Genomics Research Institute (TGen), 445 N. Fifth Street, Phoenix, AZ 85004
10 Department of Medicine, Beth Israel Deaconess Medical Center, Harvard Medical School

11 Center for Cancer Computational Biology, DanaFarber Cancer Institute

12 Institute for Systems Biology, 401 Terry Ave North, Seattle, WA 98109

13 Department of Biochemistry \& Cell Biology, Faculty of Veterinary Medicine, Utrecht University, Yalelaan 2, 3584 CM Utrecht, The Netherlands

14 Leiden Genome Technology Center, Department of Human Genetics, Leiden University Medical Center, 2300 RC Leiden, The Netherlands

15 Department of Biostatistics, University of Michigan

16 Cardiovascular Research Institute and the Department of Medicine, Division of Pulmonary, Critical Care, Sleep, and Allergy, University of California San Francisco

* These authors contributed equally. $\S$ Corresponding authors. 


\section{Abstract}

RNA-seq is increasingly employed for quantitative profiling of small RNAs (e.g., microRNAs, piRNAs, snoRNAs) in diverse sample types including isolated cells, tissues and cell-free biofluids. The accuracy and reproducibility of the multiple small RNAseq library preparation methods in use, however, have not been systematically assessed. We report systematic results obtained by a consortium of nine labs that independently sequenced reference, 'ground truth', samples of synthetic small RNAs and human plasma-derived RNA. Three commercially available library preparation methods employing adapters of defined sequence and six methods using adapters with degenerate bases were assessed. Both protocol- and sequence-specific biases were identified, including biases that reduce the ability of small RNA-seq to accurately measure adenosine-to-inosine editing in microRNAs. We report that these biases were mitigated by library preparation methods that incorporate adapters with degenerate bases. MicroRNA relative quantification between samples using small RNA-seq was found to be accurate and reproducible across laboratories and methods.

Key words: RNA-seq, small RNAs, microRNAs, exRNAs, accuracy, bias, reproducibility, RNA editing 


\section{Introduction}

RNA-seq has transformed transcriptome characterization in a wide range of biological contexts $^{1,2}$. RNA-seq can be used to sequence long reads (long RNA-seq; for example, messenger RNAs and long non- coding RNAs) and short RNAs (small RNA-seq; for example, small non-coding RNAs such as microRNAs). These applications differ in terms of the size of the targeted RNAs and by the technical methods used and the resulting biases in the quantitative data that are produced ${ }^{3}$. For example, preparation of libraries for long RNA-seq, by virtue of having sufficiently long target RNA lengths, commonly utilizes primers for direct generation of cDNA from RNA. In contrast, small RNA-seq library preparation methods typically require RNA ligation or poly-A tailing steps to overcome the challenge of performing reverse transcription and subsequent PCR amplification from extremely short (for example, 16-30 nt) target RNA sequences. Multiple approaches have been developed to overcome the challenge of uniformly and robustly generating cDNA from small RNAs for the purpose of small RNA-seq ${ }^{4-9}$. Protocols in use for small RNA-seq therefore vary more widely than those used for long RNA-seq, creating greater potential for variation from different library preparation protocols and different labs. In addition, small RNA-seq is increasingly used to study samples with very low RNA concentration, such as biofluids containing exosomes, other extracellular vesicles (EV) ${ }^{10-16}$ and RNA-protein complexes ${ }^{17-21}$. Normalization methods $\mathrm{s}^{22-24}$ that have been developed to correct for variation in long RNA-seq data are typically not well-suited for small RNA-seq data. Although performance characteristics such as reproducibility and quantitative accuracy have been well-studied for long RNA-seq ${ }^{25,26}$, only the reproducibility of a single library preparation protocol has been evaluated for small RNA-seq ${ }^{25}$.

Furthermore, the performance of different small RNA-seq methods for quantifying single-nucleotide changes in RNA sequence, such as those seen with microRNA (miRNA) editing, for example, has not been systematically examined. Yet, with the rapid accumulation of small RNA-seq data (such as, the US National Institutes of Health (NIH) short-reads archive ${ }^{27,28}$, EV-associated small RNA sequencing databases ${ }^{29-31}$, The Cancer Genome Atlas (TGCA) TCGA ${ }^{32}$, the exRNA Atlas ${ }^{33}$, etc.), meaningful, quantitative interpretation of results, especially across studies, would benefit from a systematic examination of technical bias, its effects on accuracy and of the reproducibility of small RNA-seq.

Here we report the results of a study led by investigators from the $\mathrm{NIH}$-funded Extracellular RNA Communication Consortium ${ }^{34}$ involving nine laboratories, in which a systematic multi-protocol, multi-institution assessment was carried out to assess the accuracy, reproducibility and technical bias of small RNA-seq using standardized, synthetic reference reagents as well as biologically derived reference RNA. We also 
evaluated the performance of different protocols with respect to characterizing miRNA editing and identified a library preparation approach that reduces technical bias and improves the accuracy and comparability of small RNA-seq results.

\section{Results}

\section{Study design and standard reference materials for miRNA quantification}

To evaluate the performance of small RNA-seq library preparation protocols across multiple laboratories, we developed standard reference samples as well as a standardized study design (Fig. 1). We distributed detailed instructions for library preparation and sequencing to each lab, along with four reference RNA samples (Fig. 1 and Supplementary Tables 1 and 2): one equimolar pool comprising 1,152 synthetic RNA oligonucleotides, corresponding predominantly to human miRNA sequences, as well as a small set of non-miRNA oligonucleotides of varied sequence and length (15-90 nt); two synthetic small RNA pools, called ratiometric pools SynthA and SynthB, each containing the same 334 synthetic RNAs, but in which subsets of RNAs vary in relative amount between pools $A$ and $B$ by 15 different ratios, ranging from 10:1 to 1:10; and an RNA pool isolated from human blood plasma from 11 individuals.

The common materials were distributed to nine participating research groups (L.C. Laurent, University of California at San Diego; D.J. Erle, University of California at San Francisco; I. Ghiran/ Y.E. Wang, Beth Israel Deaconess Medical Center/Dana-Farber Cancer Institute (BIDMC/DFCI); E.N.M. Nolte-'t Hoen, UUTR; J.E. Freedman, University of Massachusetts; K.Wang, Institute for System Biology (ISB); D.J. Galas, Pacific Northwest Research Institute (PNRI); K. Van Keuren-Jensen, TGen; M. Tewari, University of Michigan). Nine library preparation protocols were evaluated (Online Methods), in which at least one group prepared and sequenced quadruplicate libraries from each of the reference samples. Three of the protocols, TruSeq (Illumina), NEBNext (New England BioLabs) and CleanTag (Trilink Biotech), are commercial kits that employ adapters with invariant sequences. The remaining protocols make use of adapters with four degenerate nucleotides at the ligation ends as a strategy to reduce the bias, and we collectively refer to these as ' $4 \mathrm{~N}$ ' protocols. These six $4 \mathrm{~N}$ protocols included: a commercial kit, NEXTflex (Bioo Scientific); a recently published protocol (4N_Xu) ${ }^{35}$; and four variants of a protocol developed by members of the consortium (protocols 4N_A, 4N_B, 4N_C and 4N_D) that we collectively refer to as 'inhouse' 4N methods. The TruSeq kit served as the common reference kit for this study and was evaluated by all the groups using Illumina sequencing platforms (eight of nine groups). In addition, multiple laboratories generated libraries using the NEBNext kit (six labs) and 
the in-house protocol 4N_B (four labs), thereby allowing for standardized cross-lab comparisons for these two protocols, as well as to the Illumina TruSeq protocol.
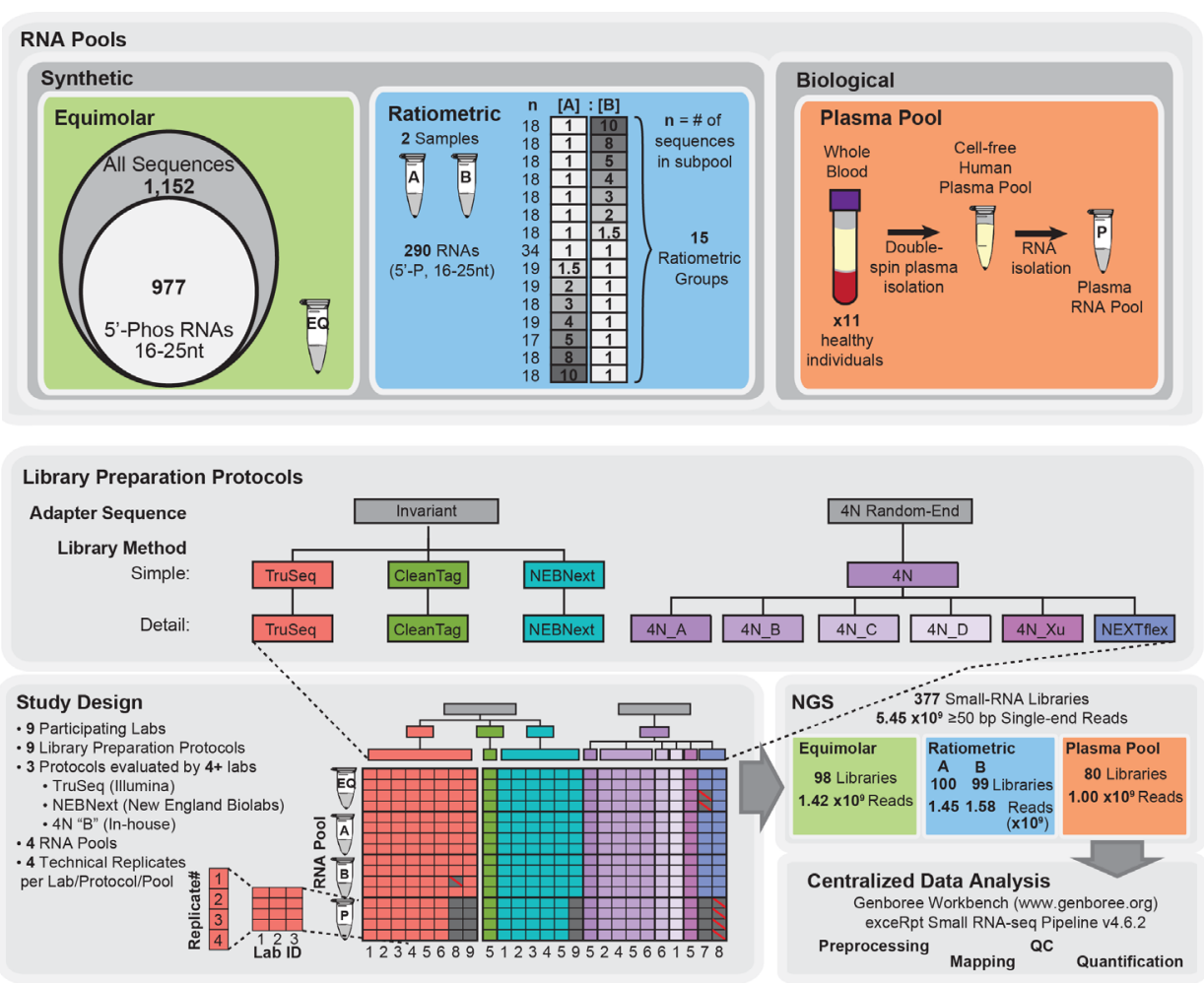

Figure 1. Overview of study design. Top, the four primary RNA pools used as common reference samples in the study are shown. Equimolar and ratiometric pools were prepared using chemically synthesized RNA oligonucleotides to establish 'ground-truth' knowledge of absolute and relative abundances, respectively. The equimolar pool consisted of 1,152 synthetic RNAs (15-90 nt) mixed at equal concentration. Downstream analyses focused on the subset of 977 16-25-nt-long RNAs with 5'-phosphorylation. The two ratiometric pools, A and B, consisted of 334 synthetic RNAs, in which subsets of RNAs were varied in relative abundance between the two pools. The RNAs were divided into 15 ratiometric subgroups. The subset of 290 16-25-nt-long RNAs were used for downstream analyses, and the number of RNAs in each ratiometric subgroup is indicated. The plasma RNA pool comprised RNA from 11 healthy males that was centrally isolated and distributed to the participating labs. Middle, nine different library preparation protocols were tested. Three commercially available kits with 'invariant' adapters and six $4 \mathrm{~N}$ degenerate-end protocols were tested. Bottom, common reference RNA pools were distributed to nine participating labs for sequencing in quadruplicate, using a standardized common protocol (TruSeq) and at least one additional method of their choice. The breakdown of the resulting libraries is depicted in the colored grid, with the Lab IDs indicated by columns, and the replicates and pools shown in rows. Solid gray blocks indicate libraries that were not attempted. Gray blocks with a diagonal red line indicate samples in which library preparation and sequencing were attempted, but were unsuccessful. Sequencing was performed by each lab independently. 
In total, the nine participating groups prepared 384 libraries for miRNA quantification analysis, of which 377 (98\%) were successfully sequenced and submitted for central analysis. The seven libraries that were not successfully prepared and sequenced included four plasma pool libraries (Lab8 4N_NEXTflex), two equimolar pool libraries (Lab7 NEXTflex) and one SynthB library (Lab8 TruSeq). Together, the nine participating groups collectively contributed 5.45 billion small RNA-seq reads to the analysis (Fig. 1). These sequencing data were centrally analyzed using the Genboree Workbench and its implementation of the Extracellular RNA Communication Consortium's exceRpt Small RNA-seq pipeline, which is specifically designed for the analysis of small RNA-seq data and uses its own alignment and quantification engine to map and quantify a range of RNAs represented in small RNA-seq data (see Supplementary Table $\mathbf{3}$ for pipeline quality control (QC) metrics). Of the 377 samples analyzed, 364 (>96\%) satisfied the minimum quality criteria (Online Methods) and were included in the analyses.

\section{Characterization of sequence-specific bias of small RNA-seq protocols}

Of the 1,152 synthetic RNAs in our equimolar pool, we focused on 9775'-phosphorylated 16-25-nt-long RNAs, which can be captured with standard small RNA-seq protocols. The efficiency of recovery of RNA sequences varied by multiple orders of magnitude depending on the protocol, confirming that small RNA-seq protocols are associated with prominent sequence-dependent $\operatorname{bias}^{4,25,36-38}(\boldsymbol{F i g . ~ 2 a , b})$ and that the bias is greater than that in long RNA-seq ${ }^{26}$. This was highly reproducible in a given protocol, both across technical replicates and laboratories using the same protocol (Fig. 2a). Libraries prepared by different labs clustered first into two groups, corresponding to methods with invariant (TruSeq, NEBNext and CleanTag) or degenerate (4N) adapters. In each of these two larger groups, the libraries then formed distinct clusters corresponding to the different protocols included in the study, indicating that the effect of the protocol bias is potentially greater than that of lab-to-lab variation. Consistent with this result, the ten most overrepresented and underrepresented sequences varied widely between protocols (Supplementary Fig. 1).

Although all of the protocols exhibited some bias, it was reduced in those using degenerate adapters (Fig. $\mathbf{2 b}$ and Supplementary Fig. 2). As one measure of this, we calculated the median percentage of sequences with a number of reads in counts per million (CPM) more than ten times above or below the expected value, for each protocol. This ranged from 41.6 to $61.5 \%$ for protocols using adapters with defined sequences (TruSeq, 41.6\%; CleanTag, 53.9\%; NEBNext, 61.5\%), and from 2.8 to $22.4 \%$ for protocols using adapters with degenerate nucleotides (4N_A, 8.9\%; 4N_B, 2.8\%; 4N_C, 12.1\%; 4N_D, 22.4\%; 4N_Xu, 7.1\%; 4N_NEXTflex, 17\%) (Fig. 2c). The in-house 4N protocols showed fewer missing sequences from the equimolar pool (Supplementary 
a
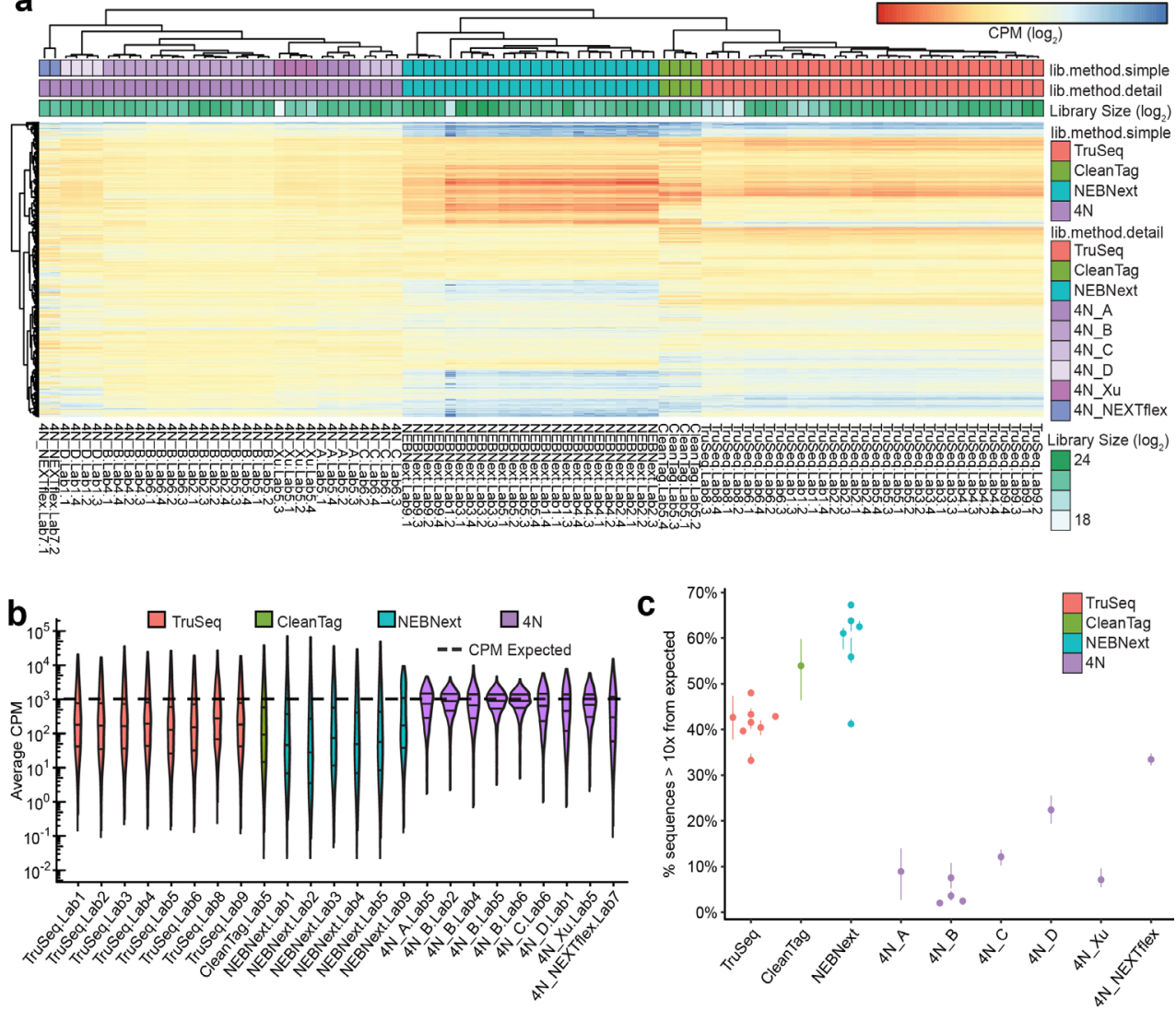

Figure 2. Equimolar pool sequencing results across multiple labs and protocols. (a) The heatmap shows expression levels for each synthetic RNA sequence (rows) across all replicate equimolar pool libraries (columns). Expression levels represent log2-scaled CPM calculated for 977 equimolar pool sequences, 16-25 nt in length and 5'-phosphorylated. Hierarchical clustering for rows and columns represents complete linkage clustering on Euclidean distances (the default setting for the $\mathrm{R}$ package, pheatmap, used for plotting). Columns are labeled at the bottom to identify replicate samples. Library size indicates the sequencing depth for each library (log2 scaled). (b) Violin plots summarize the mean CPM observed for each of the 16-25-nt, 5'-phosphorylated equimolar pool sequences (y axis, $\log 10$ scaled), as measured from equimolar pool libraries prepared by different institutions and using different library preparation protocols ( $\mathrm{x}$ axis). The width of the violins is proportional to the density of data points at each position. The horizontal lines in each violin represent the 25th, 50th and 75 th percentiles. The dashed horizontal line shows the expected CPM for each sequence in the equimolar pool (106 / 977 miRNAs = 1,023.5 CPM). Each violin plot and corresponding quantile lines summarize mean CPM values for $\mathrm{n}=977$ distinct equimolar pool sequences. The mean CPM values were calculated from $\mathrm{n}$ $=4$ technical replicate libraries for each lab/library preparation method shown, with the exception of $4 N \_N E X T f l e x . L a b 7(n=2)$. (c) The percentage of equimolar pool sequences sequenced at levels 10x higher ( $>10,235$ CPM) or 10x lower ( $<102.35$ CPM) than expected (y axis) are plotted for each lab. The dots and whiskers indicate the median and range of values, respectively, measured across the technical replicates for each lab. $n=4$ technical replicate libraries per lab/library preparation method, with the exception of $4 \mathrm{~N} \_$NEXTflex $(n=2)$. 
Table 4) and when downsampling to compare the same number of total mapped reads across protocols at varying sequencing depths (Supplementary Fig. 3). We found that, with the in-house 4N_B protocol, even when downsampling to 10,000 total mapped reads, $>90 \%$ of the miRNAs had a high probability of detection (median, 92\%; range, 78-95\%). In contrast, even with the best-performing invariant adapter protocol, TruSeq, $<50 \%$ of miRNAs had a high probability of detection (median, $46 \%$; range, $40-55 \%)$ at the same depth, indicating that the $4 \mathrm{~N} \_B$ protocol may require lower read depth to yield similar coverage as other library protocols.

We also assessed the reproducibility of small RNA cloning biases across labs by examining the rank-order of RNA sequence abundance. To do so, we calculated Spearman rank correlations for the equimolar synthetic pool counts between labs and protocols. As expected, the strongest correlations were found between technical replicates from the same lab and method (Supplementary Fig. 4). Correlations were also strong between samples generated by different labs using the same protocol (Supplementary Table 5). The somewhat lower correlation value observed for 4N_B (combined Rho value: $84 \%$; top/bottom 2\%: 0.66/0.95) can be attributed to the overall lower variation in read counts across miRNAs resulting from less cloning bias with this protocol. The reduced spread in the data limits the maximum absolute correlation coefficient values that can be obtained. This limitation notwithstanding, comparison across labs using different protocols showed much weaker correlations (Supplementary Table 5).

To dissect the source of observed bias, we evaluated the effect of several variables (5' or $3^{\prime}$ terminal bases, \%GC of the four $5^{\prime}$ or $3^{\prime}$ end bases, overall \%GC, dG [free energy], dH [enthalpy], dS [entropy] and Tm [melting temperature]) on the number of obtained reads with different library preparation protocols (Supplementary Figs. 5-13). However, none of these variables substantially explained the observed bias.

\section{Accuracy and cross-protocol concordance for relative quantification}

To investigate the accuracy of relative quantification of the same small RNAs between different samples, we designed two ratiometric pools, SynthA and SynthB, each containing the same 334 synthetic RNA sequences, but varying the relative abundance of sequences between the two pools for 15 expression ratios (Fig. 1 and Supplementary Table 2). All of the protocols that were tested showed close concordance between observed and expected ratios (Fig. 3a). We also analyzed the data using standard differential expression workflows from three commonly-used $\mathrm{R}$ packages (Edge $\mathrm{R}^{39,40}, \mathrm{DESeq} 2^{41}$ and limma/voom ${ }^{42}$ ) to determine the smallest difference in abundance that could be distinguished using small RNA-seq methods. We observed that for most protocols and for the majority of miRNAs, a difference in levels of as little 

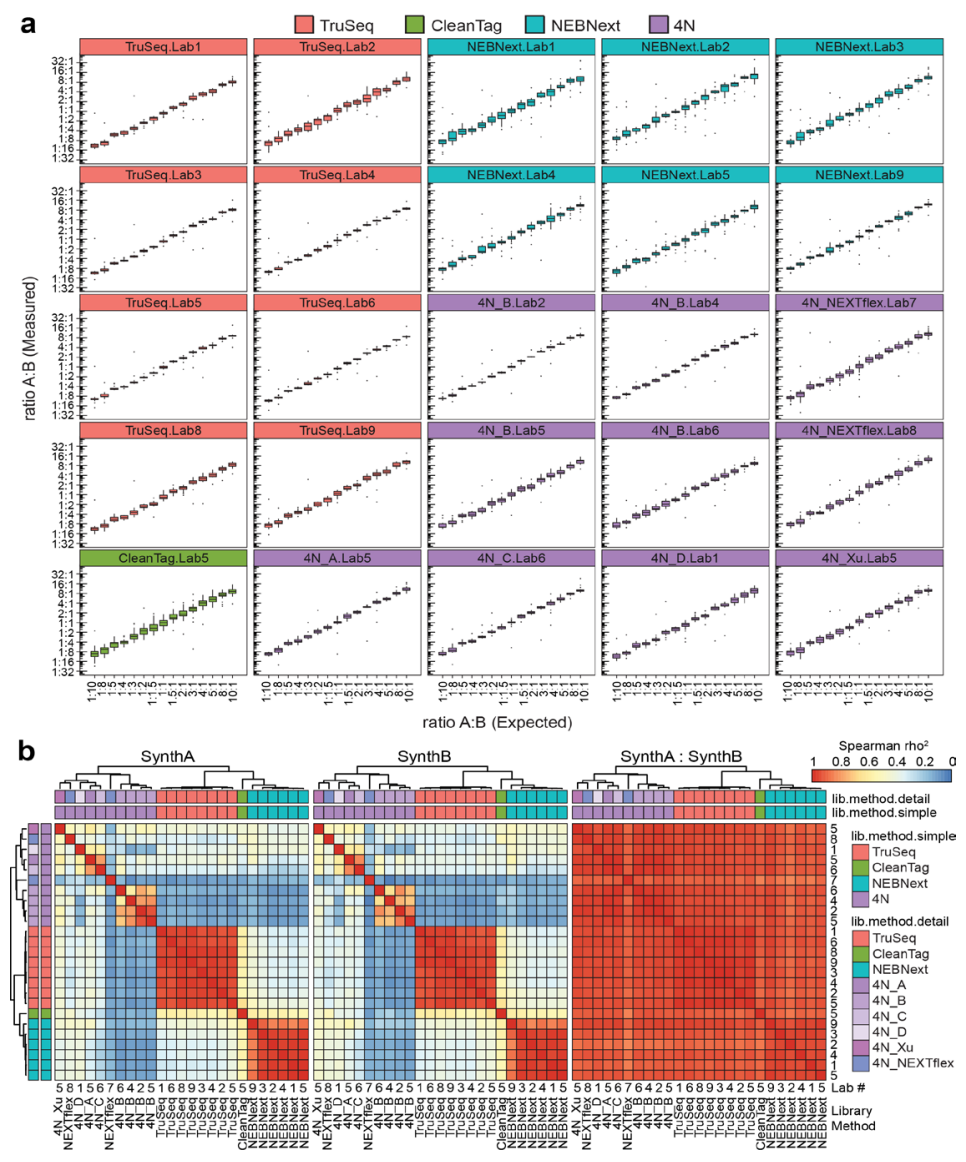

Figure 3. Small RNA-seq accuracy and cross-protocol concordance in measuring relative expression levels between samples. (a) Boxplots show the observed ratio ( $y$ axis; log2 scale) versus expected ratio ( $x$ axis) for miRNAs present in each of the SynthA and SynthB synthetic RNA subpools. Observed ratios for each miRNA were calculated as mean CPM of SynthA / mean CPM of SynthB across technical replicates for each lab and library prep method. Boxes show the median + IQR; upper/lower whiskers indicate the smallest/largest observation less than or equal to $1 \mathrm{st} / 3 \mathrm{rd}$ quartile $\pm 1.5^{*} \mathrm{IQR}$; outliers are calculated as being $<1$ st quartile $-1.5^{\text {* }}$ $I Q R$ or $>3$ rd quartile +1.5 * IQR. Mean CPM ratios were calculated from $n=4$ SynthA and $n=4$ SynthB technical replicate libraries for each lab and library preparation method shown, with the exception of TruSeq Lab8 SynthB $(n=3)$. Those miRNAs with a mean CPM of 0 in SynthA or SynthB are not plotted. The numbers of miRNA not plotted are as follows: Truseq Labs, 1, 2, 3, 4, 5, 6 and 8: 1; Lab9: 0; CleanTag Lab5: 0; NEBNext Labs, 1, 3, 5 and 9: 0; Lab4: 1; Lab2: 3; 4N_NEXTflex Lab7: 1; other 4N: 0. The number of sequences represented in each boxplot is provided in Supplementary Table 10. (b) Heatmaps show the pairwise, squared Spearman rank correlation coefficients from sequencing the SynthA and SynthB pools. Pairwise correlation coefficients were calculated on the basis of the mean CPM across technical replicates for SynthA samples (left), SynthB samples (middle) and the ratio of SynthA: SynthB (right). The mean CPM value for each ratiometric pool sequence was calculated from $n=4$ technical replicate libraries per lab, library preparation method and pool. Mean CPM values for $n=290$ ratiometric pool RNAs were used for calculating each pairwise correlation coefficient. Hierarchical clustering for rows and columns is the same for all heatmaps, and is based on the average pairwise Euclidean distances calculated from the SynthA CPM and SynthB CPM correlation matrices. Column labels indicate the lab ID and library prep method; row labels indicate only lab ID, but are presented in the same order (top to bottom) as columns (left to right). 
as 1.5-fold between the two samples could be detected (Supplementary Fig. 14). As shown in Supplementary Table 6, all of the evaluated protocols performed relatively well in detecting miRNA abundances.

We examined the rank-order of RNA sequence abundance and found that, in general, the Spearman rank correlations results obtained for the SynthA and SynthB samples were similar to those obtained for the equimolar pool: the correlation was strong when using the same protocol, but weaker across different protocols (Fig. $\mathbf{3 b}$ ). In contrast, when we analyzed the concordance of the SynthA/SynthB ratios (Fig. $\mathbf{3 b}$ ), we found a very strong correlation between labs not only when using the same protocol, but also across different protocols, confirming that relative quantification is resilient to variation in the protocol used (Supplementary Table 5).

\section{Reproducibility of small RNA-seq protocols}

To quantify intra-lab variation for each sequence, we used two metrics: the coefficient of variation (CV, 100* s.d./mean) and the quartile coefficient of dispersion (QCD, interquartile range/ average of the first and third quartile). The median CV for the equimolar pool libraries ranged from 6.18\% (TruSeq) to 23.92\% (CleanTag) for the different library preparation methods (Fig. $4 a$ and Supplementary Table 5). In addition, the median QCD was $<0.1$ for all the protocols/labs (Fig. 4a and Supplementary Table 5). We also evaluated the intra-lab variation from technical replicates of sequencing the SynthA and SynthB libraries. The calculated CV and QCD values were similar to those observed for the equimolar libraries (Supplementary Fig. 15).

To characterize the reproducibility of small RNA-seq libraries across laboratories, we focused on the three protocols (TruSeq, NEBNext and 4N_B) for which libraries were generated by at least three groups. In addition, of the six labs that generated libraries using the NEBNext protocol, two of the labs used somewhat modified conditions based on options provided by the manufacturer and were excluded from the analysis (Online Methods).

Using the results for the equimolar pool and treating each laboratory's results as one trial of the experiment, we calculated the CV and QCD for the mean CPM values for each RNA sequence across laboratories. The median CV across labs ranged from 30.42 (4N_B) to $35.28 \%$ (NEBNext) and the median QCD from 0.13 (4N_B) to 0.18 (TruSeq and NEBNext) (Fig. $\mathbf{4 b}$ and Supplementary Table 5). We confirmed that the choice of pseudo-counts for calculating CPM did not appreciably alter the CV and QCD distribution (Supplementary Fig. 16). In addition, repeating the inter-lab CV and QCD calculations using all combinations of $n=3$ labs from the TruSeq, NEBNext and 4N_B equimolar pool libraries showed that results from analysis of subsets of the data were comparable to those from analysis of the full data sets (Supplementary Fig. 17a,b). 
We also calculated across lab variation for the SynthA and SynthB pools individually and obtained median CV and QCD values that were comparable to those described for the equimolar libraries (Supplementary Table 5).
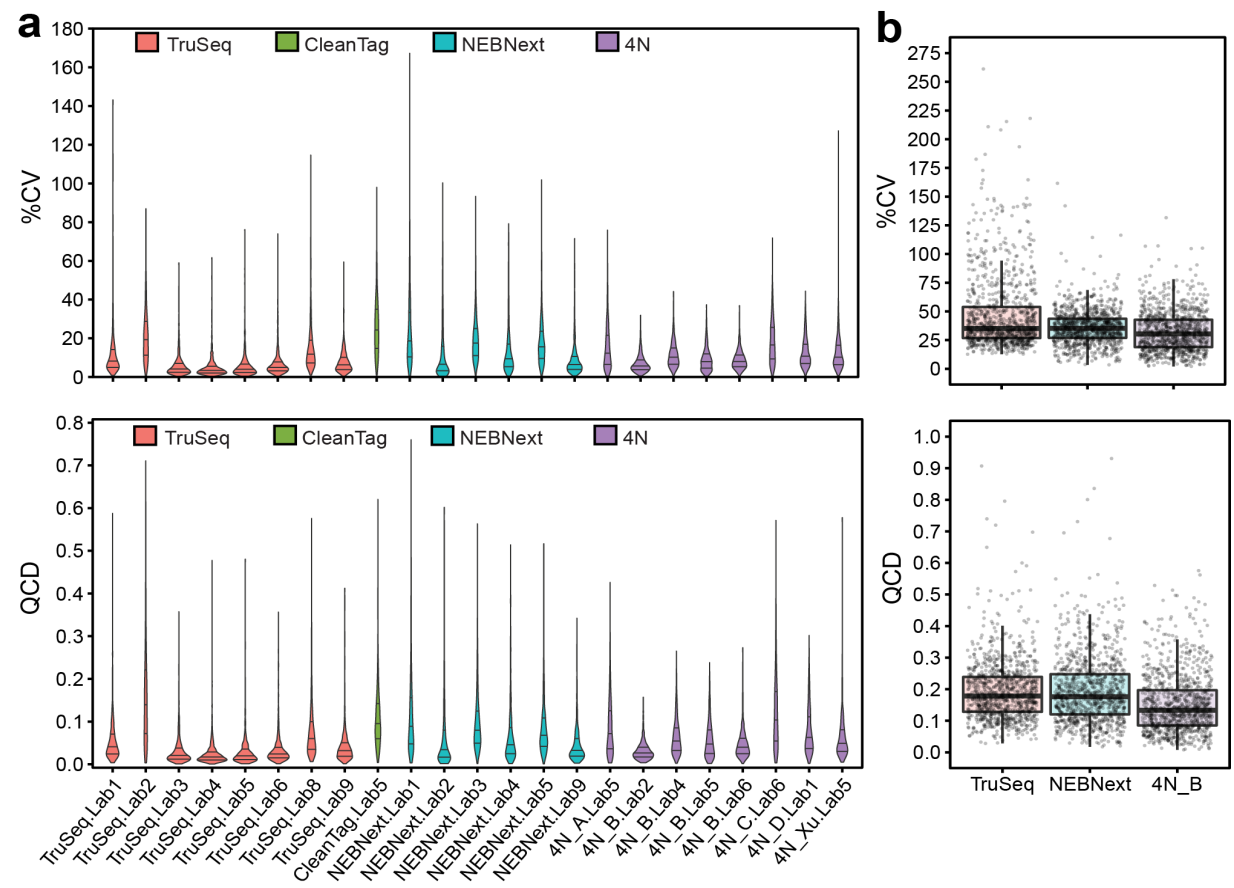

Figure 4. Reproducibility of small RNA-seq within and between labs. (a) Violin plots summarize the technical reproducibility of quantification for all equimolar pool sequences, as calculated from each lab and library preparation method. Reproducibility measurements, CV (top) and QCD (bottom) were calculated from CPM values. Horizontal lines in each violin indicate the 25th, 50th and 75th percentiles, calculated from the mean CPM values of $n=977$ equimolar pool RNA sequences. Mean CPM values were calculated from $n=4$ technical replicate libraries for each of the lab/library preparation methods shown, with the exception of TruSeq Lab1 $(n=3)$. (b) Boxplots summarize the sequence-specific reproducibility of quantification measured in equimolar pool libraries generated by different labs using the same protocol. CV (top) and QCD (bottom) values were calculated for each equimolar pool sequence across TruSeq ( $n=8$ labs), NEBNext $(\mathrm{n}=4$ labs) and 4N_B ( $\mathrm{n}=4$ labs) library preparation protocols. The mean CPM for each sequence across technical replicates ( $n=4$ technical replicates per lab/ library preparation method) was used to calculate the between-lab CV and QCD plotted here. Boxplot statistics and outliers were calculated from $C V$ or $Q C D$ values for $n=977$ equimolar pool sequences. The overlaid boxes indicate the median and IQR. Whiskers represent the $1 \mathrm{st} / 3 \mathrm{rd}$ quartile $\pm 1.5^{*} \mathrm{IQR}$. Outliers are $<1 \mathrm{st}$ quartile -1.5 * $I Q R$ or $>3$ rd quartile +1.5 * $I Q R$. 

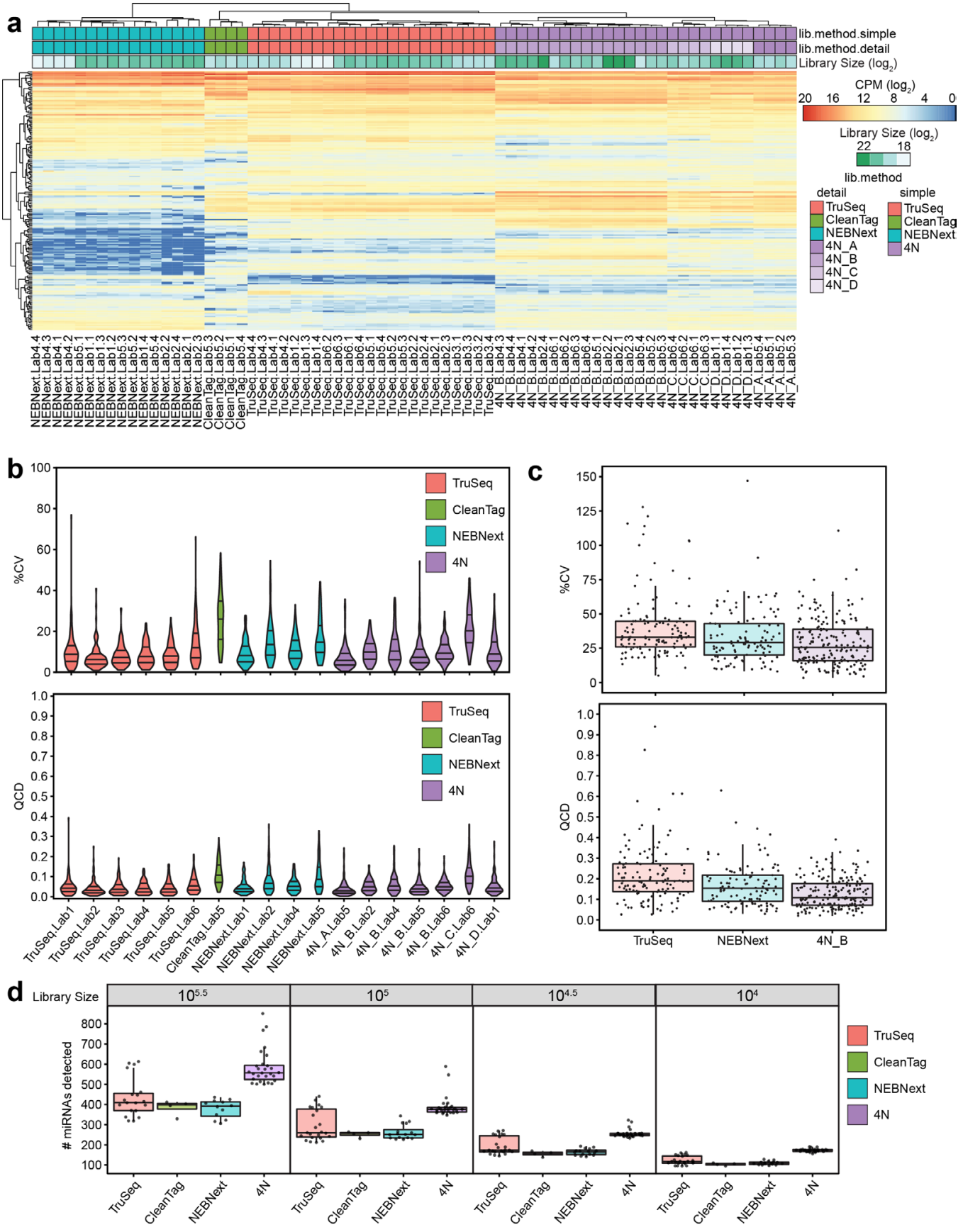
Figure 5. Small RNA-seq of reference plasma RNA by multiple laboratories using multiple library preparation protocols. (a) The heatmap shows CPM (log2 scale) for each sequence (rows) across plasma pool libraries (columns). Only mature miRNAs with a high confidence of detection are shown, requiring a minimum of 100 CPM in $90 \%$ of samples from at least one protocol (TruSeq, CleanTag, NEBNext or $4 \mathrm{~N}$ ). Hierarchical clustering for rows and columns represents complete linkage clustering on Euclidean distances. Library size indicates the sum of the mature miRNA-mapped read counts before filtering for the individual libraries (log2 scaled). (b) Violin plots summarize the technical reproducibility of quantification for miRNAs expressed in plasma pool libraries, as calculated from each lab and library preparation method. Reproducibility measurements, percent CV (top) and QCD (bottom), were calculated from CPM values. Horizontal lines within each violin indicate the 25,50 and 75 th percentiles, calculated from the mean CPM values of $n=977$ equimolar pool RNA sequences. For TruSeq Lab1 $n=3$ technical replicates; for all other lab/protocols $n=4$. (c) Boxplots summarize the between-lab reproducibility for miRNAs expressed in the plasma pool libraries using TruSeq ( $n=6$ labs), NEBNext ( $n=4$ labs) and 4N_B $(n=4$ labs) library preparation protocols. Each dot represents $C V$ or QCD calculated across labs for a single miRNA. The between-lab CV and QCD were calculated using the mean CPMs for each sequence across technical replicates for each lab. The underlying boxes show the median and IQR. Whiskers represent the $1 \mathrm{st} / 3$ rd quartile \pm 1.5 * IQR. Outliers are $<1$ st quartile -1.5 * IQR or $>3$ rd quartile $+1.5 *$ IQR. (d) Boxplots show the number of mature miRNAs detected by each protocol based on downsampling of data sets to the indicated sequencing depths. Each box summarizes number of miRNAs detected by each lab for the indicated protocol. The probability of each miRNA being detected was estimated for every sample randomly downsampled to $10^{4}, 10^{4.5}$, $10^{5}$ or $10^{5.5}$ total read counts. A miRNA was only counted as detected if the probability of detection was at least $90 \%$. Libraries with total counts less than the indicated sample size were excluded. Boxplots for $4 \mathrm{~N}$ include only in-house $4 \mathrm{~N}$ protocols (4N_A, 4N_B, 4N_C and 4N_D). The number of libraries summarized by each boxplot is as follows: 105.5 : TruSeq $=19$; CleanTag $=4$; NEBNext $=12 ; 4 \mathrm{~N}=28 ; 105,104.5$ and 104: TruSeq $=23 ;$ CleanTag $=4 ;$ NEBNext $=16 ; 4 \mathrm{~N}=28$. The underlying boxes show the median and IQR. Whiskers represent the $1 \mathrm{st} / 3 \mathrm{rd}$ quartile \pm 1.5 * IQR. Outliers are $<1$ st quartile $-1.5 *$ IQR or $>3$ rd quartile +1.5 * IQR.

\section{Performance of small RNA-seq protocols using biological samples}

We also sought to characterize the performance of small RNA-seq protocols across labs using standard reference RNA derived from biological material to assess the reproducibility and the diversity of miRNA sequences recovered.

To perform this analysis, we shipped aliquots of RNA extracted from a pool of human blood plasma from 11 donors to the participating labs for sequencing in quadruplicate (Fig. 1). We focused our analysis on miRNAs because they are well-characterized and have been extensively studied in human plasma ${ }^{43}$. Hierarchical clustering generally mirrored that from the synthetic pools, with technical replicates of the same protocol clustering most closely together (Fig. 5a), and with samples also broadly clustering according to library preparation protocol.

To evaluate the intra-lab reproducibility of plasma small RNA-seq, we calculated the CV and QCD for individual miRNA sequences across technical replicates in each lab (Fig. 5b). After applying minimum CPM filtering criteria as before, to focus on 
reliably detected miRNAs (Online Methods), we found that the median CV across the miRNAs analyzed ranged from 7.7 (TruSeq) to $24.9 \%$ (CleanTag) for different protocols. Although this degree of reproducibility seems comparable to that observed with the synthetic reference pool RNA (Fig. 4 and Supplementary Table 5), it is important to note that the filtering criteria used for plasma sequencing data were different (and generally more stringent) than for the synthetic RNA sequencing data. In addition, the median QCD was $\leq 0.1$ for all the protocols (Supplementary Table 5).

Unsupervised clustering of the plasma miRNA expression data revealed clear groups separated by preparation protocol (TruSeq, NEBNext and 4N_B), with results obtained from different labs using the same protocol clustering together (Fig. 5a). The median variability across labs measured using CV ranged from 25.7 (4N_B) to $32.9 \%$ (TruSeq) and using QCD was $<0.3$ for all protocols. (Fig. $5 c$ and Supplementary Table 5). The overall reproducibility of small RNA-seq using RNA isolated from biological samples was therefore comparable to that observed using the synthetic reference RNA samples.

To assess differences between protocols in the diversity of miRNA sequences recovered from the standard reference plasma RNA, we performed an analysis of the number of miRNAs detected by each protocol in which we plotted data from different in-house $4 \mathrm{~N}$ protocols as one group for the sake of comparison. This was done using downsampled datasets so the same total number of mature miRNA-mapping reads could be compared across protocols, at varying sequencing depths. The in-house $4 \mathrm{~N}$ protocols recovered a larger number of miRNAs than those using defined adapter sequences (Fig. 5d). In addition, an indirect assessment of miRNA diversity (that is, percent of total reads accounted for by the ten most abundant miRNAs) was consistent with the conclusion that $4 \mathrm{~N}$ protocols generate a more diverse profile of miRNAs (Supplementary Fig. 18).

\section{Evaluation of small RNA-seq in miRNA A-to-I editing}

We extended our study to evaluate the performance of different protocols for quantifying sequences exhibiting adenosine to inosine (A-to-I) miRNA editing. This naturally occurring RNA modification can alter both miRNA biogenesis and regulatory functions ${ }^{44,45}$. We designed six synthetic RNA pools, each containing ten miRNAs that have previously been reported to undergo A-to-I editing ${ }^{46,47}$. Each pool combined the unedited $(\mathrm{A})$ and edited $(\mathrm{I})$ miRNA variants in different ratios (that is, 0, 0.1, 0.5, 5, 50 and $100 \%$ edited). Each of these mixtures was then combined with a background of 277 different, unedited human miRNAs to increase complexity in the pools (Fig. 6a and Supplementary Table 7). The six pools were sequenced by three different labs, each in triplicate, using TruSeq, NEBNext and in-house 4N_B protocols (Fig. 6a). The resulting 162 libraries yielded $1.42 \times 10^{9}$ reads aligned to editing pool sequences in total, with 
a median library size of $8.22 \times 10^{6}$ (range: $1.74 \times 10^{6}$ to $29.01 \times 10^{6}$ ). All 162 libraries satisfied minimum quality criteria (Online Methods and Supplementary Table 3).

To determine the accuracy of quantifying miRNA editing in our six synthetic pools, we compared the number of reads observed for the A and I variant oligos in each library with the expected abundance based on the known composition of the pools. Inaccurate and widely varying estimates of editing levels were apparent for many miRNAs using the NEBNext and TruSeq protocols, especially for the 1, 5 and $50 \%$ editing pools (Fig. $\mathbf{6 b}$ and Supplementary Table $\mathbf{8}$ ). In contrast, the in-house protocol, $4 \mathrm{~N} \_\mathrm{B}$, proved more accurate for detecting editing levels $\geq 1 \%$. For example, in the $50 \%$ editing pool, where the edited and unedited forms of each miRNA are present at equivalent levels, the mean percent editing observed ranged from 19-98\% and $5-95 \%$ for the TruSeq and NEBNext libraries, respectively, whereas estimates were all within $10 \%$ of the expected value (43-53\%) for the 4 N_B protocol.

Aside from accuracy, we calculated across-lab reproducibility (that is, precision) of the measured edited fraction in each pool for each of the evaluated protocols using CV and QCD, which are most meaningful where there are reads in both edited and unedited categories (Supplementary Table 8). We found that precision varied as a function of known percent editing, with greater precision being observed in the 5 and $50 \%$ edited pools compared with the 0.1 and $1 \%$ pools, as expected from the higher number of edited read counts in the former pools. Across all of the tested protocols, for the majority of miRNAs, the precision of percent editing measurements was CV $<5$ $\%$ in the $50 \%$ edited pool, $<20 \%$ in the $5 \%$ edited pool and $<25 \%$ in the $1 \%$ edited pool, and QCD $<0.3$ in the $50 \%$ edited pool, $<0.4$ in the $5 \%$ edited pool and $<0.6$ in the $1 \%$ edited pool.

We evaluated the specificity and limit of detection for identifying miRNA editing by downsampling each library to $10^{6}$ reads to allow standardized comparisons across libraries. To calculate specificity, we first estimated the false positive frequency for each protocol by evaluating: the average percent edited reads observed in the $0 \%$ edited pool (that is, false positive edited read frequency) and the average percent unedited reads observed in the $100 \%$ edited pool (that is, false positive unedited read frequency). The overall median false positive rate was $0.1 \%$ across all protocols, all miRNAs, and both edited and unedited false positive calls (median false positive frequencies for individual protocols: TruSeq, 0.05\% (edited) and $0.06 \%$ (unedited); NEBNext, $0.30 \%$ (edited) and $0.14 \%$ (unedited); 4 N_B, $0.10 \%$ (edited) and $0.08 \%$ (unedited); Supplementary Table 8). This corresponds to an overall specificity across all three protocols of $99.88 \%$ for calling unedited sequences and $99.91 \%$ for calling edited sequences (Supplementary Table 8). To calculate the limit of detection (LOD), we defined detection of editing as an observed edited count that is more than 3 s.d. above the observed edited count in the $0 \%$ edited synthetic pool. For all three protocols, 
the majority of miRNAs had a limit of detection at or below the $1 \%$ edited fraction, with a few miRNAs being detectable in the $0.1 \%$ edited pool (Supplementary Table 8). It is worth noting, however, that the LOD is expected to vary based on sequencing depth, sample complexity, relative abundance of the miRNA being studied and the pipeline used for analysis.

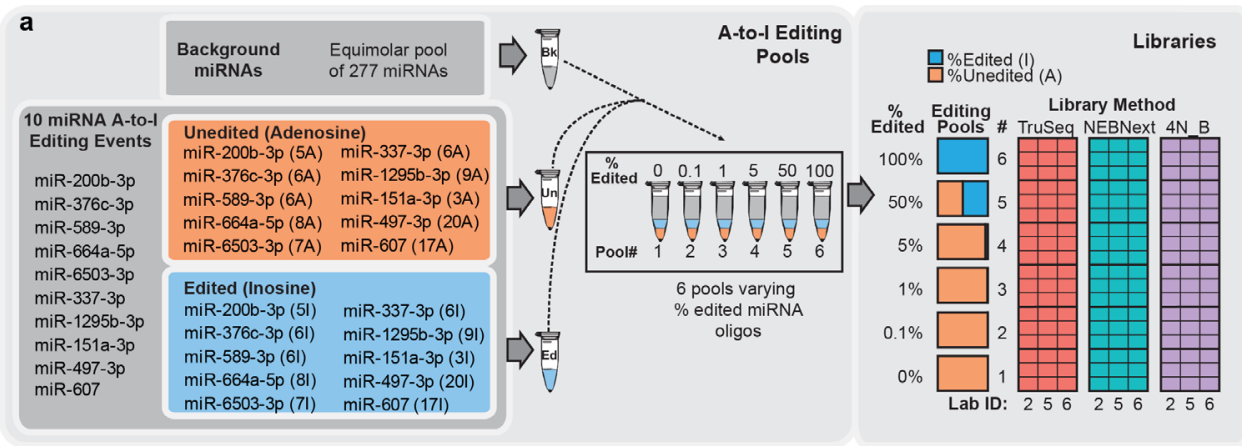

b
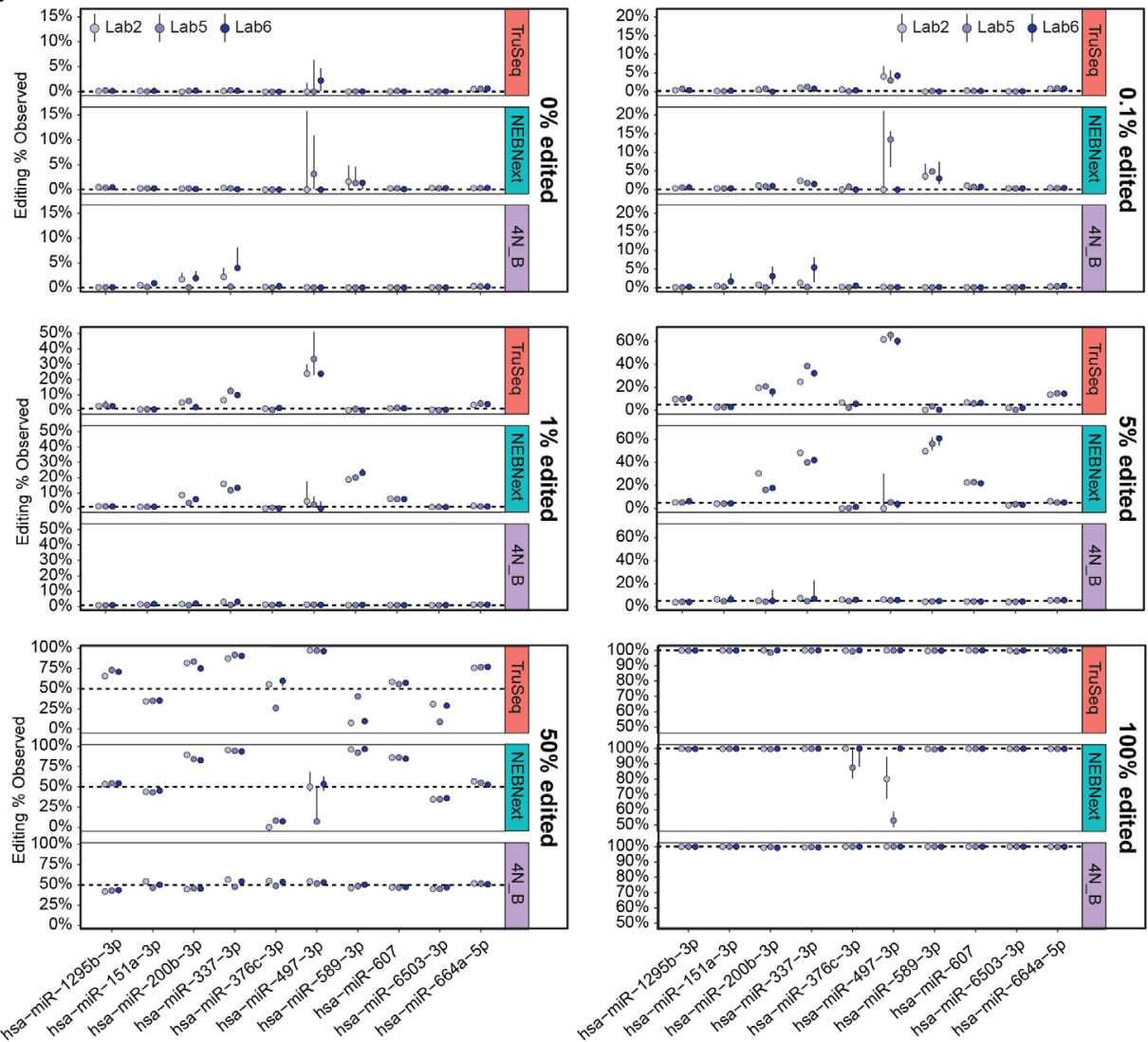
Figure 6. Library protocol performance in measuring miRNA A-to-I editing events. (a) A schematic depicting the experimental design for the miRNA A-to-I editing experiments. Left, ten miRNAs were synthesized with either an adenosine or inosine at a single position previously shown to be edited in human cells. The position, relative to the $5^{\prime}$ end of the mature miRNA, is indicated to the right of the respective miRNA IDs, along with the identity of the nucleotide. 277 other unedited human miRNAs were added at a fixed concentration to increase the background complexity of the pools. Middle, six different editing subpools were generated, using a constant amount of the background (Bk) pool and varying percentages of unedited (Un; adenosine) and edited (Ed; inosine) oligos in each pool. Right, the color-coded grid depicts the library design used in the A-to-I editing experiment. Specifically, the six editing pools were sequenced by three participating labs, using three different library preparation protocols, with each lab generating libraries in triplicate. (b) The observed percent editing ( $y$ axis) is shown for each miRNA in the six A-to-I editing pools, as measured by each of the three labs, using TruSeq, NEBNext and 4N_B protocols. The expected editing percent in each pool is both indicated to the right of each plot group and by the horizontal dotted line in each plot. The dots and whiskers represent the median and range of percent editing for each miRNA ( $x$ axis) as measured by the three labs. Individual miRNA percentage editing is shown for $n=3$ technical replicate libraries for each lab and library preparation method.

\section{Discussion}

Our results quantitatively confirm that small RNA-seq is highly affected by sequencerelated bias b $36-38,48$, which is largely protocol dependent. The observed biases were as large as $10^{4}$-fold with some commonly used commercial library preparation protocols. This sequence-dependent bias is more severe than that previously reported for long RNA-seq ${ }^{26}$, highlighting differences between the technologies and unique challenges involved in small RNA sequencing. In addition, this bias can be particularly vexing when working with low RNA input samples such as biofluids, preventing the reliable detection of some low-abundance small RNAs. The in-house $4 \mathrm{~N}$ protocols that we evaluated, which employ adapters containing degenerate bases in the ligating ends, reduced the bias on the order of 100-fold and achieved better coverage at a lower sequencing depth than the widely used commercial library preparation kits with invariant adapter sequences. The magnitude of the bias observed for some sequences when using fixed adapter protocols was so high that it is unlikely to be overcome simply by increasing sequencing depth. There were, however, differences in the results of different $4 \mathrm{~N}$ methods, suggesting that not only the use of adapters with degenerate bases, but also other factors in the protocols, such as the concentration of polyethylene glycol in ligation reactions, the time and temperature of ligations, etc., may also affect the bias. Our computational analyses of a range of sequencerelated variables (for example, $5^{\prime}$ or $3^{\prime}$ terminal nucleotides, \%GC of the four $5^{\prime}$ or $3^{\prime}$ end nucleotides, overall \%GC, dG [free energy], dH [enthalpy], dS [entropy] and Tm [melting temperature]) did not reveal strong associations, suggesting that the mechanistic basis of the bias may be complex. 
Even using the best-performing $4 \mathrm{~N}$ protocols, there is still considerable sequencerelated bias, which precludes the use of read counts alone for accurate quantification of different small RNAs in a given sample. However, despite the observed biases, we found that small RNA-seq was consistently accurate for relative quantification of a given miRNA between samples, as long as the same library preparation protocol was used for the two samples being compared, which is consistent with previous observations for mRNA sequencing ${ }^{26}$. In this sense, all of the evaluated protocols were able to distinguish samples with as little as a 1.5 -fold difference in relative abundance of most sequences examined, although the design of our ratiometric pools was such that differences smaller than 1.5 -fold could not be assessed. Reproducibility across laboratories is a crucial requirement for any experimental method used for research or clinical applications ${ }^{49,50}$. We found that for common commercial protocols as well as for our in-house $4 \mathrm{~N}$ protocol, results were reproducible between labs with a CV $\leq 20 \%$ for most sequences. Moreover, when comparing relative quantification measurements obtained by small RNA-seq across labs, the results were highly concordant even when the centers were using different protocols.

The use of a diverse pool of synthetic RNAs allowed us uniquely to evaluate sequence-specific biases and accuracy because the 'ground truth' is known. Since biological material, with a wide range of RNA species and other macromolecules, could behave differently from the synthetic RNA pools, we also characterized the diversity of miRNAs captured in a common biological sample by each protocol. We found that the in-house $4 \mathrm{~N}$ protocols detected a greater diversity of sequences than protocols using defined-sequence adapters. In addition, for a given protocol, the profile obtained for the biological sample was very reproducible between laboratories.

We believe that the data sets generated in this study can also serve as a valuable resource for benchmarking computational tools designed to facilitate and improve on RNA-seq analysis. This is important both for developing new software and for evaluating the suitability of using existing mRNA-seq algorithms for the analysis of small RNA-seq data sets. This could be particularly useful for benchmarking software developed to account for various technical biases found in mRNA-seq data ${ }^{51-54}$, as our data suggest that such biases may be different in small RNA-seq data.

We also hope that our data may facilitate the development of computational approaches for normalization of data sets generated using different library preparation protocols. Although normalization algorithms are generally not intended to account for cross-platform variation, our preliminary analysis suggests that small RNA-seq protocol-specific biases largely correlate across samples. This suggests that one may be able to account for the protocol-specific differences in sequencing bias individually for each sequence, raising the possibility of cross-protocol data normalization. We performed an initial exploration of this concept using a simple approach for 
calculating correction factors (Supplementary Note 1, Supplementary Table 9, and

Supplementary Figs. 19 and 20). Although this approach was able to make overall profiles from different protocols appear to be more similar to each other, its performance was not sufficient to be practically relevant at this time. We propose that synthetic RNA reference data, such as that generated here, can provide a foundation for the future development of more advanced computational approaches to enable accurate crossprotocol comparisons

We also assessed the ability of library protocols to measure miRNA A-to-I editing. Our results revealed that low bias protocols (that is, in-house $4 \mathrm{~N} \_B$ ) quantify editing more accurately than protocol using defined adapter sequences (that is, TruSeq and NEBNext). It is worth noting that the accuracy of editing estimates can also be affected by low sequencing coverage. Indeed, some miRNAs had very low coverage by at least one of the protocols, which contributed to the inaccuracy and variation in editing estimates. However, this lack of coverage is a consequence of technical biases in small RNA-seq, as $4 \mathrm{~N} \_$B libraries all had sufficient coverage of each sequence and, at a minimum, all libraries had depth enough for $\sim 6,000 \times$ coverage of each sequence in the pool. Thus, protocols with a higher degree of sequencing bias also have a greater potential for inaccurate estimates of editing levels, as a result of lower read coverage for some miRNAs and/ or differential preferences based on a single base difference. This is relevant to miRNA editing estimates reported in the literature, given that prior studies have commonly used the more biased protocols with fixed sequence adapters ${ }^{55}$.

\section{Acknowledgements}

We are grateful to J.S. Rozowsky, R. Kitchen, S.L. Subramanian, W. Thistlethwaite, M.B. Gerstein, A. Milosavljevic and N. Sakhanenko for facilitating access to the exceRpt pipeline and helpful conversations and suggestions. We are also grateful to P.A.C. 't Hoen for helpful discussions. We acknowledge funding support from the $\mathrm{NIH}$ Extracellular RNA Communication Common Fund grants: U01 grants HL126499 to M.T., HL126496 to D.J.G. and K.W., HL126493 to D.J.E. and P.G.W., HL126494 to L.C.L., HL126495 to J.E.F., HL126497 to I.G., and UH3 grant TR000891 to K.V.K.-J. M.D.G. acknowledges initial support from a Rio Hortega Fellowship (CM10/00084) and later from a Martin Escudero Fellowship. E.N.M.N.-`t.H. and T.A.P.D. received funding from the European Research Council under the European Union's Seventh Framework Programme (FP/2007- 2013) / ERC Grant Agreement number 337581 and from the Netherlands Organization for Scientific Research (NWO Enabling Technologies project $n$ r 435002022). Y.E.W. received funding from the Dana-Farber Strategic Plan Initiative. K.W. received funding from DOD (W911NF-10-2-0111) and DTRA (HDTRA1-13-C-0055). 
Research reported in this publication was also supported by the National Cancer Institute of the NIH under Award Number P30CA046592 by the use of the following Cancer Center Shared Resource at the University of Michigan: DNA Sequencing. D.J.G. also acknowledges a special technology support award from the Pacific Northwest Research Institute to his lab. The content is solely the responsibility of the authors and does not necessarily represent the official views of the National Institutes of Health.

\section{Author contributions}

M.D.G. designed, led and coordinated the overall study. This comprised contributions throughout the entire process, including designing, preparing and distributing synthetic RNA pools, creating detailed instructions for the participating labs, performing experiments, coordinating experimental work and communication across labs, and organizing and managing all aspects of the project. In addition, she interpreted study results and was the primary writer of the manuscript. R.M.S. led the computational analyses and data management for this study, including processing data, designing and performing data analyses, identifying and applying methods to visualize data and results, and coordinating data and metadata incoming from collaborating laboratories. He also designed the composition of the ratiometric pools, interpreted results and contributed to the manuscript by preparing the figures, drafting figure legends and writing the computational methods. A.E. contributed to experimental design and preparation of synthetic RNA pools. He also performed experimental work, interpreted results and provided comments on the manuscript. $\mathrm{He}$ also developed and contributed the core in-house $4 \mathrm{~N}$ protocol, variations of which were then used by multiple laboratories. M.T., D.J.G. and D.J.E. helped to design the study and interpret the results, along with contributions from the rest of the study team, including L.C.L., P.G.W., K.V.K.-J., I.G., Y.E.W., K.W., J.E.F., H.J., E.N.M.N.-`t.H. and H.P.J.B. contributed to design of statistical analyses and data interpretation. P.M.G., A.J.B., S.S., P.L.D.H., K.T., A.C., S.L., J.K., R.R., D.B. and T.A.P.D. carried out experiments. M.T. and D.J.G. supervised the overall study and did primary editing of the manuscript with substantial input from D.J.E. All of the authors contributed to reviewing, editing and/or providing comments on the manuscript.

\section{Competing Interests}

The spouse of L.C. Laurent is an employee of Illumina, Inc., the manufacturer of the TruSeq Small RNA Library Preparation Kit. L.C. Laurent and her spouse's equity interest in Illumina, Inc. represents $<<1 \%$ of the company. The other authors declare no competing financial interests. 


\section{References}

1. Mortazavi, A., Williams, B.A., McCue, K., Schaeffer, L. \& Wold, B. Mapping and quantifying mammalian transcriptomes by RNA-Seq. Nat. Methods 5, 621-628 (2008).

2. Wang, Z., Gerstein, M. \& Snyder, M. RNA-Seq: a revolutionary tool for transcriptomics. Nat. Rev. Genet. 10, 57-63 (2009).

3. Levin, J.Z. et al. Comprehensive comparative analysis of strand-specific RNA sequencing methods. Nat. Methods 7, 709-715 (2010).

4. Jayaprakash, A.D., Jabado, O., Brown, B.D. \& Sachidanandam, R. Identification and remediation of biases in the activity of RNA ligases in small-RNA deep sequencing. Nucleic Acids Res. 39, e141 (2011).

5. Viollet, S., Fuchs, R.T., Munafo, D.B., Zhuang, F. \& Robb, G.B. T4 RNA ligase 2 truncated active site mutants: improved tools for RNA analysis. BMC Biotechnol. 11, 72 (2011).

6. Zhang, Z., Lee, J.E., Riemondy, K., Anderson, E.M. \& Yi, R. High-efficiency RNA cloning enables accurate quantification of miRNA expression by deep sequencing. Genome Biol. 14, R109 (2013).

7. Song, Y., Liu, K.J. \& Wang, T.-H. Elimination of ligation dependent artifacts in T4 RNA ligase to achieve high efficiency and low bias microRNA capture. PLoS One 9, e94619 (2014).

8. Baran-Gale, J. et al. Addressing bias in small RNA library preparation for sequencing: a new protocol recovers microRNAs that evade capture by current methods. Front. Genet. 6, 352 (2015).

9. Sorefan, K. et al. Reducing ligation bias of small RNAs in libraries for next generation sequencing. Silence 3, 4 (2012).

10. Bellingham, S.A., Coleman, B.M. \& Hill, A.F. Small RNA deep sequencing reveals a distinct miRNA signature released in exosomes from prion-infected neuronal cells. Nucleic Acids Res. 40, 1093710949 (2012).

11. Nolte-'t Hoen, E. et al. Deep sequencing of RNA from immune cell-derived vesicles uncovers the selective incorporation of small non-coding RNA biotypes with potential regulatory functions. Nucleic Acid Res. 18, 9272-9285 (2012).

12. Huang, X. et al. Characterization of human plasma-derived exosomal RNAs by deep sequencing. BMC Genomics 14, 319 (2013).

13. Tietje, A., Maron, K.N., Wei, Y. \& Feliciano, D.M. Cerebrospinal fluid extracellular vesicles undergo age dependent declines and contain known and novel non-coding RNAs. PLoS One 9, e113116 (2014).

14. Lunavat, T.R. et al. Small RNA deep sequencing discriminates subsets of extracellular vesicles released by melanoma cells--Evidence of unique microRNA cargos. RNA Biol. 12, 810-823 (2015).

15. Tosar, J.P. et al. Assessment of small RNA sorting into different extracellular fractions revealed by highthroughput sequencing of breast cell lines. Nucleic Acids Res. 43, 5601-5616 (2015).

16. van Balkom, B.W.M., Eisele, A.S., Pegtel, D.M., Bervoets, S. \& Verhaar, M.C. Quantitative and qualitative analysis of small RNAs in human endothelial cells and exosomes provides insights into localized RNA processing, degradation and sorting. J. Extracell. Vesicles 4, 26760 (2015).

17. Burgos, K.L. et al. Identification of extracellular miRNA in human cerebrospinal fluid by next-generation sequencing. RNA 19, 712-722 (2013).

18. Bahn, J.H. et al. The landscape of microRNA, Piwi-interacting RNA, and circular RNA in human saliva. Clin. Chem. 61, 221-230 (2015).

19. Freedman, J.E. et al. Diverse human extracellular RNAs are widely detected in human plasma. Nat. Commun. 7, 11106 (2016).

20. Wecker, T. et al. MicroRNA profiling in aqueous humor of individual human eyes by next-generation sequencing. Invest. Ophthalmol. Vis. Sci. 57, 1706-1713 (2016).

21. Yuan, T. et al. Plasma extracellular RNA profiles in healthy and cancer patients. Sci. Rep. 6, 19413 (2016).

22. Bullard, J.H., Purdom, E., Hansen, K.D. \& Dudoit, S. Evaluation of statistical methods for normalization and differential expression in mRNA-Seq experiments. BMC Bioinformatics 11, 94 (2010). 
23. Risso, D., Ngai, J., Speed, T.P. \& Dudoit, S. Normalization of RNA-seq data using factor analysis of control genes or samples. Nat. Biotechnol. 32, 896-902 (2014).

24. Lin, Y. et al. Comparison of normalization and differential expression analyses using RNA-Seq data from 726 individual Drosophila melanogaster. BMC Genomics 17, 28 (2016).

25. 't Hoen, P.A.C. et al. Reproducibility of high-throughput mRNA and small RNA sequencing across laboratories. Nat. Biotechnol. 31, 1015-1022 (2013).

26. SEQC/MAQC-III Consortium. A comprehensive assessment of RNA-seq accuracy, reproducibility and information content by the Sequencing Quality Control Consortium. Nat. Biotechnol. 32, 903-914 (2014).

27. Leinonen, R., Sugawara, H., Shumway, M. \& International Nucleotide Sequence Database Collaboration The sequence read archive. Nucleic Acids Res. 39, D19-D21 (2011).

28. Kodama, Y., Shumway, M., Leinonen, R. \& International Nucleotide Sequence Database Collaboration The Sequence Read Archive: explosive growth of sequencing data. Nucleic Acids Res. 40, D54-D56 (2012).

29. Kalra, H. et al. Vesiclepedia: a compendium for extracellular vesicles with continuous community annotation. PLoS Biol. 10, e1001450 (2012).

30. Simpson, R.J., Kalra, H. \& Mathivanan, S. ExoCarta as a resource for exosomal research. J. Extracell. Vesicles 1, 18374 (2012).

31. Kim, D.-K. et al. EVpedia: an integrated database of high-throughput data for systemic analyses of extracellular vesicles. J. Extracell. Vesicles 2, 20384 (2013).

32. Weinstein, J.N. et al. The cancer genome atlas pan-cancer analysis project. Nat. Genet. 45, 1113-1120 (2013).

33. Subramanian, S.L. et al. Integration of extracellular RNA profiling data using metadata, biomedical ontologies and Linked Data technologies. J. Extracell. Vesicles 4, 27497 (2015).

34. Ainsztein, A.M. et al. The NIH Extracellular RNA Communication Consortium. The NIH Extracellular RNA Communication Consortium. J. Extracell. Vesicles 4, 27493 (2015).

35. Xu, P. et al. an improved protocol for small RNA library construction using high- definition adapters. Methods Next Gener. Seq. 2 2, http://dx.doi.org/10.1515/mngs- 2015-0001 (2015).

36. Hansen, K.D., Brenner, S.E. \& Dudoit, S. Biases in Illumina transcriptome sequencing caused by random hexamer priming. Nucleic Acids Res. 38, e131 (2010).

37. Hafner, M. et al. RNA-ligase-dependent biases in miRNA representation in deep- sequenced small RNA cDNA libraries. RNA 17, 1697-1712 (2011).

38. Fuchs, R.T., Sun, Z., Zhuang, F. \& Robb, G.B. Bias in ligation-based small RNA sequencing library construction is determined by adaptor and RNA structure. PLoS One 10, e0126049 (2015).

39. Robinson, M.D., McCarthy, D.J. \& Smyth, G.K. edgeR: a Bioconductor package for differential expression analysis of digital gene expression data. Bioinformatics 26, 139-140 (2010)

40. McCarthy, D.J., Chen, Y. \& Smyth, G.K. Differential expression analysis of multifactor RNA-Seq experiments with respect to biological variation. Nucleic Acids Res. 40, 4288-4297 (2012).

41. Love, M.I., Huber, W. \& Anders, S. Moderated estimation of fold change and dispersion for RNA-seq data with DESeq2. Genome Biol. 15, 550 (2014).

42. Law, C.W., Chen, Y., Shi, W. \& Smyth, G.K. voom: Precision weights unlock linear model analysis tools for RNA-seq read counts. Genome Biol. 15, R29 (2014).

43. Cortez, M.A. et al. MicroRNAs in body fluids: the mix of hormones and biomarkers. Nat. Rev. Clin. Oncol. 8, 467-477 (2011).

44. Yang, W. et al. Modulation of microRNA processing and expression through RNA editing by ADAR deaminases. Nat. Struct. Mol. Biol. 13, 13-21 (2006).

45. Kawahara, Y. et al. Redirection of silencing targets by adenosine-to-inosine editing of miRNAs. Science 315, 1137-1140 (2007). 
46. Wang, Y. et al. Systematic characterization of A-to-I RNA editing hotspots in microRNAs across human cancers. Genome Res. 27, 1112-1125 (2017).

47. Warnefors, M., Liechti, A., Halbert, J., Valloton, D. \& Kaessmann, H. Conserved microRNA editing in mammalian evolution, development and disease. Genome Biol. 15, R83 (2014).

48. Linsen, S.E.V. et al. Limitations and possibilities of small RNA digital gene expression profiling. Nat. Methods 6, 474-476 (2009).

49. Baker, M. 1,500 scientists lift the lid on reproducibility. Nature 533, 452-454 (2016).

50. Goodman, S.N., Fanelli, D. \& loannidis, J.P.A. What does research reproducibility mean? Sci. Transl. Med. 8, 341 ps12 (2016).

51. Risso, D., Schwartz, K., Sherlock, G. \& Dudoit, S. GC-content normalization for RNA-Seq data. BMC Bioinformatics 12, 480 (2011).

52. Hansen, K.D., Irizarry, R.A. \& Wu, Z. Removing technical variability in RNA-seq data using conditional quantile normalization. Biostatistics 13, 204-216 (2012).

53. Leek, J.T. \& Storey, J.D. Capturing heterogeneity in gene expression studies by surrogate variable analysis. PLoS Genet. 3, 1724-1735 (2007)

54. Li, S. et al. Detecting and correcting systematic variation in large-scale RNA sequencing data. Nat. Biotechnol. 32, 888-895 (2014).

55. Chu, A. et al. Large-scale profiling of microRNAs for The Cancer Genome Atlas. Nucleic Acids Res. 44, e3 (2016). 


\section{Online Methods}

\section{Experimental methods Reference samples}

A synthetic equimolar pool containing 1,152 synthetic RNA oligos was prepared in an RNase-free environment and working on ice to minimize degradation. The pool was prepared by combining (i) the miRXplore Universal Reference from Miltenyi Biotec Inc (Auburn, CA), which comprises 962 RNA oligonucleotides with sequences matching human and other miRNAs, and (ii) a set of 190 additional, custom-synthesized RNA oligonucleotides, to generate the pool in which each of the 1,152 RNA oligonucleotides is present at equimolar concentration. This latter set comprises miRNAs and nonmiRNA sequences of varied length from 15 to $90 \mathrm{nt}$, which were synthesized, HPLCpurified and quantified spectrophotometrically by IDT, Inc. (Coralville, IA). This latter set of RNA oligonucleotides is available to qualified investigators seeking to reproduce the synthetic equimolar pool for non-commercial purposes, by request of the corresponding authors (as long as supplies last). The resulting equimolar pool was aliquoted in prelabeled DNA-, DNase-, RNase-, and pyrogen-free screw cap tubes with low adhesion surface and stored immediately at $-80 \mathrm{C}$. Aliquots were distributed to the participant laboratories in overnight shipments with an abundant supply of dry ice. The complete list of RNA sequences comprising the equimolar pool is provided in

\section{Supplementary Table 1.}

Two ratiometric pools, SynthA and SynthB, containing 334 synthetic RNA oligonucleotides were designed in the coordinating lab (see computational methods) and synthesized by IDT. Subsets of these oligos were present in 15 different ratios between the two mixtures. These pools were also prepared, aliquoted and distributed to the participant centers following the same previously mentioned precautions to avoid RNA degradation. The complete list of sequences in the SynthA and SynthB pools, as well as their ratios, are provided in Supplementary Table 2.

Plasma samples from eleven healthy male donors with age ranging from 21-45 years were collected and pooled in one of the participating labs (Supplementary protocol 1). The IRB of the institution approved the study protocol to consent participants and collect samples. Informed consent was obtained from all subjects, and the samples were subsequently anonymized before distributing to participating research groups. RNA was isolated from this plasma pool (Supplementary protocol 2) in a single lab and aliquots of the purified RNA were mixed and distributed to the rest of the participant centers. 


\section{Library preparation and small RNA-seq of reference samples}

A written guideline for library preparation and sequencing was distributed to all the participant centers. The input for library preparation was 10 femtomoles of RNA for synthetic pools and $2.1 \mathrm{ul}$ of RNA for the plasma pool. Each group prepared four replicate libraries from each sample using the following small RNA library preparation protocols: Lab1 (TruSeq, NEBNext and in-house 4N_D), Lab2 (TruSeq, NEBNext and inhouse 4N_B), Lab3 (TruSeq and NEBNext), Lab4 (TruSeq, NEBNext and in-house 4N_B), Lab5 (TruSeq, CleanTag, NEBNext, in-house 4N_A, in-house 4N_B and 4N_Xu), Lab6 (TruSeq, in-house 4N_B and in-house 4N_C), Lab 7* (NEXTflex), Lab 8* (TruSeq and NEXTflex) and Lab 9* (TruSeq and NEBNext). The labs marked with an asterisk did not contribute plasma libraries. The protocols for TruSeq, CleanTag, NEBNext and NEXTflex for Illumina were performed according to the manufacturer's instructions in all labs except for NEBNext in Lab9 that performed 3' overnight ligation. Note that some manufacturers recommended dilution of the adapters when working with low input RNA (for NEBNext, adapters were diluted, 1:2 in Lab3 and Lab9 and 1:6 in Lab1, Lab2, Lab4 and Lab5; for CleanTag 1:20 dilution of the adapters was performed). NEXTflex for lon Torrent sequencing was performed as described in Supplementary protocol 3. In-house $4 \mathrm{~N}$ protocols $\mathrm{A}, \mathrm{B}, \mathrm{C}$ and D were performed as described in Supplementary protocol 4-7. 4N_Xu protocol was performed as previously described48 35. Size selection was performed using Pippin Prep instruments (in Lab1, Lab2, Lab4 and Lab6 for all protocols and Lab5 for in-house 4N_B only), 6\% acrylamide gels (in Lab3, and Lab9 for all protocols, Lab 8 for TruSeq and Lab5 for TruSeq, NEBNext, CleanTag,4N_A and 4N_Xu) or Ampure XP beads (in Lab7 and Lab8 for NEXTflex).

Single-end libraries were sequenced using the Illumina HiSeq 2500 (Lab8 and Lab9 for all the protocols and Lab5 for TruSeq, CleanTag, 4N-Xu and 4N_A), Illumina HiSeq 4000 (Lab4 for all the protocols and Lab1 for TruSeq, 4N_D and Equimolar NEBNext), Illumina NextSeq 500 (Lab2, Lab3 and Lab6 for all the protocols, Lab5 for NEBNext and in-house 4N_B and Lab1 for Ratiometric and Plasma NEBNext) or lon Torrent (Lab7) platforms (see Supplementary Table $\mathbf{3}$ which also includes information on miRNA editing libraries). All labs using Illumina sequencing performed runs specifying $\geq 50 \mathrm{bp}$ single-end reads. Details on read lengths for each library are included in Supplementary Table 3. Each laboratory was free to choose the number of samples to pool per lane, with a target of at least 8 million reads per library. FASTQ files were uploaded to the Genboree Workbench for central data analysis. 


\section{Evaluation of miRNA editing}

Ten human miRNAs previously shown in the literature to undergo adenosine-to-inosine (A-to-I) RNA editing were selected to evaluate the performance of small RNA-seq in the detecting miRNA editing. To this end, we designed six pools containing different ratios of the selected synthetic edited miRNAs and their unedited counterparts (i.e. $0 \%, 0.1 \%, 0.5 \%, 5 \%, 50 \%$ and $100 \%$ edited) plus 277 unrelated human miRNAs. All RNA oligonucleotides were synthesized by IDT (the complete list of sequences included in these pools is provided in Supplementary Table 7). The pools were prepared and aliquoted in the coordinating center and distributed to two additional labs following the same previously mentioned precautions to avoid RNA degradation. Each lab prepared three replicate libraries from 10 femtomoles of each pool using the three different small RNA library preparation protocols: TruSeq, NEBNext and in- house $4 \mathrm{~N} \_B$. The protocols for TruSeq and NEBNext were performed according to the manufacturer's instructions (note that for NEBNext, adapters were diluted 1:2). Inhouse 4N_B was performed as described in Supplementary protocol 5. Size selection was performed using the Pippin Prep. $\geq 50$ bp single-end libraries were sequenced using the Illumina NextSeq 500.

\section{Computational methods}

\section{Designing Ratiometric Pools}

290 artificial sequences were assigned at random to 8 ratiometric groups $(1,1.5,2,3$, $4,5,8$ and 10x) and to either ratiometric pool SynthA or SynthB. The ratio indicates the concentration in the assigned pool relative to the other pool. For example, a sequence in the 10x pool assigned to SynthA would be present at the base concentration in SynthB and at $10 x$ the base concentration in SynthA. To make groups of approximately equal size, assigned 8 sequences to the 8 ratiometric groups randomly, without replacement. To ensure the total amount of oligonucleotide was approximately equal in SynthA and SynthB, an even number of sequences was assigned to each ratiometric group and were distributed equally between pools, using a similar method of equallydistributed random assignment. The random assignment was performed in Excel and the complete pool composition and ratios are shown in Supplementary Table 2.

\section{Barcode splitting, FASTQ generation and data coordination}

High-throughput sequencing, demultiplexing and FASTQ file generation was performed by each participating group independently. FASTQ files were uploaded to the Genboree Workbench for centralized analysis using the ExceRpt small RNA analysis pipeline. (http://genboree.org/java-bin/workbench.jsp). 


\section{Preprocessing, mapping and read counting}

FASTQ files for the Equimolar, Ratiometric and Plasma pools were initially processed through the exceRpt small RNA-seq Pipeline (Version 4.6.2), using the batch submission tool. For details on the exceRpt pipeline and the associated processing steps, see the Genboree Workbench documentation (http://genboree.org/theCommons/projects/exrna-toolsmay2014/wiki/Small\%20RNA-seq\%20Pipeline). A brief description of parameters changed from the default settings or that differed between libraries is included below.

The exceRpt pipeline was used at the default settings whenever possible. The default for adapter trimming is "auto-detect" which identifies and trims the adapter sequence for multiple library types, and all samples were initially submitted using this functionality. For $4 \mathrm{~N}$ libraries (A, B, C, D, Xu and NEXTflex), an additional parameter was selected to indicate the degenerate sequence at the end of each adapter. The default random barcode settings were used, indicating that random 4 nt sequences are present immediately $5^{\prime}$ and $3^{\prime}$ of the insert sequence. The sequence and identity of the adapter identified by the exceRpt pipeline was confirmed in the output files. Any library with a missing or incorrect adapter identified was re-submitted to the pipeline with the adapter sequence chosen manually, and a note was added to Supplementary Table 3. For Plasma Pool libraries, sequences shorter than $18 \mathrm{nt}$ after adapter trimming were removed and not used for downstream analysis. For synthetic pools, the minimum length was changed to $15 \mathrm{nt}$, which corresponds to the length of the shortest sequences in the Equimolar and Ratiometric Pools. To quantify alignments to the full set of synthetic pool sequences, Equimolar, Ratiometric SynthA and Ratiometric SynthB libraries were mapped to a "Spike-In" sequence library uploaded to the Genboree Workbench. This spike-in library FASTA file contains a non-redundant set of sequences from the Ratiometric and Equimolar Pools (Supplementary Table 7). Adaptertrimmed and filtered reads were mapped to the spike-in index with bowtie2 using the default GenboreeWorkbench alignment parameters, except that the minimum read length was reduced to 15 . The number of reads aligning to each sequence was obtained from the "calibratormapped" output files. At the time of writing, reads mapped to the spikein sequences are removed prior to genomic alignment, so any endogenous alignment information from these samples was ignored. To quantify alignments to endogenous miRNAs, Equimolar and Plasma pool libraries were also run through the exceRpt pipeline without mapping to spike-in sequences. The default minimum read length of $18 \mathrm{nt}$ was used, along with all default alignment parameters. Reads were mapped to hg19 using the STAR alignment algorithm. Multi-mapping-adjusted read counts corresponding to mature miRNAs were used for all plasma pool analyses and for the Equimolar Pool correction factor analyses. For all other analyses with the Equimolar and Ratiometric pools, the spikein read counts from the "calibratormapped" files were used. 


\section{Sample Filtering}

Unless specifically noted in the text, only libraries meeting minimum read count requirements were considered for analysis. For the synthetic pools, an average of one million reads mapping to the "spike-in" sequences (the unique set of sequences present in the equimolar and ratiometric pools), were required across all replicate libraries. The average was taken after filtering, such that the totals were based only on 5'-phosphorylated sequences 16-25 nt in length. For the Plasma Pool samples, replicate libraries with fewer than 100,000 miRNA-mapping reads were removed. The entire sample was removed if more than one of the replicate libraries failed to pass the minimum count threshold.

\section{Equimolar Pool Analysis}

Read counts for the equimolar (and likewise for the ratiometric pools) were obtained from "calibratormapped.counts" files included in the exceRpt pipeline output for each sample file. Sample-specific information, including the contributing lab, library preparation method and replicate number were associated with the corresponding calibrator count file, and were loaded into $\mathrm{R}$ for analysis. A full list of equimolar and ratiometric sequences with additional sequence information was used as a reference to merge all input files and add zero counts, where needed. Unless specifically mentioned in the text, analysis of ratiometric and equimolar libraries was limited to sequences with a 5'-phosphate modification, 16-25nt in length. Read counts were scaled to counts-per-million (CPM) using the total counts from the filtered sequences. For plots and calculations using log-transformed values, a small count was added to avoid taking the log of zero. The adjusted CPM values were calculated using the method employed by the $\mathrm{R}$ package, Edge $\mathrm{R}^{39,40}$. This scales the user-supplied prior count ( 0.25 ; the default setting) to be proportional to the library size. The scaled prior count is calculated by multiplying the raw prior count $(0.25)$ by the sample library size divided by the mean library size across all equimolar samples and then adding this value to the raw counts for each miRNA. Library sizes are adjusted by adding $2 \times$ the scaled prior count value. Adjusted CPM values are finally calculated as ( raw.count + adjusted.prior ) * $10^{6} /$ adjusted.library.size.

\section{Determining Overrepresented and Underrepresented Sequences}

Sequences in each Equimolar pool replicate library were ranked by abundance, assigning the minimum rank value in case of ties. The 10 top and bottom-ranked sequences were determined by arranging counts in descending and ascending 
order, respectively. TruSeq, NEBNext, CleanTag and 4N libraries were each queried for sequences consistently found in the top or bottom 10, as defined by at least $75 \%$ agreement among the libraries of at least one method.

\section{Dissecting the source of bias}

The CPM obtained for each sequence of the Equimolar pool was calculated from pseudocounts, as in the Equimolar Pool Analysis described above, except that the library sizes were calculated from all equimolar pool sequences prior to filtering for length and end modifications. Sequence length, 5'and 3 ' terminal bases, \%GC of the four 5'or 3'end base, overall \%GC, dG [free energy], dH [enthalpy], dS [entropy] and $\mathrm{Tm}$ [melting temperature] were calculated from the annotated sequence. UNAFold ${ }^{56}$ (http://unafold.rna.albany.edu/) was used to obtain the $\mathrm{dG}, \mathrm{dH}, \mathrm{dS}$ and $\mathrm{Tm}$ values of each of the sequences comprising the Equimolar pool.

\section{Ratiometric Pools Analysis}

Ratiometric pool counts were initially processed as described above for equimolar pools, considering only counts for 16-25 nt sequences. The ratio of SynthA:SynthB was calculated as the ratio of the mean CPM across technical replicates in SynthA / SynthB.

\section{Ratiometric Pools: Differential Expression}

Independent differential expression workflows were run for each lab and library prep method, following a standard two-group comparison between " $\mathrm{A}$ " and " $\mathrm{B}$ " ratiometric pools. Normalization, dispersion estimation and differential expression testing was performed using three different R packages: EdgeR ${ }^{39,40}, \mathrm{DESeq}^{41}$ and limma/voom ${ }^{42}$. For EdgeR, normalization factors were calculated using the Relative Log Expression (RLE) method, and significance was calculated (after calculating common, trended and tagwise dispersion estimates) using the default settings, based on a likelihood ratio test on the null hypothesis that ratiometric sample $B-A=0$. Default settings were used for DESeq2, and significance was calculated based on a Wald Test. Significance for the limma/voom workflow was based on an empirical Bayes moderated t-test.

\section{Plasma Pool Analysis}

Comparison of plasma pool libraries was limited to mature miRNAs. Read counts for mature miRNAs were taken from "readCounts_miRNAmature_sense.txt" files provided in the exceRpt pipeline output. The read count files and associated metadata for all samples were loaded and merged in $\mathrm{R}$ for further analysis. Multi-mapping-adjusted read counts are calculated as part of the exceRpt pipeline and were used for all comparisons. The total number of unique reads mapping to miRNAs was taken from ".stats" files provided in the exceRpt pipeline output. 


\section{Downsampling Read Counts}

The $\mathrm{R}$ package Vegan, was used to simulate random downsampling of Equimolar and Plasma pool count matrices. The Vegan function, drarefy, was used to estimate the probability of detection for each sequence based on random simulations of downsampling to specified levels. For the plasma pools, downsampling was performed to four different levels $\left(10^{4}, 10^{4.5}, 10^{5}\right.$ and $\left.10^{5.5}\right)$. Equimolar pools were downsampled to six different levels ( $10^{6.5}$ down to $10^{4}$ at half-log intervals). Libraries with read counts below the specified threshold were removed. A minimum probability of 0.9 was used as the threshold for detection.

\section{Inter-protocol Bias Correction Factors: Estimation}

Equimolar pool samples were processed through the exceRpt pipeline using the same input parameters as the plasma pool libraries, in order to obtain multi-mapping, scaled read counts for mature miRNAs that were directly comparable to the plasma pool counts. Differential expression analyses were performed using the mature miRNA read counts for the equimolar pool samples, and scaling factors were calculated for each miRNA, and were taken from the resulting log2 fold-change estimates. Key assumptions used in these calculations were: that the median mapped read level calculated for a given protocol should match the median for the $4 \mathrm{~N}$ results given the same RNA input; that the comparisons of the results from a given protocol and the $4 \mathrm{~N}$ protocol are performed on data processed in the same way that biological samples are processed (i.e., using the exceRpt pipeline and its mapped read outputs). For details on limma and voom functionality and the parameters used, see the documentation for the limma package.

To summarize: correction factors were calculated for each pair of library prep. methods using the following workflow:

1. Filter out miRNAs with 0 counts in any library: For the subset of samples being tested, scaling factors are only calculated for miRNAs having at least one count in every sample of the two methods being tested.

2. Prepare miRNA count matrices for linear modeling: Use the R package, voom, to calculate precision weight estimates and normalize data to allow count data to be analyzed appropriately using the limma package. Normalization is also performed between samples such that the median miRNA expression value is the same in all samples.

3. Fit miRNA-wise linear models to account for batch (lab) effect: The ImFit function from the limma package is used to fit linear models for each miRNA, estimating coefficients for each lab+library prep method. The coefficients represent the differences in expression for each miRNA between each lab+library prep method. 
4. Estimate the log fold change between the two methods for each miRNA: Use the fitted model to calculate for each miRNA the average expression estimated using the method $A$ coefficients - the average expression estimate for the method $B$ coefficients.

The R packages limma/voom were used for read count normalization and differential expression estimates, using standard workflows suggested for RNA-seq data to account for batch (lab) effects, and then testing for the main effect of the library prep methods. For each pairwise comparison of library preparation methods, equimolar pool counts matrices were extracted and only miRNAs with $\geq 1$ read count in all samples of both methods were kept for analysis. After filtering, voom was used to normalize the count data and calculate precision weight estimates that allow count data to be appropriately tested with the linear modeling schema used in the limma package. Voom was run with the default parameters, except that read counts were additionally normalized between arrays using the "scale" method, which adjusts read counts such that the median miRNA expression value is the same in all labs. The voomtransformed data was supplied to the limma ImFit function, along with a design matrix indicating the coefficients to be estimated. Initially, coefficients were estimated for each lab+library prep method in order to model batch/lab-specific effects. The main effect of the library prep method was then calculated as the average effect of method 1 - method 2. A contrasts matrix was generated and supplied, with the fitted model, to the contrasts.fit function, followed by an empirical Bayes function to estimate the resulting statistics for each miRNA. Log2 fold-change estimates, along with $95 \% \mathrm{Cl}$ were obtained from these estimates.

\section{Inter-protocol Bias Correction Factors: Applying corrections}

The equimolar pool-derived, inter-protocol bias correction factors were applied to the corresponding plasma pool samples for testing. To apply the correction factors, count matrices for the subset of plasma pool libraries being compared were selected and were then pre-filtered and normalized in the same way as the equimolar pools in generating the correction factors, described above. MiRNAs were filtered to include only those with a) a correction factor estimated from the equimolar pool and b) at least 5 counts in every library in the subset of methods being compared. Correction factors were applied to the appropriate samples. For example, if correction factors were calculated as the log 2 fold-change between TruSeq and 4N samples (TruSeq - 4N), then the correction factors would be applied to the log2-transformed TruSeq plasma pool samples by subtracting the correction factor. For the heatmaps and density plots, corrected values were added to the original count matrix of untransformed values, and unless specifically noted in the text, normalized using quantile normalization. 


\section{miRNA Editing Analysis}

Editing libraries were trimmed of $5^{\prime}$ and $3^{\prime}$ adapters using cutadapt (version 1.9.1). Trimmed reads $\geq 16 \mathrm{nt}$ were aligned to Editing Pool sequences using bowtie2 (version 2.3.2) in local alignment mode. The first (5') 4 nt were removed from $4 \mathrm{~N}$ library reads during the alignment stage by adding the optional parameter "--trim5p 4". Read counts were calculated from alignments filtered to have a minimum MAPQ of 20 and 0 mismatches to the reference sequence within the locally-aligned region. The sum totals of the filtered read counts for each library were used to calculate read Counts Per Million (CPM). Downsampling was performed using the R package Vegan.

\section{Code and Data Availability}

Sequencing data for all experiments can be obtained from the GEO Superseries, GSE94586. Accession numbers for the four subseries are: GSE94584 (Equimolar), GSE94585 (Ratiometric A/B), GSE94582 (Human Plasma Pool) and GSE108138 (A-to-I Editing). GEO records include raw FASTQ files and processed counts from the ExceRpt pipeline. All code, metadata and processed data files required for reproducing the figures, tables and in-text statistical summaries are freely available on GitHub (https:// github.com/rspengle/CrossU01_exRNA_Manuscript2017). The repository also includes a Packrat library with a snapshot of $\mathrm{R}$ package versions used. 


\section{Supplementary Results}

\section{Exploring protocol-specific bias correction for inter-protocol data normalization}

The protocol-specific biases highlighted by our analysis of the equimolar synthetic RNA pool sequencing data represent a significant potential barrier to cross-platform data comparison and integration. Given that the sequence-specific bias is reproducible for each particular protocol across laboratories, we sought to determine whether between-protocol correction factors could be calculated and applied for inter-protocol data normalization. A detailed description of the approach for calculating these correction factors is provided in the online Methods section and a schematic is shown in Supplementary Figure 8a. Briefly, we used datasets from our equimolar synthetic pool generated with different protocols as training sets from which we calculated correction factors for each miRNA, using pairs of library preparation protocols tested in this study, setting one protocol as the "query" and the other as the "reference" (Supplementary Table 6). After applying the correction factors to the normalized read counts from the original query training set data, we found that the data much more closely approximated the reference than prior to correction (Supplementary Figure $\mathbf{1 7} \boldsymbol{b}-\boldsymbol{f}$ ). It is interesting that we could remove much of the sequencing bias from TruSeq and NEBNext abundance estimates for the synthetic pools by applying correction factors generated using 4 N_B as a reference (Supplementary Figure 17b). We next applied the correction factors to our plasma sequencing datasets and found that the correction factors also made query plasma miRNA profiles much more similar to reference profiles (Supplementary Figure 18a-d). Subsequent analysis revealed that the improvement for individual miRNAs can be variable, including both over and under correction for a number of miRNAs (data not shown). More sophisticated computational and statistical approaches will need to be developed in the future to derive and apply correction factors to account for protocol-specific quantitative differences. The data included in this study can serve as a useful reference set for the development and testing of such methods. 

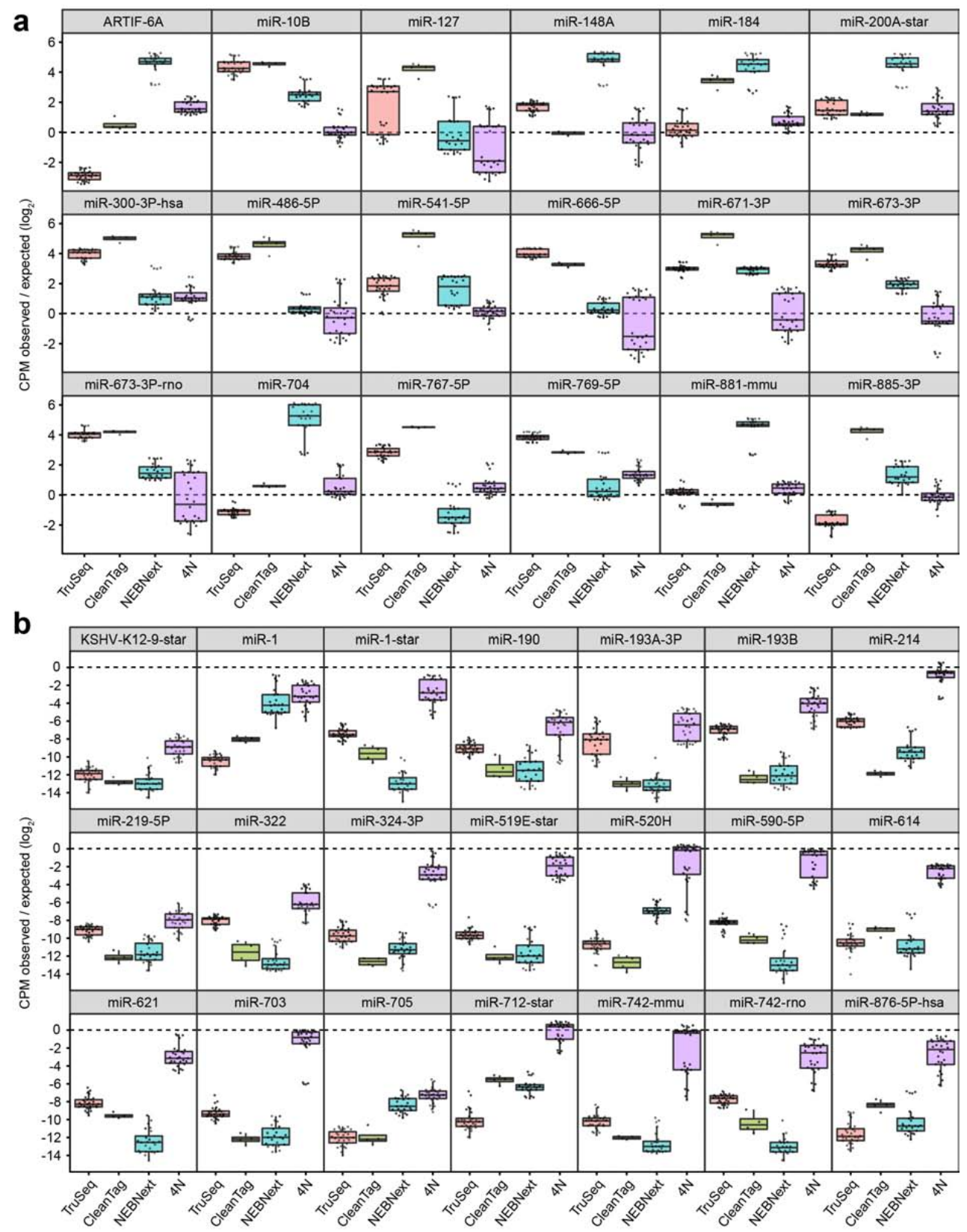


\section{Supplementary Figure 1. Examples of protocol-dependent bias in small RNA-seq.}

Boxplots summarize the $\log _{2}$ ratio of CPM measured: CPM expected $\left(10^{6} / 977 \mathrm{miRs}=1023.5\right.$ expected) (y-axis) for the indicated sequences, for all replicate TruSeq (pink), CleanTag (green), NEBNext (turquoise) and all in-house 4N (purple) Equimolar Pool libraries (x-axis). The plotted miRNAs represent those that were found to be consistently (a) over-represented, or (b) underrepresented, in at least one library preparation protocol. Individual points are overlaid representing the ratio for the individual replicate libraries. The over- and under-represented sequences plotted were among the 10 highest or 10 lowest-expressed, respectively, in $\geq 75 \%$ of TruSeq, CleanTag, NEBNext or in-house $4 \mathrm{~N}$ libraries. For both panels, over- and under- represented sequences were determined for each protocol (i.e. TruSeq, NEBNext, CleanTag and $4 \mathrm{~N}$ ) separately. Overand under- represented sequences for a particular library preparation protocol were selected on the basis of being among the top or bottom 10 , respectively, in at least $75 \%$ of libraries for a given protocol. 


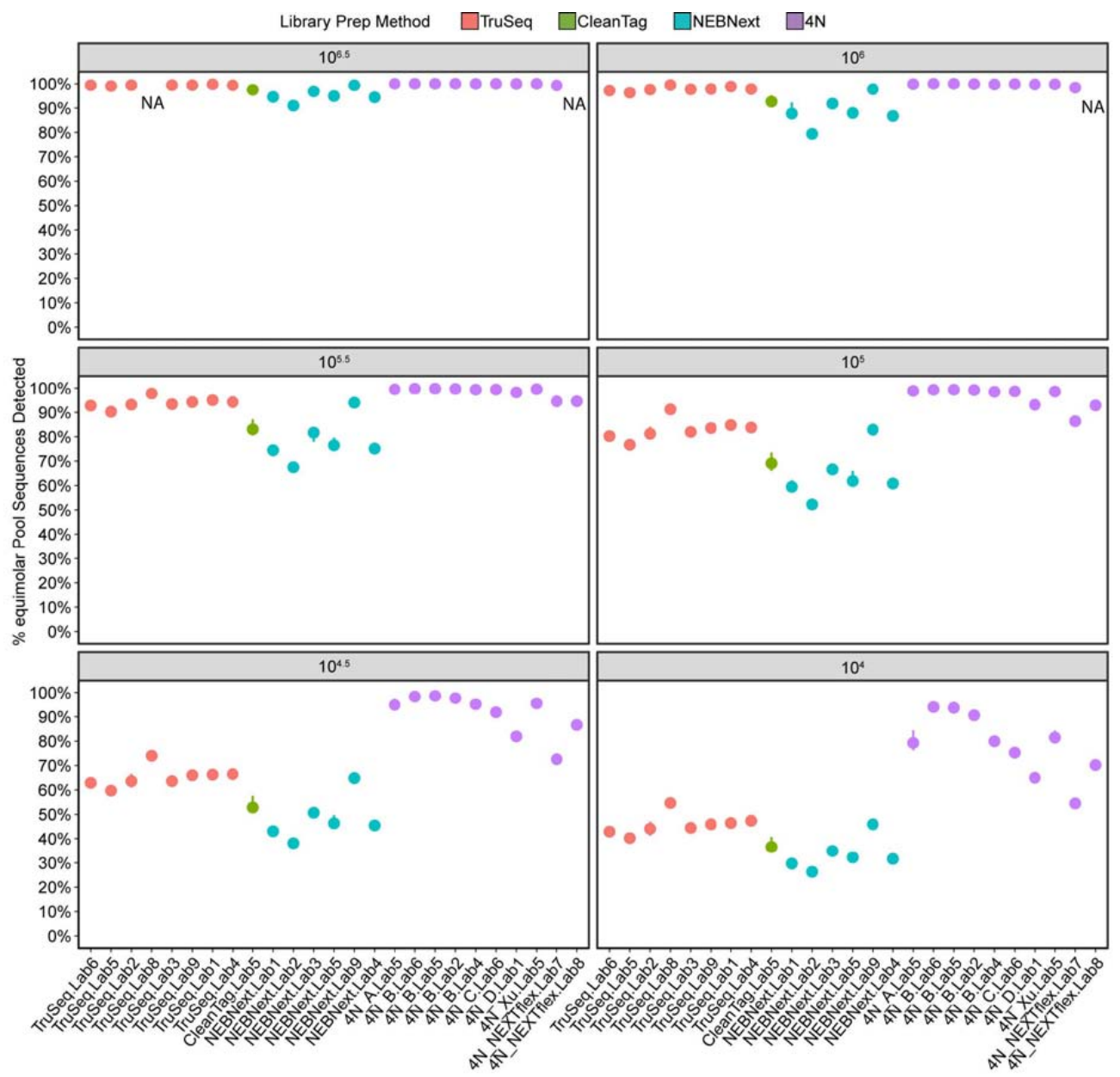

Supplementary Figure 2. Percentage of Equimolar Pool detected under different levels of simulated downsampling. The dot and whisker plots summarize the percent of sequences in the equimolar pool detected across technical replicates for each lab and method combination, and are based on probability estimates of downsampling all samples to the same level. The dots and whiskers represent the median and range of sequences detected, respectively, where "detection" was defined as a probability of detection of at least $90 \%$, as estimated using the drarefy function included in the R package Vegan. Mapped reads were first filtered, as described, and then downsampled to six different levels-from $10^{6.5}$ down to $10^{4}$ at half-log intervals (indicated in the grey boxes above each plot). Probability plots are grouped and color-coded by librarypreparation method (Truseq - pink; CleanTag - green; NEBNext - turquoise; 4N - purple). "NA" labels indicate samples where all replicate libraries had fewer total miRNA-mapped read counts than the downsampling level indicated, and therefore, were not able to be evaluated at thatlevel. 




Supplementary Figure 3. Spearman correlation for the Equimolar pool libraries. Heatmap showing the squared Spearman rank correlation coefficients calculated pairwise between equimolar pool libraries, using CPM values. Rows and columns are color coded to indicate different labs and library preparation protocols, as well as the total library size (log2-scaled), after size and sequence modification filtering, as described in the text. Hierarchical clusteringfor rows and columns represents complete linkage clustering on Euclidean distances. Row and column labels indicate the library prep method, lab ID and replicate number. 

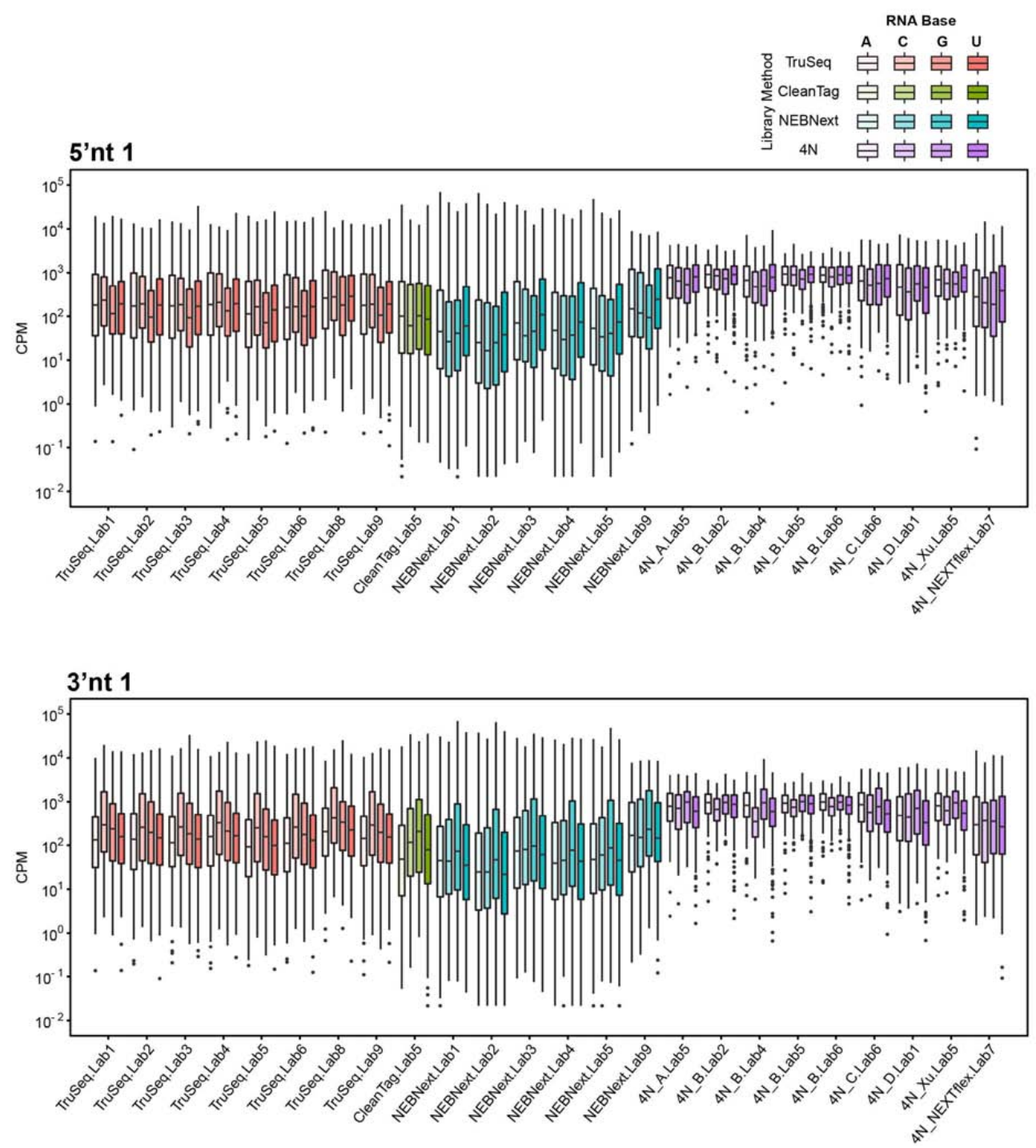

Supplementary Figure 4. Effects of 5' and 3' end nucleotide on sequence bias. Boxplots summarize (a) the 5' terminal nucleotide and (b) the 3 ' terminal nucleotide effects on mean replicate CPM ( $y$-axis) measured by each lab / library preparation protocol ( $\mathrm{x}$-axis) forEquimolar pool sequences considered in this study ( 5 ' phosphorylated, length $16-25 ; \mathrm{N}=977$ ). Boxes are colorcoded by library preparation protocol $($ TruSeq $=$ pink, CleanTag $=$ green, NEBNext $=$ turquoise, $4 \mathrm{~N}=$ purple), and shaded based on the end-nucleotide (Lightest $\rightarrow$ Darkest $=A, C, G, U)$. CPM values are plotted on a $\log _{10}$ scale. 

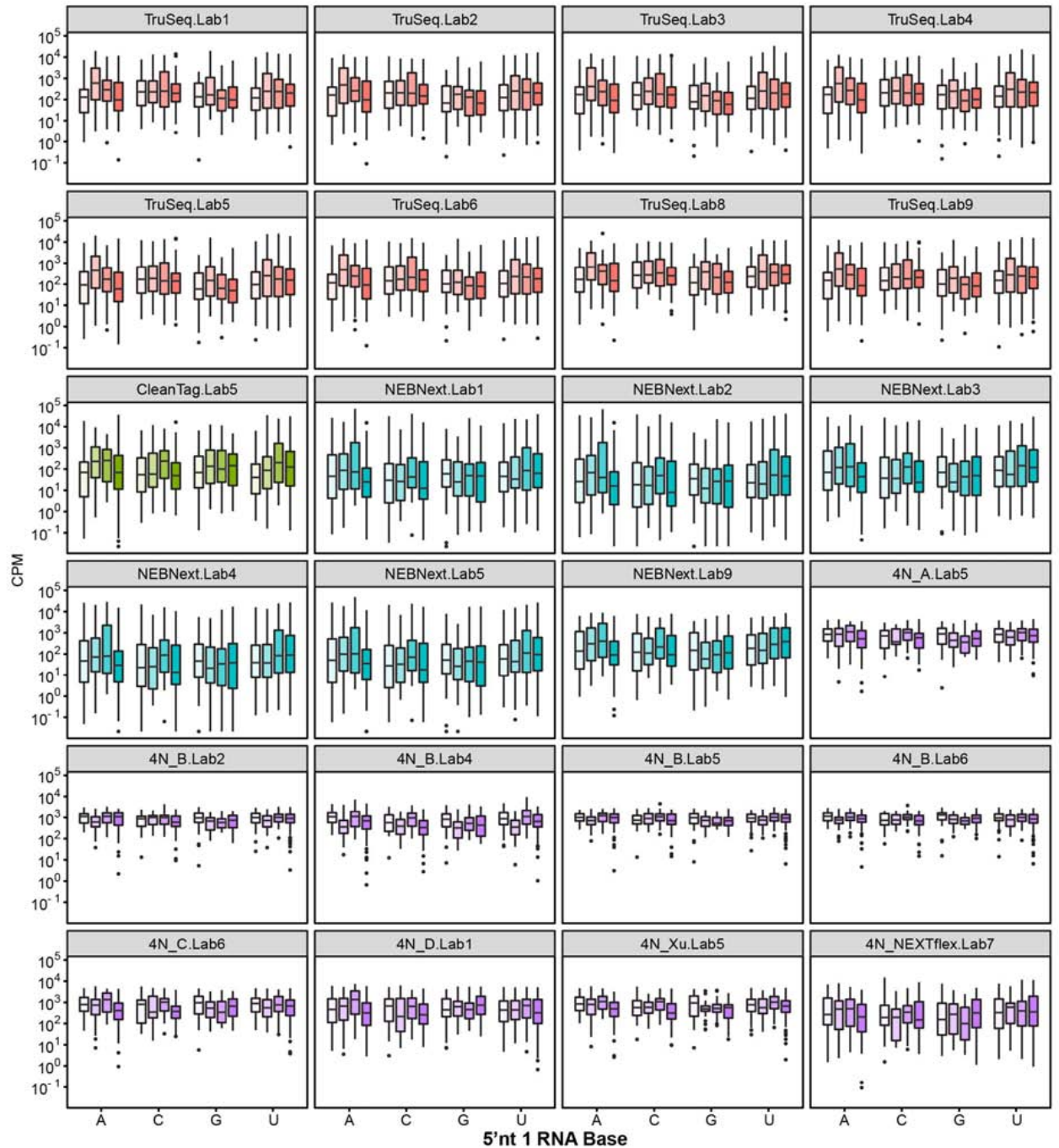

Supplementary Figure 5. Effects of the combination of end nucleotides on sequence bias. Boxplots summarize the effect of every combination of 5' and 3 ' terminal nucleotides on the mean replicate CPM(y-axis) measured by each lab / library preparation protocol (x-axis) for Equimolar pool sequences considered in this study ( $5^{\prime}$ phosphorylated, length $16-25 ; \mathrm{N}=977$ ). The $\mathrm{x}$-axis indicates the identity of the 5 ' nucleotide, and the boxes are shaded based on the 3 ' nucleotide (Lightest $\rightarrow$ Darkest $=$ A, C, G, U). Boxes are color-coded by library preparation protocol (TruSeq $=$ pink, CleanTag $=$ green, NEBNext $=$ turquoise, $4 \mathrm{~N}=$ purple) . 
S. Fig $6(A)$

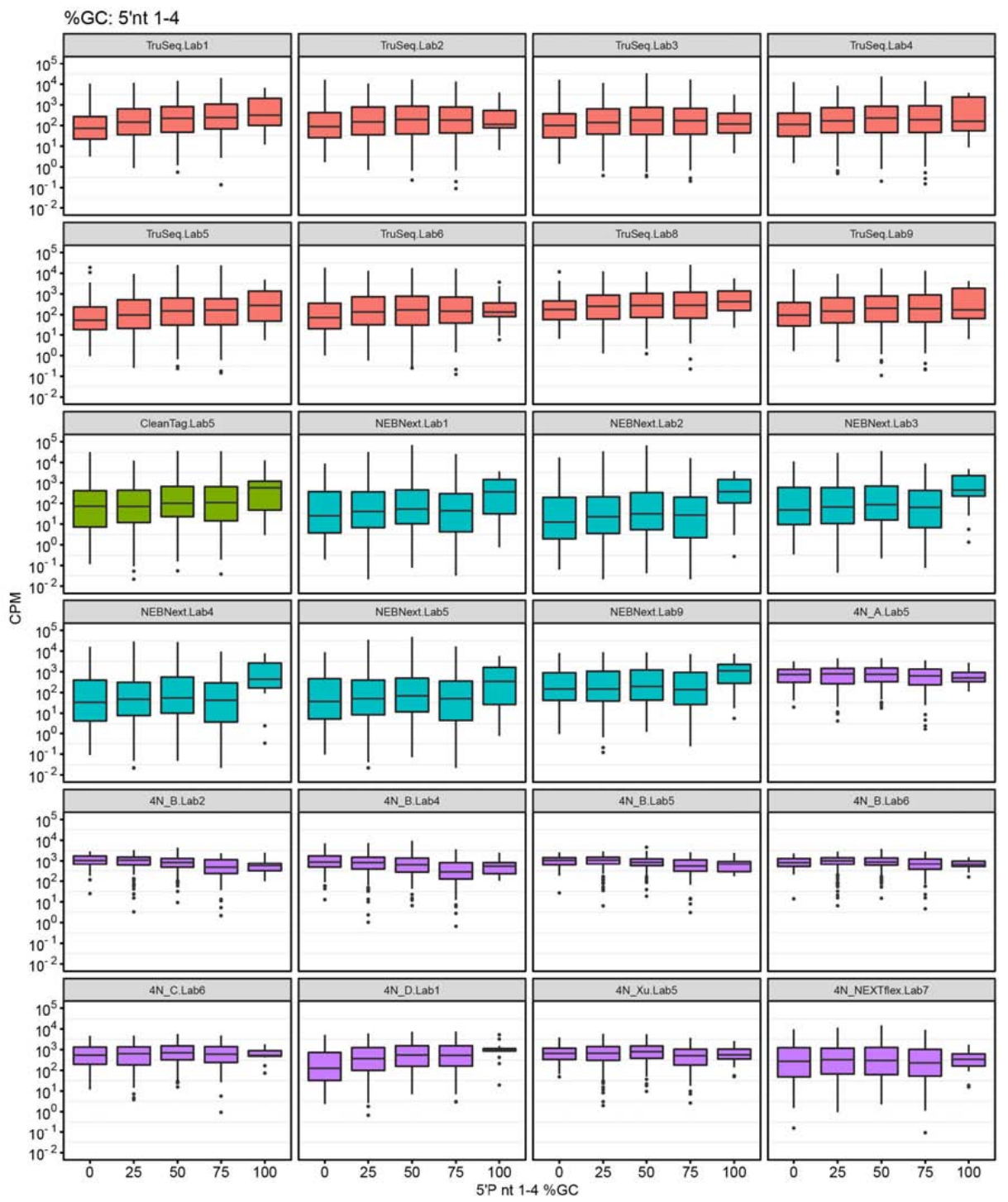


S. Fig $6(B)$

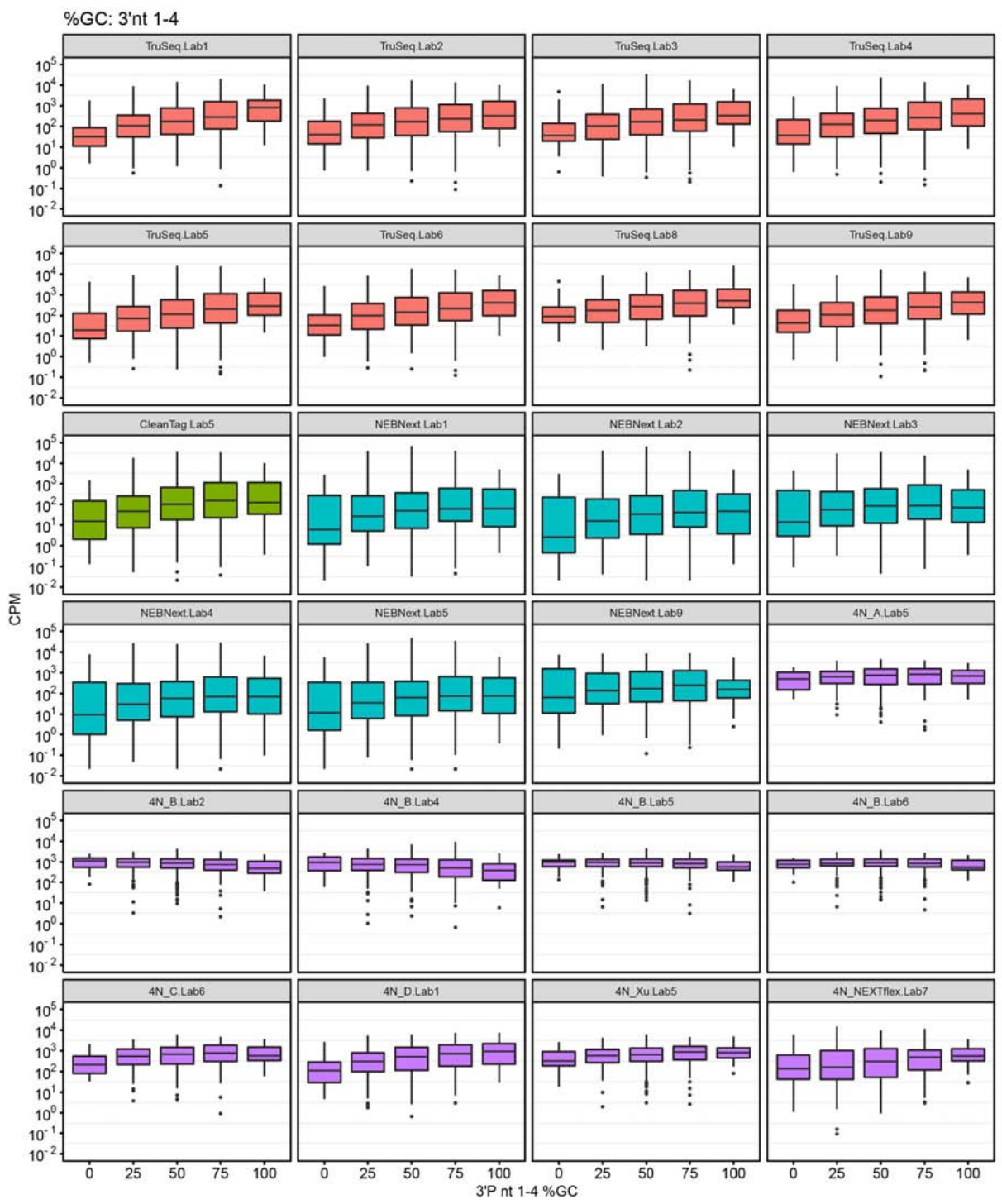

Supplementary Figure 6. Effect of the GC\% of the four end nucleotides on sequence bias. Boxplots summarize for each lab / library preparation method the mean replicate CPM obtained for Equimolar pool sequences (5' phosphorylated, length 16-25. $\mathrm{N}=977$ ) depending on the $\mathrm{GC}$ content of the (a) 5 ' four end nucleotides (\%GC = \# sequences: $0 \%=80,25 \%=339,50 \%=381$,

$75 \%=164,100 \%=13) \operatorname{or}(\mathbf{b}) 3$ 'fourendnucleotides $(\% \mathrm{GC}=$ nsequences: $0 \%=26,25 \%=$

$250,50 \%=445,75 \%=226,100 \%=30$ ). CPM values are plotted on a $\log _{10}$ scale. 

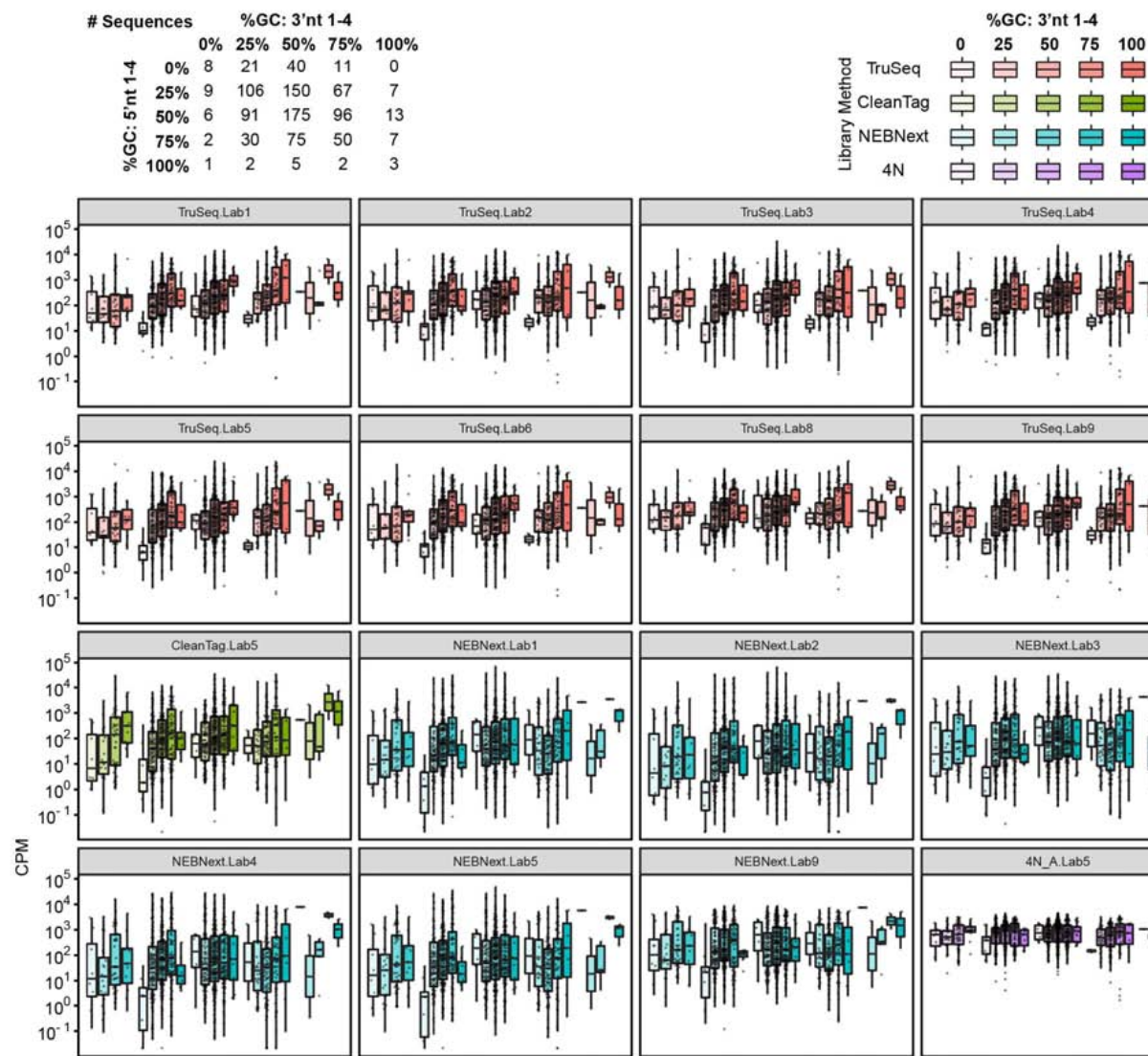

$\begin{array}{llll}25 & 50 & 75 & 100\end{array}$
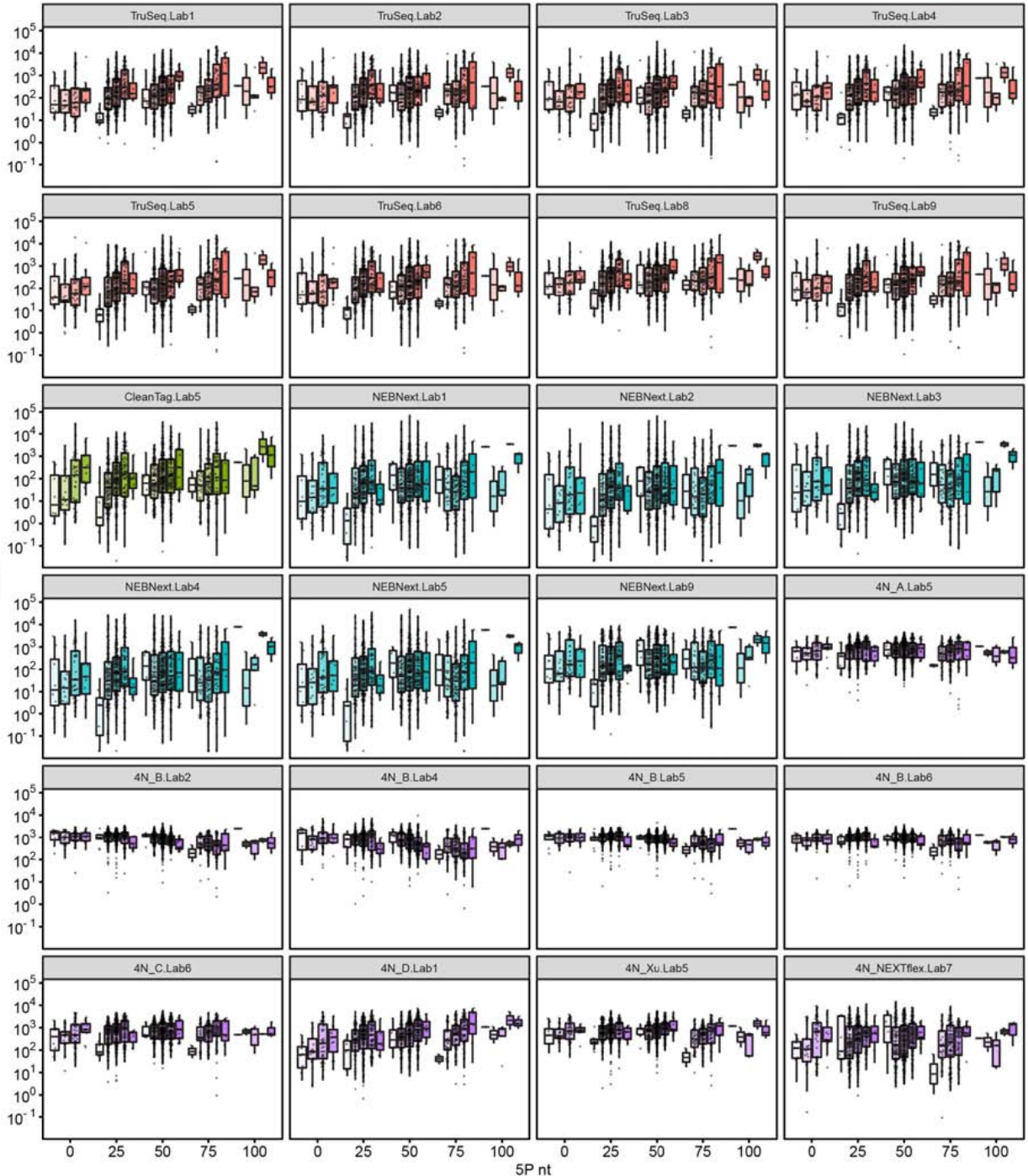

Supplementary Figure 7. Effect of the combination of GC\% of the four end nucleotides on sequence bias. Boxplots summarize the effect of every combination of \%GC content in the four 5' and 3' end nucleotides on the mean replicate CPM (y-axis) measured by each lab / library preparation protocol for the Equimolar pool sequences used in the study (5' phosphorylated, length 16-25; $\mathrm{N}=977$ ). The $\mathrm{x}$-axis indicates the \%GC content of the four 5 ' end nucleotides, and the boxes are shaded according to the \%GC content of the four 3' end-nucleotides ( Lightest $\rightarrow$ Darkest $=0$, $25,50,75,100 \%)$. Boxes are color-coded by library preparation protocol (TruSeq = pink, CleanTag = green, $\mathrm{NEBNext}=$ turquoise, $4 \mathrm{~N}=$ purple). $\mathrm{CPM}$ values are plotted on a $\log _{10}$ scale. 


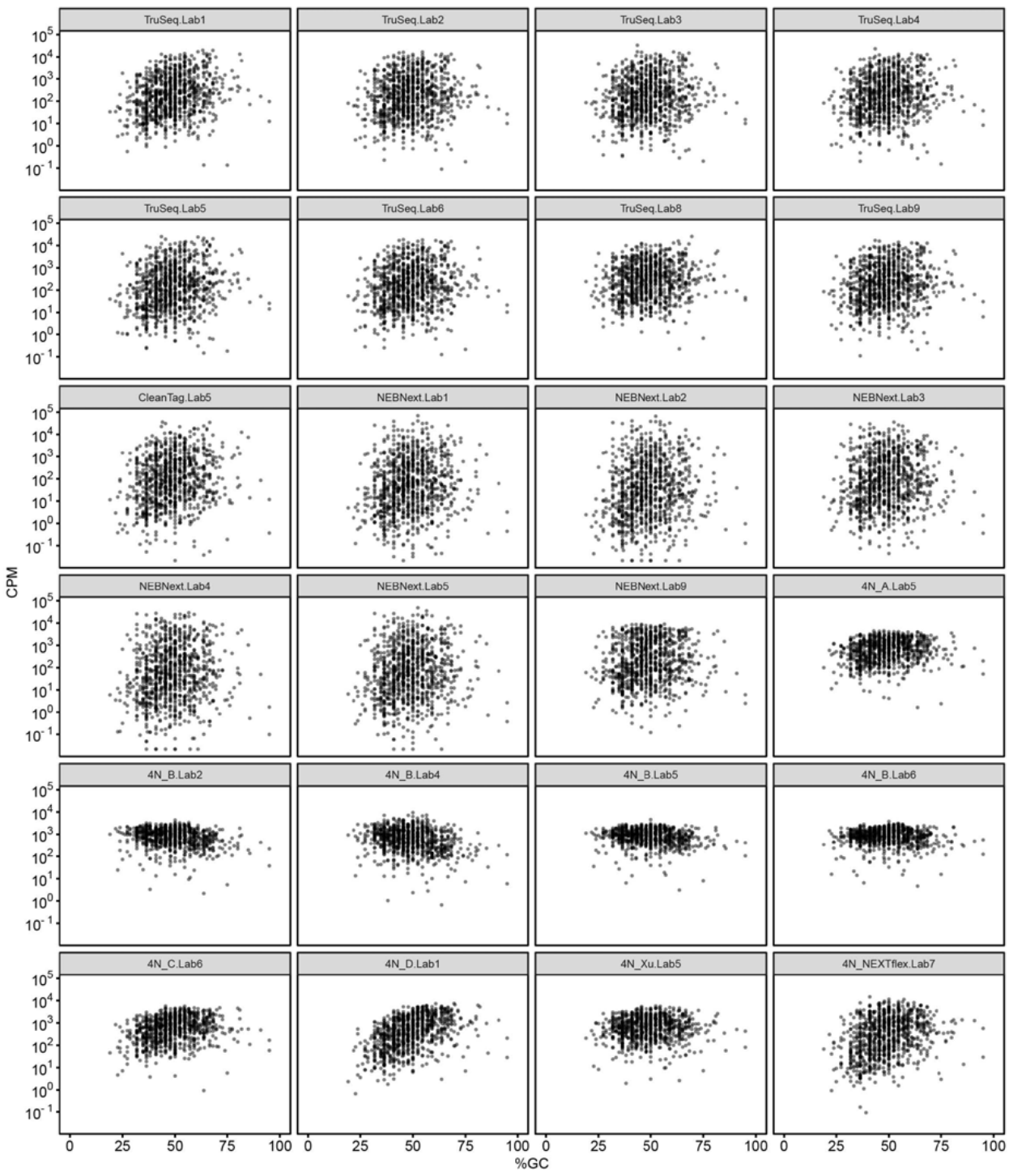

Supplementary Figure 8. Effect of the overall GC\% on sequence bias. Scatterplots show, for each lab / library preparation protocol, the mean replicate CPM (y-axis) obtained for the Equimolar pool sequences used in this study ( 5 ' phosphorylated, length $16-25 . \mathrm{N}=977$ ) as a function of the sequence $\%$ GC content. CPM values are plotted on a $\log _{10}$ scale. 


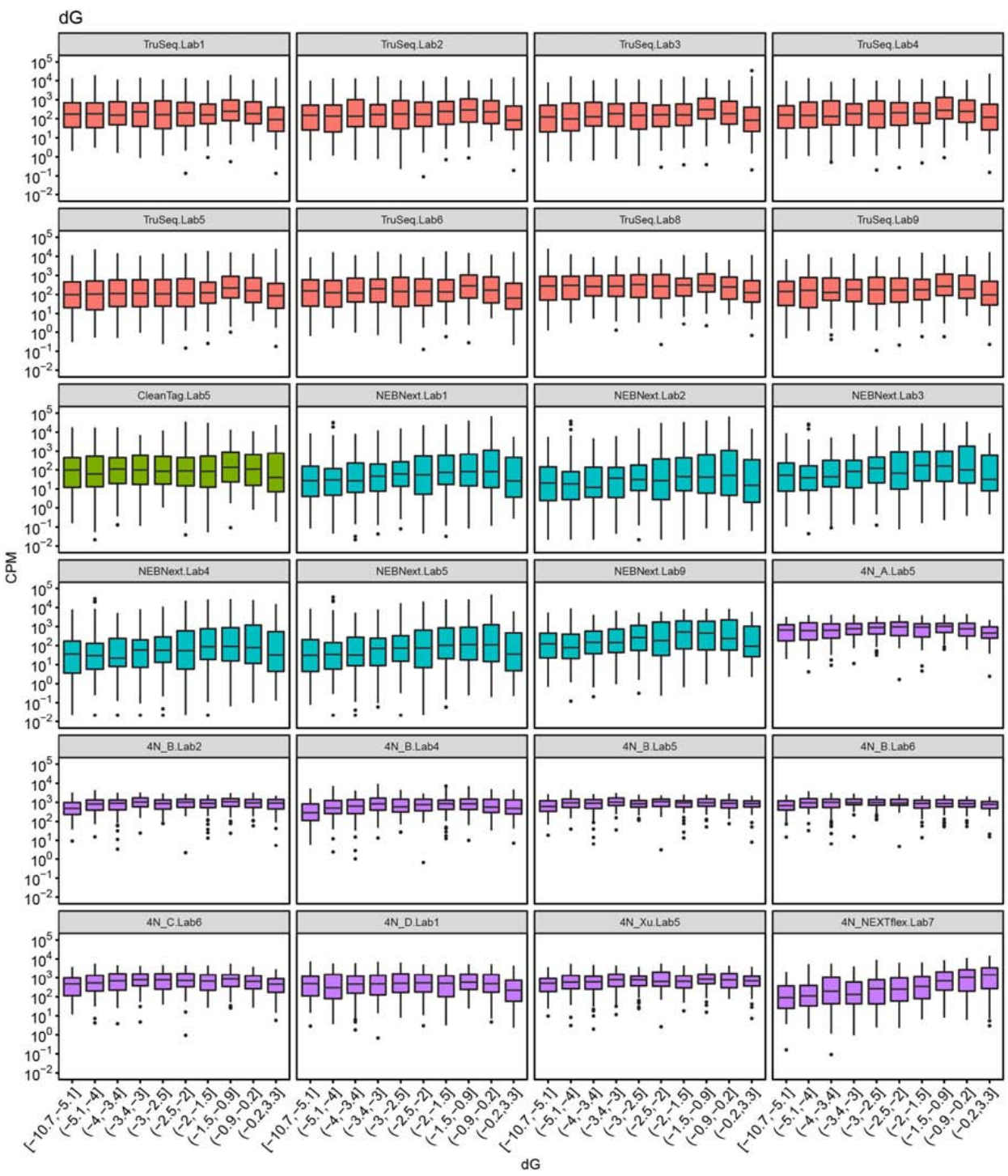

Supplementary Figure 9. Effect of free energy (dG) on sequence bias. Boxplots summarize the effect of each sequence's free energy (dG), binned into deciles, on the mean replicate CPM (y-axis) measured by each lab / library preparation protocol for the Equimolar pool sequences used in this study (5' phosphorylated, length 16-25; $\mathrm{N}=977$ ). 


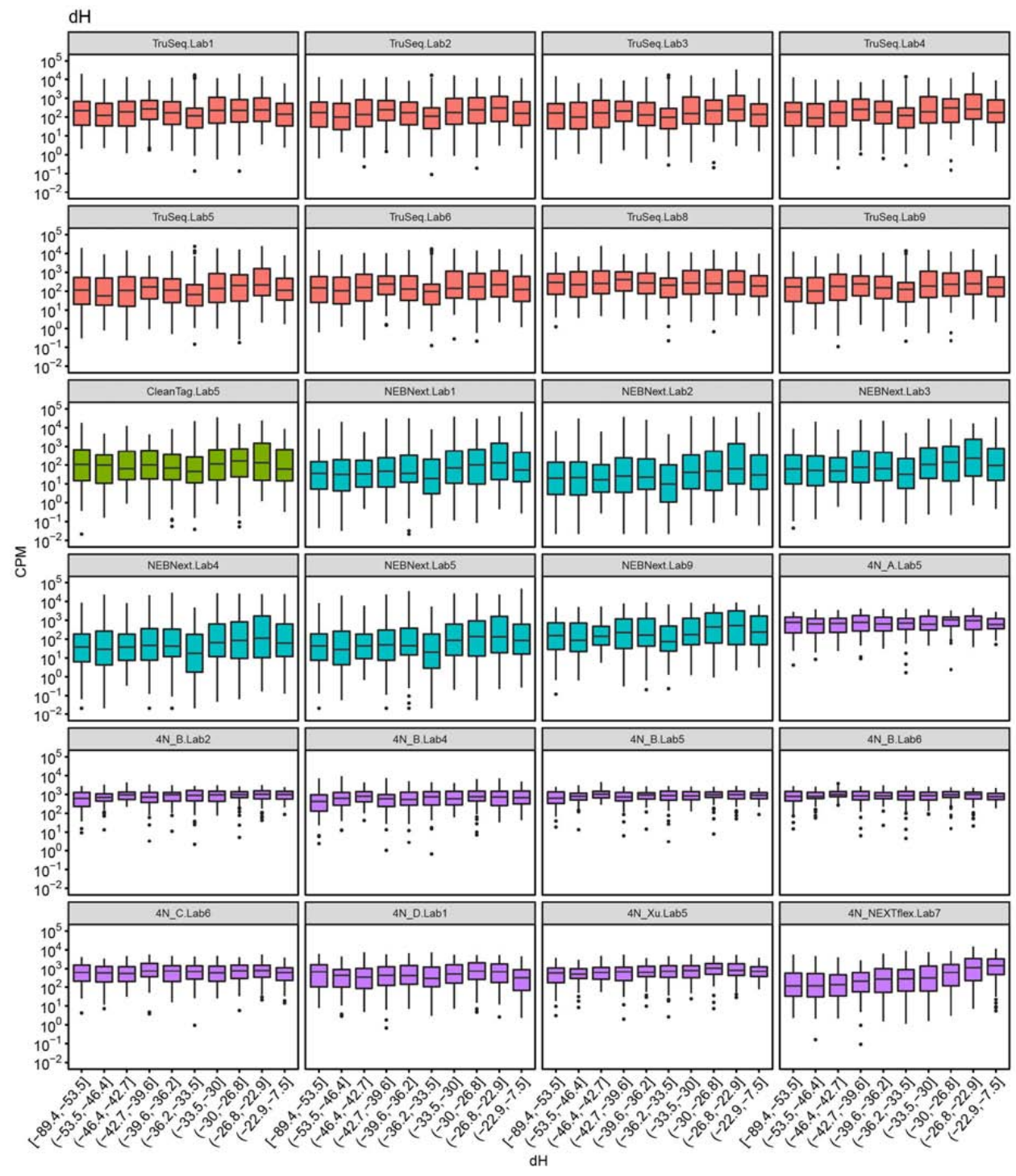

Supplementary Figure 10. Effect of enthalpy $(\mathrm{dH})$ on sequence bias. Boxplots summarize the effect of each sequence's enthalpy $(\mathrm{dH})$, binned into deciles, on the mean replicate CPM (y-axis) measured by each lab / library preparation protocol for the Equimolar pool sequences used in this study (5' phosphorylated, length $16-25 ; \mathrm{N}=977$ ). 


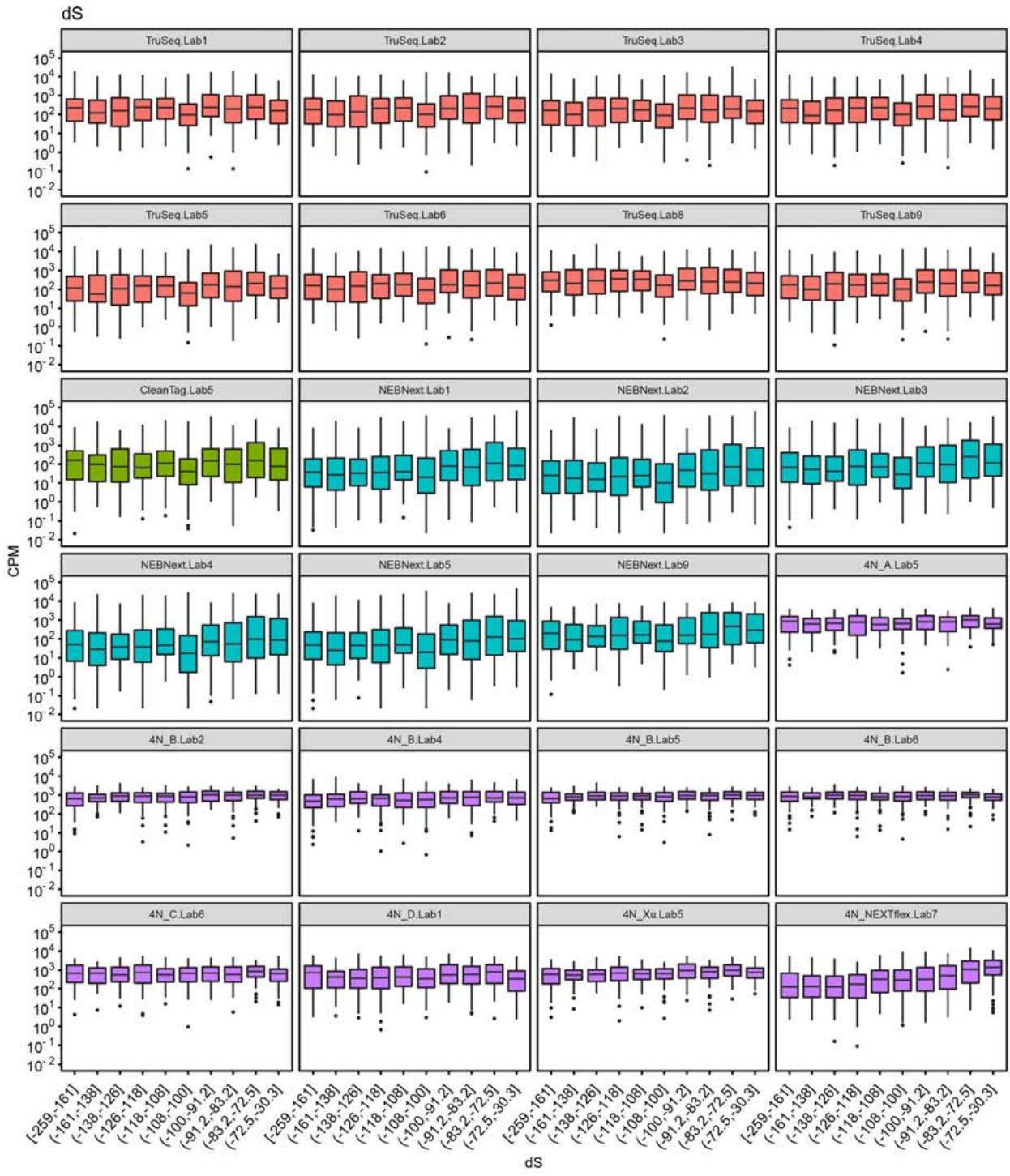

Supplementary Figure 11. Effect of entropy (dS) on sequence bias. Boxplots summarize the effect of each sequence's entropy (dS), binned into deciles, on the mean replicate CPM (y-axis) measured by each lab / library preparation protocol for the Equimolar pool sequences used in this study (5' phosphorylated, length $16-25 ; \mathrm{N}=977$ ). 


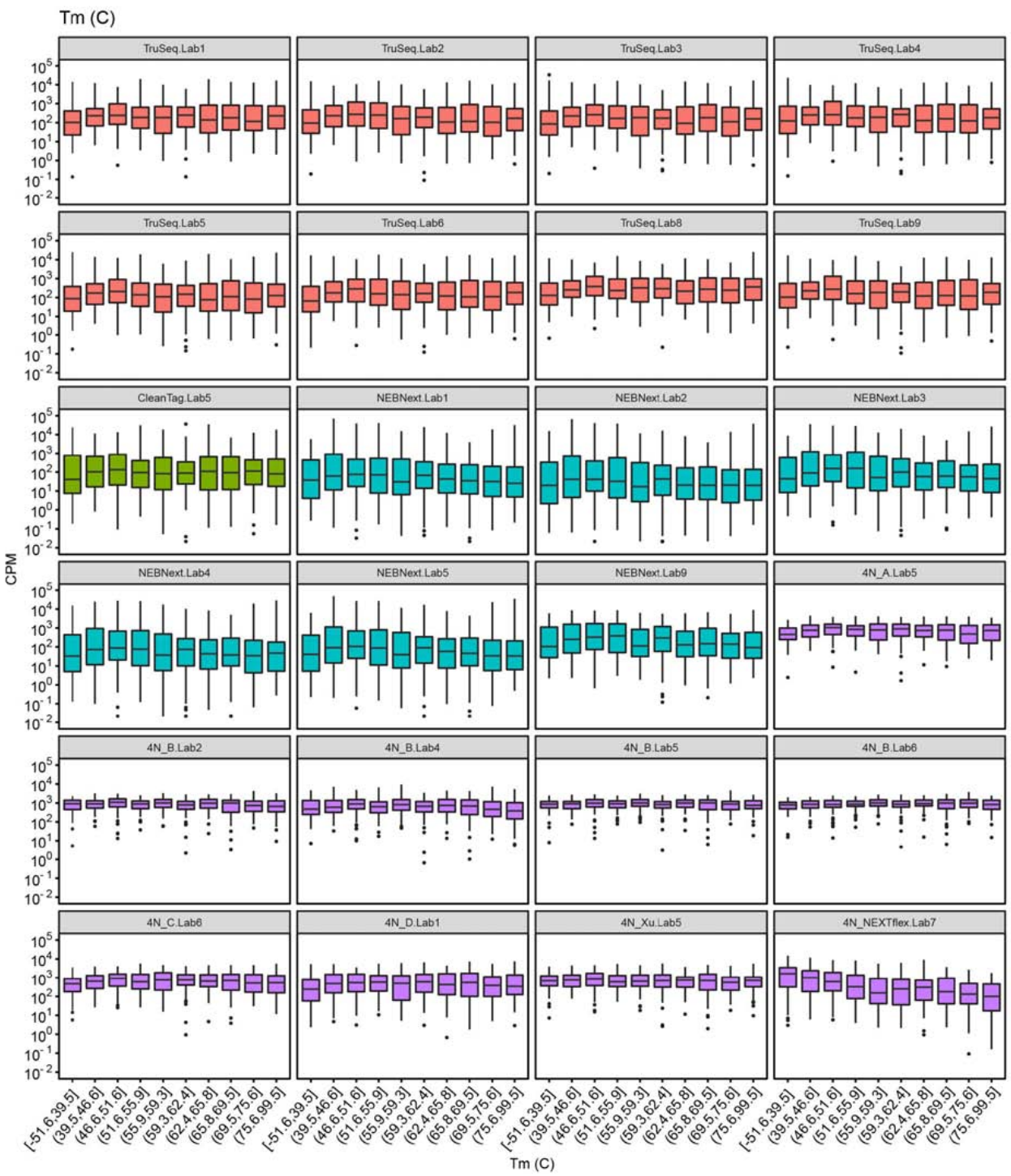

Supplementary Figure 12. Effect of melting temperature $(\mathrm{Tm})$ on sequence bias. Boxplots summarize the effect of melting temperature $(\mathrm{Tm})$, binned into deciles, on the mean replicate CPM (y-axis) measured by each lab / library preparation protocol for the Equimolar pool sequences used in this study (5' phosphorylated, length $16-25 ; \mathrm{N}=977$ ). 


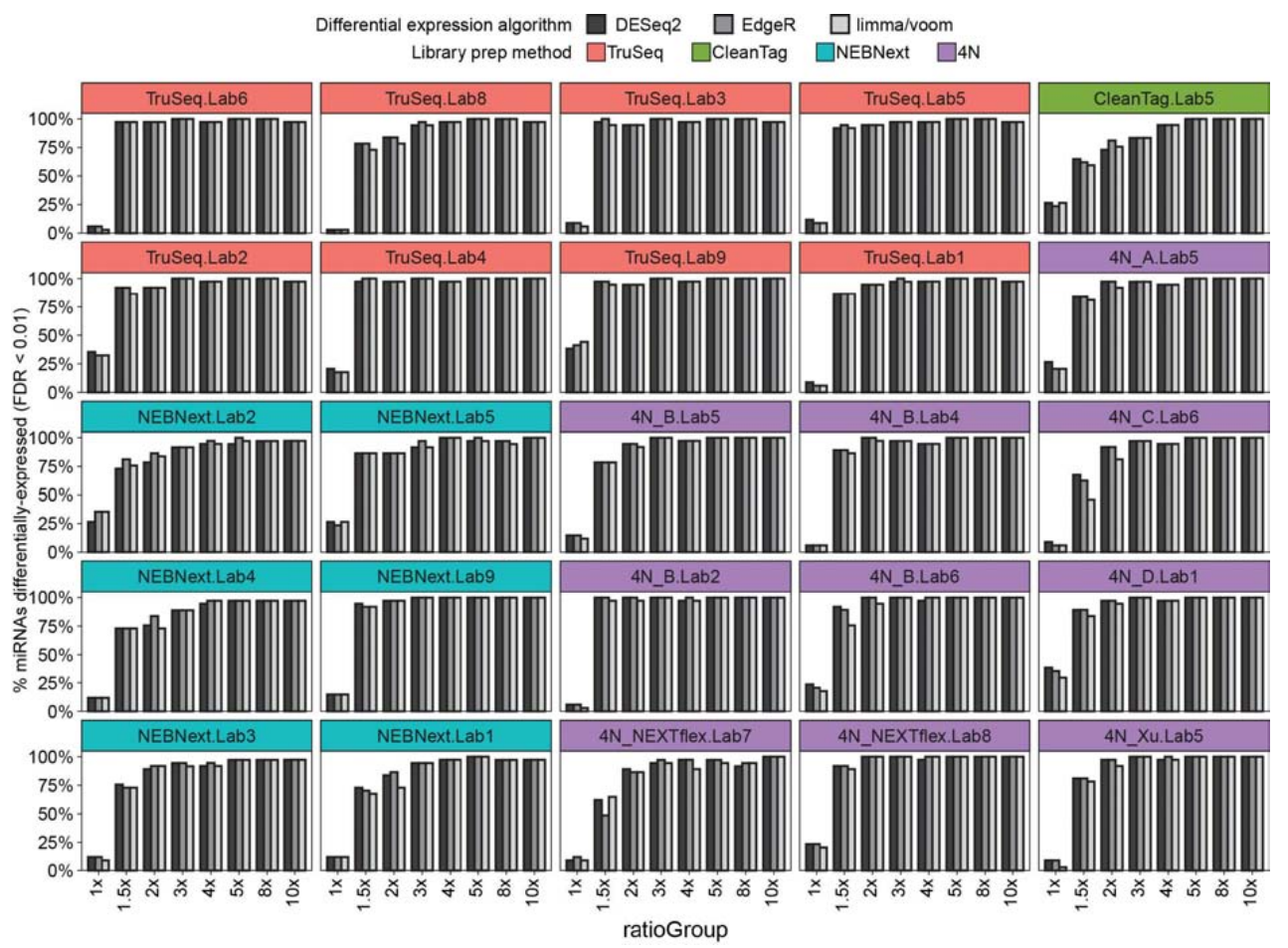

Supplementary Figure 13. Differential expression analysis with synthetic ratiometric pools. Barplots show the fraction of miRNAs in each ratiometric subpool with significantly different CPM values (FDR < 0.01) measured between the SynthA and SynthB samples. The number of miRNAs included in each of the subpools is as follows: $1 \mathrm{x}: 34 ; 1.5 \mathrm{x}: 37 ; 2 \mathrm{x}: 37 ; 3 \mathrm{x}: 36 ; 4 \mathrm{x}: 37 ; 5 \mathrm{x}: 36 ; 8 \mathrm{x}$ : 36; 10x: 37 . Tests for significant differences in abundance were performed using three different R packages commonly used for RNA-seq differential expression analysis: DESeq2 (dark grey), EdgeR (medium grey), and limma/voom (light grey) (see Methods for details). P-values are calculated based on the default tests for each of the three algorithms: likelihood ratio test (EdgeR), Wald Test (DESeq2) or empirical Bayes moderated t-test (limma) $(\mathrm{HO}=$ SynthB - Synth $A=0$, FDR $<=0.01, n=4$ per sample, except for TruSeq Lab8 SynthB where $n=3$ ). Each plot represents independent tests of differential expression for each lab + library prep method. 
RATIOMETRIC: \%CV Within-labs

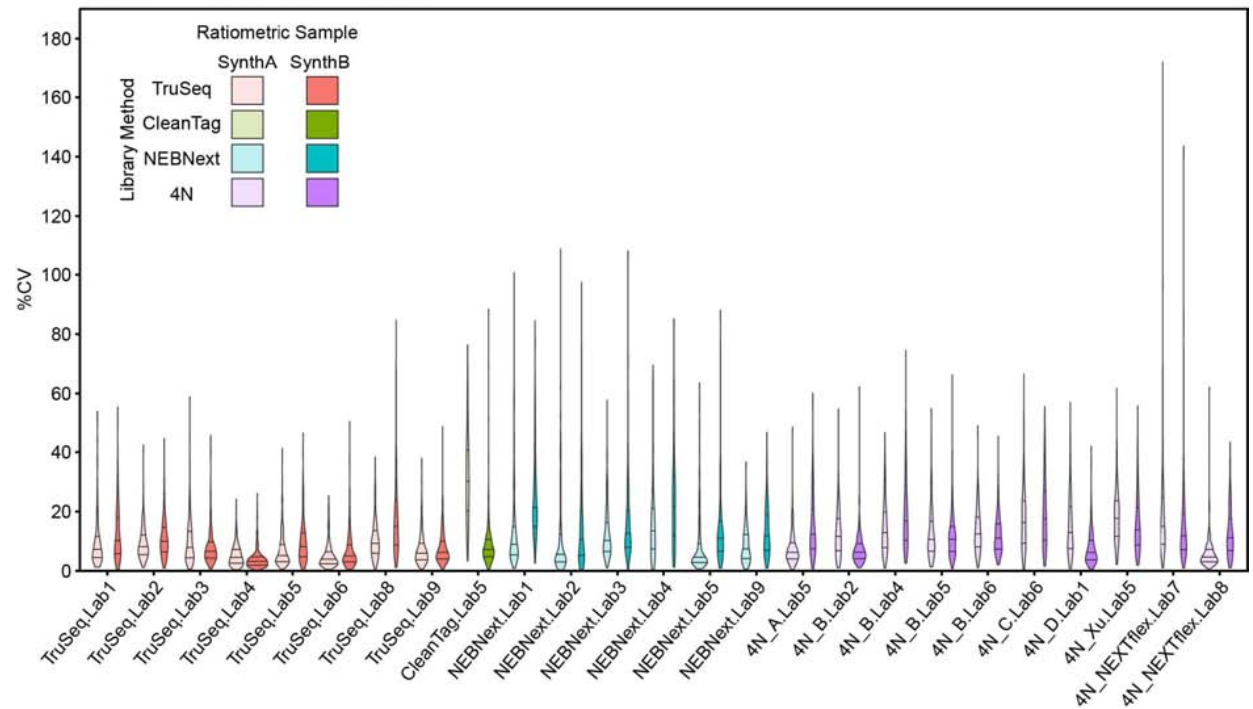

RATIOMETRIC: QCD Within-labs

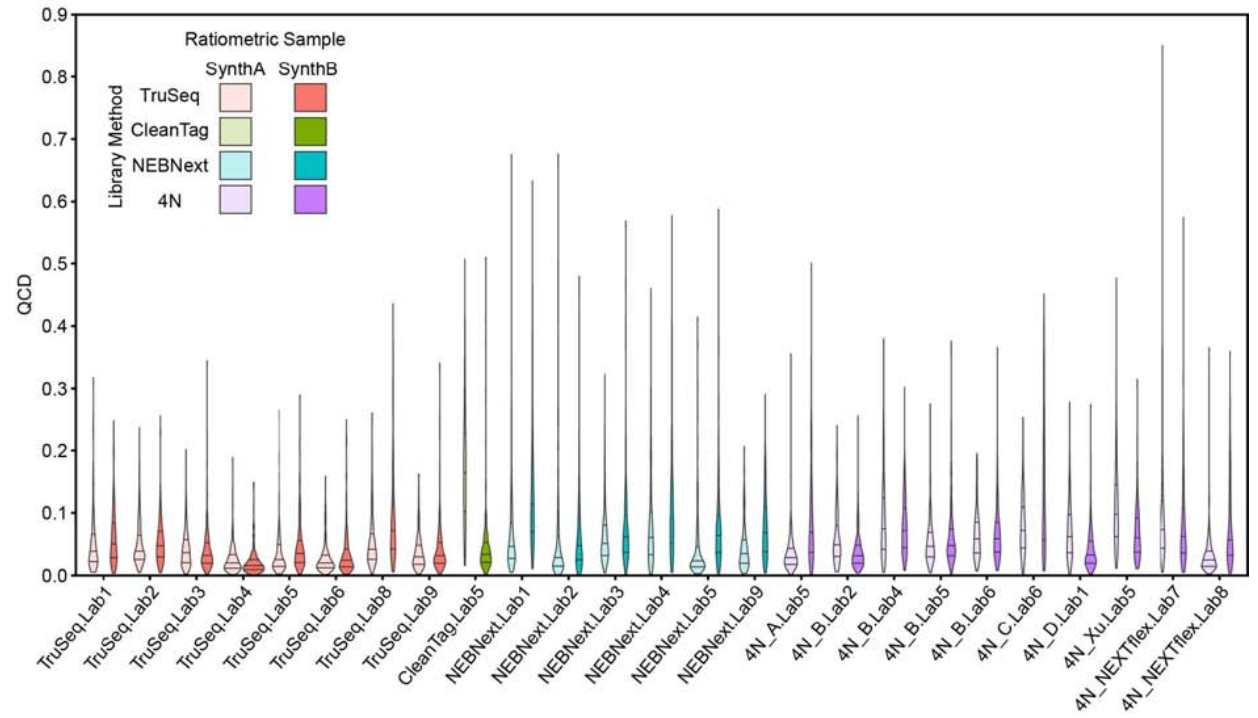

Supplementary Figure 14. Small RNA-seq accuracy in detecting differentially abundant miRNAs between ratiometric synthetic RNA pool samples SynthA and SynthB. Intralab reproducibility of CPM measurements in datasets from sequencing the SynthA and SynthB pools. Violinplotsshowthepercent\%CoefficientofVariation(CV)andquartilecoefficientof dispersion (QCD) calculated across technical replicates of the SynthA and SynthB libraries. Plots are grouped and color-coded by library preparation method (Truseq - pink; CleanTag - green; NEBNext = turquoise; $4 \mathrm{~N}=$ purple), with SynthA and SynthB libraries shown as lighter or darker shades, respectively. Horizontal lines within each violin indicate the 1st, 2nd and 3rd quartiles. 
S. Fig $15(A)$

\section{TruSeq}

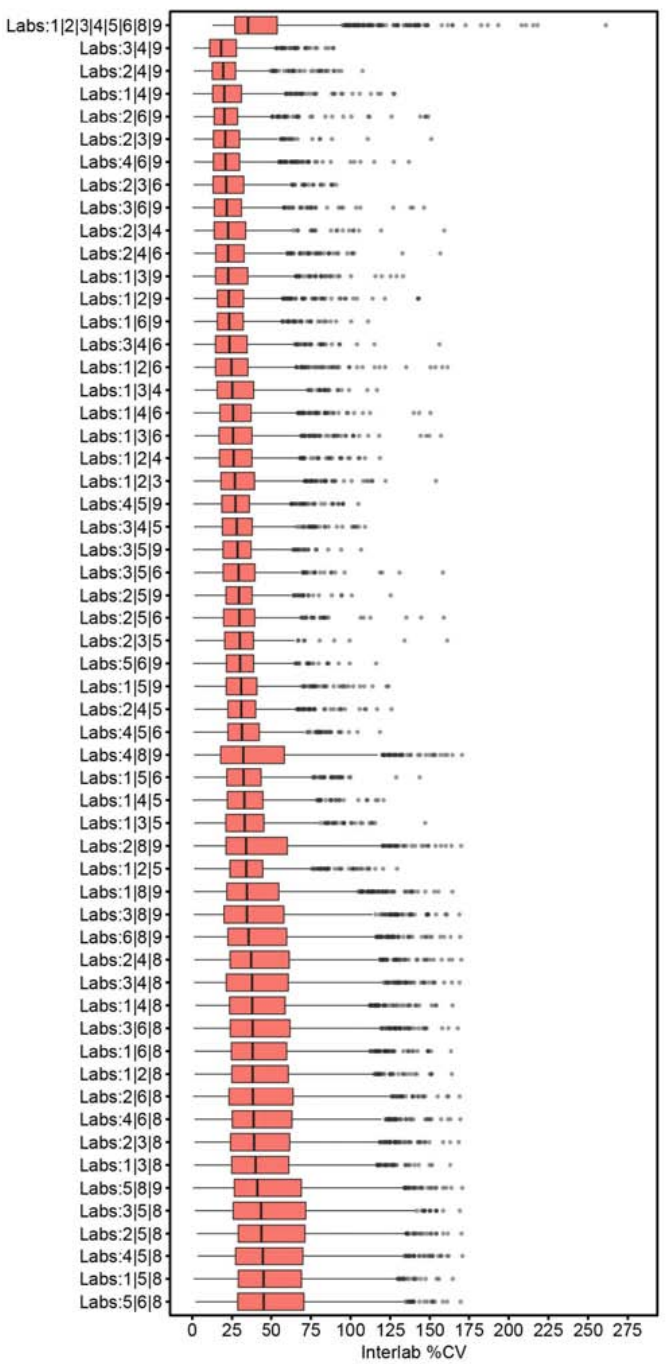

\section{NEBNext}

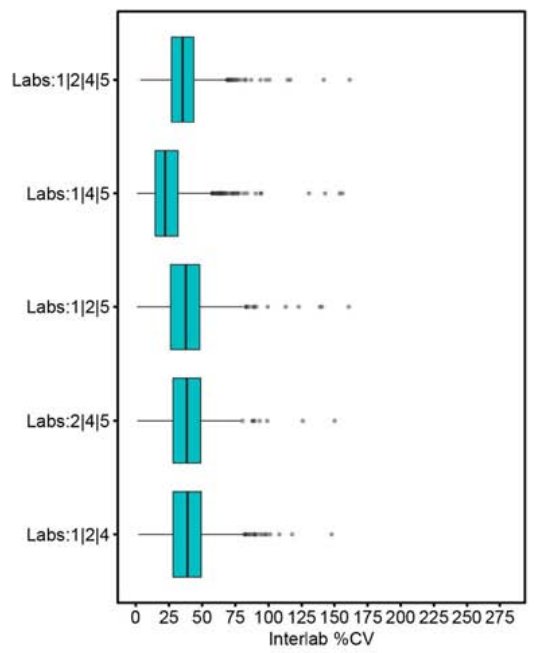

4N_B

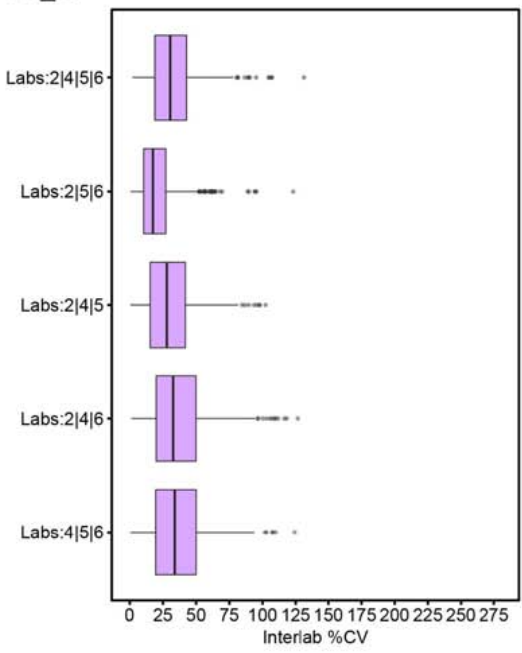




\section{TruSeq}

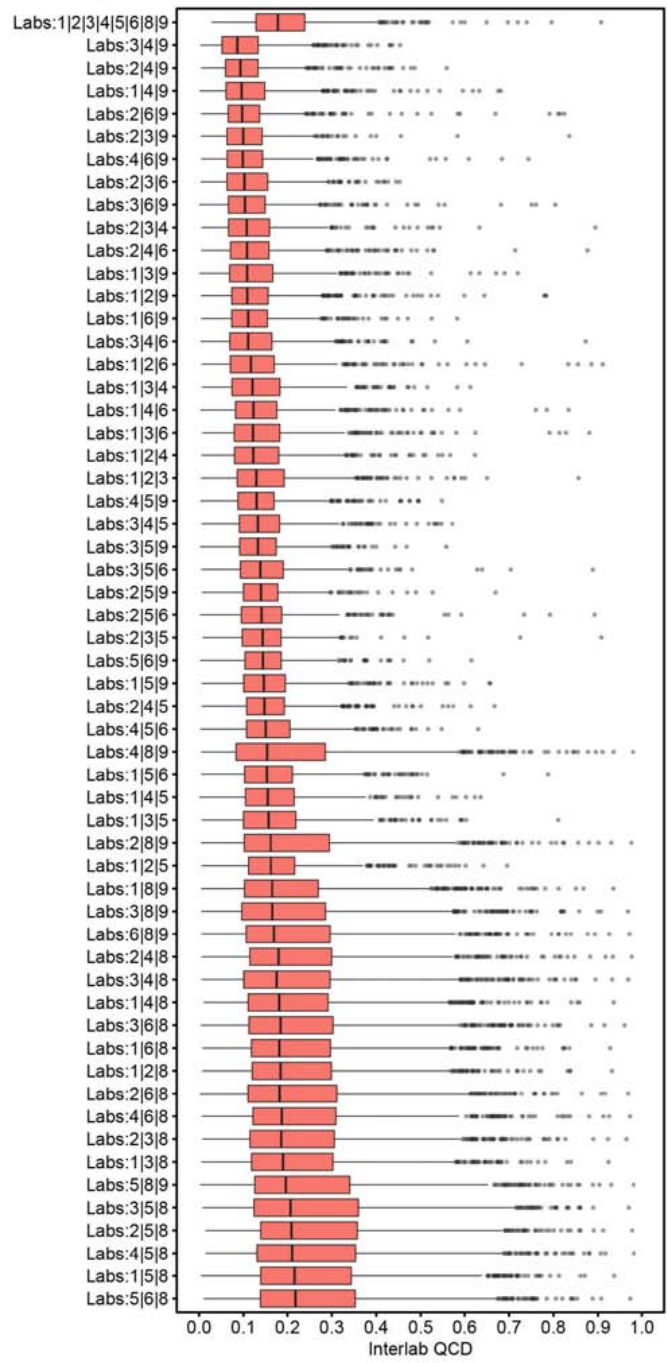

\section{NEBNext}

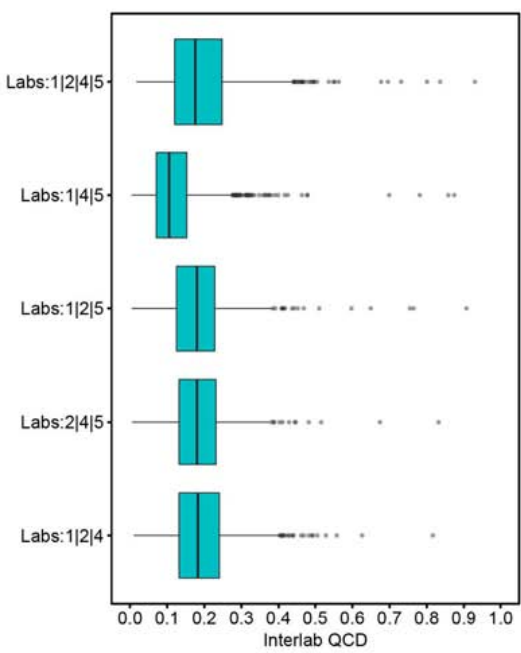

\section{N_B}

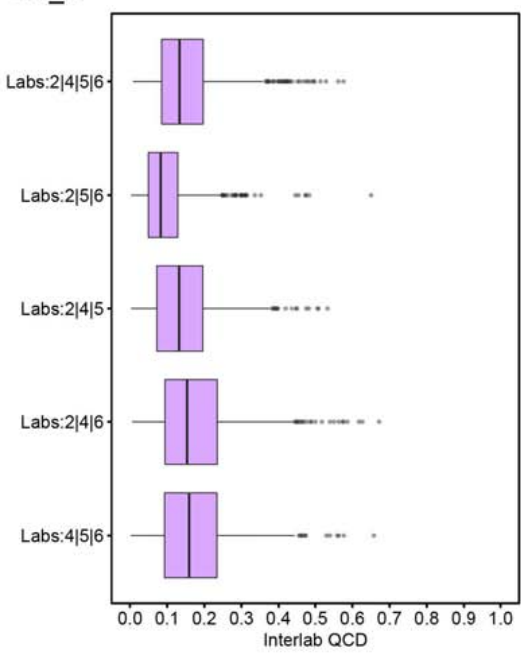

Supplementary Figure 15. Assessment of inter-lab reproducibility in smaller subsets of equimolar samples. Boxplots summarize inter-lab estimates of a) CV and b) QCD calculated using equimolar data from all three lab combinations possible for TruSeq, NEBNext or 4N_B samples. The calculations were performed as in Figure 4. A boxplot for the full set of labs used in Figure 4 is presented as the top boxplot in each figure for comparison. The $y$-axis indicates the lab identities in each group. The boxes represent the median and IQR. Whiskers represent the $1^{\text {st } /} 3^{\text {rd }}$ quartile $+/-1.5 * \mid Q R$. 


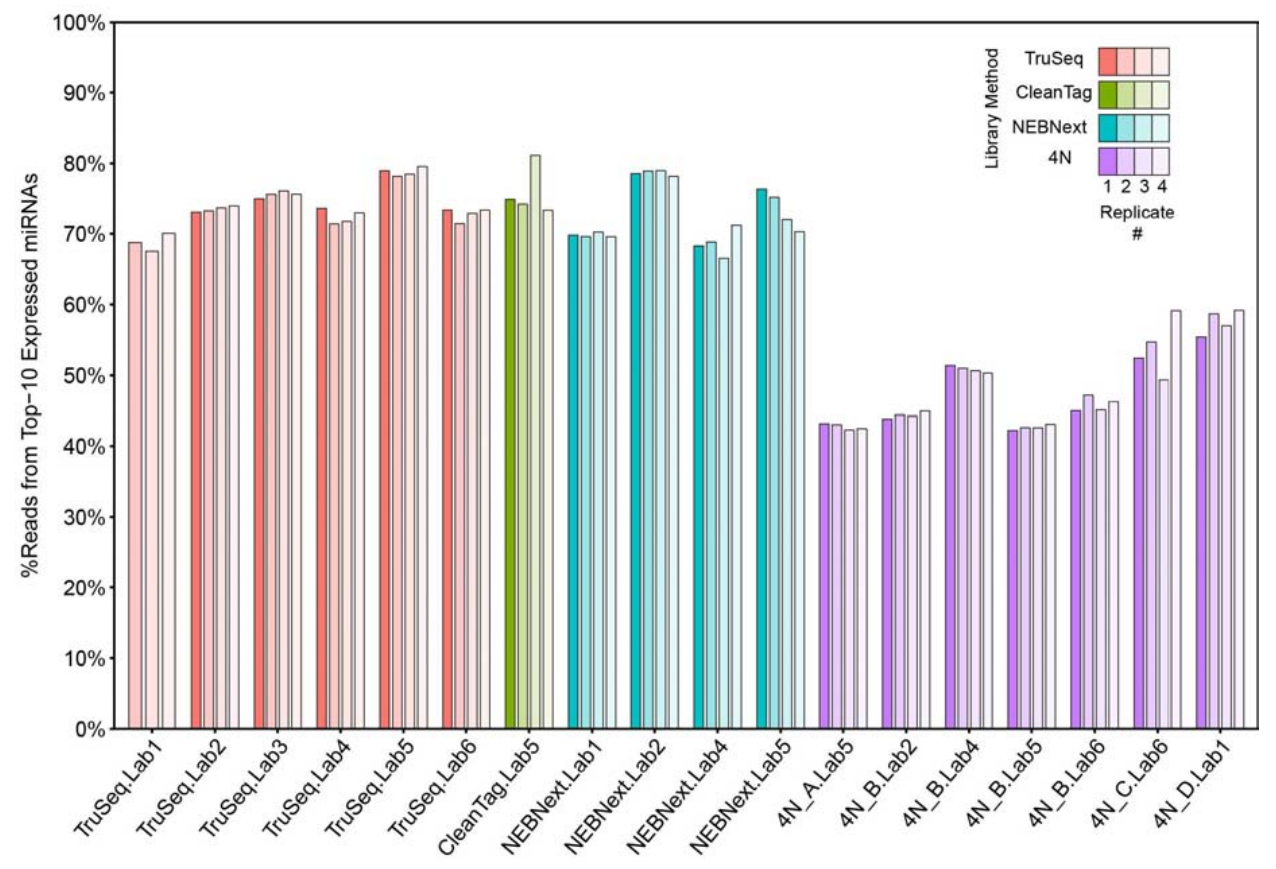

Supplementary Figure 16. Percent contribution of 10 highest-expressed miRNAs in the Plasma Pool samples. The bar plot shows the fraction of miRNA-mapping reads corresponding to the 10 miRNAs with the highest read counts sequenced in each sample. The $y$-axis shows the sum of the multi-mapping-scaled read counts for the top 10 mature miRNAs / sum of the multi- mapping-scaled read counts for all mature miRNAs. The x-axis separates distinct samples, with each bar grouped and different shades of the same color representing different technical replicates. Samples are color-coded by library preparation protocol (TruSeq - pink; CleanTag green; NEBNext - turquoise; $4 \mathrm{~N}$ - purple). Technical replicates are shaded from darkest to lightest corresponding to replicate numbers 1 through 4. 
S. Fig 17 (A)

S17a) Overview of generation and application of CFs to Training (SynthEQ) and Test (Plasma) Data.

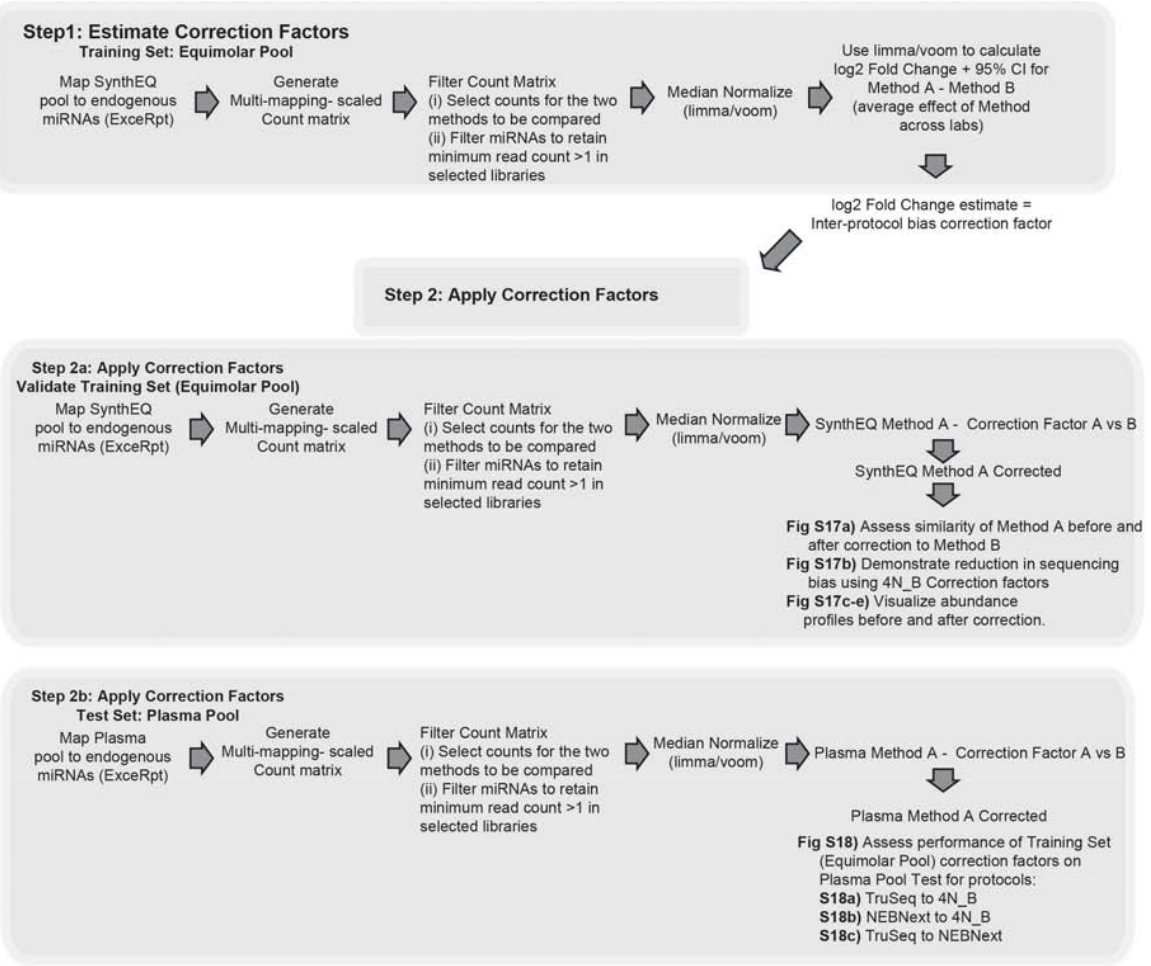

S. Fig $17(B, C)$

b
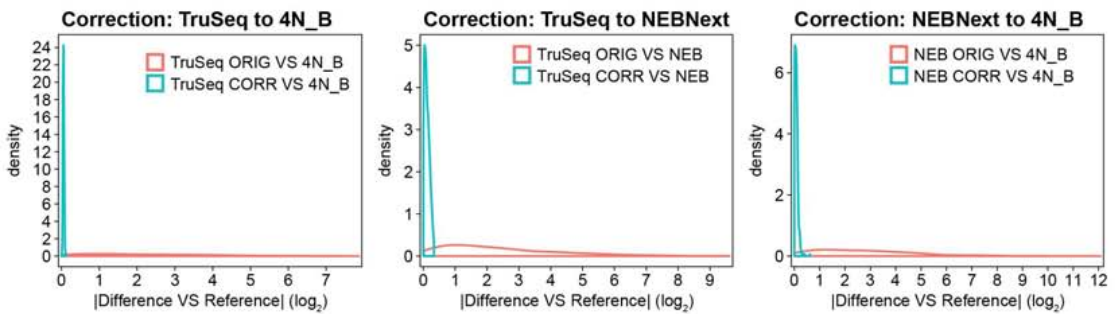

c
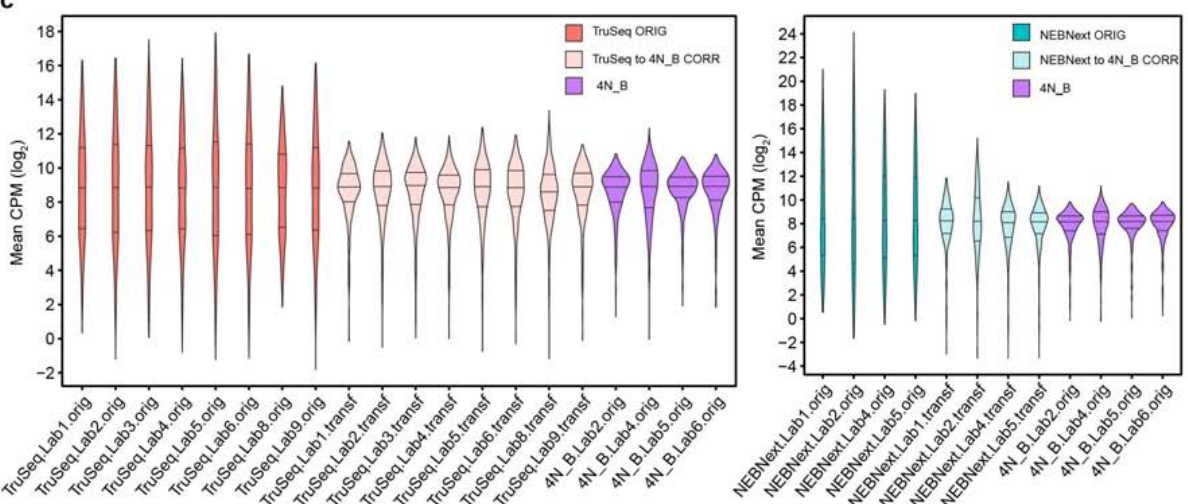
S. Fig 17 (D)

Figure S17d) Correction Factors: TruSeq to 4 N_B (SynthEQ)

CPM $\left(\log _{2}\right)$

15

50

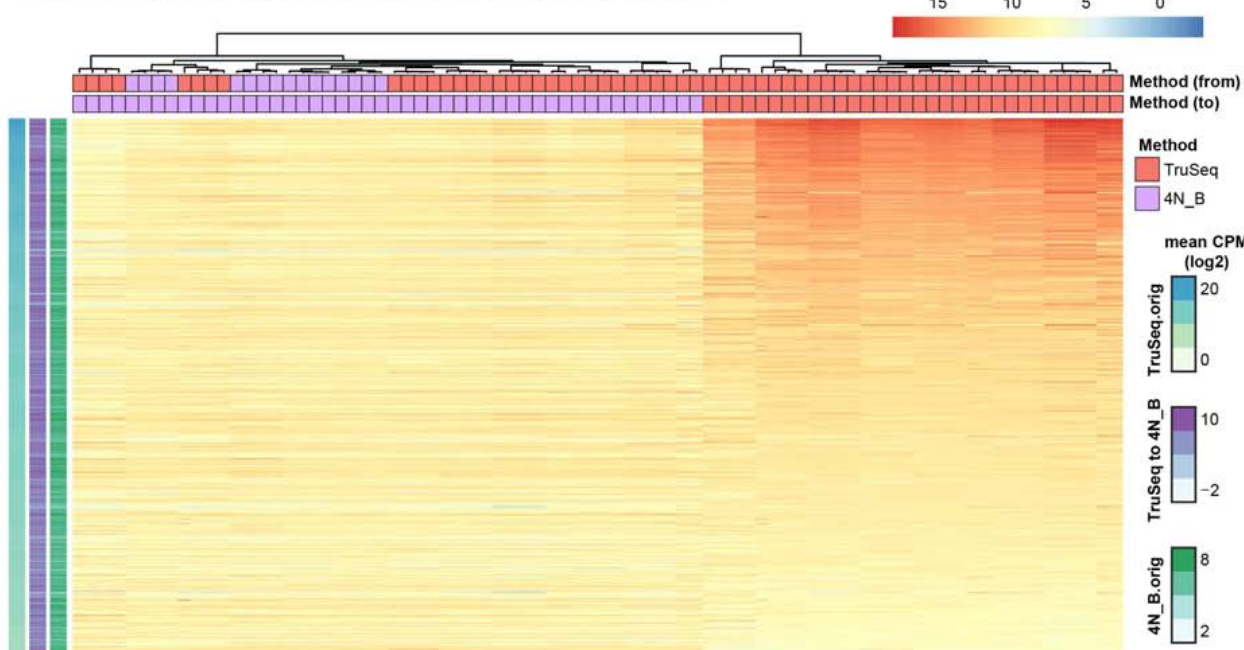

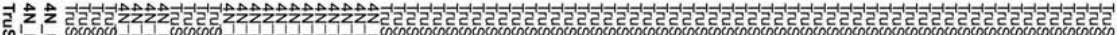

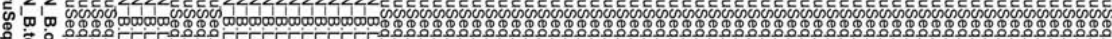

O.

फ

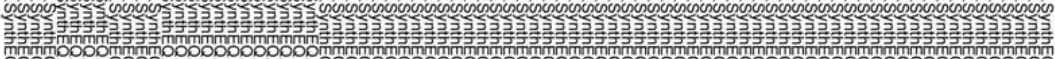

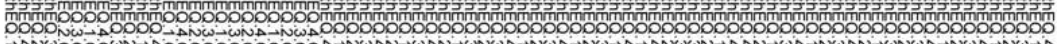

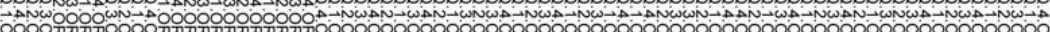

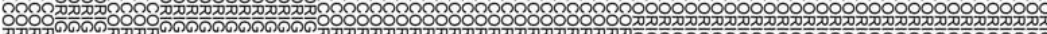

गु 
S. Fig 17 (E)

Figure S17e Correction Factors: TruSeq to NEBNext (SynthEQ)

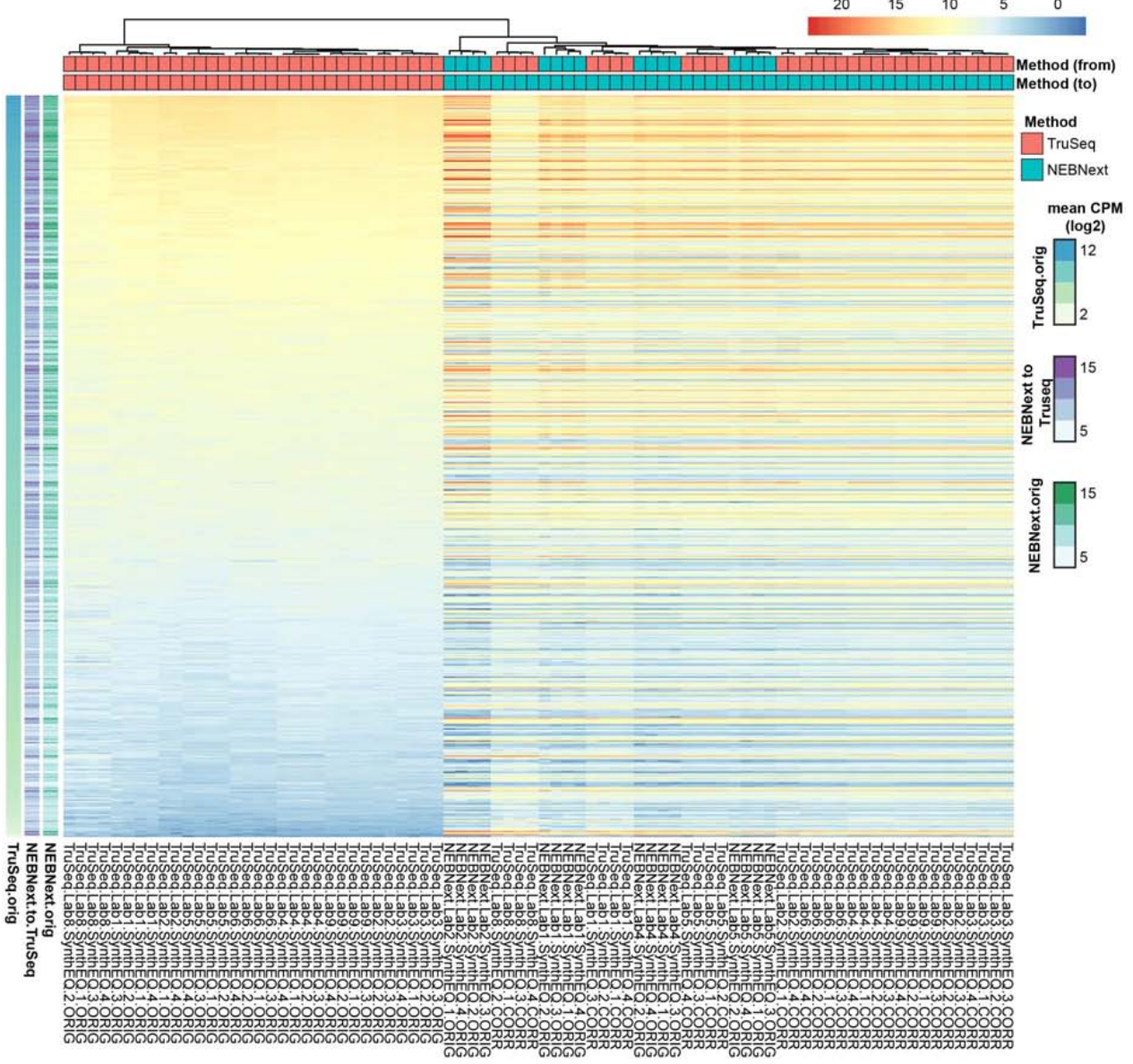


S. Fig $17(\mathrm{~F})$

Figure S17f) Correction Factors: NEBNext to 4N_B (SynthEQ)

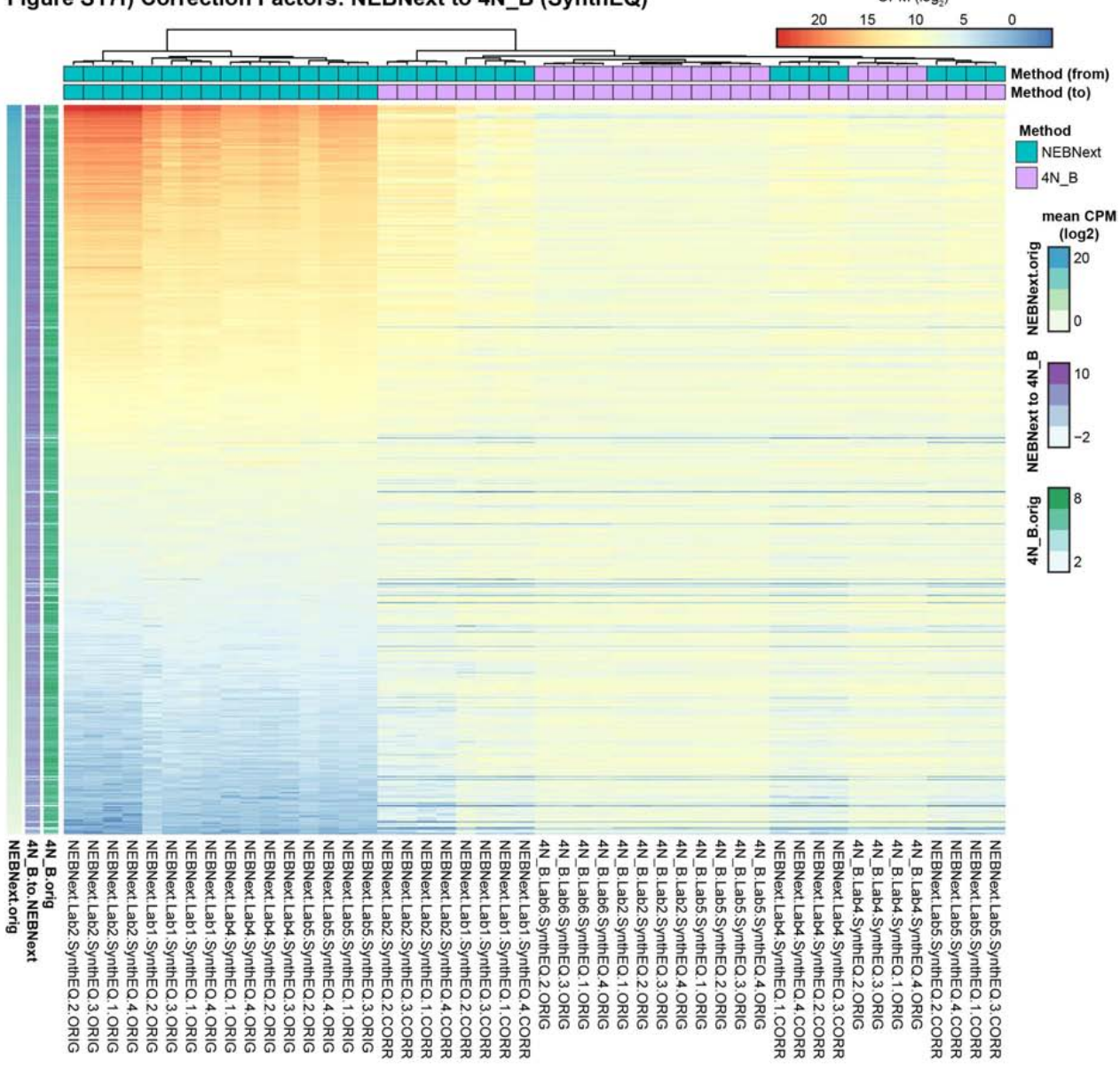


Supplementary Figure 17. Calculation of correction factors in the Equimolar pool. (a) Schematic outlining the steps used to estimate correction factors. In Step 1, correction factors are estimated using the Equimolar Pool training set. In Step 2, the correction factors are applied to either the Equimolar Pool (Step 2a; middle) or to the Plasma Pool test set (Step 2b; bottom). Supplementary figures summarizing the results for Step 2a (Supplementary Figures $8 b-f$ ) and Step $2 \mathrm{~b}$ (Supplementary Figures $8 \mathrm{a}-\mathrm{c}$ ) are indicated. (b) Probability density plots summarizing the difference in abundance estimates between an indicated protocol before correction (ORIG; pink) and after correction (CORR; turquoise), as compared to the indicated reference. The $\mathrm{x}$-axis represents the mean absolute log2-fold difference between the original or corrected miRNAs and the reference. The different plots show TruSeq versus the 4N_B reference (left); TruSeq versus the NEBNext reference (middle), and NEBNext versus the 4N_B reference (right). For NEBNext, all equimolar data in this figure were from the 4 labs which followed the most similar protocol. (C) Violin plots showing the improvement in sequence bias in the equimolar pool samples when 4N_B correction factors are applied to TruSeq (left) and NEBNext (right) libraries. The y-axis indicates the mean log2 counts-per-million (CPM) across technical replicates. The untransformed (ORIG) CPM values are shaded dark (pink-TruSeq; Turquoise- NEBNext; purple-4N_B). The light pink and light turquoise plots show the 4N_B-corrected TruSeq and NEBNext values, respectively. (d-f) Heatmaps showing the abundance (quantile- normalized log2CPM) of equimolar pool sequences before and after correction to the indicated reference, along with the untransformed reference samples. Correction factors were calculated from equimolar pools to correct (d) TruSeq to 4N_B (e) TruSeq to NEBNext and (f) NEBNext to 4N_B. Hierarchical clustering for columns (samples) represents complete linkage clustering on Euclidean distances. Rows (miRNAs) are sorted by mean miRNA abundance calculated from the untransformed source method samples (d- TruSeq; e-TruSeq; f-NEBNext), in descending order. Row-wise mean log2 abundance is provided in the heatmaps to the left of each plot, indicating the values measured for the reference method, and the source method with and without correction. 
S. Fig $18(\mathrm{~A})$
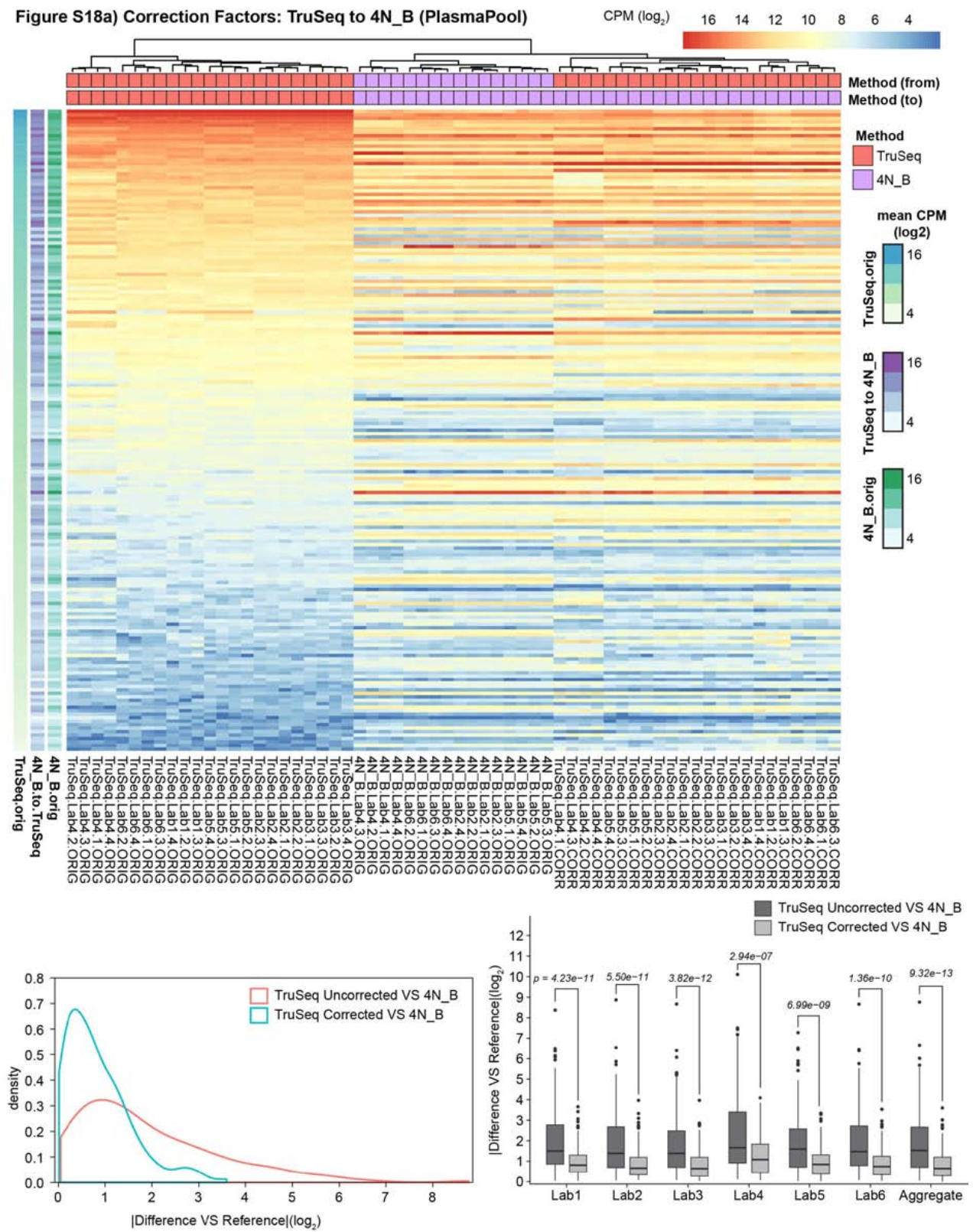
S. Fig 18 (B)

Figure S18b) Correction Factors: TruSeq to NEBNext (PlasmaPool)

CPM $\left(\log _{2}\right)$

15

10

5
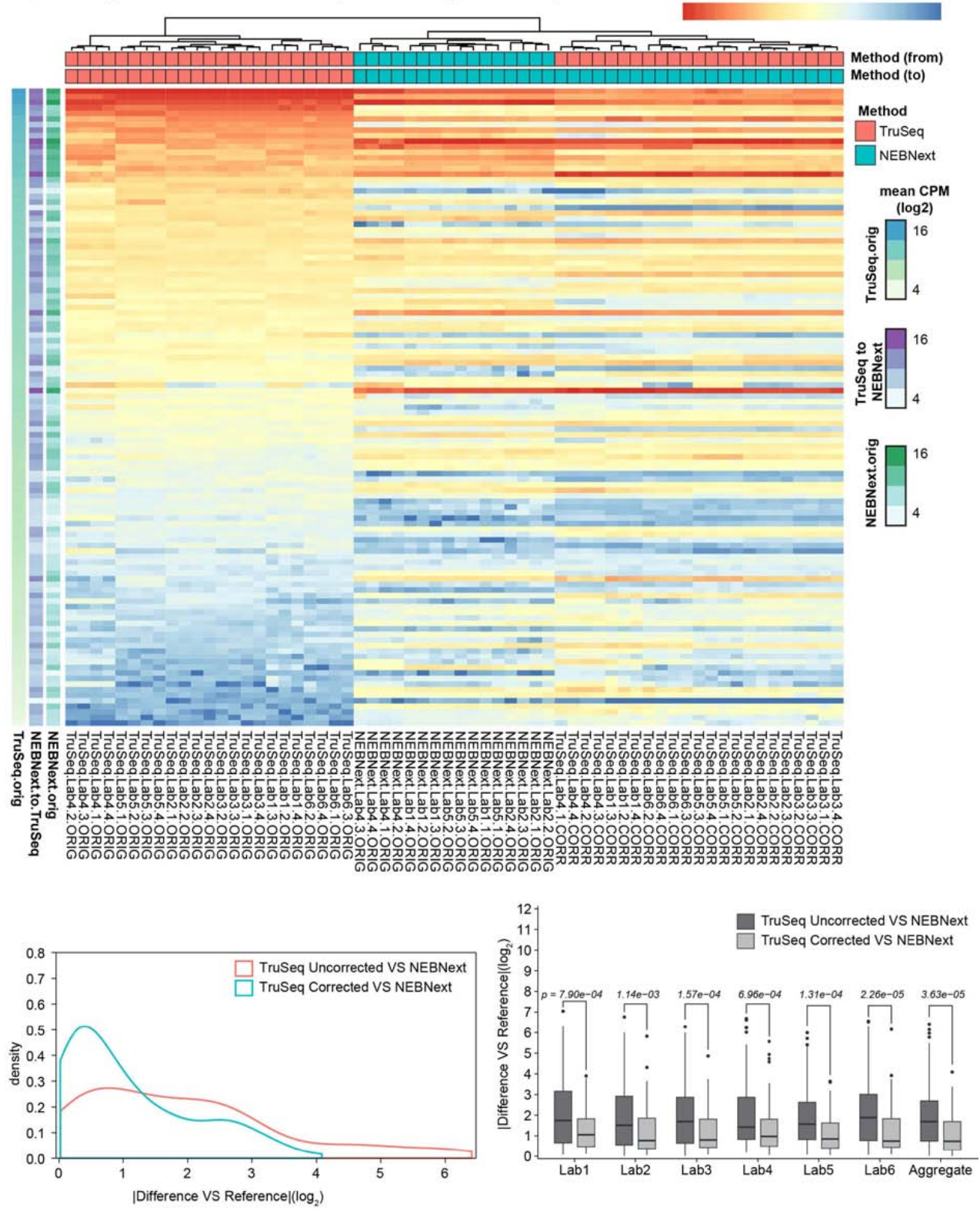
S. Fig 18 (C)

Figure S18c) Correction Factors: NEBNext to 4 N_B
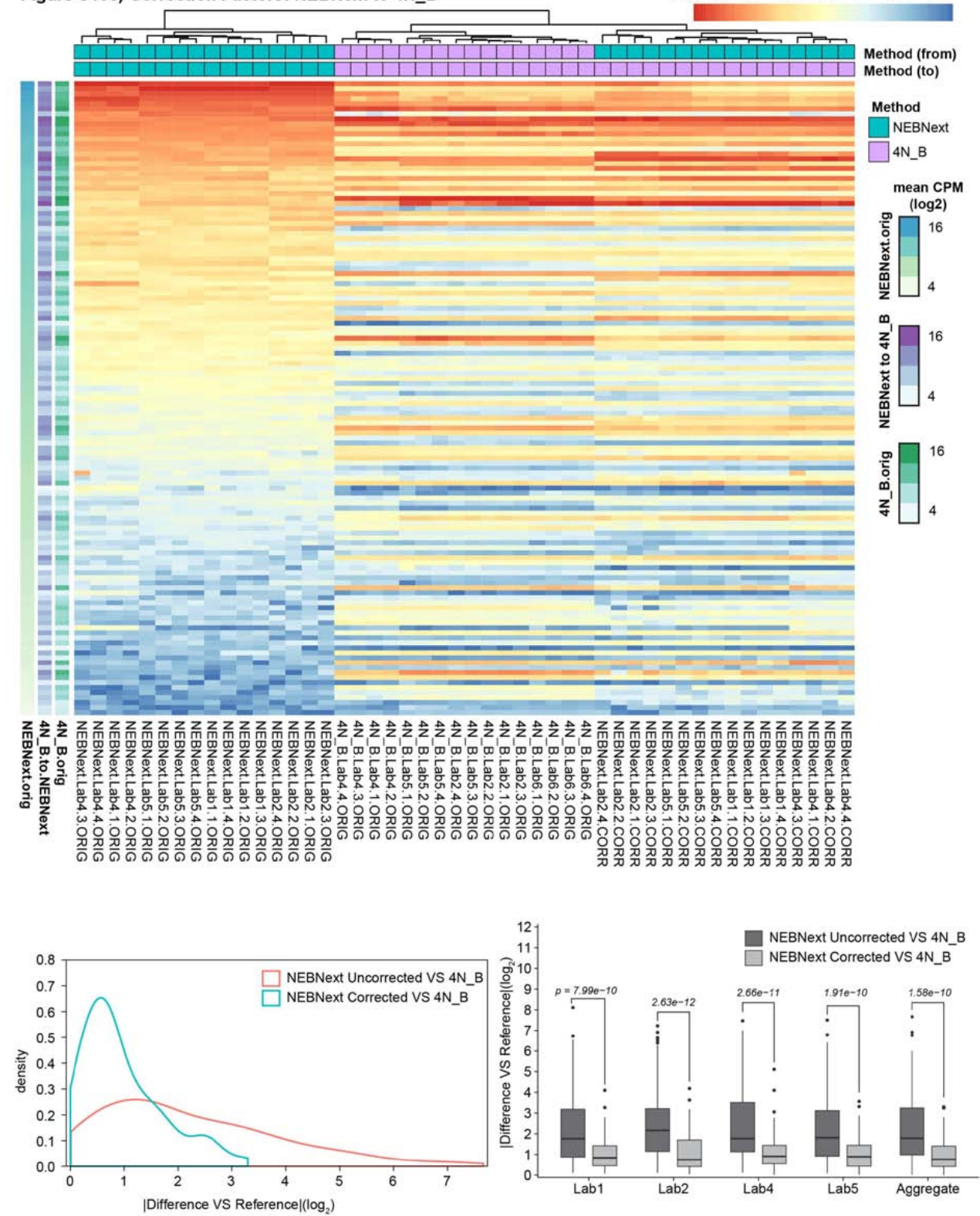
Supplementary Figure 18. Application of computational correction factors to plasma small RNA-seq datasets. Three plots summarizing the performance of inter-protocol bias correction factors calculated from the Equimolar Pool training set and applied to the Plasma Pool libraries are provided. Plots are shown for three sets of correction factors: (a) TruSeq to a 4N_B reference (b) TruSeq to NEBNext reference and (c) NEBNext to a 4 N_B reference. The same type of three summary plots are used for each comparison, and are described as follows. Top: heatmap showing the expression (quantile-normalized log2 CPM) of Plasma Pool sequences before and after correction to the indicated reference, along with those of the untransformed reference samples. Hierarchical clustering for columns (samples) represents complete linkage clustering on Euclidean distances. Rows (miRNAs) are sorted by mean miRNA abundance calculated from the untransformed source method samples (a-TruSeq; b-NEBNext; c-TruSeq). On the far left, a summary heatmap is shown, depicting the row-wise mean log2 expression for the respective reference plasma pool libraries, and the respective source libraries before and after correction. Sample IDs and annotations corresponding to plasma data before or after correction factors were applied are marked as "ORIG" and "CORR", respectively. Bottom left: probability density plots showing the distribution of average absolute log2 fold differences in miRNA CPM between the indicated reference plasma libraries and the source libraries before correction (pink line) and after correction (turquoise line) factors were applied. Bottom right: boxplots representing the average absolute log2 fold difference in CPM of RNA sequences between uncorrected (dark grey) and corrected (light grey) source plasma data, relative to the respective reference, as observed in data from each lab. For each lab, the average log2 CPM was calculated for every sequence before and after correction, and the absolute difference was calculated from the average log2 CPM measured across the respective reference libraries. Wilcoxon rank-sum tests were used to test whether the distribution of the untransformed values versus the respective reference was significantly higher than that of the transformed values versus reference. P-values for each test are indicated above each plot. The "Aggregate" plot is a boxplot representation and test corresponding to the aggregate data shown in the density plot to theleft. 


\section{Chapter 3}

\section{Technical approaches to reduce interference of Fetal Calf Serum derived RNA in the analysis of extracellular vesicle RNA from cultured cells}

Tom A.P. Driedonks, Maarten K. Nijen Twilhaar and Esther N.M. Nolte-'t Hoen*

Department of Biochemistry \& Cell Biology, Faculty of Veterinary Medicine, Utrecht University, Utrecht, The Netherlands

${ }^{*}$ corresponding author Esther N.M. Nolte-'t Hoen

Journal of Extracellular Vesicles, Vol 8, 2018 


\section{Abstract}

Fetal calf serum (FCS) is a common supplement of cell culture medium and a known source of contaminating extracellular vesicles (EV) containing RNA. Because of high sequence homology between non-coding RNA of mammalian species, residual FCSRNA in culture medium may interfere in the analysis of EV-RNA released by cultured cells. Recently, doubts have been raised as to whether commonly used protocols for depletion of FCS-EV efficiently remove FCS-RNA. Moreover, technical details in FCS-EV depletion protocols are known to vary between labs, which may lead to inter-study differences in contaminating FCS-RNA levels. Here, we investigated how technical modifications of EV-depletion protocols affect the efficiency with which bovine RNAs are depleted from FCS, and determined the contribution of contaminating bovine RNA to EV-RNA purified from cell cultures. Our data show differences in depletion efficiency between various classes of small non-coding RNA. Importantly, we demonstrate that variations in FCS-EV depletion protocols affect both the quantity and type of residual FCS-RNAs in EV-depleted medium. By using optimized FCS-EV depletion protocols combined with methods for high-grade purification of EV, a large proportion of FCS-RNA can be eliminated. However, with illustrative datasets we also demonstrate that the abundance of a specific RNA in cell culture EV can only be determined if measured relative to background levels of this RNA in medium control samples. These data highlight the need for optimization and validation of existing and novel FCS-EV depletion methods and urge for accurate descriptions of these methods in publications to increase experimental reproducibility.

Keywords: Fetal calf serum, extracellular vesicles, small non-coding RNA, in vitro cell culture, culture medium 


\section{Introduction}

The characterization of EV-enclosed RNA has gained enormous interest over the last decade, because of its involvement in cell-cell communication and potential use as biomarkers for disease (1-5). For analysis of the RNA content of EV released by in vitro cultured cells it is particularly important to deplete (EV-associated) RNAs from Fetal calf serum (FCS), which is a common supplement of cell culture medium and a known source of contaminating EV (6-9). Residual bovine small non-coding RNAs in FCS may be erroneously mapped to human or murine genomes due to high sequence homology, thereby confounding sequencing analyses of EV-RNA (10). Additionally, the presence of homologous FCS-derived RNAs in culture medium may affect RT-qPCR based quantification of RNA in EV released by cultured cells.

RNA in serum is not only enclosed in EV but can also be associated to other macromolecular structures, such as ribonucleoprotein particles (RNPs) and lipoprotein particles. These structures overlap in size and/or density with EV and may be coisolated in commonly used EV isolation procedures, such as ultracentrifugation $(9,11-$ 13). A number of studies have addressed the removal of contaminating FCS-EV and FCS-derived RNA by ultracentrifugation and/or ultrafiltration $(7,8,10,14)$, and doubts were raised on whether these methods allow effective depletion of extracellular RNA from bovine serum (10). However, it is also known that technical details in FCS-EV depletion protocols vary between labs. It is unclear to what extent differences in FCSEV depletion methods introduce unwanted variation in EV-RNA data.

Here, we investigated technical modifications of EV depletion protocols that affect the efficiency with which different classes of small non-coding RNAs are depleted from FCS. In illustrative experiments, we furthermore determined the extent to which residual FCS-RNA in EV-depleted medium affects analysis of the RNA content of cell culture-derived EV purified by density gradient ultracentrifugation. Our data show that variations in FCS EV-depletion protocols influence the quantity and type of residual FCS-derived RNA in culture medium. Additionally we show that accurate analysis of small non-coding RNAs in cell culture EV requires parallel assessment of residual levels of these RNAs in medium control samples.

\section{Materials and methods}

\section{FCS-EV depletion}

Fetal Calf Serum (FCS, lot BDC-12270, Bodinco, Alkmaar, the Netherlands) was left undiluted (100\%) or was diluted with Iscove's Modified Dulbecco's Medium (IMDM, Lonza, Verviers, Belgium) to $30 \%$ or $10 \%$ (vol/vol \%) (Figure 1A, top panel). (Diluted) 
FCS was spun overnight for $15-18 \mathrm{hrs}$ at $100,000 \times \mathrm{g}$ in a SW28 rotor (k-factor 334.2 ) or SW40 rotor (k-factor 381.5) (Beckman Coulter, Brea, CA). Supernatant was collected using a pipette, leaving at least $5 \mathrm{ml}$ (SW28 tubes) or $1.5 \mathrm{ml}$ (SW40 tubes) of fluid on top of the pellet. The pellet was resuspended in the residual fluid by pipetting up and down. This 'pellet fraction' contained all material that was depleted from the FCS and which would normally be discarded. RNA was subsequently isolated from both the pellet and the EV-depleted supernatant.

For preparation of EV-depleted medium, EV-depleted supernatant (30\% FCS in IMDM) was collected with a pipette (as described above), subsequently diluted to $10 \%$ FCS, and supplemented with 2 mM Ultraglutamine (Lonza, Verviers, Belgium), 100 IU/ $\mathrm{mL}$ penicillin and $100 \mu \mathrm{g} / \mathrm{ml}$ streptomycin (Gibco, Paisley, UK). Where indicated, EVdepleted supernatant (30\% FCS in IMDM) was not recovered by pipetting (as described above), but instead by decanting the fluid (Figure 1A, middle panel). Non-depleted medium was supplemented with $10 \%$ non-depleted FCS and the above mentioned concentrations of ultraglutamine, penicillin, and streptomycin.

\section{Cell culture}

Human Embryonic Kidney (HEK293T) cells (adherent) and murine B-lymphoblast (A20) cells (non-adherent) were grown in IMDM supplemented with 10\% FCS, 2 mM Ultraglutamine, $100 \mathrm{IU} / \mathrm{mL}$ penicillin and $100 \mu \mathrm{g} / \mathrm{ml}$ streptomycin. HEK293T cells were passaged twice a week using $0.05 \%$ trypsin/EDTA for cell detachment (Gibco, Paisley, UK). A20 cells were passaged twice a week by dilution in medium. For EVproduction, HEK293T cells were seeded in complete medium (36 - 45 million cells per batch, divided over 6 T175 flasks), and incubated for $24 \mathrm{~h}$ to attach to the flask surface. Subsequently, the monolayer was washed in 1x PBS (Gibco, Paisley, UK), after which cells were cultured in $210 \mathrm{~mL}$ EV-depleted medium per batch (prepared as described above) for $20 \mathrm{~h}$. A20 cells (56 - 79 million cells per batch, divided over 2 T75 flasks placed upright) were spun down, medium was removed from the pellet and cells were cultured in $70 \mathrm{~mL}$ EV-depleted medium for 20h. Confluency of HEK293T cells was more than $80 \%$ at harvest. Cell viability at harvest of HEK293T and A20 cells was determined by Trypan blue exclusion, which was above $90 \%$ for all cultures. All cells were maintained at $37^{\circ} \mathrm{C}$ and $5 \% \mathrm{CO} 2$ in a humidified incubator.

\section{Isolation and fluorescent labeling of EV}

EV were isolated from identical volumes of cell conditioned medium and nonconditioned control medium (Figure 1A, bottom panel) via differential centrifugation and density gradient ultracentrifugation as described previously (15). In brief, supernatants were centrifuged 2x $200 \times \mathrm{g}$ for $10 \mathrm{~min}, 2 \times 500 \times \mathrm{g}$ for $10 \mathrm{~min}, 1 \times 10,000$ $x \mathrm{~g}$ for $30 \mathrm{~min}$. Subsequently, EV were pelleted by ultracentrifugation for $65 \mathrm{~min}$ at 
$100,000 \times g$ in a SW28 rotor (k-factor 334.2) (Beckman Coulter, Brea, CA). EV pellets were resuspended in $50 \mu \mathrm{l}$ PBS $+0.2 \% \mathrm{BSA}$ (depleted from protein aggregates by overnight ultracentrifugation at $100,000 \times$ g), mixed with $1.5 \mathrm{~mL} 2.5 \mathrm{M}$ sucrose, after which a linear sucrose gradient (2.0 M - 0.4 M sucrose in PBS) was layered on top of the sample. Gradients were centrifuged $15-18 \mathrm{~h}$ at $192,000 \times \mathrm{g}$ in a SW40 rotor (k-factor 144.5) (Beckman Coulter, Brea, CA). EV-containing gradient fractions with densities of 1.12 - $1.18 \mathrm{~g} / \mathrm{ml}$ were diluted 6 times in PBS + 0.2\% EV-depleted BSA after which EV were pelleted at 192,000 $\mathrm{g}$ for $65 \mathrm{~min}$ in a SW40 rotor (k-factor 144.5). For EV quantification by high-resolution flow cytometry, 100,000 x $g$ EV-pellets were resuspended in $20 \mu \mathrm{l}$ PBS + 0.2\% BSA and labeled with 1.5 $\mu$ I PKH67 (Sigma, St. Louis, MO) in $180 \mu$ l Diluent $C$ per pellet. Labeled EVs were purified by sucrose density gradient centrifugation as described above prior to analysis by high-resolution flow cytometric analysis (see below).

\section{RNA isolation}

For RNA isolation directly from solutions (non-depleted medium, EV-depleted supernatants and pellet fractions from 100\% / 30\% / 10\% FCS dilutions), the Qiagen miRNeasy micro Serum/Plasma protocol (Qiagen, Hilden, Germany) was used. Prior to RNA isolation, supernatant samples of $30 \%$ and $100 \%$ serum conditions were diluted $3 x$ or 10x, respectively, to prevent potential interference of serum proteins and/or lipids with RNA isolation efficiency. Input volumes were $1.2 \mathrm{~mL}$ for non-depleted medium and EV-depleted FCS supernatant (with FCS concentrations equalized to 10\%), and $0.2 \mathrm{~mL}$ for pellet fractions. Volumes of reagents (Qiazol, chloroform and $100 \%$ ethanol) that are used before binding of RNA to the miRNeasy spin columns, were adjusted to sample volumes as recommended by the manufacturer. Each sample was loaded onto one miRNeasy spin column, after which regular volumes of wash buffers were used. Elution of each sample was done in $15 \mu \mathrm{l}$ RNAse free water. To isolate RNA from density gradient purified EV pellets, the miRNeasy micro kit was used according to the manufacturer's instructions without modifications (Qiagen, Hilden, Germany). RNA yields and size profile were assessed using the Agilent 2100 Bioanalyzer with Pico 6000 RNA chips (Agilent Technologies, Waldbronn, Germany).

\section{$R T-q P C R$ and cDNA synthesis}

Equal volumes of isolated RNA were used to quantify differences in specific RNA transcripts between samples. CDNA was prepared using the miScript RT2 kit (Qiagen, Hilden, Germany). Per qPCR reaction, $2 \mu$ of 10 times diluted cDNA template was used with $100 \mathrm{nM}$ primers (IDT, Leuven, Belgium) and $4 \mu \mathrm{l}$ SYBR Green Sensimix (Bioline Reagents Ltd., United Kingdom) in an $8 \mu$ l reaction. No-RT-controls confirmed the absence of genomic DNA and non-specific amplification. Cycling conditions were 
$95^{\circ} \mathrm{C}$ for $10 \mathrm{~min}$ followed by 50 cycles of $95^{\circ} \mathrm{C}$ for $10 \mathrm{~s}, 57^{\circ} \mathrm{C}$ for $30 \mathrm{~s}$ and $72^{\circ} \mathrm{C}$ for $20 \mathrm{~s}$. Subsequently, a melting curve analysis was performed.

All PCR reactions were performed on the Bio-Rad iQ5 Multicolor Real-Time PCR Detection System (Bio-Rad, Hercules, CA). Quantification cycle (Cq) values were determined using Bio-Rad CFX software using automatic baseline settings. Thresholds were set in the linear phase of the amplification curve.

\section{List of DNA oligo probes}

The following primers were used in RT-qPCR, primers $1-7$ were used in combination with the miScript universal reverse primer, primers 8 - 15 were used as pairs of forward (F) and reverse (R) primers.

\begin{tabular}{lll}
\hline $\mathbf{n r}$ & Primer name & primer sequence $\mathbf{~}^{\prime}$ to $\mathbf{3}^{\prime}$ \\
\hline 1 & bY4-F & GTGGGTTACCAGAACTTATTAA \\
\hline 2 & 7SL-F & GGAGTTCTGGGCTGTAGTGC \\
\hline 3 & miR-451-5p-F & AAACCGTTACCATTACTGAGTTT \\
\hline 4 & miR-1246-F & AAATGGATTTTTGGAGCAGG \\
\hline 5 & U1-F & CCATGATCACGAAGGTGGTTT \\
\hline 6 & miR-122-5p-F & TGGAGTGTGACAATGGTGTTTG \\
\hline 7 & miR-148a-3p-F & TCAGTGCACTACAGAACTTTGT \\
\hline 8 & hY1-F & GGCTGGTCCGAAGGTAGTGA \\
\hline 9 & hY1-R & GCAGTAGTGAGAAGGGGGGA \\
\hline 10 & hY3-F & GGCTGGTCCGAGTGCAGTGG \\
\hline 11 & hY3-R & GAAGCAGTGGGAGTGGAGAA \\
\hline 12 & hY4-F & GGCTGGTCCGATGGTAGTGG \\
13 & hY4-R & TTAGCAGTGGGGGTTGTAT \\
\hline 14 & $5 S-F$ & TCTACGGCCATACCACCCTGA \\
\hline 15 & 5S-R & GCCTACAGCACCCGGTATTCC \\
\hline
\end{tabular}

\section{High resolution flow cytometric analysis of EV}

PKH67-labeled EV were quantified by high resolution flow cytometric analysis using a BD Influx flow cytometer (BD Biosciences, San Jose, CA) with an optimized configuration, as previously described $(15,16)$. In brief, we applied threshold triggering on fluorescent signals from PKH67-labeled EV. Forward scatter (FSC) was detected with a collection angle of $15-25^{\circ}$ (reduced wide-angle (rw) FSC). Fluorescence and rw-FCS settings were calibrated using fluorescent 100- and 200-nm polystyrene beads (FluoSpheres, Invitrogen, Carlsbad, CA). Sucrose gradient fractions containing PKH67-labeled EV were diluted 142x (for A20-derived EVs) or 20x (for all other EV 
measurements) in PBS and vortexed just before measurement. This dilution factor was sufficient to avoid 'coincidence' (multiple EV arriving at the measuring spot at the same time), thereby enabling accurate quantification of EV numbers in different conditions. Moreover, samples were measured below 10,000 events per second, which is far below the limit of the electronic pulse processing speed of the BD Influx (17).

\section{EV-TRACK:}

Written details on experimental procedures have been submitted to the EV-TRACK knowledgebase (EV-TRACK ID: EV180039) (18).

\section{Results and discussion}

A range of different protocols for serum EV depletion are currently in use. To deplete EV from FCS, it was previously recommended to dilute serum prior to overnight ultracentrifugation. This reduces viscosity and increase EV depletion efficiency $(6,19)$. However, the effects of serum dilution on the depletion efficiency of different classes of (EV-associated) serum RNA have not been assessed in detail. We addressed this point by overnight ultracentrifugation of $100 \%, 30 \%$, and $10 \%$ dilutions of FCS at $100,000 \times g$, followed by RNA isolation from supernatants and pellets. For reference, RNA was isolated from medium prepared with FCS not depleted of EV ('non-depleted medium') (Figure 1A, top panel). As expected, overnight centrifugation at 100,000 $\mathrm{g}$ led to accumulation of RNA in the pellets, while the amount of RNA in the EV-depleted supernatant was reduced compared to non-depleted medium (Figure 1B). In line with earlier reports, we observed that not all FCS-derived RNA was depleted by overnight ultracentrifugation (10). We determined the distribution of FCS-RNA over EV-depleted supernatant and pellet fractions for each of the dilutions (with FCS concentrations equalized to $10 \%(\mathrm{v} / \mathrm{v})$ before RNA isolation), and observed that FCS-derived RNA pelleted more efficiently in diluted FCS suspensions (Figure 1C).

Extracellular RNA in FCS can be associated to various macromolecular structures, such as EV, lipoprotein particles, and RNP (11-13), which likely differ in sedimentation efficiency during ultracentrifugation. Moreover, the different RNA carriers in serum may be preferentially associated to different classes of small non-coding RNA. By using RT-qPCR analysis, we therefore assessed the efficiency with which various types of small non-coding RNA could be depleted from FCS. We selected example miRNAs that have been detected in multiple studies on EV-associated RNA, but which were also identified as potential FCS-derived contaminants by Wei et al (10). MiR-451-5p and miR-122-5p are mainly expressed in red blood cells and liver cells, respectively (20), whereas miR-148a-3p (an immune-related miRNA (21)) and miR-1246 (a fragment of 
A
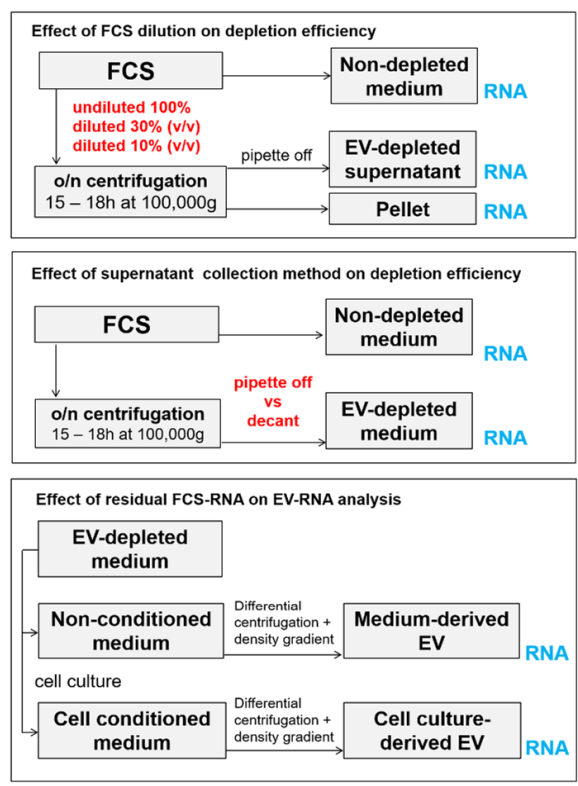

B
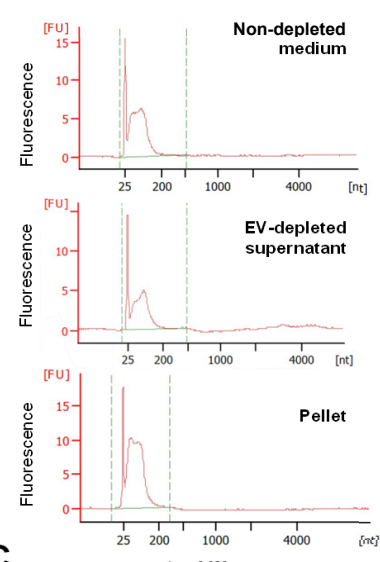

C

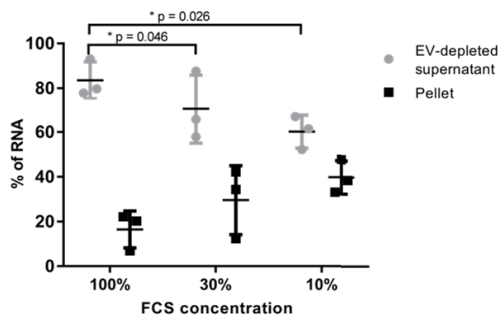

D

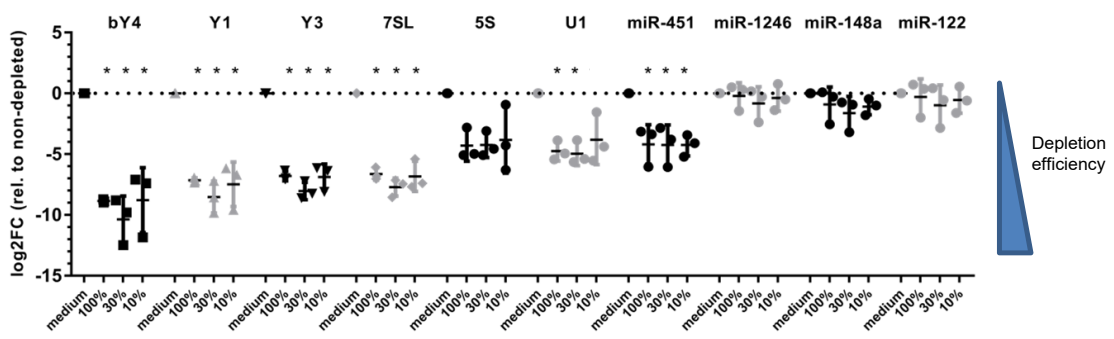

E

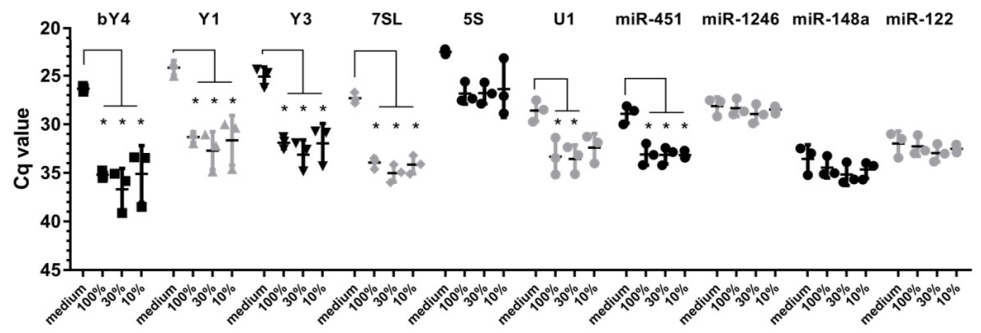

F

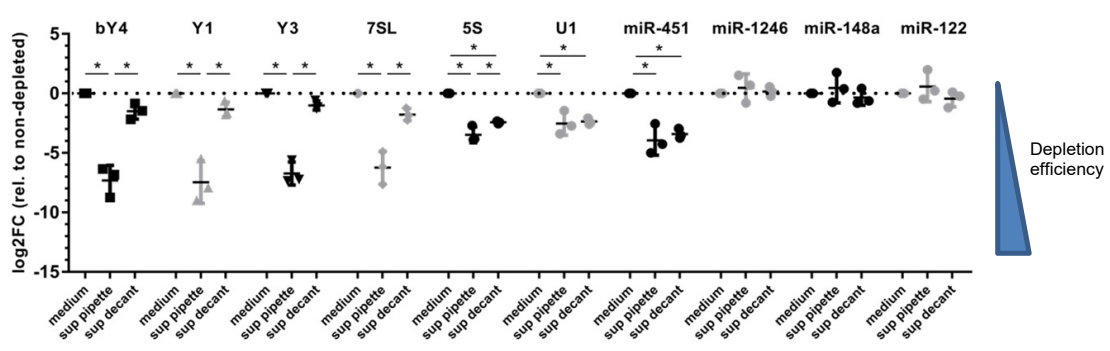


Figure 1.

A) Schematic overview of the experimental setup. Critical methodological parameters that are compared are indicated in red. Top panel: Suspensions of undiluted, $3.3 \mathrm{x}$ and $10 \mathrm{x}$ diluted FCS $(100 \%, 30 \%, 10 \%$ FCS) were depleted from EV by overnight ultracentrifugation (15 $18 \mathrm{~h})$ at $100,000 \times \mathrm{g}$. EV-depleted supernatant was recovered from ultracentrifugation tubes by pipetting. EV-depleted supernatant fractions were diluted 3x or 10x before RNA isolation to correct for differences in FCS concentration. RNA was additionally isolated from pellet fractions and non-depleted medium (containing 10\% FCS). Middle panel: a suspension of diluted FCS $(30 \%)$ was depleted from EV by overnight ultracentrifugation $(15-18 \mathrm{~h})$ at $100,000 \times \mathrm{g}$. EV depleted medium was recovered from ultracentrifugation tubes by pipetting or decanting and diluted 3 times before RNA isolation. RNA was isolated from EV-depleted supernatant and from non-depleted medium (10\% FCS). Bottom panel: EV-depleted supernatant containing $30 \%$ FCS was used to prepare EV-depleted medium in which HEK293T and A20 cells were cultured $20 \mathrm{~h}$ for EV production. Identical volumes of cell conditioned EV-depleted medium or nonconditioned EV-depleted medium were subjected to differential (ultra)centrifugation and density gradient centrifugation to purify cell-derived and/or residual bovine EV. RNA was isolated from EV-containing density fractions $(1.12-1.18 \mathrm{~g} / \mathrm{ml})$.

B) Representative Bioanalyzer Pico chip electropherograms of RNA isolated from pellet and supernatant fractions of EV-depleted medium and from non-depleted medium.

C) Suspensions of $100 \%, 30 \%$, or $10 \% \mathrm{FCS}$ were depleted from EV by overnight ultracentrifugation $(15-18 \mathrm{~h})$ at $100,000 \times \mathrm{g}$. EV-depleted supernatant was recovered from ultracentrifugation tubes by pipetting. Supernatant fractions were diluted $3 x$ and $10 x$ before RNA isolation to correct for differences in FCS concentrations. The concentrations of RNA in supernatants and pellets were assessed on Bioanalyzer. Total RNA quantity in the entire ultracentrifuge tube was calculated by adding up the total RNA quantities in pellet and supernatant fractions, corrected for the dilution factor before RNA isolation. Indicated is the distribution of RNA over supernatant and pellet fractions for each of the FCS dilutions. Mean $+/$ - SD of $n=3$ experiments, * $p<0.05$ ANOVA with Dunnett's one-sided post-hoc test (control condition: 100\% EV-depleted supernatant).

D) Equal volumes of RNA isolated from EV-depleted supernatant of different FCS dilutions (prepared as described in (C), and RNA isolated from non-depleted medium ('medium') were used for RT-qPCR analysis of the indicated RNAs. Depletion efficiencies were calculated from the raw $C q$ values shown in (E) using the $\Delta \Delta C q$ method (26) relative to non-depleted medium. Indicated are mean log2fold change values $+/-S D$ of $n=3$ experiments. Statistical differences were determined by ANOVA with Tukey HSD post-hoc test. ${ }^{*} p<0.05$. No statistical differences were found between the depletion efficiencies in different FCS dilutions.

E) Raw $\mathrm{Cq}$ values of the RT-qPCR data shown in (D). Low $\mathrm{Cq}$ values mean high abundance. Indicated are mean values $+/-\mathrm{SD}$ of $\mathrm{n}=3$ experiments. Statistical differences were determined by ANOVA with Tukey HSD post-hoc test. * $p<0.05$ No statistical differences were observed between different FCS dilutions.

F) Suspensions of $30 \%$ FCS were depleted from EV by overnight ultracentrifugation (15 - 18h) at $100,000 \times \mathrm{g}$. EV-depleted supernatants were recovered from ultracentrifugation tubes by decanting ('sup decant') or pipetting ('sup pipette'). RNA was isolated from EV-depleted supernatants collected by decanting and pipetting (equilibrated to $10 \% \mathrm{FCS}$ ) and from nondepleted medium (containing 10\% FCS). Equal volumes of RNA were used for RT-qPCR analysis of the indicated RNAs. Log2fold change levels relative to non-depleted medium were calculated as in (D). Indicated are mean values $+/-S D$ of $n=3$ experiments. Statistical differences were determined by ANOVA with Tukey HSD post-hoc test, * $p<0.05$. 
snRNA U2 (22)) are expressed in a broader range of cell types. In addition to these miRNAs, we selected several cytoplasmic and nuclear RNAs (5S rRNA, U1 snRNA, Y-RNA, $7 \mathrm{SL}$ ) that have been abundantly detected in multiple studies on EV-associated RNA $(23,24)$. The bovine and human sequences of these RNAs also show $100 \%$ homology. We compared the RNA content of EV-depleted supernatant versus non-depleted medium and observed striking differences in the efficiency with which each of the RNA types could be depleted from FCS (Figure 1D). Various members of the Y-RNA family and 7SL were efficiently depleted, with a 100-fold reduction compared to non-depleted medium. Intermediate depletion efficiencies ( 30-fold reduction) were achieved for 5S rRNA, U1 snRNA and miR-451. In contrast, no significant reduction in miRNA-122 and miR-148a was observed after EV depletion. We verified whether the calculated depletion efficiency was biased by differences in the abundance of transcripts before depletion. However, we found no correlation between the depletion efficiency depicted in Figure 1D and the abundance (Cq values) of transcripts before depletion (Figure 1E). For example, 7SL was less abundant than $5 \mathrm{~S}$ before depletion, but was depleted with higher efficiency than 5S. Additionally, miR-451 and miR-1246 were equally abundant before depletion, but miR-451 was depleted more efficiently than miR-1246. This suggests that the observed differences in depletion efficiencies are intrinsic characteristics of the studied example RNA types (and/or the macromolecular structures they are associated to), and do not depend on their abundance before depletion. These data illustrate that the FCS-RNA depletion by ultracentrifugation is variable between and even within different classes of non-coding RNAs. MiRNAs that could not be depleted by ultracentrifugation (e.g. miR-122 and miR-451, see also (10)) may be predominantly associated to specific lipoprotein particles, or to subsets of RNP and EV that do not efficiently sediment during the centrifugation step. In contrast, the RNAs that efficiently sedimented (Y-RNA and 7SL) may be predominantly associated to larger EV and/or RNP. Although FCS dilution showed a clear effect on pelleting efficiency of total FCS-RNA (Figure 1C), the depletion efficiencies of the example RNAs, as tested by RT-qPCR, were similar for all dilutions (Figure 1D). This suggests that serum contains additional types of complexed RNAs with sedimentation properties that are yet different from those observed in our data.

During ultracentrifugation, FCS-containing medium will concentrate into a proteinrich, high density fluid in which EV may become trapped. We therefore tested whether the method used for recovery of EV-depleted supernatant after ultracentrifugation affected the amount of contaminant FCS-RNA carried over to the FCS-supplemented culture medium. Although exact steps in EV depletion protocols are often not reported in publications, a widely used method to collect EV-depleted supernatant is to pour off supernatant after centrifugation (decanting method). We compared this method with a more careful approach in which supernatant was pipetted off (Figure 1A, middle 
panel). Strikingly, the depletion efficiency of several of the example non-coding RNAs was strongly reduced when the decanting method was used instead of the pipetting method (Figure 1F). Quantities of residual Y-RNA and 7SL in decanted supernatants even reversed to values similar to those observed in non-depleted medium (Figure 1F). However, other non-coding RNAs, such as 5S, U1 and miR-451, were depleted with similar efficiencies using the two methods. MiRNA-122 and miR-148a were confirmed not to be depleted after overnight centrifugation. These data indicate that small technical details in the serum EV depletion procedure strongly affect the efficiency with which different types of bovine RNAs can be depleted. Subsequently, this will have an effect on the scale and composition of the contaminating pool of bovine RNA in serum-supplemented culture medium. This urges the need to accurately describe these depletion procedures in publications to improve the reproducibility of EV-RNA studies.

The data above indicate that not all RNAs can be efficiently depleted from bovine serum. We aimed to assess the extent to which residual bovine RNAs affect the analysis of cell culture EV-associated RNA after high-grade EV purification by density gradient centrifugation. First, we analyzed the number of bovine EV remaining in EV-depleted FCS after using the most optimal protocol for EV depletion (overnight ultracentrifugation of $30 \%$ FCS, collect EV-depleted supernatant by pipetting method). Ultracentrifuged FCS or non-depleted FCS was used to prepare culture medium, after which we applied differential centrifugation followed by density gradient ultracentrifugation to purify EV from both media (Figure 1A, bottom panel). Using high resolution flow cytometry $(15,16)$, we observed that the EV depletion procedure led to $90 \%$ reduction in the number of bovine EV in culture medium. Next, we tested to what extent residual bovine (EV-associated) RNA in this EV-depleted medium influenced the analysis of EV-associated RNA released by cells cultured in this medium. We used two cell lines that differed in the quantity of released EV, because the number of EV per volume of cell culture medium will affect the relative contribution of FCS-derived RNA to the total amount of EV-RNA. A20 murine B cell lymphoma cells released six times more EV than the HEK293T human embryonic kidney, as determined by high resolution flow cytometry-based EV quantification (Figure 2B). Cell culture EV fractions contained 2.5 times (HEK293T) and 112 times (A20) more RNA than residual bovine EV present in equal volumes of non-conditioned medium (Figure $\mathbf{2 C}$ and D). Next, we used RT-qPCR to assess whether specific EV-associated RNAs released by the cells in culture could be detected above background levels of these RNAs in EV fractions of non-conditioned medium. From the example RNAs tested in Figure 1D-F, we excluded miR-122 and miR-451, which could not be detected in 293T and A20 cellular RNA (data not shown). Furthermore, we excluded Y4 and Y3 because Y4-RNA is only present in human and bovine genomes and $Y 3-R N A$ contains a number of mismatches in the murine sequence 
A

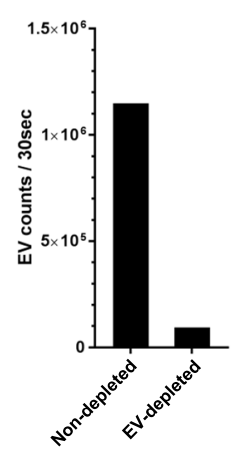

B

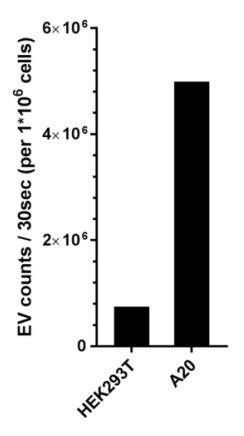

C

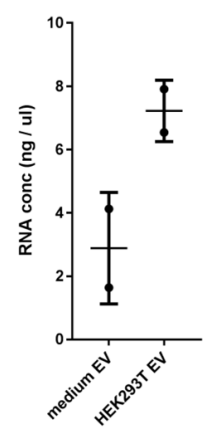

D

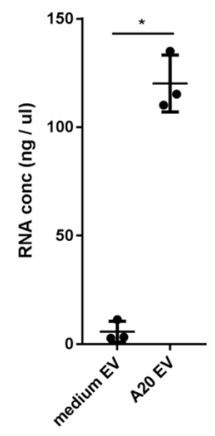

E
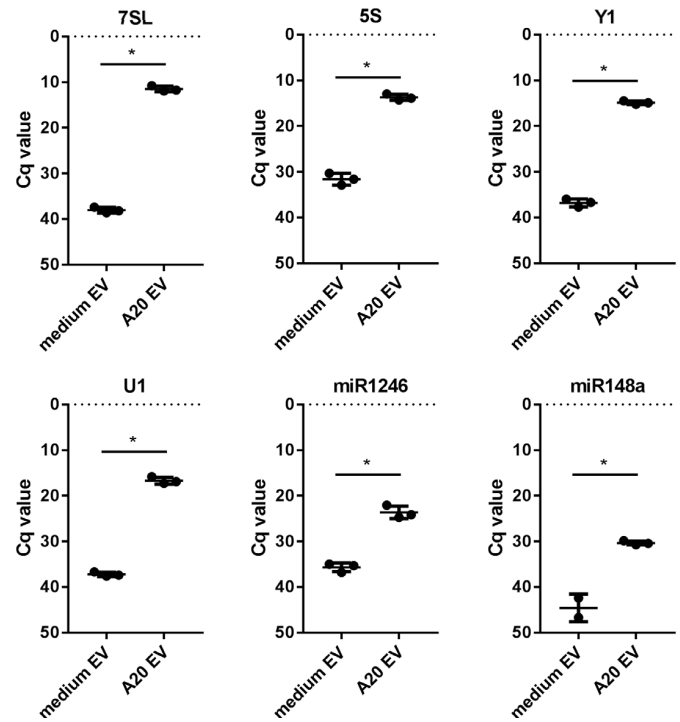

F

$7 \mathrm{SL}$

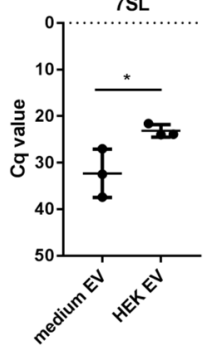

$5 S$
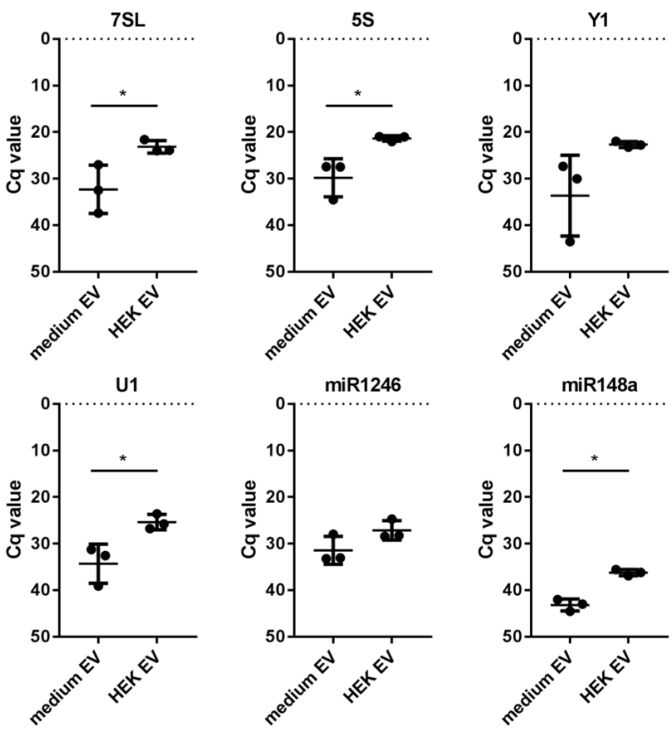
Figure 2.

A) EV-depleted medium was prepared using supernatant of ultracentrifuged solutions of $30 \%$ FCS. EV from equal volumes of EV-depleted and non-depleted medium were isolated by ultracentrifugation, fluorescently labeled with $\mathrm{PKH} 67$, and further purified by sucrose density gradient centrifugation. High-resolution flow cytometry was used to determine the number of PKH67-labeled EV. Indicated are the number of fluorescent EV detected in 30 seconds in pools of $1.12-1.18 \mathrm{~g} / \mathrm{mL}$ sucrose fractions.

B) HEK293T and A20 cells were grown 20h in EV-depleted medium (prepared from 30\% EVdepleted FCS). EV were isolated from equal volumes of HEK293T and A20 cell-conditioned supernatant, labeled with $\mathrm{PKH} 67$, and quantified using high-resolution flow cytometry. Indicated are the number of fluorescent EV in 30 seconds in pools of $1.12-1.18 \mathrm{~g} / \mathrm{mL}$ sucrose fractions, normalized to the number of cultured cells to allow direct comparison of EV-release by both cell types.

C-D) HEK293T cells orA20 cells were cultured in EV-depleted medium (prepared from 30\%-depleted FCS). EV were isolated from equal volumes of HEK293T (C) or A20 (D) conditioned medium and non-conditioned medium using differential centrifugation followed by sucrose density gradient centrifugation. RNA was isolated from EV pelleted from $1.12-1.18 \mathrm{~g} / \mathrm{mL}$ sucrose fractions. Indicated are mean RNA concentrations $+/-$ SD of $n=2$ (HEK293T) or $n=3$ (A20) replicates. Statistical differences were determined by independent samples t-test, ${ }^{*} p<0.05$.

E-F) EV-RNA isolated from A20 (E) and HEK293T (F) conditioned medium and equal volumes of non-conditioned EV-depleted medium (prepared as in D) were used for analysis of the indicated RNAs using RT-qPCR. Indicated are mean $\mathrm{Cq}$ values $+/-\mathrm{SD}$ of $\mathrm{n}=3$ replicates. Statistical differences were determined by independent samples t-test, ${ }^{*} p<0.05$.

compared to human and bovine genomes. The sequences of all other tested RNAs (7SL, 5S, Y1, U1, miR-1246 and miR-148a) were identical in all species and could be analyzed using the same primer pairs. Due to low EV numbers in HEK293T cultures, three times larger volumes of HEK293T-conditioned medium compared to A20-condition medium were used to be able to detect the EV-associated RNAs in all conditions. Since equal volumes of control non-conditioned medium were analyzed in parallel, this explains the lower Cq values for medium EV in HEK293T experiments (Figure 2F) compared to A20 experiments (Figure 2E). For A20 cell cultures, the quantified levels of all tested RNAs in cell-derived EV by far exceeded and significantly differed from the background levels in corresponding medium control samples (Figure 2E). In cultures of HEK293T cells producing low numbers of EV, on the contrary, the differences in Cq values in cell-derived EV versus medium-EV were substantially smaller (Figure 2F). For 7SL, 5S, and snRNA U1, which are abundantly present in EV, and for miR148a, which is present at low abundance in both cell culture EV and FCS-derived EV, significant differences were observed between RNA quantities in cell-derived EV and background levels in medium controls. Other EV-associated RNAs in the HEK293T cell cultures, such as Y1 and miR1246, could not be reliably detected above medium background levels. Importantly, these data show that the Cq values for each of the tested RNAs not only differ in the cell-derived EV but also in the medium control samples. This implies that 
the abundance of a specific RNA in cell culture EV can only be determined if measured relative to background levels of this RNA in medium control samples.

In conclusion, we show that optimization of FCS-EV depletion protocols and highgrade EV purification eliminates a large proportion of FCS-derived background RNA. Currently used sources of EV-depleted FCS include commercially available 'exosomedepleted FBS' and FCS depleted from EV by individual researchers using different ultracentrifugation protocols or alternative methods (14). For each of these protocols, it is necessary to identify technical parameters affecting the depletion efficiency of RNA from FCS. In addition, our data indicate that the reliability with which conserved RNAs in cell culture EV can be detected over background levels in culture medium depends on a number of different factors: 1) the quantity of EV released by the cultured cells; 2) the abundance of this RNA in these EV; and 3) the amount of this RNA that remains present in FCS-EV-depleted culture medium. Parallel processing and analysis of medium control samples is therefore essential to accurately assess the RNA content of cell culture EV. Finally, our data further underscore the importance of developing and implementing guidelines (25) for dealing with FCS-derived background RNA in EV-RNA research, and urge for accurate reporting of technical details of depletion procedures to increase experimental reproducibility (18).

\section{Acknowledgements}

We thank Dr. G. J. A. Arkesteijn for assistance on the high-resolution flow cytometric measurements. This work was supported by European Research Council under the European Union's Seventh Framework Programme [FP/2007-2013] / ERC Grant Agreement number [337581] to [ENMNtH.].

\section{Conflict of interest statement}

The authors declare no conflict of interest 


\section{References}

1. van der Grein SG, Nolte-'t Hoen ENM. "Small Talk" in the Innate Immune System via RNA-Containing Extracellular Vesicles. Front Immunol (2014) 5:1-8. doi:10.3389/fimmu.2014.00542

2. Redzic JS, Balaj L, van der Vos KE, Breakefield XO. Extracellular RNA mediates and marks cancer progression. Semin Cancer Biol (2014) 28:14-23. doi:10.1016/j.semcancer.2014.04.010

3. Cheng L, Doecke JD, Sharples RA, Villemagne VL, Fowler CJ, Rembach A, Martins RN, Rowe CC, Macaulay SL, Masters CL, et al. Prognostic serum miRNA biomarkers associated with Alzheimer's disease shows concordance with neuropsychological and neuroimaging assessment. Mol Psychiatry (2014)1-9. doi:10.1038/mp.2014.127

4. Martinez BV, Dhahbi JM, Nunez Lopez YO, Lamperska K, Golusinski P, Luczewski L, Kolenda T, Atamna H, Spindler SR, Golusinski W, et al. Circulating small non coding RNA signature in head and neck squamous cell carcinoma. Oncotarget (2015) 6:19246-19263. doi:10.18632/oncotarget.4266

5. Buschmann D, Kirchner B, Hermann S, Märte M, Wurmser C, Brandes F, Kotschote S, Bonin M, Steinlein OK Pfaffl MW, et al. Evaluation of serum extracellular vesicle isolation methods for profiling miRNAs by nextgeneration sequencing. J Extracell Vesicles (2018) 7: doi:10.1080/20013078.2018.1481321

6. Théry C, Amigorena S, Raposo G, Clayton A. Isolation and Characterization of Exosomes from Cell Culture Supernatants. Curr Protoc cell Biol (2006) Chapter 3:1-29. doi:10.1002/0471143030.cb0322s30

7. Shelke GV, Lässer C, Gho YS, Lötvall J. Importance of exosome depletion protocols to eliminate functional and RNA-containing extracellular vesicles from fetal bovine serum. J Extracell Vesicles (2014) 3:1-8. doi:10.3402/jev.v3.24783

8. Eitan E, Zhang S, Witwer KW, Mattson MP. Extracellular vesicle-depleted fetal bovine and human sera have reduced capacity to support cell growth. J Extracell Vesicles (2015) 4:1-10. doi:10.3402/jev.v4.26373

9. Mateescu B, Kowal EJK, van Balkom BWM, Bartel S, Bhattacharyya SN, Buzás El, Buck AH, de Candia P, Chow FWN, Das S, et al. Obstacles and opportunities in the functional analysis of extracellular vesicle RNA - an ISEV position paper. J Extracell Vesicles (2017) 6:1286095. doi:10.1080/20013078.2017.1286095

10. Wei Z, Batagov AO, Carter DRF, Krichevsky AM. Fetal Bovine Serum RNA Interferes with the Cell Culture derived Extracellular RNA. Sci Rep (2016) 6:31175. doi:10.1038/srep31175

11. Vickers KC, Palmisano BT, Shoucri BM, Shamburek RD, Remaley AT. MicroRNAs are transported in plasma and delivered to recipient cells by high-density lipoproteins. Nat Cell Biol (2011) 13:423-33. doi:10.1038/ ncb2210

12. Arroyo JD, Chevillet JR, Kroh EM, Ruf IK, Pritchard CC, Gibson DF, Mitchell PS, Bennett CF, PogosovaAgadjanyan EL, Stirewalt DL, et al. Argonaute2 complexes carry a population of circulating microRNAs independent of vesicles in human plasma. Proc Natl Acad Sci U S A (2011) 108:5003-8. doi:10.1073/ pnas. 1019055108

13. Driedonks TAP, van der Grein SG, Ariyurek Y, Buermans HPJ, Jekel H, Chow FWN, Wauben MHM, Buck AH, Hoen PAC, Nolte-'t Hoen ENM. Immune stimuli shape the small non-coding transcriptome of extracellular vesicles released by dendritic cells. Cell Mol Life Sci (2018)3857-3875. doi:10.1007/s00018-018-2842-8

14. Kornilov R, Puhka M, Mannerström B, Hiidenmaa H, Peltoniemi H, Siljander P, Seppänen-Kaijansinkko R, Kaur S. Efficient ultrafiltration-based protocol to deplete extracellular vesicles from fetal bovine serum. J Extracell Vesicles (2018) 7:1422674. doi:10.1080/20013078.2017.1422674

15. van der Vlist EJ, Nolte-'t Hoen ENM, Stoorvogel W, Arkesteijn GJA, Wauben MHM. Fluorescent labeling of nano-sized vesicles released by cells and subsequent quantitative and qualitative analysis by highresolution flow cytometry. Nat Protoc (2012) 7:1311-1326. doi:10.1038/nprot.2012.065

16. Nolte-'t Hoen ENM, van der Vlist EJ, Aalberts M, Mertens HCH, Bosch BJ, Bartelink W, Mastrobattista E, van Gaal EVB, Stoorvogel W, Arkesteijn GJ a, et al. Quantitative and qualitative flow cytometric analysis of nanosized cell-derived membrane vesicles. Nanomedicine (2012) 8:712-720. doi:10.1016/j. nano.2011.09.006 
17. Groot Kormelink T, Arkesteijn GJA, Nauwelaers FA, van den Engh G, Nolte-'t Hoen ENM, Wauben MHM. Prerequisites for the analysis and sorting of extracellular vesicle subpopulations by high-resolution flow cytometry. Cytom Part A (2016) 89:135-147. doi:10.1002/cyto.a.22644

18. Van Deun J, Mestdagh P, Agostinis P, Akay Ö, Anand S, Anckaert J, Martinez ZA, Baetens T, Beghein E, Bertier $L$, et al. EV-TRACK: transparent reporting and centralizing knowledge in extracellular vesicle research. Nat Methods (2017) 14:228-232. doi:10.1038/nmeth.4185

19. Momen-Heravi F, Balaj L, Alian S, Trachtenberg AJ, Hochberg FH, Skog J, Kuo WP. Impact of biofluid viscosity on size and sedimentation efficiency of the isolated microvesicles. Front Physiol (2012) 3 MAY:1-6. doi:10.3389/fphys.2012.00162

20. Kent OA, McCall MN, Cornish TC, Halushka MK. Lessons from miR-143/145: The importance of cell-type localization of miRNAs. Nucleic Acids Res (2014) 42:7528-7538. doi:10.1093/nar/gku461

21. Gonzalez-Martin A, Adams BD, Lai M, Shepherd J, Salvador-Bernaldez M, Salvador JM, Lu J, Nemazee D, Xiao C. The microRNA miR-148a functions as a critical regulator of B cell tolerance and autoimmunity. Nat Immunol (2016) 17:433-440. doi:10.1038/ni.3385

22. Baraniskin A, Nöpel-Dünnebacke S, Ahrens M, Jensen SG, Zöllner H, Maghnouj A, Wos A, Mayerle J, Munding J, Kost D, et al. Circulating U2 small nuclear RNA fragments as a novel diagnostic biomarker for pancreatic and colorectal adenocarcinoma. Int J Cancer (2013) 132:48-57. doi:10.1002/ijc.27791

23. Nolte-'t Hoen ENM, Buermans HPJ, Waasdorp M, Stoorvogel W, Wauben MHM, 't Hoen PAC. Deep sequencing of RNA from immune cell-derived vesicles uncovers the selective incorporation of small non-coding RNA biotypes with potential regulatory functions. Nucleic Acids Res (2012) 40:9272-85. doi:10.1093/nar/gks658

24. Bellingham SA, Coleman BM, Hill AF. Small RNA deep sequencing reveals a distinct miRNA signature released in exosomes from prion-infected neuronal cells. Nucleic Acids Res (2012) 40:10937-10949. doi:10.1093/nar/gks832

25. Lötvall J. Minimal experimental requirements for definition of extracellular vesicles and their functions: a position statement from the International Society for Extracellular Vesicles. (2014) 1:1-6.

26. Livak KJ, Schmittgen TD. Analysis of relative gene expression data using real-time quantitative PCR and the 2(-Delta Delta C(T)) Method. Methods (2001) 25:402-408. doi:10.1006/meth.2001.1262 


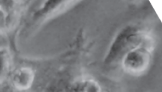
$\cos ^{2}+2$ cos ? 62,45 of $62-3$

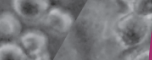
0372 s.

कैe

cisec ?eic:

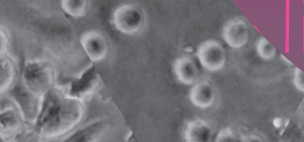

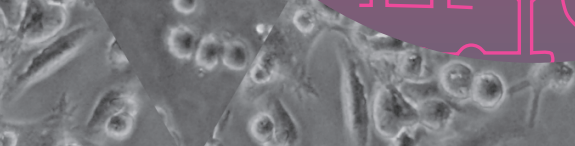

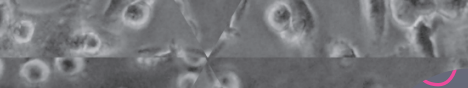

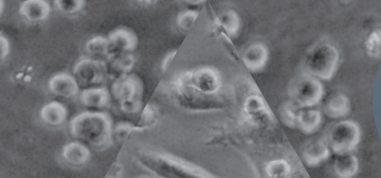

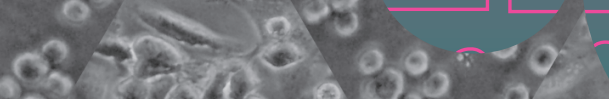

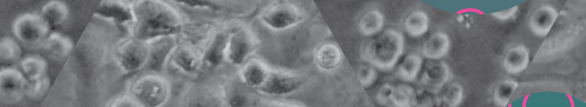
8

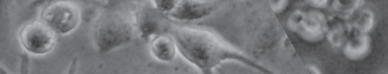

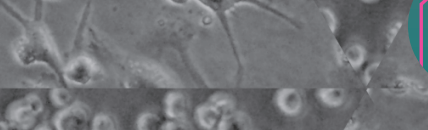

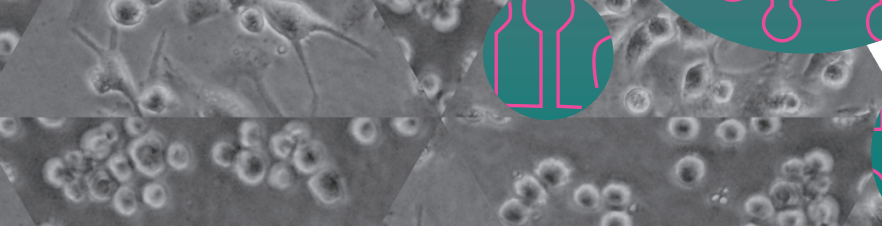
)

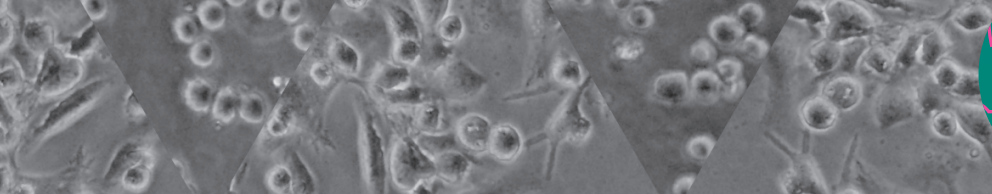
a 3 a (a)

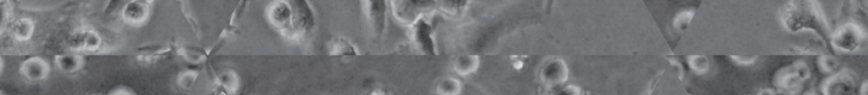

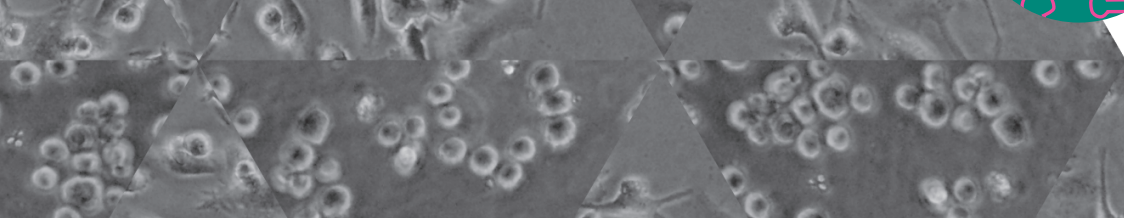

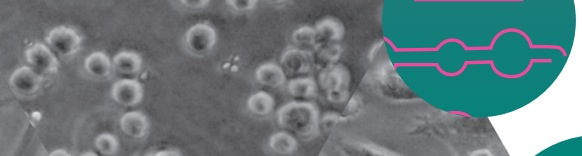

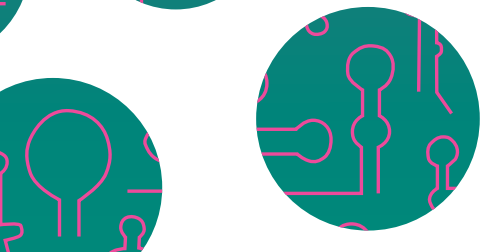

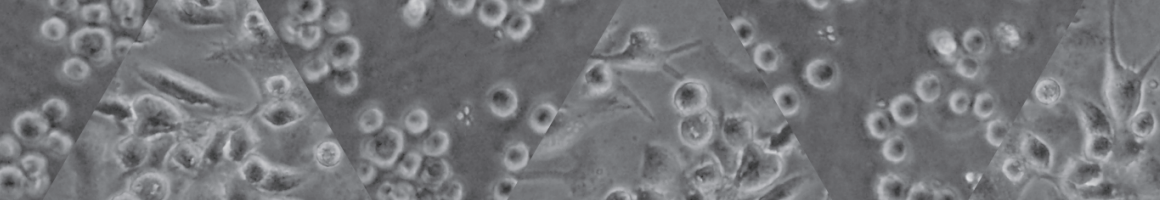
ce If

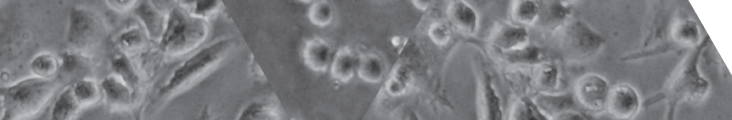

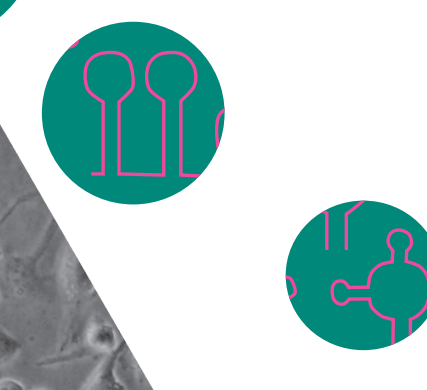




\section{Chapter 4}

\section{Immune stimuli shape the small non-coding transcriptome of extracellular vesicles released by dendritic cells}

Tom A.P. Driedonks ${ }^{1}$, Susanne G. van der Grein ${ }^{1}$, Yavuz Ariyurek ${ }^{2,3}$, Henk P.J. Buermans ${ }^{2,3}$, Henrike Jekel' ${ }^{1}$, Franklin W.N. Chow ${ }^{4}$, Marca H.M. Wauben' ${ }^{1}$ Amy H. Buck ${ }^{4}$, Peter A.C. 't Hoen², Esther N.M. Nolte-'t Hoen ${ }^{1 *}$

\footnotetext{
1 Department of Biochemistry \& Cell Biology, Faculty of Veterinary Medicine, Utrecht University, Utrecht, The Netherlands

2 Department of Human Genetics, Leiden University Medical Center, Leiden, The Netherlands 3 Leiden Genome Technology Center, Leiden University Medical Center, Leiden, The Netherlands 4 Institute of Immunology and Infection Research, Centre for Immunity, Infection and Evolution, School of Biological Sciences, University of Edinburgh, Edinburgh, UK

5 Centre for Biomolecular and Molecular Informatics, Radboud Institute for Molecular Life Sciences, Radboud University Medical Center Nijmegen, Nijmegen, The Netherlands

* To whom correspondence should be addressed. Tel: +3130 253 4336; Fax: +31 302535492 ;

Email: e.n.m.nolte@uu.nl
}

Cellular and Molecular Life Sciences, 75 : 3857-3875, 2018 


\section{Abstract}

The release and uptake of nano-sized Extracellular Vesicles (EV) is a highly conserved means of intercellular communication. The molecular composition of EV, and thereby their signaling function to target cells, is regulated by cellular activation and differentiation stimuli. EV are regarded as snapshots of cells and are therefore in the limelight as biomarkers for disease. Although research on EV-associated RNA has predominantly focused on microRNAs, the transcriptome of EV consists of multiple classes of small non-coding RNAs with potential gene regulatory functions. It is not known whether environmental cues imposed on cells induce specific changes in a broad range of EV-associated RNA classes. Here, we investigated whether immune-activating or -suppressing stimuli imposed on primary dendritic cells affected the release of various small non-coding RNAs via EV. The small RNA transcriptomes of highly pure EV populations free from ribonucleoprotein particles were analyzed by RNA sequencing and RT-qPCR. Immune stimulus-specific changes were found in the miRNA, snoRNA and Y-RNA content of EV from dendritic cells, whereas tRNA and snRNA levels were much less affected. Only part of the changes in EV-RNA content reflected changes in cellular RNA, which urges caution in interpreting EV as snapshots of cells. By comprehensive analysis of RNA obtained from highly purified EV we demonstrate that multiple RNA classes contribute to genetic messages conveyed via EV. The identification of multiple RNA classes that display cell stimulation-dependent association with EV is the prelude to unraveling the function and biomarker potential of these EV-RNAs.

\section{Abbreviations}

$\begin{array}{ll}\text { BSA: } & \text { Bovine Serum Albumin } \\ \text { DC: } & \text { Dendritic Cell } \\ \text { EV: } & \text { Extracellular Vesicles } \\ \text { LPS: } & \text { Lipopolysaccharide } \\ \text { miRNA/miR: } & \text { microRNA } \\ \text { miscRNA: } & \text { miscellaneous RNA } \\ \text { ncRNA: } & \text { non-coding RNA } \\ \text { RNP: } & \text { Ribonucleoprotein (complex) } \\ \text { rRNA: } & \text { ribosomal RNA } \\ \text { SnRNA: } & \text { small nuclear RNA } \\ \text { SnoRNA: } & \text { small nucleolar RNA } \\ \text { TLR: } & \text { Toll-like Receptor } \\ \text { tRNA: } & \text { transfer RNA }\end{array}$

Key words: Immune activation, immune suppression, small RNA sequencing, biomarker, extracellular RNA 


\section{Introduction}

Extracellular vesicles (EV) released by cells are considered as important mediators of intercellular communication [1, 2]. These 50 - $1000 \mathrm{~nm}$ sized vesicles are released by a broad range of cells and have been detected in a wide range of body fluids, such as milk, plasma, urine, and semen [1,3]. The collective term 'EV' refers to a heterogeneous population of secreted vesicles that are formed via different pathways. Exosomes are formed as intraluminal vesicles (ILVs) inside multi-vesicular endosomes (MVE) and are released upon fusion of the MVE with the plasma membrane, whereas microvesicles directly bud off from the plasma membrane [1]. These vesicle populations overlap in size and molecular composition, which currently hampers their discrimination based on biophysical or biochemical parameters [4-6].

EV are multi-component entities that transfer proteins, lipids and RNA between cells. The RNA associated with EV mainly consists of small non-coding RNA species (ncRNA), which are thought to be of major importance in altering molecular processes in the recipient cell $[3,4,7,8]$. Additionally, the RNA composition of EV can give information about the (patho)physiological status of the EV-producing cell. Since EV can be easily obtained from body fluids, such EV-associated RNAs may be used as noninvasive biomarkers for early detection of disease $[9,10]$. The molecular composition of EV is often assumed to be a 'snapshot' of the producing cell, from which the tissue type or mutational status of the parental cell can be deduced $[9,11,12]$.

Although the EV-RNA field receives major attention, with quickly rising numbers of publications on this topic, the technical difficulties to obtain pure EV remain underexposed. It is important to note that a substantial amount of extracellular RNA is not contained in EV, but is associated to ribonucleoprotein particles (RNPs), including Argonaute 2 (AGO2), or lipoprotein complexes [13-15]. These contaminating structures have been shown to co-isolate together with EV via commonly used isolation procedures, and may therefore greatly affect the outcomes of experiments $[4,5,16]$. The currently most reliable method to obtain pure EVs is by high-speed ultracentrifugation followed by density centrifugation in which EV are separated from contaminating structures based on differences in buoyant density [16]. Subsequently, analyzing the presence of common EV markers (such as CD9, CD63 and CD81) and absence of contaminant proteins should be performed to assess the purity of EV preparations used in the study $[17,18]$.

MiRNAs are the most intensely studied type of small RNA in EV, likely due to the interest in gene-regulatory functions of these RNAs [19-21]. It has been shown that changes in the activation status of the EV-producing cell lead to changes in the EVmiRNA composition [22-24]. Moreover, miRNAs in EV were shown to be functionally transferred between cells, leading to repression of genes in target cells $[25,26]$. 
It is therefore thought that differences in the miRNA content of EV underlie distinct functional effects of EV released by differentially stimulated cells [22-24]. Additionally, changes in EV-miRNA levels in plasma or serum have been associated with diseases such as cancer, rheumatoid arthritis and Alzheimer's disease [27-29].

Despite the interest in miRNAs, a larger part of EV-associated RNA consists of other ncRNA classes. We and others previously showed that EV released by cultured cells and EV present in a variety of body fluids contain many other small non-coding RNA species (20 - $300 \mathrm{nt}$ ), such as tRNA, snoRNA, snRNA, Y-RNA, Vault RNA and SRP-RNA (also named 7SL) [23, 30-35]. Some of these non-coding RNAs are relatively enriched in EV, suggesting that these RNAs are specifically shuttled into EV for release into the extracellular milieu. Inside cells, the above-mentioned non-coding RNAs are known to play a role in basic cellular processes such as translation, RNA splicing, and RNA quality surveillance. Recent studies showed that full-length and fragmented forms of these non-coding RNAs can additionally be involved in other processes including immunological signaling, gene regulation, and guiding of other RNAs and nucleases [36-41]. However, it is not known whether activation stimuli imposed on the EV-producing cell affect the levels of these ncRNA classes in EV, similar to EV-associated miRNAs. Evaluation of cell-stimulationdependent changes in EV-associated ncRNA classes is a first step in uncovering their function in EV-mediated signaling processes and their potential as biomarkers indicative of the activation status of cells.

Here, we studied how changes in the activation/differentiation status of the parental cell affect the small non-coding RNA content of EV by employing a primary immune cell model, two different types of cell stimulation, and methods yielding highly pure EV populations. For our analysis we used primary dendritic cells (DC), which are master regulators of immune responses and have been shown to communicate with various other immune cells via EV $[2,8,42]$. Additionally, the use of DC-derived EV has been proposed as a strategy for cancer immune(chemo)therapy [43-46]. Depending on external stimuli, DC can be differentiated into functionally different phenotypes that either activate or down-regulate immune responses [47]. These DC therefore present a suitable model to investigate how different external stimuli affect incorporation of a broad range of RNA classes into EV. We compared cellular and EV-associated RNA levels in control, immune-stimulating, or immune-suppressing DC conditions by using RNA deep sequencing and RT-qPCR. Our data indicate that differentiation signals imposed on dendritic cells affect the EV-associated levels of particular RNA classes, such as miRNA, snoRNA and Y-RNA, while other non-coding RNA types remain largely unchanged. Moreover, only a minor part of the stimulation-induced changes in EV-RNA content reflect changes in cellular RNA levels. This study exemplifies how comprehensive analysis of RNA obtained from highly purified EV yields candidate small ncRNAs beyond miRNAs for further exploration as biomarkers or functional entities within EV. 


\section{Materials and methods}

\section{Cell culture}

Complete culture medium was prepared by supplementing Iscove's Modified Dulbecco's Medium (IMDM, Lonza, Verviers, Belgium) with $2 \mathrm{mM}$ Ultraglutamine (Lonza, Verviers, Belgium), 10\% fetal calf serum (FCS, GE Healthcare Bio-Sciences, Pasching, Austria) $100 \mathrm{lU} / \mathrm{mL}$ penicillin and $100 \mu \mathrm{g} / \mathrm{ml}$ streptomycin (Gibco, Paisley, United Kingdom), $50 \mu \mathrm{M} \beta$-mercaptoethanol. The GM-CSF producing NIH 3 T3 cells (R1) was grown in complete medium. Primary bone marrow cells were grown in complete medium supplemented with $30 \%$ conditioned medium from R1 cells. To prepare EVdepleted medium, a mixture of $150 \mathrm{~mL}$ conditioned R1 culture supernatant and 42.5 $\mathrm{mL}$ FCS (end concentration 30\% FCS) was depleted of EV by overnight centrifugation at $100,000 \mathrm{~g}$ in a SW28 rotor ( $k$-factor 334.2) (Beckman Coulter, Brea, CA). The EVdepleted supernatant was carefully pipetted from the tubes, leaving $5 \mathrm{~mL}$ in the tubes to prevent disturbance of the pellet. This supernatant was filtered through a $0.22 \mu \mathrm{m}$ bottle top filter (Millipore, Billerica, MA) after which additional IMDM, Ultraglutamine, antibiotics and $\beta$-mercaptoethanol were added to prepare complete medium as indicated above. The efficiency of EV depletion from culture medium, as regularly assessed by high-resolution flow cytometric analysis, is on average $\sim 90 \%$. Primary bone marrow cells were flushed from the femur and tibia of $8-12$ week old C57bl/6 mice and differentiated to dendritic cells according to Lutz et al [48]. To generate tolerogenic DC, cells were treated from day 2 onward with $10 \mathrm{nM} \mathrm{1a,25-}$ dihydroxyvitaminD3 (Sigma, St Louis, MO). To generate immunogenic DC, $1 \mu \mathrm{g} / \mathrm{mL}$ lipopolysaccharide (LPS, cat. L-2630, Sigma, St. Louis, MO) was added on day 12. On day 13 , non-adherent and semi-adherent cells were recovered and cultured at $3 \times 10^{6}$ cells/dish for 20 hours in EV-depleted culture medium. Cell viability, as determined by Trypan blue exclusion, did not differ between treatments and was above $90 \%$ for all cultures. All cells were maintained at $37^{\circ} \mathrm{C}$ and $5 \% \mathrm{CO}_{2}$ in a humidified incubator. Experiments were approved by the institutional ethical animal committee at Utrecht University (Utrecht, The Netherlands).

\section{Flow cytometry}

Day 12 bone marrow DC (BMDC) were collected and labeled for 30 minutes in PBS + 1\% BSA (Bovine Serum Albumin, cat. K45-001, GE Healthcare Bio-Sciences, Pasching, Austria) containing anti-CD11C-APC (eBioscience, clone N418, $1: 400$ ), anti-MHCIIFITC (eBioscience, clone M5/114.15.2, 1 : 1500), and anti-CD40-PE (BD Biosciences, clone 3/23, $1: 200$ ), or anti-CD86-PE (eBioscience, clone GL1, $1: 200$ ) or anti-CD274-PE (PD-L1, eBioscience, clone M1H5, $1: 400$ ). As control, corresponding isotype control antibodies (eBioscience, San Diego, CA) were used. Surface labeling was analyzed 
using a FACS Calibur Flow Cytometer (BD Biosciences, San Jose, CA). Data analysis was performed using FCS Express V3 (DeNovo Software, Glendale, CA).

\section{Fluorescent labeling and purification of EV}

Conditioned cell supernatants were pooled to volumes of $100-140 \mathrm{ml}$ per condition and were subjected to differential centrifugation as described previously [49]. In brief: supernatant was sequentially centrifuged $2 \times 200 \mathrm{~g}$ for $10 \mathrm{~min}, 2 \times 500 \mathrm{~g}$ for 10 min, $1 \times 10,000 \mathrm{~g}$ for $30 \mathrm{~min}$. Next, EV were pelleted by ultracentrifugation at 100,000 g for 65 min using an SW28 rotor ( $k$-factor 334.2) (Beckman Coulter, Brea, CA). For EV quantification by high-resolution flow cytometry, pellets were resuspended in $20 \mu \mathrm{l}$ PBS $+0.2 \%$ BSA (cleared from aggregates by overnight ultracentrifugation at $100,000 \mathrm{~g}$ ) and labeled with $1.5 \mu \mathrm{l} \mathrm{PKH67} \mathrm{(Sigma,} \mathrm{St.} \mathrm{Louis,} \mathrm{MO)} \mathrm{in} 100 \mu \mathrm{l}$ Diluent C per pellet. For EV-RNA isolation, 100,000g pellets were resuspended in $50 \mu \mathrm{PBS}+0,2 \% \mathrm{BSA}$. PKH67labeled samples or samples for RNA isolation were mixed with $1.5 \mathrm{~mL} 2.5 \mathrm{M}$ sucrose, and overlaid with a linear sucrose gradient (2.0 M - 0.4 M sucrose in PBS). Gradients were centrifuged $15-18 \mathrm{~h}$ at $192,000 \mathrm{~g}$ in a SW40 rotor ( $k$-factor 144.5) (Beckman Coulter, Brea, CA). PKH67-labeled EV were used for high-resolution flow cytometric analysis (see below). Alternatively, the fractions with densities of $1.12-1.18 \mathrm{~g} / \mathrm{ml}$ (as measured by refractometry) were pooled, diluted 6 times in PBS $+0.2 \%$ EV-depleted BSA and centrifuged again at 192,000 g for 65 min in a SW40 rotor ( $k$-factor 144.5) prior to EV-RNA isolation.

\section{RNA isolation}

Small RNA was isolated from EV pellets and from $1 \times 10^{6}$ cells using the miRNeasy Micro kit according to the small RNA enrichment protocol provided by the manufacturer (Qiagen, Hilden, Germany). RNA yield and size profile were assessed using the Agilent 2100 Bioanalyzer with Pico 6000 RNA chips (Agilent Technologies, Waldbronn, Germany).

\section{Preparation of small RNA sequencing libraries}

50 ng cellular small RNA and 2 ng EV-derived small RNA was treated with DNase (Turbo DNA-free kit (Life Technologies, Carlsbad, CA)) according to the manufacturer's instructions. The DNase-treated RNA was pelleted using Pellet Paint (Merck, Darmstadt, Germany) according to manufacturer's instructions, reconstituted in milliQ (MQ) and subsequently subjected to ribosomal RNA depletion using RiboZero Gold kit (Human/ Mouse/Rat) (Illumina, San Diego, CA) according to manufacturer's instructions. This is required to deplete residual rRNA from cellular RNA samples and for comparability we subjected EV-RNA samples to the same procedure. Hereafter, RNA was pelleted with Pellet Paint and reconstituted into $6 \mu \mathrm{MQ}$. cDNA libraries were prepared using the 
NebNext smallRNA library prep kit for Illumina (New England Biolabs, Ipswich, MA), according to the manufacturer's instructions but with the following adaptations: $3^{\prime}$ adapter ligation was carried out overnight at $16^{\circ} \mathrm{C} ; \mathrm{PCR}$ amplification was done using Kapa HiFi Readymix 2x PCR mastermix (Kapa Biosystems, Wilmington, MA) using barcoded primers and the following PCR programme: 2 min at $95^{\circ} \mathrm{C}, 15$ cycles of 20 sec at $98^{\circ} \mathrm{C}, 30 \mathrm{sec}$ at $62^{\circ} \mathrm{C}, 15 \mathrm{sec}$ at $70^{\circ} \mathrm{C}$, and a final elongation step of $5 \mathrm{~min}$ at $70^{\circ} \mathrm{C}$. CDNA was purified using magnetic AMPure XP beads (Beckman Coulter, Brea, CA) and quantified using Agilent 2100 Bioanalyzer and DNA HiSensitivity chips (Agilent Technologies, Waldbronn, Germany). Adapter dimers (126 nt in size) were removed by running the cDNA on 6\% TBE gels (Life Technologies, Carlsbad, CA) for $60 \mathrm{~min}$ at $145 \mathrm{~V}$, after which cDNA products of $15-300 \mathrm{nt}$ (+ $126 \mathrm{nt}$ adapters) were cut from the gel and purified. Subsequently, all libraries were pooled at equimolar ratios and run on a 4\%-12\% TBE gel (Life Technologies, Carlsbad, CA). Size fractions of $15-25 \mathrm{nt}$ (includes miRNAs), $25-60 \mathrm{nt}, 60-80 \mathrm{nt}$ (includes tRNAs), and $80-275 \mathrm{nt}$ (each +126 nt adapters) were cut from the gel, purified and pooled in a volumetric ratio of $2: 2$ : $3: 3$. Sequencing was done using $150 \mathrm{bp}$ paired-end reads on an Illumina HiSeq 4000 machine (Illumina, San Diego, CA) at ServiceXS (Leiden, The Netherlands).

\section{RNAseq data analysis}

All data was processed as paired-end reads. Data quality was checked with FastQC and reads were processed with cutadapt (version 1.8) to remove low quality reads, clip the reads to $100 \mathrm{bp}$, remove adapter sequences. Sequences with a minimal length of $17 \mathrm{bp}$ after adapter trimming were retained to ensure high quality reads (cutadapt -q 30 -u -51 -U -51 -n1 --minimum-length 17 -a AGATCGGAAGAGCACACGTCTGAAC -A GATCGTCGGACTGTAGAACTCTGAA). Reads were mapped to the mouse genome mm10 using gsnap (v2014-12-23; gsnap --novelsplicing 1 --npaths 3 --format sam). Count tables were prepared using Htseq (v 0.6.1 p1; htseq-count --minaqual 0 --format bam --order pos --stranded yes). For miRNA count tables miRbase v20 annotation was used. Gene annotation for snoRNA, snRNA, miscRNA were retrieved from Biomart (Ensembl 84). tRNA and rRNA chromosomal positions were retrieved from the UCSC mm10 repeat mask track. Reproducibility of triplicate experiments (performed on different days) was assessed by evaluating Pearson correlation values (after logarithmic transformation of the small RNA counts).

Differential abundance was determined separately for each ncRNA class, and separately for EV and cells, using the edgeR package in R [50]. Data were normalized using the TMM method (weighted trimmed mean of M-values). Estimating the common, trended (over expression values) and tagwise dispersion, a generalized linear model, containing the effect of cell stimulation and the effect of the day-to-day variation between triplicate experiments, was fit. The loglikelihood ratio test was used 
to evaluate differential expression between treatments relative to control. P-values were adjusted for multiple testing using Benjamini and Hochberg's false discovery rate (FDR). Average fold-change over three independent experiments and standard deviation were plotted. Analysis of RNA fragments was done using the UCSC genome browser and Integrated Genome Viewer [51].

\section{Quantitative real-time PCR}

cDNA was generated from cellular or EV-derived small RNA using the miScript RT2 kit (Qiagen, Hilden, Germany). An equivalent of 20 pg RNA was used per qPCR reaction and mixed with $100 \mathrm{nM}$ primers (Isogen Life Sciences, De Meern, The Netherlands) and $4 \mu \mathrm{l}$ SYBR Green Sensimix (Bioline Reagents Ltd., United Kingdom) in an $8 \mu \mathrm{l}$ reaction. No-RT-controls confirmed the absence of genomic DNA and non-specific amplification.

Cycling conditions were $95^{\circ} \mathrm{C}$ for $10 \mathrm{~min}$ followed by 50 cycles of $95^{\circ} \mathrm{C}$ for $10 \mathrm{~s}$, $57^{\circ} \mathrm{C}$ for $30 \mathrm{~s}$ and $72^{\circ} \mathrm{C}$ for 20s. All PCR reactions were performed on the Bio-Rad iQ5 Multicolor Real-Time PCR Detection System (Bio-Rad, Hercules, CA). Quantification cycle $(\mathrm{Cq})$ values were determined using Bio-Rad CFX software using automatic baseline settings. Thresholds were set in the linear phase of the amplification curve.

\section{High resolution flow cytometric analysis of EV}

High resolution flow cytometric analysis of PKH67-labeled EV was performed using a BD Influx flow cytometer (BD Biosciences, San Jose, CA) with an optimized configuration, as previously described [49, 52]. In brief, we applied threshold triggering on fluorescence derived from PKH67-labeled EV passing the first laser. Forward scatter (FSC) was detected with a collection angle of $15-25^{\circ}$ (reduced wideangle FSC). Fluorescent 100- and 200-nm polystyrene beads (FluoSpheres, Invitrogen, Carlsbad, CA) were used to calibrate the fluorescence and rw-FSC settings. Sucrose gradient fractions containing PKH67-labeled EV were diluted 25x in PBS and vortexed just before measurement. This dilution factor was sufficient to avoid 'coincidence' (multiple EV arriving at the measuring spot at the same time), thereby allowing accurate quantitative comparison of EV numbers in different conditions. Moreover, samples were measured at maximally 10,000 events per second, which is far below the limit in the electronic pulse processing speed of the BD Influx [53].

\section{Western blotting}

Cell pellets were lysed in PBS $+1 \%$ Nonidet-P40 with protein inhibitor cocktail (Roche, Basel, Switzerland) for 15 minutes on ice. Nuclei were spun down at 16,000g for 15 min at $4^{\circ} \mathrm{C}$, supernatant was used for Western blotting. Cell lysates and $\mathrm{EV}$ were denatured in SDS-sample buffer at $100^{\circ} \mathrm{C}$ for $3 \mathrm{~min}$, separated using $12 \%$ SDS-PAGE gels, after which 
proteins were transferred onto Immobilon-P $0.45 \mu \mathrm{m}$ PVDF membranes (Millipore, Cork, Ireland). After blocking for $1-2 \mathrm{~h}$ in blocking buffer $(0.5 \%$ Cold Fish Skin Gelatin (Sigma-Aldrich, St. Louis, CA) in PBS $+0.05 \%$ Tween-20), blots were incubated overnight at $4^{\circ} \mathrm{C}$ with primary antibodies (anti-mouse-CD9 (eBioscience, clone KMC8, 1: 1000), anti-mouse-CD63 (MBL, clone D263-3, 1 : 1000), anti-mouse-Galectin-3 (eBioscience, clone M3/38, 1 : 500), anti-MHCII-p55 (GenScript, Piscataway, NJ, custom Ab raised against MHCII bèta chain peptide sequence RSQKGPRGPPPAGLLQC, $1: 5000$ ), or antimouse-beta-actin (ThermoScientific, polyclonal PA1-16889, 1 : 5000)) in blocking buffer, washed and incubated for 1 - $2 \mathrm{~h}$ with HRP-coupled secondary antibodies (Dako, cat P0450 and P0448, 1 : 5000). ECL solution (ThermoScientific, SuperSignal West Dura Extended Duration Substrate, cat. 34075) was used for detection on a Chemidoc imager (BioRad, Hercules, CA). Images were analyzed by Image Lab software (BioRad, Hercules, CA).

\section{Nanoparticle Tracking Analysis (NTA)}

EV purified by density gradient ultracentrifugation as described above were resuspended in $50 \mu \mathrm{PBS}+0.4 \%$ EV-depleted BSA. Samples were diluted 20 times in PBS (confirmed to be particle-free when analysed with same settings as used for the EV samples) before measurement on a Nanosight NS500 instrument (Malvern, Worchestershire, United Kingdom). Data acquisition and processing were performed using NTA software 3.1. Each sample was recorded for 5 times 30 seconds, 25 frames per second, camera level 14, detection threshold 5.

\section{Electron microscopy}

EV and RNP were separated by density gradient centrifugation as described above, and resuspended in $15 \mu \mathrm{l}$ PBS. $3 \mu \mathrm{l}$ aliquot of either EV or RNP were added on top of carbon-coated 300 mesh, copper grids and incubated for 2 minutes at room temperature. The grids were then washed two times in $50 \mathrm{ul} \mathrm{PBS}$ and stained with $2 \%$ uranyl acetate. Imaging was performed on a T20 electron microscope (FEI) operated at $200 \mathrm{keV}$. Images were recorded on a CCD Eagle camera (FEI).

\section{Northern blotting}

Cellular or EV-derived small RNA (between $100 \mathrm{ng}-1 \mathrm{ng}$ per lane, as indicated) was denatured in gel loading buffer II (Invitrogen) for 2 min at $70^{\circ} \mathrm{C}$ and snap-cooled on ice before loading on a denaturing 15\% PAGE gel (National Diagnostics, Nottingham, United Kingdom) as described previously[54]. RNA was transferred onto a Hybond-N Nylon membrane (Amersham Pharmacia Biotech, GE Healthcare Bio-Sciences, Pasching, Austria) and chemically crosslinked with 1-ethyl-3-(3-dimethylaminopropyl) carbodimide (Sigma) at $55^{\circ} \mathrm{C}$ for 2 hours [55]. ${ }^{32} \mathrm{P}$-labeled DNA oligo probes perfectly 
complementary to each RNA species were hybridized overnight at $42^{\circ} \mathrm{C}$ in PerfectHyb (Sigma) solution. Blots were analyzed by phosphorimaging using a Typhoon Scanner (GE Healthcare).

\section{List of DNA oligo probes $R T$-qPCR probes:}

The following forward primers were used in RT-qPCR ( $5^{\prime}$ to $\left.3^{\prime}\right)$ in combination with the miScript universal reverse primer.

$\begin{array}{ll}\text { miR-146a-5p: } & \text { TGA GAA CTG AAT TCC ATG GGT } \\ \text { miR-155-5p: } & \text { GGG TTA ATG CTA ATT GTG ATA G } \\ \text { miR-9-5p: } & \text { GGG TCT TTG GTT ATC TAG C } \\ \text { miR-10a-5p: } & \text { TAC CCT GTA GAT CCG AAT TTG TG } \\ \text { miR-27b-3p: } & \text { TTC ACA GTG GCT AAG TTC TGC } \\ \text { miR-378-5p: } & \text { ACT GGA CTT GGA GTC AGA AG } \\ \text { mY1: } & \text { GTT ATC TCA ATT GAT TGT TCA CAG TC } \\ \text { mY3: } & \text { GGC TGG TCC GAG TGC AGT GG } \\ \text { RNU1: } & \text { CCATGATCACGAAGGTGGTTT } \\ \text { RNU6: } & \text { CTCGCTTCGGCAGCACA } \\ \text { snord65: } & \text { TAGTGGTGAGCCTATGGTTTT } \\ \text { snord68: } & \text { AGTACTTTTGAACCCTTTTCCA }\end{array}$

\section{Northern oligo's}

Y1_5p:

TTG AGA TAA CTC ACT ACC TTC GGA CCA GCC

Y1_3p: GTC AAG TGC AGT AGT GAG AAG

5'-tRNA-Glu_CTC:

CCG AAT CCT AAC CAC TAG ACC ACC AG

5'-tRNA-Gly_GTT:

GCA TTG GTG GTT CAG TGG TAG AAT TCT CGC C

\section{Statistical analyses}

Differences between the numbers of EV released by LPS- and VitD3-stimulated DC versus control DC were analyzed by one-way ANOVA with Dunnett's 2-sided post-hoc test. Similar statistical testing was performed on differences in RNA yield. Differences in RNA biotypes in EV and cells were analyzed by two-tailed t-test. Differences in fold-changes of individual RNAs as measured in RT-qPCR were analyzed by one-way ANOVA. Significance was defined as $p<0.05$. Statistical tests on data not derived from RNA sequencing were done in SPSS (v24, IBM).

\section{Availability of data and materials:}

Raw data and count tables are deposited in the GEO database under accession number GSE105151. Written details on experimental procedures have been submitted to the EV-TRACK knowledgebase (EV-TRACK ID: EV170030) [5]. 


\section{Results}

Functionally distinct subsets of mouse bone marrow-derived DCs were generated by exposure of cells to LPS, thereby inducing immune-stimulatory DC (LPS-DC), or to 1a,25-dihydroxyvitaminD3, thereby inducing immune-suppressive DC (VitD3-DC) [56]. Untreated DC were used as control cells. The different DC subtypes displayed the expected differences in expression of the activation markers, with LPS-DC showing increased levels of MHC class II and CD86 expression, while VitD3-DC showed increased PD-L1/CD86 ratios [57] and resistance to LPS activation [58] (Supplementary Figure 1A-F). We isolated EV from cell culture supernatant of LPS-DC, VitD3-DC and control DC using differential (ultra)centrifugation followed by density gradient separation, as previously published by our group (Supplementary Figure 2A and [52]). Transmission electron microscopic analysis indicated that low density fractions contained 100$150 \mathrm{~nm}$ sized EV, whereas no EV were observed in high density fractions enriched in protein complexes (Supplementary Figure 2B). Our in-house developed highresolution flow cytometric method was used for high-throughput EV analysis at the single particle level $[49,52]$. The light scatter patterns induced by EV from differently stimulated DC were highly similar (Supplementary Figure $\mathbf{2 C}$ ). However, we detected differences in the number of released EV, with LPS-DC releasing more, and VitD3-DC releasing less EV than non-treated control DC (Fig. 1A). These quantitative differences were confirmed by Nanoparticle Tracking Analysis (NTA) (Supplementary Figure 2D). In addition, the NTA data indicated that the majority of EV observed in all conditions were in the 100-200 $\mathrm{nm}$ size range. Western blot analysis showed that the DC-derived EV contained variable levels of common EV proteins such as CD9, MHCII, CD63, and Galectin-3 [59], whereas abundant cellular proteins such as beta-actin were not detected (Supplementary Figure 2E). These data demonstrate that VitD3 and LPS treatment of DC differentially affect the number and protein content of EV released by these cells.

Next, we analyzed how LPS- or VitD3 treatment of DC affected the RNA content of EV released by these cells. It is important to note that extracellular RNA can be associated to either EV or nucleoprotein complexes, which may sediment at similar centrifugal force [4]. Using density gradient-based purification, we separated EV from contaminating nucleoprotein complexes. Depending on the differentiation status of the parental DC, the EV-free nucleoprotein fraction contained between $26 \%$ and $55 \%$ of total extracellular RNA released from cells (Supplementary Figure 2F-G). The total amount of EV-associated RNA released by equal numbers of control, LPS- and VitD3treated DC was different and reflected the differences in EV numbers (Figure 1A, B). To assess whether DC stimulation altered the RNA composition of EV, we prepared sequencing libraries of EV-associated and cellular small RNA from control-, LPS-, and 
VitD3-DC cultures ( $\mathrm{n}=3$ biological replicates). Sequencing libraries were prepared using an adapter-ligation based method routinely used in miRNA profiling [60]. Such a method has been frequently applied in studies to demonstrate the presence of miRNA and other small non-coding RNA types in EV (e.g.[23, 24, 31, 32, 61]). All libraries contained RNAs with lengths ranging between 20 and 300 nucleotides. Since short RNA molecules have a selective advantage during PCR amplification and preamplification on the sequencing flow-cell, leading to an apparent over-representation of short read lengths, we enriched the libraries for longer RNAs to ensure sufficient coverage of these sequences. To this end, cellular and EV-associated CDNA libraries were split into different size fractions of $15-25 \mathrm{nt}$ (includes miRNAs), $25-60 \mathrm{nt}$, 60 - 80 nt (includes tRNAs), and $80-275$ nt. Subsequently, we enriched for longer RNA molecules by pooling these size fractions in a volumetric ratio of $2: 2: 3: 3$ (for details, see Materials \& Methods). This indeed resulted in better coverage of 100-300 nt mid-size RNAs, such as full-length Vault RNA (142 nt) (Supplementary Figure 3). More than 13 million reads were obtained for each library (Supplementary Table 1). Pearson coefficients for biological replicate samples were $\geq 0.85$ for $\mathrm{EV}$, and $\geq 0.95$ for cells (Supplementary Figure 4). As expected based on previous studies [23, 30-32, 34], we detected the presence of various small RNA classes in EV (miRNA, snoRNA, snRNA, tRNA, miscRNA and rRNA). Some RNA types were enriched in EV, such as Y-RNA, Vault RNA and 7SL RNA classified as 'miscRNA', while other RNA types, such as snoRNA, were relatively less abundant in EV compared to cells (Figure 2). Since we depleted ribosomal RNA before preparation of the sequencing libraries, apparent enrichment of rRNA in EV is probably caused by differences in depletion efficiency between cells and EV. MiRNA and snRNA abundance was similar between cells and EV. The percentage of reads per RNA class between LPS-, VitD3-, or control cells and EV did not show major differences as a result of cell stimulation (Supplementary Figure 5A - B).

Next, we assessed whether, within each of the different classes, the levels of individual RNAs in EV changed as a result of cell stimulation. Hereto, read counts were normalized per RNA class, after which the fold changes in reads per million (RPM) of individual RNAs in LPS or VitD3 over control conditions were calculated. The scatter plots in Figure 3A - E display the log-fold changes induced by LPS versus VitD3 treatment for the indicated RNA classes (dashed lines correspond to a log2fold change of 1). Dot size indicates the average $(n=3)$ abundance of a transcript in EV and RNAs with non-adjusted $p<0.05$ are highlighted in black. The scatter plots of these data indicated that LPS- and VitD3-treatment affected the levels of miRNA, snoRNA, snRNA and miscRNA in EV (Figure $\mathbf{3 A}$ - E). Some of the stimulation-induced changes were similar for LPS and VitD3 conditions, while other RNAs show opposing alterations, or change only in one of the stimulation conditions. 
A

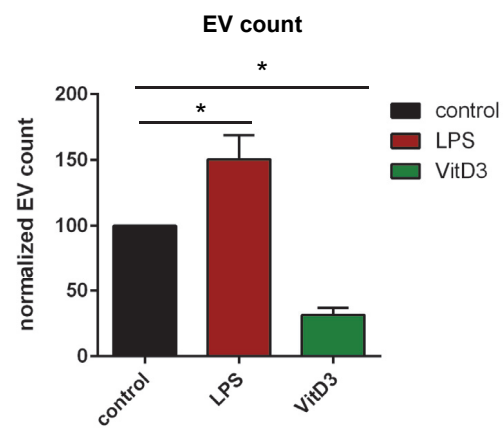

B

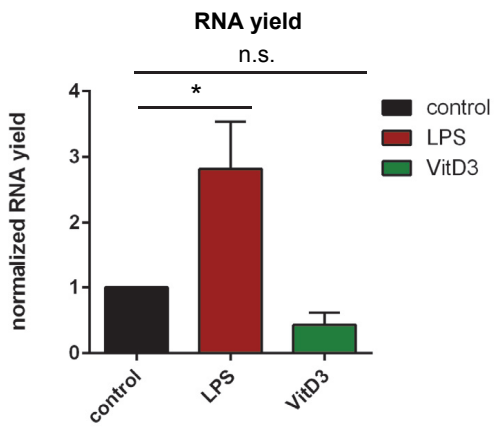

Figure 1. LPS- and VitD3-stimulated DCs release different numbers of extracellular vesicles. A) EV derived from equal numbers of control, LPS- and VitD3 treated DC were fluorescently labeled using PKH67, separated from RNPs and free dye by sucrose density centrifugation, and quantified by high-resolution flow cytometry. Indicated are the number of fluorescent events detected in 30 seconds, normalized to the control condition (mean $+/-$ s.d. of $n=3$ independent experiments, one-way ANOVA with Dunnett's 2-sided post-hoc test, ${ }^{*} p<0.05$. B) Bioanalyzerbased quantification of RNA isolated from EV derived from equal numbers of cells purified by density gradient centrifugation. Small RNA yields were normalized to the control DC condition. Indicated are the mean $+/$ - s.d. values of $n=4$ independent experiments (one-way ANOVA with Dunnett 2-sided post-hoc test, ${ }^{*} p<0.05$ )

A

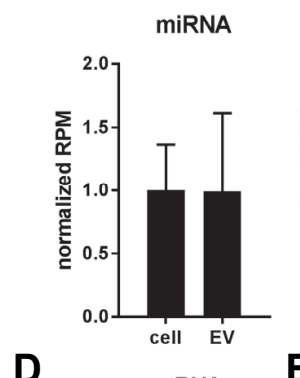

D

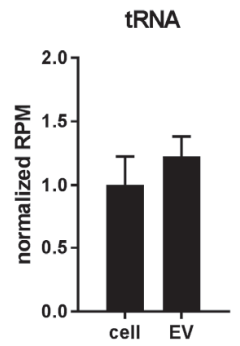

B

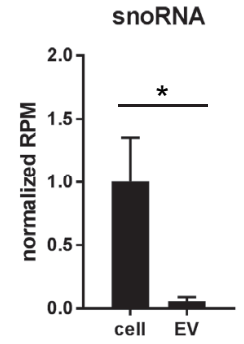

E

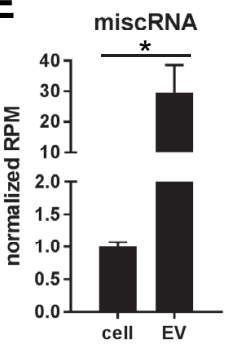

C

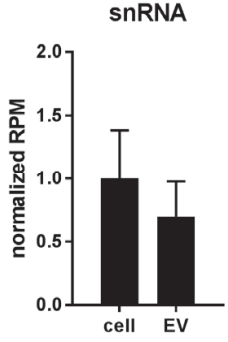

$\mathbf{F}$

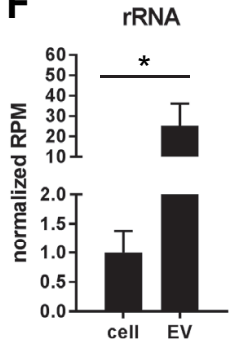

Figure 2. Cells and EV differ in the relative abundance of different small RNA classes. A-F) The relative abundance of the indicated ncRNA classes in cells versus EV was assessed by small RNAseq analysis of cellular and EV-associated RNA. Total read count for each RNA class was normalized to total library size. To compare the distribution of ncRNA classes between cells and EV, normalized RNA class read counts in EV were scaled to the normalized RNA class read counts in cellular RNA (set to 1). Indicated are mean values $+/$ - s.d. of $n=3$ independent experiments (twotailed t-test on normalized CPM values, ${ }^{*} p<0.05$ ) 
Of the tested RNA classes, tRNA levels in EV showed the lowest rate of change due to LPS or VitD3 stimulation. 97\% of tRNA reads mapped to 6 different tRNA isoacceptors (GluCTC, GlyGCC, LysCTT, GluTTC, GluCTG, and LysTTT) (Supplementary Figure 6A). Although relatively high tRNA read counts were observed in the EV-associated RNA pool (Supplementary Figure 5B), the levels of these six abundant tRNAs remained stable $(\log 2 \mathrm{FC}<1)$ in response to cellular stimulation (Supplementary Figure 6B). Besides tRNAs, the highly purified EV populations used in this study also contained substantial levels of snRNA, which is an RNA type known to be mainly confined to the nucleus. Although cell stimulation seemed to induce changes in EV-associated snRNA levels (Figure $\mathbf{3 C}$ ), many of the individual data points mapped to multicopy genes with highly similar sequences (>95\% sequence identity) corresponding to known snRNAs. Cumulative analysis of these multicopy genes indicated that $96 \%$ of snRNA reads mapped to 4 different snRNAs (U1, U2, U5, and U6) (Figure 4A), of which the levels in EV did not significantly differ between stimulation versus control conditions (Figure 4B). Thus, the levels of snRNAs and tRNAs, which are commonly detected RNA types in EV, remained constant upon different immune stimuli imposed on the EV-producing cells.

In contrast to tRNAs and snRNAs, the EV-associated levels of three other RNA types tested here, i.e. miRNAs, snoRNAs, and miscRNAs changed in response to the different stimulate imposed on the DC. We aimed to identify the RNAs differentially present in EV released by LPS- versus VitD3-treated DC, since such RNAs may underlie distinct effects of EV on recipient cells. Within the miRNA group, we observed the highest number of RNAs that differed between the LPS and VitD3 groups with a fold change $>2$ and with FDR values $<0.05$ (Figure 5A, red dots). Interestingly, for the majority (15 out of 20) of the top miRNAs that differed significantly between VitD3 and LPS conditions with lowest FDR values, DC functions have been described previously (Table 1 and [62-73]). MiRNAs known to be involved in the pro-inflammatory function of DC were enriched in LPSinduced EV, whereas EV from VitD3 treated DC were enriched in miRNAs that dampen immune stimulatory signaling cascades in DC. We validated three LPS-enriched and three VitD3-enriched RNAs by RT-qPCR. The use of stable reference transcripts in RTqPCR analysis is important to ensure reliable normalization between different samples. However, the identity and general applicability of reference transcripts stably present in EV are understudied. Here, we used our sequencing dataset to select four non-coding RNAs that were present in EV at comparable levels across all conditions, and confirmed their stability by RT-qPCR (Supplementary Figure 7A-B). We normalized our qPCR data to the geometric mean of these four genes to minimize errors caused by technical and/or biological variation [74]. Using this normalization strategy, RT-qPCR analysis confirmed the sequencing data for the tested miRNAs, although miR146-5p did not reach significance (Figure 5B). Together, these data indicate that opposing stimuli imposed on the same type of parental cell induce the release of functionally different sets of miRNAs via EV. 
A

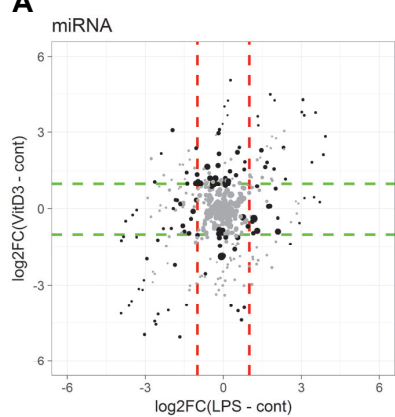

D

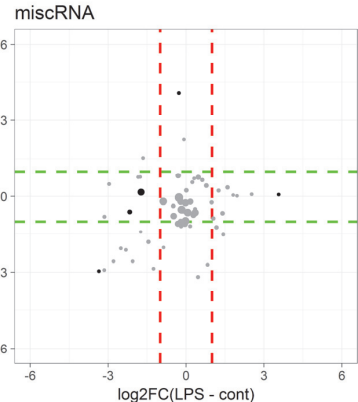

B

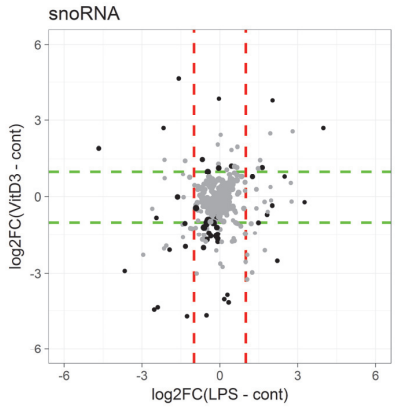

E

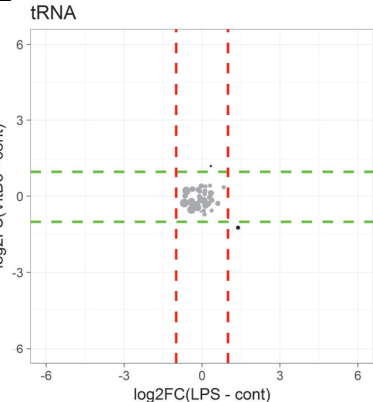

C
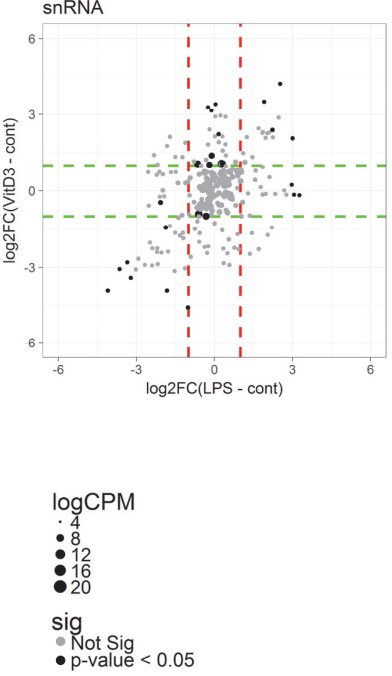

Figure 3. LPS- versus VitD3-induced changes in EV-associated RNA classes. EV-associated RNA from control, LPS, and VitD3 conditions was isolated and analyzed by RNA sequencing. Read counts for individual RNAs were normalized to the total read counts of each RNA class. A-E) LPSor VitD3-induced fold-changes and corresponding $p$-values were calculated relative to the control condition with edgeR GLM method. Data are expressed as log2fold-change in LPS-EV relative to control EV versus log2fold-change in VitD3-EV relative to control EV. Cutoffs for log2fold-changes larger or smaller than 1 are indicated with dashed lines (red = LPS, green $=$ VitD3), so all data points beyond these lines are differentially expressed with log2FC $>1$. Dot size represents the normalized abundance (logCPM) of individual RNAs. RNAs that changed with non-adjusted $p<0.05$ are highlighted in black.
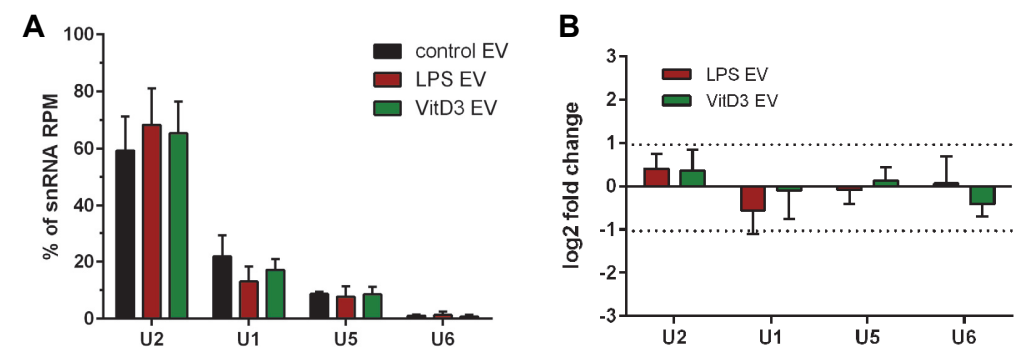

Figure 4. EV from LPS- and VitD3-stimulated DC display stable levels of abundant snRNAs. EV-associated RNA from control, LPS, and VitD3 conditions was isolated and analyzed by RNA sequencing. Read counts for individual RNAs were normalized to the total read counts of each RNA class. A) Read counts for the top-four most abundant snRNAs, constituting $96 \%$ of snRNA reads in EV-RNA, are expressed as percentage of the total snRNA read count in EV. B) Data from (A) are expressed as fold-change in the indicated snRNA levels of VitD3- or LPS-EV relative to control-EV (mean $+/$ - s.d. of $n=3$ experiments; in one-way ANOVA, no significant differences were observed) 
A
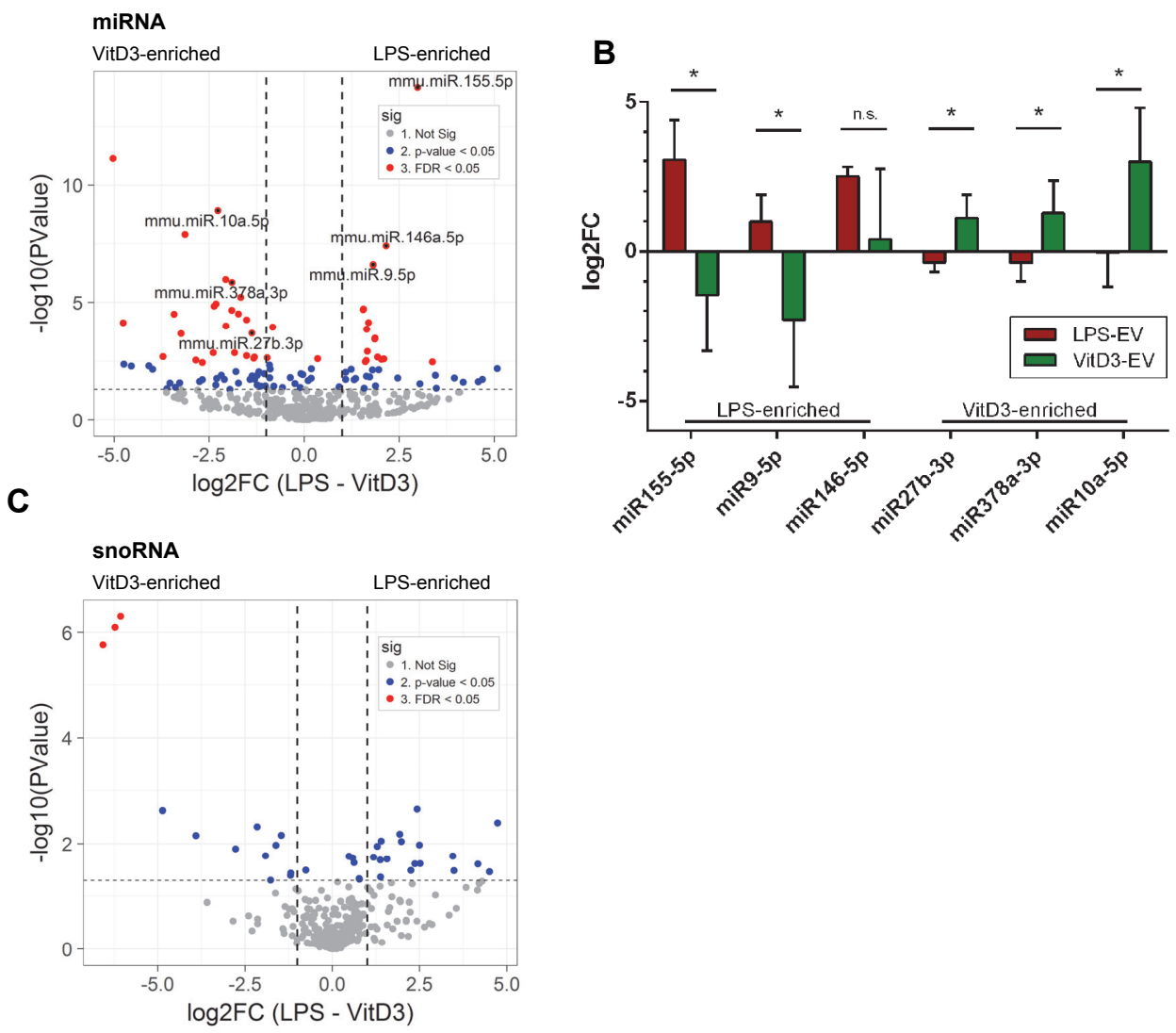

D

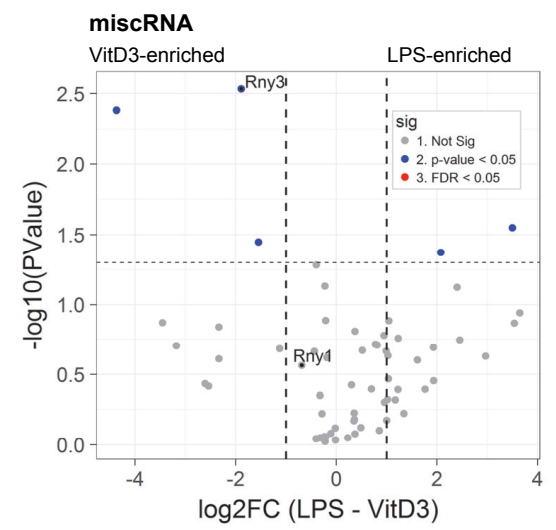

$\mathbf{E}$

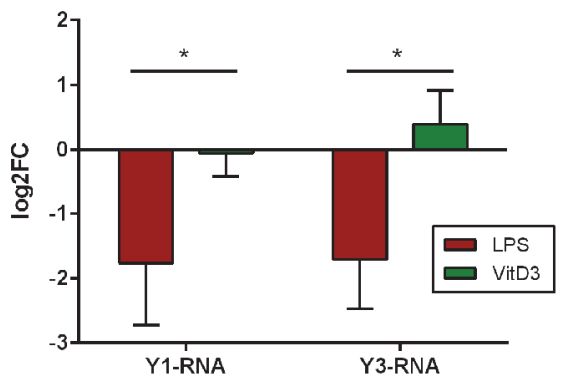


Figure 5 EV from LPS-/VitD3-stimulated DC display differences in the levels of several miRNAs, snoRNAs, and Y-RNAs. EV-associated RNA from control, LPS, and VitD3 conditions was isolated and analyzed by RNA sequencing. Read counts for individual RNAs were normalized to the total read counts of each RNA class. LPS- or VitD3-induced fold-changes and corresponding p-values were calculated relative to the control condition. A) Volcano plots of EV-associated miRNAs in LPS versus VitD3 conditions. Thresholds for 2 -fold-change and non-adjusted $p<0.05$ are indicated. Data points represent average values of $n=3$ biological replicates. Significant changes are indicated with different colours. Grey: non-significant, blue: non-adjusted p-value $<0.05$, red: FDR $<0.05$. B) RTqPCR validation of six miRNAs showing differential abundance between LPS-EV and VitD3-EV. Data are expressed as log2fold-change in LPS- and VitD3-EV compared to control-EV. Indicated are the mean $+/$ - s.d. values of $n=4$ independent experiments, one-way ANOVA, ${ }^{*}=p<0.05$. C, D) Volcano plots of EV-associated snoRNAs (C) and miscRNAs (D) in LPS versus VitD3 conditions. Thresholds for 2-fold-change and non-adjusted $p<0.05$ are indicated. Data points represent average values of $\mathrm{n}=3$ biological replicates. Significant changes are indicated in different colours. Grey: non-significant, blue: non-adjusted p-value $<0.05$, red: FDR $<0.05$. E) RT-qPCR validation of $Y 3$ and its family member $Y 1$ showing differential abundance between LPS-EV and VitD3-EV compared to control-EV. Indicated are the mean $+/$ - s.d. values of $n=4$ independent experiments, one-way ANOVA, ${ }^{*}=p<0.05$

Table 1. MicroRNAs enriched in LPS- or VitD3-EV with known functions in DC

\begin{tabular}{|c|c|c|c|c|}
\hline identifier & enriched in & FDR & Function in DC & ref $n r$ \\
\hline mmu.miR.155.5p & LPS-EV & $4.04 \mathrm{E}-12$ & Master regulator in DC maturation & [62] \\
\hline mmu.miR.708.3p & VitD3-EV & $2.21 \mathrm{E}-09$ & Downregulated in mature/activated DC & {$[63]$} \\
\hline mmu.miR.10a.5p & VitD3-EV & $2.31 \mathrm{E}-07$ & $\begin{array}{l}\text { Inhibits DC activation and Th } 1 / \mathrm{Th} 17 \text { cell immune } \\
\text { responses }\end{array}$ & [64] \\
\hline mmu.miR.146a.5p & LPS-EV & 4.53E-06 & $\begin{array}{l}\text { Down regulates IL-12p70, IL-6, and TNF-a production } \\
\text { by DC }\end{array}$ & [65] \\
\hline mmu.miR.9.5p & LPS-EV & $1.86 \mathrm{E}-05$ & $\begin{array}{l}\text { Regulatory circuitry controlling monocyte activation } \\
\text { by LPS }\end{array}$ & {$[66]$} \\
\hline mmu.miR.223.5p & VitD3-EV & $6.82 \mathrm{E}-05$ & $\begin{array}{l}\text { Repression of pro-inflammatory cytokine release by } \\
\text { DC }\end{array}$ & [67] \\
\hline mmu.miR.378a.3p & VitD3-EV & $8.24 \mathrm{E}-05$ & Upregulated in VitD3-treated DC & {$[68]$} \\
\hline mmu.miR.203.3p & VitD3-EV & 0.000328 & Upregulated in tolerogenic DC & {$[69]$} \\
\hline mmu.miR.199a.3p & VitD3-EV & 0.000484 & Upregulated in tolerogenic DC & {$[69]$} \\
\hline mmu.miR.27b.5p & VitD3-EV & 0.000578 & $\begin{array}{l}\text { Suppression of inflammatory cytokine production via } \\
\text { NF-KB }\end{array}$ & [69] \\
\hline mmu.miR.7a.5p & LPS-EV & 0.000703 & Upregulated in LPS / IFNg stimulated DC & {$[69]$} \\
\hline mmu.miR.126a.3p & VitD3-EV & 0.000946 & Reduces the responsiveness of DCs to TLR7/9 ligands & {$[70]$} \\
\hline mmu.miR.708.5p & VitD3-EV & 0.000946 & Suppresses NF-kB signaling & [71] \\
\hline mmu.miR.181b.3p & VitD3-EV & 0.001923 & Inhibition of CD40 and MHCII expression & {$[72]$} \\
\hline mmu.miR.27a.5p & VitD3-EV & 0.002511 & Suppression of inflammatory cytokine production & [73] \\
\hline
\end{tabular}

EV-associated RNA from control, LPS, and VitD3 conditions was isolated and analyzed by RNA sequencing. Read counts for individual RNAs were normalized to the total read counts of each RNA class. LPS- or VitD3-induced fold-changes and corresponding p-values were calculated relative to the control condition with edgeR GLM method. We created a top 20 list of miRNAs with the lowest FDR values. Indicated are miRNAs from this top 20 for which DC-related functions have been reported. 
Besides changes in miRNA levels, the levels of several EV-associated snoRNAs were found to differ between LPS and VitD3 conditions (Figure $\mathbf{5 C}$ ), with three H/ACA box snoRNAs (snora2b, snora32, snora55) displaying FDR levels $<0.05$. The overall low abundance of this RNA class in EV (Figure 2C) hampered reliable quantification of snoRNAs in EV by RT-qPCR (data not shown). Nevertheless, our data are a first indication that EV-associated snoRNA levels change with the activation status of parental cells. Although none of the RNAs in the miscRNA group reached FDR levels $<0.05$, analysis by RT-qPCR indicated that the levels of Y3-RNA (Rny3) and an additional member of the Y-RNA family, Y1-RNA (Rny1), significantly differed between LPS and VitD3 conditions (Figure 5D - E). In cells, the conserved family of Y-RNAs are known to bind to several different proteins that play a role in RNA quality control $[75,76]$. Members of the small non-coding Y-RNA family have frequently been detected in EV from multiple different cell types and different body fluids [30-32, 34, 35, 77-81]. We here provide evidence that Y-RNA levels in EV can be regulated by stimuli imposed on the EV-producing cell. The reduced Y-RNA levels in EV from LPS-stimulated DC compared to EV from VitD3stimulated DC (Figure 5E) indicate that the presence of Y-RNAs in EV may be indicative for the immune function of the parental cells. Overall, our data indicate that immune cell stimuli cause changes in the EV-associated levels of specific miRNAs, snoRNAs and Y-RNAs.

So far, we compared the abundance of specific RNA classes in EV by assessing the total numbers of reads mapping to non-coding RNA genes. However, also the fragmentation profile of EV-associated RNAs may be functionally important and indicative for the status of the EV producing cell. Fragmented forms of RNA have been described in EV from a wide range of cells and biological fluids [30-32, 34, 35, 77, 78, 81]. Especially Y-RNA fragments (19-35 nt) have gained attention because they were found to associate with Argonaute, suggesting scope for gene-regulatory functions $[82,83]$, and because their presence in plasma has been linked to disease $[78,84]$. We examined our data to determine whether reads mapping to Y-RNAs included these fragments in the purified DC-derived EV. Based on RNAseq data, 5' and 3' fragments of Y1-RNA were present in EV and such fragments seemed more abundant than the full-length form of this Y-RNA (Figure 6A). To confirm the full length or fragmented nature of the Y-RNA molecules, we performed Northern blot analysis. Using probes recognizing the $5^{\prime}$ and $3^{\prime}$ end of $Y 1-R N A$, we found that both $5^{\prime}$-and $3^{\prime}$ fragments and full-length Y-RNA could be detected in both cellular and EV-RNA (Figure 6B - D). Strikingly, the relative amount of full-length Y1-RNA detected by Northern blot was much higher than expected based on the sequencing coverage plots. This indicates that RNAseq-based detection of the full length form of Y1-RNA was highly inefficient. This is likely due to the $5^{\prime}$-triphosphate group of full length Y-RNAs or their strong secondary structure, which may hamper sequencing adaptor ligation and thereby 
efficient amplification and sequencing of these RNAs. Interestingly, Northern blot analysis also indicated that the levels of full-length Y1-RNA in LPS-EV were reduced, which was in accordance to the RT-qPCR analysis for full length $\mathrm{Y} 1$ (Figure 5E), while the levels of fragmented Y1-RNA in EV remained relatively stable (Figure 6D). These data urge caution in classifying fragmented and full-length forms of Y-RNA based on RNA sequencing data.

A

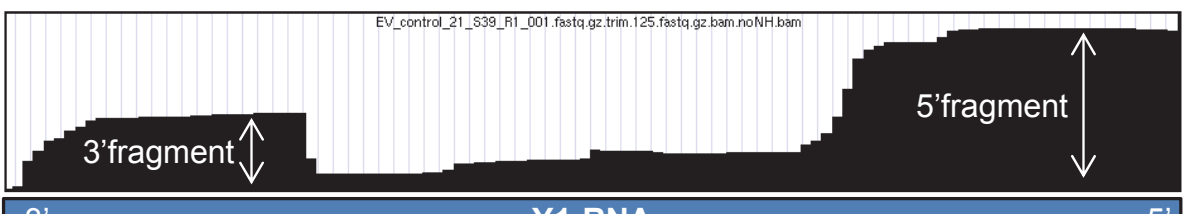

B

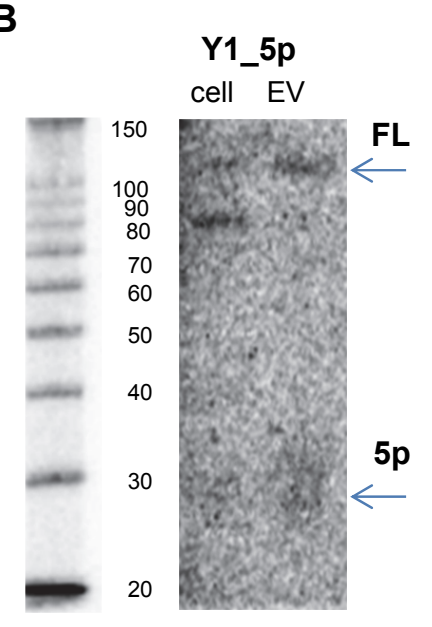

C

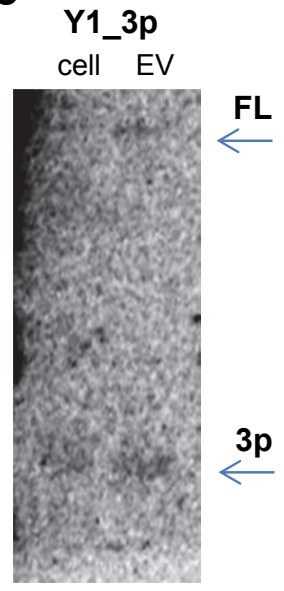

D

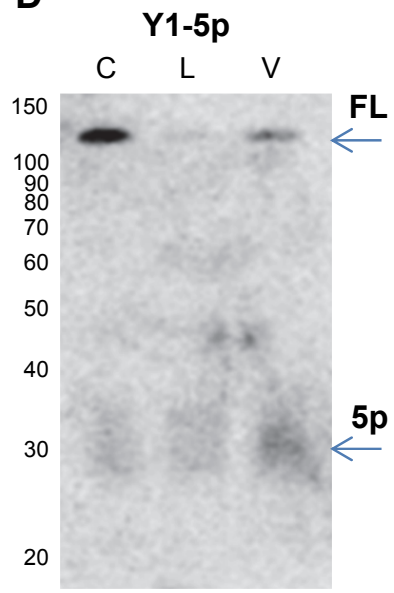

Figure 6. Full-length versus fragmented forms of Y1-RNA in cells and EV validated by Northern blot. A) Representative coverage plot of Y1-RNA as observed in EV RNA seq data (sequencing coverage depth 3,981) visualized in the UCSC genome browser. B) RNA isolated from control, LPS, and VitD3-treated cells and their EVs were analyzed by Northern blot for detection of full length (FL) and 5'fragments (5p) of Y1-RNA using Y1-5p probe. $10 \mathrm{ng}$ of smallRNA was loaded per lane. C) Northern blot detection of full length (FL) and 3'fragments (3p) of Y1-RNA using Y1$3 p$ probe. $10 \mathrm{ng}$ of smallRNA was loaded per lane. D) Northern blot detection of full-length and 5 'fragments of Y1-RNA in EV-RNA from differently stimulated DC ( $C=$ control, L=LPS, V=VitD3). 10 $\mathrm{ng}$ of smallRNA was loaded per lane. Data are representative for $\mathrm{n}=2$ independent experiments. 
A miRnA

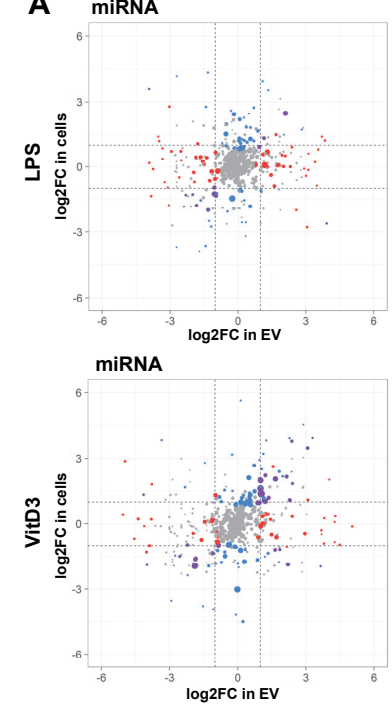

B

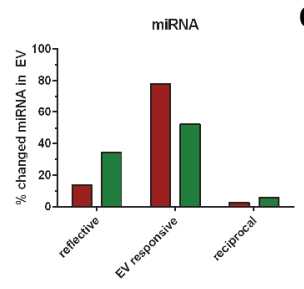

E

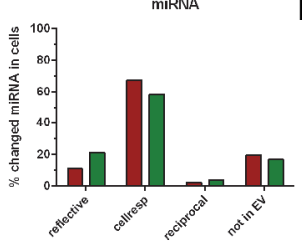

H miR155-5p-LPS miR9-5p - VitD3

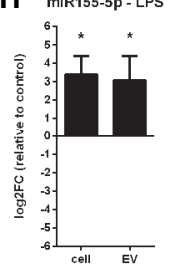

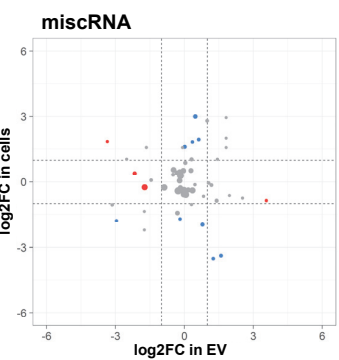

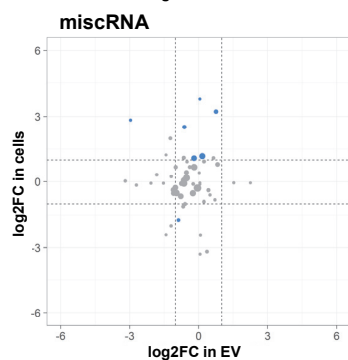

C

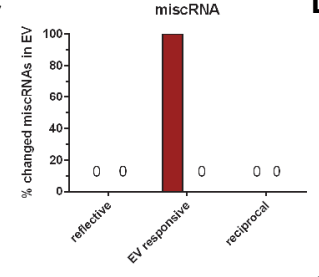

F

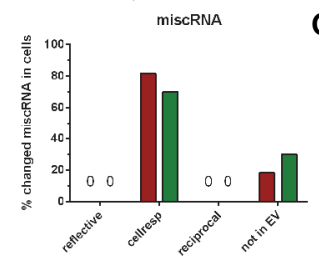

D
snoRNA

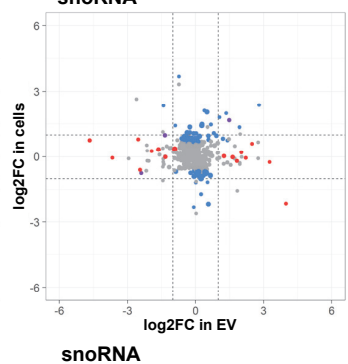

legend $\log C P M$
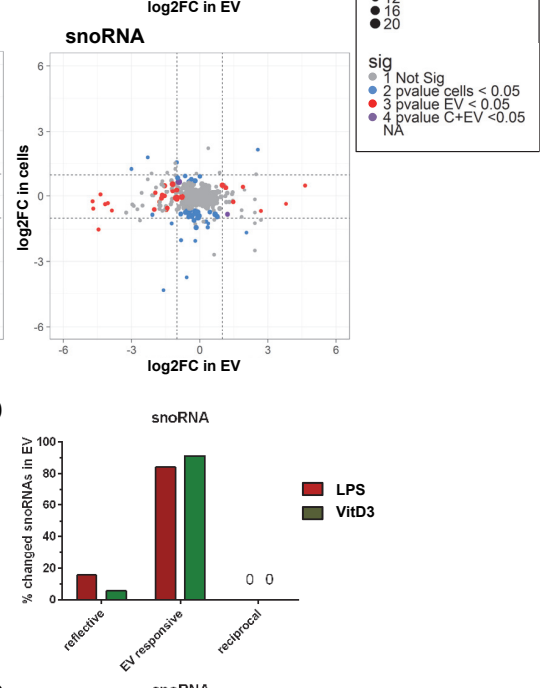

G

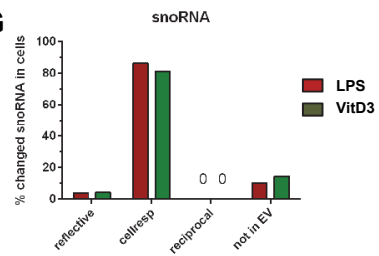

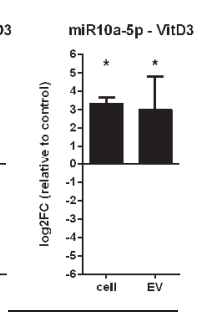
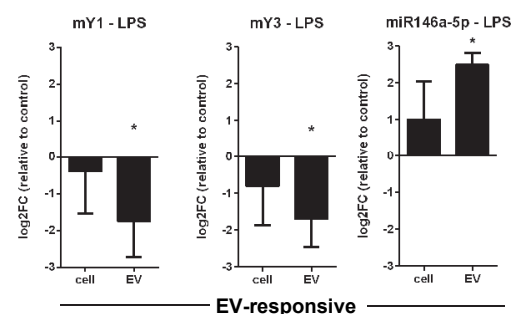
Figure 7. LPS-/VitD3-induced changes in cellular versus EV-associated RNA levels. Cellular and EV-associated RNA from control, LPS, and VitD3 conditions was isolated and analyzed by RNA sequencing. Read counts for individual RNAs were normalized to the total read counts of each RNA class. LPS- or VitD3-induced fold-changes and corresponding p-values were calculated relative to the control condition for cellular and EV-associated RNA. A) Fold-changes in EV-RNAs were plotted against the fold changes in cellular RNA in response to LPS (top graphs) and VitD3 (lower graphs) for miRNA (left), miscRNA (middle) and snoRNA (right). Dashed lines indicate log2FC larger or smaller than 1, so all data points beyond these lines are differentially expressed with $\log 2 \mathrm{FC}>1$. Colored dots indicate transcripts that changed with non-adjusted $p$-values < 0.05 in cells (blue), in EV (red), or in both cells and EV (purple), grey dots indicate unchanged transcripts. Dot size represents the normalized abundance (logCPM) of individual RNAs, mean of $n=3$ experiments. B-D) Transcripts showing significant changes in stimulated versus control EV ( $p$-value < 0.05) were selected, and fold-changes in EV versus corresponding cells were compared. Indicated are percentages of transcripts categorized as 'reflective' ( $p$-value $<0.05$ in EV and cells), 'EV-responsive' ( $p$-value $<0.05$ in EV but not in cells), and 'reciprocal' ( $p$-value $<0.05$ in cells and EV, but with opposite fold-changes). E-G) Analogous to B $-\mathrm{D}$, transcripts showing significant changes in cells $(p$-value $<0.05)$ were selected, and fold-changes in cells were compared with those in EV. Indicated are percentages of transcripts categorized as 'reflective', 'reciprocal', cell-responsive ( $p$-value $<0.05$ in cells but not in EV), or 'not in EV' (transcripts only found in cells). H) RT-qPCR validation of six genes that were found to be reflective (left panels) or EV-responsive (right panels) on EV-associated RNA and cellular RNA from control, LPS or VitD3 treated DC. Fold changes in EV and in cells were calculated relative to the control condition in cells or $\mathrm{EV}$. $\mathrm{N}=4$, one-way ANOVA, ${ }^{*}=\mathrm{p}<0.05$

Changes in the RNA content of EV have been suggested to directly reflect changes in the RNA levels of the parental cell. To test this hypothesis, we investigated whether all of the stimulation-induced changes in miRNAs, snoRNAs, and miscRNAs observed in EV reflected changes in the RNA pool of the parental cells and vice versa. After normalization within each RNA class, fold-changes in EV were plotted against the fold changes in cells for miRNA, miscRNA, and snoRNA (Figure 7A), with dashed lines indicating log2FC of 1 or -1 . Dot size indicates the average $(n=3)$ abundance of a transcript in EV, and dot coloring is used to indicate RNAs that were significantly changed in cells, EV or both. First we tested whether changes in EV-associated RNAs reflected stimulation-induced changes in cells. RNAs of which the levels in EV significantly changed (non-adjusted p-values <0.05) upon LPS or VitD3-treatment were categorized as 'reflective RNAs' ( $p<0.05$ in both cells and EV), 'EV-responsive RNAs' ( $p<0.05$ in EV, $p>0.05$ in cells), or 'reciprocal RNAs' (opposite changes in EV and cells with $\mathrm{p}<0.05$ ) (Supplementary Table 2). Of all RNAs that significantly changed in EV (set to 100\%), a minor percentage of the miRNAs and snoRNAs reflected the up/downregulation in cells or were reciprocally regulated, while most of the RNAs changed only in EV, but not (significantly) in cells (Figure 7B - D). Conversely, we aimed to assess which proportion of LPS- or VitD3-induced changes in cellular RNA was reflected in the EV-RNA content. RNAs that changed in cells upon cell stimulation 
(non-adjusted $\mathrm{p}$-values $<0.05$ ) were therefore classified as 'reflective' ( $p<0.05$ in both cells and EV), 'cell-responsive' ( $p<0.05$ in cells, $p>0.05$ in EVs), 'reciprocal' (opposite changes in EV and cells with $p<0.05$ ), or 'absent in EV' ( $p<0.05$ in cells, but not detected in EV). Comparable to what we observed in the above analysis of EV-RNA, only a minor percentage of changes in cellular miRNAs and snoRNAs was reflected in EV. The majority of the RNAs that changed in cells did not significantly change in EV (Figure 7E-G), and $10-20 \%$ of the RNAs that changed in cells were not detected in EV. We selected 3 reflective and 3 EV-responsive RNAs (Figure $\mathbf{7 H}$ ) for validation by RT-qPCR. For the reflective miRNAs miR-155, -9 , and -10 a we confirmed that the stimulation-induced fold-change in EV-associated and cellular RNA was highly similar. For the EV-responsive miRNA miR-146a and the two Y-RNAs, significant cellstimulation-induced changes were observed in EV, while cellular levels did not change significantly compared to the control conditions. Importantly, these data indicate that part of the cell stimulation-induced changes in EV-RNA content match the changes observed in cellular RNA, but that cell stimulation also induces changes in RNA levels that are only observed in cells or in EV.

Overall, the results obtained for our primary DC cultures show that the molecular messages enclosed in EV are composed of multiple RNA classes that are specifically shuttled into EV dependent on the activation status of the cell. Moreover, the stimulusinduced changes in the small RNA content of cells and EV only partly overlap.

\section{Discussion}

The data presented here broaden our view on the plasticity of the small RNA content of EV in relation to changes in the activation status of the EV-producing cell. Whereas most of the previous studies focused on the miRNA content of EV, we show that cell status-dependent changes in the RNA composition of EV also extend to other small RNA classes, which may therefore also contribute to the specific genetic messages conveyed by EV to recipient cells. The immune cell model we employed, i.e. DC that are well-known for their strong and diverse functional responsiveness to external stimuli, served two purposes. First, the cell system illustrates that diverse stimuli can induce the release of EV with variable levels of multiple RNA types, and that only some of the stimulation-induced changes in EV-associated RNAs reflect changes in the cellular RNA. Second, the data extend our knowledge on the central role of DC in raising and regulating immune responses. The identification of multiple EV-RNA types that are specifically associated with either the immune-activating or immune-suppressing status of DC is the prelude to unraveling the function of these RNAs in EV. 
So far, RNA sequencing studies in which multiple RNA classes were identified in EV employed EV isolation methods known to co-isolate extracellular protein-RNA complexes (RNP) that overlap in size with EV, e.g. ultracentrifugation and precipitationbased methods $[4,24,30,32,34,80,85,86]$. The results of our present study indicate that up to $55 \%$ of total extracellular RNA present in ultracentrifugation pellets of cell culture supernatant can be associated to RNPs. Buoyant density-based separation of EV from RNP, as employed in this study, allowed us to specifically identify RNA species that cells differentially sort into EV upon exogenous stimulation. In RNA sequencing libraries prepared from these highly pure EV we observed that the percentage of alignment to the mouse genome was lower than in cellular RNA libraries. This is likely caused by the (ultra) low input quantity of RNA in our EV sequencing libraries, which amplifies the contribution of lab-derived contaminant RNAs e.g. from commercial nucleic acid extraction kits or sample cross-contamination $[87,88]$. The percentage of alignment may also be dependent on the size range of RNAs selected for sequencing. Whereas in most published EV-RNA sequencing studies RNAs in the 20-40 nt size range were sequenced, thereby strongly selecting for microRNAs, we here sequenced RNAs of a much larger size range (20-300 nt). We previously showed that several of these larger and highly modified/structured RNAs, for which the sequencing efficiency may be compromised, are enriched in EVs compared to cellular RNA [30], which may have caused the percentages of alignment to be lower in EV compared to cells. For our studies, we selected a commercial adapter-ligation based method that has been frequently used in EV-RNA sequencing studies [23, 24, 31, 32, 61]. While optimized for miRNA analysis, it should be noted that these library preparation methods show bias in the efficiency with which sequences with base modifications, terminal triphosphate groups, and strong secondary structures are ligated and sequenced [89]. As an example, we here provide Northern blot-based evidence that the frequently reported predominance of Y-RNA fragments over full length Y-RNA in EV [30, 32, 35, 90] represents a sequencing artefact. We observed a similar discrepancy between sequencing read coverage and Northern blot analysis for tRNAs (Supplementary Figure 8). While the triphosphate group on the $5^{\prime}$-end [91] or their strong secondary structure may hamper efficient sequencing of full length Y-RNA, difficulties with sequencing of full length tRNAs are likely due to the inability of the reverse transcriptase enzyme used during sequencing library preparation to read through the highly modified tRNA structure [92]. This is corroborated by recent sequencing studies deploying reverse transcriptases that are insensitive to secondary RNA structures $[93,94]$. Together, these findings urge caution in the interpretation of RNA fragmentation based on RNA sequencing data alone. Moreover, we reduced the effect of sequencing biases on the assessment of quantitative differences in EV-RNA content by determining the fold-changes in identical transcripts between different conditions. 
Our experimental approach allowed comprehensive analysis of changes in EV-RNA classes induced by diverse immune-relevant stimuli. The most prominent stimulationinduced changes in EV-associated RNA levels were found for miRNA, Y-RNA, and snoRNAs (Fig. 3). Although detected in EV from multiple sources [23, 31, 33-35], the presence of snoRNAs in EV has been largely understudied. Here we provide evidence that the levels of EV-associated snoRNAs can be regulated by stimuli imposed on the EV producing cell. Since changes in the cellular levels of snoRNA have been associated with diseases $[36,95,96]$, our present data point to EV-associated snoRNAs as potential functional entities in EV and interesting candidate biomarkers indicative of the cellular activation status.

Numerous studies already demonstrated that miRNA levels in EV change upon disease induction or cell stimulation [22-24]. Unique aspects of our present RNAseq study are the use of two different stimuli for comparative analysis of RNA levels in highly purified EV and the parallel assessment of cellular and EV-associated RNA. The enrichment of endotoxin-responsive miRNAs, such as miR-155 and miR-146a [20, 97, 98] that we observed in EV from LPS-stimulated DC concurs with previously published semi-quantitative microarray-based data on the miRNA content of EV from stimulated DC $[22,26]$. Both miR-155 and miR-146a are known to be endotoxin responsive, but miR-155 has an immune-activating role, while miR-146a is involved in dampening of immune responses and could act as a molecular brake on inflammation [99] . It is thought that the coordinated action of these two is important in regulation of immune response [21]. Differences in the miR-155/miR-146a ratios that we observed in LPS-EV and VitD3 EV (Figure 5B) may therefore lead to different effects of these EV on immune activation. In addition, we here demonstrated that a different (i.e. immune downregulatory) type of stimulus imposed on DC led to both a reduction of EVassociated levels of these immune-activating miRNAs and an enrichment in miRNAs implicated in suppression of immune responses via modulation of multiple pathways. Examples include dampening of TLR signaling by miR-27a and miR-126a [70, 73], and downregulation of (regulators of) PI3K/Akt and NF-KB by miR-378 [100], miR708 [71] and miR-27b-3p [101], processes that have been implicated in suppression of pro-inflammatory cytokine release. Such differences in the EV miRNA content may therefore contribute to immune-activating or immune-suppressive functions of differentially stimulated DC.

Not only immune-suppressive miRNAs, but also two types of Y-RNA were found to be specifically excluded from EV released by LPS-stimulated DC (Fig. 5E). This raises the question whether $Y$-RNAs contribute to immune-related functions of EV. Until now, different types of Y-RNA ( $\mathrm{mY} 1, \mathrm{mY} 3$ in mice and hY1, hY3, hY4, hY5 in humans) have been detected in $\mathrm{EV}$ from a multitude of different cell types and in different body fluids [30-32, 34, 35, 77-81]. The extracellular presence of Y4- and Y5-RNA and fragments 
thereof has mostly been associated to cancer [32, 81, 84, 102] and coronary artery disease $[78,103]$. Immune-related functions of Y-RNAs include their capacity to trigger TLR signaling. Interestingly, Y-RNAs may vary in their specificity for different TLRs, with Y3-RNA triggering predominantly TLR3, while Y1-, Y3- and Y4-RNA trigger TLR7 [104]. On the contrary, the reduction of $Y 1$ - and Y3-RNA levels in EV released by immunestimulatory DC, shown in this study, suggest an immune downregulatory role for these EV-associated Y-RNAs. In line with this, high levels of Y-RNAs have been reported in immune-suppressive EV released by the parasite Heligmosomoides polygyrus and in seminal fluid EV for which immunosuppressive effects have been described [35, 105-107]. Overall, the observed variable presence of Y-RNA in EV from immune cells has raised further interest in unraveling the immune-related function and biomarker potential of these RNAs in EV.

With regard to the biomarker potential of EV-associated RNAs, our data also urge caution in interpreting EV as snapshots of the cell from which they arise. We found that only a subset of changes in EV-RNA reflected changes that occurred at the cellular level. The highest proportion of reflective RNAs was observed within the category of miRNAs and included prominent immune-related RNAs such as miR-155, miR-9, and miR-10-5p (Fig. 7H). For a substantial number of EV-associated miRNAs and both Y-RNA types, however, stimulation-induced changes were observed in EV, but not in cells. This could suggest that EV-levels of reflective RNAs are mainly regulated by transcription, whereas levels of EV-responsive RNAs are mainly determined by shuttling rate. For RNAs known to primarily reside in the nucleus (such as snRNAs and snoRNAs), subtle changes in the cytoplasmic pool of these RNAs may be overshadowed by the much larger nuclear pool. Comparison of EV-associated levels with cytoplasmic instead of total cellular RNA levels may therefore provide better insight in shuttling of these nuclear RNAs into EV. Interestingly, we also observed that many of the stimulationinduced changes in cellular RNA levels were not reflected in the RNA levels of EV released by these cells. This strengthens the hypothesis that cells release EV with selective sets of RNAs into the extracellular milieu. From a biomarker perspective, these data implicate that, although the presence of specific EV-RNAs may correlate with disease, the EV transcriptome is not necessarily predictive for RNA levels in the EV-producing cell.

We observed two classes of RNA, i.e. snRNA and tRNA, of which the EV-associated levels remained stable after differential stimulation of DC. These RNA classes have been commonly detected in small RNAseq studies of EV from multiple cell types and body fluids, but factors impacting their incorporation into EV had until now not been investigated. Although these data need confirmation in different cell types with various external stimuli, our data may be a first indication that tRNAs and snRNAs are constitutive components of EV. Speculatively, these RNAs may be required for basal 
scaffolding functions in EV biogenesis, or may aid the functional transfer of generegulatory RNAs. Based on their stable association with EV, these types of RNAs also have potential to be used as EV reference RNAs for RT-qPCR normalization since comparing the EV-transcriptome of differentially stimulated cells is complicated by potential differences in the number of EV, RNA yield and RNA composition. Importantly, the use of well-known stably expressed cellular RNAs is not necessarily valid for normalization of EV qPCR data due to potential differences in RNA shuttling between tested conditions. Therefore, normalization of RT-qPCR data to reference genes which are stable in EV is a reliable way to quantitate differences in individual RNAs. Here we show how RNA sequencing and RT-qPCR validation can be used to identify candidate EV-reference genes which are constitutively expressed between differently stimulated cells.

In conclusion, this study indicates that external stimuli imposed on cells can lead to changes in the levels of some EV-associated RNA classes, while leaving others unchanged. In our DC-model, two different immune-related cell stimuli led to disparate changes in the type and quantity of miRNAs, snoRNAs, and Y-RNAs in EV. Only part of the changes in RNA contents of EV reflected changes in the cellular RNA, which urges caution in interpreting EV as snapshots of cells. These findings show that differences in multiple structurally distinct RNA classes shape the EV-transcriptome. These RNA types may contribute to the functional properties of EV in cellular communication, and may be further explored as candidate EV-RNA based indicators for the immune status of the EV producing cell.

\section{Conflict of interest}

The authors declare that they have no conflict of interest.

\section{Acknowledgements}

The authors would like to thank Dr G.J.A. Arkesteijn for assistance with high resolution flow cytometric analysis of EV, Dr Z. Zeev Ben Mordehai for assistance with transmission electron microscopy, and Drs S.L. Wolin, S. Sim, and D. Staněk for valuable discussions. This work was supported by European Research Council under the European Union's Seventh Framework Programme [FP/2007-2013] / ERC Grant Agreement number [337581] to [ENMNtH.]; COST Action BM1202 Microvesicles and Exosomes in Health and Disease Short-Term Scientific Mission Grant [191116-083388] to [TAPD]; and the Human Frontiers Science Program [RGY0069] to [AHB]. 


\section{References}

1. Colombo M, Raposo G, Théry C (2014) Biogenesis, Secretion, and Intercellular Interactions of Exosomes and Other Extracellular Vesicles. Annu Rev Cell Dev Biol 30:255-289 . doi: 10.1146/annurevcellbio-101512-122326

2. van der Grein SG, Nolte-'t Hoen ENM (2014) "Small Talk" in the Innate Immune System via RNAContaining Extracellular Vesicles. Front Immunol 5:1-8 . doi: 10.3389/fimmu.2014.00542

3. Théry C, Ostrowski M, Segura E (2009) Membrane vesicles as conveyors of immune responses. Nat Rev Immunol 9:581-593. doi: 10.1038/nri2567

4. Mateescu B, Kowal EJK, van Balkom BWM, et al (2017) Obstacles and opportunities in the functional analysis of extracellular vesicle RNA - an ISEV position paper. J Extracell Vesicles 6:1286095 . doi: 10.1080/20013078.2017.1286095

5. Van Deun J, Mestdagh P, Agostinis P, et al (2017) EV-TRACK: transparent reporting and centralizing knowledge in extracellular vesicle research. Nat Methods 14:228-232 . doi: 10.1038/nmeth.4185

6. Tkach M, Kowal J, The C (2017) Why the need and how to approach the functional diversity of extracellular vesicles. Philos Trans R Soc B Biol Sci. doi: 10.1098/rstb.2016.0479

7. Camussi G, Deregibus MC, Bruno S, et al (2010) Exosomes/microvesicles as a mechanism of cell-to-cell communication. Kidney Int 78:838-848 . doi: 10.1038/ki.2010.278

8. Robbins PD, Morelli AE (2014) Regulation of immune responses by extracellular vesicles. Nat Rev Immunol 14:195-208 . doi: 10.1038/nri3622

9. Redzic JS, Balaj L, van der Vos KE, Breakefield XO (2014) Extracellular RNA mediates and marks cancer progression. Semin Cancer Biol 28:14-23 . doi: 10.1016/j.semcancer.2014.04.010

10. Revenfeld ALS, Bæk R, Nielsen MH, et al (2014) Diagnostic and prognostic potential of extracellular vesicles in peripheral blood. Clin Ther 36:830-846 . doi: 10.1016/j.clinthera.2014.05.008

11. Kahlert C, Melo SA, Protopopov A, et al (2014) Identification of doublestranded genomic dna spanning all chromosomes with mutated KRAS and P53 DNA in the serum exosomes of patients with pancreatic cancer. J Biol Chem 289:3869-3875 . doi: 10.1074/jbc.C113.532267

12. Raab-Traub N, Dittmer DP (2017) Viral effects on the content and function of extracellular vesicles. Nat Rev Microbiol 15:559-572. doi: 10.1038/nrmicro.2017.60

13. Arroyo JD, Chevillet JR, Kroh EM, et al (2011) Argonaute2 complexes carry a population of circulating microRNAs independent of vesicles in human plasma. Proc Natl Acad Sci U S A 108:5003-8. doi: 10.1073/pnas.1019055108

14. Turchinovich A, Weiz L, Langheinz A, Burwinkel B (2011) Characterization of extracellular circulating microRNA. Nucleic Acids Res 39:7223-33 . doi: 10.1093/nar/gkr254

15. Vickers KC, Palmisano BT, Shoucri BM, et al (2011) MicroRNAs are transported in plasma and delivered to recipient cells by high-density lipoproteins. Nat Cell Biol 13:423-33 . doi: 10.1038/ncb2210

16. Van Deun J, Mestdagh P, Sormunen R, et al (2014) The impact of disparate isolation methods for extracellular vesicles on downstream RNA profiling. J Extracell Vesicles 3:1-14 . doi: 10.3402/jev.v3.24858

17. Lötvall J (2014) Minimal experimental requirements for definition of extracellular vesicles and their functions: a position statement from the International Society for Extracellular Vesicles. 1:1-6

18. Vergauwen G, Dhondt B, Van Deun J, et al (2017) Confounding factors of ultrafiltration and protein analysis in extracellular vesicle research. Sci Rep 7:2704 . doi: 10.1038/s41598-017-02599-y

19. O'Connell RM, Taganov KD, Boldin MP, et al (2007) MicroRNA-155 is induced during the macrophage inflammatory response. Proc Natl Acad Sci U S A 104:1604-9 . doi: 10.1073/pnas.0610731104

20. Smyth LA, Boardman DA, Tung SL, et al (2015) MicroRNAs affect dendritic cell function and phenotype. Immunology 144:197-205 . doi: 10.1111/imm.12390

21. Mehta A, Baltimore D (2016) MicroRNAs as regulatory elements in immune system logic. Nat Rev Immunol 16:279-294. doi: 10.1038/nri.2016.40 
22. Montecalvo A, Larregina AT, Shufesky WJ, et al (2012) Mechanism of transfer of functional microRNAs between mouse dendritic cells via exosomes. Blood 119:756-66 . doi: 10.1182/blood-2011-02-338004

23. Bellingham SA, Coleman BM, Hill AF (2012) Small RNA deep sequencing reveals a distinct miRNA signature released in exosomes from prion-infected neuronal cells. Nucleic Acids Res 40:10937-10949. doi: 10.1093/nar/gks832

24. Cha DJ, Franklin JL, Dou Y, et al (2015) KRAS-dependent sorting of miRNA to exosomes. Elife 4:1-22 . doi: 10.7554/eLife.07197

25. Valadi H, Ekström K, Bossios A, et al (2007) Exosome-mediated transfer of mRNAs and microRNAs is a novel mechanism of genetic exchange between cells. Nat Cell Biol 9:654-659. doi: 10.1038/ncb1596

26. Alexander M, Hu R, Runtsch MC, et al (2015) Exosome-delivered microRNAs modulate the inflammatory response to endotoxin. Nat Commun 6:7321 . doi: 10.1038/ncomms8321

27. Taylor DD, Gercel-Taylor C (2008) MicroRNA signatures of tumor-derived exosomes as diagnostic biomarkers of ovarian cancer. Gynecol Oncol 110:13-21 . doi: 10.1016/j.ygyno.2008.04.033

28. Murata K, Yoshitomi H, Tanida S, et al (2010) Plasma and synovial fluid microRNAs as potential biomarkers of rheumatoid arthritis and osteoarthritis. Arthritis Res Ther 12:R86 . doi: 10.1186/ar3013

29. Cheng L, Doecke JD, Sharples RA, et al (2014) Prognostic serum miRNA biomarkers associated with Alzheimer's disease shows concordance with neuropsychological and neuroimaging assessment. Mol Psychiatry 1-9. doi: 10.1038/mp.2014.127

30. Nolte-'t Hoen ENM, Buermans HPJ, Waasdorp M, et al (2012) Deep sequencing of RNA from immune cell-derived vesicles uncovers the selective incorporation of small non-coding RNA biotypes with potential regulatory functions. Nucleic Acids Res 40:9272-85 . doi: 10.1093/nar/gks658

31. van Balkom BWM, Eisele AS, Pegtel DM, et al (2015) Quantitative and qualitative analysis of small RNAs in human endothelial cells and exosomes provides insights into localized RNA processing, degradation and sorting. J Extracell vesicles 4:26760 . doi: 10.3402/jev.v4.26760

32. Tosar JP, Gambaro F, Sanguinetti J, et al (2015) Assessment of small RNA sorting into different extracellular fractions revealed by high-throughput sequencing of breast cell lines. Nucleic Acids Res 1-16. doi: 10.1093/nar/gkv432

33. Yeri A, Courtright A, Reiman R, et al (2017) Total Extracellular Small RNA Profiles from Plasma, Saliva, and Urine of Healthy Subjects. Sci Rep 7:44061 . doi: 10.1038/srep44061

34. Lunavat TR, Cheng L, Kim D-K, et al (2015) Small RNA deep sequencing discriminates subsets of extracellular vesicles released by melanoma cells - evidence of unique microRNA cargos. RNA Biol 6286:00-00 . doi: 10.1080/15476286.2015.1056975

35. Vojtech L, Woo S, Hughes S, et al (2014) Exosomes in human semen carry a distinctive repertoire of small non-coding RNAs with potential regulatory functions. Nucleic Acids Res 42:7290-7304 . doi: 10.1093/nar/gku347

36. Youssef OA, Safran SA, Nakamura T, et al (2015) Potential role for snoRNAs in PKR activation during metabolic stress. Proc Natl Acad Sci U S A 112:5023-5028 . doi: 10.1073/pnas.1424044112

37. Michel Cl, Holley CL, Scruggs BS, et al (2011) Small nucleolar RNAs U32a, U33 and U35a are critical mediators of metabolic stress. Cell Metab 14:33-44. doi: 10.1016/j.cmet.2011.04.009.Small

38. Stepanov GA, Filippova JA, Komissarov AB, et al (2015) Regulatory role of small nucleolar RNAs in human diseases. Biomed Res Int 2015:206849. doi: 10.1155/2015/206849

39. Haussecker D, Huang Y, Lau A, et al (2010) Human tRNA-derived small RNAs in the global regulation of RNA silencing. RNA 16:673-695 . doi: 10.1261/rna.2000810.also

40. Ninomiya S, Kawano M, Abe T, et al (2015) Potential small guide RNAs for tRNase ZL from human plasma, peripheral blood mononuclear cells, and cultured cell lines. PLoS One 10:e0118631 . doi: 10.1371/journal.pone.0118631

41. Nabet BY, Qiu Y, Shabason JE, et al (2017) Exosome RNA Unshielding Couples Stromal Activation to Pattern Recognition Receptor Signaling in Cancer. Cell 170:352-366.e13 . doi: 10.1016/j.cell.2017.06.031

42. Tkach M, Thery C (2016) Communication by Extracellular Vesicles : where we are and where to go. Cell 164: . doi: 10.1016/j.cell.2016.01.043 
43. Zitvogel L, Regnault A, Lozier A, et al (1998) Eradication of established murine tumors using a novel cell-free vaccine: Dendritic cell-derived exosomes. Nat Med 4:594-600 . doi: 10.1038/nm0598-594

44. Pitt JM, André F, Amigorena S, et al (2016) Dendritic cell-derived exosomes for cancer therapy. J Clin Invest 126:1224-1232. doi: 10.1172/JCl81137

45. Wu T, Qi Y, Zhang D, et al (2017) Bone Marrow Dendritic Cells Derived Microvesicles for Combinational Immunochemotherapy against Tumor. Adv Funct Mater 27:1-15 . doi: 10.1002/adfm.201703191

46. Tao SC, Guo SC, Zhang CQ (2018) Modularized Extracellular Vesicles: The Dawn of Prospective Personalized and Precision Medicine. Adv Sci 1700449: . doi: 10.1002/advs.201700449

47. Raker VK, Domogalla MP, Steinbrink K (2015) Tolerogenic dendritic cells for regulatory T cell induction in man. Front Immunol 6:1-11 . doi: 10.3389/fimmu.2015.00569

48. Lutz MB, Kukutsch N, Ogilvie AL., et al (1999) An advanced culture method for generating large quantities of highly pure dendritic cells from mouse bone marrow. J Immunol Methods 223:77-92 . doi: 10.1016/S0022-1759(98)00204-X

49. van der Vlist EJ, Nolte-'t Hoen ENM, Stoorvogel W, et al (2012) Fluorescent labeling of nano-sized vesicles released by cells and subsequent quantitative and qualitative analysis by high-resolution flow cytometry. Nat Protoc 7:1311-1326. doi: 10.1038/nprot.2012.065

50. Robinson MD, McCarthy DJ, Smyth GK (2009) edgeR: A Bioconductor package for differential expression analysis of digital gene expression data. Bioinformatics 26:139-140 . doi: 10.1093/ bioinformatics/btp616

51. Robinson JT, Thorvaldsdóttir H, Winckler W, et al (2011) Integrative Genomics Viewer. Nat Biotechnol 29:24-26 . doi: 10.1038/nbt0111-24

52. Nolte-'t Hoen ENM, van der Vlist EJ, Aalberts M, et al (2012) Quantitative and qualitative flow cytometric analysis of nanosized cell-derived membrane vesicles. Nanomedicine 8:712-720 . doi: 10.1016/j.nano.2011.09.006

53. Groot Kormelink T, Arkesteijn GJA, Nauwelaers FA, et al (2016) Prerequisites for the analysis and sorting of extracellular vesicle subpopulations by high-resolution flow cytometry. Cytom Part A 89:135-147. doi: $10.1002 /$ cyto.a.22644

54. Libri V, Helwak A, Miesen P, et al (2012) Murine cytomegalovirus encodes a miR-27 inhibitor disguised as a target. Proc Natl Acad Sci U S A 109:279-284 . doi: 10.1073/pnas.1114204109

55. Pall GS, Hamilton AJ (2008) Improved northern blot method for enhanced detection of small RNA. Nat Protoc 3:1077-1084 . doi: 10.1038/nprot.2008.67

56. Piemonti L, Monti P, Sironi M, et al (2000) Vitamin D3 affects differentiation, maturation, and function of human monocyte-derived dendritic cells. J Immunol 164:4443-4451 . doi: ji_v164n9p4443 [pii]

57. Unger WWJ, Laban S, Kleijwegt FS, et al (2009) Induction of Treg by monocyte-derived DC modulated by vitamin D3 or dexamethasone: Differential role for PD-L1. Eur J Immunol 39:3147-3159. doi: 10.1002/eji.200839103

58. Penna G, Adorini L (2000) 1,25-Dihydroxyvitamin D3 Inhibits Differentiation, Maturation, Activation, and Survival of Dendritic Cells Leading to Impaired Alloreactive T Cell Activation. J Immunol 164:24052411 . doi: 10.4049/jimmunol.164.5.2405

59. Thery C, Boussac M, Veron P, et al (2001) Proteomic Analysis of Dendritic Cell-Derived Exosomes: A Secreted Subcellular Compartment Distinct from Apoptotic Vesicles. J Immunol 166:7309-7318 . doi: 10.4049/jimmunol.166.12.7309

60. 't Hoen PAC, Friedländer MR, Almlöf J, et al (2013) Reproducibility of high-throughput mRNA and small RNA sequencing across laboratories. Nat Biotechnol 31:1015-22 . doi: 10.1038/nbt.2702

61. Koppers-Lalic D, Hackenberg M, Bijnsdorp I V., et al (2014) Nontemplated nucleotide additions distinguish the small RNA composition in cells from exosomes. Cell Rep 8:1649-1658 . doi: 10.1016/j. celrep.2014.08.027

62. Dueck A, Eichner A, Sixt M, Meister G (2014) A miR-155-dependent microRNA hierarchy in dendritic cell maturation and macrophage activation. FEBS Lett 588:632-640 . doi: 10.1016/j.febslet.2014.01.009 
63. Ma Z-X, Tan X, Shen Y, et al (2015) MicroRNA expression profile of mature dendritic cell in chronic rhinosinusitis. Inflamm Res 64:885-893. doi: 10.1007/s00011-015-0870-5

64. Wu W, He C, Liu C, et al (2015) miR-10a inhibits dendritic cell activation and Th1 / Th17 cell immune responses in IBD. Gut 64:1755-1764 . doi: 10.1136/gutjnl-2014-307980

65. Park H, Huang $X$, Lu C, et al (2015) MicroRNA-146a and microRNA-146b regulate human dendritic cell apoptosis and cytokine production by targeting TRAF6 and IRAK1 proteins. J Biol Chem 290:2831-41 . doi: 10.1074/jbc.M114.591420

66. Bazzoni F, Rossato M, Fabbri M, et al (2009) Induction and regulatory function of miR-9 in human monocytes and neutrophils exposed to proinflammatory signals. Proc Natl Acad Sci U S A 106:5282-7. doi: 10.1073/pnas.0810909106

67. Zhou H, Xiao J, Wu N, et al (2015) MicroRNA-223 Regulates the Differentiation and Function of Intestinal Dendritic Cells and Macrophages by Targeting C/EBP-beta. Cell Rep 13:1149-1160 . doi: 10.1016/j. celrep.2015.09.073

68. Pedersen AW, Holmstrøm K, Jensen SS, et al (2009) Phenotypic and functional markers for 1alpha,25dihydroxyvitamin D(3)-modified regulatory dendritic cells. Clin Exp Immunol 157:48-59 . doi: 10.1111/j.1365-2249.2009.03961.x

69. Stumpfova Z, Hezova R, Meli AC, et al (2014) MicroRNA profiling of activated and tolerogenic human dendritic cells. Mediators Inflamm 2014:259689. doi: 10.1155/2014/259689

70. Agudo J, Ruzo A, Tung N, et al (2013) The miR-126-VEGFR2 axis controls the innate response to pathogen-associated nucleic acids. Nat Immunol 15:54-62 . doi: 10.1038/ni.2767

71. Baer C, Oakes CC, Ruppert AS, et al (2015) Epigenetic silencing of miR-708 enhances NF-kB signaling in chronic lymphocytic leukemia. Int J Cancer 137:1352-1361 . doi: 10.1002/ijc.29491

72. Lin J, Xia J, Tu CZ, et al (2017) H9N2 avian influenza virus protein PB1 enhances the immune responses of bone marrow-derived dendritic cells by down-regulating miR375. Front Microbiol 8:1-10. doi: 10.3389/fmicb.2017.00287

73. Lv Y, Ou-yang A, Fu L (2017) MicroRNA-27a Negatively Modulates the Inflammatory Response in Lipopolysaccharide-Stimulated Microglia by Targeting TLR4 and IRAK4. Cell Mol Neurobiol 37:195-210. doi: 10.1007/s10571-016-0361-4

74. Vandesompele J, De Preter K, Pattyn F, et al (2002) Accurate normalization of real-time quantitative RT-PCR data by geometric averaging of multiple internal control genes. Genome Biol 3: . doi: 10.1186/ gb-2002-3-7-research0034

75. Stein AJ, Fuchs G, Fu C, et al (2005) Structural insights into RNA quality control: The Ro autoantigen binds misfolded RNAs via its central cavity. Cell 121:529-539. doi: 10.1016/j.cell.2005.03.009

76. Chen X, Smith JD, Shi H, et al (2003) The Ro Autoantigen Binds Misfolded U2 Small Nuclear RNAs and Assists Mammalian Cell Survival after UV Irradiation. Curr Biol 13:2206-2211 . doi: 10.1016/j. cub.2003.11.028

77. Dhahbi JM, Spindler SR, Atamna H, et al (2013) 5'-YRNA fragments derived by processing of transcripts from specific YRNA genes and pseudogenes are abundant in human serum and plasma. Physiol Genomics 45:990-998 . doi: 10.1152/physiolgenomics.00129.2013

78. Repetto E, Lichtenstein L, Hizir Z, et al (2015) RNY-derived small RNAs as a signature of coronary artery disease. BMC Med 13:259 . doi: 10.1186/s12916-015-0489-y

79. Kaudewitz D, Skroblin P, Bender LH, et al (2015) Association of MicroRNAs and YRNAs with Platelet Function. Circ Res. doi: 10.1161/CIRCRESAHA.114.305663

80. Cambier $L$, de Couto $G$, Ibrahim A, et al (2017) Y RNA fragment in extracellular vesicles confers cardioprotection via modulation of IL-10 expression and secretion. EMBO Mol Med e201606924 . doi: 10.15252/emmm.201606924

81. Haderk F, Schulz R, Iskar M, et al (2017) Tumor-derived exosomes modulate PD-L1 expression in monocytes. Sci Immunol 2:1-12 . doi: 10.1126/sciimmunol.aah5509

82. Meiri E, Levy A, Benjamin H, et al (2010) Discovery of microRNAs and other small RNAs in solid tumors. Nucleic Acids Res 38:6234-6246 . doi: 10.1093/nar/gkq376 
83. Thomson DW, Pillman KA, Anderson ML, et al (2015) Assessing the gene regulatory properties of Argonaute-bound small RNAs of diverse genomic origin. Nucleic Acids Res 43:470-481 . doi: 10.1093/ nar/gku1242

84. Dhahbi JM, Spindler SR, Atamna H, et al (2014) Deep Sequencing of Serum Small RNAs Identifies Patterns of 5'tRNA Half and YRNA Fragment Expression Associated with Breast Cancer. Biomark Cancer 6:37-47 . doi: 10.4137/BIC.S20764.RECEIVED

85. Li CCY, Eaton SA, Young PE, et al (2013) Glioma microvesicles carry selectively packaged coding and non-coding RNAs which alter gene expression in recipient cells. RNA Biol 10:1333-44 . doi: 10.4161/ rna.25281

86. Fiskaa T, Knutsen E, Nikolaisen MA, et al (2016) Distinct Small RNA Signatures in Extracellular Vesicles Derived from Breast Cancer Cell Lines. PLoS One 11:e0161824 . doi: 10.1371/journal.pone.0161824

87. Tosar JP, Rovira C, Naya H, Cayota A (2014) Mining of public sequencing databases supports a nondietary origin for putative foreign miRNAs: underestimated effects of contamination in NGS. RNA 20:754-7 . doi: 10.1261/rna.044263.114

88. Tosar JP, Cayota A, Eitan E, et al (2017) Ribonucleic artefacts: are some extracellular RNA discoveries driven by cell culture medium components? J Extracell Vesicles 6:1-10 . doi: 10.1080/20013078.2016.1272832

89. Raabe CA, Tang T-H, Brosius J, Rozhdestvensky TS (2014) Biases in small RNA deep sequencing data. Nucleic Acids Res 42:1414-1426 . doi: 10.1093/nar/gkt1021

90. Wei Z, Batagov AO, Schinelli S, et al (2017) Coding and noncoding landscape of extracellular RNA released by human glioma stem cells. Nat Commun 8:1145 . doi: 10.1038/s41467-017-01196-x

91. Hendrick JP, Wolin SL, Rinke J, et al (1981) Ro small cytoplasmic ribonucleoproteins are a subclass of La ribonucleoproteins: further characterization of the Ro and La small ribonucleoproteins from uninfected mammalian cells. Mol Cell Biol 1:1138-1149. doi: 10.1128/MCB.1.12.1138.Updated

92. Motorin Y, Muller S, Behn-Ansmant I, Branlant C (2007) Identification of Modified Residues in RNAs by Reverse Transcription-Based Methods. In: Methods in Enzymology

93. Qin Y, Yao J, Wu DC, et al (2015) High-throughput sequencing of human plasma RNA by using thermostable group II intron reverse transcriptases. RNA 22:1-18 . doi: 10.1261/rna.054809.115.3

94. Shurtleff MJ, Yao J, Qin Y, et al (2017) Broad role for YBX1 in defining the small noncoding RNA composition of exosomes. Proc Natl Acad Sci 114:E8987-E8995 . doi: 10.1073/pnas.1712108114

95. Gee HE, Buffa FM, Camps C, et al (2011) The small-nucleolar RNAs commonly used for microRNA normalisation correlate with tumour pathology and prognosis. Br J Cancer 104:1168-1177 . doi: 10.1038/sj.bjc.6606076

96. Martens-Uzunova ES, Olvedy M, Jenster G (2013) Beyond microRNA - Novel RNAs derived from small non-coding RNA and their implication in cancer. Cancer Lett 340:201-211 . doi: 10.1016/j. canlet.2012.11.058

97. Baltimore D, Boldin MP, O'Connell RM, et al (2008) MicroRNAs: new regulators of immune cell development and function. Nat Immunol 9:839-45 . doi: 10.1038/ni.f.209

98. O'Connell RM, Kahn D, Gibson WSJ, et al (2010) MicroRNA-155 promotes autoimmune inflammation by enhancing inflammatory T cell development. Immunity 33:607-19 . doi: 10.1016/j. immuni.2010.09.009

99. Boldin MP, Taganov KD, Rao DS, et al (2011) miR-146a is a significant brake on autoimmunity, myeloproliferation, and cancer in mice. J Exp Med 208:1189-1201 . doi: 10.1084/jem.20101823

100. Ruckerl D, Jenkins SJ, Laqtom NN, et al (2012) Induction of IL-4R alpha-dependent microRNAs identifies PI3K / Akt signaling as essential for IL-4-driven murine macrophage proliferation in vivo. Blood 120:2307-2317 . doi: 10.1182/blood-2012-02-408252.The

101. Lee J-J, Drakaki A, Illiopoulos D, Struhl K (2012) MiR-27b targets PPARy to inhibit growth, tumor progression, and the inflammatory response in neuroblastoma cells. Oncogene 31:3818-3825. doi: 10.1146/annurev-immunol-032713-120240.Microglia 
4. Immune stimuli shape the small non-coding transcriptome of EV released by dendritic cells

102. Chakrabortty SK, Prakash A, Nechooshtan GAL, et al (2015) Extracellular vesicle-mediated transfer of processed and functional RNY5 RNA. RNA 1-14. doi: 10.1261/rna.053629.115.

103. Hizir Z, Bottini S, Grandjean V, et al (2017) RNY (YRNA)-derived small RNAs regulate cell death and inflammation in monocytes/macrophages. Cell Death Dis 8:e2530 . doi: 10.1038/cddis.2016.429

104. Greidinger EL, Zang Y, Martinez L, et al (2007) Differential tissue targeting of autoimmunity manifestations by autoantigen-associated Y RNAs. Arthritis Rheum 56:1589-1597 . doi: 10.1002/ art.22601

105. Skibinski G, Kelly R, Harkiss D, James K (1992) Immunosuppression by human seminal plasma extracellular organelles (prostasomes) modulate activity of phagocytic cells. Am J Reprod Immunol 28:97-103

106. Tarazona R, Delgado E, Guarnizo MC, et al (2011) Human prostasomes express CD48 and interfere with NK cell function. Immunobiology 216:41-46 . doi: 10.1016/j.imbio.2010.03.002

107. Buck AH, Coakley G, Simbari F, et al (2014) Exosomes secreted by nematode parasites transfer small RNAs to mammalian cells and modulate innate immunity. Nat Commun 5:5488 . doi: 10.1038/ ncomms6488 


\section{Supplementary Figures \& Table}

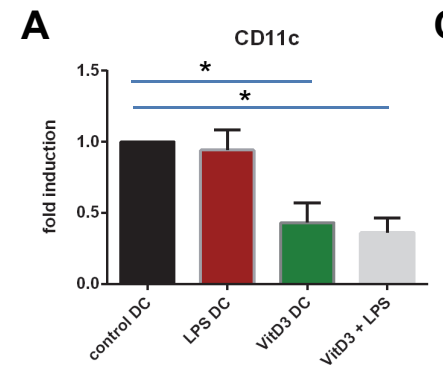

B

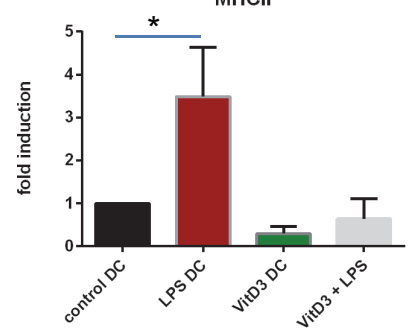

C

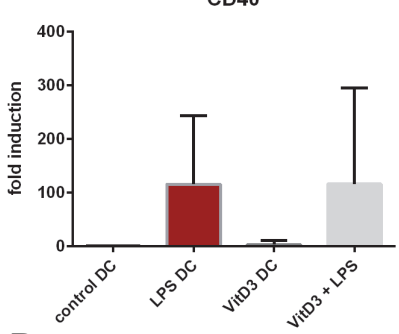

D

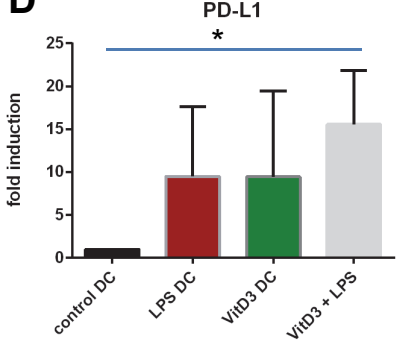

E

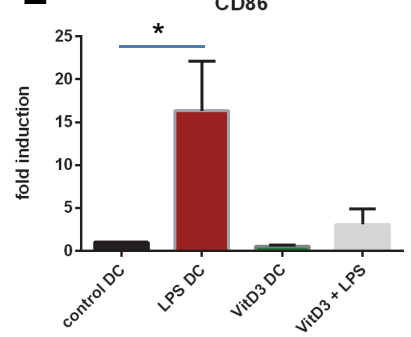

$\mathbf{F}$

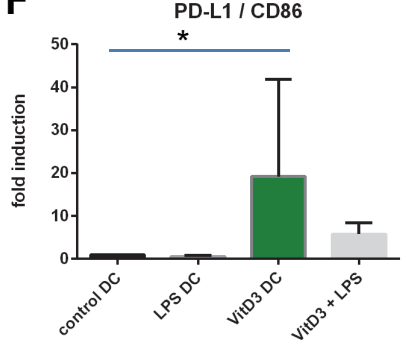

Supplementary Figure 1. Generation of immune-activating and immune-suppressive DC subsets by treatment with LPS or VitD3

DCs were left untreated (control), treated with LPS to induce immune-stimulatory DC, or treated with $1 \alpha, 25-(\mathrm{OH})_{2}-$ VitaminD $\mathrm{D}_{3}$ to induce immune-suppressive DC. A - E) Cell surface expression of CD11C, MHCII, CD40, PD-L1, and CD86 on control, LPS- or VitD3-treated DC was determined by flow-cytometry at the start of the EV-production phase. F) The ratio between PD-L1 and CD86 was determined as a measure for tolerogenicity. Data are expressed as isotype-corrected mean fluorescence intensity values, normalized against expression in control (untreated) DC cultures. Indicated are mean $+/$ - s.d. fold induction of $n=8$ independent experiments, one-way ANOVA with Dunnet's two-sided post-hoc test, ${ }^{*} p<0.05$ 
A

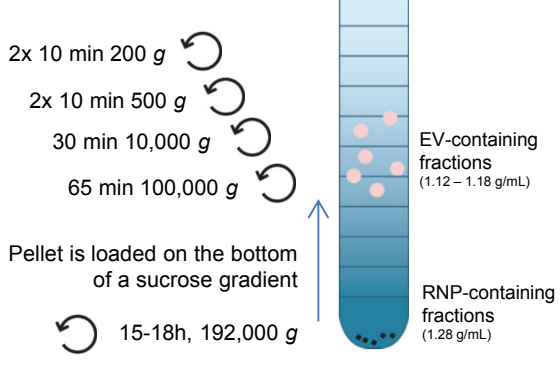

C
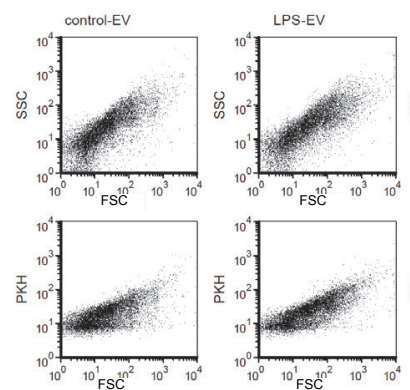

E
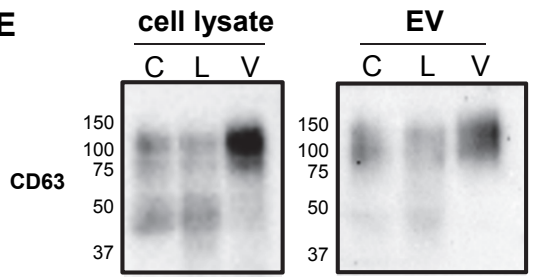

Gal3
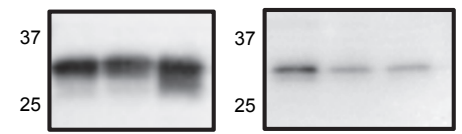

MHCII
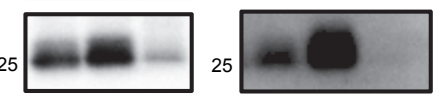

CD9
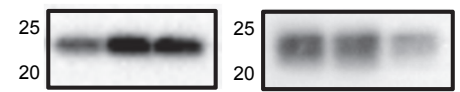

Beta

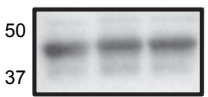

D
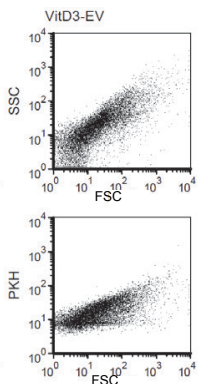

F
B
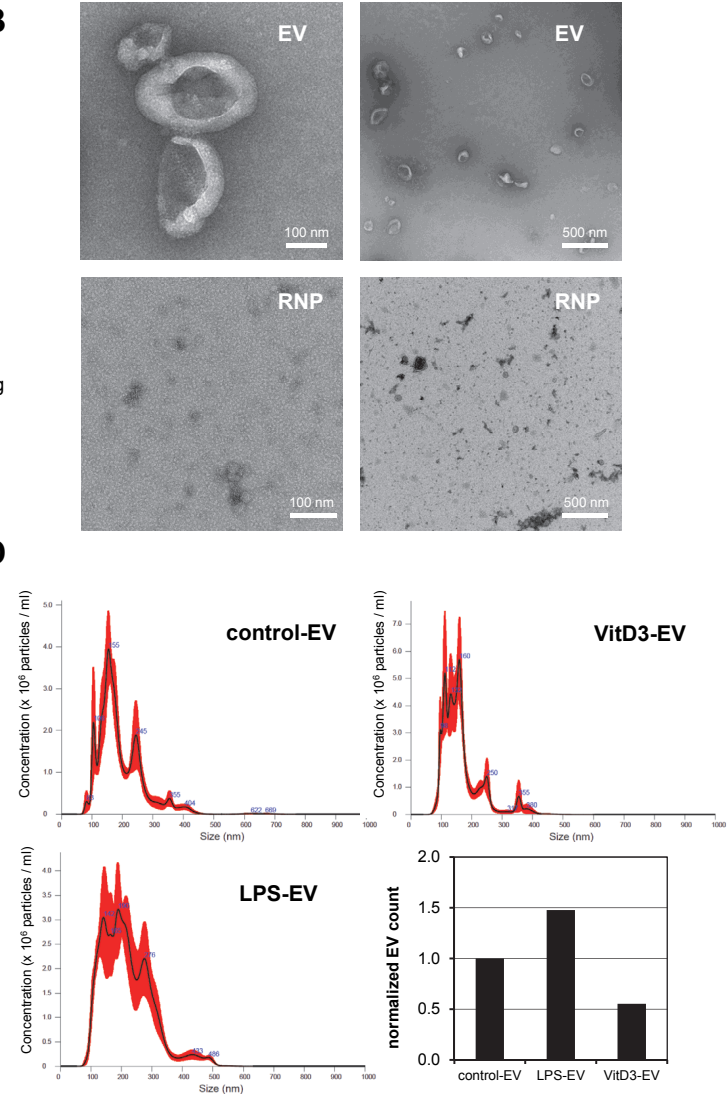

G
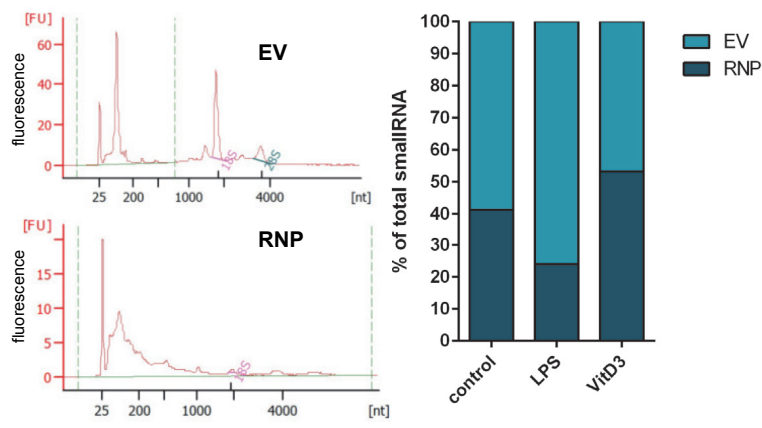


\section{Supplementary Figure 2. Isolation and characterization of dendritic cell-derived EV and}

their RNA content.

A) Schematic representation of the differential centrifugation protocol used to generate highly purified EV from cell culture supernatant. Large contaminants such as cell debris and apoptotic bodies are depleted during the low-speed centrifugation steps, after which EV are pelleted from the cleared supernatant by ultracentrifugation. Sucrose density gradient centrifugation was used to separate low density EV from high density ribonucleoprotein complexes (RNP). B) Electron microscopy images of EV isolated from $1.12-1.18 \mathrm{~g} / \mathrm{ml}$ density fractions (top panels) and from $1.28 \mathrm{~g} / \mathrm{ml}$ RNP-containing fractions (bottom panels). Scale bars represent $100 \mathrm{~nm}$ (left panels) and $500 \mathrm{~nm}$ (right panels) C) High-resolution flow cytometric analysis of purified PKH67-labeled EV $(1.14 \mathrm{~g} / \mathrm{ml})$ from differentially stimulated DC. Representative dot plots indicate no apparent differences in FSC/SSC light scatter profiles (top panels) or fluorescence intensity (PKH, bottom panels) between EV in the indicated conditions. 10,000 events are plotted per graph. D) Purified EV $(1.12-1.18 \mathrm{~g} / \mathrm{ml})$ from differentially stimulated DCs were analyzed by Nanoparticle Tracking Analysis. Indicated are the size distributions of EV in the different DC conditions and relative differences in numbers of EV released by these DC (bar graph). E) Lysates of control (C), LPStreated $(\mathrm{L})$, or VitD3-treated $(\mathrm{V})$ cells (left) and purified EV released under these conditions (right) were analyzed for the presence of CD63, Galectin-3, MHCII, CD9 and beta-actin by Western blotting. For cell lysates, an equal amount of protein was loaded for each condition. For comparison of EV protein composition, equal numbers of EV released in each condition were loaded, as based on high-resolution flow cytometric and NTA-based analysis. Beta actin was not detectable in EV, even at prolonged exposure times. F) Representative Bioanalyzer Pico 6000 electropherograms of RNA isolated from floating EV and non-floating RNP, showing differences in RNA size profiles. G) Bioanalyzer-based quantification of RNA yields from EV and RNP. Indicated are the percentages of total extracellular RNA associated to either EV or RNP (mean +/- s.d. of $n=3$ independent experiments). 
A

Vault RNA (chr18:36,801,866-36,802,008)

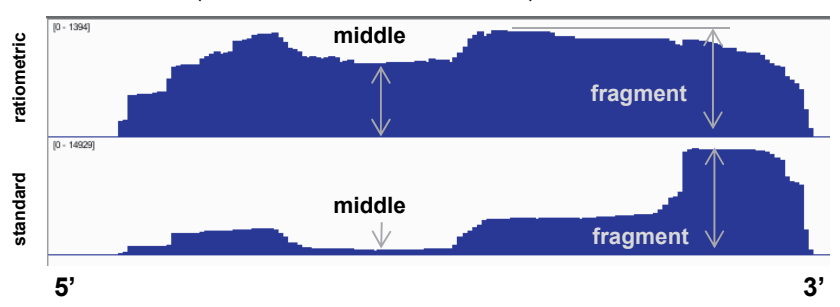

B

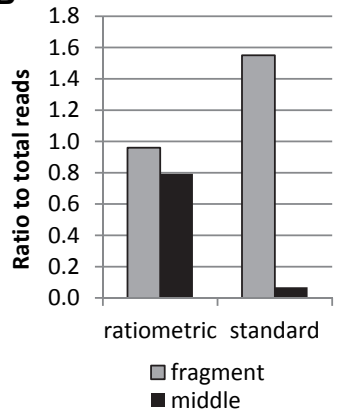

Supplementary Figure 3. Ratiometric pooling of size fractions improves RNA sequencingbased coverage of 100-300 nt mid-size RNAs

A) Representative IGV Genome Viewer plots of RNAseq read coverage of Vault-RNA after ratiometric pooling (top) or under standard library preparation conditions (bottom). B) Reads were counted for the 3' fragment and the middle part of Vault RNA, as indicated. These values were divided by the total number of reads detected for Vault RNA and compared for ratiometric versus standard conditions.

Supplementary Table 1. Library size, quality control and alignment percentages of all EV and cellular RNA libraries

Overview of the RNAseq libraries prepared for cellular and EV RNA in this study, the corresponding indices, read depth, and quality control parameters.

\begin{tabular}{lccccc}
\hline Sample & index & \# reads & \# reads pass filter & \% Pass & \% Alignment \\
\hline cell_control_S4 & 11 & $24,184,761$ & $23,206,725$ & $96 \%$ & $84.50 \%$ \\
cell_control_S7 & 14 & $16,891,584$ & $15,848,861$ & $94 \%$ & $76.80 \%$ \\
cell_control_S36 & 18 & $23,827,849$ & $21,077,194$ & $88 \%$ & $75.12 \%$ \\
cell_LPS_S5 & 12 & $19,526,758$ & $18,187,862$ & $93 \%$ & $83.50 \%$ \\
cell_LPS_S8 & 15 & $14,816,903$ & $13,494,221$ & $91 \%$ & $80.80 \%$ \\
cell_LPS_S37 & 19 & $48,426,527$ & $45,972,567$ & $95 \%$ & $85.35 \%$ \\
cell_VitD3_S6 & 13 & $15,236,904$ & $14,543,618$ & $95 \%$ & $82.90 \%$ \\
cell_VitD3_S9 & 16 & $13,004,444$ & $12,242,191$ & $94 \%$ & $76.20 \%$ \\
cell_VitD3_S38 & 20 & $38,148,808$ & $33,109,203$ & $87 \%$ & $74.31 \%$ \\
EV_cont_S10 & 17 & $15,138,107$ & $11,336,408$ & $75 \%$ & $33.60 \%$ \\
EV_cont_S13 & 20 & $18,222,150$ & $8,168,987$ & $45 \%$ & $28.30 \%$ \\
EV_control_S39 & 21 & $22,687,712$ & $15,110,524$ & $67 \%$ & $36.84 \%$ \\
EV_LPS_S11 & 18 & $16,111,933$ & $12,326,349$ & $77 \%$ & $29.30 \%$ \\
EV_LPS_S14 & 21 & $11,150,431$ & $4,269,979$ & $38 \%$ & $29.20 \%$ \\
EV_LPS_S40 & 22 & $21,884,586$ & $13,320,228$ & $61 \%$ & $30.99 \%$ \\
EV_VitD3_S12 & 19 & $16,296,162$ & $9,957,537$ & $61 \%$ & $27.30 \%$ \\
EV_VitD3_S15 & 22 & $13,273,020$ & $7,856,779$ & $59 \%$ & $26.90 \%$ \\
EV_VitD3_S41 & 23 & $15,662,964$ & $7,968,405$ & $51 \%$ & $20.31 \%$ \\
\hline
\end{tabular}



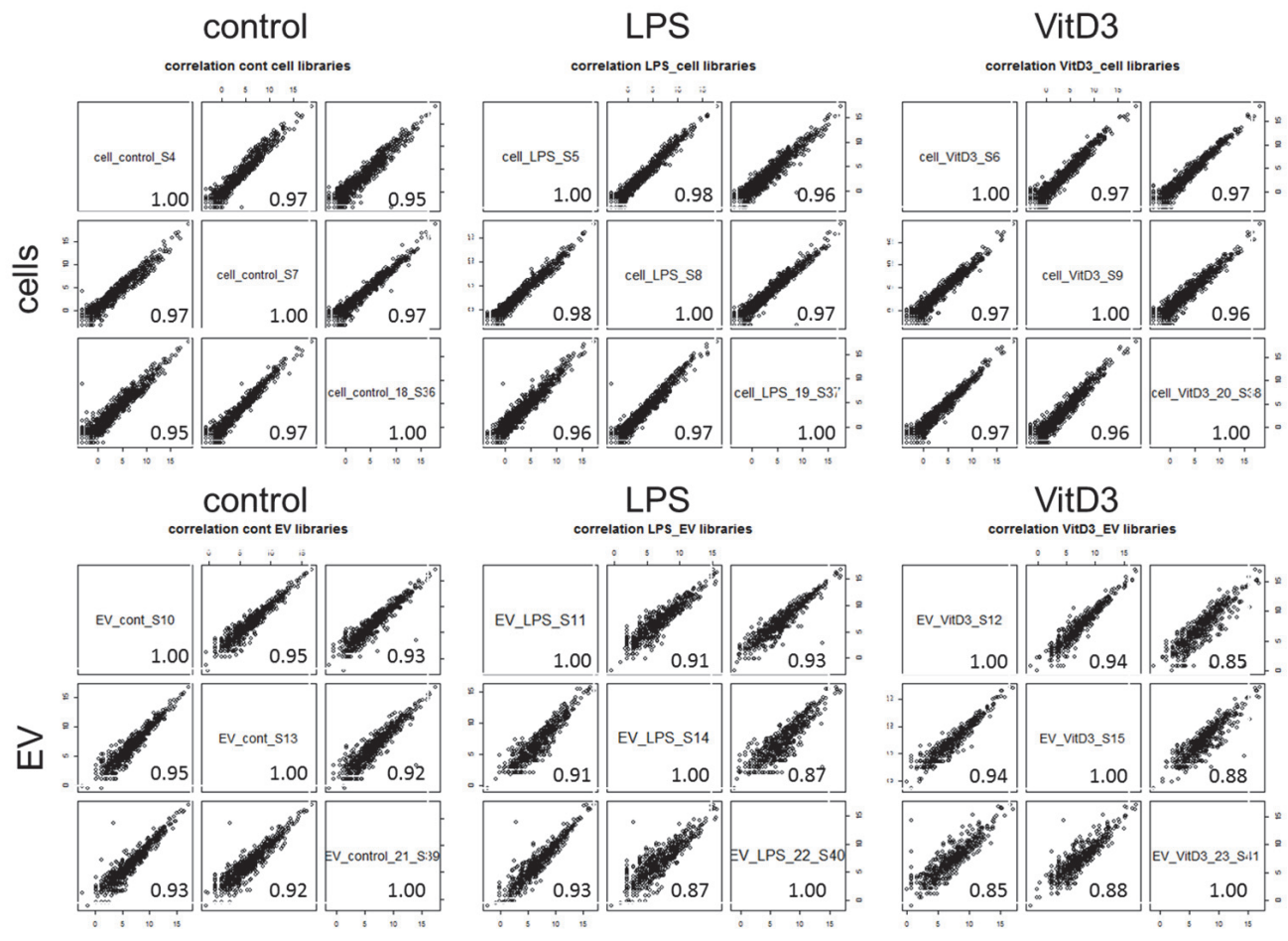

Supplementary Figure 4. Reproducibility of biological replicate libraries for cells and EV Cellular and EV-associated RNA from control, LPS, and VitD3 conditions was isolated and analyzed by RNA sequencing. The RPM for all biological replicate libraries were plotted against one another. Pearson coefficients are indicated in the plots. 
A

cellular small RNA biotype distribution

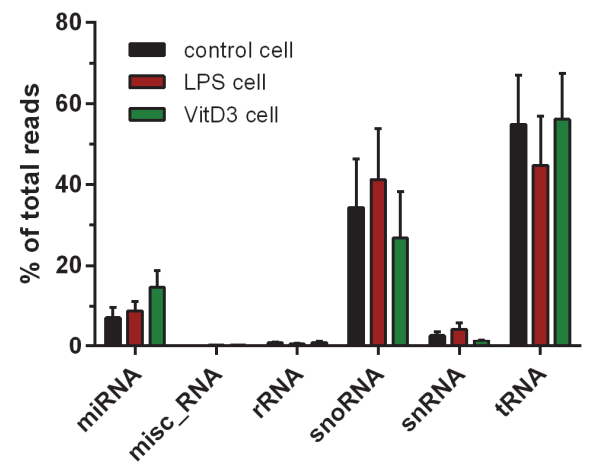

B

EV small RNA biotype distribution

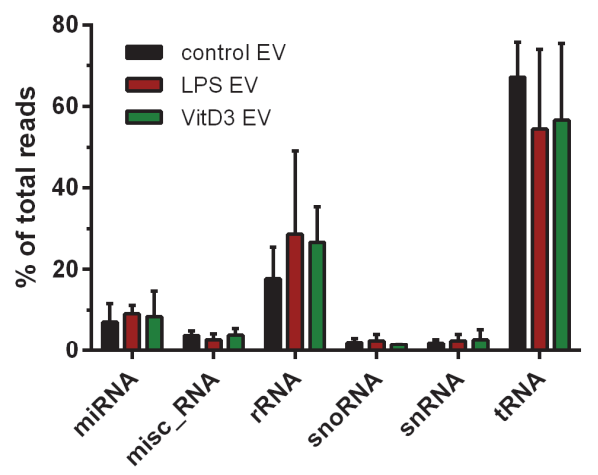

Supplementary Figure 5. RNA class distribution in cells and EV upon immune stimulation Class distribution in RNA from differently stimulated DCs (A) and corresponding EV (B) was analyzed by RNA sequencing. For each of the RNAseq libraries, total read counts for each RNA class were expressed as a percentage of the total library size. Average percentage of reads for each RNA class (of $n=3$ replicate libraries) is plotted for each of the treatments. No major changes in RNA class distribution can be observed as a result of stimuli imposed on the DCs.
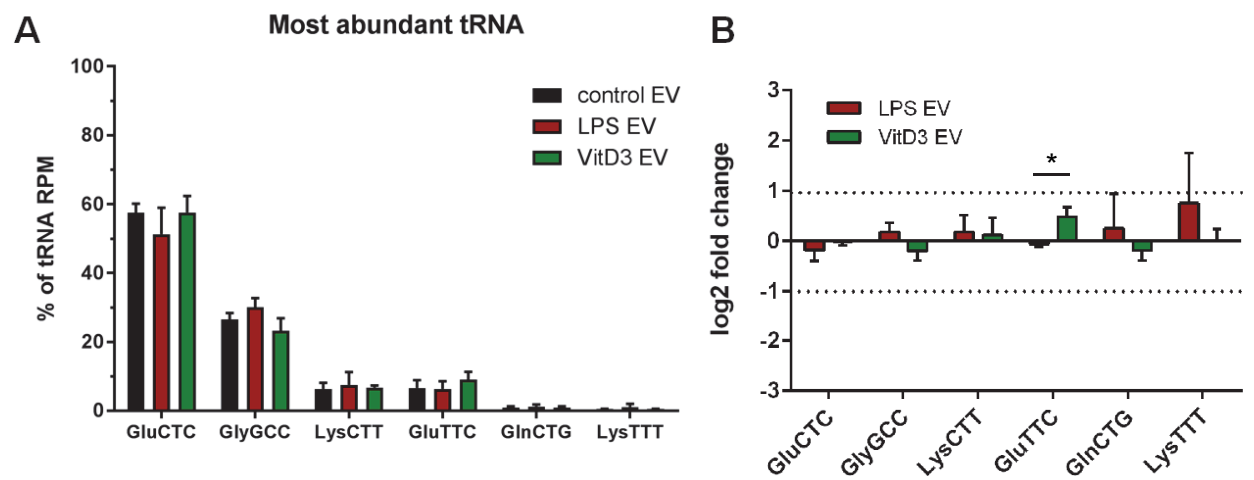

Supplementary Figure 6. EV from LPS- and VitD3-stimulated DC display stable levels of abundant tRNAs

EV-associated RNA from control, LPS, and VitD3 conditions was isolated and analyzed by RNA sequencing. Read counts for individual RNAs were normalized to the total read counts of each RNA class. A) Read counts for the top- 6 most abundant tRNAs, constituting $97 \%$ of tRNA reads in EV-RNA, are expressed as percentage of the total tRNA read count in EV. B) Data from (A) are expressed as fold-change in the indicated tRNA levels of VitD3- or LPS-EV relative to control-EV (mean $+/$ - s.d. of $n=3$ experiments, one-way ANOVA, $\left.{ }^{*} p<0.05\right)$. 
A

reference genes (seq)

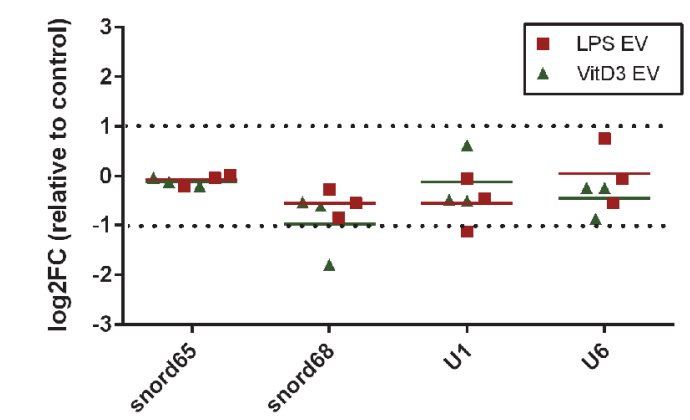

B

reference genes (qPCR)

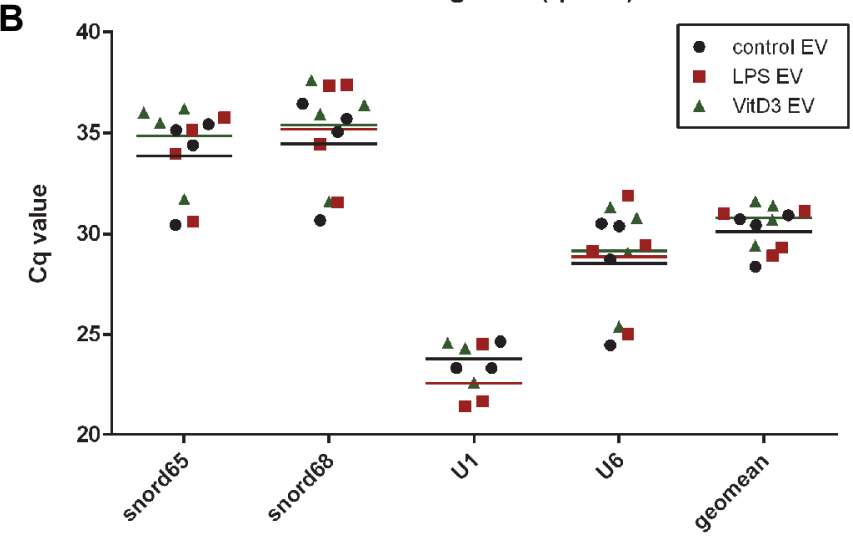

Supplementary Figure 7. Validation of reference RNAs in EV for normalization of qPCR data A) EV-RNA sequencing data were used to validate the stable presence of indicated candidate reference small RNAs in EV from differentially stimulated DC. Indicated are the LPS-and VitD3induced fold changes in EV relative to the control EV of $n=3$ independent experiments. B) RTqPCR validation of candidate reference RNAs identified in (A). RT-qPCR reactions were performed with equal RNA input from control- , LPS-, and VitD3-EV. Indicated are individual and mean Cq values of snord65, snord68, U1 and U6 from $3-4$ independent experiments. The geometric mean of these four reference genes, as shown on the right side of $(B)$ was used as normalization value for EV qPCR data. 
A

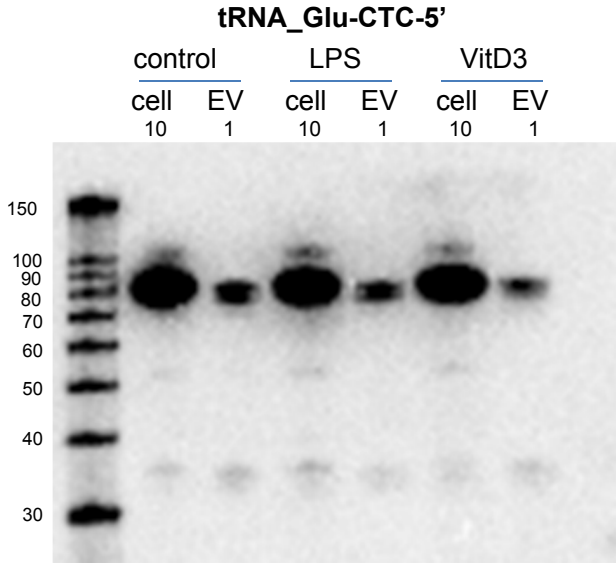

B

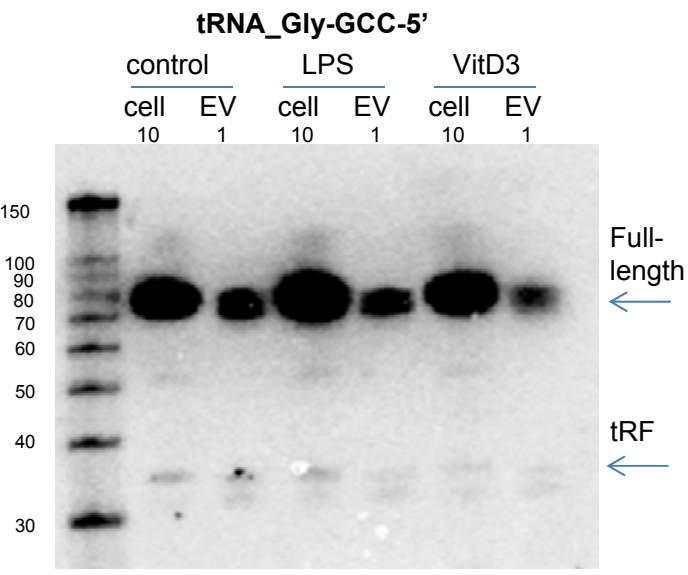

C

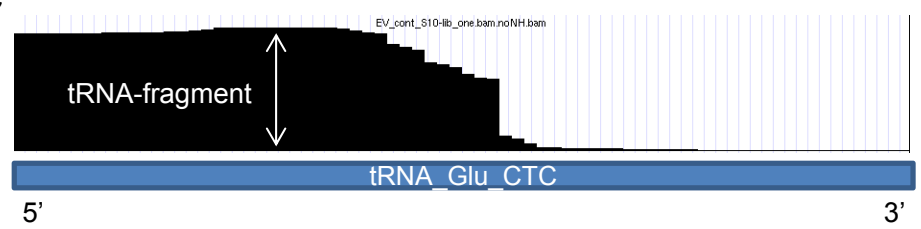

Supplementary Figure 8. Northern blot analysis to validate the ratio of full-length versus fragmented forms of tRNA-Glu-CTC and tRNA-Gly-GCC in cells and EV

A) RNA isolated from control, LPS, and VitD3-treated cells and their EVs were analysed by Northern blot for detection of tRNA-Glu-CTC in using 5'tRNA-Glu probe B) The blot in (A) was stripped and reprobed for tRNA-Gly-GCC using 5'tRNA-Gly probe. Full length tRNA and tRNA fragments (tRF) are indicated with arrows. $10 \mathrm{ng}$ of cellular smallRNA and $1 \mathrm{ng}$ of EV-smallRNA were loaded per lane. Data are representative for $n=2$ independent experiments.

C) Representative coverage plot of tRNA-Glu-CTC as observed in EV RNA seq data (sequencing coverage depth 463,870 ) visualized in the UCSC Genome Browser. 
$\log _{0}$ ar ar 098 ? 58 8364 e $)^{2}(50$

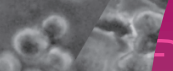
(3) (1)

(e)

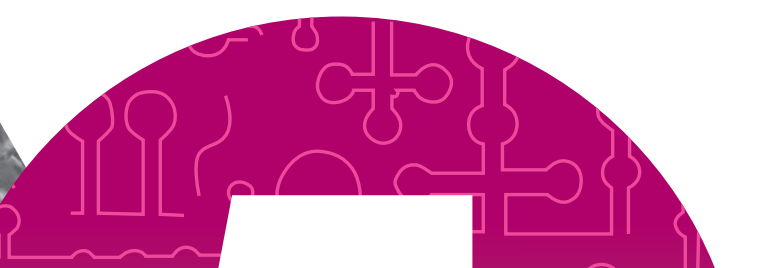

$-3$

$\cos \theta 6$

जece

8 ${ }_{0} c$ $45^{\circ} i^{\circ} 0^{\circ}$

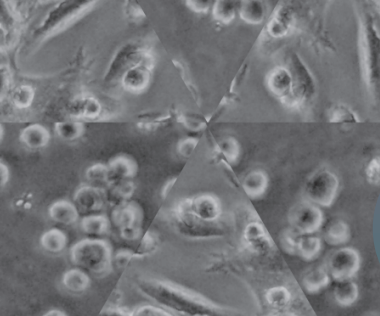

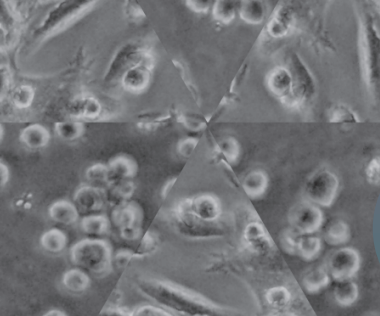
$\frac{6}{8}$

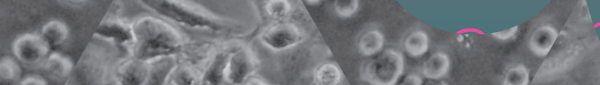

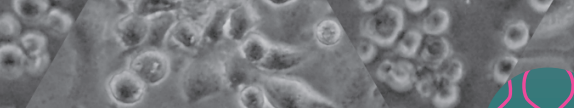

$$
e^{9} \text { as } ?^{2} \text { ? }
$$

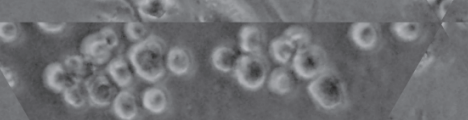
Q 10

(2) c

3

(6)

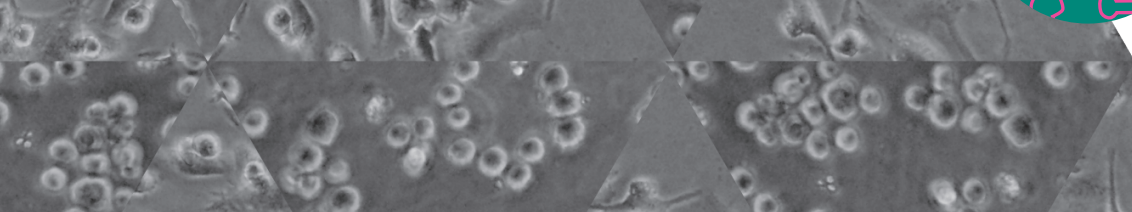
का

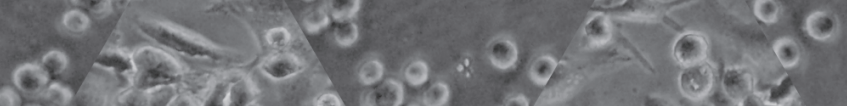
6)

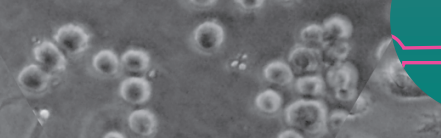


Chapter 5

Circulating Y-RNAs in extracellular vesicles and ribonucleoprotein complexes; implications for the immune system

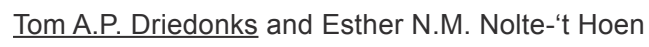

Frontiers in Immunology, 9:3164, 2019 


\section{Abstract}

The exchange of extracellular vesicles (EV) between immune cells plays a role in various immune regulatory processes. EV are nano-sized lipid bilayer-enclosed structures that contain a multitude of proteins and small non-coding RNA molecules. Of the various RNA classes present in EV, miRNAs have been most intensively studied because of their known gene-regulatory functions. These miRNAs constitute only a minor part of all EV-enclosed RNA, whereas other 20-200 nt sized non-coding RNAs were shown to be abundantly present in EV. Several of these mid-sized RNAs perform basic functions in cells, but their function in EV remains elusive. One prominent class of mid-sized extracellular RNAs associated with EV are the Y-RNAs. This family of highly conserved non-coding RNAs discovered as RNA component of circulating ribonucleoproteins targeted by autoantibodies in serum from Systemic Lupus Erythematosus and Sjögren's Syndrome patients. Y-RNA has been implicated in cellular processes such as DNA replication and RNA quality control. In recent years, Y-RNA has been abundantly detected in EV from multiple different cell lines and biofluids, and also in murine and human retroviruses. Accumulating evidence suggests that EV-associated Y-RNA may be involved in a range of immune-related processes, including inflammation, immune suppression, and establishment of the tumor microenvironment. Moreover, changes in plasma levels of extracellular Y-RNA have been associated with various diseases. Recent studies have aimed to address the mechanisms underlying their release and function. We for example showed that the levels of EV-associated Y-RNA released by immune cells can be regulated by Toll-like receptor (TLR) signaling. Combined, these data have triggered increased interest in extracellular Y-RNAs. In this review, we provide an overview of studies reporting the occurrence of extracellular Y-RNAs, as well as signaling properties and immune-related functions attributed to these RNAs. We list RNA-binding proteins currently known to interact with Y-RNAs and evaluate their occurrence in EV. In parallel, we discuss technical challenges in assessing whether extracellular Y-RNAs are contained in ribonucleoprotein complexes or EV. By integrating the current knowledge on extracellular Y-RNA we further reflect on the biomarker potential of Y-RNA and their role in immune cell communication and immunopathology.

Key words: Y-RNA, extracellular vesicles, exosomes, ribonucleoprotein complexes, immune signaling, biomarker 


\section{Introduction}

Extracellular vesicles (EV) are 50 - $1000 \mathrm{~nm}$ sized lipid bilayer-enclosed vesicles containing proteins and nucleic acids (1), which are released by virtually all cells. All living cells, including archaea, bacteria, and eukaryotes release EV, which suggests that the release of EV is a conserved mechanism of cellular communication $(2,3)$. EV have been found in many body fluids and have been implicated in several diseases, including immune-related disorders, cancer, neurological disorders and cardiovascular diseases (4-7). Characterizing the protein, lipid, and RNA content of EV is an active area of research. One of the major topics in the field is to delineate how differences in $\mathrm{EV}$ composition relate to differences in their function, and to determine whether differences in the protein / RNA content of EV can be used as biomarkers for disease.

It has been shown that EV-enclosed RNAs can be functionally transferred to target cells (8-11). Many studies have focused on elucidating the miRNA composition of EVs because of their known effects on gene regulation. However, miRNAs only constitute a minor percentage of EV-enclosed RNA. In contrast, the majority of EV-RNA consists of other types of small- to mid-sized non-coding RNAs (20-200 nt) (12-16). Of these RNAs, Y-RNA attracted attention because this conserved RNA has been detected in EV from many different cell types and in various vertebrate species (12-14,16-18). Moreover, Y-RNAs are highly abundant in body fluids, such as blood and seminal fluid $(19,20)$. Recent data indicate that as much as $67 \%$ of sequencing reads in plasma samples of healthy donors map to Y-RNA (19). There are also indications that the levels of Y-RNA in body fluids could correlate with disease $(21,22)$. Research on the regulation of Y-RNA sorting into EV and the function of EV-associated Y-RNA is in its early days. Our laboratory recently showed that incorporation of Y-RNA in EV released by dendritic cells is regulated by immunogenic and tolerogenic stimuli imposed on these cells (16). Initial studies on the function of EV-enclosed Y-RNA reported proand anti-inflammatory effects (23-25). Given the increasing interest in and number of publications on extracellular Y-RNA we took the initiative to compile an inventory of data and assess the inter-study comparability of discoveries in this field. In this review, we provide an overview of reports describing the occurrence of extracellular Y-RNA in EVs from various cell types and biofluids, as well as its signaling properties and potential immune-related functions. After introducing general aspects of EVassociated RNA and the role of Y-RNA inside cells, we summarize current knowledge on Y-RNA association with EV and with extracellular ribonucleoprotein complexes. In addition, we provide an overview of protein partners of Y-RNA that have also been detected in EV and may therefore be involved in sorting these RNAs into EV. Finally, we provide an overview of the proposed functions of extracellular Y-RNA and reflect on its biomarker potential. Key steps in the Y-RNA life cycle, putative pathways for Y-RNA 
release into the extracellular space, and ideas on the function of Y-RNA transferred to target cells are illustrated in Figure 1.

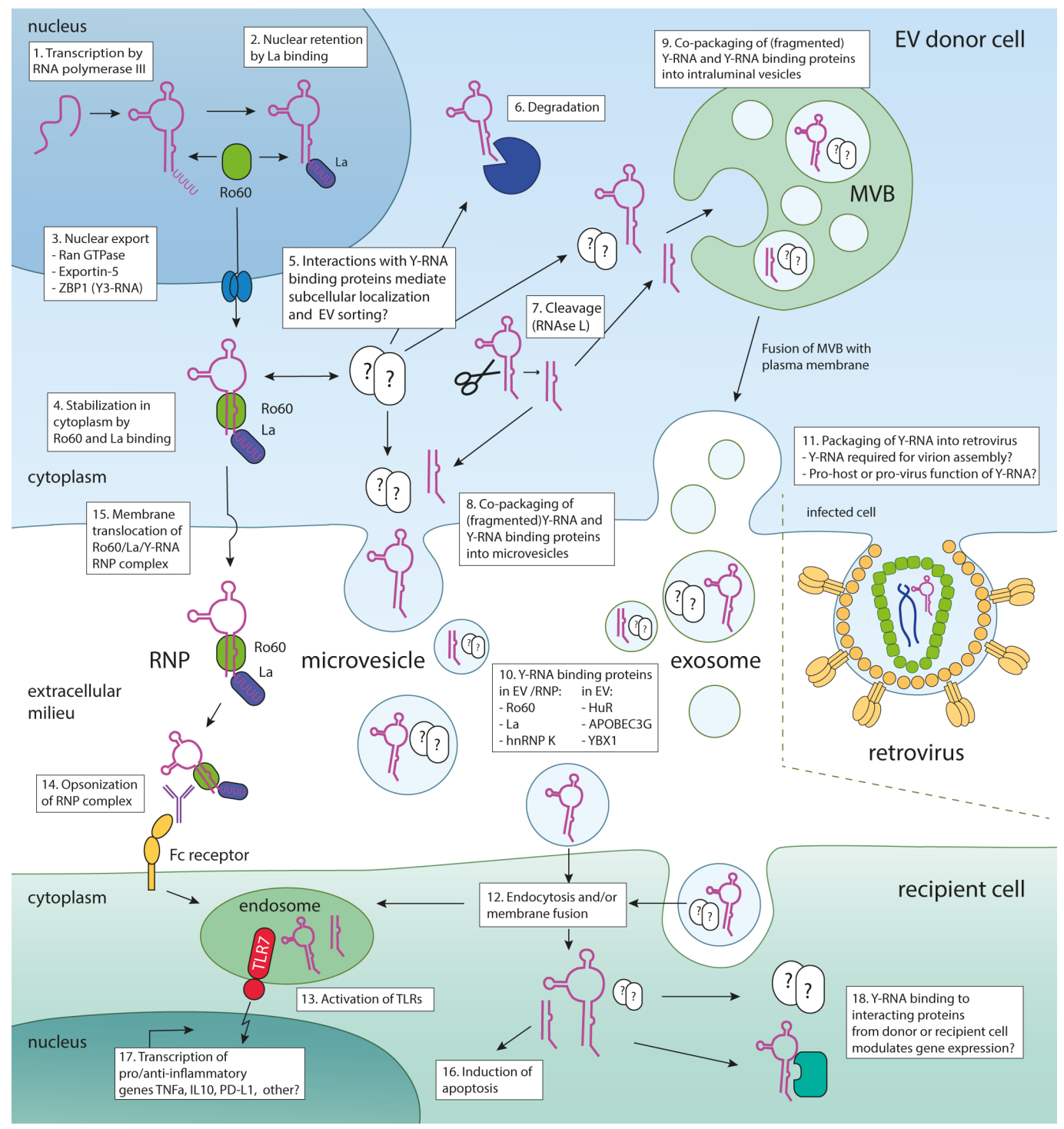


Figure 1. Model illustrating the Y-RNA life cycle, putative pathways for Y-RNA release into the extracellular space, and ideas on the function of Y-RNA transferred to target cells.

Various steps in the process of Y-RNA transport within cells and between cells are indicated numerically. Upon transcription (1), newly generated Y-RNA may remain in the nucleus through binding of La (2). Alternatively, upon binding of Ro60, it can be transported into the cytoplasm by Ran GTPase, Exportin-5 and/or ZBP1 (3). In the cytoplasm, Ro60 binding stabilizes Y-RNA (4). Y-RNA can also bind to various other RNA-binding proteins (for instance the Y-RNA binding proteins summarized in Table 3) that may influence its subcellular localization and/or fate (5). Y-RNA may be degraded (6), or be cleaved into fragments by RNAse $L$ (7). Both full-length and fragmented Y-RNA are packaged into EV, either via passive engulfment of Y-RNA by budding membranes, or through protein-mediated shuttling towards sites of EV biogenesis (such as the plasma membrane (8) or late endosomes / multivesicular bodies (9)). Certain proteins known to bind Y-RNA are co-packaged into EV, but others may only serve to shuttle Y-RNA to the sites of EV biogenesis. The Y-RNA binding proteins from Table 3 that have been found in extracellular space, associated with EV and/or RNP are listed in (10). In the case of retrovirus infected cells, Y-RNA may be additionally released from cells by incorporation into virions (11). EV can be taken up by recipient cells by endocytosis and/or membrane fusion (12). Y-RNA may be delivered to the endosome, where it may activate TLRs (13). TLR triggering also occurs after uptake of opsonized Y-RNA/Ro60/La RNP complexes (14) which may be released from cells after translocation across the cellular membrane (15). Naked Y-RNA has been shown to induce apoptosis (16). TLR triggering of Y-RNA drives the transcription of various pro- and anti-inflammatory cytokines (17). On a more speculative note, transferred Y-RNA could affect the function of recipient cells through the action of Y-RNA binding proteins present in recipient cells or co-transferred by the EV (18). For example, binding to translation enhancer proteins, such as HuR and HuD, may alter mRNA stability and translation efficiency.

\section{Intercellular communication via EV-associated RNA}

EV constitute a unique way in which molecular messages are exchanged between cells. Upon transfer, the lipids, proteins, and RNA associated to EV can modify the function of recipient cells $(1,3,26-28)$. EV are either formed by inward budding into multivesicular bodies, which upon fusion with the plasma membrane are released as exosomes, or by direct budding off the plasma membrane (microvesicles) $(1,3)$. Exosomes and microvesicles cannot be separated using currently available methods, and are therefore collectively referred to as EV. EV are heterogeneous in size and molecular composition, but unique molecular markers to distinguish biologically distinct EV subtypes are yet to be discovered. Various classes of RNA have been detected in EV, including mRNA, IncRNA, circRNA, and small non-coding RNA. Most of the EV-RNA consists of small non-coding RNA types, such as miRNA, tRNA, rRNA, snoRNA, Y-RNA, SRP-RNA (7SL), and Vault RNA $(12,13)$.

It is important to realize that not all extracellular RNA is associated with EV. Other macromolecular structures in the extracellular milieu, such as ribonucleoproteins (RNPs) and lipoprotein particles, also contain RNA (16,29-31). These other structures overlap in size and/or density with EV and are therefore frequent contaminants in EV 
preparations $(32,33)$. The degree to which some of these contaminants co-isolate with EV depends on the fluid used as source of EV and the applied EV isolation method. The most widely used techniques are ultracentrifugation, size exclusion chromatography (SEC), and density gradient centrifugation, in which particles are separated based on mass, size, or buoyant density, respectively. Ultrafiltration-based methods concentrate particles by molecular weight. Precipitation-based methods, on the contrary, concentrate all macromolecules in solution. The advantages and disadvantages of available methods have been extensively reviewed $(32,34)$. Sequential application of methods that separate particles based on size and on density likely yields the purest EV preparations.

It has been demonstrated that both the protein- and miRNA composition of EV can change upon exogenous stimuli imposed on EV-producing cells $(9,13,16)$. These changes in the 'molecular message' that is conveyed via EV can lead to alterations in the function of EV-recipient cells. We have previously shown that, in addition to miRNA, the levels of Y-RNA and snoRNA in EV are regulated by exogenous stimuli imposed on the EV-producing immune cells (16). Importantly, the activation-induced changes in EV-RNA composition we observed only partly reflected changes in cellular RNA, which suggests that the cell stimuli triggered mechanisms for sorting of specific RNA types into EV (16).

Several in vitro and in vivo studies have demonstrated that intercellular transfer of EVassociated miRNA and mRNA leads to changes in recipient cell function (8,10,32,35-37). For example, EV-mediated transfer of miR-155 and miR-146a from wildtype dendritic cells to recipient cells deficient for these miRNAs modulated the response of these recipient cells to lipopolysaccharide (LPS). Transfer of miR-155 into miR-155 negative recipient cells increased IL6 release via repression of SHIP1 and BACH1, while transfer of miR-146a dampened this LPS response by repression of TRAF6 and IRAK1 (10). Functional transfer of mRNA was evidenced by demonstrating that EV-associated mRNA derived from in vitro cultured mast cells could be translated in recipient cells (8). In vivo evidence for EV-mediated transfer of mRNA was provided by the use of Cre-Lox mouse models. Hematopoietic cells or tumor cells expressing Cre-recombinase were shown to release EV containing Cre-mRNA, which induced recombination-mediated expression of floxed fluorescent reporter genes in recipient cells at local or distant sites $(36,38)$. The functional effects of other RNA classes, which compose the major part of all EV-RNA, are beginning to be unveiled. The experimental approaches used to study miRNA transfer may serve as a basis to gain understanding of how other EV-associated RNA classes affect recipient cell behavior, but these RNAs likely exert their functions via mechanisms other than base-pairing with RNA targets. Although many questions remain to be answered, EV-mediated transfer of RNA appears to be a common, frequent, and adaptable process that cells employ to communicate with other cells. 


\section{Intracellular location and function of Y-RNAs}

In order to unravel the role of Y-RNA in EV, it is important to understand the function of Y-RNA inside cells. Y-RNAs have been studied for many years and multiple comprehensive reviews are available on this topic (39-44). Y-RNAs were initially discovered as RNA components of circulating ribonucleoprotein (RNP) autoantigens Ro60 and La in serum from lupus patients (45). These RNP are major targets for autoantibodies in rheumatic diseases such as Systemic Lupus Erythematosus (SLE) and Sjögren's Syndome (SS) $(46,47)$. Y-RNAs are well conserved through evolution and have been found in all vertebrate species $(48,49)$, and related ncRNAs have been found in some bacteria (44) and in nematodes $(50,51)$. Although the nematode ncRNAs called "stem-bulge RNAs" resemble Y-RNA because of their stem-loop structure (51), they differ from bona fide $Y$ RNAs in that they have not been found complexed with Ro60 in cells (50). The human genome encodes four different Y-RNAs (hY1, hY3, hY4 and hY5) while only two different Y-RNAs exist in rodents ( $m Y 1$ and mY3) (52). All Y-RNAs contain a long stem, formed by basepairing the $5^{\prime}$ and $3^{\prime}$ ends, that contains the Ro60 binding site, but individual Y-RNAs differ slightly in their primary and secondary structures (53).

Y-RNAs are transcribed in the nucleus by RNA polymerase III (54) (Figure 1, box 1). Binding of La to the $3^{\prime}$ oligo-uridine tail of Y-RNA mediates its nuclear retention and protects $\mathrm{Y}$-RNA from $3^{\prime}$ to $5^{\prime}$ exonucleolytic degradation $(55,56)$ (Figure 1, box 2). Binding of Ro60 to the stem region of Y-RNA enhances nuclear export (55), which is mediated by Ran GTPase and exportin-5 (57) (Figure 1, box 3). Y3-RNA can also be exported via an alternative pathway through binding of Ro60/Y3-RNA to zipcode binding protein (ZBP1), enabling export via exportin-1 (alternatively named CRM1) (58). It is not fully understood whether Y-RNA is transported from the nucleus in complex to La, or whether La reassociates to Y-RNA after nuclear export. Binding of Ro60 stabilizes Y-RNA in the cytoplasm (Figure 1, box 4), as knockout of Ro60 was shown to drastically reduce Y-RNA levels (59). The loop of Y-RNA is known to interact with various other proteins including nucleolin (60), polypyrimidine tract-binding protein (PTB / hnRNP I) (61), and zipcode-binding protein 1 (ZBP1) (62). It has been proposed that interactions with these proteins could affect the localization and/or function of Y-RNA (43) (Figure 1, box 5). Conversely, Y-RNA can influence the localization of Y-RNA binding proteins, since siRNA-mediated knockdown of Y-RNA leads to nuclear accumulation of Ro60 (63).

Various housekeeping functions of Y-RNA have been described, such as involvement in DNA replication (43) and quality control of non-coding RNA $(64,65)$. The effects of Y-RNA on DNA replication were first observed in cell-free reactions, in which addition of purified Y-RNA subtypes increased the percentage of dividing nuclei (66). siRNA- 
mediated knockdown of Y1-RNA in cells was sufficient to reduce the percentage of cells in S-phase, during which DNA replication takes place (66). In a later study, association of Y-RNA with chromatin was shown to increase 2-4 fold during S-phase and to decrease during $\mathrm{G} 1$ phase and mitosis, which suggests an association with the origin replication complex (ORC) (67). It has been shown that a specific sequence in the Y-RNA stem was sufficient to increase DNA replication in cell-free reactions (68). However, Ro60 knockout cells that contain $\sim 30$-fold lower Y-RNA levels did not show reduced growth rates (59). The exact molecular mechanisms by which Y-RNA affects DNA replication therefore remain unresolved.

Y-RNAs are involved in regulating the degradation of misfolded RNAs through its interaction with Ro60 (47,64,69). Misfolded RNAs that contain a 3' single-stranded end and adjacent helices can bind Ro60 $(70,71)$. This has been shown for $5 \mathrm{~S}$ rRNA in Xenopus (72) and for U2 snRNA in mouse embryonic stem cells (59), and suggested for a wider variety of structured RNAs (70). Structural analyses revealed that the single-stranded tail of the misfolded RNAs extend through the Ro60 cavity, while helices bind on its outer surface (70). Y-RNAs sterically blocked binding of misfolded RNAs to Ro60, thereby regulating the RNA quality control function of Ro60 (64). A similar mechanism has also been demonstrated for the bacterial ortholog of Ro (ro-sixty related, Rsr) (71). In the bacterium Deinococcus radiodurans Y-RNA tethers Rsr to the exonuclease PNPase, thereby forming a RNA-degrading RNP complex resembling the eukaryotic exosome (71). It was proposed that tethering to Rsr potentiates PNPase to specifically degrade structured RNAs. Although mammalian PNPases localize inside mitochondria, it has been proposed that Y-RNAs could potentially tether Ro60 to other proteins involved in RNA metabolism, including exoribonucleases, helicases or RNA chaperones (40).

Interestingly, it was recently discovered in neuronal cells that Y3-RNA can act as a molecular sponge for the enhancer protein HuD (ELAVL4) (73). HuD can enhance gene expression by binding and stabilizing the 3 ' untranslated regions (UTRs) of specific mRNAs involved in motor neuron differentiation and axonogenesis. This activity is counteracted by $\mathrm{Y} 3-\mathrm{RNA}$ binding to $\mathrm{HuD}$, which leads to changes in HuD localization and reduced expression of the involved mRNAs (73). Moreover, dysregulation of Y-RNA binding to HuD has been found to cause alternative splicing in neurons of Alzheimer patients (74).

In cells, Y-RNA does not only occur in its full length form, but has also been shown to be cleaved into specific fragments of $25-35 \mathrm{nt}$. This cleavage, which is carried out by the enzyme RNase L (75) (Figure 1, box 7) occurs in response to UV irradiation or by polyl:Cmediated activation of the innate immune system $(76,77)$. Because $Y$-RNA fragments arise from conserved ends of the Y-RNA hairpin and have comparable sizes to miRNAs, it was proposed that Y-RNA fragments function similar to miRNAs (78). Although interactions of Y-RNA fragments with Argonaute have been demonstrated, mRNA reporter constructs could not be repressed by Y-RNA / Argonaute complexes (79). 
Taken together, the highly conserved family of Y-RNAs interacts with, and regulates the localization and activity of various RNA-binding proteins involved in basic cell functions.

\section{Y-RNAs are abundantly present in the extracellular milieu}

RNA sequencing studies aiming to characterize the small transcriptome of EV have indicated that cells release Y-RNAs into the extracellular milieu (12-18,20,24,25,31,80). There is now strong evidence that Y-RNAs are abundantly present both in supernatants of multiple in vitro cultured primary and immortalized cell types, as well as in various biofluids (see Table 1). In fact, Y-RNA was found to be the most abundant non-coding RNA species in plasma from healthy individuals (19). Multiple studies reported a strong enrichment of Y-RNA in EV relative to intracellular levels, which suggests that the shuttling of Y-RNA into EV is highly efficient $(12-14,25,31)$. 


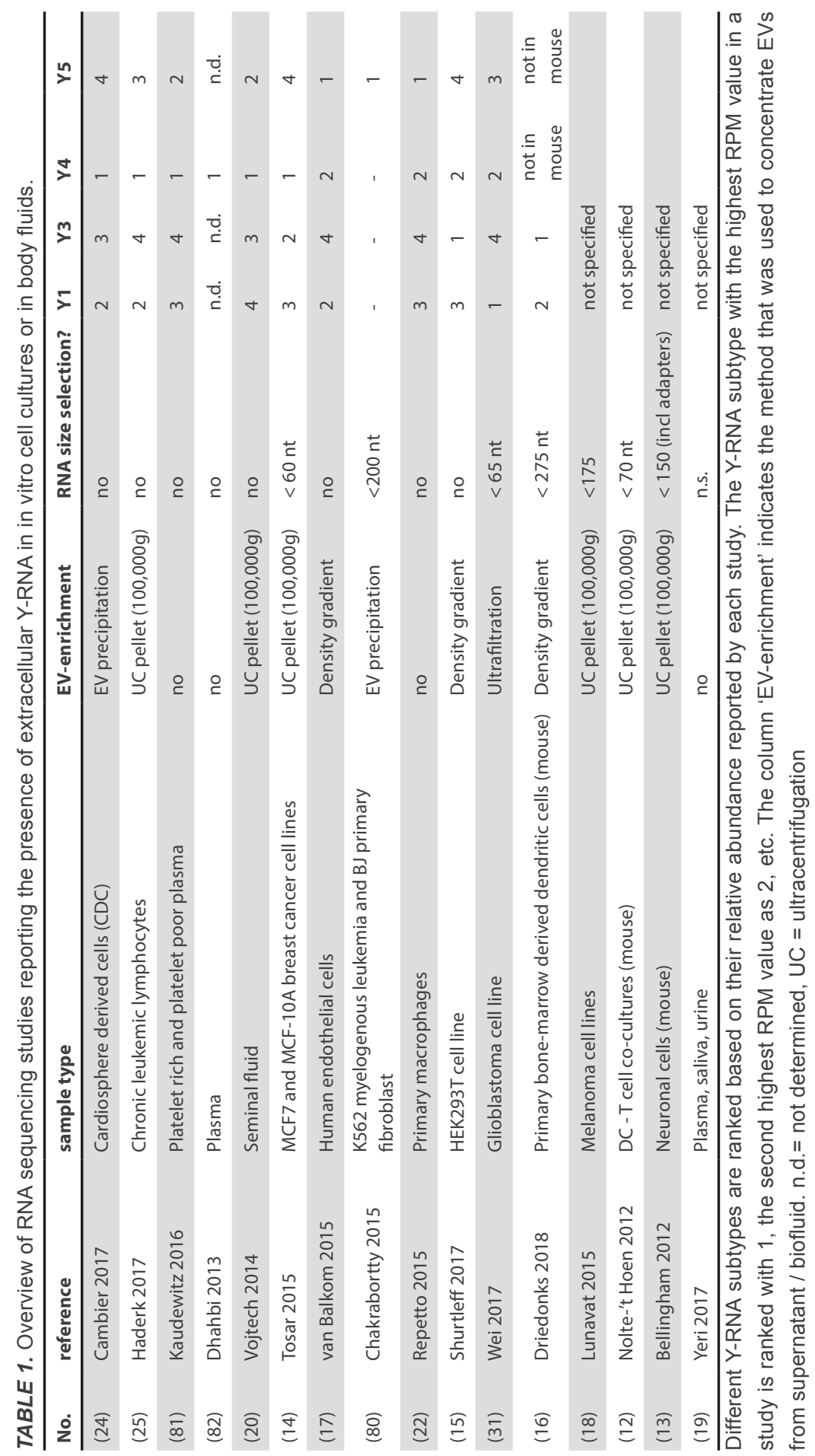


The frequent detection of Y-RNAs in the external milieu of cells suggests that release of Y-RNAs from cells is a common and ubiquitous process. We compared the abundance of Y-RNA subtypes reported in each of the RNA sequencing studies and ranked these from 1 (highest) to 4 in Table 1. Although differences exist between studies that used different cell types or EV-purification methods, $Y 4$ is most abundantly detected in the extracellular milieu. A number of studies indicate that differences exist between the relative abundance of Y-RNA subtypes inside cells and those released by these cells into the extracellular milieu $(14,25,31)$, which supports subtype-specific differences in Y-RNA release.

The data in Table 1 and 2 indicate that different size selections were applied during sequencing library generation for extracellular Y-RNA detection. Several of the studies primarily focused on miRNA detection and therefore applied a narrow size selection ( $<65 \mathrm{nt}$ ). This hampers detection of longer transcripts such as full-length Y-RNA, which are $83-110 \mathrm{nt}$ in size. The sequencing approach in these studies may therefore bias towards detection of Y-RNA fragments $(12,14,19,31)$. However, Y-RNA fragments have also been detected in sequencing studies where no size selection was applied (Table 2) $(17,19,20,25,80-82)$. Most studies show that the extracellular Y-RNA fragments derive from both the $5^{\prime}$ and $3^{\prime}$ arms of the Y-RNA hairpin and that they can be categorized in defined lengths of $\sim 21 \mathrm{nt}, \sim 30 \mathrm{nt}$ and $\sim 40 \mathrm{nt}$ (Table 2). Fragments of the $5^{\prime}$ arm of the Y-RNA hairpin were generally found to be more abundant than the 3 ' fragments. Although these data suggest that Y-RNA fragments are frequently released from cells, reliable detection of full-length Y-RNA in these studies may have been hampered by technical limitations. Y-RNA forms complex RNA structures that are known to negatively influence cDNA synthesis efficacy and to introduce bias in deep sequencing. Reverse transcriptases may not efficiently read through these complex RNA structures, leading to overestimation of fragmented non-coding RNA in sequencing data $(83,84)$. This is corroborated by recent sequencing studies deploying reverse transcriptases that are insensitive to secondary structures, which detected mostly full-length Y-RNAs (and other structured ncRNA such as tRNA) $(15,85)$. By using Northern blot analysis, we also recently confirmed that EV contain mostly full-length Y-RNA and only a small amount of 19 - 35 nt fragments (16). This urges caution in drawing conclusions on the presence of Y-RNA fragments in EV based on RNA sequencing data (16). Taken together, both full-length and fragmented forms of extracellular Y-RNA are abundantly detected in body fluids and in culture supernatant of various cell lines. 


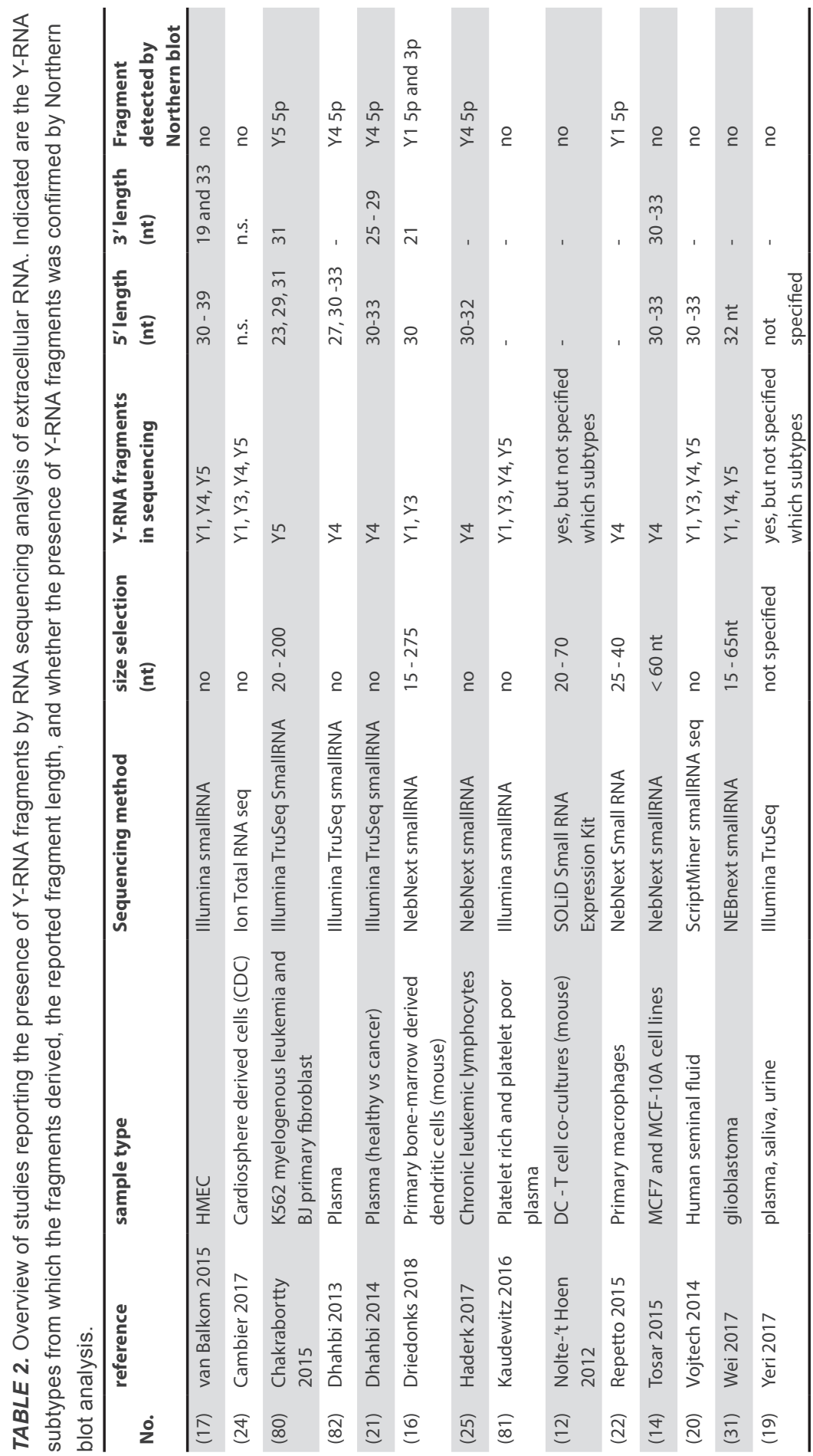




\section{Y-RNA binding proteins identified in EV}

Several different proteins are known to interact with Y-RNA inside cells and determine its function or localization. Additionally, protein binding may shield motifs in Y-RNA which may trigger cellular RNA sensors. For instance, the La-protein potentially shields the triphosphate moiety (56), which may prevent activation of RIG-I (86). Ro60 covers the stem-motif (87), which may prevent activation of dsRNA sensor TLR-3. In the context of EV release, protein partners of Y-RNA may be involved in shuttling of the RNA into $\mathrm{EV}$ and in functional effects of transferred Y-RNA in target cells. It is largely unknown which protein partners are associated with Y-RNA in EV and whether this differs between EV of different cellular origin (Figure 1, box 8 and 9). We therefore composed a list of known Y-RNA protein partners and evaluated whether these proteins have been detected in EV by searching public databases of mass spectrometry data of EVassociated proteins (Vesiclepedia) (88). The list of known Y-RNA protein partners can be found in Table 3. Most of these proteins have been identified by immunoprecipitation with antibodies against RNA binding proteins followed by Y-RNA detection, or by using tagged Y-RNA molecules to pull down proteins from cell lysates that directly interact with this RNA (RNA affinity purification). Studies that initially discovered the interaction between an RNA-binding protein and Y-RNA subtypes, as well as later studies further validating this interaction have been listed in Table 3. Twenty-three proteins have been reported to directly interact with Y-RNA. Ro60 and La, which are the best characterized protein partners of $Y$-RNA, have been discussed in chapter 2. Many of the other Y-RNA-binding proteins (hnRNP I, hnRNP K, RoBPI, ZBP1, YBX1, YBX3, ELAVL1 (HUR), CPSF1, CPSF2, FIPL1 SYMPK, and HuD) function in processing or splicing of mRNA transcripts. Several of these proteins mediate $3^{\prime}$ end processing of human histone-H3 mRNA in conjunction with a truncated form of Y3-RNA called $Y 3^{* *}(89)$. As mentioned earlier, the protein HuD is specifically expressed in neuronal cells where it enhances translation efficiency by stabilizing the mRNAs of mTORC1responsive genes, which is counteracted by Y3-RNA binding to HuD (73). Similarly, the related protein HuR, also known to bind Y3-RNA, can bind AU-rich elements in mRNA transcripts. Via this mechanism, HuR was for example shown to influence cytokine production, evidenced by increased interferon- $\beta$ expression in synoviocytes of arthritis patients, and reduced production of inflammatory cytokines including TNFa and TGF $\beta$ in LPS-treated macrophages $(90,91)$. Two other proteins, MOV10 and Argonaute, are important players in miRNA-mediated gene silencing. Additionally, a number of Y-RNA interacting proteins are involved in virus infection or innate immunity, such as MOV10, APOBEC, IFIT5, SYMPK, YBX1. Interestingly, not all proteins were found to interact with all four human Y-RNA subtypes. This suggests specialized functions for different Y-RNA subtypes, dependent on their associated proteins. 


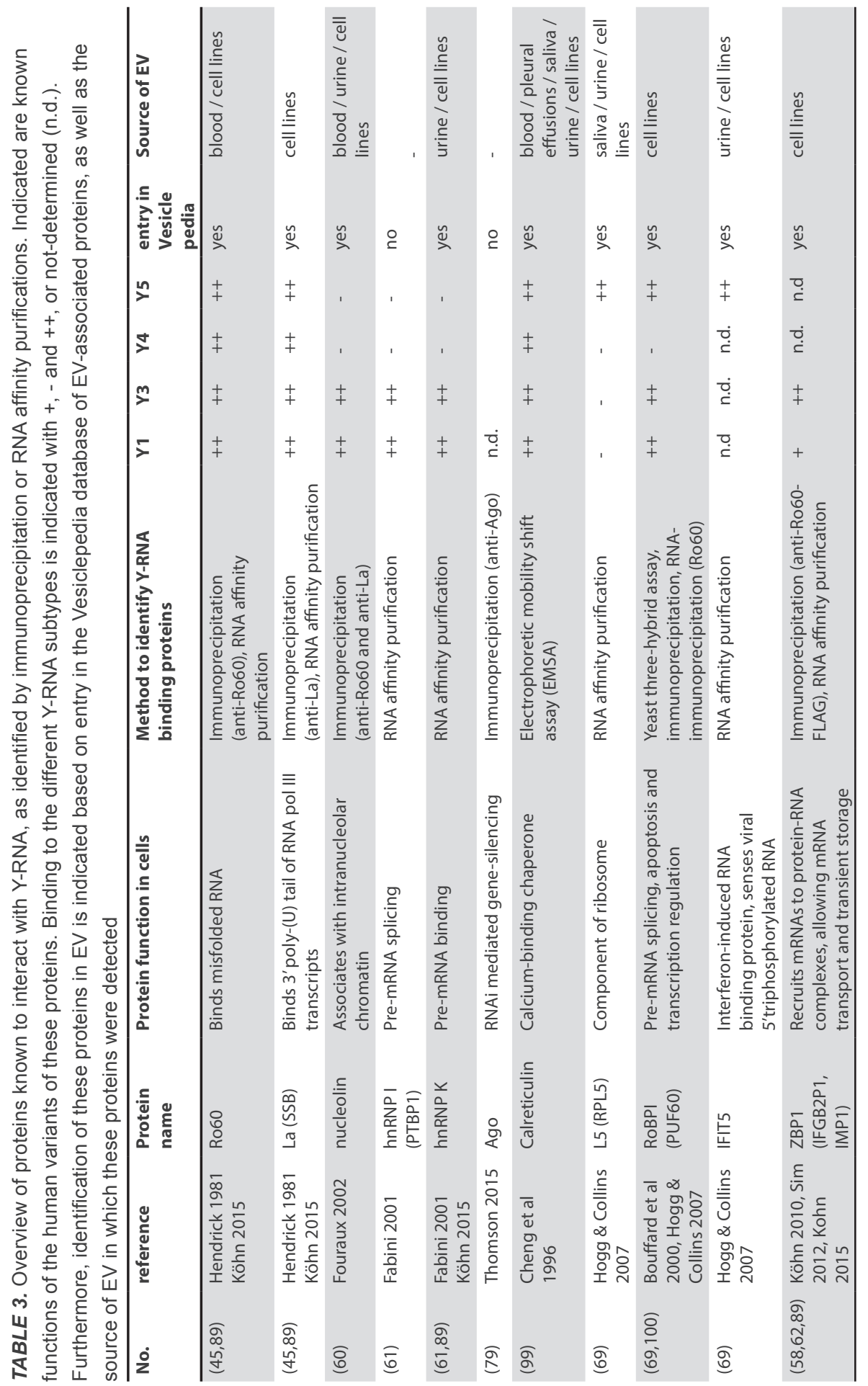




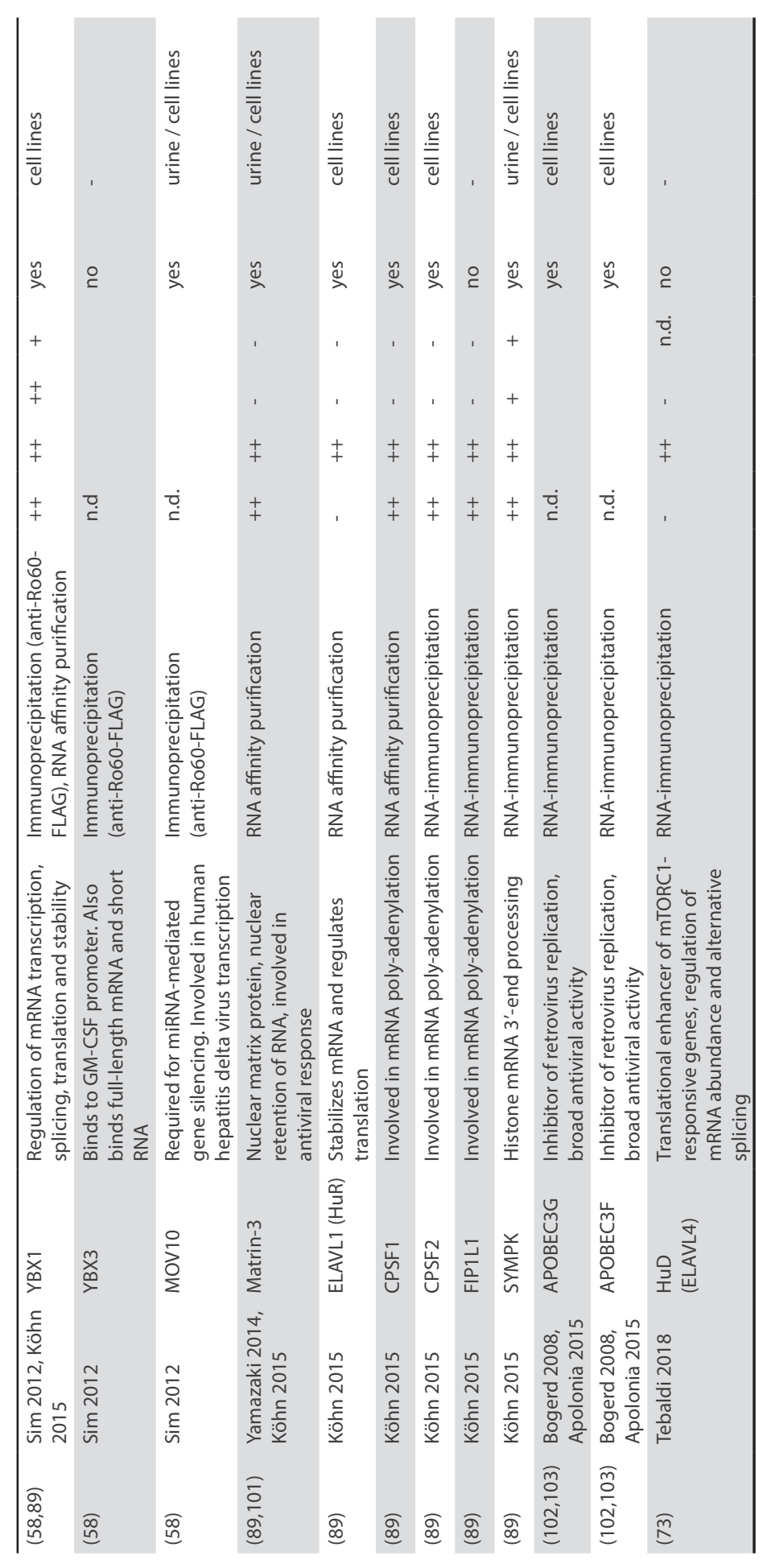


Next, we searched Vesiclepedia (www.microvesicles.org), a repository for extracellular vesicle proteomics data (88), to investigate which of the known Y-RNA binding proteins have been detected in EV. Interestingly, 18 out of 23 known Y-RNA binding proteins were reportedly present in EV from various cell types (Table 3 ). In addition, 10 of these proteins have been detected in EV from biofluids such as blood and urine. Of note, most entries in Vesiclepedia are based on mass-spectrometry, which may be prone to false-positive identification of proteins due to its high sensitivity. Therefore we additionally searched the literature to determine whether the presence of these proteins in EV has been validated by Western blot detection. This was the case for 6 proteins: Ro60 (20,31), La (31), hnRNP K (92), YBX1 (93), APOBEC3G (94), and ELAV1 (HuR) $(95,96)$. In studies reporting the presence of Ro60 (20,31), La (31), and hnRNP K (92), EV had only been enriched by ultracentrifugation/ultrafiltration. These proteins may therefore be associated to EV or RNP or both. Association of HuR and APOBEC3G to EV was convincingly demonstrated using EV purification by density gradient centrifugation $(94,96)$. Additionally, it was shown that lipid membrane-enclosed YBX1 was protected from protease degradation, indicating that this protein is found inside EV (93).

We noticed that several of the Y-RNA-binding proteins detected in EV have previously been implicated in sorting of miRNAs into EV. ELAV1 (HuR), for example, dissociates miRNA-122 from AGO2/mRNA complexes in hepatocytes and drives subsequent expulsion of miR-122 from the cell via EV (96). Furthermore, YBX1 was shown to package miR-223 into EV from HEK293T cells (97). Initial evidence suggests that YBX1 also plays a role in sorting Y-RNA into EV (15). Knockout of this protein in HEK293T cells resulted in a reduced packaging of Y-RNA into EV. However, disruption of YBX1 did not completely abolish Y-RNA packaging, suggesting the involvement of additional proteins in this process. Moreover, YBX1 knockout also affected the packaging of other small non-coding RNAs such as tRNAs and Vault RNA, which suggests a more general function in EV-RNA packaging. Delineating the mechanisms underlying sorting of RNAs into EV is an area of intense research. Identification of proteins that specifically interact with EV-RNAs of interest, as performed above for Y-RNA, is a starting point to investigate potential involvement in sorting these RNAs into EV. Besides the assumed involvement of RBP in this process, sorting of RNAs into EV may also be influenced by the presence of specific motifs, modifications, or structures in RNA, post-translational modifications in RBP, and local enrichment of RNA close to membrane compartments (reviewed in (32)). RNA sorting into EV may additionally be modulated by signaling processes triggered in the parental cells. There is strong evidence that miRNA sorting is influenced by cell stimulation $(9,16)$. Our own laboratory recently showed that EV-mediated release of Y-RNA is influenced by immune-related stimuli imposed on EV-producing cells (16). The EV-associated changes in Y-RNA were not reflected in 
cellular Y-RNA levels, which suggests that the Y-RNA shuttling rate, rather than the transcriptional level of Y-RNA, is modulated by these stimuli. Condition-dependent changes in the levels of extracellular Y-RNA have also been observed in vivo. Physical exercise was shown to increase the levels of circulating Y4-RNA, while Y1, Y3, and Y5 were decreased relative to resting conditions (98). Further research is needed to evaluate whether regulation of Y-RNA shuttling to the extracellular space is driven by differential expression or localization of Y-RNA binding proteins.

In conclusion, a large number of proteins known to interact with Y-RNA have been detected in EV. Some of these proteins may be involved in sorting of Y-RNA into EV, but the underlying mechanisms should be further explored (Figure 1, box 10). The copresence of Y-RNA and Y-RNA binding proteins in EV also highlights the need to study the functional effects of EV-associated Y-RNA in the context of its protein partners.

\section{Y-RNA and viruses}

Extracellular Y-RNA has not only been detected in EV and RNP, but also in various retroviruses such as murine leukemia virus (MLV) and human immunodeficiency virus (HIV) (104-108) (Figure 1, box 11). These viruses incorporate not only Y-RNA, but also various other host-derived non-coding RNAs, such as tRNA and 7SL. The presence of extracellular Y-RNA in both retroviruses and EV is interesting because both structures are formed via overlapping biogenesis routes $(109,110)$. In addition, several Y-RNA binding proteins that have been identified in EV also interact with retroviral RNA. In the case of HIV, these proteins included YBX1, hnRNP K, PTBP1, Nucleolin, and Matrin-3 (111). This raises the interesting question whether there is overlap in mechanisms underlying the sorting of RNAs into EV and retroviruses.

Retroviruses use specific host tRNAs to prime reverse transcription, which is a key step in the retroviral life cycle (112). In addition, encapsidated host non-coding RNAs may mediate packaging of antiviral proteins, such as the antiviral cytidine deaminase APOBEC3G into virions (113). It has been hypothesized that newly synthesized host RNAs, including Y-RNAs that have not been bound by Ro60, may act as a scaffold for virion assembly $(105,106,114)$. Moreover, it has been suggested that Y-RNA may benefit the host via potential triggering of TLR7 in newly infected cells, thereby initiating an antiviral immune response (107). Additionally, it was reported that many packaged RNAs, including Y-RNA, can mediate APOBEC packaging which leads to mutations in the viral genome or restricts retrotransposition (103). It remains to be investigated whether these Y-RNA-driven processes only occur during virus infection and whether we could learn from retroviruses to further delineate the function of Y-RNA in EV. 


\section{The role of extracellular Y-RNA in immune regulation}

The high abundance of Y-RNA in EVs and RNPs raises the question whether extracellular Y-RNAs have signaling functions when transferred to target cells (Figure 1, box 12). In general, it is technically challenging to assess the role of individual RNAs in EVmediated effects because EV mediate simultaneous transfer of multiple proteins, lipids and RNAs to target cells. Until now, functional transfer of EV-associated miRNAs have been addressed either by using target cells with luciferase reporter constructs containing the 3'UTR of the mRNA target, or by investigating EV released by miRNA knockout cells, or by assessing the effects of transfecting synthetic analogues of the RNA of interest into target cells (reviewed in (32)). It is unlikely that, upon transfer to target cells, Y-RNA functions similar to miRNA, as it has been shown that Y-RNA fragments bound to Ago2 were unable to repress reporter mRNAs (79). In addition, the effects of Y-RNA in EV may differ from those elicited by Y-RNA-containing RNP. Although the number of studies addressing the effects of extracellular Y-RNA are limited, the majority of these studies described effects of $Y$-RNA on immune regulation. Interestingly, both pro- and anti-inflammatory effects have been described, which will be discussed in more detail below.

Table 4 summarizes the immune-related effects that have been reported for extracellular Y-RNA subtypes in various experimental settings. Some of these studies specifically addressed the function of Y-RNA containing RNP $(23,115,116)$, whereas others focused on the function of EV-associated RNA $(24,25)$. In the recent study by Haderk et al. it was shown that $Y 4$ and 5 '-fragments of $Y 4$ were abundantly present in EV released by B cell leukemia cells. These EV not only induced inflammatory effects, such as the release of CCL2, CCL4 and IL6 by monocytes, but also induced PD-L1 expression on these cells, which inhibits T-cell activation (117). To investigate whether the EV-induced effects were mediated by $Y 4$, monocytes were transfected with synthetic homologues of full-length $Y 4$ or fragments thereof. Full length $Y 4$, but not $Y 4$ fragments, induced similar pro- and anti-inflammatory effects in monocytes as those observed after incubation with EV. Based on these data it was suggested that Y-RNA in tumor EV could contribute to establishing a favorable tumor microenvironment via suppressing the immune system (25).

Y4-RNA containing EV have also been implicated in myocardial infarctions (24). Cardiosphere-derived cells $(C D C)$ can reduce damage during myocardial infarction by modulating inflammatory responses via an unknown mechanism. It was found that CDC-EVs contain a relatively large percentage of Y-RNAs, and that one specific 5' fragment of Y4-RNA was particularly abundant in CDC-EV compared to normal human dermal fibroblasts. Evidence was provided that EV could transfer Y4-fragments to bone-marrow derived macrophages, and that transfection of this Y4-fragment 


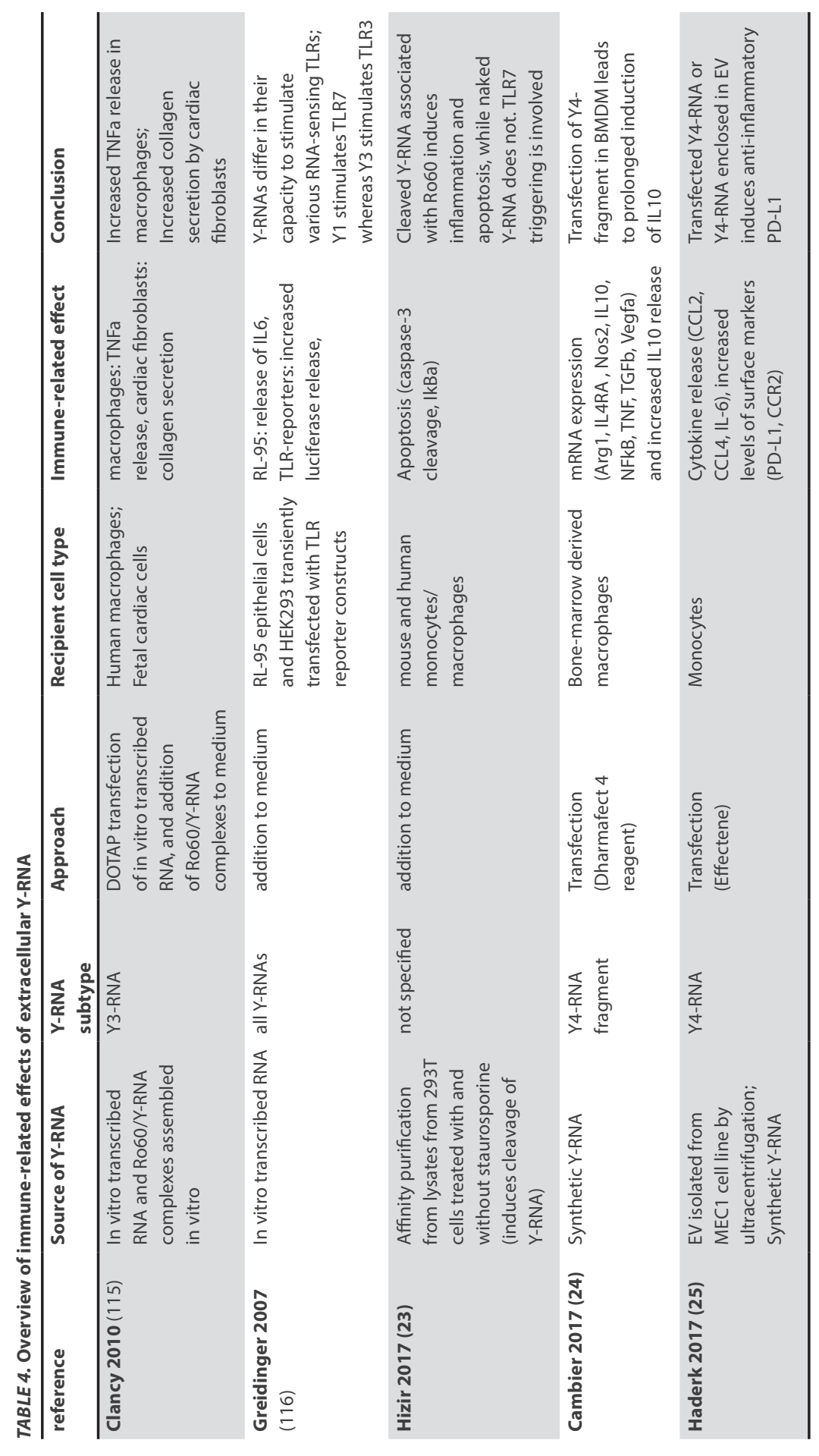


into macrophages resulted in strong and prolonged upregulation of IL 10 , and to a lesser extent TNFa. Additionally, administration of this Y4-fragment in vivo induced IL10 release and reduced damage in a myocardial infarction model in rats (24). Thus, the abundance of Y4-fragments in CDC-EVs correlated with the potency of these RNA fragments to mitigate damage after myocardial infarction.

The function of EV-associated Y-RNA has until now been addressed by transfecting Y-RNA (fragments) into target cells as a model for EV-mediated transfer of these RNAs. Although this may currently be the most feasible approach, several limitations can be identified. The naked, synthetic RNAs employed in these studies are not complexed to proteins, whereas RBP may play a role in the function of truly EV-associated Y-RNAs. Additionally, the route of uptake of lipofected RNA complexes may be different from EV-enclosed RNAs, resulting in delivery of the Y-RNA to subcellular locations other than those reached after EV-mediated delivery.

A few other studies provide indirect support for a role of EV-associated Y-RNA in immune-modulatory processes. For instance, Y-RNAs and tRNAs are particularly abundant in seminal plasma EV (prostasomes) (20), which are known to confer immunesuppressive effects leading to reduced rejection of sperm cells (118). Similarly, EV released by the parasite Heligmosomoides polygyrus contain high levels of nematode stem-bulge RNAs (which are related in sequence to $Y$ RNAs) and suppress cytokine release in mice (119). Furthermore, in our latest study, we demonstrate that EV released from dendritic cells with an immune-suppressive function are more enriched in Y-RNA than EV released by dendritic cells with an immune-activating phenotype (16).

While EV-associated Y-RNAs seem to induce a range of different immune-related effects, circulating RNP containing Y-RNA predominantly induce immune activation. Many of these effects reportedly depend on TLR-mediated triggering (Figure 1, box 13). However, Y-RNA subtypes differ in their capacity to trigger different TLRs. Y3-RNA predominantly triggers TLR3, while Y1, Y3 and Y4 trigger TLR7 (116). Whereas unbound Y-RNA may trigger TLR signaling, Y-RNA bound to protein partners such as Ro60 and La has a reduced stimulatory potential, likely because these proteins shield dsRNA hairpin structure and 5' triphosphate group that are ligands for TLR and other pattern recognition receptors. In support of this idea, Clancy et al. showed that Ro60-associated Y3-RNA, in contrast to naked Y3-RNA, does not induce TNFa release in macrophages (115). The pro-inflammatory effects of Y-RNA-Ro60 complexes in autoimmune diseases such as SLE and SS are likely explained by binding of auto-antibodies to these RNP. Opsonization of Ro60-associated Y3-RNA by anti-Ro60 lgG was shown to be required for stimulation of TNFa release by macrophages, supporting a role for FcyR in this process (115) (Figure 1, box 14). However, not only FcyR-mediated triggering, but also RNA-mediated triggering of TLR7 contributed to the inflammatory effects elicited by the RNP (115). It is not known whether exposed Y-RNA-Ro60/La complexes only 
occur as RNP or whether these complexes are also present on the surface of EV. During apoptosis of fibroblasts, Y3-RNA was shown to drive the translocation of Ro60 to the outer leaflet of the plasma membrane (120) (Figure 1, box 15). Upon opsonization with anti-Ro60 antibodies, these apoptotic fibroblasts induced TNFa release in macrophages in a TLR7 dependent manner (120). Since apoptotic cells release various types of EV as well as apoptotic bodies (121), it is possible that some of these EV display surface-exposed Y-RNA-Ro60 complexes. In fact, it is known that EV associate with autoantibodies in several autoimmune diseases, thereby forming proinflammatory complexes that contribute to disease (reviewed in (122)).

Besides triggering inflammation in SLE and SS, extracellular Y-RNA complexes have also been demonstrated to induce apoptosis in atherosclerosis (23) and cancer (80). In atherosclerosis, lipoproteins accumulating in arteries can lead to activation of macrophages and subsequent apoptosis induction in these cells. In vitro cultured macrophages treated with lipids release increased levels of fragmented Y-RNA into the medium (22). Hizir et al showed that affinity purified Y-RNA fragments/Ro60 RNPs from apoptotic HEK293T cells induced cell death in macrophages (Figure 1, box 16). Y-RNA fragment-containing RNP released by macrophages could therefore contribute to a negative feed-back loop in which more and more macrophages in the lipid-rich environment die by apoptosis. In the context of cancer, it was shown that EV released by myelogenous leukemia cell lines contain high levels of fragmented Y5-RNA (80). Not only these EV, but also deproteinized total RNA from these EV and synthetic Y5 fragments were shown to induce apoptosis in healthy cells, but not in cancer cells. Via this mechanism, Y5-fragments in EV could favor cancer cell proliferation and invasion of tissues.

The studies described above suggest that the functional effects of extracellular Y-RNAs depend on both the macromolecular structure to which it is associated and the conditions under which the Y-RNA is released. In addition to the TLR-mediated effects of Y-RNA that have been reported to date (Figure 1, box 17), Y-RNA may also mediate functional effects via their interacting proteins (Figure 1, box 18). This highlights the importance of separating EV from RNP in studies addressing the function of EVassociated RNA $(32,123)$

\section{Biomarker potential of extracellular Y-RNA}

The abundance of circulating Y-RNA in body fluids has triggered interest in the potential use of Y-RNA as biomarker for disease. Increased levels of Y-RNA have been observed in the circulation of cancer patients $(14,21,25,31)$. In breast cancer patients, the abundance of $3^{\prime}$ Y-RNA fragments was higher than in healthy controls (21). A more recent study on chronic lymphocytic leukemia (CLL) reported the increased abundance of Y4-RNA in serum from CLL patients compared to healthy controls (25). However, these studies 
were performed with small groups of patients and the data currently lack power to confirm the suitability of Y-RNA (fragments) as biomarkers for cancer. Whereas there is no evidence that EV from tumor cells are more enriched in Y-RNA than their nontumorigenic counterparts, most tumor cells release relatively high numbers of EV $(14,25)$. The cancer-related increase in circulating Y-RNA may therefore be explained by increased numbers of tumor cell-derived EV in the circulation. Alternatively, other cell types may react to the presence of the tumor by increasing cellular export of Y-RNA.

Increased levels of circulating extracellular Y-RNA have also been observed in the context of atherosclerosis and coronary artery disease. Repetto and colleagues observed a higher number of $5^{\prime} \mathrm{Y} 1$-fragments in the blood of ApoE-/- mice used as a model for atherosclerosis (22). Likewise, increased levels of circulating 5'-Y1 were observed in a cohort of 43 men with stable coronary artery disease (CAD), as compared to 106 age-matched healthy men. These data were validated in an independent subcohort including 220 patients versus 408 controls. In 45 CAD patients an increased abundance of Y4-RNA 5'-fragments was observed (22). This raises the question of which cells are the main producers of extracellular Y-RNA fragments present in the circulation. The suggested candidates include macrophages (23) and platelets (81). However, it is important to note that pre-analytical variables can strongly affect characterization of extracellular RNA in plasma. Plasma samples are commonly contaminated by platelets, which may disintegrate during freezing (34), thereby releasing their internal (RNA) content. Indeed, plasma miRNA levels were shown to correlate with platelet counts prior to freezing (124). Thus, there is an urgent need for standardization of sample collection, storage conditions and sample processing for reliable assessment of Y-RNA and other extracellular RNAs present in body fluids.

In conclusion, differences in circulating Y-RNA may be further explored as biomarkers for disease, but it is critically important to evaluate and standardize the various methods used to isolate different carriers of Y-RNA in body fluids. Additionally, acquisition of knowledge on how disease-associated changes in cells affect the release of Y-RNAs will help to better understand their biomarker potential.

\section{Concluding remarks}

Current data suggest that the family of Y-RNAs does not only play a role in intracellular processes to maintain cell function, but also acts as versatile intercellular messengers. Various studies have indicated that extracellular transport of Y-RNA is a highly efficient process employed by many different cell types. Additionally, Y-RNA is one of the most abundant extracellular non-coding RNAs in human plasma. Such extracellular RNA can occur in RNP or in EV. One potential trigger that regulates extracellular release of Y-RNA is TLR signaling. Moreover, currently available data suggest functional involvement of extracellular Y-RNA in various immune-related processes. Y-RNAs can bind to 
several different proteins. We here provided an overview of Y-RNA binding proteins that occur inside cells and in Y-RNA-containing RNP or EV released by cells into the extracellular milieu. We propose that binding to these proteins not only determines how Y-RNA regulates cellular processes, but may also drive their sorting into EV and could be essential for functional effects of Y-RNA transferred to recipient cells (Figure 1). Partly based on currently available data, we envision that Y-RNA may affect the function of recipient cells via different mechanisms. These include direct effects of Y-RNA, such as activation of RNA sensors (e.g. TLRs), leading to the release of pro- and anti-inflammatory cytokines. Additional effects may be mediated by Y-RNA binding proteins, many of which function in regulation of transcription and translation. Initial data suggest that levels of extracellular Y-RNA may correlate with disease. However, more research is needed as to how Y-RNA release is altered in diseased cells and how this affects other cells in order to delineate the contribution of extracellular Y-RNA in (immune-related) diseases and to correctly interpret its applicability as a disease biomarker.

\section{Acknowledgements}

We would like to thank Dr. S. Wolin for critically reading and commenting on this manuscript. Although we tried to provide a comprehensive overview of existing literature on extracellular Y-RNA, the authors apologize to those colleagues who were not cited.

\section{Conflict of interest statement}

The authors declare no conflict of interest.

\section{Funding disclosure}

This work was supported by European Research Council under the European Union's Seventh Framework Programme [FP/2007-2013] / ERC Grant Agreement number [337581] to EN. 


\section{References}

1. Colombo M, Raposo G, Théry C. Biogenesis, Secretion, and Intercellular Interactions of Exosomes and Other Extracellular Vesicles. Annu Rev Cell Dev Biol (2014) 30:255-289. doi:10.1146/annurevcellbio-101512-122326

2. Yáñez-Mó M, Siljander PRM, Andreu Z, Zavec AB, Borràs FE, Buzas El, Buzas K, Casal E, Cappello F, Carvalho J, et al. Biological properties of extracellular vesicles and their physiological functions. $J$ Extracell Vesicles (2015) 4:1-60. doi:10.3402/jev.v4.27066

3. van Niel G, D'Angelo G, Raposo G. Shedding light on the cell biology of Extracellular Vesicles. Nat Rev Mol Cell Biol (2018) 19:213-228. doi:10.1038/nrm.2017.125

4. Robbins PD, Dorronsoro A, Booker CN. Regulation of chronic inflammatory and immune processes by extracellular vesicles. J Clin Invest (2016) 126:1173-1180. doi:10.1172/JCl81131.tions

5. Sato S, Weaver AM. Extracellular vesicles: important collaborators in cancer progression. Essays Biochem (2018) 62:149-163. doi:10.1042/EBC20170080

6. Quek C, Hill AF. The role of extracellular vesicles in neurodegenerative diseases. Biochem Biophys Res Commun (2017) 483:1178-1186. doi:10.1016/j.bbrc.2016.09.090

7. Osteikoetxea X, Németh A, Sódar BW, Vukman KV., Buzás El. Extracellular vesicles in cardiovascular disease: Are they Jedi or Sith? J Physiol (2016) 594:2881-2894. doi:10.1113/JP271336

8. Valadi H, Ekström K, Bossios A, Sjöstrand M, Lee JJ, Lötvall JO. Exosome-mediated transfer of mRNAs and microRNAs is a novel mechanism of genetic exchange between cells. Nat Cell Biol (2007)

9:654-659. doi:10.1038/ncb1596

9. Montecalvo A, Larregina AT, Shufesky WJ, Stolz DB, Sullivan MLG, Karlsson JM, Baty CJ, Gibson GA, Erdos G, Wang Z, et al. Mechanism of transfer of functional microRNAs between mouse dendritic cells via exosomes. Blood (2012) 119:756-66. doi:10.1182/blood-2011-02-338004

10. Alexander M, Hu R, Runtsch MC, Kagele DA, Mosbruger TL, Tolmachova T, Seabra MC, Round JL, Ward DM, O'Connell RM. Exosome-delivered microRNAs modulate the inflammatory response to endotoxin. Nat Commun (2015) 6:7321. doi:10.1038/ncomms8321

11. Tkach M, Thery C. Communication by Extracellular Vesicles : where we are and where to go. Cell (2016) 164: doi:10.1016/j.cell.2016.01.043

12. Nolte-'t Hoen ENM, Buermans HPJ, Waasdorp M, Stoorvogel W, Wauben MHM, 't Hoen PAC. Deep sequencing of RNA from immune cell-derived vesicles uncovers the selective incorporation of small non-coding RNA biotypes with potential regulatory functions. Nucleic Acids Res (2012) 40:9272-85. doi:10.1093/nar/gks658

13. Bellingham SA, Coleman BM, Hill AF. Small RNA deep sequencing reveals a distinct miRNA signature released in exosomes from prion-infected neuronal cells. Nucleic Acids Res (2012) 40:10937-10949. doi:10.1093/nar/gks832

14. Tosar JP, Gambaro F, Sanguinetti J, Bonilla B, Witwer KW, Cayota A. Assessment of small RNA sorting into different extracellular fractions revealed by high-throughput sequencing of breast cell lines. Nucleic Acids Res (2015)1-16. doi:10.1093/nar/gkv432

15. Shurtleff MJ, Yao J, Qin Y, Nottingham RM, Temoche-Diaz MM, Schekman R, Lambowitz AM. Broad role for YBX1 in defining the small noncoding RNA composition of exosomes. Proc Natl Acad Sci (2017) 114:E8987-E8995. doi:10.1073/pnas.1712108114

16. Driedonks TAP, van der Grein SG, Ariyurek Y, Buermans HPJ, Jekel H, Chow FWN, Wauben MHM, Buck AH, Hoen PAC, Nolte-'t Hoen ENM. Immune stimuli shape the small non-coding transcriptome of extracellular vesicles released by dendritic cells. Cell Mol Life Sci (2018)3857-3875. doi:10.1007/s00018018-2842-8

17. van Balkom BWM, Eisele AS, Pegtel DM, Bervoets S, Verhaar MC. Quantitative and qualitative analysis of small RNAs in human endothelial cells and exosomes provides insights into localized RNA processing, degradation and sorting. J Extracell vesicles (2015) 4:26760. doi:10.3402/jev.v4.26760 
18. Lunavat TR, Cheng L, Kim D-K, Bhadury J, Jang SC, Lässer C, Sharples RA, López MD, Nilsson J, Gho YS, et al. Small RNA deep sequencing discriminates subsets of extracellular vesicles released by melanoma cells evidence of unique microRNA cargos. RNA Biol (2015) 12:810-823. doi:10.1080/15476286.2015.1056975

19. Yeri A, Courtright A, Reiman R, Carlson E, Beecroft T, Janss A, Siniard A, Richholt R, Balak C, Rozowsky J, et al. Total Extracellular Small RNA Profiles from Plasma, Saliva, and Urine of Healthy Subjects. Sci Rep (2017) 7:44061. doi:10.1038/srep44061

20. Vojtech L, Woo S, Hughes S, Levy C, Ballweber L, Sauteraud RP, Strobl J, Westerberg K, Gottardo R, Tewari $\mathrm{M}$, et al. Exosomes in human semen carry a distinctive repertoire of small non-coding RNAs with potential regulatory functions. Nucleic Acids Res (2014) 42:7290-7304. doi:10.1093/nar/gku347

21. Dhahbi JM, Spindler SR, Atamna H, Boffelli D, Martin DIK. Deep Sequencing of Serum Small RNAs Identifies Patterns of $5^{\prime}$ tRNA Half and YRNA Fragment Expression Associated with Breast Cancer. Biomark Cancer (2014) 6:37-47. doi:10.4137/BIC.S20764.RECEIVED

22. Repetto E, Lichtenstein L, Hizir Z, Tekaya N, Benahmed M, Ruidavets J-B, Zaragosi L-E, Perret B, Bouchareychas L, Genoux A, et al. RNY-derived small RNAs as a signature of coronary artery disease. BMC Med (2015) 13:259. doi:10.1186/s12916-015-0489-y

23. Hizir Z, Bottini S, Grandjean V, Trabucchi M, Repetto E. RNY (YRNA)-derived small RNAs regulate cell death and inflammation in monocytes/macrophages. Cell Death Dis (2017) 8:e2530. doi:10.1038/cddis.2016.429

24. Cambier L, de Couto G, Ibrahim A, Echavez AK, Valle J, Liu W, Kreke M, Smith RR, Marbán L, Marbán E. Y RNA fragment in extracellular vesicles confers cardioprotection via modulation of IL-10 expression and secretion. EMBO Mol Med (2017)e201606924. doi:10.15252/emmm.201606924

25. Haderk F, Schulz R, Iskar M, Cid LL, Worst T, Willmund KV, Schulz A, Warnken U, Seiler J, Benner A, et al. Tumor-derived exosomes modulate PD-L1 expression in monocytes. Sci Immunol (2017) 2:eaah5509. doi:10.1126/sciimmunol.aah5509

26. van der Grein SG, Nolte-'t Hoen ENM. "Small Talk" in the Innate Immune System via RNA-Containing Extracellular Vesicles. Front Immunol (2014) 5:1-8. doi:10.3389/fimmu.2014.00542

27. Tkach M, Kowal J, The C. Why the need and how to approach the functional diversity of extracellular vesicles. Philos Trans R Soc B (2017) 373: doi:10.1098/rstb.2016.0479

28. Gho YS, Lee C. Emergent properties of extracellular vesicles: A holistic approach to decode the complexity of intercellular communication networks. Mol Biosyst (2017) 13:1291-1296. doi:10.1039/c7mb00146k

29. Arroyo JD, Chevillet JR, Kroh EM, Ruf IK, Pritchard CC, Gibson DF, Mitchell PS, Bennett CF, PogosovaAgadjanyan EL, Stirewalt DL, et al. Argonaute2 complexes carry a population of circulating microRNAs independent of vesicles in human plasma. Proc Natl Acad Sci U S A (2011) 108:5003-8. doi:10.1073/ pnas. 1019055108

30. Vickers KC, Palmisano BT, Shoucri BM, Shamburek RD, Remaley AT. MicroRNAs are transported in plasma and delivered to recipient cells by high-density lipoproteins. Nat Cell Biol (2011) 13:423-33. doi:10.1038/ ncb2210

31. Wei Z, Batagov AO, Schinelli S, Wang J, Wang Y, El Fatimy R, Rabinovsky R, Balaj L, Chen CC, Hochberg F, et al. Coding and noncoding landscape of extracellular RNA released by human glioma stem cells. Nat Commun (2017) 8:1145. doi:10.1038/s41467-017-01196-x

32. Mateescu B, Kowal EJK, van Balkom BWM, Bartel S, Bhattacharyya SN, Buzás El, Buck AH, de Candia P, Chow FWN, Das $\mathrm{S}$, et al. Obstacles and opportunities in the functional analysis of extracellular vesicle RNA - an ISEV position paper. J Extracell Vesicles (2017) 6:1286095. doi:10.1080/20013078.2017.1286095

33. Van Deun J, Mestdagh P, Sormunen R, Cocquyt V, Vermaelen K, Vandesompele J, Bracke M, De Wever $\mathrm{O}$, Hendrix A. The impact of disparate isolation methods for extracellular vesicles on downstream RNA profiling. J Extracell Vesicles (2014) 3:1-14. doi:10.3402/jev.v3.24858

34. Witwer KW, Buzás El, Bemis LT, Bora A, Lässer C, Lötvall J, Nolte-'t Hoen EN, Piper MG, Sivaraman S, Skog J, et al. Standardization of sample collection, isolation and analysis methods in extracellular vesicle research. J Extracell vesicles (2013) 2:1-25. doi:10.3402/jev.v2i0.20360 
35. Ridder K, Sevko A, Heide J, Dams M, Rupp A-K, Macas J, Starmann J, Tjwa M, Plate KH, Sültmann $\mathrm{H}$, et al. Extracellular vesicle-mediated transfer of functional RNA in the tumor microenvironment. Oncoimmunology (2015) 4:e1008371. doi:10.1080/2162402X.2015.1008371

36. Zomer A, Maynard C, Verweij FJ, Kamermans A, Schäfer R, Beerling E, Schiffelers RM, de Wit E, Berenguer J, Ellenbroek SIJ, et al. In Vivo Imaging Reveals Extracellular Vesicle-Mediated Phenocopying of Metastatic Behavior. Cell (2015) 161:1046-1057. doi:10.1016/j.cell.2015.04.042

37. Lai CP, Kim EY, Badr CE, Weissleder R, Mempel TR, Tannous B a, Breakefield XO. Visualization and tracking of tumour extracellular vesicle delivery and RNA translation using multiplexed reporters. Nat Commun (2015) 6:7029. doi:10.1038/ncomms8029

38. Ridder K, Keller S, Dams M, Rupp A-K, Schlaudraff J, Del Turco D, Starmann J, Macas J, Karpova D, Devraj $K$, et al. Extracellular Vesicle-Mediated Transfer of Genetic Information between the Hematopoietic System and the Brain in Response to Inflammation. PLoS Biol (2014) 12:e1001874. doi:10.1371/journal. pbio.1001874

39. Reinisch KM, Wolin SL. Emerging themes in non-coding RNA quality control. Curr Opin Struct Biol (2007) 17:209-214. doi:10.1016/j.sbi.2007.03.012

40. Wolin SL, Belair C, Boccitto M, Chen X, Sim S, Taylor DW, Wang H-W. Non-coding Y RNAs as tethers and gates: Insights from bacteria. RNA Biol (2013) 10:1602-8. doi:10.4161/rna.26166

41. Hall AE, Turnbull C, Dalmay T. Y RNAs: Recent developments. Biomol Concepts (2013) 4:103-110. doi:10.1515/bmc-2012-0050

42. Köhn M, Pazaitis N, Hüttelmaier S. Why YRNAs? About Versatile RNAs and Their Functions. Biomolecules (2013) 3:143-56. doi:10.3390/biom3010143

43. Kowalski MP, Krude T. Functional roles of non-coding Y RNAs. Int J Biochem Cell Biol (2015) 66:20-29. doi:10.1016/j.biocel.2015.07.003

44. Sim S, Wolin SL. Bacterial Y RNAs: Gates, Tethers, and tRNA Mimics. Microbio/ Spectr (2018) 6:1-14. doi:10.1128/microbiolspec.RWR-0023-2018

45. Hendrick JP, Wolin SL, Rinke J, Lerner MR, Steitz JA. Ro small cytoplasmic ribonucleoproteins are a subclass of La ribonucleoproteins: further characterization of the Ro and La small ribonucleoproteins from uninfected mammalian cells. Mol Cell Biol (1981) 1:1138-1149. doi:10.1128/MCB.1.12.1138. Updated

46. von Mühlen CA, Tan EM. Autoantibodies in the diagnosis of systemic rheumatic diseases. Semin Arthritis Rheum (1995) 24:323-358. doi:10.1016/S0049-0172(95)80004-2

47. Chen X, Wolin SL. The Ro 60 kDa autoantigen: Insights into cellular function and role in autoimmunity. J Mol Med (2004) 82:232-239. doi:10.1007/s00109-004-0529-0

48. Pruijn GJM, Wingens PAETM, Peters SLM, Thijssen JPH, Van Venrooij WJ. Ro RNP associated Y RNAs are highly conserved among mammals. Biochim Biophys Acta - Gene Struct Expr (1993) 1216:395-401. doi:10.1016/0167-4781(93)90006-Y

49. Mosig A, Guofeng M, Stadler BMR, Stadler PF. Evolution of the vertebrate Y RNA cluster. Theory Biosci (2007) 126:9-14. doi:10.1007/s12064-007-0003-y

50. van Horn DJ, Eisenberg D, O'Brien CA, Wolin SL. Caenorhabditis elegans embryos contain only one major species of Ro RNP. RNA (1995) 1:293-303.

51. Boria I, Gruber AR, Tanzer A, Bernhart SH, Lorenz R, Mueller MM, Hofacker IL, Stadler PF. Nematode sbRNAs: Homologs of vertebrate Y RNAs. J Mol Evol (2010) 70:346-358. doi:10.1007/s00239-010-9332-4

52. Perreault J, Noël JF, Brière F, Cousineau B, Lucier JF, Perreault JP, Boire G. Retropseudogenes derived from the human Ro/SS-A autoantigen-associated hY RNAs. Nucleic Acids Res (2005) 33:2032-2041. doi:10.1093/nar/gki504

53. van Gelder CWG, Thijssen JPHM, Klaassen ECJ, Sturchler C, Krol A, van Venrooij WJ, Pruijn GJM. Common structural features of the Ro RNP associated hY1 and hY5 RNAs. Nucleic Acids Res (1994)

22:2498-2506. doi:10.1093/nar/22.13.2498 
54. Wolin SL, Steitz JA. Genes for two small cytoplasmic Ro RNAs are adjacent and appear to be singlecopy in the human genome. Cell (1983) 32:735-744. doi:10.1016/0092-8674(83)90059-4

55. Simons FHM, Rutjes SA, Van Venrooij WJ, Pruijn GJM. The interactions with Ro60 and La differentially affect nuclear export of hY1 RNA. RNA (1996) 2:264-273.

56. Wolin SL, Cedervall T. The La Protein. Annu Rev Biochem (2002) 71:375-403. doi:10.1146/annurev.biochem. Copyright

57. Rutjes SA, Lund E, van der Heijden A, Grimm C, van Venrooij WJ, Pruijn GJ. Identification of a novel cis-acting RNA element involved in nuclear export of hY RNAs. RNA (2001) 7:741-752. doi:10.1017/ S1355838201002503

58. Sim S, Yao J, Weinberg DE, Niessen S, Yates JR, Wolin SL. The zipcode-binding protein ZBP1 influences the subcellular location of the Ro 60-kDa autoantigen and the noncoding Y3 RNA. RNA (2012) 18:100-10. doi:10.1261/rna.029207.111

59. Chen X, Smith JD, Shi H, Yang DD, Flavell RA, Wolin SL. The Ro Autoantigen Binds Misfolded U2 Small Nuclear RNAs and Assists Mammalian Cell Survival after UV Irradiation. Curr Biol (2003) 13:2206-2211. doi:10.1016/j.cub.2003.11.028

60. Fouraux MA, Bouvet P, Verkaart S, Van Venrooij WJ, Pruijn GJM. Nucleolin associates with a subset of the human Ro ribonucleoprotein complexes. J Mol Biol (2002) 320:475-488. doi:10.1016/500222836(02)00518-1

61. Fabini G, Raijmakers R, Hayer S, Fouraux MA, Pruijn GJM, Steiner G. The Heterogeneous Nuclear Ribonucleoproteins I and K Interact with a Subset of the Ro Ribonucleoprotein-associated Y RNAs in Vitro and in Vivo. J Bio/ Chem (2001) 276:20711-20718. doi:10.1074/jbc.M101360200

62. Köhn M, Lederer M, Wächter K, Hüttelmaier S. Near-infrared (NIR) dye-labeled RNAs identify binding of ZBP1 to the noncoding Y3-RNA. RNA (2010) 16:1420-8. doi:10.1261/rna.2152710

63. Sim S, Weinberg DE, Fuchs G, Choi K, Chung J, Wolin SL. The Subcellular Distribution of an RNA Quality Control Protein, the Ro Autoantigen, Is Regulated by Noncoding Y RNA Binding. Mol Biol Cell (2009) 20:1555-1564. doi:10.1091/mbc.E08

64. Stein AJ, Fuchs G, Fu C, Wolin SL, Reinisch KM. Structural insights into RNA quality control: The Ro autoantigen binds misfolded RNAs via its central cavity. Cell (2005) 121:529-539. doi:10.1016/j. cell.2005.03.009

65. Chen X, Sim S, Wurtmann EJ, Feke A, Wolin SL. Bacterial noncoding Y RNAs are widespread and mimic tRNAs. RNA (2014) 20:1715-24. doi:10.1261/rna.047241.114

66. Christov CP, Gardiner TJ, Szüts D, Krude T. Functional requirement of noncoding Y RNAs for human chromosomal DNA replication. Mol Cell Biol (2006) 26:6993-7004. doi:10.1128/MCB.01060-06

67. Kheir EGA, Krude T. Non-coding Y RNAs associate with early replicating euchromatin concordantly with the origin recognition complex (ORC). J Cell Sci (2017)jcs.197566. doi:10.1242/jcs. 197566

68. Gardiner TJ, Christov CP, Langley AR, Krude T. A conserved motif of vertebrate Y RNAs essential for chromosomal DNA replication. RNA (2009) 15:1375-1385. doi:10.1261/rna.1472009

69. Hogg JR, Collins K. Human Y5 RNA specializes a Ro ribonucleoprotein for 5 S ribosomal RNA quality control service Human Y5 RNA specializes a Ro ribonucleoprotein for $5 S$ ribosomal RNA quality control. Genes Dev (2007)3067-3072. doi:10.1101/gad.1603907

70. Fuchs G, Stein AJ, Fu C, Reinisch KM, Wolin SL. Structural and biochemical basis for misfolded RNA recognition by the Ro autoantigen. Nat Struct Mol Biol (2006) 13:1002-1009. doi:10.1038/nsmb1156

71. Chen X, Taylor DW, Fowler CC, Galan JE, Wang HW, Wolin SL. An RNA degradation machine sculpted by Ro autoantigen and noncoding RNA. Cell (2013) 153:166-177. doi:10.1016/j.cell.2013.02.037

72. Shi H, O'Brien CA, van Horn DJ, Wolin SL. A misfolded form of 5 S rRNA is complexed with the Ro and La autoantigens. RNA (1996) 2:769-784.

73. Tebaldi T, Zuccotti P, Peroni D, Köhn M, Gasperini L, Potrich V, Bonazza V, Dudnakova T, Rossi A, Sanguinetti G, et al. HuD Is a Neural Translation Enhancer Acting on mTORC1-Responsive Genes and Counteracted by the Y3 Small Non-coding RNA. Mol Cell (2018) 71:256-270. doi:10.1016/j.molcel.2018.06.032 
74. Scheckel C, Drapeau E, Frias MA, Park CY, Fak J, Zucker-Scharff I, Kou Y, Haroutunian V, Ma'ayan A, Buxbaum $J \mathrm{D}$, et al. Regulatory consequences of neuronal ELAV-like protein binding to coding and non-coding RNAs in human brain. Elife (2016) 5:1-35. doi:10.7554/eLife.10421

75. Donovan J, Rath S, Kolet-Mandrikov D, Korennykh A. Rapid RNase L-driven arrest of protein synthesis in the dsRNA response without degradation of translation machinery. RNA (2017) 23:1660-1671.

76. Rutjes SA, van der Heijden A, Utz PJ, van Venrooij WJ, Pruijn GJ. Rapid nucleolytic degradation of the small cytoplasmic Y RNAs during apoptosis. J Biol Chem (1999) 274:24799-807. doi:10.1074/ jbc.274.35.24799

77. Nicolas FE, Hall AE, Csorba T, Turnbull C, Dalmay T. Biogenesis of Y RNA-derived small RNAs is independent of the microRNA pathway. FEBS Lett (2012) 586:1226-30. doi:10.1016/j.febslet.2012.03.026

78. Verhagen APM, Pruijn GJM. Are the Ro RNP-associated Y RNAs concealing microRNAs? Y RNA-derived miRNAs may be involved in autoimmunity. Bioessays (2011) 33:674-82. doi:10.1002/bies.201100048

79. Thomson DW, Pillman KA, Anderson ML, Lawrence DM, Toubia J, Goodall GJ, Bracken CP. Assessing the gene regulatory properties of Argonaute-bound small RNAs of diverse genomic origin. Nucleic Acids Res (2015) 43:470-481. doi:10.1093/nar/gku1242

80. Chakrabortty SK, Prakash A, Nechooshtan GAL, Hearn S, Gingeras TR. Extracellular vesicle-mediated transfer of processed and functional RNY5 RNA. RNA (2015) 21:1-14. doi:10.1261/rna.053629.115.

81. Kaudewitz D, Skroblin P, Bender LH, Barwari T, Willeit P, Pechlaner R, Sunderland NP, Willeit K, Morton A, Armstrong PC, et al. Association of MicroRNAs and YRNAs with Platelet Function. Circ Res (2016) 118:420-432. doi:10.1161/CIRCRESAHA.114.305663

82. Dhahbi JM, Spindler SR, Atamna H, Boffelli D, Mote P, Martin DIK. 5'-YRNA fragments derived by processing of transcripts from specific YRNA genes and pseudogenes are abundant in human serum and plasma. Physio/ Genomics (2013) 45:990-998. doi:10.1152/physiolgenomics.00129.2013

83. Motorin Y, Muller S, Behn-Ansmant I, Branlant C. "Identification of Modified Residues in RNAs by Reverse Transcription-Based Methods," in Methods in Enzymology, 21-53. doi:10.1016/S0076-6879(07)25002-5

84. Raabe CA, Tang T-H, Brosius J, Rozhdestvensky TS. Biases in small RNA deep sequencing data. Nucleic Acids Res (2014) 42:1414-1426. doi:10.1093/nar/gkt1021

85. Qin Y, Yao J, Wu DC, Nottingham RM, Mohr S, Hunicke-Smith S, Lambowitz AM. High-throughput sequencing of human plasma RNA by using thermostable group II intron reverse transcriptases. RNA (2015) 22:1-18. doi:10.1261/rna.054809.115.3

86. Hornung V, Ellegast J, Kim S, Brzózka K, Jung A, Kato H, Poeck H, Akira S, Conzelmann K-K, Schlee M, et al. 5'-Triphosphate RNA is the Ligand for RIG-I. Science (80-) (2006) 314:994-997. doi:10.1126/ science. 1132505

87. Pruijn GJM, Slobbe RL, van Venrooij WJ. Analysis of Protein - Rna Interactions Within Ro Ribonucleoprotein Complexes. Nucleic Acids Res (1991) 19:5173-5180.

88. Kalra H, Simpson RJ, Ji H, Aikawa E, Altevogt P, Askenase P, Bond VC, Borràs FE, Breakefield X, Budnik V, et al. Vesiclepedia: A Compendium for Extracellular Vesicles with Continuous Community Annotation. PLoS Biol (2012) 10:8-12. doi:10.1371/journal.pbio.1001450

89. Köhn M, Ihling C, Sinz A, Krohn K, Hüttelmaier S. The Y3** ncRNA promotes the $3^{\prime}$ end processing of histone mRNAs. Genes Dev (2015) 29:1998-2003. doi:10.1101/gad.266486.115

90. Katsanou V, Papadaki O, Milatos S, Blackshear PJ, Anderson P, Kollias G, Kontoyiannis DL. HuR as a negative posttranscriptional modulator in inflammation. Mol Cell (2005) 19:777-789. doi:10.1016/j. molcel.2005.08.007

91. Herdy B, Karonitsch T, Vladimer Gl, Tan CSH, Stukalov A, Trefzer C, Bigenzahn JW, Theil T, Holinka J, Kiener HP, et al. The RNA-binding protein HUR/ELAVL1 regulates IFN- $\beta$ mRNA abundance and the type I IFN response. Eur J Immunol (2015) 45:1500-1511. doi:10.1002/eji.201444979

92. Welton JL, Khanna S, Giles PJ, Brennan P, Brewis IA, Staffurth J, Mason MD, Clayton A. Proteomics Analysis of Bladder Cancer Exosomes. Mol Cell Proteomics (2010) 9:1324-1338. doi:10.1074/mcp. M000063-MCP201 
93. Frye BC, Halfter S, Djudjaj S, Muehlenberg P, Weber S, Raffetseder U, En-Nia A, Knott H, Baron JM, Dooley $\mathrm{S}$, et al. Y-box protein-1 is actively secreted through a non-classical pathway and acts as an extracellular mitogen. EMBO Rep (2009) 10:783-789. doi:10.1038/embor.2009.81

94. Khatua AK, Taylor HE, Hildreth JEK, Popik W. Exosomes Packaging APOBEC3G Confer Human Immunodeficiency Virus Resistance to Recipient Cells. J Virol (2009) 83:512-521. doi:10.1128/ JVI.01658-08

95. Collino F, Deregibus MC, Bruno S, Sterpone L, Aghemo G, Viltono L, Tetta C, Camussi G. Microvesicles derived from adult human bone marrow and tissue specific mesenchymal stem cells shuttle selected pattern of miRNAs. PLoS One (2010) 5: doi:10.1371/journal.pone.0011803

96. Mukherjee K, Ghoshal B, Ghosh S, Chakrabarty Y, Shwetha S, Das S, Bhattacharyya SN. Reversible HuRmicroRNA binding controls extracellular export of miR122 and augments stress response. EMBO Rep (2016) 17:11841203. doi:10.15252/embr

97. Shurtleff MJ, Temoche-Diaz MM, Karfilis KV., Ri S, Schekman R. Y-box protein 1 is required to sort microRNAs into exosomes in cells and in a cell-free reaction. Elife (2016) 5:1-23. doi:10.7554/ eLife.19276

98. Shah R, Yeri AS, Das A, Courtright-Lim A, Ziegler O, Gervino E, Ocel J, Quintero Pinzon P, Wooster L, Shields Bailey C, et al. Small RNA-seq during acute maximal exercise reveal RNAs involved in vascular inflammation and cardiometabolic health. Am J Physiol - Hear Circ Physiol (2017) 313:ajpheart.00500.2017. doi:10.1152/ajpheart.00500.2017

99. Cheng ST, Nguyen TQ, Yang YS, Capra JD, Sontheimer RD. Calreticulin binds hYRNA and the 52-kDa polypeptide component of the Ro/SS-A ribonucleoprotein autoantigen. J Immunol (1996) 156:448491. Available at: http://www.ncbi.nlm.nih.gov/pubmed/8666824

100. Bouffard P, Barbar E, Brière F, Boire G. Interaction cloning and characterization of RoBPI, a novel protein binding to human Ro ribonucleoproteins. RNA (2000) 6:66-78. doi:10.1017/S1355838200990277

101. Yamazaki F, Kim HH, Lau P, Hwang CK, luvone PM, Klein D, Clokie SJH. pY RNA1-s2: a highly retinaenriched small RNA that selectively binds to Matrin 3 (Matr3). PLoS One (2014) 9:e88217. doi:10.1371/ journal.pone.0088217

102. Bogerd HP, Cullen BR. Single-stranded RNA facilitates nucleocapsid: APOBEC3G complex formation. RNA (2008) 14:1228-36. doi:10.1261/rna.964708

103. Apolonia L, Schulz R, Curk T, Rocha P, Swanson CM, Schaller T, Ule J, Malim MH. Promiscuous RNA binding ensures effective encapsidation of APOBEC3 proteins by HIV-1. PLoS Pathog (2015) 11:e1004609. doi:10.1371/journal.ppat.1004609

104. Onafuwa-Nuga AA, King SR, Telesnitsky A. Nonrandom packaging of host RNAs in moloney murine leukemia virus. J Virol (2005) 79:13528-37. doi:10.1128/JVI.79.21.13528-13537.2005

105. Garcia EL, Onafuwa-Nuga A, Sim S, King SR, Wolin SL, Telesnitsky A. Packaging of host mY RNAs by murine leukemia virus may occur early in Y RNA biogenesis. J Virol (2009) 83:12526-34. doi:10.1128/ JVI.01219-09

106. Eckwahl MJ, Sim S, Smith D, Telesnitsky A, Wolin SL. A retrovirus packages nascent host noncoding RNAs from a novel surveillance pathway. Genes Dev (2015) 29:646-657. doi:10.1101/gad.258731.115

107. Telesnitsky A, Wolin SL. The host RNAs in retroviral particles. Viruses (2016) 8:1-15. doi:10.3390/ v8080235

108. Eckwahl MJ, Arnion H, Kharytonchyk S, Zang T, Bieniasz PD, Telesnitsky A, Wolin SL. Analysis of the human immunodeficiency virus-1 RNA packageome. RNA (2016)1-11. doi:10.1261/rna.057299.116

109. Booth AM, Fang Y, Fallon JK, Yang J-M, Hildreth JEK, Gould SJ. Exosomes and HIV Gag bud from endosome-like domains of the T cell plasma membrane. J Cell Bio/ (2006) 172:923-35. doi:10.1083/ jcb.200508014

110. Nolte-'t Hoen E, Cremer T, Gallo RC, Margolis LB. Extracellular vesicles and viruses: Are they close relatives? Proc Natl Acad Sci (2016)201605146. doi:10.1073/pnas.1605146113 
111. Stake M, Singh D, Singh G, Marcela Hernandez J, Kaddis Maldonado R, Parent LJ, Boris-Lawrie K. HIV-1 and two avian retroviral 5' untranslated regions bind orthologous human and chicken RNA binding proteins. Virology (2015) 486:307-320. doi:10.1016/j.virol.2015.06.001

112. Freed EO. HIV-1 replication. Somat Cell Mol Genet (2001) 26:13-33. Available at: http://www.ncbi.nIm. nih.gov/pubmed/12465460

113. Wang T, Tian C, Zhang W, Luo K, Sarkis PTN, Yu L, Liu B, Yu Y, Yu X-F. 7SL RNA mediates virion packaging of the antiviral cytidine deaminase APOBEC3G. J Virol (2007) 81:13112-24. doi:10.1128/JVI.00892-07

114. Eckwahl MJ, Telesnitsky A, Wolin SL. Host RNA packaging by retroviruses: A newly synthesized story. MBio (2016) 7:1-8. doi:10.1128/mBio.02025-15

115. Clancy RM, Alvarez D, Komissarova E, Barrat FJ, Swartz J, Buyon JP. Ro60-Associated SingleStranded RNA Links Inflammation with Fetal Cardiac Fibrosis via Ligation of TLRs: A Novel Pathway to Autoimmune-Associated Heart Block. J Immunol (2010) 184:2148-2155. doi:10.4049/ jimmunol.0902248

116. Greidinger EL, Zang Y, Martinez L, Jaimes K, Nassiri M, Bejarano P, Barber GN, Hoffman RW. Differential tissue targeting of autoimmunity manifestations by autoantigen-associated $Y$ RNAs. Arthritis Rheum (2007) 56:1589-1597. doi:10.1002/art.22601

117. Riley JL. PD-1 signaling in primary T cells. Immunol Rev (2009) 229:114-125. doi:10.1111/j.1600065X.2009.00767.X

118. Skibinski G, Kelly R, Harkiss D, James K. Immunosuppression by human seminal plasma - extracellular organelles (prostasomes) modulate activity of phagocytic cells. Am J Reprod Immunol (1992) 28:97103.

119. Buck AH, Coakley G, Simbari F, McSorley HJ, Quintana JF, Le Bihan T, Kumar S, Abreu-Goodger C, Lear $M$, Harcus $Y$, et al. Exosomes secreted by nematode parasites transfer small RNAs to mammalian cells and modulate innate immunity. Nat Commun (2014) 5:5488. doi:10.1038/ncomms6488

120. Reed JH, Sim S, Wolin SL, Clancy RM, Buyon JP. Ro60 requires Y3 RNA for cell surface exposure and inflammation associated with cardiac manifestations of neonatal lupus. J Immunol (2013) 191:110116. doi:10.4049/jimmunol.1202849

121. Caruso S, Poon IKH. Apoptotic cell-derived extracellular vesicles: More than just debris. Front Immunol (2018) 9: doi:10.3389/fimmu.2018.01486

122. Buzás El, Tóth EÁ, Sódar BW, Szabó-Taylor KÉ. Molecular interactions at the surface of extracellular vesicles. Semin Immunopathol (2018) 40:453-464. doi:10.1007/s00281-018-0682-0

123. Van Deun J, Mestdagh P, Agostinis P, Akay Ö, Anand S, Anckaert J, Martinez ZA, Baetens T, Beghein E, Bertier $L$, et al. EV-TRACK: transparent reporting and centralizing knowledge in extracellular vesicle research. Nat Methods (2017) 14:228-232. doi:10.1038/nmeth.4185

124. Mitchell AJ, Gray WD, Hayek SS, Ko YA, Thomas S, Rooney K, Awad M, Roback JD, Quyyumi A, Searles CD. Platelets confound the measurement of extracellular miRNA in archived plasma. Sci Rep (2016) 6:1-11. doi:10.1038/srep32651 


\section{Chapter 6}

\section{Y-RNA subtype ratios in plasma extracellular vesicles are cell type-specific and are candidate biomarkers for inflammatory diseases}

T.A.P. Driedonks ${ }^{1}$, S. Mol ${ }^{1,2}$, S. de Bruin ${ }^{3}$, A.L. Peters ${ }^{4}$, X.G. Zhang ${ }^{1}$, M.F.S. Lindenbergh ${ }^{1}$,

B.M. Beuger ${ }^{5}$, A.D. van Stalborch ${ }^{6}$, T. Spaan ${ }^{7}$, E.C. de Jong ${ }^{2}$, E. van der Vries ${ }^{7}$, C. Margadant ${ }^{6}$, R. van Bruggen ${ }^{5}$, A.P.J. Vlaar ${ }^{3}$, T. Groot Kormelink², E.N.M. Nolte-'t Hoen ${ }^{1 *}$

1 Dept. Biochemistry \& Cell Biology, Fac. Veterinary Medicine, Utrecht University, The Netherlands

2 Dept. Experimental Immunology, Amsterdam University Medical Centers, Location AMC, University of Amsterdam, Amsterdam, The Netherlands

3 Dept. Intensive Care, Amsterdam University Medical Centers, location AMC , Amsterdam, The Netherlands

4 Dept. Anesthesiology, University Medical Center Utrecht, Utrecht, The Netherlands

5 Dept. of Blood Cell Research, Sanquin Research, and Landsteiner Laboratory, Amsterdam University Medical Centers, Location AMC, Amsterdam, The Netherlands
6 Molecular Cell Biology lab, Dept. of Molecular and Cellular Hemostasis, Sanquin Research, and Landsteiner Laboratory, Amsterdam University Medical Centers, Location AMC, Amsterdam, The Netherlands

7 Dept. of Infectious Diseases \& Immunity, Division of Virology, Faculty of Veterinary Medicine, Utrecht University, Utrecht, The Netherlands

Submitted 


\section{Abstract}

Major efforts are made to characterize the presence of microRNA (miRNA) and messenger RNA in blood plasma to discover novel disease-associated biomarkers. MiRNAs in plasma are associated to several types of macromolecular structures, including extracellular vesicles (EV), lipoprotein particles (LPP) and ribonucleoprotein particles (RNP). RNAs in these complexes are recovered at variable efficiency by commonly used EV- and RNA isolation methods, which causes biases and inconsistencies in miRNA quantitation. Besides miRNAs, various other non-coding RNA species are contained in EV and present within the pool of plasma extracellular RNA. Members of the Y-RNA family have been detected in EV from various cell types and are among the most abundant non-coding RNA types in plasma. We previously showed that shuttling of full-length Y-RNA into EV released by immune cells is modulated by microbial stimulation. This indicated that Y-RNAs could contribute to the functional properties of EV in immune cell communication and that EV-associated Y-RNAs could have biomarker potential in immune-related diseases. Here, we investigated which macromolecular structures in plasma contain full length Y-RNA and whether the levels of three Y-RNA subtypes in plasma (Y1, Y3 and $Y 4$ ) change during systemic inflammation. Our data indicate that the majority of full length Y-RNA in plasma is stably associated to EV. Moreover, we discovered that EV from different blood-related cell types contain cell type specific Y-RNA subtype ratios. Using a human model for systemic inflammation, we show that the neutrophil-specific $Y 4 / Y 3$ ratios and $P B M C$-specific $Y 3 / Y 1$ ratios were significantly altered after induction of inflammation. The plasma Y-RNA ratios strongly correlated with the number and type of immune cells during systemic inflammation. Cell type specific 'Y-RNA signatures' in plasma EV can be determined without prior enrichment for $\mathrm{EV}$, and may be further explored as simple and fast test for diagnosis of inflammatory responses or other immune-related diseases. 


\section{Introduction}

Body fluids contain a plethora of RNA molecules that have been released by cells. Macromolecular structures in plasma that contain extracellular RNAs (exRNAs) include extracellular vesicles (EV), lipoprotein particles (LPP) and ribonucleoprotein complexes (RNPs) (1-3). In recent years, the major focus has been on characterizing the RNA content of EV. EV are nano-sized membrane vesicles that are involved in transferring transmembrane and cytosolic proteins, lipids, and RNA between different cells $(4,5)$. The release and molecular composition of EV change depending on the activation or differentiation status of the EV-producing cell. EV have therefore not only been implicated as important mediators of intercellular communication, but also as biomarkers for disease (6-12). Numerous studies addressed the cell type-specific profiles of EV-associated RNA and how such profiles change due to cell signaling, oncogenic transformation, or other disease-related processes (13-19). Most of this research has focused on the microRNA (miRNA) and messenger RNA (mRNA) content of EV because of their known functions in gene expression. Changes in EV-associated levels of these RNA types in plasma or serum have been demonstrated for diseases such as cancer, rheumatoid arthritis and neurodegenerative diseases $(10,20,21)$. Since recent years, there has been growing awareness that separation of EV, LPP, and RNP is technically challenging due to their overlap in size and buoyant density. The levels of contamination with LPP and RNP in EV preparations obtained with the commonly used EV-isolation methods are variable. This introduces biases in EV-miRNA biomarker studies and reduces inter-study reproducibility of data $(22,23)$.

Our previous work indicated that EV also contain less well-known types of noncoding RNA $(19,24)$. One of these RNA types, Y-RNA, has been abundantly detected in EV from several different cell types (reviewed in (25)). Y-RNAs are highly conserved non-coding RNAs with sizes of $\sim 100 \mathrm{nt}$. Inside cells, these RNAs are involved in various basic cellular processes such as DNA replication $(26,27)$ and RNA quality control $(28,29)$. The human genome encodes four Y-RNA subtypes $(30)$, which are hairpin-like structures with homologous stem regions and subtype-specific loop sequences (31). Although several research groups proposed that EV mainly contain specific fragments of Y-RNA, our recent data demonstrate that this is a common artefact in small RNA sequencing procedures (19). Difficulties to ligate the adapters to the $5^{\prime}$-triphosphate group of full length Y-RNAs and the strict size selection applied during small RNA library preparation strongly bias towards detection of fragmented Y-RNAs (32). Using Northern blotting, we provided evidence that most of the Y-RNA in EV from cultured primary dendritic cells was in the full length form (19). Moreover, we showed that the dendritic cells could regulate the amount of full length Y-RNA sorted into EV in response to immune-activating or immune-suppressing stimuli (19), similar to the 
regulated incorporation of specific miRNAs $(13,33,34)$. This suggests that Y-RNAs can contribute to the functional properties of EV in immune cell communication and that EV-associated Y-RNAs could have biomarker potential in immune-related diseases.

Y-RNA is not only present at high levels in EV from several cell lines, but is also among the most abundant RNA types in plasma of healthy individuals $(35,36)$. The levels of circulating Y-RNA have been proposed to correlate with cancer and coronary artery disease $(37,38)$, but these studies were biased towards detection of Y-RNA fragments. It is currently unknown whether full length Y-RNAs in plasma are associated to both EV, LPP, and RNP, similar to what has been shown for miRNAs (1-3). It is also unknown which blood-related cell types can release Y-RNA containing EV into the circulation and whether the levels of full length Y-RNA in plasma are regulated by immune-related stimuli.

We here show that the majority of full length Y-RNA in plasma is stably associated to EV. Interestingly, we found that EV released by different blood-related cell types contain unique quantitative ratios of different Y-RNA subtypes. In response to TLR-mediated stimulation, several of these cell types showed increased release of Y-RNA containing EV, but the cell type-specific Y-RNA subtype ratios remained stable under these conditions. In a human endotoxemia model for systemic inflammation, we observed significant alterations in the Y-RNA content within the total pool of plasma EV. A substantial increase was observed in the neutrophil-specific Y-RNA subtype ratio and this correlated tightly with the increase in number of neutrophils that occurred during inflammation. Detection of Y-RNA subtype ratios in plasma EV can be determined without prior enrichment for $\mathrm{EV}$ and may be further explored as simple and fast test for diagnosis of inflammatory responses or other immune-related diseases.

\section{Materials and methods}

\section{Clinical samples}

Plasma samples of the human endotoxemia study were obtained under approval of the Academic Medical Center Medical Ethical Committee and are according to the Declaration of Helsinki, including Good Clinical Practice $(39,40)$. This study has been registered at the Dutch Trial Register (NTR4455). All enrolled volunteers provided written informed consent before enrolment. Two volunteers displayed aberrant cytokine levels at either $\mathrm{t}=0$ or $\mathrm{t}=2$ and were excluded from all further analyses.

Blood and plasma from healthy volunteers was obtained following approval of the Medical Ethical Committees of Utrecht Medical Centre, Amsterdam Medical Centre and Sanquin Research. All volunteers provided written informed consent, the experiments abide by the Declaration of Helsinki principles for human research ethics. 


\section{Plasma collection and fractionation}

During the human endotoxemia study, plasma samples were collected as described previously $(39,40)$. In brief, arterial blood samples were collected in two tubes with $0.11 \mathrm{M}$ sodium citrate (Vacutainer, Becton Dickinson). Samples were collected directly before infusion of LPS and before infusion of the transfusion product and every 2 hours thereafter until 6 hours after transfusion. Tubes were centrifuged at 1,500 $\mathrm{g}$ for $10 \mathrm{~min}$ at $20^{\circ} \mathrm{C}$, the supernatant was centrifuged again at $1,550 \mathrm{~g}$ for $20 \mathrm{~min}$, plasma was frozen at $-80^{\circ} \mathrm{C}$ until analysis.

For preparation of all other plasma samples from healthy donors, blood was collected in the morning by venipuncture with a $21 \mathrm{G}$ needle into a citrate tube (Greiner Vacuette $9 \mathrm{NC} \mathrm{NaC} \mathrm{3,2 \% ),} \mathrm{and} \mathrm{was} \mathrm{processed} \mathrm{within} 30$ minutes after collection. Tubes were centrifuged at 2,500 g for $15 \mathrm{~min}$ at RT, supernatant was pipetted off using a plastic Pasteur pipette. Supernatant was centrifuged again 3,000 g for $15 \mathrm{~min}$, supernatant was collected and frozen directly at $-80^{\circ} \mathrm{C}$ in $0.5 \mathrm{~mL}$ aliquots in Eppendorf LoBind Tubes.

For fractionation of plasma (Figure 1B), $0.5 \mathrm{~mL}$ plasma was thawn at RT and fractionated on a qEV Classic size exclusion column (Izon Science, Christchurch, New Zealand) eluted with 1x PBS (Gibco, Paisley, UK). $0.5 \mathrm{~mL}$ fractions were collected manually. Fractions $7-12$ ('early') and fractions $17-24$ ('late') were pooled into two SW40 tubes and were centrifuged for $65 \mathrm{~min}$ at 100,000 $\mathrm{g}$ (k-factor: 381.5 ). $90 \%$ of the supernatant ('sup') was removed by pipetting and stored at $4{ }^{\circ} \mathrm{C}$, and the last $10 \%$ was decanted, after which the pellets were resuspended in $50 \mu \mathrm{l} \mathrm{PBS}+0.2 \%$ EV-depleted BSA (which was cleared of aggregates by overnight ultracentrifugation at 100,000 g). Resuspended pellets were overlaid with sucrose density gradients $(2.5 \mathrm{M}-0.4 \mathrm{M})$ and centrifuged for $15-18 \mathrm{~h}$ at $192,000 \mathrm{~g}$ in a SW40 rotor (k-factor 144.5). High-density $(1.25 \mathrm{~g} / \mathrm{mL}$, 'hi dens') and intermediate density $(1.11-1.18 \mathrm{~g} / \mathrm{mL}$, 'int dens') fractions were diluted four times in PBS $+0.2 \%$ EV-depleted BSA and ultracentrifuged for $65 \mathrm{~min}$ at $192,000 \mathrm{~g}$ in a SW40 rotor (k-factor 144.5). Pellets were resuspended in $60 \mu \mathrm{l}$ PBS, divided into three aliquots which were subjected to different enzymatic treatments. Stored 100,000 g supernatants were concentrated on PBS-washed Amicon Ultra

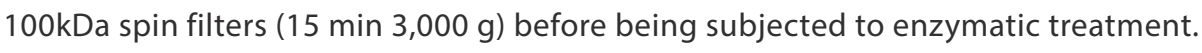




\section{Protease, RNase and detergent treatments}

Each of the plasma fractions was subjected to treatment with combinations of detergent, protease and RNase according to the scheme below.

Table 1. Overview of enzymatic treatments on plasma fractions

\begin{tabular}{l|lll}
\hline Treatment & Untreated & Protease + RNase & Protease + RNase + detergent \\
\hline NP-40 & - & - & + \\
Proteinase K & - & + & + \\
RNase A & - & + & + \\
\hline
\end{tabular}

First, 1\% NP-40 detergent or an equal volume of PBS was added and samples were incubated $15 \mathrm{~min}$ on ice. Next, $45 \mathrm{ng} / \mathrm{mL}$ Proteinase K (Roche, Basel, Switzerland) or an equivalent volume of PBS was added and incubated $30 \mathrm{~min}$ at $37^{\circ} \mathrm{C}$, after which the reaction was stopped by adding $5 \mathrm{mM}$ PMSF (Roche, Basel, Switzerland) an incubation on ice for $10 \mathrm{~min}$. Finally, $8 \mathrm{U} / \mathrm{mL}$ RNase A (Roche, Basel, Switzerland) or an equivalent volume of PBS was added, incubated at $37^{\circ} \mathrm{C}$ for $30 \mathrm{~min}$. Immediately hereafter, the RNA isolation procedure was started by adding $700 \mu$ l Qiazol (Qiagen, Hilden, Germany).

\section{Blood cell culture and medium preparation}

\section{Preparation of EV-depleted media}

EV were depleted from fetal calf serum (FCS) for preparation of EV-depleted culture medium (41). In brief, FCS was diluted to $30 \%$ and centrifuged overnight at 100,000 $\mathrm{g}$ in a SW28 rotor (k-factor 334.2). Supernatants were recovered by pipetting, filtered through a $0.45 \mu \mathrm{m}$ bottle top filter (Millipore, Leiden, NL). EGM-2 medium contained only $2 \%$ of serum and was thus ultracentrifuged as complete medium overnight at $100,000 \mathrm{~g}$ in a SW28 rotor (k-factor 334.2). Supernatants were recovered by pipetting, filtered through a $0.45 \mu \mathrm{m}$ bottle top filter (Millipore, Leiden, NL) before use.

\section{PBMC}

Blood was drawn with a $21 \mathrm{G}$ needle into a $10 \mathrm{ml}$ Lithium Hep Green Vacutainer (Beckton Dickinson, Franklin Lakes, NJ), layered onto a $15 \mathrm{~mL}$ FicollPaque (GE, Piscataway, NJ) cushion and centrifuged for 20 minutes at $560 \mathrm{~g}$. The plasma layer was discarded, after which the PBMC interphase was removed with a plastic Pasteur pipette and mixed with 20 mL RPMI-1640 (Gibco, Paisley, UK). Cells were washed 3x by centrifugation for 10 minutes at $560 \mathrm{~g}$. PBMC were seeded in RPMI-1640 with 10\% EV-depleted FCS, $100 \mathrm{IU} / \mathrm{mL}$ penicillin and $100 \mathrm{\mu g}$ streptomycin (Gibco, Paisley, UK) at $1 \mathrm{E} 6$ cells / $\mathrm{ml}$ for EV-production. PBMC were checked for neutrophil contamination by flow cytometry (see below) which was $<3 \%$ for all donors. Where indicated, cells were stimulated with 
$1 \mathrm{\mu g} / \mathrm{mL}$ LPS (O111-B4, cat L2630, Sigma-Aldrich, St. Louis, MO). EV were produced in 4h with 12 - 20x10E6 PBMC, after which medium was collected for EV isolation. Cell viability was checked by Trypan blue exclusion and was $>95 \%$ for all cultures.

\section{Neutrophils}

Blood was drawn with a Vacutainer Eclipse needle into a Vacuette sodium heparine $9 \mathrm{ml}$ tube (Greiner Bio-One, Alphen aan de Rijn, Netherlands). Blood was diluted with HBSS (Sigma Aldrich H6136) after which granulocytes and erythrocytes were separated from PBMC by density gradient centrifugation on $20 \mathrm{ml}$ Lymphoprep (STEMcell Technologies, Köln, Germany). The granulocyte/erythrocyte pellet was subsequently transferred to a new $50 \mathrm{~mL}$ tube followed by erythrocyte lysis in ice-cold erythrocyte lysis buffer $(0.155 \mathrm{M}$ $\mathrm{NH}_{4} \mathrm{Cl}$ (Sigma), $1 \mathrm{mM} \mathrm{KHCO}$ (Merck), $80 \mu \mathrm{M}$ EDTA (Merck) in Milli-Q) in 2 consecutive rounds. The final cell pellet was washed in PBS, centrifuged $8 \mathrm{~min}$ at $400 \mathrm{~g}$ and seeded in IMDM + ultraglutamine (Gibco) supplemented with 1\% EV-depleted FCS (SigmaAldrich, F7524), and $86 \mu \mathrm{g} / \mathrm{mL}$ gentamycin (Duchefa, DUC586714) at $1 \mathrm{E} 6$ cells / $\mathrm{mL}$ for

EV production. Purity of isolated cells was determined on flow cytometry (see below). Neutrophils were positive for CD15 and contained less than 3\% PBMC (determined by FCS / SSC detection). Where indicated, cells were stimulated with $100 \mathrm{ng} / \mathrm{mL}$ LPS (O111-B4, cat L2360, Sigma-Aldrich, St. Louis, MO). EV were produced in $2 \mathrm{~h}$ with 9x10E6 neutrophils, after which medium was collected for EV isolation. Cell viability after culture was checked by PI or DAPI staining, which was $>90 \%$ for all cultures.

\section{Platelets}

Whole blood was collected in 3.2\% trisodium citrate Vacuette tubes (Greiner, Kremsmünster, Austria) and was centrifuged at $160 \mathrm{~g}$ for 15 minutes at RT without a brake. Platelet-rich plasma (PRP) was transferred into a new tube containing 1/10 volume of Acid-Citrate-Dextrose (ACD; $38 \mathrm{mM}$ citric acid, $75 \mathrm{mM}$ sodium citrate, $135 \mathrm{mM}$ dextrose). PRP was then centrifuged at $400 \mathrm{~g}$ for $15 \mathrm{~min}$ at room temperature with brake level 1. Afterwards, the supernatant was discarded and the pellet was resuspended in modified Tyrode's buffer (10 mM HEPES, $137 \mathrm{mM} \mathrm{NaCl}, 2.8 \mathrm{mM} \mathrm{KCl}, 12 \mathrm{mM} \mathrm{NaHCO}_{3}$ ) with 5.55 mM D-glucose, pH 6.5. Then, prostaglandin 12 (PGI2, Cayman Chemical Company) was prediluted into modified Tyrode's buffer ( $\mathrm{pH} 6.5)$ ) and added to a final concentration of 0.5 $\mu \mathrm{M}$, after which the previous centrifugation step was repeated immediately. Finally, the platelets were resuspended in modified Tyrode's, supplemented with $5.55 \mathrm{mM} \mathrm{D-glucose,}$ $\mathrm{pH}$ 7.4. Platelet suspensions were counted using the CELL-DYN Emerald System (Abbott, Green Oaks, IL) and adjusted to 200x10E6 platelets/mL in $1 \mathrm{~mL}$ for EV production. Where indicated, platelets were stimulated with 1 Mg/ml LPS (O111-B4, cat L2360, Sigma-Aldrich, St. Louis). After $2 \mathrm{~h}$ of culture, $1 \mathrm{ml}$ platelet suspension was collected, topped up with 10 $\mathrm{mL}$ PBS + 1\% EV-depleted BSA and subjected to EV isolation. 


\section{HUVEC}

Three pools of Human Umbilical Vein Endothelial cells (HUVEC) were obtained from Lonza (Verviers, Belgium). Cells were thawn and cultured for 5 passages before being seeded at $0.55 \mathrm{E} 6$ cells $/ \mathrm{cm}^{2}$ in EV-depleted EGM-2 complete medium with $2 \mathrm{mM}$ L-glutamine, $100 \mathrm{U} / \mathrm{ml}$ pen/strep in porcine skin-gelatin coated culture dishes for EV-production from 9x10E6 cells. Where indicated, HUVEC were stimulated with $1 \mu \mathrm{g} / \mathrm{ml}$ LPS (O55-B5, cat L2880, Sigma-Aldrich, St. Louis). After 4h, cell viability was checked by LIVE/DEAD (>95\% for all cultures) (ThermoScientific), medium was collected and subjected to EV-isolation.

\section{Red blood cells}

$9 \mathrm{~mL}$ of blood was collected into a Vacuette Natrium Heparine tube (Greiner Bio-One). Red blood cells were isolated by the following centrifugation steps (42): $15 \mathrm{~min}$ at 1000 $\mathrm{rpm}, 5 \mathrm{~min}$ at $2500 \mathrm{rpm}, 5 \mathrm{~min}$ at $2500 \mathrm{rpm}$. After isolation, red blood cells were incubated for $2 \mathrm{~h}$ in HEPES buffer (132 mM NaCl, 20 mM HEPES, $6 \mathrm{mM} \mathrm{KCl}, 1 \mathrm{mM} \mathrm{MgSO}$, $1.2 \mathrm{mM}$ K2HPO4, (Sigma-Aldrich, Spruce, USA) supplemented with $1 \mathrm{mM} \mathrm{Ca} 2+$ and $1 \mathrm{mg} / \mathrm{ml}$ glucose), EV were produced from 2x10E9 red blood cells in $1 \mathrm{~mL}$. Where indicated, RBC were stimulated with $1 \mu \mathrm{g} / \mathrm{ml}$ LPS (O111-B4, cat L2360, Sigma-Aldrich, St. Louis). After $2 \mathrm{~h}, 10 \mathrm{~mL}$ PBS $+1 \%$ EV-depleted BSA was added to the supernatants which were then subjected to EV isolation.

\section{Flow cytometry}

$5 \times 10$ E4 to $1 \times 10$ E5 cells were labeled for 30 minutes in $18 \mu \mathrm{l}$ to $100 \mu \mathrm{l}$ PBS $+1 \%$ BSA containing one or combinations of the following antibodies: anti-CD16-PECy7 (Biolegend; 1:1000; 3G8), anti-CD62L-APCCy7 (Biolegend; 1:25; Greg-56), anti-CD63-APC (Biolegend; 1:100; H5C6), anti-CD66b-PE (Biolegend; 1:100; G10F5), DAPI (Sigma-Aldrich), anti-CD3PacificBlue (Beckman Coulter, 1 : 50, UCHT-1), anti-CD14-FITC (Miltenyi, 1:100, Tük 4), anti-ICAM-1-AlexaFluor-405 (SantaCruz, 1:100, sc-107-af405), an in-house VHH against P-selectin (1 :125, B10.6, (43)), or Annexin-V (250 ug/ml, VPS diagnostics, cat. nr. A705). Surface labeling of cells was measured on a FACSCanto (BD Biosciences) or a LSR-II (BD Biosciences) flow cytometer relative to unlabeled controls.

\section{EV isolation and fluorescent labeling}

Cells were incubated in their respective media for $2-4 \mathrm{~h}$, after which EV were isolated as described before (19). Supernatants were centrifuged at $4^{\circ} \mathrm{C}$, twice for $10 \mathrm{~min}$ at $200 \mathrm{~g}$, twice for $10 \mathrm{~min}$ at $500 \mathrm{~g}$, and $30 \mathrm{~min}$ at $10,000 \mathrm{~g}$ in a SW40 rotor (k-factor 2773.9). Supernatant was carefully pipetted off and transferred to new SW40 tubes, EV were pelleted at 100,000 $\mathrm{g}$ for $65 \mathrm{~min}$ (k-factor 381.5). EV pellets were resuspended in $20 \mu \mathrm{l}$ PBS + 0.2\% EV-depleted BSA and labeled with $1.5 \mu \mathrm{l} \mathrm{PKH67} \mathrm{(Sigma-Aldrich)} \mathrm{in} 180 \mu \mathrm{l}$ Diluent $C$, the reaction was stopped by adding $100 \mu \mathrm{I}$ EV-depleted medium. Labeled 
EV were overlaid with a sucrose density gradient as described above and centrifuged for $15-18 \mathrm{~h}$ at 192,000g (k-factor 144.5). Equal volumes of non-conditioned culture medium were processed in parallel to quantify background levels of residual mediumderived RNA (41).

\section{High resolution flow cytometry}

High-resolution flow cytometric analysis of PKH67-labeled EV was performed on a BD Influx flow cytometer (BD Biosciences, San Jose, CA) with an optimized configuration for small particle analysis as previously described $(44,45)$. We applied fluorescence threshold triggering to discriminate PKH67 labeled EV from non-fluorescent noise signals. Forward scatter (FSC) was detected at a 15 - 25 degree collection angle. Fluorescent polystyrene 100 and $200 \mathrm{~nm}$ beads (FluoSpheres, Invitrogen, Carlsbad, CA) were used to calibrate the fluorescence and reduced width-FSC settings before each measurement. Sucrose gradient fractions were diluted 10 - 20 times in PBS and vortexed just before measurement. Samples were measured at maximally 10,000 events per second, which is far below the electronic pulse processing limit of the BD Influx (46). Serial dilutions of peak fractions were included to control for potential 'invisible swarm' effects (47).

\section{Immunoblotting}

EV produced by equal numbers of stimulated and unstimulated cells were purified as described before, and resuspended in $30 \mu \mathrm{l} 2 \mathrm{x}$ SDS sample buffer. Cell lysates were prepared in PBS $+1 \%$ NP-40 and Complete Mini Protease inhibitor cocktail tables (Roche, Basel, Switzerland) and incubation for 15 min on ice. Nuclei were spun down at $16,000 \mathrm{~g}$ for $15 \mathrm{~min}$. Protein content of cleared cell lysates was measured by BCA assay (ThermoScientific), and equal amounts of cell lysate proteins were used in immunoblotting. Equal volumes of fractionated plasma samples were mixed with $2 x$ SDS sample buffer. Samples were incubated at $100^{\circ} \mathrm{C}$ for $3 \mathrm{~min}$ for detection of CD9, for $10 \mathrm{~min}$ for detection of ApoAl and ApoB100, and were not heated for detection of CD63 and CD81. Proteins were separated on a 10\% SDS-PAGE gel (6\% gel for ApoB100), after which proteins were transferred onto an Immobilon-P $0.45 \mu \mathrm{m}$ PVDF membrane (Millipore, Cork, Ireland). After blocking for $1-2 \mathrm{~h}$ in blocking buffer $(0.5 \%$ Cold Fish Skin Gelatin in PBS $+0.05 \%$ Tween-20), blots were incubated overnight at $4^{\circ} \mathrm{C}$ with one of the following primary antibodies: anti-human-CD9 (BioLegend, 1 : 1000, clone HI9a), anti-human-CD63 (BD Bioscience, 1 : 500, clone H5C6) , anti-human-CD81 (SantaCruz, 1 : 1000, clone B-11), anti-human-ApoAl (SantaCruz, 1 : 1000, clone sc376818), anti-humanApoB100 (Biotechne, 1 : 1000, clone AF3260), or anti-human-Calnexin (Transduction Labs, 1 : 1000, clone 37) in blocking buffer, washed three times in PBS-Tween, and incubated for 1 - $2 \mathrm{~h}$ with HRP-coupled secondary antibodies (Dako, $1: 5000$ - $1: 10$ 000). Blots were washed again three times in PBS-Tween, followed by two washes in 
plain PBS and incubation with ECL solution (ThermoScientific, SuperSignal West Dura Extended Duration Substrate, cat. 34075). Blots were analyzed using a BioRad Chemidoc imager (BioRad, Hercules, CA) and Image Lab software.

\section{RNA analysis}

RNA was isolated from cell culture EV isolates using the miRNeasy micro kit according to the manufacturer's guidelines (Qiagen, Hilden, Germany). RNA was isolated from the $100 \mu \mathrm{l}$ plasma aliquots according to the miRNeasy micro serum/plasma guidelines. RNA was eluted in $15 \mu \mathrm{l}$ RNase-free water supplied with the kit. The Bioanalyzer Pico 6000 kit (Agilent, Waldbronn, Germany) was used to quantify the RNA. cDNA was prepared using the miScript RT II kit (Qiagen, Hilden, Germany) using equal input volumes of RNA (12 $\mu$ l per reaction) and HiFlex buffer. Quantification of specific RNA molecules by qPCR was done in $8 \mu \mathrm{l}$ reactions, containing $2 \mu \mathrm{l}$ of 10x diluted cDNA template, $4 \mu \mathrm{l}$ of SYBR Green SensiMix PCR master mix (BioLine Reagents Ltd., UK) and $100 \mathrm{nM}$ primers (IDT, Leuven, Belgium). Cycling conditions were $95^{\circ} \mathrm{C}$ for $10 \mathrm{~min}$ followed by 50 cycles of $95^{\circ} \mathrm{C}$ for $10 \mathrm{~s}, 57^{\circ} \mathrm{C}$ for $30 \mathrm{~s}$ and $72^{\circ} \mathrm{C}$ for $20 \mathrm{~s}$, run on a BioRad CFX384 machine (BioRad, Hercules, CA). Cq values were determined at a threshold of 300 fluorescent units, data analysis was done in BioRad CFX Manager.

Isolation of mRNA from cells was performed using the MN Nucleospin RNA kit (Macherey-Nagel, Düren, Germany). RNA was eluted in $60 \mu \mathrm{l}$ RNase-free water and quantified by Nanodrop 2000 (ThermoScientific). cDNA was prepared using the RevertAid cDNA kit with random hexamer primers (ThermoScientific) and $\mathrm{qPCR}$ reactions were set up as described previously. Cycling conditions were $95^{\circ} \mathrm{C}$ for $10 \mathrm{~min}$ followed by 40 cycles of $95^{\circ} \mathrm{C}$ for $10 \mathrm{~s}, 60^{\circ} \mathrm{C}$ for $30 \mathrm{~s}$ and $72^{\circ} \mathrm{C}$ for $20 \mathrm{~s}$, run on a BioRad CFX96 machine (BioRad, Hercules, CA). Data analysis was done in BioRad CFX Manager.

The following primers combinations were used $(1-8$ were combined with miScript Universal reverse primer, primers $9-16$ were matched forward and reverse primers):

\section{Statistics}

Statistical analyses were done in SPSS (v24, IBM) or R Studio (v 3.5.2) with ggplot2 and ggally. The Kolmogorov-Smirnov test was used to assess the normality of the data. Various statistical tests were used which are indicated in the respective figure legends. p-values $<0.05$ were considered statistically significant. Y-RNA abundance ratios were calculated from the differences in $\mathrm{Cq}$ value $(\mathrm{dCq})$ between individual Y-RNA subtypes. For example: $\mathrm{Y} 4 / \mathrm{Y} 3=\mathrm{dCq}_{\mathrm{Y} 3 \mathrm{Y} 4}=\mathrm{Cq}_{\mathrm{Y} 3}-\mathrm{Cq}_{\mathrm{Y} 4}$. The resultant values represent the relative abundance between two Y-RNA subtypes, for example: a Y4/Y3 ratio of zero means that both Y-RNA subtypes are present in equal amounts; a ratio of 1 means $Y 4$ is twice as abundant as $\mathrm{Y} 3$; a ratio of -1 means $\mathrm{Y} 4$ is two times less abundant as $\mathrm{Y} 3$. Receiveroperator characteristics curve was plotted using the $\mathrm{pROC}$ plugin for $\mathrm{R}(48)$. 
Table 2. qPCR primers

\begin{tabular}{|c|c|c|}
\hline No & Primer name & Sequence $\left(5^{\prime}-3^{\prime}\right)$ \\
\hline 1 & Y1_loop-F & GATCGAACTCCTTGTTCTACTC \\
\hline 2 & Y3_loop-F & AGATTTCTTTGTTCCTTCTCCACTC \\
\hline 3 & Y4_loop-F & GTGTCACTAAAGTTGGTATACAAC \\
\hline 4 & Y5_loop-F & GTTAAGTTGATTTAACATTGTCTC \\
\hline 5 & hsa-miR-150-5p & TCTCCCAACCCTTGTACCAGTG \\
\hline 6 & hsa-miR-21-5p & TAGCTTATCAGACTGATGTTGA \\
\hline 7 & hsa-miR-122-5p & TGGAGTGTGACAATGGTGTTTG \\
\hline 8 & hsa-miR-16-5p & TAGCAGCACGTAAATATTGGCG \\
\hline 9 & hGAPDH-F & TGCACCACCAACTGCTTAGC \\
\hline 10 & hGAPDH-R & GGCATGGACTGTGGTCATGAG \\
\hline 11 & hActin-F & CCTTCCTGGGCATGGAGTCCTG \\
\hline 12 & hActin-R & GGAGCAATGATCTTGATCTTC \\
\hline 13 & hIL-6-F & AACCTGAACCTTCCAAAGATGG \\
\hline 14 & hIL-6-R & TCTGGCTTGTTCCTCACTACT \\
\hline 15 & hTNFa-F & ATGAGCACTGAAAGCATGATCC \\
\hline 16 & hTNFa-R & GAGGGCTGATTAGAGAGAGGTC \\
\hline
\end{tabular}

\section{Results}

\section{Full length Y-RNA in human plasma is predominantly associated to EV}

Previously, it was shown that miRNAs in plasma are associated to both EV, RNP, and LPP $(1-3)$. We here investigated whether full length Y-RNAs in human plasma also associate to these three types of macromolecular structures. We first assessed whether all four Y-RNA subtypes could be detected in unfractionated plasma. To reliably distinguish between the different Y-RNA subtypes, we designed primers complementary to the loop sequences, which are unique to each of the 4 Y-RNA subtypes. RNA was isolated directly from $100 \mu \mathrm{l}$ human plasma, followed by RT-qPCR-based quantification of all Y-RNA subtypes. Selected miRNAs known to be abundantly present in plasma (miR16-5p, miR-21-5p, miR-122-5p and miR-150-5p) (49) were included for comparison (Figure 1A). Y1, Y3 and Y4 were as abundant as the highly abundant miRNAs, whereas the levels of Y5 were relatively low. Next, we separated EV, RNP and LPP based on differences in size and buoyant density using a combination of isolation methods (Figure 1B). We used size-exclusion chromatography to separate large EV and (lipo) protein particles ('early fractions') from small (lipo) protein particles and soluble proteins ('late fractions'). These 'early' and 'late' fractions were subjected to ultracentrifugation at $100,000 \mathrm{~g}$ to separate pelletable from non-pelletable structures. Non-pelletable 
a

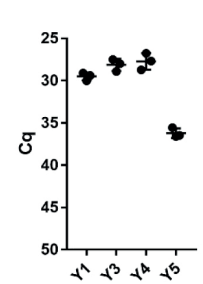

C
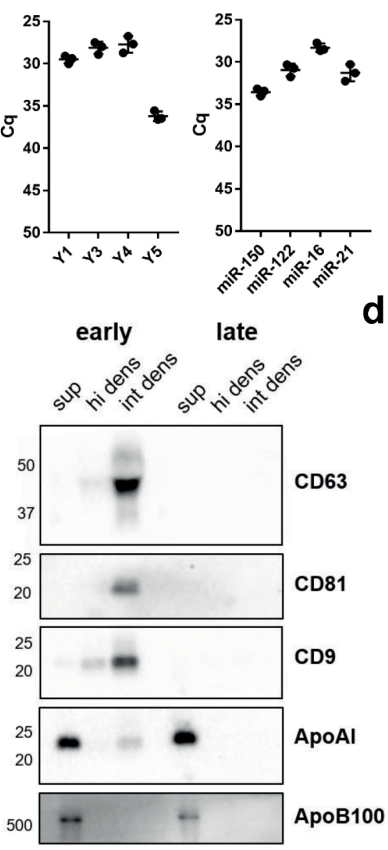

b

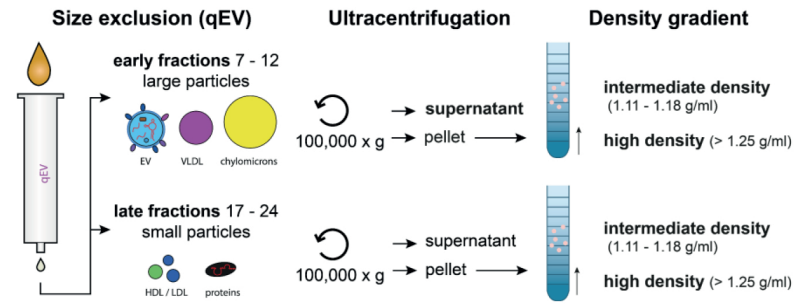

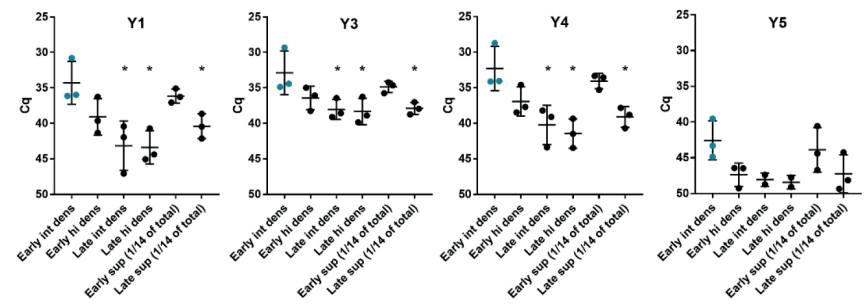
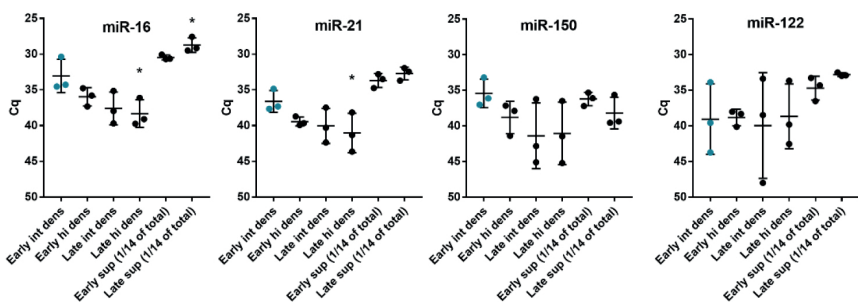

Figure 1. Distribution of full-length Y-RNA subtypes over RNA carriers in plasma with different sizes and densities

A) RNA was isolated from $100 \mu$ unfractionated plasma, after which Y-RNA subtypes and selected miRNAs were quantified by RT-qPCR. Indicated are raw $\mathrm{Cq}$ values, with lower $\mathrm{Cq}$ values indicating higher transcript abundance. B) Schematic overview of the plasma fractionation protocol. Plasma was fractionated by size exclusion chromatography into 'early' and 'late' fractions, which were ultracentrifuged at $100,000 \mathrm{~g}$ to separate pelletable from non-pelletable structures. Non-pelletable structures ('sup') were concentrated by ultrafiltration, whereas pelleted particles were separated into particles with densities characteristic for EV (intermediate density, 'int dens') and particles with a higher density ('hi dens') by using density gradient ultracentrifugation. C) Plasma fractions obtained in B were immunoblotted for the common EV proteins CD9, CD63 and CD81, and LPP-associated proteins ApoAl and ApoB100. Data are representative of $n=2$ experiments with different plasma donors. D) RNA was isolated from all plasma fractions, after which the Y-RNA subtypes and selected miRNAs in these fractions were quantified by RT-qPCR. Note that data from supernatant fractions represent equivalents of $1 / 14^{\text {th }}$ of the initial plasma input. * $p<0.05$, ANOVA with Dunnett's twosided post-hoc test was used to determine enrichment of RNA relative to the 'early int dens' fraction. 
structures ('sup') were concentrated by ultrafiltration, and the pelleted particles were separated into particles with densities characteristic for EV (intermediate density, 'int dens') and particles with a higher density ('hi dens'), by using density gradient ultracentrifugation. The resulting six fractions were assessed for the presence of the common EV markers CD9, CD63, and CD81, and the lipoprotein markers ApoAl and ApoB100 (Figure 1C). Consistent with their reported association with differently sized lipoproteins, ApoAl (present in $75-1200 \mathrm{~nm}$ chylomicrons and $5-12 \mathrm{~nm} \mathrm{HDL}$ ) and ApoB100 (present in 30-80 nm VLDL, 25-35 nm ILDL, and 18 -25 nm LDL) were detected in the early and late supernatant fractions, and were not efficiently pelleted in line with their low density $(50,51)$. Common EV-associated proteins were predominantly detected on larger particles (present in the early SEC fractions) that sedimented at $100,000 \mathrm{~g}$ and displayed intermediate buoyant densities. This is in accordance with the typical size $(50-300 \mathrm{~nm})$ and buoyant density $(1.11-1.18 \mathrm{~g} / \mathrm{ml})$ of EV. Additionally, a faint signal for CD9 was detected in the early supernatant fraction, suggesting the presence of a EV-subset that did not sediment efficiently during ultracentrifugation. Next, we compared the six fractions for the presence of full length Y-RNA subtypes and selected miRNAs by RT-qPCR (Figure 1D). All Y-RNA subtypes were comparably distributed over the different plasma fractions. Y-RNAs were mainly detected in the early intermediate 'EV' fraction and early supernatant fractions, while they were almost absent in the late supernatant fraction. The distribution of miRNAs, on the contrary, varied per miRNA species. MiR-16, and miR-21 were most abundantly detected in early and late 'LPP' fractions, and to a lesser extent in the early 'EV' fraction. MiR-122 was exclusively detected in the 'LPP' supernatant fractions. The distribution of miR-150 was similar to Y-RNAs, with high levels present in the early intermediate 'EV' fraction. These data show that full length Y-RNAs are predominantly detected in plasma fractions that contain EV.

Due to the overlap in size and density of different extracellular RNA carriers, the enrichment methods used above did not allow for strict separation of EV from all LPP subsets and RNP. Additional methods were needed to confirm the presence of Y-RNA in plasma EV. We therefore combined our method for plasma fractionation with protease/RNase treatments. MiRNAs and miRNA-protein complexes inside EV are thought to be protected against degradation by these enzymes by the EV-membrane and will only become sensitive to these enzymes upon detergent lysis. In contrast, degradation of the protein scaffold of RNP and LPP by proteases will render the RNAs associated to these structures sensitive to RNases (2). We selected the two plasma fractions containing the highest levels of Y-RNA ('early intermediate density' and 'early sup') and an additional fraction in which high levels of miRNAs were detected ('late sup') for treatment with different combinations of RNase, protease, and detergent (Figure 2). As expected, all Y-RNA subtypes in the early intermediate (EV) fractions 
were resistant to protease + RNase treatment, while addition of detergent led to complete degradation of the enclosed Y-RNAs (Figure 2A). Also the tested miRNAs in this fraction were protected unless detergent was added (Figure 2B). Importantly, we observed that all Y-RNA present in the lipoprotein-enriched early SEC fractions ('early sup') was also protected against protease/RNase treatment unless detergent was added. For miRNAs present in this fraction, however, we observed miRNA-specific differences in the sensitivity to protease/RNase degradation, with miR-16, -21, and -122 being sensitive and miR-150 being protected from degradation. All of the tested miRNAs were also present in the lipoprotein-enriched late SEC fractions ('late sup'), but miRNA present in this fraction was sensitive to enzymatic degradation. These data strengthen the conclusion that the 'early intermediate density' fractions contain a pure population of EV that protect enclosed miRNAs and Y-RNAs against degradation. In contrast, the miRNAs present in the lipoprotein-enriched late SEC fractions, which were almost devoid of Y-RNAs, were fully sensitive to degradation by protease/RNase treatment. Furthermore, the lipoprotein-enriched early SEC fractions likely contain a mix of miRNA-containing LPPs and a low number of miRNA/Y-RNA containing EV that did not pellet efficiently during the $100,000 \mathrm{~g}$ centrifugation step. Indeed, our western blot data indicated that this fraction is not only enriched in apolipoproteins but also contains a low amount of the EV marker protein CD9 (Figure 1C). Together, our data indicate that full-length Y-RNAs in plasma, unlike miRNAs, are primarily associated to and protected by EV.

\section{Blood-associated cell types release EV with cell type-specific Y-RNA signatures}

EV in plasma are likely derived from multiple cell types. It is currently unknown whether a broad range of blood-related cell types has the capacity to release EV containing full length Y-RNA into the extracellular space. To investigate this, we isolated EV from in vitro cultures of human red blood cells (RBC), neutrophils, peripheral blood mononuclear cells (PBMC, which consist of T cells, B cells, and monocytes), and endothelial cells (HUVEC). We additionally prepared EV from platelets since these are known to be abundantly present in blood plasma (52). We aimed to compare the number of EV released by these different cell types within a similar time frame. Because primary neutrophils are short-lived in culture (53), EV from all different blood-related cell types were collected after a short culturing period of 2-4 hrs. EV were purified by differential centrifugation followed by density gradient ultracentrifugation. By using high-resolution flow cytometric quantification of EV $(44,45)$, we observed that all cells released EV within the given culture period, although the number of released EV per cell strongly differed between cell types (Figure 3A). Next, we used RT-qPCR to assess whether EV from all selected blood cells contained full length Y-RNAs (Figure 3B). Y-RNA subtypes Y1, Y3 
a

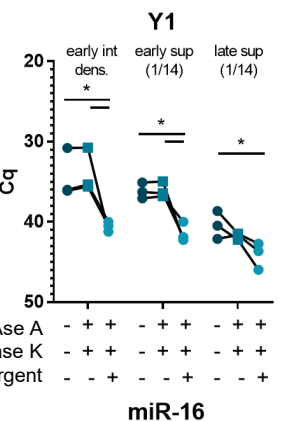

b

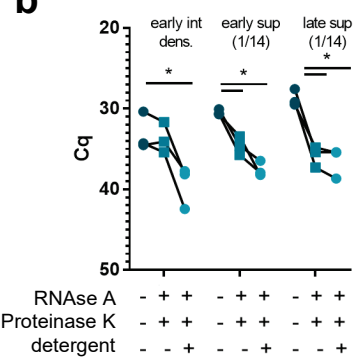

Y3

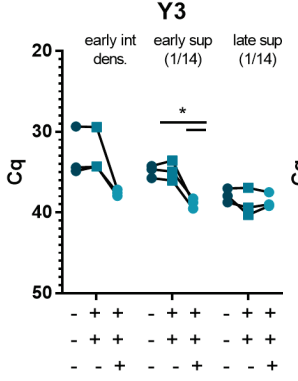

miR-21

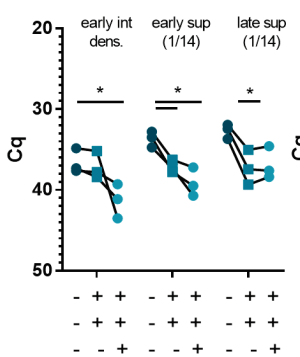

Y4

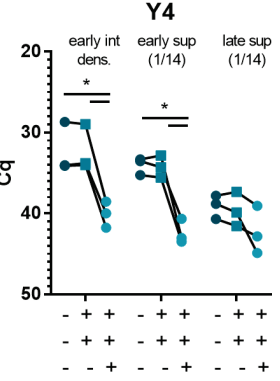

miR-150

$207 \underset{\substack{\text { early int } \\ \text { dens. }}}{\substack{\text { early sup } \\(1 / 4)}} \underset{(1 / 14)}{\text { late sup }}$

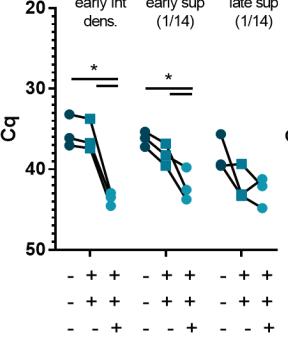

Y5
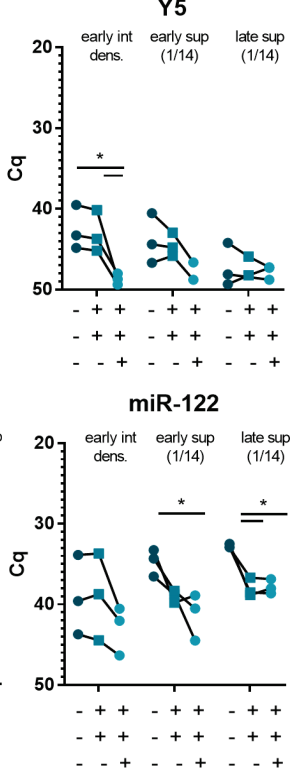

Figure 2 - Full length Y-RNA in plasma is protected from enzymatic degradation. EV-enriched ('early int dens') and LPP-enriched ('early sup' and 'late sup') plasma fractions from Figure 1B were subjected to treatment with detergent, protease, and RNase as indicated. Subsequently, RNA was isolated from each of the treated fractions and RT-qPCR was used to quantify the indicated RNA transcripts. A) Quantification of Y-RNA subtypes, indicated are raw Cq values obtained in the different treatment conditions. Data from $n=3$ individual plasma donors are shown, ${ }^{*} p<0.05$, ANOVA with Tukey's HSD post-hoc test. B) Quantification of selected miRNAs, indicated are raw $\mathrm{Cq}$ values obtained in the different treatment conditions. Data from $n=3$ individual plasma donors are shown, ${ }^{*} p<0.05$, ANOVA with Tukey's HSD post-hoc test.

and Y4 were abundantly detected in EV from all cell types. Y5 levels were substantially lower and were excluded in subsequent computational analyses because of the low reliability with which such low-abundance transcripts can be quantified. Across the different cell types, we found a statistically significant log-linear relationship between Y-RNA abundance and EV number, which indicates that cells that release more EV also release more Y-RNA (Figure $\mathbf{3 C}$ ). Furthermore, we observed remarkable differences in the relative abundance of Y-RNA subtypes in EV from various cell types (Figure 3D). Notably, neutrophil-derived EV were unique in their high levels of $Y 4$ and low levels of Y3. In contrast, PBMC derived EV and platelet-derived EV contained high levels of both Y4 and Y3, but low levels of Y1. To further explore these cell-specific Y-RNA profiles, we calculated abundance-ratios between each of the Y-RNA subtypes, which we defined as the log2fold difference between individual Y-RNA subtypes (Figure 3E). Based on these calculations, we conclude that EV from neutrophils uniquely displayed high Y4/ 
Y3 and negative $Y 3 / Y 1$ ratios. PBMC- and platelet-EV were characterized by high $Y 3 / Y 1$ ratios but $Y 4 / Y 3$ ratios around zero. In EV from HUVEC and RBC, on the contrary, none of the Y-RNA subtype ratios deviated significantly from zero. Overall, our results indicate that blood-related cell types differ in the amount of EV-associated Y-RNA they release. In addition, our data illustrate that EV released by different blood cells vary in Y-RNA subtype composition and therefore have a specific 'Y-RNA signature'.

\section{LPS stimulation of neutrophils affects the number, but not the characteristic Y-RNA subtype ratios of EV}

Next, we investigated whether the Y-RNA load and signature of EV released by the different cell types changed after microbial stimulation. To this end, the different blood cells were cultured for 2-4 hrs in the presence or absence of LPS (Supplementary Figure 1A-B).Within this short time frame, neutrophils from all donors responded to LPS exposure as indicated by a clear downregulation of CD62L, but did not yet show the phenotype of fully activated neutrophils (CD16low CD63high). PBMC showed increased expression of TNFa and IL- 6 mRNA (Supplementary Figure 1B). HUVEC and platelets showed a modest upregulation of activation markers, whereas RBC did not respond to LPS stimulation (Supplementary Figure 1A). In response to LPS, neutrophils from all donors showed a rapid and strong, but variable increase in the number of released EV, as quantified by high-resolution flow cytometry (Figure 4A) and western blot analysis of the common EV proteins CD9 and CD63 (Supplementary Figure 1C). The expected increase in EV release by PBMC due to LPS stimulation (54) was also observed, but differed by only 1.2-fold at this early time point (Figure 4A). Under the selected conditions, no increase in the number of released EV was observed for the other cell types. For LPS-stimulated neutrophils, but not for PBMC, we additionally observed a significant increase in the total amount of Y-RNA released via EV (Figure 4B), which is in line with the previously observed correlations between EV counts and Y-RNA levels (Figure $\mathbf{3 C}$ ). Importantly, our data indicated that the cell type specific Y-RNA subtype ratios did not change in response to LPS stimulation (Figure $\mathbf{4 C}$ ). Together, the data indicated that LPS stimulation of specific blood-related cell types can increase the total amount of EV-associated Y-RNA by increased sorting of Y-RNA into EV and/or by increased release of EV. However, the cell type specific Y-RNA signatures remain stable under these conditions.

\section{Systemic inflammation induces alterations in the Y-RNA subtype ratios in the total pool of plasma EV}

Next, we investigated whether the Y-RNA signatures could also be identified in complex biofluids such as plasma. We hypothesized that the Y-RNA subtype ratios in blood are influenced by the presence of different cell types in the circulation 
a

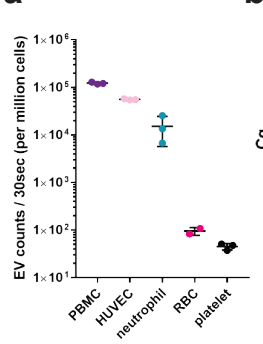

C

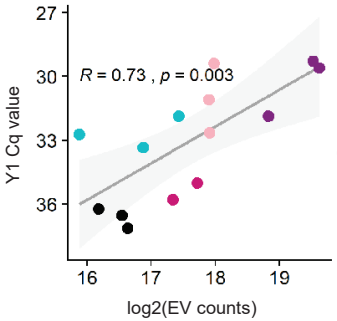

d

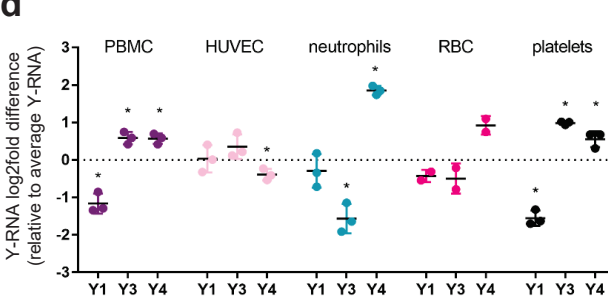

b

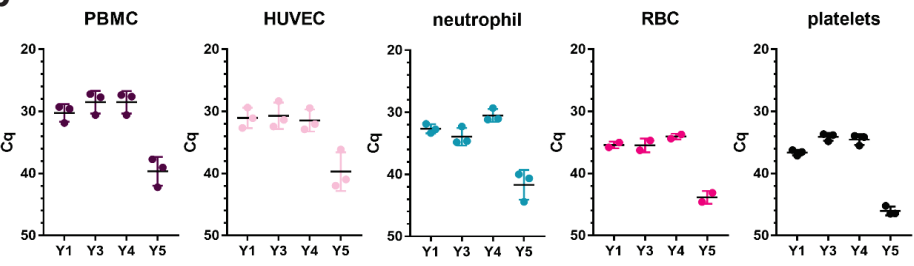

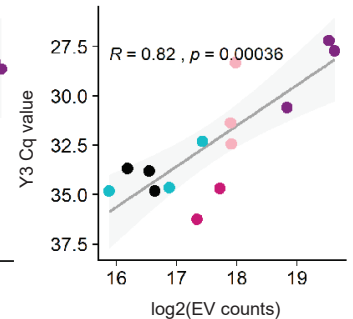

e
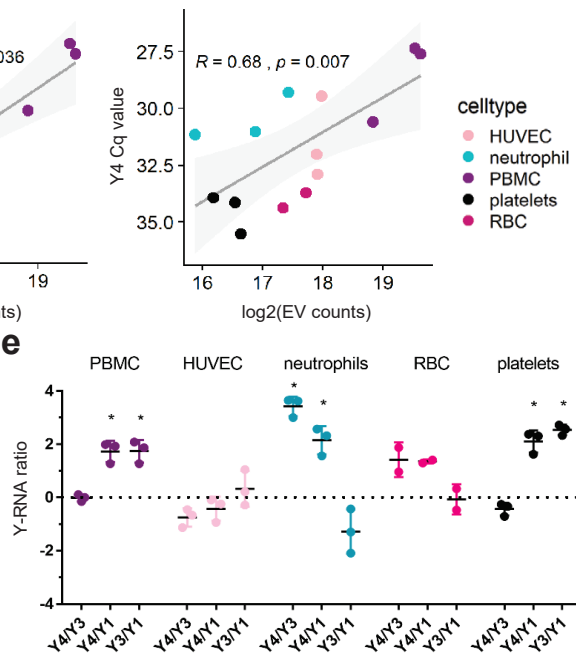

Figure 3. Blood-associated cell types release EV with cell type-specific Y-RNA signatures. A) Various blood-related cell types were cultured in EV-depleted medium or buffer for $2 \mathrm{~h}$ (Neutrophils, RBC and platelets) or $4 \mathrm{~h}$ (PBMC and HUVEC). High-resolution flow cytometry was used to quantify PKH67-labeled EV. Indicated are the number of EV calculated per million EVproducing cells. EV counts of $n=3$ replicates with different cell donors are shown ( $n=2$ for $R B C)$. B) RNA was isolated from the EV-containing fractions of all cell types. The abundance of Y-RNA subtypes was quantified by RT-qPCR. Cq values of $n=3$ experiments with different cell donors are shown, $R B C n=2$. C) Linear relationships were determined between the log2-transformed $E V$ counts shown in (A) and the $\mathrm{Cq}$ values for different $\mathrm{Y}$-RNA subtypes shown in (B). Colored dots indicate the respective cell types $($ HUVEC $=$ pink, neutrophil $=$ turquoise, $\mathrm{PBMC}=$ purple, $\mathrm{RBC}=$ magenta, platelets $=$ black). Linear regression was performed, and trendlines are shown in black. $95 \%$ confidence intervals are indicated in grey. Pearson correlation coefficients (R) and $p$-values of the correlations are indicated. Data from $n=3$ experiments with different cell donors are shown. D) Relative differences in Y-RNA subtype composition were determined based on the data in (B). Indicated are the differences between the $\mathrm{Cq}$ value of individual $\mathrm{Y}$-RNA subtypes relative to the average $\mathrm{Cq}$ value of $\mathrm{Y} 1, \mathrm{Y} 3$ and $\mathrm{Y} 4$ measured for the $\mathrm{EV}$ from the respective cell type. Enrichment of individual Y-RNA subtypes was calculated by Student's t-test, with * $p<0.05, n=3$ experiments on individual cell donors. E) Ratios between the different Y-RNA subtypes were calculated from the data in B. ${ }^{*} p<0.05$, deviation from zero calculated by Student's t-test, $n=3$ experiments on individual cell donors. 
that produce EV with characteristic Y-RNA signatures. To test this hypothesis, we investigated changes in human plasma Y-RNA ratios after injection of healthy volunteers with LPS, which induces a rapid change in neutrophil and lymphocyte numbers (55). This experimental human endotoxemia model is often used to study systemic inflammatory processes, such as those occurring during sepsis $(56,57)$. We analyzed plasma samples of an earlier conducted study, in which plasma was collected from volunteers before and 2 or 6 hours after infusion with LPS $(39,40)$. Clinical responses in these volunteers were characterized by an increase in body temperature and heart rate, and increased levels of circulating inflammatory cytokines $(39,40)$. In addition, induction of systemic endotoxemia led to increased numbers of neutrophils and decreased numbers of lymphocytes and monocytes in the circulation, similar to what has been observed during sepsis $(39,40,55,58)$. The preferential localization of circulating Y-RNAs in EV (Figure 1 and 2) allowed us to determine Y-RNA subtype ratios in plasma without prior isolation of EV. We isolated total RNA from plasma obtained before and 2 hrs after infusion of LPS. A slight increase in average RNA concentration could be observed at $\mathrm{t}=2$, with considerable variability between individuals (Supplementary Figure 2A). We subsequently quantified the levels of Y-RNA subtypes by RT-qPCR and calculated the different Y-RNA ratios (Figure 5A). Substantial changes were detected in cell-specific Y-RNA ratios in whole plasma 2 hrs after induction of inflammation. We observed a highly significant increase in the $Y 4 / Y 3$ ratio $(p=0.001)$ and a significant decrease in the $Y 3 / Y 1$ ratio $(p=0.03)$, while the $Y 4 / Y 1$ ratio did not change $(p=0.078)$. The increase in $Y 4 / Y 3$ ratio, which is specific for neutrophil-derived EV (Figure 3E) accompanied the previously observed increase in number of neutrophils (2.2-fold increase, $p=0.001$ ) in the $t=2$ blood samples (39). Similarly, the decrease in $\mathrm{Y} 3 / \mathrm{Y} 1$ ratio, which may indicate reduced numbers of $\mathrm{EV}$ released by PBMC (Figure 3E) accompanied the observed decrease in numbers of lymphocytes (2.2-fold decrease, $\mathrm{p}=4.2 \mathrm{E}-9$ ) and/or monocytes (19-fold decrease, $\mathrm{p}=$ 2.3E-10) (39). To further investigate this, we determined Pearson's correlations between the Y-RNA subtype ratios and cell numbers within the combined $t=0$ and $t=2$ datasets (see Supplementary Figure 2B and $\mathbf{3}$ for a full overview of these correlations). Importantly, we found a strong correlation between the neutrophil specific $\mathrm{Y} 4 / \mathrm{Y} 3$ ratios and the blood neutrophil counts $(R=0.52, p=0.0024)$ (Figure 5B). The decrease in $Y 3$ / Y1 ratios correlated with a decrease in monocyte numbers, but these data did not reach statistical significance (Figure $\mathbf{5 C}$ ). Besides changes in immune cell counts, the levels of pro- and anti-inflammatory cytokines are highly increased during systemic endotoxemia and sepsis $(55,59)$. We observed that the neutrophil-specific $Y 4 / Y 3$ ratio in plasma strongly correlated with levels of the pro-inflammatory cytokines IL- 6 ( $R=0.51$, $\mathrm{p}=0.0028), \mathrm{IL}-8(\mathrm{R}=0.43 \mathrm{p}=0.013)$ and also with TNFa $(\mathrm{R}=0.35, \mathrm{p}=0.05)$, whereas the $\mathrm{Y} 3 / \mathrm{Y} 1$ ratio negatively correlated with levels of the anti-inflammatory cytokine IL-10 $(R=-0.39$, 
a

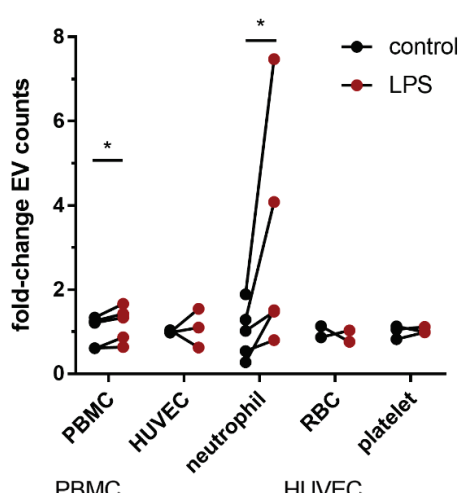

b

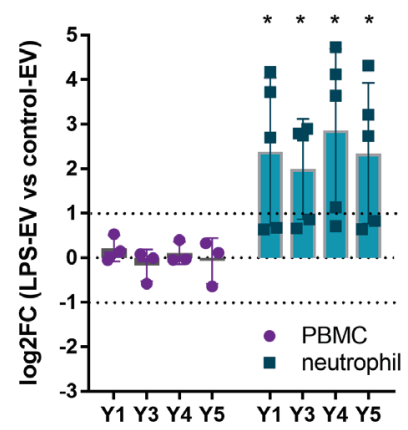

neutro

RBC

platelet

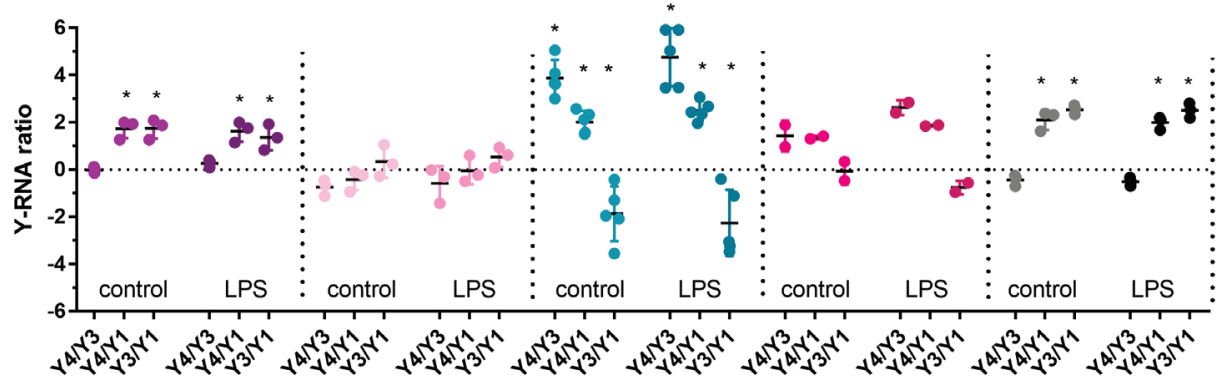

Figure 4. LPS stimulation changes the quantity of EV-associated Y-RNA by specific blood-related cell types, while cell-type specific Y-RNA signatures remain stable. PBMC, HUVEC, neutrophils, RBC and platelets were cultured for 2-4h in EV-depleted medium or buffer as described in Figure 3, in the presence or absence of LPS. EV were purified, labeled with PKH67 and quantified by high-resolution flow cytometry as in Figure 3. A) The fold-difference in EV-release was calculated for each specific cell type, relative to the unstimulated condition. $N=5$ PBMC and neutrophil, $n=3$ platelets and HUVEC, $n=2$ RBC, ${ }^{*} p<0.05$ Wilcoxon signed ranks test. B) RNA was isolated from the neutrophil and PBMC-derived EV purified in (A), after which Y-RNA subtypes were quantified by RT-qPCR. Indicated is the increase in EV-associated Y-RNA release from equal numbers of LPS-stimulated versus unstimulated cells, calculated as a log2fold difference. $\mathrm{N}=5$ neutrophils, $\mathrm{n}=3$ PBMC, ${ }^{*} \mathrm{p}<0.05$, deviation from zero calculated by one-sample Wilcoxon signed ranks test. C) Y-RNA subtype ratios were determined for EV released by equal numbers of LPS-stimulated and unstimulated cells as described previously. $\mathrm{N}=5$ neutrophils, $\mathrm{n}=3$ PBMC, ${ }^{*} p<0.05$, deviation from zero calculated by Student's t-test. 
$\mathrm{p}=0.029$ ) (Figure 5D). This raised the question whether the observed changes in Y-RNA subtype ratios were mostly determined by the increased number of circulating immune cells or by the increased levels of cytokines that may influence the release of EV and/ or their molecular composition. We therefore also analyzed plasma samples collected $6 \mathrm{hrs}$ after LPS infusion. At this time point, body temperature, heart rate and cytokine levels had returned to baseline levels (39). Additionally, while monocyte counts were increased to normal levels, neutrophil counts remained elevated on $t=6$. We isolated RNA from total plasma collected at $\mathrm{t}=6$ and observed that the RNA concentrations at this time point had returned to values that were slightly lower than those observed on $t=0$ (Supplementary Figure $\mathbf{2 C}$ ). Importantly, we observed that the $Y 4 / Y 3$ ratio at $\mathrm{t}=6$ was still highly increased relative to $\mathrm{t}=0$ (Figure $\mathbf{5 E}$ ). Moreover, the $\mathrm{Y} 4 / \mathrm{Y} 3$ levels still correlated strongly with the neutrophil counts at this time point (Figure 5F). In contrast, the other correlations observed at $\mathrm{t}=2$ were not observed at $\mathrm{t}=6$ (Supplementary Figure 2D and 4). The $Y 3 / Y 1$ levels, which were decreased on $t=2$, returned to control levels at $\mathrm{t}=6$ (Figure 5E), thereby following the pattern for monocyte counts (Figure 5G). This indicated that the observed alterations in Y-RNA subtype ratios predominantly correlated with the numbers of circulating immune cells, but not with inflammatory cytokine levels.

We performed receiver-operator characteristics (ROC) analysis (48) of the Y4/ Y3 ratios determined in this endotoxemia model to explore whether plasma $Y 4 / Y 3$ ratio values could discriminate individuals in the early and late (resolving) phases of inflammation from healthy individuals (Figure 6). The Y4/Y3 ratio data of both $t=0$ versus $t=2$, and $t=0$ versus $t=6$ yielded excellent AUC values of 0.82 and 0.88 , respectively. This suggests that increased $Y 4 / Y 3$ ratio values accurately mark the past or present occurrence of systemic inflammatory processes.

Taken together, we have shown that Y-RNA in plasma is predominantly localized in EV. Furthermore, we discovered that EV from different blood cells contain cell type specific Y-RNA subtype ratios. Systemic inflammation induced significant alterations in neutrophil- and PBMC-specific Y-RNA ratios observed in plasma. The detected changes in Y-RNA subtype ratios strongly correlated with the inflammation-induced changes in the number of circulating neutrophils and monocytes. These immune cell type specific 'Y-RNA signatures' in plasma EV can be determined without prior enrichment for EV, and may be further explored as biomarkers for inflammatory responses or other immune-related diseases. 

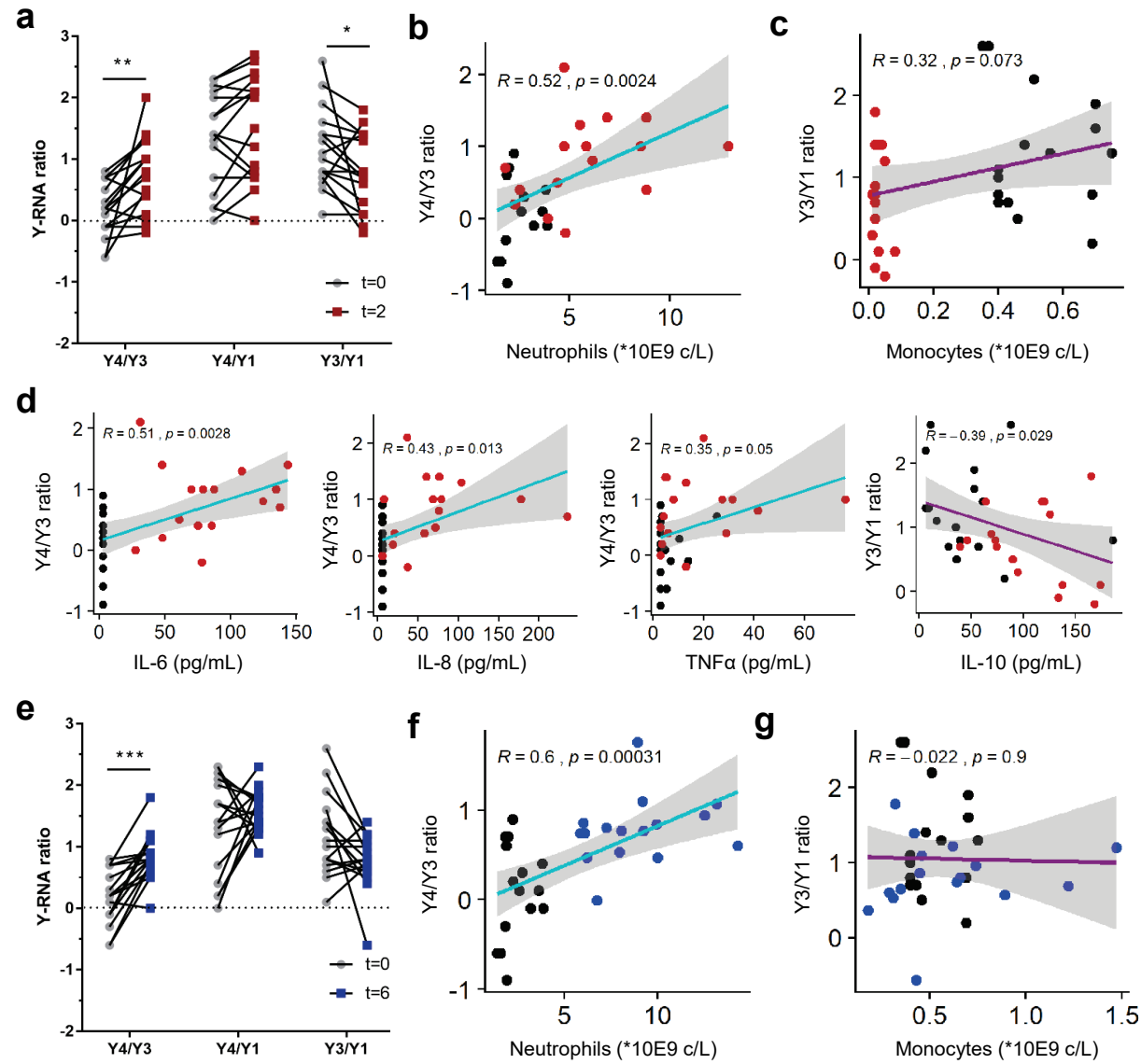

Figure 5. Y-RNA subtype ratios in total plasma change during systemic endotoxemia. 16 healthy volunteers were infused with LPS and plasma was collected before and 2 or 6 hours after LPS infusion. RNA was isolated from $100 \mu \mathrm{l}$ plasma obtained from each individual at $\mathrm{t}=0$, $t=2$, and $t=6$, after which Y-RNA subtypes were quantified by RT-qPCR. A) Y-RNA ratios were calculated as described before, differences in Y-RNA ratios between $t=0$ and $t=2$ were tested by Wilcoxon signed ranks test on $n=16$ plasma samples, ${ }^{*} p \leq 0.05,{ }^{* *} p \leq 0.01$. B) Linear correlations between neutrophils and $\mathrm{Y} 4 / \mathrm{Y} 3$ ratios in the volunteers at $\mathrm{t}=0$ (black dots) and $\mathrm{t}=2$ (red dots). Trendlines are shown in turquoise and the grey area depicts $95 \%$ confidence intervals. Pearson correlation coefficient $(R)$ and significance $(p)$ are shown based on $n=16$ volunteers. C) Linear correlation between monocytes and $Y 3 / Y 1$ ratios in the volunteers at $t=0$ and $t=2$. Trendlines are shown in purple and the grey area depicts 95\% confidence intervals. Pearson correlation coefficient $(R)$ and significance $(p)$ are shown based on $n=16$ volunteers. D) Linear correlations between concentrations of IL6, IL8, TNF a and the $Y 4 / Y 3$ ratio ratios at $t=0$ (black dots) and $t=2$ (red dots), and between the Y3/Y1 ratio and IL10. Trendlines are shown in turquoise (Y4/Y3) and purple (Y3/Y1), grey areas depict $95 \%$ confidence intervals. Pearson correlation coefficient $(R)$ and significance $(p)$ are shown based on $n=16$ volunteers. E) Y-RNA ratios were calculated as described before, differences in Y-RNA ratios between $\mathrm{t}=0$ and $\mathrm{t}=6$ were tested by Wilcoxon signed ranks test on $n=16$ plasma samples, ${ }^{* * *} p \leq 0.001$. F) Linear correlations between neutrophils and $Y 4 / Y 3$ ratios in the volunteers at $t=0$ (black dots) and $t=6$ (blue dots), similar to $B$. G) Linear correlation between monocytes and $Y 3 / Y 1$ ratios in the volunteers at $t=0$ (black dots) and $t=6$ (blue dots), similar to $B$. 


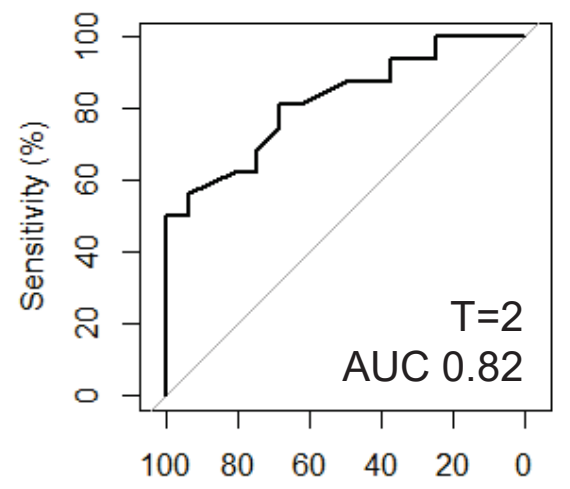

Specificity (\%)

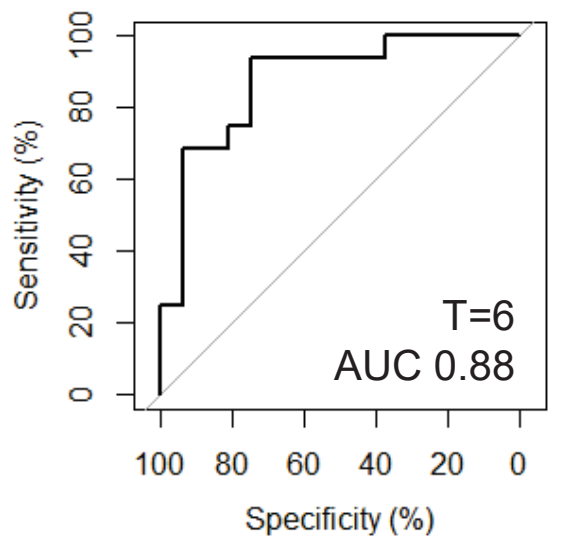

Figure 6. ROC curves of Y-RNA ratios. Y-RNA was quantified in RNA isolated from $100 \mu$ l plasma obtained from 16 volunteers before, $2 \mathrm{~h}$ and $6 \mathrm{~h}$ after LPS infusion. Y-RNA ratios were calculated as described before, after which receiver operator characteristic (ROC) curves of the $Y 4 / Y 3$ ratios were determined with $\mathrm{t}=0$ versus $\mathrm{t}=2$ data (left), and $\mathrm{t}=0$ versus $\mathrm{t}=6$ data (right). Area-under-curve $(A \cup C)$ is indicated, diagonal line represents no predictive value (AUC 0.5).

\section{Discussion}

Several studies have reported the abundant presence of Y-RNA in cell culture EV and body fluids $(24,32,35,36,41,60-64)$. Yet, the function and biomarker potential of extracellular Y-RNAs is an understudied research area. We therefore have limited knowledge on the presence of full length versus fragmented versions of Y-RNA in the extracellular space, the type of macromolecular structures to which extracellular Y-RNA is associated, and how this varies between different Y-RNA subtypes. Here, we performed extensive fractionation and characterization of macromolecular structures in plasma to show that the majority of full length Y-RNA is present in EV. This is importantly different from most of the studied miRNAs in plasma, which are known to distribute over both EV, LPP and RNP (1-3). In addition, we show that EV released by various blood-related cell types differed in Y-RNA subtype composition. This cell typespecific 'Y-RNA signature' remained stable upon LPS-stimulation of these cells. In a human endotoxemia model for systemic inflammation, we obtained proof-of-concept that the number of circulating immune cells correlates with the abundance of cell type-specific Y-RNA subtype ratios in plasma. The abundance of full length Y-RNAs in body fluids, their stability due to association with EV, and the immune cell type specific Y-RNA signatures may render these non-coding RNAs interesting candidate biomarkers for immune-related diseases. 
In the field of exRNA research there is an increasing awareness that extracellular miRNAs are distributed over different macromolecular complexes, including EV, LPP and RNP $(5,23,51,65)$. These different RNA carriers overlap in size and buoyant density (51), which complicates strict separation of the different particles. The different types of EV isolation methods that are currently in use vary largely in the level of contamination with LPP and RNP $(22,66-68)$. Moreover, the currently used RNA isolation kits exhibit variable recovery efficiencies for RNAs enclosed in each of the different macromolecular complexes (EV, LPP, RNP) (22). All of these factors complicate the accurate characterization of carrier-specific RNA profiles and cause inter-study and inter-laboratory variation in exRNA analysis. In this study, plasma was fractionated by consecutive application of size-exclusion chromatography and density gradient centrifugation. This is currently considered the most optimal method for separation of EV and LPP/RNP (51). Yet, our data indicate that this approach did not achieve complete separation of EV and LPP/RNP. This is illustrated by some residual ApoAl (found in chylomicrons and HDL (50)) in the EV fractions. This may be due to incomplete separation of EV and LPP using this approach, or because LPP can physically interact with EV (69). In addition, the non-pelletable fractions that were most enriched in the LPP proteins ApoAI and ApoB100 contained some residual CD9, which may point towards a subset of EV that are not efficiently pelleted at 100,000 g. Another approach to distinguish between EV- or LPP/RNP-associated RNA is to test for sensitivity to Protease/RNase treatment. Arroyo et al. applied enzymatic treatment of unfractionated plasma to demonstrate that plasma contained both miRNAs that are insensitive to protease/RNase treatment, likely due to enclosure in EV, and miRNAs that are sensitive to these enzymes (2). In our current study, we built on this approach by performing size- and density-based enrichment for EV and LPP/ RNP prior to applying the enzymatic treatment. Our data illustrate that the early SEC fractions not only contained EV-associated miRNAs that were resistant to enzymatic degradation, but also LPP/RNP-associated miRNAs that were sensitive to degradation. All of the full length Y-RNA in plasma, on the contrary, was protected by lipid membranes, supporting a predominant localization inside EV. In previous publication it was reported that Y-RNA in plasma and in culture medium of glioblastoma cells was associated to RNP complexes $(61,65,70)$. However, the glioblastoma studies were focused on detection of Y-RNA fragments and the data indicated that such fragments were more abundant in RNP than in EV and microvesicles $(61,65)$. The study that identified RNP-associated Y-RNA in plasma employed plasma samples collected from individuals with autoimmune diseases $(70,71)$. It is known that plasma sample of these patients contain more Y-RNA containing RNP and higher levels of anti-Ro60 antibodies compared to healthy controls (72). Our current data show that in healthy plasma the full length Y-RNA molecules are predominantly associated to EV. 
The human genome encodes 4 different Y-RNAs, but little is known about functional differentiation of these Y-RNA subtypes inside cells. In many exRNA sequencing studies, the data were biased towards detection of Y-RNA fragments derived from the Y-RNA stem regions that are highly conserved between the Y-RNA subtypes (25). The use of loop-specific primers, employed in our current study, allowed discrimination of the full length forms of the different Y-RNA subtypes. The cell type specific Y-RNA ratio signatures were most pronounced for EV from neutrophils (high $\mathrm{Y} 4 / \mathrm{Y} 3$ - low $\mathrm{Y} 3$ / $\mathrm{Y} 1$ ) and PBMC (high $\mathrm{Y} 3 / \mathrm{Y} 1$ - high $\mathrm{Y} 4 / \mathrm{Y} 1$ ). We used a human systemic endotoxemia model to provide proof-of-concept that Y-RNA subtype ratios in plasma can change during systemic inflammation. This model system is commonly used to mimic the inflammatory response during sepsis $(39,56,57)$. The inflammatory responses induced by LPS infusion recapitulate those during sepsis, including major changes in circulating immune cells and inflammatory cytokines $(39,55)$. Our data provide strong evidence that the abundant presence of specific immune cell types, such as neutrophils, can cause changes in the plasma Y-RNA composition. The neutrophil-specific Y4/Y3 ratio was increased at 2 and 6 hrs after induction of systemic inflammation and correlated well with the number of neutrophils in the circulation. An earlier study reported a strong increase in neutrophil-derived large microparticles in plasma of sepsis patients (73). In our current study, we focused on small EV and demonstrate in primary neutrophil cultures that in vitro LPS stimulation induces a large and rapid increase in the number of small EV released by these cells. Neutrophils are the most abundant immune cells in blood and are among the first to migrate and respond to infection $(74,75)$. The increased $Y 4 / Y 3$ ratio we observed during systemic inflammation may therefore be caused by both the larger number of neutrophils that release EV and the activation-induced increase in EV release per neutrophil. Besides the changes in neutrophil-specific Y-RNA signatures, we observed a decrease in the Y3/Y1 ratio during inflammation. Our in vitro experiments indicated that EV from both PBMC and platelets were characterized by high $Y 3 / Y 1$ ratios. Although we cannot exclude that EV from platelets contribute to the changes in $Y 3 / Y 1$ ratios during inflammation, we observed that the $Y 3 / Y 1$ levels in the endotoxemia plasma samples correlated with the monocyte counts at $\mathrm{t}=2$ and $\mathrm{t}=6$. Future research is needed to further establish the monocyte-specificity of $Y 3 / Y 1$ ratios in $E V$ and the use of plasma $Y 3 / Y 1$ ratios as indicators of monocyte-driven disease processes.

Based on our data, we argue that Y-RNA subtype ratios represent an interesting set of biomarkers to be further explored as biomarkers in the context of infection and inflammation. Detection of EV-associated full length Y-RNAs may provide several advantages over detection of miRNAs, which have until now been a primary focus in EV-RNA biomarker research (22,76-78). First, our data indicate that full length Y-RNAs are predominantly present in EV. As a result, they are stable and resistant to enzymes 
that degrade proteins and RNAs, which reduces variability in their detection. Second, quantification of EV-associated full length Y-RNA in plasma does not require initial enrichment or purification of EV. Quantification of Y-RNA ratios can therefore be achieved by simply isolating total RNA from unprocessed plasma. This circumvents introduction of variation caused by variability in efficiency or purity of EV isolation. Third, Y-RNA subtype ratios are based on the relative abundance of two transcripts in the same sample, which circumvents the need for RT-qPCR normalization or spike-in controls, which may further increase its detection accuracy.

Assessment of Y-RNA subtype ratios could potentially be used in diagnosis and monitoring of inflammatory conditions such as sepsis syndrome, for which early biomarkers are an unmet need (79-81). Assessment of Y-RNA subtype ratios such as the $\mathrm{Y} 4 / \mathrm{Y} 3$ ratio could be useful in diagnosis of sepsis because these ratios showed sufficient discriminatory potential between inflammatory and healthy plasma at the early activation of systemic inflammation and in the later resolution phase. At the later timepoint in our endotoxemia model, neutrophil counts and $\mathrm{Y} 4 / \mathrm{Y} 3$ ratios were still high whereas the level of proinflammatory cytokines had returned to normal levels. This suggests that neutrophils and their EV may play a role in both the induction and the resolution of inflammation. In support of this, EV have been shown to exert both pro- and anti-inflammatory roles (reviewed in (82)). EV from activated neutrophils can cause tissue damage in the lungs (83), whereas anti-inflammatory effects include the reduction of phagocytosis and increasing LPS-resistance in dendritic cells (84). Compared to the volunteers in the endotoxemia study, sepsis patients are much more heterogeneous with regard to the initial site of infection, causative organisms, and the overall health status of the patient (85). We propose that combined assessment of Y-RNA subtype ratios and other clinical parameters may provide novel opportunities for stratification of sepsis patient populations, or give additional information on the disease stadium. In case of local instead of systemic inflammation, neutrophils migrate into the inflamed tissues. Neutrophil-derived EV levels in the circulation may remain high under these conditions based on the idea that EV can cross epithelial and endothelial barriers $(86,87)$. Future research is needed to evaluate the applicability of Y-RNA subtype based diagnosis in diseases involving local tissue inflammation, such as in rheumatoid arthritis.

In conclusion, our data demonstrate that full length Y-RNAs in plasma are stably associated to EV. Moreover, we provide proof-of-concept that that Y-RNA ratios in pools of plasma EV are indicative for the number and type immune-related cells during systemic inflammation. Hence, EV-associated Y-RNAs provide an abundant and stable source of candidate biomarkers for infectious and inflammatory diseases. 


\section{Acknowledgements}

The authors would like to thank Dr. G.J.A. Arkesteijn for the assistance with high resolution flow cytometric analysis of EV, and E.G.F. Borg for assistance with the flow cytometric analyses.

\section{Funding}

This work was supported by the European Research Council under the European Union's Seventh Framework Programme [FP/2007-2013] / ERC Grant Agreement number [337581] to [ENMNtH]; a fundamental research grant of the Dutch Arthritis Society [Project number 17-1-403] to [SM]; a Postdoc Stipend of the Amsterdam Infection and Immunity Institute to [TGK]; grants of the Dutch Thrombosis Foundation [TSN 2017-01] and Netherlands Organization for Scientific Research [ZonMW Veni 016.146.160] to [CM]; a grant of the China Scholarship Council [201606300042] to [XGZ]; and a grant of the Netherlands Organization for Scientific Research (NWO) grant number [022.004.018a] to [MFSL].

\section{Conflict of interests}

The authors declare that they have no conflict of interest 


\section{References}

1. Vickers KC, Palmisano BT, Shoucri BM, Shamburek RD, Remaley AT. MicroRNAs are transported in plasma and delivered to recipient cells by high-density lipoproteins. Nat Cell Biol (2011) 13:423-33. doi:10.1038/ncb2210

2. Arroyo JD, Chevillet JR, Kroh EM, Ruf IK, Pritchard CC, Gibson DF, Mitchell PS, Bennett CF, PogosovaAgadjanyan EL, Stirewalt DL, et al. Argonaute2 complexes carry a population of circulating microRNAs independent of vesicles in human plasma. Proc Natl Acad Sci U S A (2011) 108:5003-8. doi:10.1073/ pnas. 1019055108

3. Turchinovich A, Weiz L, Langheinz A, Burwinkel B. Characterization of extracellular circulating microRNA. Nucleic Acids Res (2011) 39:7223-33. doi:10.1093/nar/gkr254

4. van Niel G, D'Angelo G, Raposo G. Shedding light on the cell biology of Extracellular Vesicles. Nat Rev Mol Cell Biol (2018) 19:213-228. doi:10.1038/nrm.2017.125

5. Mateescu B, Kowal EJK, van Balkom BWM, Bartel S, Bhattacharyya SN, Buzás El, Buck AH, de Candia P, Chow FWN, Das S, et al. Obstacles and opportunities in the functional analysis of extracellular vesicle RNA - an ISEV position paper. J Extracell Vesicles (2017) 6:1286095. doi:10.1080/20013078.2017.1286095

6. Redzic JS, Balaj L, van der Vos KE, Breakefield XO. Extracellular RNA mediates and marks cancer progression. Semin Cancer Biol (2014) 28:14-23. doi:10.1016/j.semcancer.2014.04.010

7. Revenfeld ALS, Bæk R, Nielsen MH, Stensballe A, Varming K, Jørgensen M. Diagnostic and prognostic potential of extracellular vesicles in peripheral blood. Clin Ther (2014) 36:830-846. doi:10.1016/j. clinthera.2014.05.008

8. De Toro J, Herschlik L, Waldner C, Mongini C. Emerging roles of exosomes in normal and pathological conditions: New insights for diagnosis and therapeutic applications. Front Immunol (2015) 6:1-12. doi:10.3389/fimmu.2015.00203

9. Tofaris GK. A Critical Assessment of Exosomes in the Pathogenesis and Stratification of Parkinson's Disease. J Parkinsons Dis (2017) 7:569-576. doi:10.3233/JPD-171176

10. Buzas El, György B, Nagy G, Falus A, Gay S. Emerging role of extracellular vesicles in inflammatory diseases. Nat Rev Rheumatol (2014) 10:356-64. doi:10.1038/nrrheum.2014.19

11. Zijlstra C, Stoorvogel W. Prostasomes as a source of diagnostic biomarkers for prostate cancer. J Clin Invest (2016) 126:1144-1151. doi:10.1172/JCl81128

12. Quek C, Hill AF. The role of extracellular vesicles in neurodegenerative diseases. Biochem Biophys Res Commun (2017) 483:1178-1186. doi:10.1016/j.bbrc.2016.09.090

13. Montecalvo A, Larregina AT, Shufesky WJ, Stolz DB, Sullivan MLG, Karlsson JM, Baty CJ, Gibson GA, Erdos $G$, Wang Z, et al. Mechanism of transfer of functional microRNAs between mouse dendritic cells via exosomes. Blood (2012) 119:756-66. doi:10.1182/blood-2011-02-338004

14. Cha DJ, Franklin JL, Dou Y, Liu Q, Higginbotham JN, Beckler MD, Weaver AM, Vickers K, Prasad N, Levy S, et al. KRAS-dependent sorting of miRNA to exosomes. Elife (2015) 4:1-22. doi:10.7554/eLife.07197

15. Rak J. Extracellular vesicles - biomarkers and effectors of the cellular interactome in cancer. Front Pharmacol (2013) 4 MAR:1-14. doi:10.3389/fphar.2013.00021

16. Thompson AG, Gray E, Heman-Ackah SM, Mäger I, Talbot K, El Andaloussi S, Wood MJ, Turner MR. Extracellular vesicles in neurodegenerative disease-pathogenesis to biomarkers. Nat Rev Neurol (2016) 12:346-357. doi:10.1038/nrneurol.2016.68

17. Nabet BY, Qiu Y, Shabason JE, Wu TJ, Yoon T, Kim BC, Benci JL, DeMichele AM, Tchou J, Marcotrigiano J, et al. Exosome RNA Unshielding Couples Stromal Activation to Pattern Recognition Receptor Signaling in Cancer. Cell (2017) 170:352-366.e13. doi:10.1016/j.cell.2017.06.031

18. Datta Chaudhuri A, Dastgheyb RM, Yoo SW, Trout A, Talbot CC, Hao H, Witwer KW, Haughey NJ. TNFa and IL-1 $\beta$ modify the miRNA cargo of astrocyte shed extracellular vesicles to regulate neurotrophic signaling in neurons. Cell Death Dis (2018) 9: doi:10.1038/s41419-018-0369-4 
19. Driedonks TAP, van der Grein SG, Ariyurek Y, Buermans HPJ, Jekel H, Chow FWN, Wauben MHM, Buck AH, Hoen PAC, Nolte-'t Hoen ENM. Immune stimuli shape the small non-coding transcriptome of extracellular vesicles released by dendritic cells. Cell Mol Life Sci (2018)3857-3875. doi:10.1007/s00018018-2842-8

20. Katsuda T, Kosaka N, Ochiya T. The roles of extracellular vesicles in cancer biology: Toward the development of novel cancer biomarkers. Proteomics (2014) 14:412-425. doi:10.1002/pmic.201300389

21. Cheng L, Doecke JD, Sharples RA, Villemagne VL, Fowler CJ, Rembach A, Martins RN, Rowe CC, Macaulay SL, Masters CL, et al. Prognostic serum miRNA biomarkers associated with Alzheimer's disease shows concordance with neuropsychological and neuroimaging assessment. Mol Psychiatry (2014)1-9. doi:10.1038/mp.2014.127

22. Buschmann D, Kirchner B, Hermann S, Märte M, Wurmser C, Brandes F, Kotschote S, Bonin M, Steinlein OK, Pfaffl MW, et al. Evaluation of serum extracellular vesicle isolation methods for profiling miRNAs by next-generation sequencing. J Extracell Vesicles (2018) 7: doi:10.1080/20013078.2018.1481321

23. Srinivasan S, Yeri A, Cheah PS, Chung A, Danielson K, De Hoff P, Filant J, Laurent CD, Laurent LD, Magee $\mathrm{R}$, et al. Small RNA Sequencing across Diverse Biofluids Identifies Optimal Methods for exRNA Isolation. Cell (2019) 177:446-462.e16. doi:10.1016/j.cell.2019.03.024

24. Nolte-'t Hoen ENM, Buermans HPJ, Waasdorp M, Stoorvogel W, Wauben MHM, 't Hoen PAC. Deep sequencing of RNA from immune cell-derived vesicles uncovers the selective incorporation of small non-coding RNA biotypes with potential regulatory functions. Nucleic Acids Res (2012) 40:9272-85. doi:10.1093/nar/gks658

25. Driedonks TAP, Nolte-'t Hoen ENM. Circulating Y-RNAs in Extracellular Vesicles and Ribonucleoprotein Complexes; Implications for the Immune System. Front Immunol (2019) 9:1-15. doi:10.3389/ fimmu.2018.03164

26. Christov CP, Gardiner TJ, Szüts D, Krude T. Functional requirement of noncoding Y RNAs for human chromosomal DNA replication. Mol Cell Biol (2006) 26:6993-7004. doi:10.1128/MCB.01060-06

27. Gardiner TJ, Christov CP, Langley AR, Krude T. A conserved motif of vertebrate Y RNAs essential for chromosomal DNA replication. RNA (2009) 15:1375-1385. doi:10.1261/rna.1472009

28. Sim S, Weinberg DE, Fuchs G, Choi K, Chung J, Wolin SL. The Subcellular Distribution of an RNA Quality Control Protein, the Ro Autoantigen, Is Regulated by Noncoding Y RNA Binding. Mol Biol Cell (2009)

20:1555-1564. doi:10.1091/mbc.E08

29. Fuchs G, Stein AJ, Fu C, Reinisch KM, Wolin SL. Structural and biochemical basis for misfolded RNA recognition by the Ro autoantigen. Nat Struct Mol Biol (2006) 13:1002-1009. doi:10.1038/nsmb1156

30. Perreault J, Noël JF, Brière F, Cousineau B, Lucier JF, Perreault JP, Boire G. Retropseudogenes derived from the human Ro/SS-A autoantigen-associated hY RNAs. Nucleic Acids Res (2005) 33:2032-2041. doi:10.1093/nar/gki504

31. Rutjes SA, van der Heijden A, Utz PJ, van Venrooij WJ, Pruijn GJ. Rapid nucleolytic degradation of the small cytoplasmic Y RNAs during apoptosis. J Bio/ Chem (1999) 274:24799-807. doi:10.1074/ jbc.274.35.24799

32. Vojtech L, Woo S, Hughes S, Levy C, Ballweber L, Sauteraud RP, Strobl J, Westerberg K, Gottardo R, Tewari $\mathrm{M}$, et al. Exosomes in human semen carry a distinctive repertoire of small non-coding RNAs with potential regulatory functions. Nucleic Acids Res (2014) 42:7290-7304. doi:10.1093/nar/gku347

33. Alexander M, Hu R, Runtsch MC, Kagele DA, Mosbruger TL, Tolmachova T, Seabra MC, Round JL, Ward DM, O'Connell RM. Exosome-delivered microRNAs modulate the inflammatory response to endotoxin. Nat Commun (2015) 6:7321. doi:10.1038/ncomms8321

34. Zhang Y, Liu D, Chen X, Li J, Li L, Bian Z, Sun F, Lu J, Yin Y, Cai X, et al. Secreted Monocytic miR150 Enhances Targeted Endothelial Cell Migration. Mol Cell (2010) 39:133-144. doi:10.1016/j. molcel.2010.06.010 
35. Yeri A, Courtright A, Reiman R, Carlson E, Beecroft T, Janss A, Siniard A, Richholt R, Balak C, Rozowsky J, et al. Total Extracellular Small RNA Profiles from Plasma, Saliva, and Urine of Healthy Subjects. Sci Rep (2017) 7:44061. doi:10.1038/srep44061

36. Godoy PM, Bhakta NR, Barczak AJ, Cakmak H, Fisher S, Mackenzie TC, Patel T, Price RW, Smith J, Woodruff PG, et al. Large Differences in Small RNA Composition Between Human Biofluids. Cell Rep (2018) 25:1346-1358. doi:10.2139/ssrn.3155656

37. Dhahbi JM, Spindler SR, Atamna H, Boffelli D, Martin DIK. Deep Sequencing of Serum Small RNAs Identifies Patterns of 5'tRNA Half and YRNA Fragment Expression Associated with Breast Cancer. Biomark Cancer (2014) 6:37-47. doi:10.4137/BIC.S20764.RECEIVED

38. Repetto E, Lichtenstein L, Hizir Z, Tekaya N, Benahmed M, Ruidavets J-B, Zaragosi L-E, Perret B, Bouchareychas L, Genoux A, et al. RNY-derived small RNAs as a signature of coronary artery disease. BMC Med (2015) 13:259. doi:10.1186/s12916-015-0489-y

39. Peters AL, van Hezel ME, Cortjens B, Tuip-de Boer AM, van Bruggen R, de Korte D, Jonkers RE, Bonta PI, Zeerleder SS, Lutter R, et al. Transfusion of 35-Day Stored RBCs in the Presence of Endotoxemia Does Not Result in Lung Injury in Humans*. Crit Care Med (2016) 44:e412-e419. doi:10.1097/CCM.0000000000001614

40. Peters AL, van Hezel ME, Klanderman RB, Tuip-de Boer AM, Wiersinga WJ, van der Spek AH, van Bruggen R, de Korte D, Juffermans NP, Vlaar APJ. Transfusion of 35-day-stored red blood cells does not alter lipopolysaccharide tolerance during human endotoxemia. Transfusion (2017) 57:1359-1368. doi:10.1111/trf.14087

41. Driedonks TAP, Nijen-Twilhaar MK, Nolte-'t Hoen ENM. Technical approaches to reduce interference of Fetal calf serum derived RNA in the analysis of extracellular vesicle RNA from cultured cells. J Extracell Vesicles (2018) 8: doi: 10.1080/20013078.2018.1552059

42. Burger P, Hilarius-Stokman P, Korte D De, Berg TKVan Den, Bruggen RVan. CD47 functions as a molecular switch for erythrocyte phagocytosis. Blood (2012) 119:5512-5522. doi:10.1182/blood-201110-386805

43. Schmitt C, Abt M, Ciorciaro C, Kling D, Jamois C, Schick E, Solier C, Benghozi R, Gaudreault J. First-in-man Study with Inclacumab, a Human Monoclonal Antibody Against P-selectin. J Cardiovasc Pharmacol (2015) 65:611-619. doi:10.1097/FJC.0000000000000233

44. van der Vlist EJ, Nolte-'t Hoen ENM, Stoorvogel W, Arkesteijn GJA, Wauben MHM. Fluorescent labeling of nanosized vesicles released by cells and subsequent quantitative and qualitative analysis by high-resolution flow cytometry. Nat Protoc (2012) 7:1311-1326. doi:10.1038/nprot.2012.065

45. Nolte-'t Hoen ENM, van der Vlist EJ, Aalberts M, Mertens HCH, Bosch BJ, BartelinkW, Mastrobattista E, van Gaal EVB, Stoorvogel W, Arkesteijn GJ a, et al. Quantitative and qualitative flow cytometric analysis of nanosized cellderived membrane vesicles. Nanomedicine (2012) 8:712-720. doi:10.1016/j.nano.2011.09.006

46. Groot KormelinkT, Arkesteijn GJA, Nauwelaers FA, van den Engh G, Nolte-'t Hoen ENM, Wauben MHM. Prerequisites for the analysis and sorting of extracellular vesicle subpopulations by high-resolution flow cytometry. Cytom Part A (2016) 89:135-147. doi:10.1002/cyto.a.22644

47. Libregts SFWM, Arkesteijn GJA, Németh A, Nolte-'t Hoen ENM, Wauben MHM. Flow cytometric analysis of extracellular vesicle subsets in plasma: impact of swarm by particles of non-interest. J Thromb Haemost (2018) 16:1423-1436. doi:10.1111/jth.14154

48. Robin X, Turck N, Hainard A, Tiberti N, Lisacek F, Sanchez J-C, Müller M. pROC: an open-source package for R and S+ to analyze and compare ROC curves. BMC Bioinformatics (2011) 12: doi:10.1007/s00134-009-1641-y

49. Williams Z, Ben-Dov IZ, Elias R, Mihailovic A, Brown M, Rosenwaks Z, TuschlT. Comprehensive profiling of circulating microRNA via small RNA sequencing of cDNA libraries reveals biomarker potential and limitations. Proc Natl Acad Sci (2013) 110:4255-4260. doi:10.1073/pnas.1214046110

50. Feingold K, Grunfeld C. "Introduction to Lipids and Lipoproteins," in Endotext, eds. K.Feingold, B. Anawalt, A. Boyce (MDtext.com). Available at: https://europepmc.org/books/ NBK305896;jsessionid=FC86076A4DE6F06B7F048A4DFF4C7F05\%0A 
51. Karimi N, Cvjetkovic A, Jang SC, Crescitelli R, Hosseinpour Feizi MA, Nieuwland R, Lötvall J, Lässer C. Detailed analysis of the plasma extracellular vesicle proteome after separation from lipoproteins. Cell Mol Life Sci (2018)1-14. doi:10.1007/s00018-018-2773-4

52. Arraud N, Linares R, Tan S, Gounou C, Pasquet JM, Mornet S, Brisson AR. Extracellular vesicles from blood plasma: Determination of their morphology, size, phenotype and concentration. J Thromb Haemost (2014) 12:614-627. doi:10.1111/jth.12554

53. Sabroe I, Prince LR, Jones EC, Horsburgh MJ, Foster SJ, Vogel SN, Dower SK, Whyte MKB. Selective Roles for Toll-Like Receptor (TLR)2 and TLR4 in the Regulation of Neutrophil Activation and Life Span. J Immunol (2014) 170:5268-5275. doi:10.4049/jimmunol.170.10.5268

54. Wang J, Williams JC, Davis BK, Jacobson K, Doerschuk CM, Ting JP-Y, Mackman N. Monocytic microparticles activate endothelial cells in an IL-1b dependent manner. Blood (2011) 118:2366-2374. doi:10.1182/blood-2011-01-330878

55. Lowry SF. Human endotoxemia: A model for mechanistic insight and therapeutic targeting. Shock (2005) 24:94-100. doi:10.1097/01.shk.0000191340.23907.a1

56. Andreasen A, Krabbe K, Krogh-Madsen R, Taudorf S, Pedersen B, Moller K. Human endotoxinemia as a model of systemic inflammation. Curr Med Chem (2008) 15:1697-1705.

57. van Lier D, Geven C, Leijte GP, Pickkers P. Experimental human endotoxemia as a model of systemic inflammation. Biochimie (2018)1-8. doi:10.1016/j.biochi.2018.06.014

58. Hotchkiss RS, Monneret G, Payen D. Sepsis-induced immunosuppression: From cellular dysfunctions to immunotherapy. Nat Rev Immunol (2013) 13:862-874. doi:10.1038/nri3552

59. Netea MG, Van Der Meer JWM, Van Deuren M, Kullberg BJ. Proinflammatory cytokines and sepsis syndrome: Not enough, or too much of a good thing? Trends Immunol (2003) 24:254-258. doi:10.1016/S1471-4906(03)00079-6

60. Bellingham SA, Coleman BM, Hill AF. Small RNA deep sequencing reveals a distinct miRNA signature released in exosomes from prion-infected neuronal cells. Nucleic Acids Res (2012) 40:10937-10949. doi:10.1093/nar/gks832

61. Wei Z, Batagov AO, Schinelli S, Wang J, Wang Y, El Fatimy R, Rabinovsky R, Balaj L, Chen CC, Hochberg F, et al. Coding and noncoding landscape of extracellular RNA released by human glioma stem cells. Nat Commun (2017) 8:1145. doi:10.1038/s41467-017-01196-x

62. Tosar JP, Gambaro F, Sanguinetti J, Bonilla B, Witwer KW, Cayota A. Assessment of small RNA sorting into different extracellular fractions revealed by high-throughput sequencing of breast cell lines. Nucleic Acids Res (2015)1-16. doi:10.1093/nar/gkv432

63. Shurtleff MJ, Yao J, Qin Y, Nottingham RM, Temoche-Diaz MM, Schekman R, Lambowitz AM. Broad role for YBX1 in defining the small noncoding RNA composition of exosomes. Proc Natl Acad Sci (2017) 114:E8987-E8995. doi:10.1073/pnas.1712108114

64. Lässer C, Shelke GV, Yeri A, Kim D-K, Crescitelli R, Raimondo S, Sjöstrand M, Gho YS, Van Keuren Jensen K, Lötvall J. Two distinct extracellular RNA signatures released by a single cell type identified by microarray and next-generation sequencing. RNA Biol (2017) 14:58-72. doi:10.1080/15476286.2016.12 49092

65. Jeppesen DK, Fenix AM, Franklin JL, Higginbotham JN, Zhang Q, Zimmerman LJ, Liebler DC, Ping J, Liu Q, Evans R, et al. Reassessment of Exosome Composition. Cell (2019) 177:428-445.e18. doi:10.1016/j. cell.2019.02.029

66. Van Deun J, Mestdagh P, Sormunen R, Cocquyt V, Vermaelen K, Vandesompele J, Bracke M, De Wever O, Hendrix A. The impact of disparate isolation methods for extracellular vesicles on downstream RNA profiling. J Extracell Vesicles (2014) 3:1-14. doi:10.3402/jev.v3.24858

67. Karttunen J, Heiskanen M, Navarro-Ferrandis V, Das Gupta S, Lipponen A, Puhakka N, Rilla K, Koistinen A, Pitkänen A. Precipitation-based extracellular vesicle isolation from rat plasma co-precipitate vesicle-free microRNAs. J Extracell Vesicles (2018) 8: doi:10.1080/20013078.2018.1555410 
68. Théry C, Witwer KW, Aikawa E, Alcaraz MJ, Anderson JD, Andriantsitohaina R, Antoniou A, Arab T, Archer F, Atkin-Smith GK, et al. Minimal information for studies of extracellular vesicles 2018 (MISEV2018): a position statement of the International Society for Extracellular Vesicles and update of the MISEV2014 guidelines. J Extracell Vesicles (2018) 8:1535750. doi:10.1080/20013078.2018.1535750

69. Sódar BW, Kittel Á, Pálóczi K, Vukman KV, Osteikoetxea X, Szabó-Taylor K, Németh A, Sperlágh B, Baranyai T, Giricz Z, et al. Low-density lipoprotein mimics blood plasma-derived exosomes and microvesicles during isolation and detection. Sci Rep (2016) 6:24316. doi:10.1038/srep24316

70. Hendrick JP, Wolin SL, Rinke J, Lerner MR, Steitz JA. Ro small cytoplasmic ribonucleoproteins are a subclass of La ribonucleoproteins: further characterization of the Ro and La small ribonucleoproteins from uninfected mammalian cells. Mol Cell Biol (1981) 1:1138-1149. doi:10.1128/MCB.1.12.1138. Updated

71. Clancy RM, Alvarez D, Komissarova E, Barrat FJ, Swartz J, Buyon JP. Ro60-Associated SingleStranded RNA Links Inflammation with Fetal Cardiac Fibrosis via Ligation of TLRs: A Novel Pathway to Autoimmune-Associated Heart Block. J Immunol (2010) 184:2148-2155. doi:10.4049/ jimmunol.0902248

72. Garberg H, Jonsson R, Brokstad KA. The serological pattern of autoantibodies to the Ro52, Ro60, and La48 autoantigens in primary Sjögren's syndrome patients and healthy controls. Scand J Rheumatol (2005) 34:49-55. doi:10.1080/03009740510017940

73. Nieuwland R, Berckmans RJ, McGregor S, Boing AN, Romijn FP, Westendorp RG, Hack CE, Sturk A. Cellular origin and procoagulant properties of microparticles in meningococcal sepsis. Blood (2000) 95:930-935. doi:10.1182/blood.v98.10.3143

74. Ng LG, Ostuni R, Hidalgo A. Heterogeneity of neutrophils. Nat Rev Immunol (2019) 4531200:255-265. doi:10.1038/s41577-019-0141-8

75. Rosales C. Neutrophil: A cell with many roles in inflammation or several cell types? Front Physiol (2018) 9:1-17. doi:10.3389/fphys.2018.00113

76. Vasilescu C, Rossi S, Shimizu M, Tudor S, Veronese A, Ferracin M, Nicoloso MS, Barbarotto E, Popa M, Stanciulea O, et al. MicroRNA fingerprints identify miR-150 as a plasma prognostic marker in patients with sepsis. PLoS One (2009) 4: doi:10.1371/journal.pone.0007405

77. Ma Y, Liu Y, Hou H, Yao Y, Meng H. MiR-150 predicts survival in patients with sepsis and inhibits LPSinduced inflammatory factors and apoptosis by targeting NF-KB1 in human umbilical vein endothelial cells. Biochem Biophys Res Commun (2018) 500:828-837. doi:10.1016/j.bbrc.2018.04.168

78. Benz F, Roy S, Trautwein C, Roderburg C, Luedde T. Circulating MicroRNAs as biomarkers for sepsis. Int J Mol Sci (2016) 17: doi:10.3390/ijms17010078

79. Reinhart K, Bauer M, Riedemann NC, Hartog CS. New approaches to sepsis: Molecular diagnostics and biomarkers. Clin Microbiol Rev (2012) 25:609-634. doi:10.1128/CMR.00016-12

80. Terrasini N, Lionetti V. Exosomes in critical illness. Crit Care Med (2017) 45:1054-1060. doi:10.1097/ CCM.00000000000002328

81. Raeven P, Zipperle J, Drechsler S. Extracellular vesicles as markers and mediators in sepsis. Theranostics (2018) 8:3348-3365. doi:10.7150/thno.23453

82. Groot KormelinkT, Mol S, de Jong EC, Wauben MHM. The role of extracellular vesicles when innate meets adaptive. Semin Immunopathol (2018)1-14. doi:10.1007/s00281-018-0681-1

83. Genschmer KR, Russell DW, Lal C, Szul T, Bratcher PE, Noerager BD, Abdul Roda M, Xu X, Rezonzew G, Viera L, et al. Activated PMN Exosomes: Pathogenic Entities Causing Matrix Destruction and Disease in the Lung. Cell (2019) 176:113-126.e15. doi:10.1016/j.cell.2018.12.002

84. Eken C, Gasser O, Zenhaeusern G, Oehri I, Hess C, Schifferli JA. Polymorphonuclear neutrophil-derived ectosomes interfere with the maturation of monocyte-derived dendritic cells. J Immunol (2008)

180:817-24. doi:10.4049/jimmunol.180.2.817 
6. Y-RNA subtype ratios in plasma extracellular vesicles are cell type specific

85. Angus DC, van der Poll T. Severe sepsis and septic shock. N Engl J Med (2013) 369:703-707. doi:10.1007/978-81-322-0535-7_88

86. Alvarez-Erviti L, Seow Y, Yin H, Betts C, Lakhal S, Wood MJA. Delivery of siRNA to the mouse brain by systemic injection of targeted exosomes. Nat Biotechnol (2011) 29:306-309. doi:10.1038/nbt.1807

87. EL Andaloussi S, Mäger I, Breakefield XO, Wood MJ. Extracellular vesicles: biology and emerging therapeutic opportunities. Nat Rev Drug Discov (2013) 12:347-357. doi:10.1038/nrd3978 
a
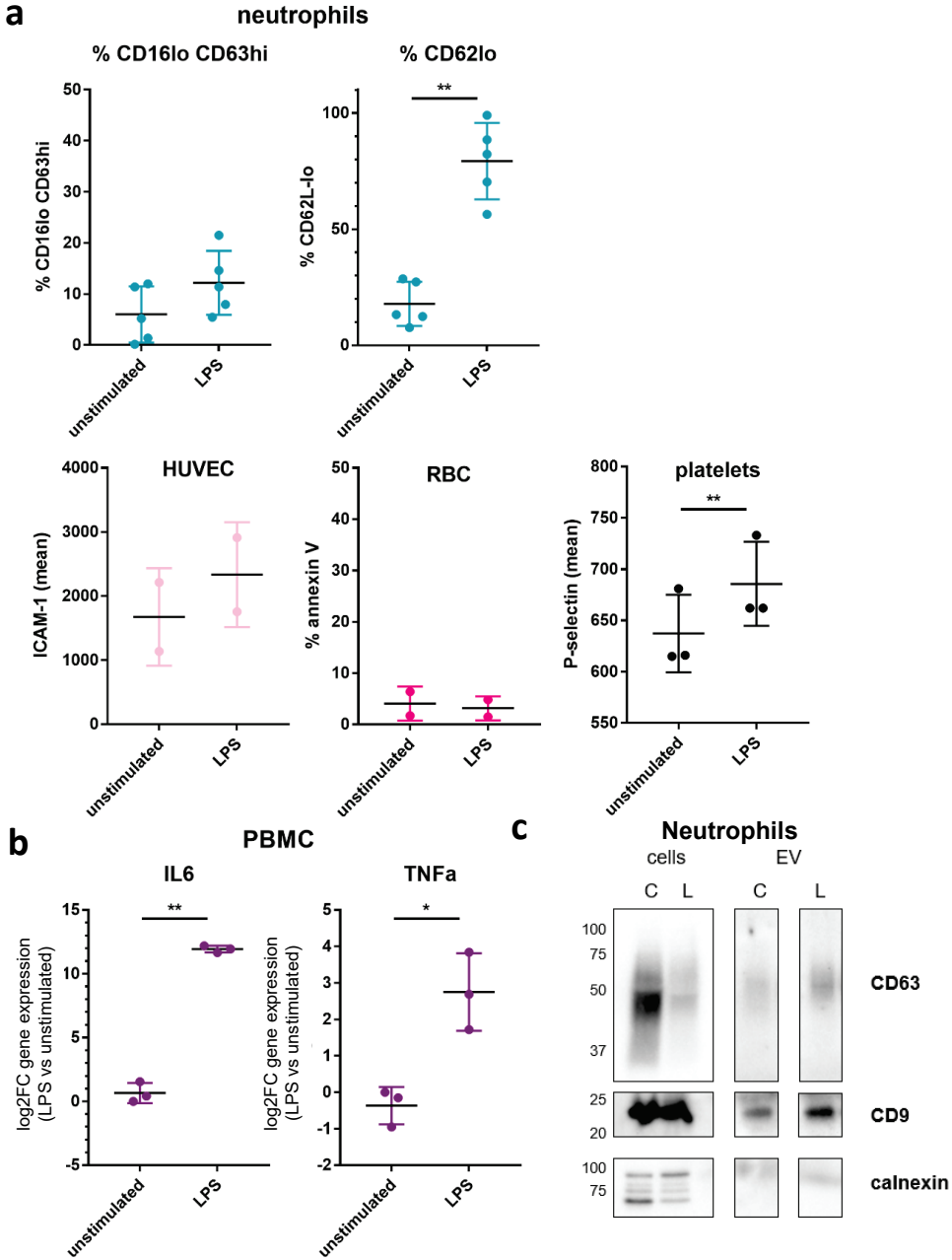

Supplementary Figure 1. Responses of blood cells to treatment with LPS. A) Neutrophils, HUVEC, RBC and platelets were cultured in EV-depleted medium for 2-4h in the presence or absence of LPS. Activation was measured by flow cytometric analysis of cell type specific activation markers, gates were set relative to unstained cells. Neutrophil activation (top) was measured as $\%$ CD16-lo/CD63hi and \% CD62L-lo cells, RBC activation was measured as \% annexin-V positive cells. HUVEC and platelet activation was measured as the geomean fluorescence of ICAM-1 or P-selectin, respectively. ${ }^{*} p<0.05$, ${ }^{* *}<p ~ 0.01$, Student's paired samples t-test B) PBMC were cultured in EV-depleted medium for $4 \mathrm{~h}$ in the presence or absence of LPS. Total cellular RNA was isolated and RT-qPCR was performed for the quantification of IL-6 and TNFa mRNA. Gene expression was shown as log2fold change in LPS stimulated cells versus unstimulated cells. $n=3$ experiments, ${ }^{*} p<0.05$, Student's paired samples t-test. C) Neutrophils were cultured in EVdepleted medium in the absence (C) or presence of LPS (L). EV were purified by differential centrifugation and density gradient centrifugation. Equal amounts of cellular proteins, or proteins from EV produced by equal numbers of cells were run on a SDS-PAGE gel and immunoblotted with antibodies to CD63, CD9 and Calnexin. 
a

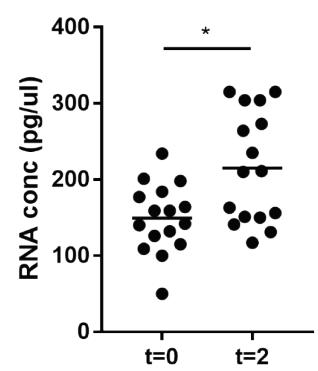

C

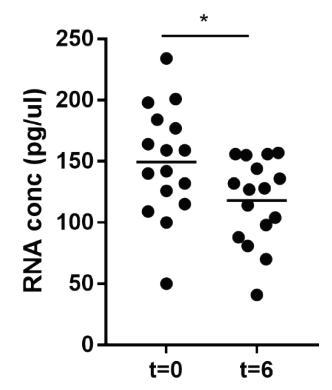

b

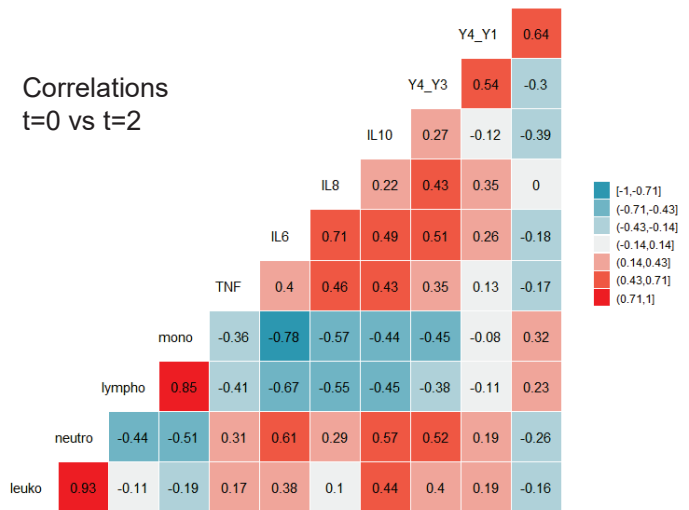

d

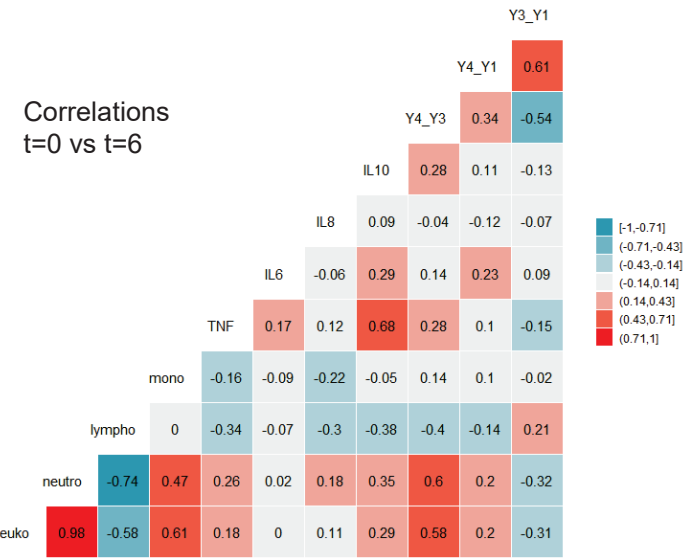

Supplementary Figure 2. The total RNA concentration in plasma is increased during endotoxemia. 16 healthy volunteers were infused with LPS and plasma was collected before, two and six hours after infusion. RNA was isolated from $100 \mu$ plasma. A) Isolated RNA was quantified on a Bioanalyzer Pico chip. Depicted are the RNA concentrations for all volunteers at $t=0$ and $\mathrm{t}=2$. * $\mathrm{p}<0.05$, Student's paired t-test. B) Circulating immune cells and cytokine levels were previously determined in Peters et al 2016. Pearson's correlations between all measured immune cells, cytokine concentrations in plasma, and $Y-R N A$ ratios at $t=0$ and $t=2$ in 16 volunteers were calculated. Correlation heatmap shows the correlation coefficient between the different immune cells, cytokines and Y-RNA ratios. Positive correlations in shades of red, negative correlations in shades of blue. C) Isolated RNA was quantified on a Bioanalyzer Pico chip. Depicted are the RNA concentrations for all volunteers at $t=0$ and $t=6 .{ }^{*} p<0.05$, Student's paired t-test $D$ ) Correlation heatmap showing the Pearson's correlations between all measured immune cells, cytokine concentrations in plasma, and $\mathrm{Y}-\mathrm{RNA}$ ratios at $\mathrm{t}=0$ and $\mathrm{t}=6$ in 16 volunteers, similar to $\mathrm{B}$. 

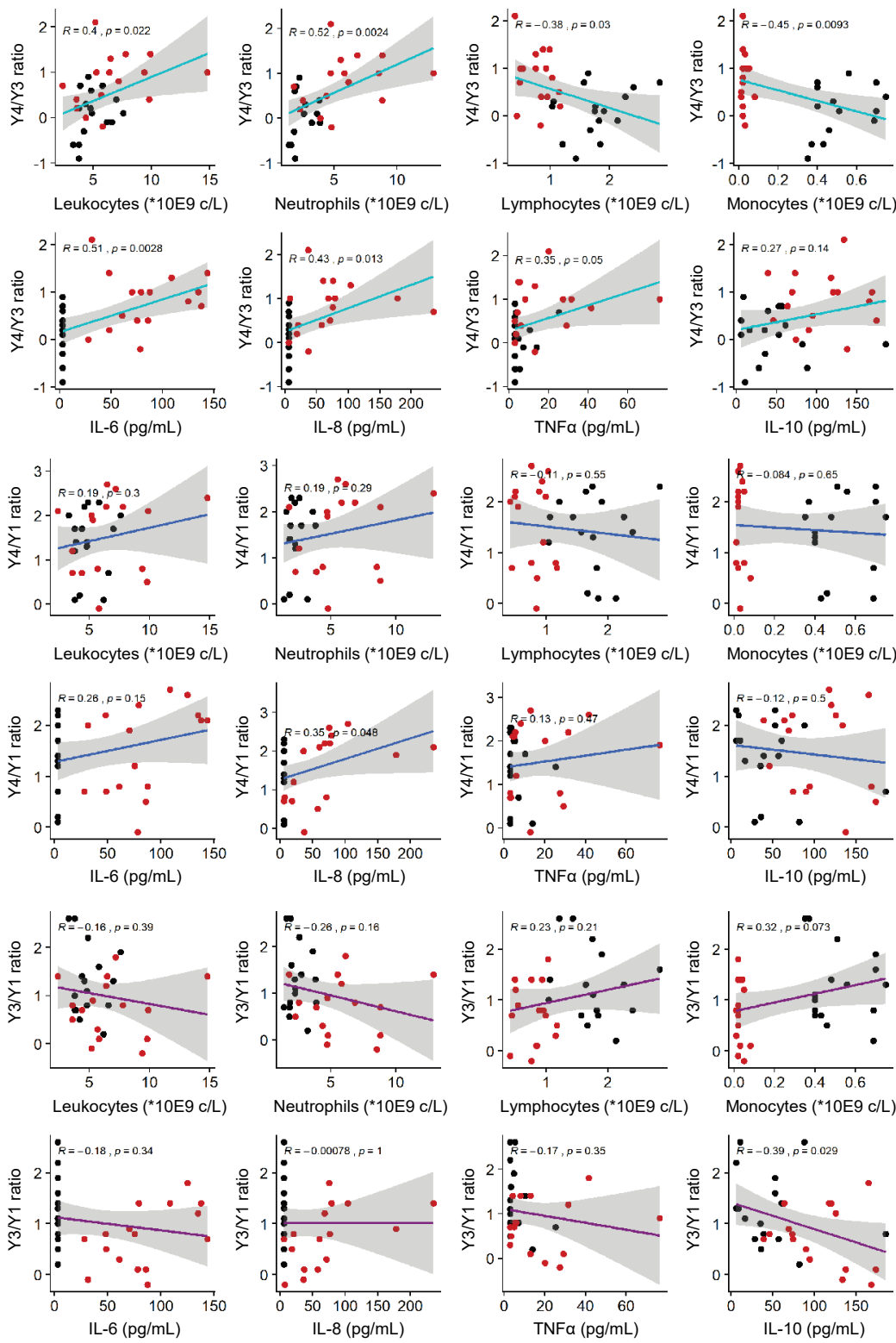

Supplementary Figure 3. All linear correlations between immune cells, cytokines and Y-RNA ratios between $t=0$ and $t=2$. 16 healthy volunteers were infused with LPS and plasma was collected before, and two hours after infusion. RNA was isolated from $100 \mu \mathrm{l}$ plasma. Circulating immune cells and cytokine levels were previously determined in Peters et al 2016. Linear correlations between all circulating immune cells and Y-RNA ratios in the volunteers at $\mathrm{t}=0$ (black dots) and $\mathrm{t}=2$ (red dots). Trendlines are shown in turquoise and the grey area depicts $95 \%$ confidence intervals. Pearson correlation coefficient (R) and significance $(p)$ are shown based on $n=16$ volunteers. 

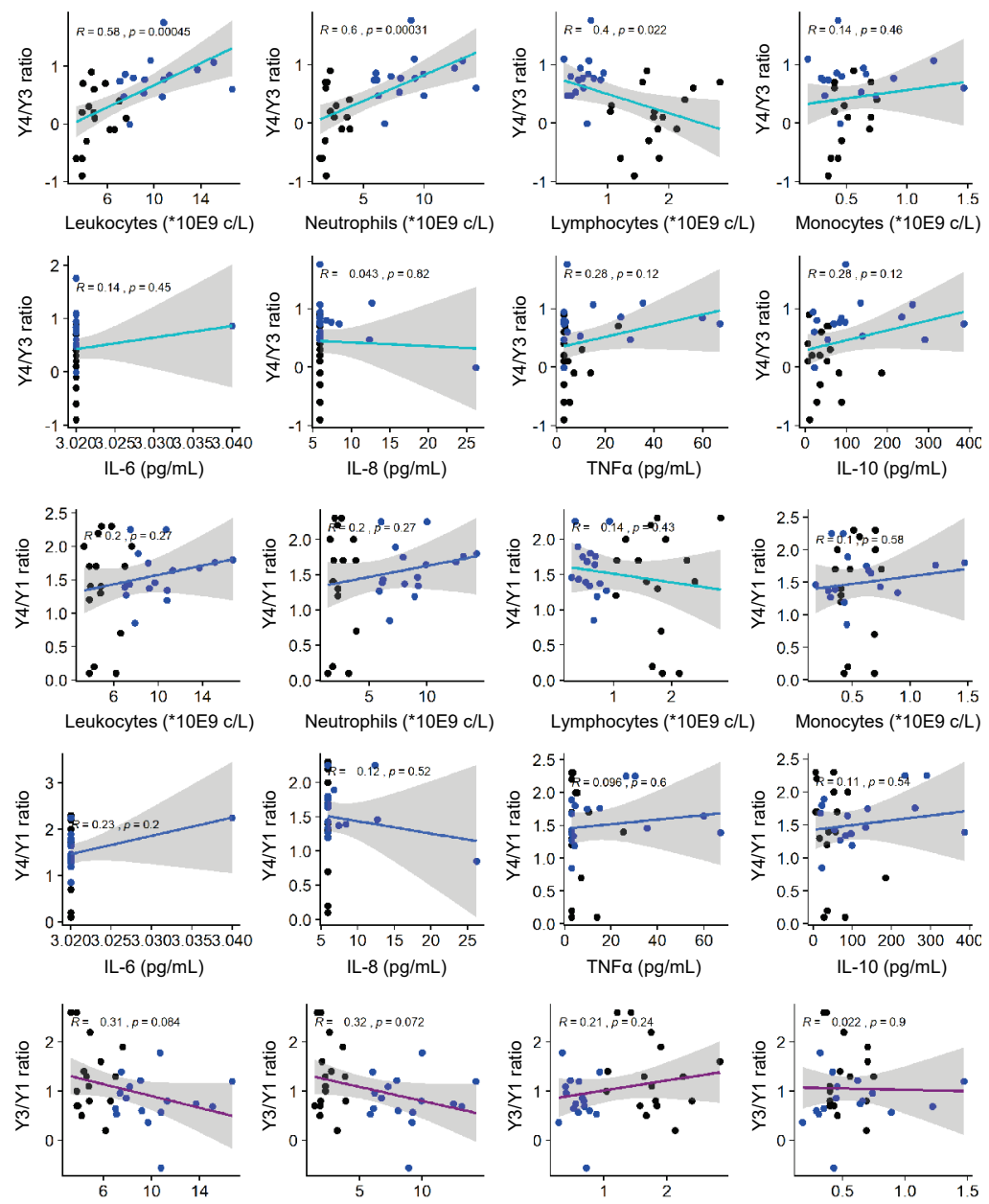

Leukocytes (*10E9 c/L)

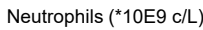

Lymphocytes ( $\left.{ }^{*} 10 \mathrm{E} 9 \mathrm{c} / \mathrm{L}\right)$
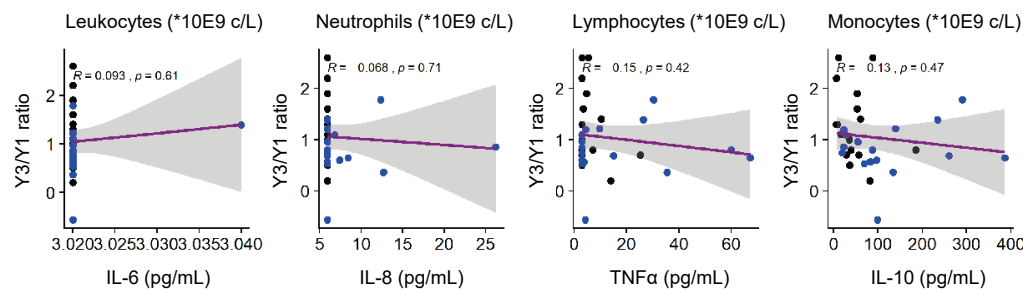

Supplementary Figure 4. All linear correlations between immune cells, cytokines and Y-RNA ratios between $\mathrm{t}=\mathbf{0}$ and $\mathrm{t}=\mathbf{6}$. 16 healthy volunteers were infused with LPS and plasma was collected before, and six hours after infusion. RNA was isolated from $100 \mu$ plasma. Circulating immune cells and cytokine levels were previously determined in Peters et al 2016. Linear correlations between all circulating immune cells and Y-RNA ratios in the volunteers at $t=0$ (black dots) and $t=6$ (blue dots). Trendlines are shown in turquoise and the grey area depicts $95 \%$ confidence intervals. Pearson correlation coefficient $(R)$ and significance $(p)$ are shown based on $n=16$ volunteers. 
संद्व $08 \%$

$\log ^{\circ} \mathrm{c}$

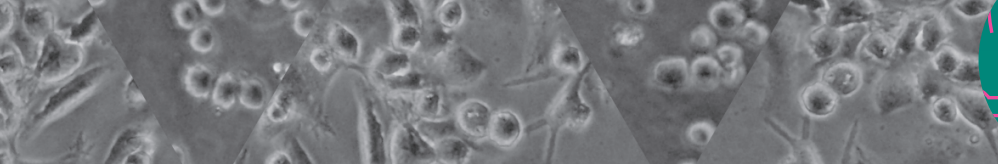
$\left.0^{\circ} \cos ^{\circ}\right)^{\circ}$

$2^{\circ} \mathrm{g}$

c

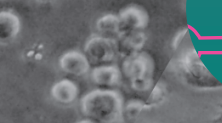
$30^{\circ} \mathrm{s}$

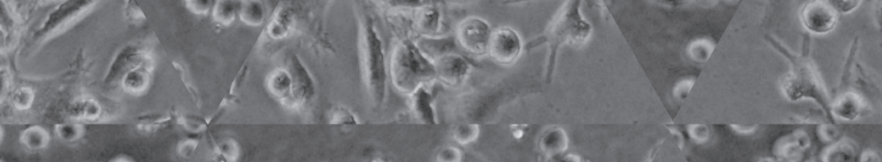

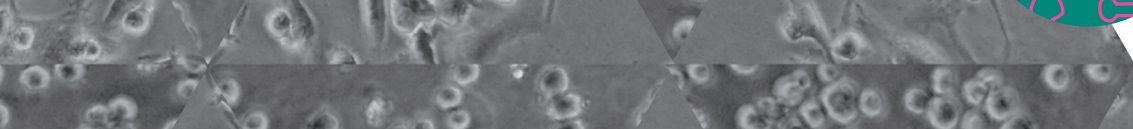

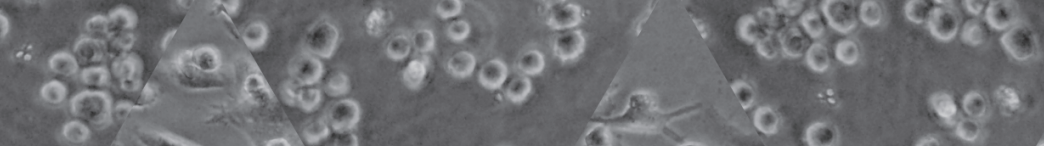

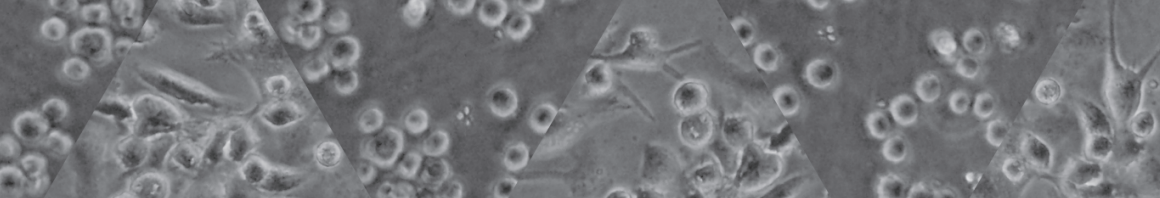

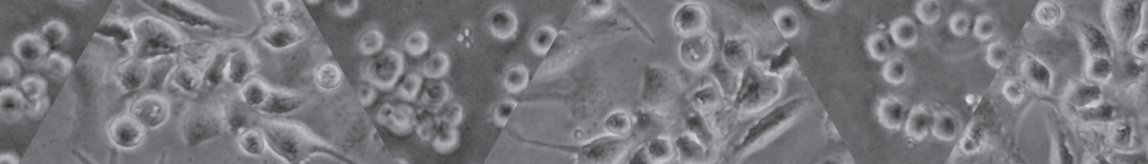

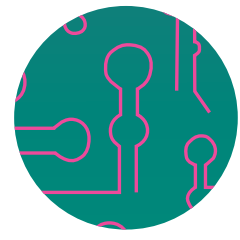
Wasero $=$ 
Chapter 7

The potential role of Y-RNA binding proteins in TLRinduced sorting of Y-RNAs into extracellular vesicles

T.A.P. Driedonks and E.N.M. Nolte-'t Hoen

Dept. Biochemistry \& Cell Biology, Fac. Veterinary Medicine, Utrecht University, The Netherlands

In preparation 


\section{Abstract}

Various small non-coding RNA species have been detected in extracellular vesicles (EV), Most of the previously performed studies have focused on the miRNA content of EV. However, other types of non-coding RNAs are more abundantly present in EV. Y-RNA has been detected in EV from various different cell types and body fluids. This small non-coding RNA type is evolutionary conserved and, in conjunction with interacting proteins, plays a role in maintaining cellular homeostasis. When associated to EV, Y-RNA has been implicated in immune-regulatory functions, and has been proposed as biomarker for cancer and coronary artery disease. Our previous work has shown that the levels of Y-RNA in EV released by primary dendritic cells were modulated by Toll-Like Receptor (TLR) signaling. This type of stimulation did not affect the cytoplasmic levels of Y-RNA, suggesting that Y-RNA shuttling into EV is a regulated process. It is currently unknown which mechanisms control the shuttling of Y-RNA into EV, but this process likely involves RNA-binding proteins (RBP). In cells, Y-RNA interacts with various RBP and some of these proteins have previously been detected in EV. We investigated whether these RBP could drive the TLR-mediated increase in Y-RNA shuttling into EV released by macrophages. Hereto, we tested whether Y-RNA binding proteins colocalized with Y-RNA in subcellular compartments and in EV. Our data indicate that TLR stimulation led to increased sorting of Y-RNA and Ro60 into EV, whereas other Y-RNA binding proteins were not detected in EV. In TLR-stimulated cells, Y-RNA levels were slightly but significantly increased in the endosomal compartments where EV biogenesis takes place. However, we did not observe increased recruitment of Ro60 to this compartment. Instead, we show that two Y-RNA binding proteins that are known to stabilize mRNAs, HuR and hnRNP K, translocated from the late endosomes to ER in response to TLR triggering. Based on these data, we speculate that RBP-mediated export of Y-RNA via EV is a mechanism to fine-tune the function of EV-producing cells, e.g. by changing the localization and function of Y-RNA binding proteins involved in translation. 


\section{Introduction}

Various types of non-coding RNA (ncRNA) have been discovered in EV $(1,2)$. Of these RNA types, miRNA have been studied most intensively because of their known effects on gene expression. In conjunction with the RISC complex, miRNAs bind to mRNAs in a sequence-specific manner, which leads to mRNA cleavage or translational repression (3). It has been shown that EV-mediated transfer of miRNAs functionally represses target mRNAs in recipient cells (4-6). Besides miRNAs, EV contain various other ncRNA types, such as Y-RNA, 7SL (SRP-RNA), tRNA, and snRNA $(1,2)$. In cells, many of these ncRNAs function as structural components of protein complexes involved in housekeeping processes, such as mRNA splicing, RNA quality control, and protein translation (7-10). It is largely unknown whether all of these ncRNA types contribute to cell-cell signaling via EV.

One of the ncRNA types that has been abundantly detected in EV released by different cell types and in various biofluids is Y-RNA (reviewed in (11)). This RNA is transcribed by RNA polymerase III and is evolutionary conserved among mammals (12). A structurally homologous stem-bulge RNAs have been found in nematodes (13) and bacteria (14). The human genome contains four different Y-RNA subtypes (Y1, Y3, Y4 and Y5), which all form stem-loop structures composed of a conserved stem and a variable loop region (15). Y-RNA acts as a scaffold for various proteins $(9,11,16)$. Of these proteins, Ro60 and La have been studied most intensively (9,17-19). Ro60 stabilizes cytoplasmic Y-RNA by binding its stem region, whereas La protects the $3^{\prime}$ oligo-uridine tail from exonucleic degradation. Binding by La additionally shields the $5^{\prime}$ triphosphate motif of Y-RNA (19), which is a ligand for the innate immune receptor RIG-I (20). Besides these two 'canonical' Y-RNA binding proteins, RNA affinity purification and RNA co-immunoprecipitation have revealed a wide range of other Y-RNA binding proteins $(11,16)$, which mostly bind to specific oligopyrimidine loops present in $\mathrm{Y} 1$ and $\mathrm{Y} 3 \mathrm{RNA}$. There are various examples of how Y-RNA affects protein localization (9). For instance, binding of Y-RNA to Ro60 is required to retain Ro60 in the cytoplasm (21). Binding of the foreign DNA binding protein ZBP1 (22) to Ro60/Y-RNA complexes in the nucleus mediates nuclear export (23). Although La complexed to Y-RNA is predominantly present in the nucleus (24), a minor fraction of La additionally localizes to the cytoplasm, potentially after cleavage of its nuclear localization signal $(19,25)$.

Besides influencing protein localization, Y-RNA binding can also influence the activity of its protein partners. For instance, Y-RNA regulates the binding of misfolded RNAs to the central cavity of Ro60, which has an important role in RNA degradation $(26,27)$. Furthermore, depletion of Y-RNA was shown to increase the affinity of enhancer protein HuD for the $3^{\prime}$ UTR region of its mRNA targets, thereby increasing 
mRNA stability and translation (28). Apart from these roles in RNA quality control and regulation of translation, Ro60 and Y-RNA have been implicated in various stress responses and DNA replication (reviewed in (9)), although the mechanisms underlying these processes remain to be uncovered. Together, this illustrates how cellular Y-RNA serves as a protein scaffold that regulates various cell functions.

A major question in the EV field is how specific RNA transcripts are packaged into EV, and how the activation status of cells controls these processes. The dominant idea is that RNAs are actively incorporated into EV by RNA binding proteins (29). Various proteins have been implicated in packaging miRNAs into EV, such as hnRNP A2B1, SYNCRIP, HuR and YBX1 (30-34). It is not understood how these miRNA-sorting processes are modulated by the activation status of cells. There is also limited knowledge on the mechanisms underlying incorporation of other small non-coding RNAs into EV.

We recently demonstrated that TLR-activation of primary dendritic cells not only modulated the EV miRNA composition, but also controlled the incorporation of Y-RNAs into EV (11,35). Interestingly, TLR stimulation led to changes in EV-associated Y-RNA, whereas intracellularY-RNA levels remained constant. This suggests that alterations in the amount of Y-RNA packaged in EV is not determined by intracellular levels of Y-RNA, but may be caused by changes in local concentrations of Y-RNA and Y-RNA binding proteins at sites of EV-biogenesis. In our current study, we designed an experimental approach to investigate which Y-RNA binding proteins drive enhanced shuttling of Y-RNAs into EV released by TLR-activated macrophages. We employed human THP1 monocytic cells differentiated into macrophages, which respond readily to TLR-activation but do not exhibit the donor variations observed in cultures of in vitro matured monocyte-derived dendritic cells or macrophages. In our approach, we performed parallel assessment of the colocalization of Y-RNAs with various Y-RNA binding proteins in subcellular compartments that are involved in EV biogenesis and in EV released by the cells. To identify proteins involved in Y-RNA sorting, we monitored TLR-induced changes in the subcellular localization of Y-RNA and various Y-RNA binding proteins. While multiple Y-RNA binding proteins were expressed by THP-1 cells, Ro60 was the only Y-RNA binding protein present at detectable levels in EV released by these cells. Inside cells, Y-RNA and Ro60 localized to late endosomal compartments where EV can be formed. TLR stimulation led to a small but significant increase in Y-RNA localization in the endosomal compartment. Interestingly, other Y-RNA binding proteins known to stabilize mRNAs for enhanced translation relocalized from late endosomes to ER upon TLR stimulation. We propose that specific Y-RNA binding proteins mediate sorting of Y-RNA into EV. Moreover, we speculate that enhanced release of Y-RNA via EV upon cellular activation may lead to changes in the localization and function of Y-RNA binding proteins involved in translation in the EV-releasing cells. 


\section{Materials and methods}

\section{Cells and EV production}

The human monocytic cell line THP1 was a kind gift from M. Boes at UMC Utrecht and was maintained in IMDM (Gibco, Paisley, UK) supplemented with 10\% FCS (Bodinco, Alkmaar, The Netherlands), $2 \mathrm{mM}$ ultraglutamine and $100 \mathrm{U} / \mathrm{ml}$ penicillin / streptomycin (Gibco, Paisley, UK). EV-depleted FCS was prepared as described before (36). FCS was diluted to $30 \%$ in IMDM and ultracentrifuged at 100,000 g (k-factor 334.2) for 15$18 \mathrm{~h}$ in a SW28 rotor. Medium was removed by pipetting until 10\% was left on top of the pellet, filtered in a $0.45 \mu \mathrm{m}$ bottle top filter. EV-depleted FCS was subsequently diluted to $10 \%$ and supplemented with ultraglutamine, penicillin and streptomycin (as described above) to make EV-depleted medium. Cells were sub-cultured by dilution twice per week. For all experiments, cells were seeded at 0.5 million cells $/ \mathrm{ml}$. The time-course experiment was done in 6-well dishes, all other experiments were done in T75 and T175 flasks. Cells were differentiated into a macrophage phenotype by treatment with $10 \mathrm{nM}$ phorbol-12-myristate-13-acetate (PMA, Sigma, St. Louis, MO) for $24 \mathrm{~h}$. Medium was removed, after which cells were washed with $3 \mathrm{ml}$ PBS (Gibco, Paisley, UK) and replenished with EV-depleted medium. Where indicated, cells were stimulated with $5 \mu \mathrm{g} / \mathrm{ml}$ Pam3CSK4 (EMC Microcollections, Tübingen, Germany). Subcellular fractionation was done after $4 \mathrm{~h}$ of culture, the culturing times of the timecourse experiment are indicated in Figure 2A. Cells were cultured for $20-24 \mathrm{~h}$ for EV production. Viability of cells was checked by Trypan Blue (Sigma, Sigma, St. Louis, MO) exclusion and was above $95 \%$ for all experiments.

\section{EV purification}

EV were purified by differential ultracentrifugation as described before (35). In brief, supernatant was collected and centrifuged $2 \times 10 \mathrm{~min}$ at $200 \mathrm{~g}, 2 \times 10 \mathrm{~min}$ at $500 \mathrm{~g}$, and $1 \times 30 \mathrm{~min}$ at $10,000 \mathrm{~g}$. Supernatant was carefully removed by pipetting, not disturbing the $10,000 \mathrm{~g}$ pellet, and EV were pelleted from this supernatant by centrifugation at 100,000 $\mathrm{g}$ for $65 \mathrm{~min}$ in a SW28 (k-factor 334.2) or SW40 (k-factor 280.3) rotor. EV pellets were resuspended in $20 \mu \mathrm{l}$ PBS $+0.2 \%$ BSA (previously depleted of particles by overnight centrifugation at 100,000g), and labeled with $1.5 \mu \mathrm{l}$ fluorescent dye PKH67 in $100 \mu$ liluent C (Sigma, St. Louis, MO). Labeled EV were mixed with 2.5 $\mathrm{M}$ sucrose, overlaid with a sucrose density gradient (2.0 M - 0.4 M sucrose in PBS) and centrifuged $15-18 \mathrm{~h}$ at $192,000 \mathrm{~g}$ in a SW40 rotor (k-factor 144.5). EV in sucrose fractions were quantified using high-resolution flow cytometry, or were pelleted from the fractions with densities of $1.11-1.18 \mathrm{~g} / \mathrm{ml}$ by dilution in PBS $+0.2 \%$ BSA followed by centrifugation for $65 \mathrm{~min}$ at $192,000 \mathrm{~g}$ in a SW40 rotor (k-factor 144.5). 


\section{High-resolution flow-cytometry}

Sucrose density fractions were diluted 20x in PBS just before flow-cytometric quantification on a BD Influx (BD Biosciences, San Jose, CA) with an optimized configuration for small particle detection $(37,38)$. In brief, fluorescence based threshold triggering was applied, and forward-scatter (FSC) was detected at a $15-25$ degree collection angle. Fluorescent 100 and $200 \mathrm{~nm}$ polystyrene beads (Fluospheres, Invitrogen, Carlsbad, CA) were used to calibrate the instrument before measurement. Fluorescent events were collected in a 30 second timeframe for all samples and were measured at event rates below 10,000 events/sec, which is below the limit of the electronic pulse processing of the BD Influx (39).

\section{Subcellular fractionation}

For the time course experiment, stimulated and unstimulated cells were washed and detached with PBS $+2 \mathrm{mM}$ EDTA at timepoints indicated in Figure 2A. Cells were pelleted $1 \mathrm{~min}$ at $10,000 \mathrm{~g}$, lysed in PBS $+1 \%$ Triton-X100 for 5 min on ice, after which nuclei were spun down $5 \mathrm{~min}$ at $16,000 \mathrm{~g}$ at $4^{\circ} \mathrm{C}$. Supernatant was collected, centrifuged again to remove any residual nuclei, mixed with $700 \mu \mathrm{l}$ Qiazol and stored at $-80^{\circ} \mathrm{C}$ until RNA was isolated. Subcellular fractionation performed using a protocol based on Gibbings et al $(40,41)$. Four hours after activation with Pam3CSK4, cells were harvested from flasks using a rubber cell scraper. 5 million cells per condition were pelleted at $200 \mathrm{~g}$ for $10 \mathrm{~min}$ at $4^{\circ} \mathrm{C}$, washed in ice-cold PBS and incubated for 10 minutes in ice-cold hypotonic homogenization buffer $(0.083 \mathrm{M}$ sucrose, $10 \mathrm{mM}$ HEPES, $1 \mathrm{mM}$ EGTA) supplemented with the following inhibitors: Complete Mini protease inhibitor cocktail (Roche, Basel, Switzerland), 200 MM MG132 proteasome inhibitor (Calbiochem, Darmstadt Germany), $8 \mathrm{mM}$ vanadyl ribonucleoside (New England Biolabs, Ipswitch, MA), $8 \mu \mathrm{l}$ RNAse Out (Invitrogen, Carlsbad, CA). Cells were homogenized by 40 passages through a $26 \mathrm{G}$ needle using a $1 \mathrm{~mL}$ syringe. Trypan blue exclusion was performed on the homogenates to ensure comparable numbers of cells were disrupted. Nuclei and large cell debris were removed by centrifugation for $5 \mathrm{~min}$ at $1000 \mathrm{~g}$ at $4^{\circ} \mathrm{C}$, after which the supernatant was subjected to a second centrifugation step to ensure all debris was removed. The post-nuclear cytosolic fraction was loaded on top of a $5-40 \%$ Optiprep (Axis-Shield, Dundee, UK) gradient in $78 \mathrm{mM} \mathrm{KCl}, 4 \mathrm{mM}$ $\mathrm{MgCl}_{2}, 8.4 \mathrm{mM} \mathrm{CaCl}, 10 \mathrm{mM} \mathrm{EGTA}, 50 \mathrm{mM}$ HEPES- $\mathrm{NaOH}$ pH 7.0 prepared in $5 W 60$ tubes. Tubes were spun in a SW60 rotor at 100,000 g (32,000 rpm k-factor 159.6) for $18-20 \mathrm{~h}$ at $4^{\circ} \mathrm{C}$ to separate the subcellular organelles according to their buoyant density. Subsequently, $300 \mu \mathrm{l}$ fractions were collected. Of these fractions $50 \mu$ aliquots were pooled for RNA isolation (fractions $1+2+3,4+5,6+7,8+9$ and 10+11+12). The remaining $250 \mu \mathrm{l}$ was pooled for TCA precipitation using the following protocol: sodium-deoxycholate (Sigma, St. Louis, MO) was added to the fractions at $125 \mathrm{\mu g} /$ 
$\mathrm{ml}$, after which $10 \%$ tricholoroacetic acid (final concentration) was added. Samples were incubated on ice for $15 \mathrm{~min}$, protein precipitates were pelleted for $10 \mathrm{~min}$ at $16,000 \mathrm{~g}$ in a cooled epifuge. Supernatant was removed gently, after which $0.5 \mathrm{ml}$ $-20^{\circ} \mathrm{C}$ acetone was added. Samples were incubated at $-20^{\circ} \mathrm{C}$ for $10 \mathrm{~min}$ followed by centrifugation for $10 \mathrm{~min}$ at $16,000 \mathrm{~g}$ at $4^{\circ} \mathrm{C}$. Supernatant was removed and $0.5 \mathrm{ml}$ $-20^{\circ} \mathrm{C}$ acetone was added followed by centrifugation for $10 \mathrm{~min}$ at $16,000 \mathrm{~g}$ at $4^{\circ} \mathrm{C}$ to wash. Supernatant was taken off, pellets were left to dry to the air until all acetone had evaporated. Pellets were resuspended in $30 \mu \mathrm{l} 2 \mathrm{x}$ SDS sample buffer and $5 \mu \mathrm{l} 1 \mathrm{M}$ Tris $\mathrm{pH} 8.8$ was added to adjust the $\mathrm{pH}$.

\section{Western blot}

Protein samples were denatured for 3 minutes at $100^{\circ} \mathrm{C}$ in $2 x$ SDS sample buffer and separated on a 10\% SDS-PAGE gel, followed by protein transfer onto $0.45 \mu \mathrm{m}$ Immobilon-P PVDF membranes (Millipore, Cork, Ireland). After blocking for $>1 \mathrm{~h}$ in blocking buffer $(0.5 \%$ Cold Fish Skin Gelatin (Sigma-Aldrich, St. Louis, MO) in PBS + $0.05 \%$ Tween-20), blots were incubated overnight at $4^{\circ} \mathrm{C}$ with primary antibodies rabbit-anti-YBX1 (Cell Signaling, 4202, 1:1000), rabbit-anti-Lamp-1 (SantaCruz, sc-5571, 1: 1000), mouse-anti-hnRNP K (SantaCruz, sc-28380, 1:5000), mouse-anti-Calnexin (Transduction Labs, clone 37, 1 : 1000), mouse-anti-HuR (SantaCruz, sc-5261, 1:1000), mouse-anti-Ro60 (a kind gift from S.L. Wolin, 1:50), rabbit-anti-La (Cell Signaling, 5034, 1:1000), mouse-anti-CD9 (BioLegend, HI9a, 1:1000), mouse-anti-CD63 (Abcam, ab59479, 1:1000), rabbit-anti-Histone-H3 (Cell Signaling, 9715, 1:1000), mouse-antiCD81 (SantaCruz, clone B-11, 1:1000), mouse-anti-EEA1 (Transduction Labs, clone 14/ EEA1, 1:1000), mouse-anti-gm130 (BD, clone 35/GM130, $1: 500$ ), and rabbit-anti-Tsg101 (reduced conditions with 100 mM DTT, Sigma, T5951, $1: 1000$ ) in blocking buffer. Blots were washed and incubated for 1 - $2 \mathrm{~h}$ with HRP-coupled secondary antibodies (Dako, cat P0450 and P0448, 1 : 5000). ECL solution (ThermoScientific, SuperSignal West Dura Extended Duration Substrate, cat. 34075) was used for detection on a Chemidoc imager (BioRad, Hercules, CA). Images were analyzed by Image Lab software (BioRad, Hercules, CA).

\section{RNA isolation, cDNA synthesis and RT-qPCR}

mRNA was isolated from cell pellets $4 \mathrm{~h}$ after stimulation using the Nucleospin RNA kit (Macherey-Nagel, Düren, Germany) according to the manufacturer's instructions. cDNA was prepared using the RevertAid cDNA kit (ThermoScientific) following the manufacturer's instructions and using random hexamer primers.

Small non-coding RNA was isolated from pelleted cells, EV, or from pooled subcellular fractions using the miRNeasy micro kit (Qiagen, Hilden, Germany). $700 \mu \mathrm{l}$ Qiazol was added to pellets or to $150 \mu$ l pooled Optiprep fractions, after which the 
manufacturer's instructions were followed. Quantification of the eluted RNA was done by Bioanalyzer Pico 6000 (Agilent, Waldbronn, Germany). cDNA synthesis was done using the miScript RT II kit (Qiagen, Hilden, Germany) according to the manufacturer's instructions, RNA input was normalized according to the concentration of $20-300$ nt small RNA. CDNA was diluted 10x in MQ water, and $2 \mu$ diluted CDNA template was used for amplification of target RNAs in qPCR with $0.8 \mu \mathrm{M}$ primers (IDT, Leuven, Belgium) in SensiMix PCR master mix (BioLine, United Kingdom). The following PCR program was used for amplification of mRNA: $95^{\circ} \mathrm{C} 10 \mathrm{~min}$, followed by 40 cycles of $10 \mathrm{~s}$ at $95^{\circ} \mathrm{C}, 20 \mathrm{~s}$ at $60^{\circ} \mathrm{C}$ and $45 \mathrm{~s}$ at $72^{\circ} \mathrm{C}$. For small RNA we used the following PCR program: $95^{\circ} \mathrm{C} 10 \mathrm{~min}$, followed by 50 cycles of $10 \mathrm{~s}$ at $95^{\circ} \mathrm{C}, 20 \mathrm{~s}$ at $57^{\circ} \mathrm{C}$ and $30 \mathrm{~s}$ at $72^{\circ} \mathrm{C}$. Melt curves were run from $65-95^{\circ} \mathrm{C}$ after each PCR. All qPCRs were performed on a Biorad CFX96 or CFX384 machine (BioRad, Hercules, CA). Normalization of small RNA qPCR data was done relative to U6 snRNA in line with earlier studies $(35,42)$, and to beta-actin for mRNA qPCR data.

The following PCR primers were used, primers 1 - 7 were used in combination with the miscript universal reverse primer, primers $8-17$ were used as pairs of specific forward and reverse primers:

Table 1. qPCR primers

\begin{tabular}{|c|c|c|}
\hline No & Primer name & Sequence $\left(5^{\prime}-3^{\prime}\right)$ \\
\hline 1 & Y1 loop-F & GATCGAACTCCTTGTTCTACTC \\
\hline 2 & Y3 loop-F & AGATTTCTTTGTTCCTTCTCCACTC \\
\hline 3 & Y4 loop-F & GTGTCACTAAAGTTGGTATACAAC \\
\hline 4 & Y5 loop-F & GTTAAGTTGATTTAACATTGTCTC \\
\hline 5 & miR-146a & TGAGAACTGAATTCCATGGGT \\
\hline 6 & U6-F & CTCGCTTCGGCAGCACA \\
\hline 7 & $7 S L-F$ & GGAGTTCTGGGCTGTAGTGC \\
\hline 8 & $5 S-F$ & TCTACGGCCATACCACCCTGA \\
\hline 9 & $5 S-R$ & GCCTACAGCACCCGGTATTCC \\
\hline 10 & $18 \mathrm{~S}-\mathrm{F}$ & CGCGGTTCTATTTTTGTTGGT \\
\hline 11 & $18 S-R$ & AGTCGGCATCGTTTATGGTT \\
\hline 12 & hActin-F & CCTTCCTGGGCATGGAGTCCTG \\
\hline 13 & hActin-R & GGAGCAATGATCTTGATCTTC \\
\hline 14 & hIL-6-F & AACCTGAACCTTCCAAAGATGG \\
\hline 15 & hIL-6-R & TCTGGCTTGTTCCTCACTACT \\
\hline 16 & hTNFa-F & ATGAGCACTGAAAGCATGATCC \\
\hline 17 & hTNFa-R & GAGGGCTGATTAGAGAGAGGTC \\
\hline
\end{tabular}




\section{Microscopy}

Microscopic images were taken on a Olympus CK2 light microscope with a Leica camera at 200x magnification.

\section{Statistical analysis}

Statistical analyses were done in SPSS v24, p-values $<0.05$ were considered statistically significant.

\section{Results}

To study how TLR stimulation affected the trafficking of Y-RNA inside cells and into EV, we used the human monocytic cell line THP1, which we differentiated into macrophages. First, we assessed how TLR-stimulation of THP1 cells affected the number and Y-RNA content of EV released by these cells. THP1 cells differentiated into macrophages were stimulated with the TLR2/1 ligand Pam3CSK4. Treatment with this TLR ligand more potently activated the THP1 cells than LPS treatment (data not shown). Four hours after TLR activation, the cells displayed morphological changes characteristic for cellular activation (43) (Supplementary Figure 1A). Other hallmarks of cellular activation were the increased levels of mRNA for the inflammatory cytokines IL-6 and TNFa, which could readily be observed at four hours after TLR activation (Supplementary Figure 1B). We collected EV produced during overnight incubation of stimulated and non-stimulated cells. EV were purified from cell culture supernatant by differential ultracentrifugation followed by density gradient purification and quantified by high-resolution flow cytometry (Figure 1A). Pam3CSK4-stimulated cells released over 2-fold more EV than unstimulated cells, while the buoyant density of these EV was similar (Figure 1 A,B). In addition, we observed a stimulation-induced increase in the release of common EV-associated proteins CD9, CD63 and CD81, as determined by western blotting.(Figure 1C). We previously observed that TLR4 stimulation of primary dendritic cells led to changes in the amount of Y-RNA in EV, while intracellular Y-RNA levels remained constant (35). For various endotoxin-responsive miRNAs, however, we observed that stimulation-induced changes in EV-associated miRNA levels reflected the changes in the intracellular miRNA levels (35). To evaluate whether RNA levels in EV were similarly regulated in Pam3CSK4-stimulated THP1 cells, we quantified changes in Y-RNA subtypes and endotoxin responsive miRNAs in stimulated and unstimulated THP1 cells and their released EV, using RT-qPCR analysis (Figure 1D-F). Pam3CSK4-activation of THP1 cells led to an increase in the amount of Y1, Y3 and Y4 per EV released by these cells (Figure 1D). In contrast, the intracellular levels of these Y-RNA subtypes remained unchanged after TLR-stimulation (Figure 1E), which is in line with our earlier findings in DC (35). As expected, the levels of the TLR-responsive miR-146a were 


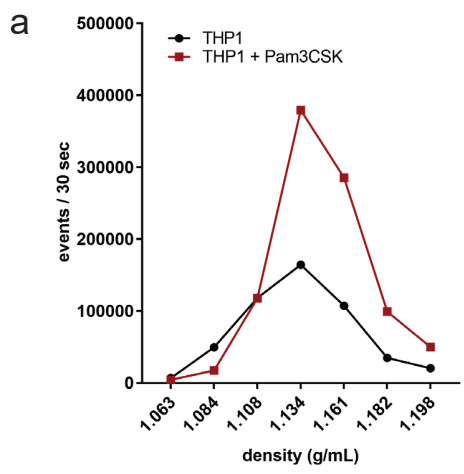

d

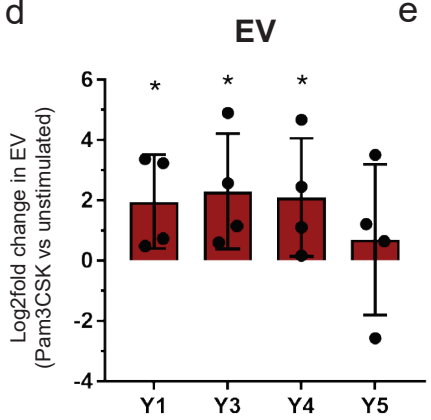

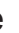

b

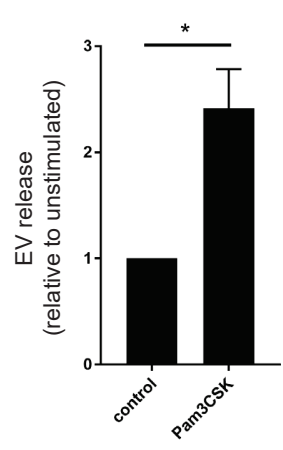

C

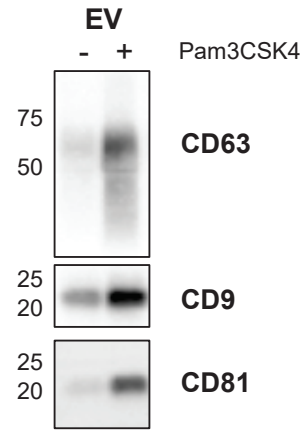

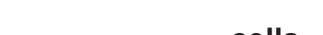

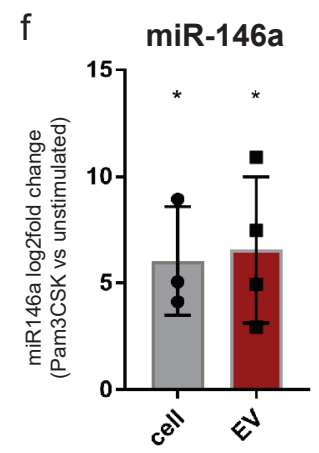

Figure 1. Pam 3CSK stimulation of THP1 macrophages increases the release of EV-associated Y-RNA. THP1 macrophages cultured in EV-depleted medium were stimulated with Pam3CSK4 or left unstimulated. After overnight incubation, EV were purified by differential ultracentrifugation, labelled with PKH67 fluorescent dye, and floated into a sucrose density gradient. A) High resolution flow cytometry based quantification of EV in the different sucrose density fractions. Indicated are the numbers of detected events within the fixed time frame of 30 seconds. Black line: unstimulated THP1, red line: Pam3CSK stimulated THP1. Distribution of EV over different density fractions is representative for $n=3$ experiments. $B$ ) Relative quantification of $E V$ in the peak fractions (1.11 -1.18 $\mathrm{g} / \mathrm{ml}$ ) as determined by high resolution flow cytometry. Data are normalized to the unstimulated condition for each experiment. * $p<0.05$, Student's paired $t$ test, $n=3$ independent experiments. C) EV from unstimulated and Pam3CSK4 stimulated THP1 cells were purified from equal volumes of culture supernatant and were analyzed by Western blot for the presence of CD63, CD9, and CD81. Data are representative for $n=3$ independent experiments. D) RNA was isolated from EV released by unstimulated and Pam3CSK4 stimulated THP1 cells. An equal amount of RNA per condition was used as input in RT-qPCR analysis of all Y-RNA subtypes. Log2fold differences in Y-RNA between EV from Pam3CSK4 stimulated versus unstimulated cells are depicted. U6 RNA was used for normalization. * $p<0.05$, Student's $t$ test, $n=4$ independent experiments. E) Cellular RNA from unstimulated and Pam3CSK4 stimulated THP1 cells was used for RT-qPCR analysis of all Y-RNA subtypes. Log2fold differences in Y-RNA in Pam3CSK4 stimulated versus unstimulated cells are depicted. U6 RNA was used for normalization. * $p<0.05$, Student's test, $\mathrm{n}=3$ independent experiments. F) RT-qPCR analysis of miR-146a in cellular and EV RNA as in (D and $E) .{ }^{*} p<0.05$, Student's t test, $n=3$ independent experiments. 
strongly upregulated in cells (44) and to a similar extent in EV (Figure 1F). Taken together, TLR-stimulation of THP1 macrophages increases both the number of released EV and the amount of $\mathrm{Y} 1, \mathrm{Y} 3$ and $\mathrm{Y} 4$ per released EV. In addition, the data suggest that Y-RNA levels associated to EV are regulated independently of cellular Y-RNA levels.

The specific increase in EV-associated Y-RNA after TLR stimulation may be caused by an increase in the efficiency with which cytoplasmic Y-RNA is incorporated into EV. Alternatively, cellular Y-RNA levels are upregulated early after activation, causing a parallel increase in Y-RNA incorporation into EV, but return to baseline levels at the time of RNA isolation. To investigate this latter possibility, we performed a time course experiment in which cellular RNA was isolated at different timepoints after activation (Figure 2A). In this experiment, we specifically isolated Y-RNA from the cytoplasm to exclude that a much larger pool of co-isolated nuclear Y-RNA prevents detection of changes in the cytoplasmic pool of Y-RNA, which is likely the main source of Y-RNA in $\mathrm{EV}$. We confirmed that cytoplasmic fractions were largely devoid of nuclear proteins (Supplementary Figure 2A). Using RT-qPCR analysis, we observed that cytoplasmic Y-RNA levels did not change in response to Pam3CSK-stimulation throughout the time course (Figure 2B). In contrast, the cytoplasmic levels of miR-146a, which we included for comparison, were significantly increased at timepoint zero (two hours after activation) and increased steadily for the following $24 \mathrm{~h}$ (in line with $(44,45)$ ). This suggests that processes other than transcriptional regulation steer enhanced Y-RNA sorting into EV upon TLR stimulation.

Sorting of RNAs into EV is likely mediated by proteins that specifically interact with these RNAs. We here investigated whether proteins known to interact with Y-RNAs were present in EV from THP1 cells, and whether increased levels of these proteins were sorted into EV upon TLR-stimulation. We recently made a list of known Y-RNA binding proteins (11), which we compared to EV-associated proteins catalogued in Vesiclepedia (46). This resulted in the following shortlist of Y-RNA binding proteins that are potentially associated to EV: Ro60, La, hnRNP K, YBX1, HuR (ELAVL1) and APOBEC3G. Unfortunately, APOBEC3G could not be tested because antibodies against this protein were not available to us. We observed that all other selected proteins, as well as CD9 and CD81, were present in lysates of THP1 cells (Figure 3A). To assess which of these proteins were packaged into EV, we purified EV from supernatants of activated and non-activated THP1 macrophages by differential centrifugation followed by density gradient purification. Non-floating RNP complexes, which resided in the bottom fractions of the gradient, were recovered as well. Equal numbers of EV, as quantified by high-resolution flow cytometry, were loaded per lane. EV from activated and nonactivated THP1 contained comparable levels of CD9, while CD81 levels were higher in EV from unstimulated cells (Figure 3B). Of all tested Y-RNA binding proteins, only Ro60 was detected in the purified population of THP1-derived EV, but not in RNP. Western 
a

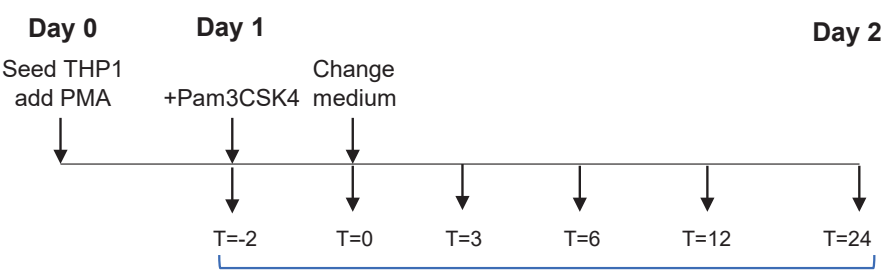

b
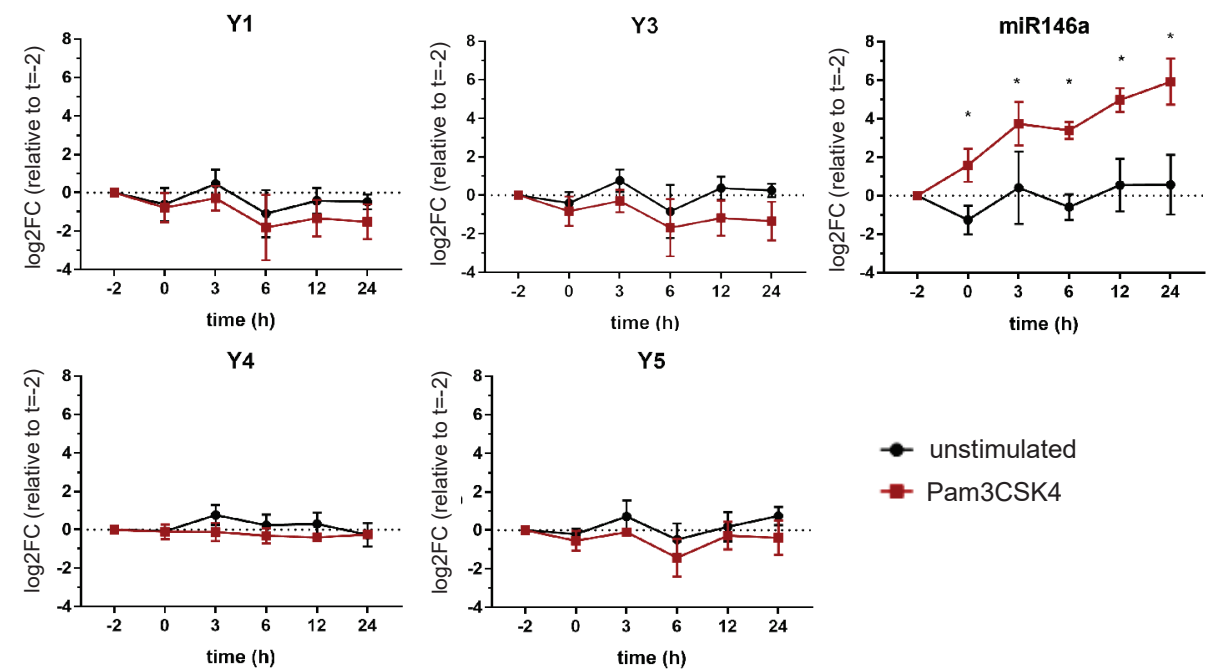

$\rightarrow$ Pam3CSK4

Figure 2. Y-RNA levels in the cytosol remain stable during Pam3CSK4-stimulation. A) Schematic overview of the experiment. THP1 macrophages were stimulated with Pam3CSK4 or left untreated. Two hours later $(\mathrm{t}=0)$, culture medium was removed and wells were washed with PBS, after which cells were incubated in EV-depleted medium for the indicated amounts of time. At each timepoint, cells were detached, lysed in 1\% Triton-X100, and nuclei were removed by centrifugation after which RNA was isolated from the cytoplasm. B) Quantification of Y-RNA subtypes and miR-146a by RT-qPCR analysis of the RNA samples obtained in (A). Log2fold changes in RNA expression were calculated relative to the RNA abundance at the beginning of the time course $(t=-2)$. Black line: unstimulated, red line: Pam3CSK4 stimulated. ${ }^{*} p<0.05$, paired $t$ test, $\mathrm{n}=3$ independent experiments.

blot detection of Ro60 in EV yielded a second band with lower molecular weight, which may represent a cleavage product of Ro60. Even after prolonged exposure of the blots, the other Y-RNA binding proteins could not be detected in EV (data not shown). Because the lysate was prepared from a large number of EV (calculated to contain 1x10E9 EV per lane, based on high resolution flow cytometric analysis) and because La, YBX1, HuR and hnRNP K could be readily detected in cell lysates, our data suggest that these proteins are not at all or in very low quantities shuttled into EV from THP1 cells. Importantly, we found that EV released by stimulated THP1 cells 
were more enriched in Ro60 compared to EV from non-stimulated cells. Together, the data indicate that Ro60 is the most prominent Y-RNA binding protein present in EV from THP1 cells and that TLR2-mediated activation of these cells results in increased shuttling of both Y-RNA and Ro60 into EV.

Next, we questioned how cellular activation could lead to increased packaging of Y-RNA and Ro60 into EV. We hypothesized that cellular activation increases the selective recruitment of Y-RNA/Ro60 complexes to sites of EV biogenesis, such as the multivesicular endosome (MVE), thereby increasing the local availability of such complexes for incorporation into EV. To investigate this, we employed a subcellular fractionation approach, adapted from Gibbings et al. $(40,41)$. This method allows the separation of cytosolic organelles by density gradient centrifugation and parallel analysis of both proteins and small ncRNA in these organelles. We first investigated the subcellular distribution of various organelle-specific proteins and RNAs under nonstimulated conditions. Post-nuclear cytosol was fractionated overnight on a 5\%-40\% Optiprep gradient and the different density fractions were analyzed for the presence of marker proteins for endoplasmic reticulum (ER), Golgi apparatus, early endosomes, and MVE (Figure 4A). The ER-associated protein Calnexin and Golgi marker gm130 were detected in high-density fractions. The endolysosomal-associated proteins CD63 and Lamp-1 $(47,48)$ were enriched in intermediate density fractions, consistent with earlier reports $(42,49)$. The early endosome markers EEA1 and Tsg101 (ESCRT-I) were expectedly enriched in low density fractions $(49,50)$. Next, we evaluated the presence of specific ncRNA types known to be associated with the different organelles. Bioanalyzer measurement indicated that peaks of large ribosomal RNAs (18S and 28S) were most abundant in the high density fractions $(1.20 \mathrm{~g} / \mathrm{ml}-1.13 \mathrm{~g} / \mathrm{ml})$, while most small RNA was found in intermediate and low density fractions $(<1.09 \mathrm{~g} / \mathrm{ml})$ (Supplementary Figure 2B). By RT-qPCR analysis (Figure 4B), we confirmed that the ribosomal 18S RNA, which is often excluded from EV, was mainly present in the high density ER fractions. The ribosomal 5S RNA and the signal-recognition particle complex associated 7SLRNA (7), which are often detected in EV, were present in both the ER and MVE fractions. U6-snRNA is a nuclear RNA that also localizes to cytoplasmic RNP complexes called U-bodies (51). This RNA was previously found to be abundantly present in EV (35). We here observed that U6 was predominantly detected in the lighter fractions enriched in endosomal and endolysosomal markers. These data confirmed that our fractionation approach separates ncRNAs and organelles to distinct densities. Next, we quantified the abundance of different Y-RNA subtypes in the subcellular fractions (Figure 4C). The highest levels of all Y-RNA subtypes were detected in the fractions enriched in the endolysosomal proteins Lamp-1 and CD63, and a considerable amount coincided with the Tsg101 and EEA1 positive fractions (1.09 - $1.05 \mathrm{~g} / \mathrm{ml}$ ). Both CD63 and Tsg101 have been implicated in EV biogenesis. Furthermore, the distribution of the Y-RNA 
binding proteins Ro60 and La overlapped with the distribution of Y-RNA subtypes (Figure 4D). The other Y-RNA binding proteins displayed a wider distribution over the density fractions. HuR and hnRNP K localized to the fractions of $1.20-1.09 \mathrm{~g} / \mathrm{ml}$ and thus partly co-fractionated with Y-RNAs. In contrast, YBX1 was predominantly found in high density fractions and did not overlap with Y-RNA. Overall, the data indicate that Y-RNAs and the Y-RNA binding proteins Ro60, La, HuR and hnRNP K at least partially co-fractionate with the endosomal/MVE-proteins CD63 and Tsg101, suggesting that Y-RNAs and several of their protein binding partners are physically associated with sites of EV biogenesis (52,53).

a

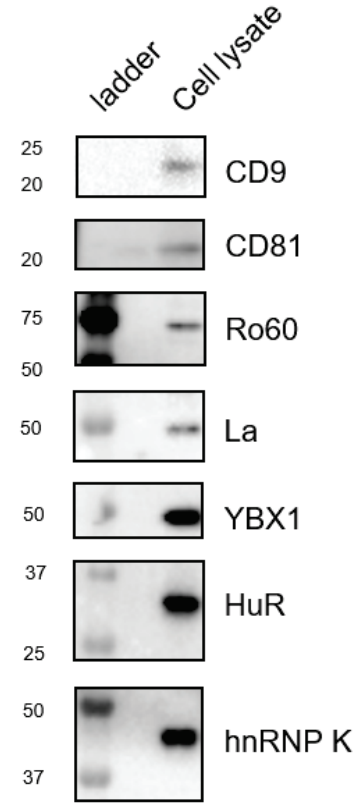

b

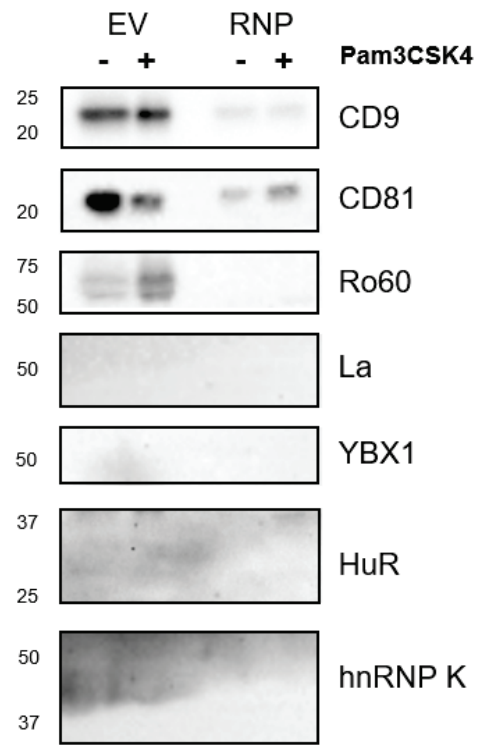

Figure 3. Effects of Pam3CSK4 stimulation on cellular and EV-associated levels of tetraspanins and Y-RNA binding proteins. THP1 cells grown in EV-depleted medium were stimulated with Pam3CSK4 or were left unstimulated. EV were purified by density gradient centrifugation. Cells and EV were assessed for the presence of tetraspanins commonly detected in EV (CD9 and CD81) and Y-RNA binding proteins (Ro60, La, YBX1, HuR, and hnRNP K) A) Western blot indicating the expression of the indicated proteins in THP1 macrophages. Data are representative of $n=3$ independent experiments $B$ ) Western blot indicating protein levels in equal numbers of EV and RNP derived from stimulated or unstimulated THP1 cells. Prolonged exposure did not yield positive signals for the other tested proteins. Data are representative of $n=3$ independent experiments. 
a

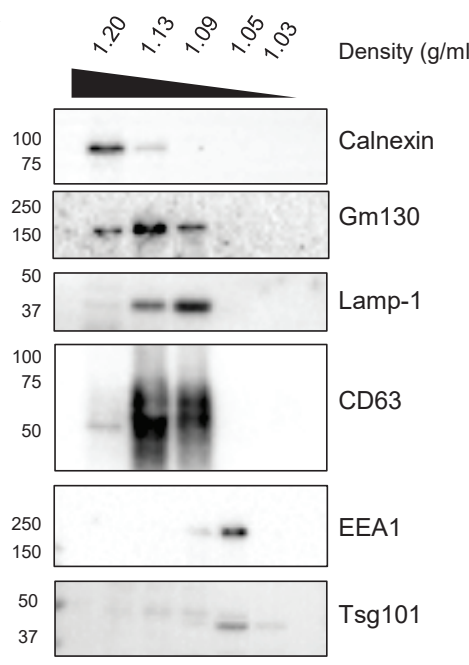

b

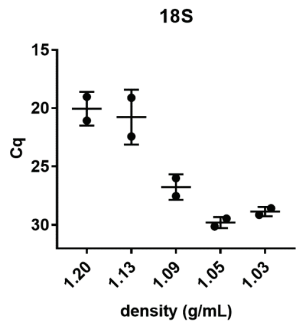

$7 \mathrm{SL}$

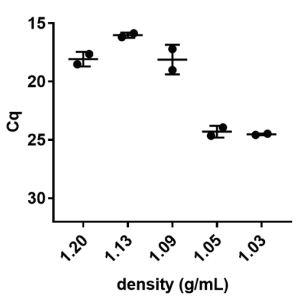

$5 S$

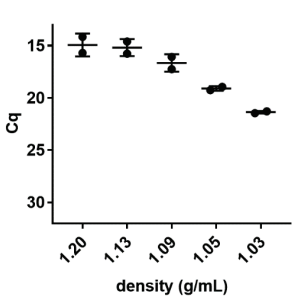

U6

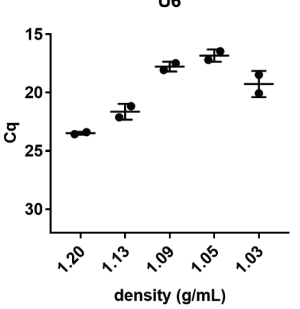

C
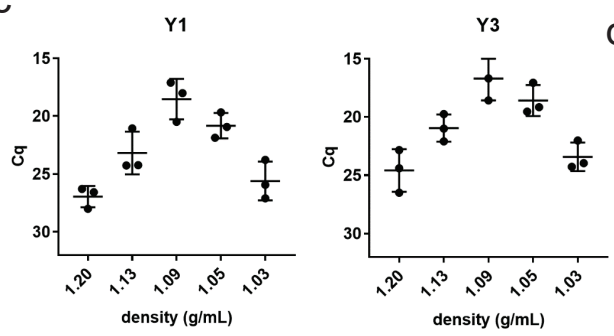

d
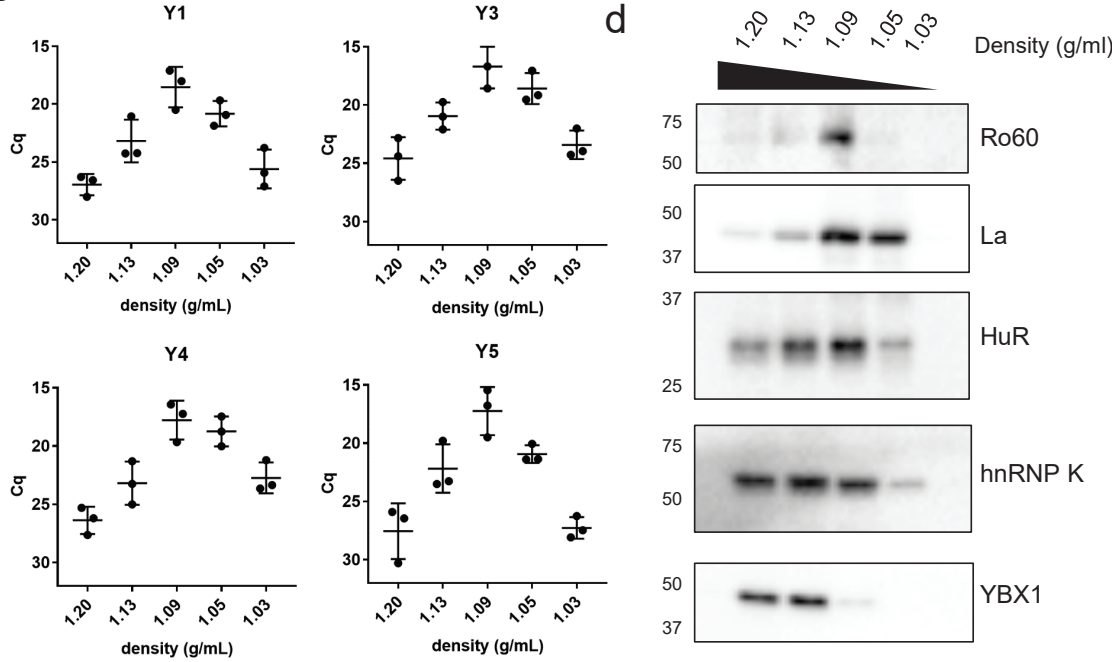

Figure 4. Co-fractionation of Y-RNA and selective Y-RNA binding proteins in subcellular fractions enriched for endolysosomal markers. Post-nuclear lysates of THP-1 cells were separated on a 5-40\% Optiprep gradient, yielding density fractions enriched in different subcellular compartments. RNA was isolated directly from the density gradient fractions and analyzed by RTqPCR. Proteins were retrieved by TCA precipitation and analyzed by Western blotting. A) Western blot analysis of the ER-associated protein Calnexin, Golgi marker gm130, endolysosomal proteins Lamp-1 and CD63, and early endosome markers EEA1 and Tsg101 in the different subcellular fractions. Gm130, EEA1 and Tsg101 were tested once, data for Calnexin, Lamp-1 and CD63 are representative of $n=3$ independent experiments. B) RT-qPCR based quantification of various noncoding RNAs in the subcellular density fractions. Raw $\mathrm{Cq}$ values are shown, lower values indicate higher RNA abundance. Data from $n=2$ independent experiments are shown. C) RT-qPCR based quantification of all Y-RNA subtypes in the subcellular density fractions. Raw Cq values are shown, lower values indicate higher RNA abundance. Data from $n=3$ independent experiments are shown. D) Western blot analysis of various proteins with reported association to Y-RNA in the different subcellular fractions. Data are representative of $n=3$ independent experiments. 
Next, we determined whether the subcellular localization of Y-RNA and Y-RNA binding proteins changed after TLR-activation. Cells were stimulated with Pam3CSK4 for $4 \mathrm{~h}$, and subcellular fractionation was carried out as described before. At this timepoint, cells were notably activated (Figure 1) and we assumed that changes in subcellular localization of proteins and RNA leading to increased Y-RNA shuttling had taken place. We aimed to quantify TLR signaling-induced changes in protein and RNA distribution over the subcellular fractions. To compare the protein distributions in stimulated and unstimulated conditions, we expressed the signal intensity of each band as a percentage of the total abundance in all fractions of each condition. For RNAs quantified by RTqPCR, we quantified the abundance of RNAs in each fraction relative to the average $\mathrm{Cq}$ in unstimulated fractions. No differences were observed in the distribution of the ER- and MVE-associated proteins Calnexin, Lamp-1 and CD63 between Pam3CSK4-stimulated and unstimulated cells (Supplementary Figure 3 ). The distribution of the Y-RNAs was nearly identical between unstimulated and stimulated cells. However, a small but significant enrichment of $Y 1, Y 3$ and $Y 5$ was observed in the $1.05 \mathrm{~g} / \mathrm{ml}$ fraction during stimulation (Figure 5A). In contrast, miR-146a did not localize to a specific fraction, but was robustly upregulated in all fractions of activated cells (Supplementary Figure 4). We then determined whether the localization of Y-RNA binding proteins changed during activation (Figure 5B and C). The subcellular fractionation of Ro60, La, and YBX1 did not differ between stimulated and unstimulated cells. These data therefore do not provide a lead for the potential role of Ro60 in the increased sorting of Y-RNA into EV upon cellular activation. Interestingly, however, we observed a remarkable shift in the localization of HuR and hnRNP $\mathrm{K}$ during cellular activation. In activated cells, the abundance of HuR decreased in the $1.09 \mathrm{~g} / \mathrm{ml}$ fractions and simultaneously increased in the $1.20 \mathrm{~g} /$ $\mathrm{ml}$ fractions. A similar shift in distribution was observed for hnRNP K, although these data did not reach significance. These data suggest that TLR-activation drives the partial relocalization of HuR and hnRNP $K$ from subcellular locations rich in Y-RNAs to ER which contains low levels of Y-RNA. HuR and hnRNP $K$ are known to function as mRNA-binding proteins at the ER. For HuD, which is the neuron-specific homolog of HuR, it was found that this function can only be exerted when Y-RNA is not bound (28). Based on these data, we speculate that removal of Y-RNA from cells via EV may regulate the localization and function of mRNA stabilizing proteins, and thereby regulate the translation of specific proteins. 
a
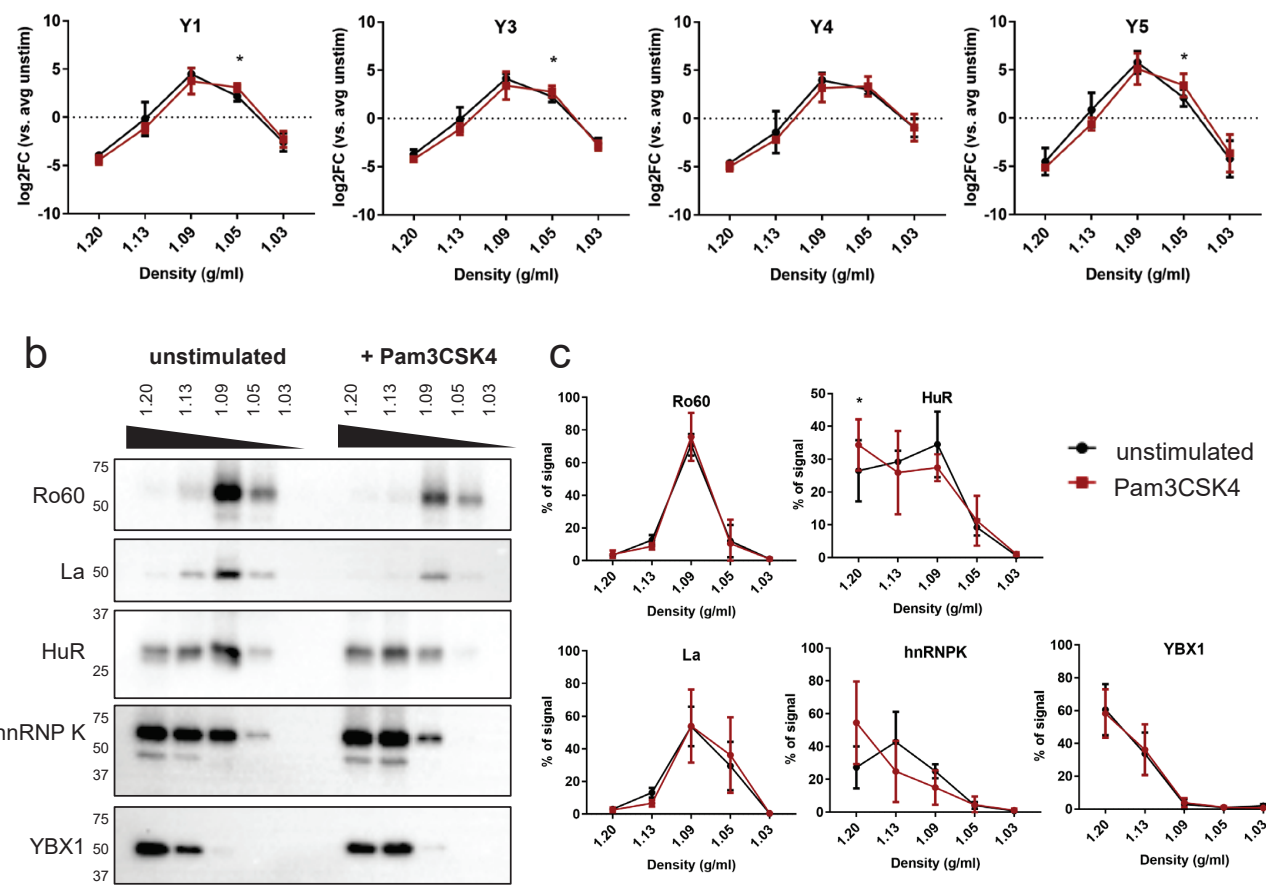

- unstimulated

$\rightarrow$ Pam3CSK4
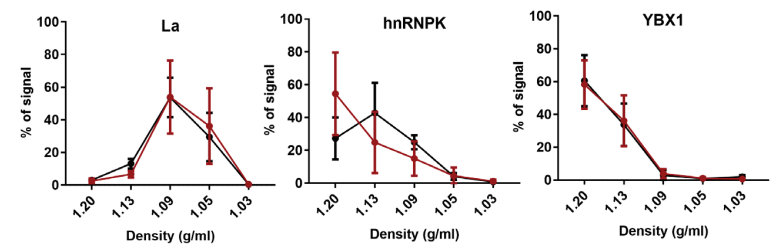

Figure 5. Pam3CSK4-stimulation causes a partial shift of HuR and hnRNP $K$ from endolysosomal towards ER-containing fractions. THP1 macrophages were left unstimulated or were treated with Pam3CSK4 for 4 hours. Post-nuclear lysates of THP-1 cells were separated on a 5-40\% Optiprep gradient, yielding density fractions enriched in different subcellular compartments. RNA was isolated directly from the density gradient fractions and analyzed by RT-qPCR. Proteins were retrieved by TCA precipitation and analyzed by Western blotting. A) RTqPCR based quantification of the distribution of Y-RNA subtypes in different density fractions. To analyze stimulation-induced changes in both the distribution of RNAs over the different fractions and changes in RNA levels, data are expressed as the log2fold change relative to the average $\mathrm{Cq}$ value in fractions of unstimulated cells. Black line: unstimulated, red line: stimulated. * $p<$ 0.05 , paired t test, $n=3$ independent experiments. B) Western blot analysis of different Y-RNA binding proteins in subcellular fractions of Pam3CSK4 stimulated and unstimulated THP1 cells. Data are representative for $n=3$ independent experiments. C) Quantification of band intensities shown in (C). The abundance of the indicated proteins in the different fractions was calculated as a percentage of the total signal over all fractions in each condition. Black line: unstimulated, red line: stimulated. * $p<0.05$, paired t test, $n=3$ independent experiments. 


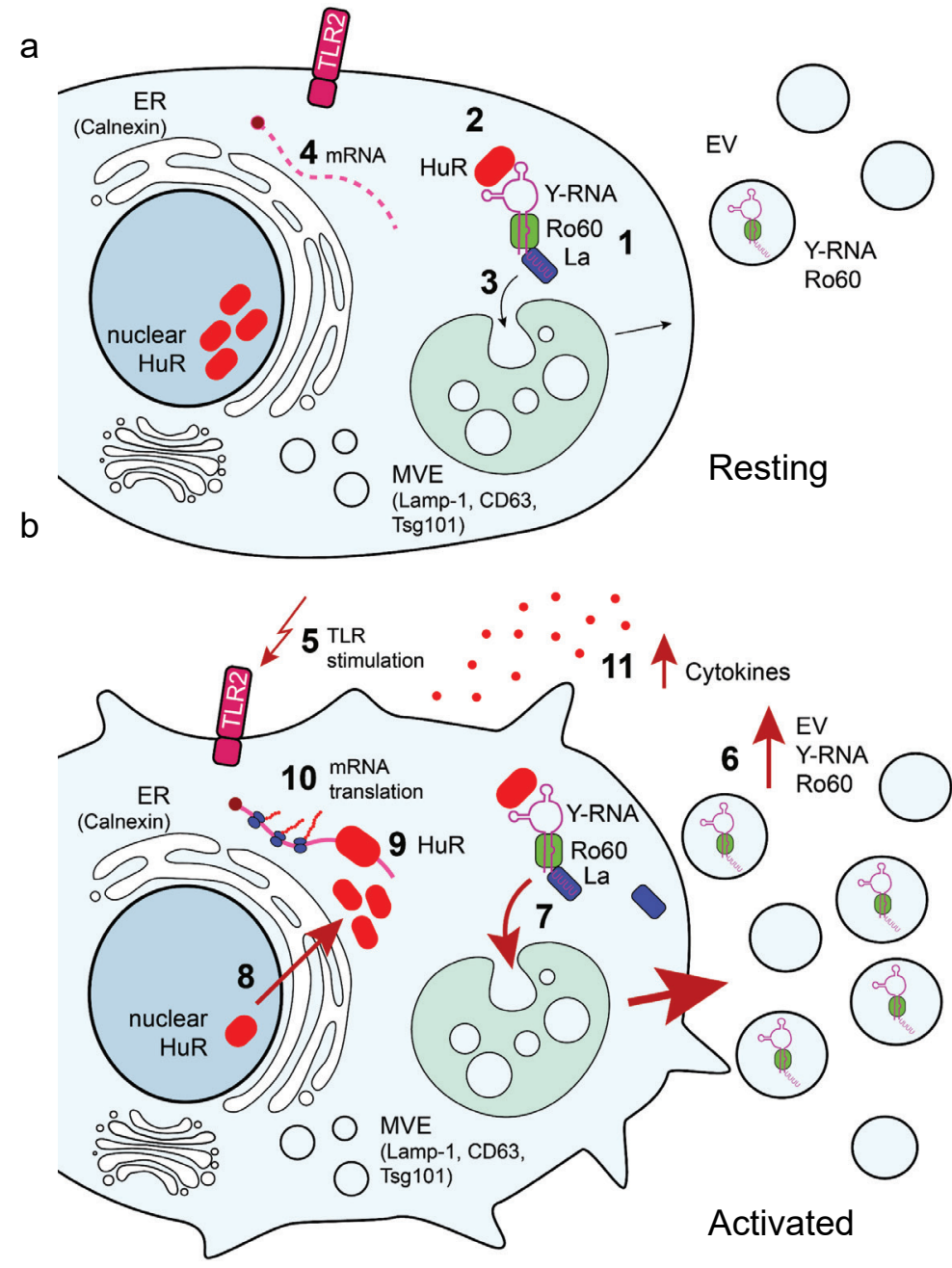

Figure 6. Hypothetical model illustrating how Pam3CSK4 -induced Y-RNA export via EV may affect translocation of mRNA binding proteins, such as HuR. A) In the resting state, Y-RNA is localized at or near the endolysosomal system and is stabilized by Ro60 and La (1). The loop structure is available for binding of other Y-RNA binding proteins, such as HuR (2). The packaging efficiency of Y-RNA into EV and the number of Y-RNA-containing EV released by the cells are low in this condition (3). Cytokine mRNAs are not bound by enhancers, are thus relatively unstable and not translated (4). B) Cellular activation by TLR stimulation (5) increases the number of EV that are released (6) as well as the incorporation efficiency of Y-RNA into EV (7) via mechanisms that are yet unknown. While Ro60 is incorporated into EV together with Y-RNA, La is not. TLR-stimulation also increases the translocation of HuR to the cytoplasm (8). The increased export of Y-RNA via EV reduces the amount of available binding sites for HuR. Together, this results in increased HuR binding to AU-rich elements on cytokine mRNAs (9), which leads to their stabilization and enhances their translation (10). This results in a subsequent increase in cytokine release (11). Taken together, Y-RNA shuttling may thus regulate mRNA translation in the EV-producing cell. 


\section{Discussion}

Several studies have investigated which RNA binding proteins are involved in the shuttling of miRNA into EV (30-34) Nevertheless, other ncRNA species are more abundantly present in EV than miRNA $(1,2)$. For instance, Y-RNA is abundantly detected in EV from various cell types and in biofluids (reviewed in (11)). In this study, we have investigated in more detail whether and how TLR-activation leads to increased incorporation of Y-RNA into EV. Using a human monocytic cell line, we have shown that Pam3CSK4-stimulation increased the Y-RNA content in EV, while cellular Y-RNA levels remained unchanged. This finding suggested that $Y$-RNA packaging is an active process that is potentially mediated by proteins interacting with Y-RNA. Such proteins may be incorporated into EV together with Y-RNA, or may deliver Y-RNA to sites of EV biogenesis by transient RNA-protein interactions. We tested a number of proteins known to interact with Y-RNA for their presence in EV. Only Ro60 could be detected in EV, while YBX1, HuR, hnRNP K, and La were only detected in cell lysates and not in EV isolates. Although not detected as EV-cargo, these proteins may be involved in selective recruitment of Y-RNA/ Ro60 complexes to the EV biogenesis site in the producing cells. Using subcellular fractionation we observed co-fractionation of Y-RNA with Ro60, La, HuR and hnRNP K in fractions that were positive for endolysosomal proteins, suggesting these proteins may be involved in Y-RNA delivery to EV-generating compartments. By comparing Pam3CSK4-stimulated versus unstimulated cells, we only observed a minor increase in the localization of Y-RNA to endolysosomal fractions. Interestingly, we observed substantial relocalization of the Y-RNA binding protein $\mathrm{HuR}$, which is known to regulate mRNA translation and mRNA stability, towards ER-fractions.

Subcellular fractionation is commonly used to assess changes in protein localization within cells $(34,40,42,49,50)$. In our current study we used this approach to obtain clues on where Y-RNA and Y-RNA binding proteins localize inside cells and how their localization may change upon cellular activation. A limitation of this approach is that co-fractionation of components in the same density fraction does not necessarily mean that these components are spatially close together in the cell. Ideally, the presumed colocalization of Y-RNA, the Y-RNA binding proteins and endososomal markers should be substantiated by fluorescence microscopy. However, the sensitivity of detecting small ncRNAs by fluorescent in situ hybridization is generally low and simultaneous detection of proteins is technically difficult (54). Using subcellular fractionation, we here demonstrated that Y-RNA primarily localized to fractions enriched for CD63 and Tsg101. This calls for further research to establish whether Y-RNA is shuttled into EV via both ESCRT independent (CD63 (52)) and ESCRT dependent (Tsg101 (53)) biogenesis routes. 
Although we observed co-fractionation of various Y-RNA binding proteins with endolysosomal proteins, only Ro60 was incorporated into EV. This was not expected since in our assessment we only included Y-RNA binding proteins that were previously reported to be present in EV preparations (11). In these studies, EV had been prepared from a very wide range of biological specimens (sperm (55), glioblastoma cells (56), bladder cancer cells (57), macrophages (58), T cells (59), mesenchymal stem cells (60) and liver cells (34)). EV from these sources may differ in protein composition, and Y-RNA binding proteins found in one particular type of EV may not necessarily be present in EV from another source. An alternative explanation is that the EV populations described in these earlier studies were contaminated with non-EV-associated proteins, because in many of these studies crude preparations of $100,000 \mathrm{~g}$ pelleted $\mathrm{EV}$ were used. A recent study confirmed that many RBP that were previously thought to be present in EV may rather be associated to non-vesicular structures (61). This was shown for YBX1, but may additionally be the case for a number of other Y-RNA binding proteins analyzed in our current study.

Interestingly, western blot analysis of Ro60 in our high-grade purified population of EV revealed two distinct bands, one of which could be a cleavage product of Ro60. A similar, smaller-sized Ro60 band was observed in the $1.09 \mathrm{~g} / \mathrm{ml}$ subcellular fractions (Figure 4D, 5B), while Ro60 in the whole cell lysates was visible as a single band the expected height (Figure $\mathbf{3}$ ). This pattern is reminiscent of the cleavage of the C.elegans Ro-homolog ROP-1 that occurs during larval development, and is carried out by an aspartic protease (62). It has been reported that these proteases may be packaged into EV (63), but cleavage may additionally occur in the cytosol. Nevertheless, it is unlikely that Ro60 cleavage is a strict requirement for incorporation into EV because uncleaved Ro60 was also detected in EV. In contrast to Ro60, La was not packaged into EV. This is remarkable, since La associates to the $3^{\prime}$-oligouridine tail of Y-RNA and is therefore expected to be loaded into EV together with Ro60 and Y-RNA. We speculate that La dissociates from Y-RNA upon cleavage of Y-RNA in between the $3^{\prime}$-stem and the 3 '-oligouridine tail. A similar cleavage reaction occurs during tRNA maturation, when the La-associated 3 '-oligouridine tail is cleaved by tRNAse Z $(64,65)$. Further research is needed to investigate a potential role for $L a$ in restricting Y-RNA packaging into EV and to establish whether and how Ro60 is actively involved in sorting of Y-RNA into EV.

Unexpectedly, we did not observe detectable incorporation of HuR and hnRNP K in EV released by macrophages, although HuR and members of the hnRNP family have been implicated in miRNA shuttling into EV. For instance, HuR was shown to drive the shuttling of miR-122 in into EV from serum-starved liver cells (34). Additionally, hnRNP A2B1, hnRNP Q (SYNCRIP) and other hnRNP types have also been shown to play a role in miRNA shuttling $(30,33,66)$. The involvement of HuR and hnRNP $\mathrm{K}$ in shuttling of other ncRNA species into EV has not been studied previously. Although we did 
not observe any incorporation of HuR and hnRNP K into EV, we observed that TLR stimulation induced a partial relocalization of HuR and hnRNP K from endolysosomal fractions towards ER-fractions. Because this shift did not reach significance for hnRNP $\mathrm{K}$, we will only elaborate on the potential effects on HuR function in relation to our observations. It is known that HuR can bind to AU-rich elements on mRNAs and control their translation (67-69). This kind of posttranscriptional regulation may enable rapid induction of protein expression from pre-formed mRNAs, as is for example required for induction of cytokine responses $(68,69)$. HuR has been shown to enhance the translation of TNFa (70), and IFN- $\beta$ (71). HuR is mainly localized in the nucleus, but is also known to shuttle between nucleus and cytoplasm (72). LPS stimulation of macrophages was shown to induce the translocation of HuR from the nucleus to the cytoplasm (73). HuR was previously found to cofractionate with Alix (34), which is a common component of endolysosomal compartments (74). The association of HuR with cellular Y-RNA has been shown by two studies $(16,28)$. Our results suggest that TLR activation increases the expulsion of Y-RNA via EV, and additionally induces the recruitment of HuR to the ER (Figure 6). It is an outstanding question whether the observed shift in HuR to ER is a direct cause of the increased Y-RNA export from cells, or whether these are two independent events caused by TLR-activation. A recent study provides support for the idea that removal of Y-RNA causes changes in enhancer protein localization and function. Tebaldi et al. have shown in neuronal cells that siRNA-mediated depletion of Y3-RNA affects the subcellular localization of the enhancer protein HuD (28). HuD and HuR are both members of the Hu family of RBP and are highly homologous in sequence and function. The relocalization of HuD from cytosol to the ER reported by Tebaldi et al. seems similar to what we observed for HuR. Furthermore, it was shown that Y3-binding to HuD induced dissociation of this protein from the UTRs of AREcontaining mRNAs, thereby inhibiting the mRNA stabilizing effect of HuD. This led to a reduction of the expression of proteins encoded by these mRNAs (28). HuD has a stronger affinity for $\mathrm{Y} 3$ than for AU-rich element-containing RNAs, which illustrates how Y-RNA can outcompete mRNA binding by HuD (28). Our current findings are consistent with these data and we speculate that enhanced release of Y-RNA via EV may lead to changes in the localization and function of Y-RNA binding proteins that regulate translation of specific mRNAs, such as those encoding cytokines (Figure 6). In this model, increased Y-RNA export via EV reduces the availability of binding sites for HuR, which then bind and stabilize mRNAs at the ER, thereby enhancing their translation. This would imply that EV-mediated export of cellular RNAs is not only a means of intercellular communication, but may additionally play a role in fine-tuning the function of EV-producing cells. This idea is supported by a recent study showing that the release of tRNA fragments into EV is required for appropriate $T$ cell activation (75). Removal of RNAs via release in EV may be a more rapid way to dispose of RNAs 
than enzymatic degradation, because small RNAs are less accessible to cellular RNA degradation pathways due to their highly structured nature and association with RBP (76). Such a mechanism could for example underly the rapid upregulation of cytokine release by innate immune cells (68) and memory T cells (77). These cells contain large numbers of pre-formed cytokine-encoding mRNAs, which are intrinsically unstable due to AU-rich elements (67). These mRNAs may be stabilized and translated during immune activation by the binding of enhancer proteins like HuR (70).

It is important to note that the increased export of Y-RNA via EV did not result in a detectable decrease in (sub)cellular Y-RNA levels. This may seem at odds with our hypothesis that increased Y-RNA export reduces the amount of binding sites for HuR. Nevertheless, it is highly likely that only a subset of cellular Y-RNA binds to HuR. It is possible that only this subset of Y-RNA molecules is exported, which leads to the observed relocalization of HuR, while the majority of Y-RNA is associated to other proteins and remains in the cell. To further test this hypothesis, it is important to acquire more knowledge on the stoichiometry of how many Y-RNA molecules are located in the different cellular compartments, how many of these are bound to HuR, and how many are released into EV.

Taken together, our findings shed light on the potential role of RNA binding proteins in sorting of Y-RNAs into EV. Ro60 and Y-RNA localize to endosomal compartments where EV biogenesis takes place and are both present in released EV, suggesting that Ro60 plays a role in shuttling of Y-RNA into EV. Other Y-RNA binding proteins may recruit Ro60-bound Y-RNA to sites of EV-biogenesis but are subsequently retained in the cell. Furthermore, we speculate that increased export of Y-RNA via EV upon TLR stimulation may regulate the mRNA stabilizing function of enhancer proteins, which is controlled by binding of Y-RNAs.

\section{Acknowledgements}

The authors would like to thank Dr. G.J.A. Arkesteijn for assistance with the highresolution flow cytometric measurements, Dr. S.L. Wolin for kindly providing us with the Ro60 antibody, and Dr. R. Wubbolts for helpful discussions and critical reading of the manuscript. 


\section{References}

1. Nolte-'t Hoen ENM, Buermans HPJ, Waasdorp M, Stoorvogel W, Wauben MHM, 't Hoen PAC. Deep sequencing of RNA from immune cell-derived vesicles uncovers the selective incorporation of small non-coding RNA biotypes with potential regulatory functions. Nucleic Acids Res (2012) 40:9272-85. doi:10.1093/nar/gks658

2. Bellingham SA, Coleman BM, Hill AF. Small RNA deep sequencing reveals a distinct miRNA signature released in exosomes from prion-infected neuronal cells. Nucleic Acids Res (2012) 40:10937-10949. doi:10.1093/nar/gks832

3. He L, Hannon GJ. MicroRNAs: small RNAs with a big role in gene regulation. Nat Rev Genet (2004) 5:522-31. doi:10.1038/nrg1379

4. Alexander M, Hu R, Runtsch MC, Kagele DA, Mosbruger TL, Tolmachova T, Seabra MC, Round JL, Ward DM, O'Connell RM. Exosome-delivered microRNAs modulate the inflammatory response to endotoxin. Nat Commun (2015) 6:7321. doi:10.1038/ncomms8321

5. Mittelbrunn M, Gutiérrez-Vázquez C, Villarroya-Beltri C, González S, Sánchez-Cabo F, González MÁ, Bernad A, Sánchez-Madrid F. Unidirectional transfer of microRNA-loaded exosomes from T cells to antigen-presenting cells. Nat Commun (2011) 2:282. doi:10.1038/ncomms1285

6. Montecalvo A, Larregina AT, Shufesky WJ, Stolz DB, Sullivan MLG, Karlsson JM, Baty CJ, Gibson GA, Erdos $G$, Wang Z, et al. Mechanism of transfer of functional microRNAs between mouse dendritic cells via exosomes. Blood (2012) 119:756-66. doi:10.1182/blood-2011-02-338004

7. Akopian D, Shen K, Zhang X, Shan S. Signal Recognition Particle: an essential protein targeting machine. Annu Rev Biochem (2013) 30:311-328. doi:10.1016/j.cll.2010.01.003.Lyme

8. Matera AG, Wang Z. A day in the life of the spliceosome. Nat Rev Mol Cell Biol (2014) 15:108-21. doi:10.1038/nrm3742

9. Boccitto M, Wolin SL. Ro60 and Y RNAs: structure, functions, and roles in autoimmunity. Crit Rev Biochem Mol Biol (2019) 0:1-20. doi:10.1080/10409238.2019.1608902

10. Anderson P, Ivanov P. TRNA fragments in human health and disease. FEBS Lett (2014) 588:4297-4304. doi:10.1016/j.febslet.2014.09.001

11. Driedonks TAP, Nolte-'t Hoen ENM. Circulating Y-RNAs in Extracellular Vesicles and Ribonucleoprotein Complexes; Implications for the Immune System. Front Immunol (2019) 9:1-15. doi:10.3389/ fimmu.2018.03164

12. Pruijn GJM, Wingens PAETM, Peters SLM, Thijssen JPH, Van Venrooij WJ. Ro RNP associated Y RNAs are highly conserved among mammals. Biochim Biophys Acta - Gene Struct Expr (1993) 1216:395-401. doi:10.1016/0167-4781(93)90006-Y

13. van Horn DJ, Eisenberg D, O'Brien CA, Wolin SL. Caenorhabditis elegans embryos contain only one major species of Ro RNP. RNA (1995) 1:293-303.

14. Chen X, Wurtmann EJ, Van Batavia J, Zybailov B, Washburn MP, Wolin SL. An ortholog of the Ro autoantigen functions in 23S rRNA maturation in D. radiodurans. Genes Dev (2007) 21:1328-1339. doi:10.1101/gad.1548207

15. van Gelder CWG, Thijssen JPHM, Klaassen ECJ, Sturchler C, Krol A, van Venrooij WJ, Pruijn GJM. Common structural features of the Ro RNP associated hY1 and hY5 RNAs. Nucleic Acids Res (1994) 22:2498-2506. doi:10.1093/nar/22.13.2498

16. Köhn M, Ihling C, Sinz A, Krohn K, Hüttelmaier S. The Y $3^{* *}$ ncRNA promotes the $3^{\prime}$ end processing of histone mRNAs. Genes Dev (2015) 29:1998-2003. doi:10.1101/gad.266486.115

17. Kowalski MP, Krude T. Functional roles of non-coding Y RNAs. Int J Biochem Cell Biol (2015) 66:20-29. doi:10.1016/j.biocel.2015.07.003

18. Chen X, Wolin SL. The Ro $60 \mathrm{kDa}$ autoantigen: Insights into cellular function and role in autoimmunity. J Mol Med (2004) 82:232-239. doi:10.1007/s00109-004-0529-0

19. Wolin SL, Cedervall T. The La Protein. Annu Rev Biochem (2002) 71:375-403. doi:10.1146/annurev. biochem.Copyright 
20. Hornung V, Ellegast J, Kim S, Brzózka K, Jung A, Kato H, Poeck H, Akira S, Conzelmann K-K, Schlee M, et al. 5'-Triphosphate RNA is the Ligand for RIG-I. Science (80-) (2006) 314:994-997. doi:10.1126/science.1132505

21. Sim S, Weinberg DE, Fuchs G, Choi K, Chung J, Wolin SL. The Subcellular Distribution of an RNA Quality Control Protein, the Ro Autoantigen, Is Regulated by Noncoding Y RNA Binding. Mol Biol Cell (2009) 20:1555-1564. doi:10.1091/mbc.E08

22. Takaoka A, Wang Z, Choi MK, Yanai H, Negishi H, Ban T, Lu Y, Miyagishi M, Kodama T, Honda K, et al. DAl (DLM-1/ZBP1) is a cytosolic DNA sensor and an activator of innate immune response. Nature (2007) 448:501-505. doi:10.1038/nature06013

23. Sim S, Yao J, Weinberg DE, Niessen S, Yates JR, Wolin SL. The zipcode-binding protein ZBP1 influences the subcellular location of the Ro 60-kDa autoantigen and the noncoding Y3 RNA. RNA (2012) 18:100-10. doi:10.1261/rna.029207.111

24. Simons FHM, Rutjes SA, Van Venrooij WJ, Pruijn GJM. The interactions with Ro60 and La differentially affect nuclear export of hY1 RNA. RNA (1996) 2:264-273.

25. Romero V, Fellows E, Jenne DE, Andrade F. Cleavage of La protein by granzyme H induces cytoplasmic translocation and interferes with La-mediated HCV-IRES translational activity. Cell Death Differ (2009) 16:340-348. doi:10.1038/cdd.2008.165

26. Stein AJ, Fuchs G, Fu C, Wolin SL, Reinisch KM. Structural insights into RNA quality control: The Ro autoantigen binds misfolded RNAs via its central cavity. Cell (2005) 121:529-539. doi:10.1016/j. cell.2005.03.009

27. Fuchs G, Stein AJ, Fu C, Reinisch KM, Wolin SL. Structural and biochemical basis for misfolded RNA recognition by the Ro autoantigen. Nat Struct Mol Biol (2006) 13:1002-1009. doi:10.1038/nsmb1156

28. Tebaldi T, Zuccotti P, Peroni D, Köhn M, Gasperini L, Potrich V, Bonazza V, Dudnakova T, Rossi A, Sanguinetti G, et al. HuD Is a Neural Translation Enhancer Acting on mTORC1-Responsive Genes and Counteracted by the Y3 Small Non-coding RNA. Mol Cell (2018) 71:256-270. doi:10.1016/j.molcel.2018.06.032

29. Mateescu B, Kowal EJK, van Balkom BWM, Bartel S, Bhattacharyya SN, Buzás El, Buck AH, de Candia P, Chow FWN, Das S, et al. Obstacles and opportunities in the functional analysis of extracellular vesicle RNA - an ISEV position paper. J Extracell Vesicles (2017) 6:1286095. doi:10.1080/20013078.2017.1286095

30. Villarroya-Beltri C, Gutiérrez-Vázquez C, Sánchez-Cabo F, Pérez-Hernández D, Vázquez J, Martin-Cofreces N, Martinez-Herrera DJ, Pascual-Montano A, Mittelbrunn M, Sánchez-Madrid F. Sumoylated hnRNPA2B1 controls the sorting of miRNAs into exosomes through binding to specific motifs. Nat Commun (2013) 4:2980. doi:10.1038/ncomms3980

31. Cha DJ, Franklin JL, Dou Y, Liu Q, Higginbotham JN, Beckler MD, Weaver AM, Vickers K, Prasad N, Levy S, et al. KRAS-dependent sorting of miRNA to exosomes. Elife (2015) 4:1-22. doi:10.7554/eLife.07197

32. Shurtleff MJ, Temoche-Diaz MM, Karfilis KV., Ri S, Schekman R. Y-box protein 1 is required to sort microRNAs into exosomes in cells and in a cell-free reaction. Elife (2016) 5:1-23. doi:10.7554/eLife.19276

33. Santangelo L, Giurato G, Cicchini C, Montaldo C, Mancone C, Tarallo R, Battistelli C, Alonzi T, Weisz A, Tripodi M. The RNA-Binding Protein SYNCRIP Is a Component of the Hepatocyte Exosomal Machinery Controlling MicroRNA Sorting. Cell Rep (2016) 17:799-808. doi:10.1016/j.celrep.2016.09.031

34. Mukherjee K, Ghoshal B, Ghosh S, Chakrabarty Y, Shwetha S, Das S, Bhattacharyya SN. Reversible HuRmicroRNA binding controls extracellular export of miR122 and augments stress response. EMBORep

(2016) 17:11841203. doi:10.15252/embr

35. Driedonks TAP, van der Grein SG, Ariyurek Y, Buermans HPJ, Jekel H, Chow FWN, Wauben MHM, Buck AH, Hoen PAC, Nolte-'t Hoen ENM. Immune stimuli shape the small non-coding transcriptome of extracellular vesicles released by dendritic cells. Cell Mol Life Sci (2018)3857-3875. doi:10.1007/s00018-018-2842-8

36. Driedonks TAP, Nijen-Twilhaar MK, Nolte-'t Hoen ENM. Technical approaches to reduce interference of Fetal calf serum derived RNA in the analysis of extracellular vesicle RNA from cultured cells. J Extracell Vesicles (2018) 8: doi:10.1080/20013078.2018.1552059 
37. van der Vlist EJ, Nolte-'t Hoen ENM, Stoorvogel W, Arkesteijn GJA, Wauben MHM. Fluorescent labeling of nano-sized vesicles released by cells and subsequent quantitative and qualitative analysis by highresolution flow cytometry. Nat Protoc (2012) 7:1311-1326. doi:10.1038/nprot.2012.065

38. Nolte-'t Hoen ENM, van der Vlist EJ, Aalberts M, Mertens HCH, Bosch BJ, Bartelink W, Mastrobattista E, van Gaal EVB, Stoorvogel W, Arkesteijn GJ a, et al. Quantitative and qualitative flow cytometric analysis of nanosized cell-derived membrane vesicles. Nanomedicine (2012) 8:712-720. doi:10.1016/j. nano.2011.09.006

39. Groot Kormelink T, Arkesteijn GJA, Nauwelaers FA, van den Engh G, Nolte-'t Hoen ENM, Wauben MHM. Prerequisites for the analysis and sorting of extracellular vesicle subpopulations by high-resolution flow cytometry. Cytom Part A (2016) 89:135-147. doi:10.1002/cyto.a.22644

40. Gibbings DJ, Ciaudo C, Erhardt M, Voinnet O. Multivesicular bodies associate with components of miRNA effector complexes and modulate miRNA activity. Nat Cell Biol (2009) 11:1143-1149. doi:10.1038/ncb1929

41. Gibbings DJ. "Continuous density gradients to study Argonaute and GW182 complexes associated with the endocytic pathway," in Methods in Molecular Biology, 63-76. doi:10.1016/j.cub.2011.05.020

42. Squadrito ML, Baer C, Burdet F, Maderna C, Gilfillan GD, Lyle R, Ibberson M, De Palma M. Endogenous RNAs Modulate MicroRNA Sorting to Exosomes and Transfer to Acceptor Cells. Cell Rep (2014) 8:1432-1446. doi:10.1016/j.celrep.2014.07.035

43. van Helden SFG, van Leeuwen FN, Figdor CG. Human and murine model cell lines for dendritic cell biology evaluated. Immunol Lett (2008) 117:191-197. doi:10.1016/j.imlet.2008.02.003

44. Taganov K, Boldin M. NF-kB-dependent induction of microRNA miR-146, an inhibitor targeted to signaling proteins of innate immune responses. Proc ... (2006) Available at: http://www.pnas.org/ content/103/33/12481.short [Accessed June 5, 2015]

45. Mann M, Mehta A, Zhao JL, Lee K, Marinov GK, Garcia-Flores Y, Baltimore D. An NF-kB-microRNA regulatory network tunes macrophage inflammatory responses. Nat Commun (2017) 8: doi:10.1038/ s41467-017-00972-z

46. Kalra H, Simpson RJ, Ji H, Aikawa E, Altevogt P, Askenase P, Bond VC, Borràs FE, Breakefield X, Budnik V, et al. Vesiclepedia: A Compendium for Extracellular Vesicles with Continuous Community Annotation. PLoS Biol (2012) 10:8-12. doi:10.1371/journal.pbio.1001450

47. Kobayashi T, Vischer UM, Rosnoblet C, Lebrand C, Lindsay M, Parton RG, Kruithof EKO, Gruenberg J. The Tetraspanin CD63/lamp3 Cycles between Endocytic and Secretory Compartments in Human Endothelial Cells. Mol Biol Cell (2013) 11:1829-1843. doi:10.1091/mbc.11.5.1829

48. Huotari J, Helenius A. Endosome maturation. EMBO J (2011) 30:3481-500. doi:10.1038/emboj.2011.286

49. Huotari J, Meyer-Schaller N, Hubner M, Stauffer S, Katheder N, Horvath P, Mancini R, Helenius A, Peter M. Cullin-3 regulates late endosome maturation. Proc Natl Acad Sci (2012) 109:823-828. doi:10.1073/ pnas. 1118744109

50. Mckenzie AJ, Hoshino D, Hong NH, Coffey RJ, Patton JG, Weaver AM, Mckenzie AJ, Hoshino D, Hong $\mathrm{NH}$, Cha DJ, et al. KRAS-MEK Signaling Controls Ago2 Sorting into Report KRAS-MEK Signaling Controls Ago2 Sorting into Exosomes. Cell Rep (2016) 15:1-10. doi:10.1016/j.celrep.2016.03.085

51. Liu J-L, Gall JG. U bodies are cytoplasmic structures that contain uridine-rich small nuclear ribonucleoproteins and associate with P bodies. Proc Natl Acad Sci (2007) 104:11655-11659. doi:10.1073/pnas.0704977104

52. van Niel G, Charrin S, Simoes S, Romao M, Rochin L, Saftig P, Marks MS, Rubinstein E, Raposo G. The Tetraspanin CD63 Regulates ESCRT-Independent and -Dependent Endosomal Sorting during Melanogenesis. Dev Cell (2011) 21:708-721. doi:10.1016/j.devcel.2011.08.019

53. Colombo M, Moita C, van Niel G, Kowal J, Vigneron J, Benaroch P, Manel N, Moita LF, Théry C, Raposo $G$. Analysis of ESCRT functions in exosome biogenesis, composition and secretion highlights the heterogeneity of extracellular vesicles. J Cell Sci (2013) 126:5553-65. doi:10.1242/jcs.128868 
54. Lei Z, van Mil A, Xiao J, Metz CHG, van Eeuwijk ECM, Doevendans PA, Sluijter JPG. MMISH: Multicolor microRNA in situ hybridization for paraffin embedded samples. Biotechnol Reports (2018) 18:e00255. doi:10.1016/j.btre.2018.e00255

55. Vojtech L, Woo S, Hughes S, Levy C, Ballweber L, Sauteraud RP, Strobl J, Westerberg K, Gottardo R, Tewari $\mathrm{M}$, et al. Exosomes in human semen carry a distinctive repertoire of small non-coding RNAs with potential regulatory functions. Nucleic Acids Res (2014) 42:7290-7304. doi:10.1093/nar/gku347

56. Wei Z, Batagov AO, Schinelli S, Wang J, Wang Y, El Fatimy R, Rabinovsky R, Balaj L, Chen CC, Hochberg F, et al. Coding and noncoding landscape of extracellular RNA released by human glioma stem cells. Nat Commun (2017) 8:1145. doi:10.1038/s41467-017-01196-x

57. Welton JL, Khanna S, Giles PJ, Brennan P, Brewis IA, Staffurth J, Mason MD, Clayton A. Proteomics Analysis of Bladder Cancer Exosomes. Mol Cell Proteomics (2010) 9:1324-1338. doi:10.1074/mcp. M000063-MCP201

58. Frye BC, Halfter S, Djudjaj S, Muehlenberg P, Weber S, Raffetseder U, En-Nia A, Knott H, Baron JM, Dooley $\mathrm{S}$, et al. Y-box protein-1 is actively secreted through a non-classical pathway and acts as an extracellular mitogen. EMBORep (2009) 10:783-789. doi:10.1038/embor.2009.81

59. Khatua AK, Taylor HE, Hildreth JEK, Popik W. Exosomes Packaging APOBEC3G Confer Human Immunodeficiency Virus Resistance to Recipient Cells. J Virol (2009) 83:512-521. doi:10.1128/ JVI.01658-08

60. Collino F, Deregibus MC, Bruno S, Sterpone L, Aghemo G, Viltono L, Tetta C, Camussi G. Microvesicles derived from adult human bone marrow and tissue specific mesenchymal stem cells shuttle selected pattern of miRNAs. PLoS One (2010) 5: doi:10.1371/journal.pone.0011803

61. Jeppesen DK, Fenix AM, Franklin JL, Higginbotham JN, Zhang Q, Zimmerman LJ, Liebler DC, Ping J, Liu Q, Evans R, et al. Reassessment of Exosome Composition. Cell (2019) 177:428-445.e18. doi:10.1016/j. cell.2019.02.029

62. Labbé J, Burgess J. ROP-1, an RNA quality-control pathway component, affects Caenorhabditis elegans dauer formation. Proc Natl Acad Sci U S A (2000) 97:13233-13238. doi:10.1073/pnas.230284297

63. Kanninen KM, Bister N, Koistinaho J, Malm T. Exosomes as new diagnostic tools in CNS diseases. Biochim Biophys Acta - Mol Basis Dis (2016) 1862:403-410. doi:10.1016/j.bbadis.2015.09.020

64. Vogel A, Schilling O, Späth B, Marchfelder A. The tRNase Z family of proteins: Physiological functions, substrate specificity and structural properties. Biol Chem (2005) 386:1253-1264. doi:10.1515/ BC.2005.142

65. Hasler D, Meister G. From tRNA to miRNA: RNA-folding contributes to correct entry into noncoding RNA pathways. FEBS Lett (2016) 590:2354-2363. doi:10.1002/1873-3468.12294

66. Statello L, Maugeri M, Garre E, Nawaz M, Wahlgren J, Papadimitriou A, Lundqvist C, Lindfors L, Colle A, Sunnerhagen $\mathrm{P}$, et al. Identification of RNA-binding proteins in exosomes capable of interacting with different types of RNA: RBP-facilitated transport of RNAs into exosomes. PLoS One (2018) 13:1-30. doi:10.1371/journal.pone.0195969

67. Mayr C. Regulation by 3 -Untranslated Regions. Annu Rev Genet (2017) 51:171-194.

68. Turner M, Díaz-Muñoz MD. RNA-binding proteins control gene expression and cell fate in the immune system. Nat Immunol (2018) 19:120-129. doi:10.1038/s41590-017-0028-4

69. Ostareck DH, Ostareck-Lederer A. RNA-Binding Proteins in the Control of LPS-Induced Macrophage Response. Front Genet (2019) 10:1-10. doi:10.3389/fgene.2019.00031

70. Tiedje C, Ronkina N, Tehrani M, Dhamija S, Laass K, Holtmann H, Kotlyarov A, Gaestel M. The p38/MK2Driven Exchange between Tristetraprolin and HuR Regulates AU-Rich Element-Dependent Translation. PLoS Genet (2012) 8: doi:10.1371/journal.pgen.1002977

71. Herdy B, Karonitsch T, Vladimer Gl, Tan CSH, Stukalov A, Trefzer C, Bigenzahn JW, Theil T, Holinka J, Kiener HP, et al. The RNA-binding protein HuR/ELAVL1 regulates IFN- $\beta$ mRNA abundance and the type I IFN response. Eur J Immunol (2015) 45:1500-1511. doi:10.1002/eji.201444979 
72. Fan XC, Steitz JA. Overexpression of HuR, a nuclear-cytoplasmic shuttling protein, increases the in vivo stability of ARE-containing mRNAs. EMBOJ (1998) 17:3448-3460.

73. Ke Y, Han Y, Guo X, Wen J, Wang K, Jiang X, Tian X, Ba X, Boldogh I, Zeng X. PARP1 promotes gene expression at the post-transcriptiona level by modulating the RNA-binding protein HuR. Nat Commun (2017) 8:1-15. doi:10.1038/ncomms14632

74. Baietti MF, Zhang Z, Mortier E, Melchior A, Degeest G, Geeraerts A, Ivarsson Y, Depoortere F, Coomans C, Vermeiren E, et al. Syndecan-syntenin-ALIX regulates the biogenesis of exosomes. Nat Cell Biol (2012) 14:677-685. doi:10.1038/ncb2502

75. Chiou NT, Kageyama R, Ansel KM. Selective Export into Extracellular Vesicles and Function of tRNA Fragments during T Cell Activation. Cell Rep (2018) 25:3356-3370.e4. doi:10.1016/j.celrep.2018.11.073

76. Frankel LB, Lubas M, Lund AH. Emerging connections between RNA and autophagy. Autophagy (2017) 13:3-23. doi:10.1080/15548627.2016.1222992

77. Salerno F, Engels S, van den Biggelaar M, van Alphen FPJ, Guislain A, Zhao W, Hodge DL, Bell SE, Medema JP, von Lindern M, et al. Translational repression of pre-formed cytokine-encoding mRNA prevents chronic activation of memory T cells. Nat Immunol (2018) 19:828-837. doi:10.1038/s41590018-0155-6 
a

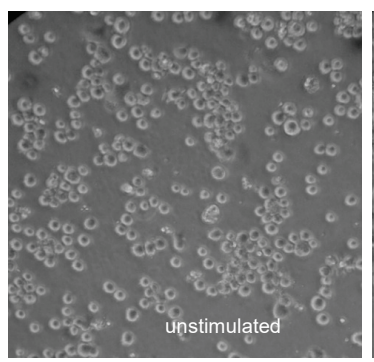

b

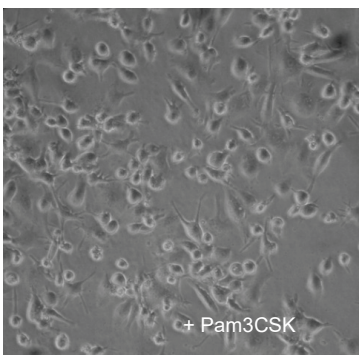

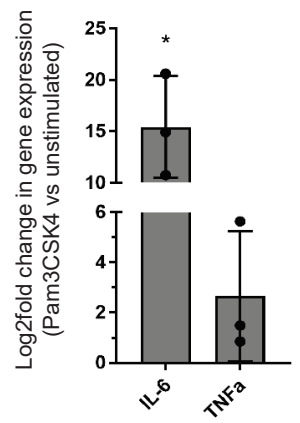

Supplementary Figure 1. Pam3CSK4-stimulated THP1 macrophages display morphological changes and increased cytokine expression. A) Brightfield microscopical image of unstimulated (left) and Pam3CSK4 stimulated THP1 cells at 200x magnification. B) Cellular RNA was isolated 4 hours after addition of Pam3CSK4. Quantification of mRNAs for IL-6 and TNFa was performed by RT-qPCR analysis. Indicated are log2 fold changes of gene expression in the stimulated versus unstimulated condition, normalized to beta-actin mRNA. * $p<0.05$, Student's $t$ test, $n=3$ independent experiments. 
a

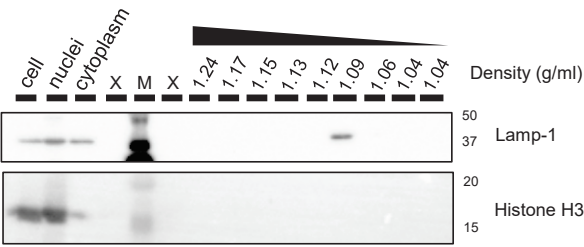

b
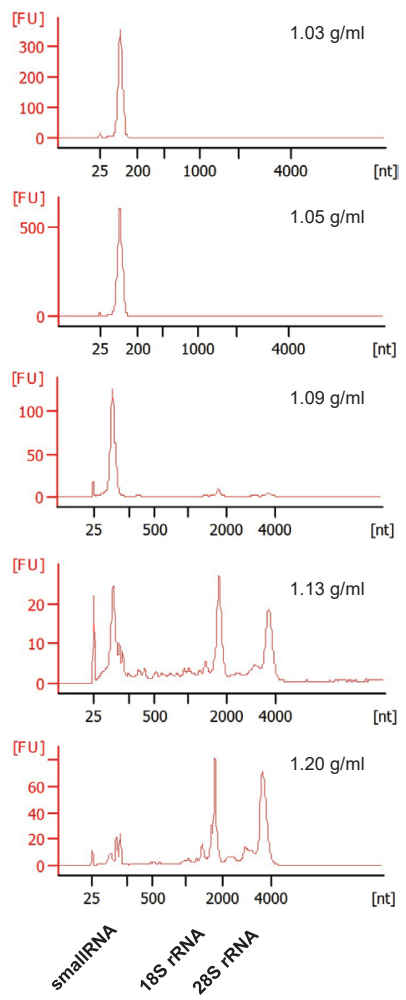

Supplementary Figure 2. THP1 subcellular fractions contain distinct RNA profiles and are devoid of nuclear proteins. Unstimulated THP1 cells were homogenized and the postnuclear cytosolic extract was loaded onto a 5-40\% Optiprep gradient. Different density fractions were recovered which were used for RNA isolation directly from the fractions, and proteins were retrieved by TCA precipitation and analyzed by Western blotting. A) Bioanalyzer profiles of RNA isolated from the different THP1 subcellular fractions. Each peak represents RNAs of a specific size, indicated in arbitrary fluorescent units (FU). Small RNA, 18S rRNA and 28S rRNA peaks are indicated, as well as the fraction densities. Data are representative for $n=3$ independent experiments B) Western blot analysis of subcellular density fractions for the presence of the endolysosomal protein Lamp-1 and the nuclear protein Histone H3. Total cell lysate, nuclei pellet and post-nuclear cytosolic extracts are included as controls. 


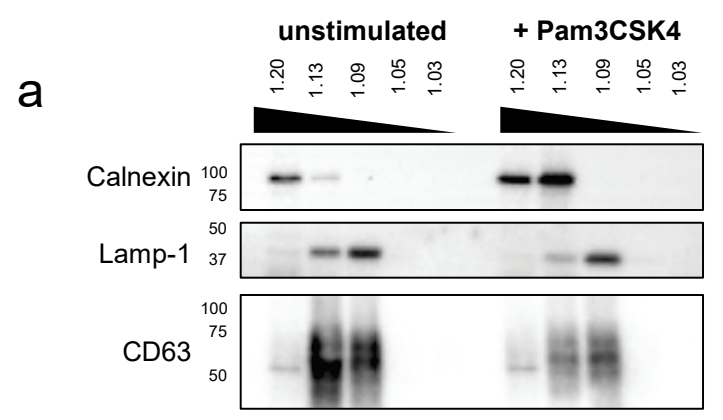

b
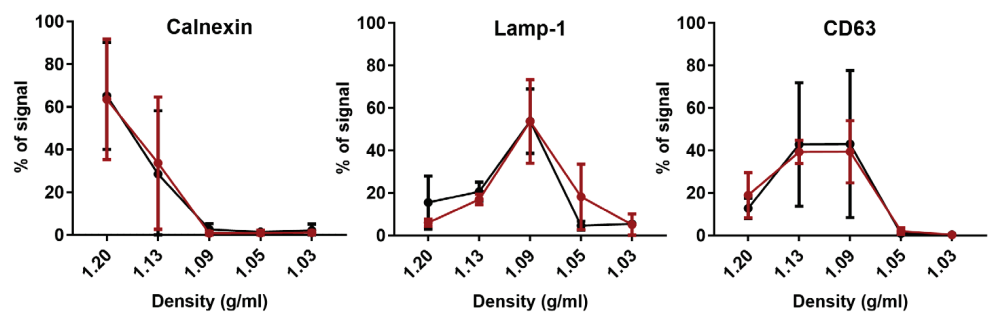

\begin{abstract}
Supplementary Figure 3. Pam3CSK4-stimulation does not change the subcellular distribution of ER- and endolysosome-associated proteins. Pam3CSK4 stimulated or unstimulated THP1 cells were homogenized and the post-nuclear cytosol was loaded onto a $5-40 \%$ Optiprep gradient. Density gradient fractions were subjected to TCA precipitation and proteins were analyzed by Western blotting. A) Western blot showing the distribution of Calnexin (ER), and Lamp-1 and CD63 (endolysosomes) in subcellular fractions isolated from stimulated and unstimulated THP1 cells, representative for $n=3$ independent experiments. B) Quantification of band intensities shown in (A). The relative abundance of each protein was calculated as a percentage of the total signal over all fractions. Black line: unstimulated, red line: stimulated. ${ }^{*} p$ $<0.05$, paired t test, $\mathrm{n}=3$ independent experiments.
\end{abstract}




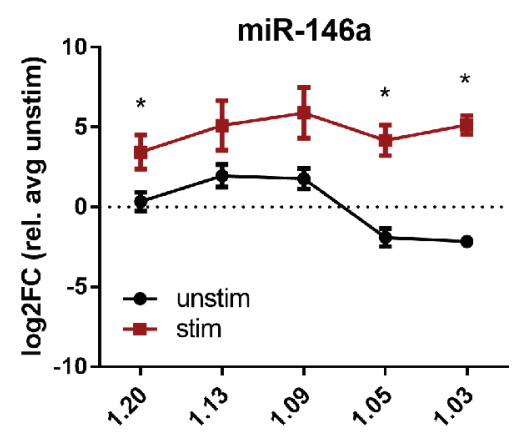

Density $(\mathrm{g} / \mathrm{ml})$

Supplementary Figure 4. Pam3CSK4-stimulation increases miR-146a levels in all subcellular fractions. THP1 macrophages were left unstimulated or were treated with Pam3CSK4 for 4 hours. Post-nuclear lysates of THP-1 cells were separated on a 5-40\% Optiprep gradient, yielding density fractions enriched in different subcellular compartments. RNA was isolated directly from the density gradient fractions, followed by RT-qPCR based quantification of miR-146a in different density fractions. To analyze stimulation-induced changes in both the distribution of RNAs over the different fractions and changes in RNA levels, data are expressed as the log2fold change relative to the average $\mathrm{Cq}$ value in fractions of unstimulated cells. Black line: unstimulated, red line: stimulated. ${ }^{*} p<0.05$, paired $t$ test, $n=3$ independent experiments. 
Chapter 8

General Discussion 
The discovery that extracellular vesicles (EV) can transfer RNA between cells has provided new perspectives in our understanding of intercellular communication (1). It has become clear that in response to environmental signals or mutations associated with diseases, the RNA content of EV released by these cells is modified (2-5). This likely also translates into changes in the effects that these EV have on recipient cells. The discovery that these 'intercellular messages' can be detected in biofluids, and thus may be used to detect diseases, has further sparked interest in EV-associated RNA as biomarkers (6-13). Numerous studies addressed how the RNA profiles of EV change due to cell signaling, oncogenic transformation, or other disease-related processes $(3,4,14-18)$. However, most of these studies have focused on changes in miRNA content, whereas EV are known to contain a wealth of other non-coding RNA species (2,18-24). Many of these RNAs are more abundant in EV than miRNAs, and could therefore be important players in intercellular communication. Furthermore, these other RNA species may provide opportunities as novel disease biomarkers, which may be more easily detected because of their abundance. In this thesis, we explored whether the incorporation of these other non-coding RNA species in EV may be modulated by exogenous stimuli imposed on cells. In this work we predominantly studied EV released by antigen presenting immune cells, such as dendritic cells (DC) and macrophages, as well as EV from other immune cells such as neutrophils, both of which are known to robustly respond to immune-activating stimuli in order to initiate or regulate immune responses. First, we addressed a number of technical issues that affect reliable quantification of small non-coding RNA in EV from cultured cells and biofluids. We optimized and validated protocols for RNA sequencing (Chapter 2), and addressed the depletion of contaminant RNA species from fetal calf serum (FCS) (Chapter 3). We used this knowledge to characterize changes in the small non-coding RNA content of EV released by DC upon treatment with either immune stimulating or immune suppressive stimuli (Chapter 4). Besides expected differences in miRNA content, we discovered that the incorporation of Y-RNA into EV was changed in LPSstimulated DC. This finding sparked our interest, as numerous studies have abundantly detected Y-RNA in EV, and ideas on a role for Y-RNA in immune regulation and their biomarker potential are gaining ground within the field of extracellular RNA research. This motivated us to make an inventory of published data on extracellular Y-RNA and compare the various studies in this field (Chapter 5). Subsequently, we explored the presence of Y-RNA in blood plasma and investigated whether Y-RNA subtype ratios in plasma can be used as indicators of inflammatory processes (Chapter 6). Finally, we investigated how Y-RNA binding proteins may affect Y-RNA shuttling in immune cells under homeostatic and immune stimulatory conditions (Chapter 7) to learn more about the processes that underly the incorporation of Y-RNA into EV. 


\section{Technical considerations for reliable isolation and characterization of EV-RNA}

\section{Identification and reduction of small RNA sequencing bias}

Small RNA sequencing is increasingly used to obtain a comprehensive, quantitative profile of extracellular small RNA in studies on the role of EV-RNA in intercellular communication and for biomarker discovery (25). However, major challenges in small RNA sequencing are the various sources of bias that are introduced during the generation of sequencing libraries, such as ligation bias and PCR amplification bias $(26,27)$. To ensure the comparability of RNA sequencing results obtained in different labs, in-depth knowledge about the accuracy and reproducibility of this technique is important to guide reliable biomarker discovery. It has been suggested that small RNA sequencing library preparation methods are generally reproducible between labs (28). However, this study did not address whether comparable biases are introduced by the different commercial small RNA sequencing kits that are available. Furthermore it was not known to what extent these kits capture the full complexity of RNA transcripts in a sample, and whether they allow accurate estimation of the abundance of each RNA transcript.

To address these questions, we had the opportunity to take part in a multi-center study for comparison of commercially available small RNA sequencing methods, led by the exRNA Communication Consortium (ERCC) of the National Institutes of Health. In this study, a standardized reference sample of 1152 synthetic miRNAs (in equimolar and ratiometric pools) was analyzed in several different laboratories using different small RNA sequencing methods to assess whether these methods allow for accurate absolute and relative quantification of miRNAs (Chapter 2, (29)). Strikingly, large protocol-dependent biases were observed in the absolute read counts for each of the miRNAs. Although all individual miRNAs were equally abundant in the equimolar RNA pool, numbers of read counts differed greatly between individual miRNAs, and differed between the same miRNA quantified with different protocols. This bias could be partially resolved using $4 \mathrm{~N}$-adapters containing unique molecular barcodes. In this procedure, each transcript was tagged with a unique barcode which allowed to computationally correct for differences in PCR-amplification between transcripts. While this approach resolved the bias that is introduced during PCR-based library amplification, other sources of bias could not be resolved. We assessed whether these biases could be attributed to differences in the physical properties of the RNA transcripts, such as differences in 5'- and 3'-terminal nucleotides, GC-content, free energy $(d G)$, enthalpy $(d H)$, entropy $(d S)$, or melting temperature $(T m)$. Ideally, these properties may be used to predict and computationally correct for sequencing biases. However, none of these parameters had an unambiguous effect on their detection by 
RNA sequencing, indicating that computational correction of sequencing bias based on these parameters may not be feasible. These findings have important implications for the interpretation of RNA sequencing data. Although our data indicated that sequencing counts do not correlate with the absolute RNA abundance, various studies select RNAs with high sequencing counts for follow-up experiments, assuming that these are the most abundant (and therefore important) transcripts in their samples (30-32). It would be advisable to validate the absolute abundance of non-coding RNAs of interest by additional methods, such as RT-qPCR. Despite difficulties with quantification of transcripts based on read counts, our study showed that relative differences in the abundance of the same miRNA type in different samples can be accurately assessed, provided that the samples have been prepared using the same library preparation protocol (Chapter 2, (29)). In conclusion, these results indicate that although biases interfere in the sequencing efficiency of small RNA transcript, the relative abundance of the same RNA transcripts in different samples can be reliably measured.

\section{Characterization of fragmented and full-length forms of the same transcripts}

In addition to biases that affect the quantification of RNA transcripts, other types of biases can affect the identification and characterization of RNA transcripts. During bioinformatic analysis of RNA sequencing data, transcripts are 'mapped' onto genomic data, which can be visualized in so-called 'coverage plots'. These plots show which regions of an RNA transcript are found in a sequencing library, and whether the entire length of a transcript has been detected or only a particular region. This is of importance because many small non-coding RNAs can be cleaved into fragments, and therefore occur in the transcriptome in fragmented and full length forms. It is important to distinguish between these two forms, because fragmented RNAs often have other functions in cells than their full-length counterparts (33). For example, fragments of vault RNA and specific snoRNA fragments can repress the translation of mRNAs (34-36). Furthermore, specific tRNA fragments have been implicated in initiation of translation (37), in enhancing translation of mRNAs encoding ribosomal proteins (38), and in protection against endogenous retrotransposons (39). Interestingly, fragmented forms of various RNA types have been detected in EV, which may imply that these fragments play a role in intercellular communication (19-21,40). Also in the case of Y-RNA, various studies have reported the presence of Y-RNA cleavage fragments and tRNA fragments in EV (18-21,40-42). Indeed, it is known that Y-RNA can be cleaved under specific circumstances, such as apoptosis, poly-l:C stimulation or viral infection (43-45). However, technical limitations of the standard small RNA-seq protocols used in EV studies hamper accurate assessment of full length form of Y-RNA 
and tRNA. First, the overrepresentation of RNA fragments in RNA sequencing studies may be caused by size selection of sequencing libraries. This is a common procedure in standard miRNA sequencing protocols, which enriches for miRNAs while excluding longer-sized RNAs, such as full-length Y-RNAs and tRNAs. Second, posttranscriptional modifications on these RNA transcripts may hamper the adapter ligation efficiency (46). Furthermore, the strong secondary structures of Y-RNAs and tRNAs may hamper reverse transcription of the full-length transcripts, causing preferential detection of fragments of these RNAs. These technical problems can be mitigated by library preparation at high temperatures using thermostable reverse transcriptases $(24,47)$. Indeed, studies employing these protocols have indicated that the majority of Y-RNA and tRNA in plasma and EV from HEK293T cells is full-length $(24,47)$. Northern blotting can be employed as an alternative method to accurately assess the size of RNA transcripts. In this procedure, RNA is separated on size by gel electrophoresis, followed by detection of specific transcripts by hybridization of detection oligonucleotides. Via this method, we found that the majority of Y-RNA in EV from in vitro cultured primary DC is full-length (Chapter 4, (18)). Furthermore, cleavage of Y-RNA was not increased in EV-RNA or cellular RNA from LPS-stimulated DC (Chapter 4, (18)). Additionally, using RT-qPCR primers against the loop region of Y-RNA (which is not present in fragmented Y-RNA) we have confirmed the predominant presence of full length Y-RNA in EV, thereby corroborating the data from Qin et al ((47), and Chapter 6). These findings contrast sharply the conclusions of several other studies, in which it was stated that most Y-RNA in EV and RNP is fragmented $(23,42,48)$. However, all of these studies employed a narrow size-selection during library preparation, which does not allow the detection of full-length Y-RNA (which is $83-110 \mathrm{nt}$ in size). This is in contrast to the studies by Shurtleff and Qin, where no size selection was applied and in which $>75 \mathrm{nt}$ paired end sequencing was performed. Although our RNA sequencing data did show predominant coverage at the $5^{\prime}$ and $3^{\prime}$ ends of Y-RNA, we have shown by Northern blot that DC-EV predominantly contain full-length Y-RNA and tRNA (Chapter 4, (18)), thereby corroborating the data from Shurtleff et al.

Another point of discussion is how Y-RNA is distributed across EV and RNP. We have shown that the full-length $Y$-RNA in plasma is protected from degradation by proteinase $\mathrm{K}$ and RNAse $\mathrm{A}$, indicating that Y-RNA is predominantly present in EV (Chapter 6). In in vitro cultures of primary DC (Chapter 4) and immortalized B-cells (Driedonks, unpublished data) we have consistently found enrichment of Y-RNA in EV fractions compared to RNP fractions. Jeppesen et al., on the contrary, noted that most Y-RNA released by glioblastoma cells is fragmented and is predominantly associated with RNP (48). This confirmed the data of another study in which EV from a glioblastoma cell line were analyzed (23). The Jeppesen study also showed that in cultures of breast cancer cells, Y-RNA is equally distributed over EV and in RNP (48). These data suggests 
that the high release of RNP-associated Y-RNA may be characteristic of glioblastoma, but cannot be generalized for other cell types (48) or body fluids. Summarizing, there is considerable discussion on the type of macromolecular structures in the extracellular space to which non-coding RNAs, such as tRNA and Y-RNA, are associated and on the fragmentation status of these RNAs. Differences in the conclusions drawn in multiple studies on this topic are likely caused by differences in biological material and in the employed methods for RNA sequencing.

Taken together, accurate and reproducible small RNA sequencing results can be obtained through differential expression analysis between EV-RNA samples prepared in parallel using the same kits and by employing size selection that spans the entire size range of small RNAs. For subsequent RT-qPCR validation, full-length RNA transcripts can be specifically detected using oligonucleotide primers that recognize regions specifically present in the full-length transcript and not in fragments thereof (Chapter 6 and 7). Verification of RNA fragments and accurate size determination can be performed using Northern blot (Chapter 4). Together, these complementary techniques can be combined to obtain a comprehensive picture on the small noncoding RNA content of EV.

\section{Reducing background levels of contaminant RNAs in fetal bovine serum}

Specific technical challenges are encountered when analyzing EV-RNA derived from in vitro cultured cells. The medium in which cells are grown is commonly supplemented with fetal calf serum (FCS), which is an abundant source of RNA-containing EV. These EV are commonly depleted by overnight ultracentrifugation $(49,50)$, and it was previously proposed that this procedure sufficiently depletes FCS-derived RNA from culture medium (51). However, a more recent study highlighted that a considerable amount of FCS-derived RNA remains detectable in culture medium after prolonged ultracentrifugation $(52,53)$. Importantly, these residual bovine small non-coding RNAs may interfere with the analysis of EV-RNA due to high sequence similarity with human and murine small non-coding RNAs $(54,55)$. We investigated whether the depletion efficiency of RNA-carriers in FCS could be improved via technical optimization of ultracentrifugation protocols (Chapter 3, (56)). We found that dilution of FCS prior to ultracentrifugation increased the pelleting efficiency of RNA-containing structures, likely because it decreases the viscosity of the medium $(50,57)$. In addition, not disturbing the pellet by leaving $10 \%$ of volume on top of the pellet by pipetting off the EV-depleted resulted a low degree of RNA contamination, in comparison to decanting of the supernatant (Chapter 3, (56)). Nevertheless, we observed that not all non-coding RNA was depleted by ultracentrifugation (Chapter 3, (56)). This is in line with the observed association of non-coding RNA to different macromolecular 
structures in plasma and serum, such as ribonucleoprotein complexes (RNP) and lipoprotein particles (LPP) ((58-60), Chapter 6). Lipoprotein particles are highly abundant in serum and plasma. LDL, VLDL and chylomicrons have a low density which hampers their efficient sedimentation (Chapter 6). HDL particles are much smaller than EV, but overlap in buoyant density and may thus be partly depleted from FCS by prolonged ultracentrifugation. The presence of residual low-density and high density LPP may explain the observations by Lehrich et al., who reported that a high number of particles remained in FCS after depletion by overnight ultracentrifugation (61), and wrote a critical letter to the Journal of Extracellular Vesicles regarding our study (62). Their observations may be explained by the fact that particles were quantified using nanoparticle tracking analysis (NTA), which does not allow discrimination between EV and other types of particles, such as LPP $(63,64)$. By using a combination of methods aiming to obtain high-grade purified EV and EV quantification by high-resolution flow cytometry, we have shown that $95 \%$ of FCS-derived EV were depleted using our optimized protocol (Chapter 3, (56)). In addition, we have shown that FCS-derived RNAs in EV-depleted culture medium were not co-purified with density gradient purified EV (Chapter 3, (56)). Furthermore, we demonstrated the importance of including medium controls to quantify the background levels of contaminant RNAs in purified EV samples. This does not only allow estimation of contaminant RNA derived from culture medium, but also addresses contaminant RNAs that are introduced via laboratory reagents used in sample processing $(53,65)$. Therefore, addressing background RNA contamination using procedural controls is important, irrespective of the depletion method or medium alternative that is used. The use of EV-depleted medium controls is already recommended for studies on the biological effects of EV (66), and will hopefully be implemented for EV-RNA analysis in the next versions of the MISEV guidelines.

\section{RNAs associated with lipoproteins and RNPs in plasma may confound EV-RNA biomarker studies}

In addition to the challenges that LPP-associated RNAs pose in depletion of contaminant RNA from FCS, LPP-associated RNAs in human plasma also complicate the search for EV-RNA biomarkers ((58-60), Chapter 6). It has been estimated that only $38 \%$ of miRNA in plasma is EV-enclosed (67), and LPP were estimated to be 100 fold more abundant in plasma than EV (68). As stated in the previous paragraphs, LPP are highly heterogeneous in size, and the buoyant density of HDL overlaps with that of EV (69), which complicates the separation of EV and LPP. Both HDL and LDL contain miRNAs (reviewed in (70)) and were recently shown to additionally contain tRNA-fragments (71), but other types of RNA have not (yet) been characterized in LPP. In principle, both LPP-associated RNA and EV-associated RNA may be explored for 
their biomarker potential, although EV-RNA biomarkers have a number of advantages based on our current knowledge. For example, we have shown that RNAs associated to EV that are purified from plasma are more resistant to degradation by external proteases and RNAses than RNAs associated to purified LPP/RNP (Chapter 6). We recently validated these findings in total, unfractionated plasma. In line with our earlier findings (Chapter 6), specific EV-associated RNAs, such as Y-RNA, were resistant to degradation when proteases and RNAses were added to full plasma, whereas non-EV associated RNAs were degraded under these conditions (Driedonks, unpublished data). This suggests that EV-associated RNA biomarkers are more resistant to degradation by proteases and RNAses that may circulate in plasma. Another advantage of EVRNA biomarkers may be that changes in EV-RNA more directly resemble changes in (diseased) cells, as these changes are directly caused by (patho)physiological changes in EV-producing cells. Therefore, profiling EV-specific RNAs in plasma may provide a more direct 'window' through which the disease state of cells can be assessed. This was exemplified by a study on RNA biomarkers for classical Hodgkin lymphoma, which showed that RNA isolated from purified plasma EV outperformed RNA isolated from total plasma in distinguishing between diseased individuals versus healthy controls (72). The specificity of RNA profiling may be increased further by enrichment for cell-specific or tissue-specific EV subsets. This allows the selective quantification of RNA that is released from a specific cell type, e.g. from brain-derived EV to diagnose neurodegenerative diseases (73). Although this illustrates the specific advantages that EV-RNAs have in biomarker applications, LPP-RNA may also contain valuable disease related information, as it has been shown that the RNA composition of LPP in plasma can also change during disease. For example, it was shown that the levels of LPPassociated miR-223 were altered during atherosclerosis (60). Furthermore, HDL- and LDL-associated miRNAs have been found to be dysregulated in hypercholesterolemia and heart diseases (reviewed in (70)). Additionally, the rate of uptake of LPP in the liver by scavenger receptor $\mathrm{BI}$ (SR-BI) affects the levels of LPP-associated miRNAs in plasma (71). Thus LPP may be of interest in these conditions, and the relation of LPP-RNA to other diseases may be discovered in the future. It should be noted that, besides disease-related changes, the LPP concentration in plasma also depends on the prandial status of individuals $(74,75)$. As a result, LPP levels in plasma strongly fluctuate, which may cause large diurnal variability in the levels of LPP-associated miRNA present in plasma. Summarizing, LPP may provide a source of biomarkers, for example for liver conditions and heart diseases, but the diurnal variability and lower stability of LPP associated RNAs are points of attention.

Finally, it is important to note that many studies that aim to assess changes in the RNA content of plasma EV are confounded by co-isolated LPP structures. As we illustrated in Chapter 1, many EV-purification methods may co-purify contaminant LPP. In addition, 
the level of contamination with LPP differs per EV-enrichment method, which may result in inter-study and inter-laboratory differences in the RNA pool recovered from plasma EV preparations (76). Furthermore, it was shown that LDL can associate to the EV, which further complicates the complete separation of LPP and EV from plasma (75). The current consensus is that combinations of size- and density-based separation techniques are considered as most effective to separate LPP and EV $((67,68)$, Chapter 6). Nevertheless, this approach is labor-intense and largely impractical for processing large numbers of patient samples. Therefore, many plasma biomarker studies have employed direct RNA purification from plasma $(76,77)$. Although this may be a practically feasible approach, the simultaneous isolation of RNA from both EV and LPP in total plasma may decrease the sensitivity with which disease-related biomarkers may be detected (72). Therefore, to assess the utility of a proposed RNA biomarker, it is important to determine whether this marker is associated to EV, LPP or RNP, as we have shown in Chapter 6. RNA markers that are exclusively associated with EV, LPP or RNP may subsequently be profiled in unfractionated plasma for practical reasons.

Summarizing, these points illustrate that LPP-associated RNAs form a considerable point of attention for liquid biopsy EV-RNA profiling, since they are extremely abundant in plasma, may co-purify with EVs, are more sensitive to degradation than EV, and their levels can greatly fluctuate throughout the day. In addition, technical parameters in EV-enrichment procedures may greatly affect the outcome of studies characterizing extracellular RNA in biofluids and in EV from in vitro cultured cells. As this awareness is growing in the EV-research field, initiatives are being developed to optimize and standardize technologies for EV-RNA isolation and characterization in order to move this exciting field forward (67).

\section{Immune-stimuli change the levels of various small non-coding RNA types in EV released by immune cells}

While the miRNA content of EV is the current point of focus in the EV-research field, EV contain a wide range of other small non-coding RNA molecules $(2,18-21,42,78)$. Importantly, these other RNA types are generally more abundant than miRNAs and have been reported to be enriched in EV compared to cells $(18-21,78)$, which suggests that these molecules may be specifically recruited into EV for release into the extracellular milieu. While changes in EV-miRNA content have been reported in the context of various cellular stimuli $(2-5,79)$, effects of cellular stimulation on the levels of other non-coding RNAs in EV are largely unexplored. Furthermore, our knowledge on processes that underlie activation-induced changes in RNA shuttling into EV are still limited. To address these topics, we have focused on EV-RNA released by antigen 
presenting immune cells, such as dendritic cells and macrophages, and other immune cells such as neutrophils, which are known to robustly respond to immune-activating stimuli (Chapter 4, 6 and 7). We hypothesized that differentially incorporated noncoding RNAs may have a functional role in cellular communication, or may be explored further in the context of disease biomarkers.

As a starting point, we explored the small non-coding RNA transcriptome of EV from primary DC (Chapter 4, (18)). DC are master regulators of immune responses, and can differentiate into functionally different phenotypes depending on immuneactivating or immune-tolerizing signals they encounter in the environment. Every type of immune cell is equipped with pattern recognition receptors (PRRs), such as Toll-like receptors (TLRs) and the cytosolic RNA sensors RIG-I and MDA-5 $(80,81)$. These sensors allow cells to recognize specific molecular patterns that are found on pathogens such as bacteria and viruses. For example, TLR4 recognizes lipopolysaccharide (LPS), which is a component of the outer membrane of Gram-negative bacteria, leading to the activation of a signaling cascade. TLR-activation in DC is critical for the production of inflammatory cytokines that stimulate $T$ cells and B cells to mount an immune response. Furthermore, triggering of PRRs results in increased antigen presentation on MHC molecules and exposure of costimulatory molecules like CD40 and CD86 (82). Apart from these immune-activating stimuli, various other stimuli have a tolerizing effect on DCs, such as 1,25a-vitamin-D3 (the biologically active form of Vitamin D3) and the anti-inflammatory cytokine IL-10 (83). These ligands bind to the vitamin-D receptor and IL-10 receptors, respectively, and render the DCs insensitive to subsequent activation by TLR ligands $(82,84-86)$. Furthermore tolerogenic DC induce the differentiation of $\mathrm{T}$ regulatory cells via the release of anti-inflammatory cytokine IL-10 (82,83). Thus, environmental stimuli cause DC to take on functionally distinct phenotypes. Importantly, the EV that are released by DC contribute to the signaling to other immune cells (87-91). Furthermore, the EV released by DC may have therapeutic potential $(92,93)$. It was attempted to use EV from immature DC to induce T cellmediated immune responses against cancer (94), and EV from IL-10 treated DC were shown to suppress inflammation in an arthritis model (95). How these functionally distinct phenotypes affect the RNA content of the EV is not fully understood. Therefore, we compared the RNA composition of EV released by unstimulated DC, immunogenic DC (stimulated with LPS), and tolerogenic DC (stimulated with 1,25a-vitamin-D3) using small RNA sequencing. As expected, we observed that the different stimuli imposed on the DC led to selective enrichment of specific miRNA subsets in EV released by these cells (Chapter 4, (18)). EV released by immunogenic DC contained higher levels of the endotoxin-responsive miRNAs miR-155 and miR-146a $(96,97)$, while we observed enrichment of miRNAs with immune-suppressive functions in EV from tolerogenic DC, such as miR-10a and miR-27a $(98,99)$. Furthermore, we observed that only a small 
percentage of the stimulation-induced changes in EV-miRNAs reflected the changes in the cellular levels of these miRNAs. Besides these changes in miRNA content, the treatments imposed on the DC led to changes in the Y-RNA and snoRNA content of the released EV (Chapter 4, (18)). Effects of immune-related stimuli on levels of snoRNA and Y-RNA in EV have not been described before. The changes observed in EV-associated snoRNAs did not reflect those observed in cells, suggesting that snoRNA levels in EV are regulated independently from their cellular levels. Alterations in snoRNA expression in human cells can affect various vital cellular processes, such as cancer, viral infection and neurodegenerative disease (100). While changes in snoRNA levels in cells have mostly been reported in relation with cancer (101), they have not been investigated in the context of immune cells. Nevertheless, the functional involvement of snoRNAs in the development of disease has not been addressed. Furthermore, since snoRNAs are generally retained in cells $(18-20,78)$, it remains to be investigated whether the low levels of snoRNA in EV may have functional relevance in intercellular communication.

\section{TLR-stimulation affects the incorporation of Y-RNA into EV from various immune cells}

In contrast to snoRNAs, Y-RNAs were abundantly detected in EV (Chapter 4). We discovered that the incorporation of Y-RNA into EV was influenced by TLR-stimulation of dendritic cells (Chapter 4). We additionally observed that TLR-stimulation of altered the release of Y-RNA in EV from macrophages (Chapter 7) and neutrophils (Chapter 6). Interestingly, the TLR-activation induced changes in EV-associated Y-RNA levels were different for these different cell types. Namely, TLR-activation decreased the Y-RNA content of DC-EV (Chapter 4), it increased the Y-RNA content of THP1-macrophage EV (Chapter 7), and did not change the net amount of Y-RNA of neutrophil-EV (although it increased the overall EV release) (Chapter 6). These differences in Y-RNA release in response to TLR-stimulation might be due to intrinsic biological differences between these cell types. Additionally, they might be explained by differences in experimental setup and/or differences in the type of TLR ligands we used in these studies. Whereas in Chapter 4, we only started the EV production after TLR stimulation, in Chapter 6 and 7 EV production and TLR stimulation were done simultaneously. Despite these experimental differences, we consistently observed that cellular Y-RNA levels remained stable after TLR-stimulation (Chapters 4 and Chapter 7). This suggested that incorporation of Y-RNA in EV is an active, protein mediated process which can be regulated by external stimuli. A number of proteins have been identified which are involved in the packaging of miRNAs into EV, such as hnRNP A2B1 and hnRNP Q, HuR, and YBX1 (102-105). The efficiency with which these 
proteins are incorporated into EV may depend on posttranslational modifications. For example, the incorporation of hnRNP A2B1 into EV from T cells seems to be controlled by SUMOylation (102). In addition, the incorporation of these miRNA-binding proteins may additionally be regulated through interactions with other proteins. It was recently shown that the shuttling of hnRNP A2B1-bound miRNAs (miR-17 and miR-93) is controlled by caveolin-1, which under hyperoxic conditions becomes phosphorylated, binds to hnRNP A2B1, and is exported into EV in complex with hnRNP A2B1 (106). Furthermore, it was suggested that the shuttling of other non-coding RNA species, such as Y-RNA, into EV was mediated by YBX1 (24). Nevertheless, we did not observe this protein in THP1 macrophage EV, nor did YBX1 co-localize with Y-RNA in subcellular compartments isolated by gradient fractionation (Chapter 7). For other proteins that are known to be associated with Y-RNA (summarized in Chapter 5, (107)), we did observe co-localization with Y-RNA in subcellular compartments isolated by density gradient fractionations. Furthermore, various proteins that are involved in EV biogenesis, such as Tsg101 (ESCRT-I) (108) and CD63 (109), also co-localized with Y-RNA in these subcellular compartments. This suggests that Y-RNA may be closely associated to sites of biogenesis within the cell. We observed that the release of Y-RNA was increased in EV from TLR-activated macrophages, which coincided with increased levels of Ro60 in EV. It is known that Ro60 is required for stabilization of Y-RNA in the cytosol (110), and may thus additionally stabilize Y-RNA in EV. Nevertheless, the increased incorporation of Y-RNA and Ro60 could not be explained by changes in their local concentration at the sites of EV-biogenesis. Based on our findings, it may be hypothesized that immediately after packaging of Y-RNA and Ro60 molecules into EV, they are directly replenished by other Y-RNA and Ro60 molecules. This would mean that immune stimuli would increase the total flux of Y-RNA from the nucleus and other sites in the cytosol into MVB, where they are more effectively packaged. Taken together, our results indicate that the RNA composition of EV does not completely correspond to that of the EV producing cell (Chapter $\mathbf{4}$ and 7). Although a number of RNA-shuttling proteins have been discovered, we do not yet understand how their activity is controlled by the activation status of the EV-producing cell. Answering these questions in future studies will allow us to better understand how the EV-transcriptome relates to the transcriptional profiles of their parental cells.

\section{Potential functions of EV-enclosed Y-RNA in recipient cells}

The observation that the incorporation of Y-RNA into EV is regulated by TLR-activation of various immune cells suggests it may be have a role in immune regulatory processes. Indeed, Y-RNA is emerging as a non-coding RNA type with emerging immune-related 
functions (summarized in Chapter 5, (107)). These functions likely depend on the type of macromolecular structure to which the Y-RNA is associated, and on whether this Y-RNA is fragmented or full-length. Previous studies indicate that RNP-associated Y-RNA predominantly mediate inflammatory effects, while anti-inflammatory effects have been proposed for EV-associated Y-RNA (Chapter 5, (107)). For example, an EVassociated 5'-fragment of Y4-RNA has been implicated in the induction of the immune suppressive cytokine IL-10 when transfected into macrophages (32). In addition, fulllength Y4-RNA was shown to activate TLR7 when transfected into monocytes, leading to the production of various chemokines and the expression of PD-L1, a ligand for the immune inhibitory receptor PD1 (111). Although transfection is currently the most feasible method to assess the effects of single RNA components of EV, this delivery route may differ from Y-RNA that is transferred by EV. Importantly, transfected synthetic RNA may activate cellular RNA sensors, for instance TLR7 which is specifically activated by single-stranded RNA (such as synthetic Y-RNA). In contrast, RNA in EV may be associated to proteins that shield off parts of the RNA, thereby preventing the activation of RNA sensors in the cell. This is exemplified by a recent study by Nabet et al. who found that the non-coding RNA 7SL in EV from activated stromal cells is not shielded by proteins and can trigger cytosolic RNA sensor RIG-I in breast cancer cells (16).The exact mechanisms by which Y-RNA elicits an effect in recipient cells remain to be uncovered. These might include activation of cellular RNA sensors (such as TLR7 (111)), or binding of proteins that affect mRNA translation (112). It has been shown that Y3-RNA in neuronal cells acts as a molecular scavenger for enhancer protein HuD, which enhances the translation of various proteins involved in neuronal development (112). Thus, increased Y3-levels induce the dissociation of HuD from mRNAs, thereby downregulating their translation. We have observed that increased Y-RNA export from cells via EV was accompanied by a translocation of the related protein HuR from endolysosomal compartments toward ER (Chapter 7). Similar to what was proposed by Tebaldi et al., our data therefore also suggest that local Y-RNA levels influence the localization and potentially the function of mRNA stabilizing proteins. In Chapter 7, we mainly discussed how Y-RNA export via EV could influence the function of the EVproducing cell and we did not speculate on the effects of Y-RNA in recipient cells. However, it may be hypothesized that Y-RNA transferred into recipient cells via EV scavenge away proteins that stabilize mRNA, thereby regulating their translation or stability. More research is needed to more accurately evaluate the effects of Y-RNA on recipient cells, and to compare the functional effects of EV-versus RNP-associated Y-RNA released from the same cell. 


\section{Potential of Y-RNA based biomarkers for diagnosis of immune-mediated inflammatory diseases}

Y-RNAs or Y-RNA fragments have been suggested as biomarkers in coronary artery disease and cancer $(113,114)$. These studies did not address to which structures Y-RNA in plasma was associated, and were biased towards the detection of Y-RNA fragments. Based on our findings that TLR triggering led to changes in the Y-RNA content of EV, we investigated whether EV-associated Y-RNAs in plasma could serve as indicators for ongoing inflammatory processes (Chapter 6). Interestingly, we discovered that EV from different blood-related cell types contained specific Y-RNA subtype ratios. EV from neutrophils, for example, contained a unique $Y 4 / Y 3$ ratio which was not detected in EV from other tested cell types. Furthermore, EV release by neutrophils was rapidly (within $2 \mathrm{hrs}$ ) upregulated after LPS stimulation, while the Y4/Y3 ratio in these EV remained stable. In a human model for systemic endotoxemia (115), where the numbers of circulating neutrophils increase upon LPS injection, we observed a positive correlation between the numbers of neutrophils and the $Y 4 / Y 3$ ratio in total plasma. This observed change in circulating Y-RNA subtype levels may be caused by increased numbers of neutrophils in blood and perhaps also by increased EV release by these neutrophils. Our data provided proof-of-concept that systemic inflammation can change the overall Y-RNA composition of human plasma, and suggested that the Y4/Y3 ratio correlates with neutrophil recruitment during inflammatory conditions (Chapter 6). The endotoxemia model that we used is a common model for studying sepsis $(116,117)$. It will be interesting to explore whether Y-RNA subtype ratio determination in plasma could also aid in the diagnosis of sepsis. Sepsis is characterized by a systemic inflammatory response which is caused by microbial infection and may lead to multi-organ failure (118). This condition is very heterogeneous with regard to the sites of infection, causative microbes, and the general health status of the patient (119). Diagnostic criteria include fever, elevated heart rate, elevated or decreased leukocyte counts, aberrant hemodynamic and organ-dysfunction variables, but the most definitive diagnosis is made through overnight microbial culture. Through the development of novel biomarkers, diagnosis times could be decreased which may lead to better care. Many cases of sepsis start with only subtle symptoms, which are likely caused by local inflammation (120). Novel biomarkers for sepsis that are sensitive enough to be of diagnostic value at these early timepoints are still heavily sought after (121-123). Several studies to date have investigated differences in plasma miRNAs during sepsis, few of which may be translated to clinical use (124). Recently, EV were proposed to have diagnostic potential, because their levels increase in inflammatory conditions and they may be functionally involved in the pathophysiology of sepsis (123). Neutrophils are one of the first cell types to respond to pathogens by infiltrating 
infected tissues and controlling the infection. Since we have shown that neutrophils rapidly and strongly increase their EV release upon treatment with LPS (Chapter 6), the neutrophil-associated Y-RNA ratio might be sensitively detected already during the local inflammation phase of sepsis. Another reason why it is interesting to specifically monitor neutrophil-EV in plasma of patient with inflammatory conditions is that neutrophil EVs have been functionally implicated in immune-suppression (reviewed in(125)) and tissue damage (126). Nevertheless, in order to utilize this neutrophilspecific Y-RNA ratio as marker for levels of neutrophil EV, a number of questions need to be addressed. For example, do $\mathrm{Y} 4 / \mathrm{Y} 3$ ratios in plasma indeed correlate with neutrophil-specific EV protein markers? Is analysis of the Y-RNA subtype ratio in plasma sensitive enough to detect inflammatory processes in local tissues? How well does this biomarker candidate perform in an actual cohort of sepsis cases? Can Y-RNA subtype ratios additionally be used to detect other inflammatory diseases involving neutrophils, such as rheumatoid arthritis? Taken together, our findings provide support for the diagnostic value of Y-RNA ratios in inflammatory conditions, and their biomarker potential may be investigated further.

\section{Future perspectives}

The great technological advances in RNA-sequencing and the increased interest in EVRNA have led to many novel insights in the role of EV-RNA in health and disease and their applicability as biomarkers. Nevertheless, how the disease status of cells causes disease-related changes in circulating RNA in plasma is an important question that has remained largely unanswered. In my personal opinion, we need to increase our understanding of how diseases affect the incorporation of RNA into EV to evaluate changes that are observed in total biofluids. It is evident that diagnosis of diseases can be made based on aberrant levels of circulating RNA in plasma. However, understanding what these aberrant levels say about the disease condition of the parental cell will be of additional value, for example in prognosis or prediction of disease outcome, and may furthermore aid in patient stratification (e.g. into stages of disease progression). Furthermore, these insights will additionally increase our understanding of how aberrant RNA levels in EV functionally contribute to this disease. Importantly, our experiments have shown that changes in EV-RNA do not necessarily represent changes observed in cells (Chapter 4, 7). Thus, based on their RNA content, EV are not simple 'snapshots' of cells as was proposed by Skog et al. (6). It has become clear that protein-mediated processes play an important role in the specific shuttling of various RNA species into EV, as a number of initial studies have shown $(4,24,102,103,105,106)$. There are several important questions that need 
to be addressed in future studies. For instance, what determines the specificity of RNA binding proteins for certain RNA species? How does the disease/activation status of cells control the sorting activity of these proteins? It is often assumed that the RNA content of plasma changes only because of EV from diseased cells. However, it is also important to address whether the RNA cargo of EV from healthy cells is also modulated due to the presence of diseased cells. Furthermore, more attention should be paid to whether putative RNA biomarkers are associated with EV, RNP, LPP, or a combination of these structures, as these structures greatly influence the stability of the RNAs.

Strikingly, out of 150,000 proposed biomarkers, which include disease-associated proteins, lipids, metabolites and RNA both in tissues and biofluids, only 100 have become routinely used in the clinic (127). Although these cases do not just include EVbased biomarkers, these numbers are a cause of concern. The lack of standardization of sample collection and matching of people from which these specimens are taken were named as reasons for this high failure rate $(127,128)$. On the positive side, this leaves much room for EV-researchers to do better. Worldwide standardization efforts of biofluid collection and sample analysis will be required to reduce the variability and improve the quality of EV-research. Important groundwork has already been done by the EV-TRACK and MISEV2018 initiatives $(66,129)$, and new taskforces aiming to improve the reproducibility of EV work are in preparation (67). The last decade of research has highlighted the applicability of RNA-based 'liquid biopsies' in prevention and monitoring of a broad range of diseases. The current challenge is to better understand the complex systems that underlie differential RNA shuttling in biofluids, so that we can correctly interpret changes in plasma RNA in the context of disease. 


\section{References}

1. Valadi H, Ekström K, Bossios A, Sjöstrand M, Lee JJ, Lötvall JO. Exosome-mediated transfer of mRNAs and microRNAs is a novel mechanism of genetic exchange between cells. Nat Cell Biol (2007) 9:654-659. doi:10.1038/ncb1596

2. Bellingham SA, Coleman BM, Hill AF. Small RNA deep sequencing reveals a distinct miRNA signature released in exosomes from prion-infected neuronal cells. Nucleic Acids Res (2012) 40:10937-10949. doi:10.1093/nar/gks832

3. Montecalvo A, Larregina AT, Shufesky WJ, Stolz DB, Sullivan MLG, Karlsson JM, Baty CJ, Gibson GA, Erdos $G$, Wang Z, et al. Mechanism of transfer of functional microRNAs between mouse dendritic cells via exosomes. Blood (2012) 119:756-66. doi:10.1182/blood-2011-02-338004

4. Cha DJ, Franklin JL, Dou Y, Liu Q, Higginbotham JN, Beckler MD, Weaver AM, Vickers K, Prasad N, Levy S, et al. KRAS-dependent sorting of miRNA to exosomes. Elife (2015) 4:1-22. doi:10.7554/eLife.07197

5. Mckenzie AJ, Hoshino D, Hong NH, Coffey RJ, Patton JG, Weaver AM, Mckenzie AJ, Hoshino D, Hong NH, Cha DJ, et al. KRAS-MEK Signaling Controls Ago2 Sorting into Report KRAS-MEK Signaling Controls Ago2 Sorting into Exosomes. Cell Rep (2016) 15:1-10. doi:10.1016/j.celrep.2016.03.085

6. Skog J, Würdinger T, van Rijn S, Meijer DH, Gainche L, Curry WT, Carter BS, Krichevsky AM, Breakefield XO. Glioblastoma microvesicles transport RNA and proteins that promote tumour growth and provide diagnostic biomarkers. Nat Cell Biol (2008) 10:1470-1476. doi:10.1038/ncb1800

7. Redzic JS, Balaj L, van der Vos KE, Breakefield XO. Extracellular RNA mediates and marks cancer progression. Semin Cancer Biol (2014) 28:14-23. doi:10.1016/j.semcancer.2014.04.010

8. Revenfeld ALS, Bæk R, Nielsen MH, Stensballe A, Varming K, Jørgensen M. Diagnostic and prognostic potential of extracellular vesicles in peripheral blood. Clin Ther (2014) 36:830-846. doi:10.1016/j. clinthera.2014.05.008

9. De Toro J, Herschlik L, Waldner C, Mongini C. Emerging roles of exosomes in normal and pathological conditions: New insights for diagnosis and therapeutic applications. Front Immunol (2015) 6:1-12. doi:10.3389/fimmu.2015.00203

10. Tofaris GK. A Critical Assessment of Exosomes in the Pathogenesis and Stratification of Parkinson's Disease. J Parkinsons Dis (2017) 7:569-576. doi:10.3233/JPD-171176

11. Buzas El, György B, Nagy G, Falus A, Gay S. Emerging role of extracellular vesicles in inflammatory diseases. Nat Rev Rheumatol (2014) 10:356-64. doi:10.1038/nrrheum.2014.19

12. Zijlstra C, Stoorvogel W. Prostasomes as a source of diagnostic biomarkers for prostate cancer. J Clin Invest (2016) 126:1144-1151. doi:10.1172/JCl81128

13. Quek C, Hill AF. The role of extracellular vesicles in neurodegenerative diseases. Biochem Biophys Res Commun (2017) 483:1178-1186. doi:10.1016/j.bbrc.2016.09.090

14. Rak J. Extracellular vesicles - biomarkers and effectors of the cellular interactome in cancer. Front Pharmacol (2013) 4 MAR:1-14. doi:10.3389/fphar.2013.00021

15. Thompson AG, Gray E, Heman-Ackah SM, Mäger I, Talbot K, El Andaloussi S, Wood MJ, Turner MR. Extracellular vesicles in neurodegenerative disease-pathogenesis to biomarkers. Nat Rev Neurol (2016) 12:346-357. doi:10.1038/nrneurol.2016.68

16. Nabet BY, Qiu Y, Shabason JE, Wu TJ, Yoon T, Kim BC, Benci JL, DeMichele AM, Tchou J, Marcotrigiano J, et al. Exosome RNA Unshielding Couples Stromal Activation to Pattern Recognition Receptor Signaling in Cancer. Cell (2017) 170:352-366.e13. doi:10.1016/j.cell.2017.06.031

17. Datta Chaudhuri A, Dastgheyb RM, Yoo SW, Trout A, Talbot CC, Hao H, Witwer KW, Haughey NJ. TNFa and IL-1 $\beta$ modify the miRNA cargo of astrocyte shed extracellular vesicles to regulate neurotrophic signaling in neurons. Cell Death Dis (2018) 9: doi:10.1038/s41419-018-0369-4

18. Driedonks TAP, van der Grein SG, Ariyurek Y, Buermans HPJ, Jekel H, Chow FWN, Wauben MHM, Buck AH, Hoen PAC, Nolte-'t Hoen ENM. Immune stimuli shape the small non-coding transcriptome of extracellular vesicles released by dendritic cells. Cell Mol Life Sci (2018)3857-3875. doi:10.1007/s00018-018-2842-8 
19. Nolte-'t Hoen ENM, Buermans HPJ, Waasdorp M, Stoorvogel W, Wauben MHM, 't Hoen PAC. Deep sequencing of RNA from immune cell-derived vesicles uncovers the selective incorporation of small non-coding RNA biotypes with potential regulatory functions. Nucleic Acids Res (2012) 40:9272-85. doi:10.1093/nar/gks658

20. van Balkom BWM, Eisele AS, Pegtel DM, Bervoets S, Verhaar MC. Quantitative and qualitative analysis of small RNAs in human endothelial cells and exosomes provides insights into localized RNA processing, degradation and sorting. J Extracell vesicles (2015) 4:26760. doi:10.3402/jev.v4.26760

21. Tosar JP, Gambaro F, Sanguinetti J, Bonilla B, Witwer KW, Cayota A. Assessment of small RNA sorting into different extracellular fractions revealed by high-throughput sequencing of breast cell lines. Nucleic Acids Res (2015)1-16. doi:10.1093/nar/gkv432

22. Lässer C, Shelke GV, Yeri A, Kim D-K, Crescitelli R, Raimondo S, Sjöstrand M, Gho YS, Van Keuren Jensen K, Lötvall J. Two distinct extracellular RNA signatures released by a single cell type identified by microarray and next-generation sequencing. RNA Biol (2017) 14:58-72. doi:10.1080/15476286.2016.1249092

23. Wei Z, Batagov AO, Schinelli S, Wang J, Wang Y, El Fatimy R, Rabinovsky R, Balaj L, Chen CC, Hochberg F, et al. Coding and noncoding landscape of extracellular RNA released by human glioma stem cells. Nat Commun (2017) 8:1145. doi:10.1038/s41467-017-01196-x

24. Shurtleff MJ, Yao J, Qin Y, Nottingham RM, Temoche-Diaz M, Schekman R, Lambowitz AM. A broad role for YBX1 in defining the small non-coding RNA composition of exosomes. PNAS (2017)8987-8995. doi:10.1101/160556

25. Das S, Ansel KM, Bitzer M, Breakefield XO, Charest A, Galas DJ, Gerstein MB, Gupta M, Milosavljevic A, McManus MT, et al. The Extracellular RNA Communication Consortium: Establishing Foundational Knowledge and Technologies for Extracellular RNA Research. Cell (2019) 177:231-242. doi:10.1016/j. cell.2019.03.023

26. Jayaprakash AD, Jabado O, Brown BD, Sachidanandam R. Identification and remediation of biases in the activity of RNA ligases in small-RNA deep sequencing. Nucleic Acids Res (2011) 39:e141. doi:10.1093/nar/ gkr693

27. Raabe CA, Tang T-H, Brosius J, Rozhdestvensky TS. Biases in small RNA deep sequencing data. Nucleic Acids Res (2014) 42:1414-1426. doi:10.1093/nar/gkt1021

28. 't Hoen PAC, Friedländer MR, Almlöf J, Sammeth M, Pulyakhina I, Anvar SY, Laros JFJ, Buermans HPJ, Karlberg O, Brännvall M, et al. Reproducibility of high-throughput mRNA and small RNA sequencing across laboratories. Nat Biotechnol (2013) 31:1015-22. doi:10.1038/nbt.2702

29. Giraldez MD, Spengler RM, Etheridge A, Godoy PM, Barczak AJ, Srinivasan S, De Hoff PL, Tanriverdi K, Courtright A, Lu S, et al. Comprehensive multi-center assessment of small RNA-seq methods for quantitative miRNA profiling. Nat Biotechnol (2018) doi:10.1038/nbt.4183

30. Jenjaroenpun P, Kremenska Y, Nair VM, Kremenskoy M, Joseph B, Kurochkin I V. Characterization of RNA in exosomes secreted by human breast cancer cell lines using next-generation sequencing. PeerJ (2013) 1:e201. doi:10.7717/peerj.201

31. Huang X, Yuan T, Tschannen M, Sun Z, Jacob H, Du M, Liang M, Dittmar RL, Liu Y, Liang M, et al. Characterization of human plasma-derived exosomal RNAs by deep sequencing. BMC Genomics (2013) 14:319. doi:10.1186/1471-2164-14-319

32. Cambier L, de Couto G, Ibrahim A, Echavez AK, Valle J, Liu W, Kreke M, Smith RR, Marbán L, Marbán E. Y RNA fragment in extracellular vesicles confers cardioprotection via modulation of IL-10 expression and secretion. EMBO Mol Med (2017)e201606924. doi:10.15252/emmm.201606924

33. Tuck AC, Tollervey D. RNA in pieces. Trends Genet (2011) 27:422-432. doi:10.1016/j.tig.2011.06.001

34. Esteller M. Non-coding RNAs in human disease. Nat Rev Genet (2011) 12:861-74. doi:10.1038/nrg3074

35. Persson H, Kvist A, Vallon-Christersson J, Medstrand P, Borg A, Rovira C. The non-coding RNA of the multidrug resistance-linked vault particle encodes multiple regulatory small RNAs. Nat Cell Biol (2009)

11:1268-71. doi:10.1038/ncb1972 
36. Hussain S, Sajini A a, Blanco S, Dietmann S, Lombard P, Sugimoto Y, Paramor M, Gleeson JG, Odom DT, Ule J, et al. NSun2-mediated cytosine-5 methylation of vault noncoding RNA determines its processing into regulatory small RNAs. Cell Rep (2013) 4:255-61. doi:10.1016/j.celrep.2013.06.029

37. Ivanov P, Emara MM, Villen J, Gygi SP, Anderson P. Angiogenin-Induced tRNA Fragments Inhibit Translation Initiation. Mol Cell (2011) 43:613-623. doi:10.1016/j.molcel.2011.06.022

38. Kim HK, Fuchs G, Wang S, Wei W, Zhang Y, Park H, Roy-Chaudhuri B, Li P, Xu J, Chu K, et al. A transferRNA-derived small RNA regulates ribosome biogenesis. Nature (2017) doi:10.1038/nature25005

39. Schorn AJ, Gutbrod MJ, Leblanc C, Martienssen R. LTR-Retrotransposon Control by tRNA-Derived Small RNAs. Cell (2017) 170:61-71.e11. doi:10.1016/j.cell.2017.06.013

40. Vojtech L, Woo S, Hughes S, Levy C, Ballweber L, Sauteraud RP, Strobl J, Westerberg K, Gottardo R, Tewari $\mathrm{M}$, et al. Exosomes in human semen carry a distinctive repertoire of small non-coding RNAs with potential regulatory functions. Nucleic Acids Res (2014) 42:7290-7304. doi:10.1093/nar/gku347

41. Chiou NT, Kageyama R, Ansel KM. Selective Export into Extracellular Vesicles and Function of tRNA Fragments during T Cell Activation. Cell Rep (2018) 25:3356-3370.e4. doi:10.1016/j.celrep.2018.11.073

42. Godoy PM, Bhakta NR, Barczak AJ, Cakmak H, Fisher S, Mackenzie TC, Patel T, Price RW, Smith J, Woodruff PG, et al. Large Differences in Small RNA Composition Between Human Biofluids. Cell Rep (2018) 25:1346-1358. doi:10.2139/ssrn.3155656

43. Rutjes SA, van der Heijden A, Utz PJ, van Venrooij WJ, Pruijn GJ. Rapid nucleolytic degradation of the small cytoplasmic Y RNAs during apoptosis. J Biol Chem (1999) 274:24799-807. doi:10.1074/ jbc.274.35.24799

44. Nicolas FE, Hall AE, Csorba T, Turnbull C, Dalmay T. Biogenesis of Y RNA-derived small RNAs is independent of the microRNA pathway. FEBS Lett (2012) 586:1226-30. doi:10.1016/j. febslet.2012.03.026

45. Donovan J, Rath S, Kolet-Mandrikov D, Korennykh A. Rapid RNase L-driven arrest of protein synthesis in the dsRNA response without degradation of translation machinery. RNA (2017) 23:1660-1671.

46. Motorin Y, Muller S, Behn-Ansmant I, Branlant C. "Identification of Modified Residues in RNAs by Reverse Transcription-Based Methods," in Methods in Enzymology, 21-53. doi:10.1016/500766879(07)25002-5

47. Qin Y, Yao J, Wu DC, Nottingham RM, Mohr S, Hunicke-Smith S, Lambowitz AM. High-throughput sequencing of human plasma RNA by using thermostable group II intron reverse transcriptases. RNA (2015) 22:1-18. doi:10.1261/rna.054809.115.3

48. Jeppesen DK, Fenix AM, Franklin JL, Higginbotham JN, Zhang Q, Zimmerman LJ, Liebler DC, Ping J, Liu Q, Evans R, et al. Reassessment of Exosome Composition. Cell (2019) 177:428-445.e18. doi:10.1016/j. cell.2019.02.029

49. Théry C, Regnault A, Garin J, Wolfers J, Zitvogel L, Ricciardi-Castagnoli P, Raposo G, Amigorena S. Molecular Characterization of Dendritic Cell-derived Exosomes: Selective Accumunlation of the Heat Shock Protein hsc73. J Cell Biol (1999) 147:599-610. doi:10.1083/jcb.147.3.599

50. Théry C, Amigorena S, Raposo G, Clayton A. Isolation and Characterization of Exosomes from Cell Culture Supernatants. Curr Protoc cell Biol (2006) Chapter 3:1-29. doi:10.1002/0471143030.cb0322s30

51. Shelke GV, Lässer C, Gho YS, Lötvall J. Importance of exosome depletion protocols to eliminate functional and RNA-containing extracellular vesicles from fetal bovine serum. J Extracell Vesicles (2014) 3:1-8. doi:10.3402/jev.v3.24783

52. Wei Z, Batagov AO, Carter DRF, Krichevsky AM. Fetal Bovine Serum RNA Interferes with the Cell Culture derived Extracellular RNA. Sci Rep (2016) 6:31175. doi:10.1038/srep31175

53. Tosar JP, Cayota A, Eitan E, Halushka MK, Witwer KW. Ribonucleic artefacts: are some extracellular RNA discoveries driven by cell culture medium components? Jextracell Vesicles (2017) 6:1-10. doi:10.1080/ 20013078.2016 .1272832

54. Li SC, Chan WC, Hu LY, Lai CH, Hsu CN, Lin W chang. Identification of homologous microRNAs in 56 animal genomes. Genomics (2010) 96:1-9. doi:10.1016/j.ygeno.2010.03.009 
55. Griffiths-Jones S, Saini HK, van Dongen S, Enright AJ. miRBase: tools for microRNA genomics. Nucleic Acids Res (2008) 36:D154-8. doi:10.1093/nar/gkm952

56. Driedonks TAP, Nijen-Twilhaar MK, Nolte-'t Hoen ENM. Technical approaches to reduce interference of Fetal calf serum derived RNA in the analysis of extracellular vesicle RNA from cultured cells. J Extracell Vesicles (2018) 8: doi:10.1080/20013078.2018.1552059

57. Momen-Heravi F, Balaj L, Alian S, Trachtenberg AJ, Hochberg FH, Skog J, Kuo WP. Impact of biofluid viscosity on size and sedimentation efficiency of the isolated microvesicles. Front Physiol (2012) 3 MAY:1-6. doi:10.3389/fphys.2012.00162

58. Turchinovich A, Weiz L, Langheinz A, Burwinkel B. Characterization of extracellular circulating microRNA. Nucleic Acids Res (2011) 39:7223-33. doi:10.1093/nar/gkr254

59. Arroyo JD, Chevillet JR, Kroh EM, Ruf IK, Pritchard CC, Gibson DF, Mitchell PS, Bennett CF, PogosovaAgadjanyan EL, Stirewalt DL, et al. Argonaute2 complexes carry a population of circulating microRNAs independent of vesicles in human plasma. Proc Natl Acad Sci U S A (2011) 108:5003-8. doi:10.1073/ pnas.1019055108

60. Vickers KC, Palmisano BT, Shoucri BM, Shamburek RD, Remaley AT. MicroRNAs are transported in plasma and delivered to recipient cells by high-density lipoproteins. Nat Cell Biol (2011) 13:423-33. doi:10.1038/ ncb2210

61. Lehrich BM, Liang Y, Khosravi P, Federoff HJ, Fiandaca MS. Fetal Bovine Serum-Derived Extracellular Vesicles Persist within Vesicle-Depleted Culture Media. Int J Mol Sci (2018) 19: doi:10.3390/ijms19113538

62. Lehrich BM, Liang Y, Fiandaca MS. Response to "Technical approaches to reduce interference of Fetal calf serum derived RNA in the analysis of extracellular vesicle RNA from cultured cells." J Extracell Vesicles (2019) 8:1599681. doi:10.1080/20013078.2019.1599681

63. Gardiner C, Ferreira YJ, Dragovic RA, Redman CW., Sargent IL. Extracellular vesicle sizing and enumeration by nanoparticle tracking analysis. J Extracell Vesicles (2013) 2:19671. doi:10.3402/jev. v2i0.19671

64. Driedonks TAP, Nolte-'t Hoen ENM. Response to letter to the editor. J Extracell Vesicles (2019) 8:1599682. doi:10.1080/20013078.2019.1599682

65. Heintz-Buschart A, Yusuf D, Kaysen A, Etheridge A, Fritz J V., May P, de Beaufort C, Upadhyaya BB, Ghosal A, Galas DJ, et al. Small RNA profiling of low biomass samples: identification and removal of contaminants. BMC Biol (2018) 16:52. doi:10.1186/s12915-018-0522-7

66. Théry C, Witwer KW, Aikawa E, Alcaraz MJ, Anderson JD, Andriantsitohaina R, Antoniou A, Arab T, Archer F, Atkin-Smith GK, et al. Minimal information for studies of extracellular vesicles 2018 (MISEV2018): a position statement of the International Society for Extracellular Vesicles and update of the MISEV2014 guidelines. J Extracell Vesicles (2018) 8:1535750. doi:10.1080/20013078.2018.1535750

67. Clayton A, Buschmann D, Byrd JB, Carter DRF, Cheng L, Compton C, Daaboul G, Devitt A, Falcon-Perez JM, Gardiner C, et al. Summary of the ISEV workshop on extracellular vesicles as disease biomarkers, held in Birmingham, UK, during December 2017. J Extracell Vesicles (2018) 7: doi:10.1080/20013078.2018 .1473707

68. Karimi N, Cvjetkovic A, Jang SC, Crescitelli R, Hosseinpour Feizi MA, Nieuwland R, Lötvall J, Lässer C. Detailed analysis of the plasma extracellular vesicle proteome after separation from lipoproteins. Cell Mol Life Sci (2018)1-14. doi:10.1007/s00018-018-2773-4

69. Feingold K, Grunfeld C. "Introduction to Lipids and Lipoproteins," in Endotext, eds. K. Feingold, B. Anawalt, A. Boyce (MDtext.com). Available at: https://europepmc.org/books/ NBK305896;jsessionid=FC86076A4DE6F06B7F048A4DFF4C7F05\%0A

70. Michell DL, Vickers KC. Lipoprotein carriers of microRNAs. Biochim Biophys Acta-Mol Cell Biol Lipids (2016) 1861:2069-2074. doi:10.1016/j.bbalip.2016.01.011

71. Allen RM, Zhao S, Solano MAR, Michell DL, Shyr Y, Sethupathy P, Linton MF, Graf GA, Sheng Q. Bioinformatic analysis of endogenous and exogenous small RNAs on lipoproteins. J Extracell Vesicles (2018) 7: doi:10.1080/20013078.2018.1506198 
72. van Eijndhoven MAJ, Zijlstra JM, Groenewegen NJ, Drees EEE, van Niele S, Baglio SR, Koppers-Lalic D, van der Voorn H, Libregts SFWM, Wauben MHM, et al. Plasma vesicle miRNAs for therapy response monitoring in Hodgkin lymphoma patients. JCl Insight (2016) 1:1-16. doi:10.1172/jci.insight.89631

73. Mustapic M, Eitan E, Werner JK, Berkowitz ST, Lazaropoulos MP, Tran J, Goetzl EJ, Kapogiannis D. Plasma extracellular vesicles enriched for neuronal origin: A potential window into brain pathologic processes. Front Neurosci (2017) 11:1-12. doi:10.3389/fnins.2017.00278

74. Witwer KW, Buzás El, Bemis LT, Bora A, Lässer C, Lötvall J, Nolte-'t Hoen EN, Piper MG, Sivaraman S, Skog J, et al. Standardization of sample collection, isolation and analysis methods in extracellular vesicle research. J Extracell vesicles (2013) 2:1-25. doi:10.3402/jev.v2i0.20360

75. Sódar BW, Kittel Á, Pálóczi K, Vukman KV, Osteikoetxea X, Szabó-Taylor K, Németh A, Sperlágh B, Baranyai T, Giricz Z, et al. Low-density lipoprotein mimics blood plasma-derived exosomes and microvesicles during isolation and detection. Sci Rep (2016) 6:24316. doi:10.1038/srep24316

76. Buschmann D, Kirchner B, Hermann S, Märte M, Wurmser C, Brandes F, Kotschote S, Bonin M, Steinlein OK, Pfaffl MW, et al. Evaluation of serum extracellular vesicle isolation methods for profiling miRNAs by next-generation sequencing. J Extracell Vesicles (2018) 7: doi:10.1080/20013078.2018.1481321

77. Srinivasan S, Yeri A, Cheah PS, Chung A, Danielson K, De Hoff P, Filant J, Laurent CD, Laurent LD, Magee R, et al. Small RNA Sequencing across Diverse Biofluids Identifies Optimal Methods for exRNA Isolation. Cell (2019) 177:446-462.e16. doi:10.1016/j.cell.2019.03.024

78. Lunavat TR, Cheng L, Kim D-K, Bhadury J, Jang SC, Lässer C, Sharples RA, López MD, Nilsson J, Gho YS, et al. Small RNA deep sequencing discriminates subsets of extracellular vesicles released by melanoma cells - evidence of unique microRNA cargos. RNA Biol (2015) 12:810-823. doi:10.1080/1547 6286.2015 .1056975

79. Squadrito ML, Baer C, Burdet F, Maderna C, Gilfillan GD, Lyle R, Ibberson M, De Palma M. Endogenous RNAs Modulate MicroRNA Sorting to Exosomes and Transfer to Acceptor Cells. Cell Rep (2014)

8:1432-1446. doi:10.1016/j.celrep.2014.07.035

80. Takeuchi O, Akira S. Pattern Recognition Receptors and Inflammation. Cell (2010) 140:805-820. doi:10.1016/j.cell.2010.01.022

81. Barbalat R, Ewald SE, Mouchess ML, Barton GM. Nucleic Acid Recognition by the Innate Immune System. Annu Rev Immunol (2011) 29:185-214. doi:doi:10.1146/annurev-immunol-031210-101340

82. Banchereau J, Briere F, Caux C, Davoust J, Lebecque S, Liu Y, Pulendran B, Palucka K. Immunobiology of Dendritic Cells. Annu Rev Immunol (2000) 18:767-811.

83. Raker VK, Domogalla MP, Steinbrink K. Tolerogenic dendritic cells for regulatory T cell induction in man. Front Immunol (2015) 6:1-11. doi:10.3389/fimmu.2015.00569

84. Penna G, Adorini L. 1 ,25-Dihydroxyvitamin D3 Inhibits Differentiation, Maturation, Activation, and Survival of Dendritic Cells Leading to Impaired Alloreactive T Cell Activation. J Immunol (2000)

164:2405-2411. doi:10.4049/jimmunol.164.5.2405

85. Piemonti L, Monti P, Sironi M, Fraticelli P, Leone BE, Dal Cin E, Allavena P, Di Carlo V. Vitamin D3 affects differentiation, maturation, and function of human monocyte-derived dendritic cells. J Immunol (2000) 164:4443-4451. doi:ji_v164n9p4443 [pii]

86. Adorini L, Penna G, Giarratana N, Uskokovic M. Tolerogenic dendritic cells induced by vitamin D receptor ligands enhance regulatory $T$ cells inhibiting allograft rejection and autoimmune diseases. $J$ Cell Biochem (2003) 88:227-233. doi:10.1002/jcb.10340

87. Lindenbergh MFS, Stoorvogel W. Antigen Presentation by Extracellular Vesicles from Professional Antigen-Presenting Cells. Annu Rev Immunol (2018) 36:annurev-immunol-041015-055700. doi:10.1146/annurev-immunol-041015-055700

88. Robbins PD, Morelli AE. Regulation of immune responses by extracellular vesicles. Nat Rev Immunol (2014) 14:195-208. doi:10.1038/nri3622

89. Nolte-'t Hoen ENM, Buschow SI, Anderton SM, Stoorvogel W, Wauben M. Activated T cells recruit exosomes secreted by dendritic cells via LFA-1 Brief report Activated T cells recruit exosomes secreted by dendritic cells via LFA-1. Blood (2009) 113:1977-1981. doi:10.1182/blood-2008-08-174094 
90. Alexander M, Hu R, Runtsch MC, Kagele DA, Mosbruger TL, Tolmachova T, Seabra MC, Round JL, Ward DM, O'Connell RM. Exosome-delivered microRNAs modulate the inflammatory response to endotoxin. Nat Commun (2015) 6:7321. doi:10.1038/ncomms8321

91. Tkach M, Kowal J, Zucchetti AE, Enserink L, Jouve M, Lankar D, Saitakis M, Martin-Jaular L, Théry C. Qualitative differences in T-cell activation by dendritic cell-derived extracellular vesicle subtypes. EMBO J (2017)e201696003. doi:10.15252/embj.201696003

92. Pitt JM, André F, Amigorena S, Soria J-C, Eggermont A, Kroemer G, Zitvogel L. Dendritic cell-derived exosomes for cancer therapy. J Clin Invest (2016) 126:1224-1232. doi:10.1172/JCl81137

93. Robbins PD, Dorronsoro A, Booker CN. Regulation of chronic inflammatory and immune processes by extracellular vesicles. (2016) 126:1173-1180. doi:10.1172/JCl81131.tions

94. Zitvogel L, Regnault A, Lozier A, Wolfers J, Flament C, Tenza D, Ricciardi-Castagnoli P, Raposo G, Amigorena S. Eradication of established murine tumors using a novel cell-free vaccine: Dendritic cellderived exosomes. Nat Med (1998) 4:594-600. doi:10.1038/nm0598-594

95. Kim S-H, Lechman ER, Bianco N, Menon R, Keravala A, Nash J, Mi Z, Watkins SC, Gambotto A, Robbins PD. Exosomes Derived from IL-10-Treated Dendritic Cells Can Suppress Inflammation and CollagenInduced Arthritis. J Immunol (2014) 174:6440-6448. doi:10.4049/jimmunol.174.10.6440

96. O'Connell RM, Kahn D, Gibson WSJ, Round JL, Scholz RL, Chaudhuri AA, Kahn ME, Rao DS, Baltimore D. MicroRNA-155 promotes autoimmune inflammation by enhancing inflammatory $T$ cell development. Immunity (2010) 33:607-19. doi:10.1016/j.immuni.2010.09.009

97. Boldin MP, Taganov KD, Rao DS, Yang L, Zhao JL, Kalwani M, Garcia-Flores Y, Luong M, Devrekanli A, Xu J, et al. miR-146a is a significant brake on autoimmunity, myeloproliferation, and cancer in mice. J Exp Med (2011) 208:1189-1201. doi:10.1084/jem.20101823

98. Wu W, He C, Liu C, Cao AT, Xue X, Evans-Marin HL, Sun M, Fang L, Yao S, Pinchuk IV, et al. miR-10a inhibits dendritic cell activation and Th1 / Th17 cell immune responses in IBD. Gut (2015) 64:17551764. doi:10.1136/gutjnl-2014-307980

99. LV Y, Ou-yang A, Fu L. MicroRNA-27a Negatively Modulates the Inflammatory Response in Lipopolysaccharide-Stimulated Microglia by Targeting TLR4 and IRAK4. Cell Mol Neurobio/ (2017) 37:195-210. doi:10.1007/s10571-016-0361-4

100. Stepanov GA, Filippova JA, Komissarov AB, Kuligina EV, Richter VA, Semenov DV. Regulatory role of small nucleolar RNAs in human diseases. Biomed Res Int (2015) 2015:206849. doi:10.1155/2015/206849

101. Martens-Uzunova ES, Olvedy M, Jenster G. Beyond microRNA - Novel RNAs derived from small non-coding RNA and their implication in cancer. Cancer Lett (2013) 340:201-211. doi:10.1016/j. canlet.2012.11.058

102. Villarroya-Beltri C, Gutiérrez-Vázquez C, Sánchez-Cabo F, Pérez-Hernández D, Vázquez J, MartinCofreces N, Martinez-Herrera DJ, Pascual-Montano A, Mittelbrunn M, Sánchez-Madrid F. Sumoylated hnRNPA2B1 controls the sorting of miRNAs into exosomes through binding to specific motifs. Nat Commun (2013) 4:2980. doi:10.1038/ncomms3980

103. Mukherjee K, Ghoshal B, Ghosh S, Chakrabarty Y, Shwetha S, Das S, Bhattacharyya SN. Reversible HuRmicroRNA binding controls extracellular export of miR122 and augments stress response. EMBO Rep (2016) 17:11841203. doi:10.15252/embr

104. Shurtleff MJ, Temoche-Diaz MM, Karfilis KV., Ri S, Schekman R. Y-box protein 1 is required to sort microRNAs into exosomes in cells and in a cell-free reaction. Elife (2016) 5:1-23. doi:10.7554/ eLife. 19276

105. Santangelo L, Giurato G, Cicchini C, Montaldo C, Mancone C, Tarallo R, Battistelli C, Alonzi T, Weisz A, Tripodi M. The RNA-Binding Protein SYNCRIP Is a Component of the Hepatocyte Exosomal Machinery Controlling MicroRNA Sorting. Cell Rep (2016) 17:799-808. doi:10.1016/j.celrep.2016.09.031

106. Lee H, Li C, Zhang Y, Zhang D, Otterbein LE, Jin Y. Caveolin-1 selectively regulates microRNA sorting into microvesicles after noxious stimuli. (2019) 
107. Driedonks TAP, Nolte-'t Hoen ENM. Circulating Y-RNAs in Extracellular Vesicles and Ribonucleoprotein Complexes; Implications for the Immune System. Front Immunol (2019) 9:1-15. doi:10.3389/ fimmu.2018.03164

108. Colombo M, Moita C, van Niel G, Kowal J, Vigneron J, Benaroch P, Manel N, Moita LF, Théry C, Raposo G. Analysis of ESCRT functions in exosome biogenesis, composition and secretion highlights the heterogeneity of extracellular vesicles. J Cell Sci (2013) 126:5553-65. doi:10.1242/jcs.128868

109. van Niel G, Charrin S, Simoes S, Romao M, Rochin L, Saftig P, Marks MS, Rubinstein E, Raposo G. The Tetraspanin CD63 Regulates ESCRT-Independent and -Dependent Endosomal Sorting during Melanogenesis. Dev Cell (2011) 21:708-721. doi:10.1016/j.devcel.2011.08.019

110. Sim S, Weinberg DE, Fuchs G, Choi K, Chung J, Wolin SL. The Subcellular Distribution of an RNA Quality Control Protein, the Ro Autoantigen, Is Regulated by Noncoding Y RNA Binding. Mol Biol Cell (2009) 20:1555-1564. doi:10.1091/mbc.E08

111. Haderk F, Schulz R, Iskar M, Cid LL, Worst T, Willmund KV, Schulz A, Warnken U, Seiler J, Benner A, et al. Tumor-derived exosomes modulate PD-L1 expression in monocytes. Sci Immunol (2017) 2:eaah5509. doi:10.1126/sciimmunol.aah5509

112. Tebaldi T, Zuccotti P, Peroni D, Köhn M, Gasperini L, Potrich V, Bonazza V, Dudnakova T, Rossi A, Sanguinetti G, et al. HuD Is a Neural Translation Enhancer Acting on mTORC1-Responsive Genes and Counteracted by the Y3 Small Non-coding RNA. Mol Cell (2018) 71:256-270. doi:10.1016/j. molcel.2018.06.032

113. Repetto E, Lichtenstein L, Hizir Z, Tekaya N, Benahmed M, Ruidavets J-B, Zaragosi L-E, Perret B, Bouchareychas L, Genoux A, et al. RNY-derived small RNAs as a signature of coronary artery disease. BMCMed (2015) 13:259. doi:10.1186/s12916-015-0489-y

114. Dhahbi JM, Spindler SR, Atamna H, Boffelli D, Martin DIK. Deep Sequencing of Serum Small RNAs Identifies Patterns of 5'tRNA Half and YRNA Fragment Expression Associated with Breast Cancer. Biomark Cancer (2014) 6:37-47. doi:10.4137/BIC.S20764.RECEIVED

115. Peters AL, van Hezel ME, Cortjens B, Tuip-de Boer AM, van Bruggen $R$, de Korte $D$, Jonkers RE, Bonta PI, Zeerleder SS, Lutter R, et al. Transfusion of 35-Day Stored RBCs in the Presence of Endotoxemia Does Not Result in Lung Injury in Humans*. Crit Care Med (2016) 44:e412-e419. doi:10.1097/ CCM.00000000000001614

116. Lowry SF. Human endotoxemia: A model for mechanistic insight and therapeutic targeting. Shock (2005) 24:94-100. doi:10.1097/01.shk.0000191340.23907.a1

117. van Lier D, Geven C, Leijte GP, Pickkers P. Experimental human endotoxemia as a model of systemic inflammation. Biochimie (2018)1-8. doi:10.1016/j.biochi.2018.06.014

118. Hotchkiss RS, Monneret G, Payen D. Sepsis-induced immunosuppression: From cellular dysfunctions to immunotherapy. Nat Rev Immunol (2013) 13:862-874. doi:10.1038/nri3552

119. Angus DC, van der Poll T. Severe sepsis and septic shock. N Engl J Med (2013) 369:703-707. doi:10.1007/978-81-322-0535-7_88

120. Fry DE. Sepsis, systemic inflammatory response, and multiple organ dysfunction: The mystery continues. Am Surg (2012) 78:1-8.

121. Reinhart K, Bauer M, Riedemann NC, Hartog CS. New approaches to sepsis: Molecular diagnostics and biomarkers. Clin Microbiol Rev (2012) 25:609-634. doi:10.1128/CMR.00016-12

122. Terrasini N, Lionetti V. Exosomes in critical illness. Crit Care Med (2017) 45:1054-1060. doi:10.1097/ CCM.00000000000002328

123. Raeven P, Zipperle J, Drechsler S. Extracellular vesicles as markers and mediators in sepsis. Theranostics (2018) 8:3348-3365. doi:10.7150/thno.23453

124. Benz F, Roy S, Trautwein C, Roderburg C, Luedde T. Circulating MicroRNAs as biomarkers for sepsis. Int J Mol Sci (2016) 17: doi:10.3390/ijms17010078

125. Groot Kormelink T, Mol S, de Jong EC, Wauben MHM. The role of extracellular vesicles when innate meets adaptive. Semin Immunopathol (2018)1-14. doi:10.1007/s00281-018-0681-1 
8. General Discussion

126. Genschmer KR, Russell DW, Lal C, Szul T, Bratcher PE, Noerager BD, Abdul Roda M, Xu X, Rezonzew G, Viera L, et al. Activated PMN Exosomes: Pathogenic Entities Causing Matrix Destruction and Disease in the Lung. Cell (2019) 176:113-126.e15. doi:10.1016/j.cell.2018.12.002

127. Poste G. Bring on the biomarkers. Nature (2011) 469:156-157. doi:10.1038/469156a

128. Diamandis EP. Cancer biomarkers: Can we turn recent failures into success? J Natl Cancer Inst (2010) 102:1462-1467. doi:10.1093/jnci/djq306

129. Van Deun J, Mestdagh P, Agostinis P, Akay Ö, Anand S, Anckaert J, Martinez ZA, Baetens T, Beghein E, Bertier $L$, et al. EV-TRACK: transparent reporting and centralizing knowledge in extracellular vesicle research. Nat Methods (2017) 14:228-232. doi:10.1038/nmeth.4185 
8 


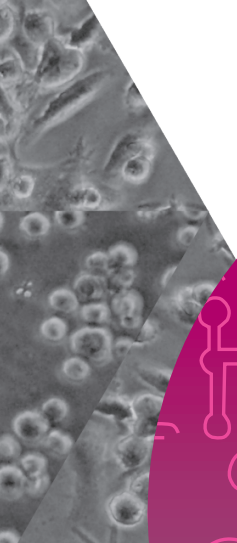
$\cos ^{2}+2$ 628 ?

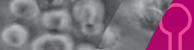

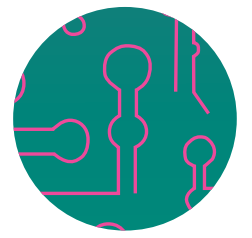

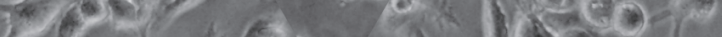


Appendix

Nederlandse samenvatting

Curriculum Vitae

List of Publications

Dankwoord 


\section{Nederlandse samenvatting voor leken}

\section{Extracellulaire vesicles spelen een rol in cel-cel communicatie}

Extracellulaire vesicles (EV) zijn 50 tot 1000 nanometer kleine 'membraanblaasjes', welke bestaan uit een lipide membraan, eiwitten en RNA. Deze membraanblaasjes worden uitgescheiden door vrijwel alle typen cellen in het lichaam, en zijn daarnaast aanwezig in verschillende lichaamsvloeistoffen zoals melk, urine, speeksel en bloedplasma. EV worden uitgescheiden door de ene cel, en opgenomen door een andere cel. Via deze EV worden signaalstoffen tussen cellen getransporteerd. Je zou EV kunnen vergelijken met een 'cellulaire pakketjesdienst' die eiwitten en RNA uitwisselt tussen cellen, wat op verschillende manieren processen in ontvangende cellen beïnvloedt. Behalve via EV wisselen cellen ook op andere manieren signalen uit, zoals via direct contact tussen receptoren op het celmembraan, en via uitgescheiden signaalstoffen zoals cytokines. Deze twee laatstgenoemde routes worden al jarenlang intensief onderzocht. Daarentegen is de rol van EV in de communicatie tussen cellen relatief kort geleden ontdekt. We willen beter begrijpen welke rol deze vesicles spelen in de signalering tussen cellen.

\section{Extracellulaire vesicles worden via verschillende routes gevormd in de cel}

De term EV omvat een zeer diverse populatie membraanblaasjes die zich afsplitsen van cellulaire membranen. Deze blaasjes kunnen op twee verschillende plaatsen in de cel gevormd worden. De zogenaamde 'microvesicles' worden gevormd door uitstulping en afsplitsing ('budding') van het celmembraan. De zogenaamde 'exosomen' worden gevormd door instulping en afsplitsing van het membraan van het endosoom, een compartiment binnenin de cel. Deze 'bubbels-in-een-bubbel' ('intraluminale vesicles') komen vrij wanneer het endosoom fuseert met het plasmamembraan. Tijdens de vorming van deze beide typen vesicles worden specifieke moleculen verpakt (bijvoorbeeld bepaalde eiwitten en bepaalde RNA typen), om te worden uitgezonden naar andere cellen. Ook al worden microvesicles en exosomen op verschillende plaatsen in de cel gevormd, op dit moment is het niet mogelijk deze verschillend gevormde vesicles van elkaar te onderscheiden nadat zij zijn uitgescheiden. Exosomen en microvesicles hebben namelijk een soortgelijke grootte, dichtheid en samenstelling. Omdat er dus geen unieke eigenschappen zijn waaraan je een exosoom of microvesicle kan herkennen, en in de meeste studies een combinatie van microvesicles en exosomen wordt onderzocht, gebruiken we bij voorkeur de collectieve term Extracellulaire Vesicles (EV). 


\section{De samenstelling van EV verandert onder invloed van veranderingen in de activatiestatus van cellen}

Interessant genoeg is de moleculaire inhoud van EV niet altijd hetzelfde. Onder invloed van externe factoren, zoals stress of als gevolg van een ziekteproces, kan een 'geactiveerde' cel EV uitscheiden met een inhoud die afwijkt van EV van een nietgeactiveerde cel. Door deze afwijkende inhoud hebben EV van geactiveerde cellen mogelijk een ander effect op ontvangende cellen dan EV van niet-geactiveerde cellen. Daarnaast kunnen afwijkingen in de samenstelling van EV in lichaamsvloeistoffen wijzen op de aanwezigheid van bepaalde ziekten, waardoor EV interessant zijn om als biomarker te worden toegepast. Hiervoor is het belangrijk om te weten welke veranderingen in EV samenhangen met een bepaalde ziekte, en welke cellulaire processen hierbij een rol spelen. Hoe de inhoud van EV verandert onder invloed van een bepaalde cellulaire stimulus staat centraal in dit proefschrift. Deze veranderingen kunnen zowel invloed hebben op de functie van deze EV, als wel nieuwe inzichten opleveren over een mogelijke toepassing als biomarker.

\section{Functies van verschillende typen RNA in cellen}

Met name de RNA inhoud van EV heeft de laatste jaren sterk in de belangstelling gestaan. RNA is net als DNA een drager van genetische informatie, en fungeert als een kopie van genen die in het DNA liggen opgeslagen. Omdat EV-RNA centraal staat in dit proefschrift zal ik kort wat achtergrond geven over verschillende typen RNA en hun functie in de cel. Het meest bekende type RNA is het zogenaamde messenger RNA ('boodschapper RNA', mRNA), hetgeen fungeert als blauwdruk voor de productie van eiwitten. Genen die in het DNA liggen, worden 'gekopieerd' (transcriptie) naar RNA, en op basis van deze RNA-kopieën worden vervolgens eiwitten geproduceerd (translatie). Deze eiwitten zijn de 'onderdelen' van een cel, die ervoor zorgen dat deze kan functioneren. Naar schatting bestaat een cel uit wel 10.000 verschillende eiwitten, die tezamen vrijwel alle processen in de cel uitvoeren. Voor het optimaal functioneren van een cel moet elk eiwit op het juiste moment in de juiste hoeveelheid geproduceerd worden; met de juiste efficiëntie zijn functie vervullen; en ook weer op tijd worden afgebroken of stopgezet als zij niet meer nodig is. Je kunt je wel voorstellen dat een cel dus een enorm complex geheel is, waarin zich talrijke processen tegelijkertijd afspelen die strak gereguleerd moeten worden om te kunnen functioneren.

Behalve eiwit-coderende mRNAs bestaat er ook een groep niet-coderende RNAs ('non-coding RNAs', (ncRNAs)) die andere essentiële functies vervullen in de cel. Veel ncRNAs vormen samen met verschillende eiwitten grote RNA-eiwitcomplexen die bepaalde biochemische processen uitvoeren. Deze complexen zou je kunnen vergelijken met minuscule 'nano-robotjes' die bepaalde functies vervullen. Het ribosoom bijvoorbeeld bestaat uit wel 50 verschillende eiwitten en 4 verschillende 
ribosomale RNAs (rRNA), en produceert alle eiwitten in de cel aan de hand van de aanwezige mRNAs. De zogenaamde tRNAs zorgen voor de aanvoer van aminozuren die nodig zijn voor de productie van eiwitten. Daarnaast zorgen de snoRNAs voor de correcte vorming van ribosomale RNAs, en spelen de snRNAs een rol bij de vorming van functionele mRNAs. De Y-RNAs spelen mogelijk een rol bij de afbraak van foutief gevormde mRNAs. Verder spelen de zogenaamde microRNAs (miRNAs) een rol in de regulatie van gen-expressie. Deze extreem korte RNAs kunnen binden aan mRNAs, wat verhindert dat deze mRNAs worden getransleerd tot eiwitten, of leidt tot de afbraak van mRNAs. Deze voorbeelden illustreren dat de verschillende typen non-coding RNAs belangrijk zijn in allerlei processen die belangrijk zijn voor correcte eiwitexpressie.

\section{EV bevatten verschillende soorten non-coding RNA}

Behalve dat deze ncRNAs belangrijke functies vervullen in cellen, is gebleken dat veel van deze ncRNAs ook zeer efficient in EVs terecht komen om te worden verzonden naar andere cellen. Waarom dit gebeurt, en of de ncRNAs in EV ook een functie vervullen wanneer zij worden opgenomen door ontvangende cellen, is grotendeels onbekend. Met name de miRNAs in EV zijn afgelopen jaren intensief onderzocht, omdat hun effect op gen-expressie grotendeels bekend is. Er is bijvoorbeeld aangetoond dat miRNAs via EV van de ene naar de andere cel getransporteerd kunnen worden. Er is ook aangetoond dat deze overgedragen miRNAs inderdaad de gen-expressie van hun specifieke target mRNAs kunnen remmen. Verder is aangetoond dat de activatie/ ziekte-status van cellen invloed heeft op de miRNA inhoud van EV die deze cellen uitscheiden. Zo verschilt bijvoorbeeld de miRNA inhoud van EV uit kankercellen van die van gezonde cellen. Ook verschilt de miRNA samenstelling in plasma van patiënten met verschillende ziektes zoals kanker, de ziekte van Alzheimer en rheumatoide arthritis. Behalve miRNAs bevatten EV ook allerlei andere ncRNA typen, zoals tRNA, Y-RNA, snRNA en snoRNA. Sommige hiervan zijn zelfs in hogere mate aanwezig dan de meeste miRNAs. Deze ncRNAs in EV dragen mogelijk bij aan de communicatie tussen cellen, maar dit is nog niet in detail onderzocht. Verder is ook onbekend of hun aanwezigheid in EV beïnvloed kan worden door ziekten of activatie van EVproducerende cellen. Welke veranderingen in ncRNA samenstelling samenhangen met welke ziekte is een belangrijke vraag om in de toekomst te beantwoorden. Op basis hiervan kunnen in de toekomst in een paar druppels bloed de aanwezigheid van ziekte en ziektestadium worden bepaald, wat kan leiden tot betere diagnoses, prognoses en patiënt-specifieke behandelingen. 


\section{In dit proefschift}

Een eerste stap om te onderzoeken of andere typen ncRNA in EV, naast miRNAs, een rol spelen in cel-cel communicatie, is om te bepalen of hun aanwezigheid in EV beïnvloed wordt door de activatiestatus van EV-producerende cellen. Daarom hebben we onderzocht of veranderingen in de activatiestatus van gekweekte immuuncellen invloed hebben op de incorporatie van verschillende typen ncRNAs in EV. Omdat onbekend is welke RNAs in EV veranderen door deze stimuli, hebben we gebruik gemaakt van een geavanceerde techniek waarmee alle RNAs in een EV of cel bepaald kunnen worden, genaamd RNA sequencing. Behalve dat deze techniek belangrijk is voor het beantwoorden van fundamentele onderzoeksvragen, wordt RNA-sequencing ook steeds vaker gebruikt om afwijkingen in EV-RNA in lichaamsvloeistoffen te bepalen, bijvoorbeeld in relatie tot ziekten. RNA-sequencing is een extreem gevoelige methode, maar is ook vatbaar voor systematische meetfouten. Om deze meetfouten in kaart te brengen hebben wij deelgenomen aan een grote studie die is opgezet door de National Institutes of Health $(\mathrm{NIH}$, de Amerikaanse overheidsinstelling die veel fundamenteel gezondheidsonderzoek uitvoert), beschreven in Hoofdstuk 2. In deze studie zijn 1152 verschillende microRNAs gemaakt in het lab die als referentie samples zijn gebruikt om de betrouwbaarheid en de nauwkeurigheid van RNA sequencing methoden te vergelijken tussen verschillende methoden uitgevoerd in 9 verschillende laboratoria. Uit deze studie is gebleken dat RNA sequencing data sterk afwijken van de daadwerkelijke samenstelling van een RNA sample. Sommige RNA moleculen werden zeer efficiënt gedetecteerd, en andere juist veel minder. Daarnaast bleken verschillende sequencing methoden elk een andere afwijking te geven. Hierdoor is het onmogelijk om data te vergelijken die met verschillende methodes verkregen zijn. Data gegenereerd met dezelfde methode in verschillende laboratoria was wel goed vergelijkbaar. Daarnaast bleek uit onze resultaten dat relatieve verschillen in RNAs tussen samples zeer nauwkeurig konden worden bepaald. Dus, RNA-sequencing geeft beperkt inzicht in de absolute samenstelling van een RNA sample, maar kan wel goed worden gebruikt om verschillen in de hoeveelheid van RNAs tussen samples te bepalen.

In Hoofdstuk 3 hebben we een ander technisch probleem onderzocht wat belangrijk is voor het betrouwbaar bepalen van de RNA inhoud van EV van gekweekte cellen. Deze cellen worden namelijk gekweekt in medium waaraan foetaal kalfsserum (fetal calf serum, FCS) wordt toegevoegd. Dit serum bevat allerlei voedingsstoffen en groeifactoren die nodig zijn om cellen te laten groeien in plastic petrischaaltjes. Echter, net als andere lichaamsvloeistoffen bevat FCS ook EV waar RNA in zit. Het RNA in FCS$\mathrm{EV}$ is niet te onderscheiden van EV van de cellen die worden onderzocht. Doorgaans worden de FCS-EV verwijderd door middel van ultracentrifugatie, wat voor de meeste studies voldoende is. Er is echter kort geleden aangetoond dat na ultracentrifugatie 
nog allerlei FCS-RNA achterblijft. Omdat het RNA in FCS niet te onderscheiden is van het EV-RNA van gekweekte cellen, kan dit de meting van EV-RNA verstoren. Daarom hebben we in Hoofdstuk 3 onderzocht welke technische optimalisaties de hoeveelheid achtergebleven FCS-RNA kan verminderen, en in hoeverre het achtergebleven FCSRNA terecht komt in gezuiverde EV uit gekweekte cellen. Hierin hebben wij gevonden dat sommige typen RNA wel degelijk efficiënt verwijderd kunnen worden door middel van ultracentrifugatie. Daarentegen worden andere RNA typen niet efficiënt verwijderd en blijven inderdaad achter in het kweekmedium. Desalniettemin bleken veel van deze achtergebleven RNAs niet detecteerbaar in opgezuiverde EV. Op basis van onze resultaten hebben wij aanbevelingen opgesteld voor experimentele controles om de hoeveelheid achtergebleven FCS-RNA in opgezuiverd EV-RNA te bepalen, en dit te rapporteren in publicaties. Verder hebben wij de meest optimale methode gebruikt in onze verdere studies waarin EV van gekweekte cellen onderzocht werden.

In Hoofdstuk 4 hebben we onderzocht hoe de RNA inhoud van EV verandert ten gevolge van verschillende immuun-gerelateerde stimuli. In deze studie hebben we gebruik gemaakt van gekweekte dendritische cellen (DC), welke een belangrijke rol spelen in de aansturing van het immuunsysteem. Door middel van immuunactiverende (LPS, een bestanddeel van bacteriën wat een sterke immuun activerende werking heeft) en immuun-remmende (Vitamine D3) stoffen toe te voegen aan deze cellen, verandert de cel-status en functie van deze DC. Op basis van de opgedane kennis uit Hoofdstukken $\mathbf{2}$ en $\mathbf{3}$ hebben wij RNA sequencing gebruikt om te bepalen hoe de RNA inhoud verschilt tussen EV geproduceerd door LPS- en VitD3-gestimuleerde en door ongestimuleerde DC. Naast verwachte verschillen in miRNA inhoud bleken de EV ook te verschillen in de hoeveelheid Y-RNA en snoRNA die zij bevatten. Met name de veranderingen in Y-RNA waren interessant omdat dit type RNA in hoge mate aanwezig is in EV van verschillende typen cellen, en het mogelijk een rol speelt in regulatie van processen in het immuunsysteem. Onze resultaten waren een eerste indicatie dat naast miRNAs ook andere typen RNA kunnen veranderen onder invloed van cel-stimulatie. Daarnaast hebben we de veranderingen in het EV-RNA vergeleken met de veranderingen in het RNA in de cellen. Het werd voorheen aangenomen dat het EV-RNA een volledige afspiegeling is van EV-producerende cellen. Echter, uit onze gegevens bleek dat slechts een klein deel van de veranderingen in EV-RNA overeenkwamen met die in cellulair RNA. Verschillende typen RNA, waaronder Y-RNA, vertoonden verschillen in EV terwijl in cellen geen verschil was in de hoeveelheid van deze RNAs. Dit suggereert dat het merendeel van het RNA in EV op een zeer specifieke wijze verpakt wordt in deze vesicles.

Omdat het Y-RNA zo veelvuldig is gevonden in verschillende studies naar EVRNA, en het mogelijk een rol speelt in immuun-regulatie hebben we hier een literatuuronderzoek naar gedaan. In Hoofdstuk $\mathbf{5}$ bespreken we de functies die 
Y-RNA vervult in de cellen en welke studies Y-RNA hebben aangetoond in EV. In Hoofdstuk 4 hebben we gezien dat de hoeveelheid Y-RNA die in EV verpakt wordt, niet beïnvloed wordt door de hoeveelheid Y-RNA in de cel. Dit is een aanwijzing dat bepaalde eiwitten betrokken zijn bij de verpakking van Y-RNA in EV. Om uit te zoeken welke eiwitten hierbij een rol zouden kunnen spelen, hebben we een overzicht gemaakt van de eiwitten waarvan is aangetoond dat ze kunnen binden aan Y-RNA. Vervolgens hebben we bepaald of deze eiwitten al eerder in EV zijn aangetoond. Dit zou hints kunnen geven over welke eiwitten verantwoordelijk zijn voor de verpakking van Y-RNA in EV. Verder hebben we verschillende studies vergeleken die effecten van Y-RNA beschrijven in relatie tot het immuunsysteem. Interessant genoeg worden er zowel immuun-activerende als immuun-onderdrukkende functies van extracellulair Y-RNA beschreven. Mogelijk hangt dit af van of Y-RNA in een EV zit of is gebonden aan een extracellulair eiwitcomplex.

We hebben in Hoofdstuk 4 gezien dat de hoeveelheid Y-RNA in EV afhankelijk is van immuun-stimulatie, waardoor het mogelijk bruikbaar kan zijn als biomarker voor ontstekingsziekten. Daarnaast is bekend dat Y-RNA in hoge mate aanwezig is in bloedplasma. Plasma bevat verschillende RNA-bevattende structuren, waaronder EV, maar ook lipoproteïne partikels (LPP) en RNA-eiwitcomplexen. Het is onbekend welke structuren in plasma Y-RNA bevatten. In Hoofdstuk 6 hebben we verschillende structuren in plasma van elkaar gescheiden op basis van grootte en soortelijk gewicht. Y-RNA bleek met name in EV te lokaliseren, terwijl verschillende miRNAs ook in LPP en eiwitcomplexen gevonden werden. We hebben aangetoond dat Y-RNA omsloten is door het EV-membraan en daardoor bestand is tegen afbraak door verschillende enzymen, terwijl miRNAs in LPP daardoor afgebroken werden. Er zijn 4 subtypen bekend van het Y-RNA (Y1, Y3, Y4 en Y5) en deze zijn alle vier aan te tonen in het bloed. Vervolgens hebben we bestudeerd of EV van verschillende typen bloedcellen deze Y-RNA subtypen bevatten. Hierbij viel op dat de EV van verschillende celtypen andere hoeveelheden van Y-RNA subtypen bevatten. De EV van de meest voorkomende immuuncel in bloed, de zgn. neutrofiel, bevatte relatief veel Y4-RNA en weinig Y3RNA. Deze unieke hoge Y4/Y3 ratio zagen we niet in EV van andere bloedcellen (witte bloedcellen, rode bloedcellen, bloedplaatjes en endotheliale cellen). Neutrofielen bleken in korte tijd zeer veel EV uit te scheiden wanneer zij geactiveerd raakten door LPS, terwijl andere celtypen niet reageerden in deze tijd. Vervolgens hebben we gekeken of we dit effect van immuunactivatie dat we zagen in kweekbakjes, ook terug zouden vinden als een verandering in circulerend Y-RNA in humaan plasma. Hiervoor hebben we gebruik gemaakt van een studie waarbij vrijwilligers geïnjecteerd zijn met LPS. Dit leidde tot een sterke ontstekingsreactie en veranderingen in immuuncelaantallen in het bloed. Van deze vrijwilligers is op verschillende tijdpunten na injectie plasma afgenomen. In dit plasma hebben we de hoeveelheid van verschillende Y-RNAs 
gemeten. Interessant genoeg zagen we dat de $\mathrm{Y} 4 / \mathrm{Y} 3$ ratio in plasma verhoogd was tijdens en na de ontstekingsreactie, en dat deze ratio correleerde met een verhoging in circulerende neutrofielen. Dit duidt erop dat de $Y 4 / Y 3$ ratio mogelijk verband houdt met EV uitgescheiden door geactiveerde neutrofielen. De toepasbaarheid van de Y4/ Y3 ratio als cel type-specifieke biomarker kan verder worden onderzocht in relatie tot ontstekingsziekten zoals sepsis of arthritis.

Uit de voorgaande hoofstukken is gebleken dat Y-RNA mogelijk belangrijk is voor de signalering tussen immuuncellen en mogelijk kan worden ingezet als biomarker voor ontstekingsziekten. Om beter te begrijpen welke cellulaire processen invloed hebben op de sortering van Y-RNA naar EV, hebben we in Hoofdstuk 7 onderzocht welke eiwitten hierin een rol zouden kunnen spelen. Hiervoor hebben we gebruik gemaakt van een aanverwant type immuuncel, de macrofaag. Net als dendritische cellen en neutrofielen reageren macrofagen sterk op de aanwezigheid van ziekteverwekkers. Deze macrofagen hebben we gestimuleerd met een ander type bacteriële component, genaamd Pam3CSK4. Als gevolg van activatie produceerden zij meer EV waarin zich een hogere concentratie Y-RNA bevond. We hebben onderzocht of de Y-RNA bindende eiwitten die we in Hoofdstuk $\mathbf{5}$ gevonden hadden, in deze EV te detecteren waren. Dit was alleen het geval voor het belangrijkste Y-RNA-bindende eiwit Ro60, terwijl andere eiwitten niet gedetecteerd werden in EV. Het is mogelijk dat deze eiwitten de Y-RNA moleculen alleen afleveren op de plaats waar EV gevormd worden, maar zelf niet verpakt worden in deze EV. Deze hypothese hebben we onderzocht door structuren waar EV gevormd worden te scheiden van andere structuren in de cel. We vonden een hoge concentratie Y-RNA op plaatsen waar EV gevormd werden. Hier bevonden zich ook meerdere Y-RNA bindende eiwitten die we in Hoofdstuk 5 hadden gevonden. Om Y-RNA efficiënter te verpakken, zou je je voor kunnen stellen dat de lokale concentratie Y-RNA waar EVs gevormd worden verhoogd moet zijn. Activatie leidde tot een licht verhoogde Y-RNA concentratie op de plek waar EV gevormd worden, maar gaf geen duidelijke verplaatsing van de meeste Y-RNA bindende eiwitten. Dus, het is onwaarschijnlijk dat deze eiwitten direct een rol spelen in de verpakking van Y-RNA in EV. We zagen wél een verschuiving van een Y-RNA bindend eiwit genaamd HuR. De lokalisatie van dit eiwit veranderde van Y-RNA bevattende fracties naar het endoplasmisch reticulum, waar eiwitten geproduceerd worden. Het is bekend dat HuR de aanmaak van signaaleiwitten zoals cytokinen kan bevorderen. Onze data suggereren dat de toegenomen Y-RNA verpakking in EV de lokalisatie van HuR zou kunnen beïnvloeden. Dit zou betekenen dat het verpakken van Y-RNA in EV niet alleen dient om een signaal naar andere cellen te verzenden, maar ook een manier is om processen in de EV-producerende cel aan te sturen. 


\section{Conclusie}

In dit proefschrift hebben we een aantal experimentele procedures onderzocht en geoptimaliseerd die belangrijk zijn voor een betrouwbare isolatie en karakterisatie van EV-RNA. Onze vindingen kunnen bijdragen aan betrouwbaardere data en betere reproduceerbaarheid, en zijn belangrijk voor zowel biomarker-studies als fundamentele celbiologische vraagstukken. Verder hebben we in verschillende immuuncellen aangetoond dat activerende stimuli niet alleen de miRNA-samenstelling van EV beïnvloeden, maar ook de hoeveelheid Y-RNA die in EV wordt verpakt. Dit is een aanwijzing dat Y-RNA mogelijk bijdraagt aan de signalering tussen immuuncellen. Daarnaast blijkt uit onze resultaten dat Y-RNA potentie heeft als nieuwe biomarker voor ontstekingsziekten.

Uit onze resultaten blijkt ook dat veranderingen in EV-RNA slechts ten dele overeen komen met veranderingen in de cel. Dit wijst erop dat de RNA inhoud van EV geen directe weerspiegeling is van zijn cel van oorsprong. Verschillende onderzoeken hebben laten zien dat afwijkingen in EV-RNA in lichaamsvloeistoffen voldoende kunnen zijn voor een diagnose. Desalniettemin kunnen we meer te weten komen over wat deze afwijkingen zeggen over het type en de conditie van de cel als we de onderliggende mechanismen beter begrijpen. Dit kan leiden tot betere classificaties van ziektestadia, hetgeen kan leiden tot betere diagnoses, prognoses en een beter afgestemde behandeling voor patiënten. 


\section{Curriculum Vitae}

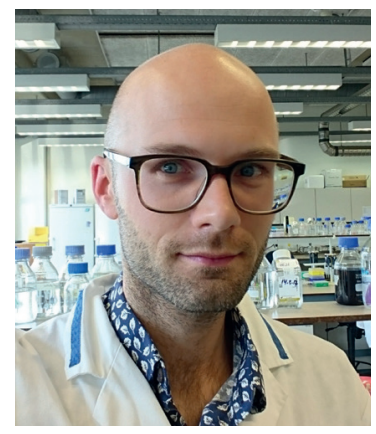

Tom Adrianus Paulus Driedonks was born on November $1^{\text {st }}$ 1986 in Boxtel, the Netherlands. In 2005, he graduated from the Jacob Roelandslyceum (pre-university education) in Boxtel. In September 2005, he started his university study Biotechnology at Wageningen University and Research Centre, and obtained his Bachelor degree in November 2008. He then became a full-time board member and Treasurer of student association JV Unitas, before continuing his studies in September 2009. He conducted his Master thesis at the Laboratory of Virology of Wageningen University under supervision of dr. Stefan Metz and dr. Gorben Pijlman. Here, he employed the baculovirus expression system to produce a virus-like particle vaccine against Chikungunya virus in insect cells. He then conducted an internship at Fundacíon Ciencia para la Vida in Santiago, Chile, under supervision of dr. Nicolás Cifuentes-Muñoz and dr. Nicole Tischler. Here, he studied the Andes virus fusion protein through pseudotyped lentiviral vectors and cell-cell fusion assays. After obtaining his Masters degree in October 2011, he worked as research technician investigating novel recombinant virus-like particle vaccines and subunit vaccines against respiratory syncytial virus (RSV) and human immunodeficiency virus (HIV) in the team of dr. Hans Langedijk and prof. dr. Jörn Stitz at Crucell N.V. (currently Janssen Vaccines) in Leiden.

In November 2014, Tom started his PhD project at the Department of Biochemistry \& Cell Biology at Utrecht University under supervision of dr. Esther Nolte-'t Hoen and prof. dr. Marca Wauben. He studied how immune-related stimuli imposed on immune cells cause changes in the small non-coding RNA content of extracellular vesicles. During his PhD, Tom was awarded a short-term scientific mission grant from the COST Action 'Microvesicles and Exosomes in Health And Disease' to perform experiments in the lab of dr. Amy Buck at the University of Edinburgh. He also took part in a large inter-laboratory study on small RNA sequencing methods lead by the exRNA Communications Consortium (ERCC) of the National Institutes of Health. Furthermore, Tom took part in the Educational Committee of the Infection and Immunity Graduate school. The research conducted during his PhD is described in this thesis and was published in peer-reviewed scientific journals. He will continue his research as postdoctoral researcher at the Johns Hopkins Medical School in Baltimore, MD, USA. 


\section{List of publications}

This thesis

Driedonks TAP and Nolte-'t Hoen ENM, The potential role of $Y$-RNA binding proteins in $T L R$-induced sorting of Y-RNAs into extracellular vesicles Manuscript in preparation

Driedonks TAP, Mol S, de Bruin S, Peters AL, Zhang XG, Lindenbergh MFS, Beuger BM, van Stalborch AD, Spaan T, de Jong EC, van der Vries E, Margadant $C$, van Bruggen R, Vlaar APJ, Groot Kormelink T, Nolte-'t Hoen ENM, Y-RNA subtype ratios in plasma extracellular vesicles are cell type-specific and are candidate biomarkers for inflammatory diseases

Manuscript submitted

Driedonks TAP, Nolte-'t Hoen ENM, Circulating Y-RNA in extracellular vesicles and ribonucleoprotein complexes; implications for the immune system

Frontiers in Immunology, Vol 9, January 2019

Driedonks TAP, Nijen-Twilhaar MK, Nolte-'t Hoen ENM, Technical approaches to reduce interference of Fetal Calf Serum derived RNA in the analysis of extracellular vesicle RNA from cultured cells

Journal of Extracellular Vesicles, Vol. 8, December 2018

Driedonks TAP, van der Grein SG, Ariyurek Y, Buermans HPJ, Jekel H, Chow FWN, Wauben MHM, Buck AH, 't Hoen PAC, Nolte-'t Hoen ENM, Immune stimuli shape the small noncoding transcriptome of extracellular vesicles released by dendritic cells

Cellular and Molecular Life Sciences, Vol 75, May 2018

Giraldez MD, Spengler RM, Etheridge A, Godoy PM, Barczak AJ, Srinivasan S, De Hoff PL, Tanriverdi K, Courtright A, Lu S, Khoory J, Rubio R, Baxter D, Driedonks TAP, Buermans HPJ, Nolte-'t Hoen ENM, Jiang H, Wang K, Ghiran I, Wang Y, Van Keuren-Jensen K, Freedman JE, Woodruff PG, Laurent LC, Erle DJ, Galas DJ, Tewari M, Comprehensive multi-center assessment of small RNA-seq methods for quantitative miRNA profiling Nature Biotechnology, May 2018

Other publications

Driedonks TAP, Nolte-'t Hoen ENM, Response to letter to the editor Journal of Extracellular Vesicles, Vol. 8., April 2019 
Lindenbergh MFS, Koerhuis DGJ, Borg EGF, van 't Veld EM, Driedonks TAP, Wubbolts R, Stoorvogel W, Boes M, Bystander T-cells support clonal T-cell activation by controlling the release of dendritic cell-derived immune-stimulatory extracellular vesicles Frontiers in Immunology, Vol. 10, March 2019

Théry C, Witwer KW, (...), Driedonks TAP (...), Zuba-Surma EK (2019), Minimal information for studies of extracellular vesicles 2018 (MISEV2018): a position statement of the international society for extracellular vesicles and update of the MISEV2014 guidelines Journal of Extracellular Vesicles, Vol. 8, Nov 2018

Van Herwijnen MJC, Driedonks TAP, Snoek BL, Kroon AMT, Kleinjan M, Jorritsma R, Pieterse CMJ, Nolte-'t Hoen ENM, Wauben MHM, Abundantly present miRNAs in milkderived extracellular vesicles are conserved between mammals Frontiers in Nutrition, Vol. 5, September 2018

Mateescu B, Kowal EJK, (...), Driedonks TAP, (...), Nolte-'t Hoen ENM, Obstacles and opportunities in the functional analysis of extracellular vesicle RNA - an ISEV position paper Journal of Extracellular Vesicles, Vol. 6, Mar 2017

van Deun J,(...), Driedonks TAP, (...), Hendrix A, EV-TRACK: transparent reporting and centralizing knowledge in extracellular vesicle research

Nature Methods, Vol 14, Issue 3, 2017

\section{Conferences}

\section{Oral presentations}

Decoding the genetic messages exchanged by cells via extracellular vesicles

Science for Life 2017, Utrecht

Differences and similarities in full-length and fragmented non-coding RNA biotypes in EV from differentially stimulated dendritic cells

ISEV 2017, Toronto

Immunogenic vs tolerogenic dendritic cells release extracellular vesicles that differ in small non-coding RNA content

Veterinary Science Day 2016, Driebergen

Infection and Immunity Graduate School PhD Retreat 2016, Leersum

ISEV 2016, Rotterdam 


\section{Poster presentations}

$Y$-RNA is a common non-coding RNA component of extracellular vesicles and a potential biomarker for immune-related conditions

EMBO | EMBL Symposium: The Non-Coding Genome, Heidelberg, 2019

$T L R$-stimulation affects the non-coding RNA composition of extracellular vesicles released by immune cells

Veterinary Science Day, Bunnik, 2018

I\&I 25 years Symposium, Utrecht, 2018

Comparison of Fetal Calf Serum EV depletion protocols indicates differences in depletion efficiency of miRNAs and other RNA classes

NLSEV 2018, Amsterdam

ISEV 2018, Barcelona

Immunogenic vs tolerogenic dendritic cells release extracellular vesicles that differ in small non-coding RNA content

Dutch Immunology Winter School (NVVI) 2015, Noordwijkerhout

\section{Other accomplishments}

\section{Prizes and grants}

- COST-MeHaD Short Term Scientific Mission Grant

- Best Oral presentation - Infection and Immunity Graduate School PhD Retreat 2016

International activities

- Two-week exchange to Edinburgh for short-term scientific mission in the lab of dr. Amy Buck

- Take part as only international collaborator in inter-lab sequencing study lead by the exRNA consortium of the NIH

\section{Teaching activities}

- Supervision of two master students and two higher laboratory education students

- Designing, organizing and supervising 2.5-day research practicals for Bachelor students 


\section{Courses attended}

- Writing successful grant proposals (online course, Utrecht University)

- Writing a scientific paper (6x half-day writing course, Utrecht University)

- RNA-seq data analysis (3 day training course, Leiden University Medical Center)

- Online database resources for non-coding RNA (one day, at EMBL-EBI, Hinxton, UK)

- Advanced immunology (7 day theoretical course, Utrecht University)

\section{Other activities}

- Chairman during poster session at ISEV2018, Barcelona

- Organization of Pubquiz part of the 25 year Anniversary of the Infection \& Immunity Graduate School

- Member of the Infection and Immunity Educational Committee (September 2016 - February 2019)

- In charge of audiovisual equipment at ISEV 2015 RNA seminar in Utrecht

- Organizer of departmental scientific meetings 
"The solution often turns out more beautiful than the puzzle" Richard Dawkins 


\section{Dankwoord}

Zo, het zit erop! Nu kan ik terecht zeggen dat alle geruchten over het doen van een PhD op waarheid berusten: het is zwaar, experimenten werken nooit precies zoals je ze plant, resultaten zijn nooit duidelijk genoeg, het is nooit af en zie de boel dan maar eens gepubliceerd te krijgen... Maar wat wás het de moeite waard en wat heb ik veel geleerd! Wetenschap bedrijven is een teamsport, je kunt nooit alles zelf en dat hoeft ook niet. Ik heb uit eerste hand gezien hoe waardevol het is om technieken, kennis en klinische samples te delen om uiteindelijk tot een gezamenlijk eindproduct te komen. Ik wil hier graag iedereen bedanken die ik de afgelopen 5 jaar heb leren kennen tijdens dit avontuur in 'Vesicletopia' en die direct of indirect heeft meegeholpen aan de totstandkoming van dit proefschrift.

Esther, ik begin bij jou. Wat ben ik enorm dankbaar dat je me deze PhD positie hebt gegund en dat je me al die jaren de juiste kant op hebt geholpen. In het begin moest ik wat wennen aan je kritische blik, maar ik ben gaan inzien dat dit een belangrijke voorwaarde is voor het doen van kwalitatief hoogstaand onderzoek. Onder druk ontstaan vaak de mooiste dingen! Ik denk met veel plezier terug aan de vele werkbesprekingen, waarin we zowel in het diepste detail op ruwe data konden puzzelen, maar waarin ook aandacht was voor het opzetten van 'argumentatiebomen' voor papers én voor persoonlijke ontwikkeling. Ik waardeer het enorm hoe je me altijd hebt gestimuleerd om te blijven nadenken over het nut van een experiment en hoe dit bijdraagt aan een stuk, in plaats van als een kip zonder kop experimenten te draaien. Jouw mantra 'welke vraag wil je hiermee beantwoorden' komt in een gouden lijstje op mijn volgende werkplek. Naast 'gericht proeven doen', was jouw talent voor het opzetten van samenwerkingen een eye-opener waar ik ook in de toekomst nog veel aan zal hebben. Ook jouw aansporing aan het begin van het laatste jaar om 'zelf de drijvende kracht achter je onderzoek te zijn' heb ik ter harte genomen. Hopelijk blijkt dit uit de laatste twee experimentele hoofdstukken die allebei in korte tijd tot stand zijn gekomen en daarnaast interessante inzichten hebben opgeleverd. Ik wil je enorm bedanken voor alle support, ik weet zeker dat we elkaar tegen zullen blijven komen in het kleine EV-veld!

Marca, ontzettend bedankt dat je me zo hebt gesteund tijdens dit project. Ik kon altijd rekenen op enorm veel enthousiasme en support wanneer ik updates gaf over de laatste ontwikkelingen binnen mijn project. Daarnaast heb je ook mijn kijk op het schrijfproces van papers veranderd: toen ik stond te klagen over de eindeloze stroom commentaar op één van de manuscripten heb je me laten inzien dat schrijven teamwerk is, en dat het krijgen van veel correcties niet wil zeggen dat je niet kunt 
schrijven. Sindsdien heb ik met steeds meer plezier correcties doorgevoerd, want als ik die eerste versie niet schrijf is er ook niks om op te schieten. Ik heb gemerkt dat je alleen zo tot een mooi eindresultaat komt. En wat was het tof om samen de barricades op te gaan bij de March for Science! Enorm bedankt voor je aanstekelijke enthousiasme, we houden contact!

Susanne, bedankt dat je al deze jaren mijn partner-in-crime in het DC-EV project bent geweest. Waar onze projecten eigenlijk naar elkaar hadden moeten groeien, was de werkelijkheid nogal weerbarstig. Wat hebben we een hoop ellende gehad met celkweken die om uiteenlopende redenen niet meer wilden groeien. Gelukkig konden we onze vele frustraties kwijt bij elkaar en wisten we elkaar goed te supporten bij het uitschrobben van incubators. Je bent een echte doorzetter en ik ben blij dat jouw promotiedatum inmiddels ook in de planning zit. Fijn dat we op het allerlaatst onze experimentele lijntjes nog aan elkaar konden knopen. Natuurlijk ook heel erg bedankt dat je me als paranimf wil ondersteunen tijdens mijn verdediging. Enorm veel succes met de allerlaatste loodjes, jouw titel is straks dubbel en dwars verdiend!

Henrike, bedankt voor alle technische ondersteuning die ik de opstartfase van jou heb gehad. Er viel enorm veel uit te zoeken en te optimaliseren en jij pakte alles aan; lentivirale transducties, SEC kolommetjes gieten, Western blots optimaliseren, RNA isolatie kits vergelijken, EV isolatie methodes fine-tunen en nog veel meer. Ik kan me ook nog een enorme DC-kweek herinneren met wel 80 kweekschaaltjes die je in je uppie hebt opgewerkt om een recordhoeveelheid RNA te verkrijgen. Daar heb ik heel wat sequencing en Northern blots op kunnen doen! Enorm bedankt voor alles wat je hebt gedaan en veel geluk in de toekomst.

Martijn, wat heb ik een hoop gelachen met jou ('Kun je zien dat ik geplast heb?', 'unas apapas, unas peperas'). Daarnaast is volgens mij het halve repertoire van Jiskefet voorbij gekomen de afgelopen jaren. Als de melk-EVs ooit uitgemolken raken, kun je altijd nog cabaretier worden. Maar naast alle flauwekul hebben we ook nog serieuze wetenschap bedreven. Ik vond het een mooie uitdaging om gezamenlijk uit te puzzelen hoe die miRNAs in melk-EVs hun functionele effecten konden verklaren. En laten we vooral ons paper in Frontiers in Nutrition niet vergeten ;-) ! Bedankt ook voor al jouw adviezen op basis van jouw ervaring als postdoc en natuurlijk ook voor de vele Vinex-Vesicle BBQs!

Tom, TGK, Tommeehhh, ik was altijd erg onder de indruk van jouw gedrevenheid, dat heeft aanstekelijk gewerkt en ervoor gezorgd dat ik zelf ook wel wat harder door kon werken. Daarnaast heb ik de vele gezamenlijke uurtjes in de celkweek en de hulp met de muizen erg gewaardeerd. En wat is het tof dat er zelfs na jouw vertrek nog een 
mooie samenwerking is ontstaan waar een mooi hoofdstuk uit is voortgevloeid. Ik wens je veel succes in Amsterdam, we gaan elkaar ongetwijfeld nog vaak tegenkomen op allerlei ISEV meetings en andere gelegenheden.

Sten, zelfs de meest simpele werkbespreking-presentatie kreeg van jou altijd een thematisch sausje met een persoonlijke touch. En natuurlijk moeten alle grafiek-assen op dezelfde schaal staan en moet alles netjes worden uitgelijnd! Dat geldt natuurlijk ook voor de Influx, wat een geweldig apparaat maar wat kun je daar een hoop aan uitlijnen, optimaliseren en tweaken...man man man man! Bedankt ook voor alle hele goede én minder goede muzikale tips (du weisst?) die ik van je heb gekregen. Verder moet ik helaas bekennen dat ik nog steeds niet weet in welke drie Italiaanse dorpjes nou de échte mozzarella gemaakt wordt! Heel veel succes in Cambridge en houdoe war!

Marijke, jij was voor mij het levende bewijs dat alles goed komt in het laatste jaar van je promotie. Daar heb ik me al die jaren mee op de been gehouden, gelukkig ging ook bij mij alles op het laatst in een stroomversnelling. Bedankt voor alle gezelligheid in het lab, met jouw zonnige (what's in a name?) persoonlijkheid is het altijd feest. STOP!....en door! Ik wens je heel veel succes in Maastricht, hopelijk kun je daar nog even aanblijven en mooie resultaten behalen.

Marthe, bedankt dat ik gebruik mocht maken van jouw expertise op het gebied van humane cellen! Daar heb ik zeker veel van geleerd en ik ben blij dat ik jóu dan weer kon helpen met qPCRs. Het was ook altijd leuk om de verhalen over jouw sportieve weekendprestaties te horen, maar of ik me zelf nou ooit aan een triathlon ga wagen... ik weet het nog niet. Wat een geweldig idee trouwens om een hele middag 'They're Taking The Hobbits to Isengard' op repeat op vol volume in het lab te draaien, ik ben blij dat we dit hebben doorgezet. En mocht ik in de toekomst nog iets patenteerbaars uitvinden, dan weet ik je zeker te vinden!

Ellen, grappig dat ik jou al tijdens mijn eerste sollicitatie hier tegenkwam en dat het meteen klikte. Het was altijd fijn om met jou de laatste lab-roddels te delen en kleine irritaties te ventileren. Ook kon ik met kleine technische vragen altijd bij jou terecht. Ik weet zeker dat jouw georganiseerde stijl van werken bij Genmab in de smaak gaat vallen. Enneehm, gaan we nog een keer koffie drinken daar?

Janneke, met jou was het ook altijd gezellig: wadlopen, dinner parties, zelfs salsadansen, jij bent overal voor in. En ook bedankt dat je op Koos hebt gepast in een van onze vakanties, gelukkig heb je nu zelf twee lieve poezebeesten om plat te 
knuffelen. Ik klop zeker bij je aan als ik van plan ben een hele grote beursaanvraag te doen, maar ook om gewoon bij te buurten natuurlijk.

Marije, bedankt voor alle ondersteuning door de jaren heen. Alle bestellingen, uitgevulde FCS, R1 sup, ingevroren beenmerg, schoonmaakroosters, boze mailtjes... Zonder jouw bezielende leiding zou het lab allang in elkaar gestort zijn. En daarnaast run je ook nog allerlei experimenten, ben je te porren voor poster presentaties en uitwisseling naar Sydney; jij bent geen technician maar een duizendpoot! Heel veel succes in de komende jaren, ik weet zeker dat het goed gaat komen.

Kyra, toen je hier als stagiair binnen kwam maakte je al een vliegende start, heel indrukwekkend. Met jouw proefschrift gaat het ongetwijfeld helemaal goed komen, gezien jouw vrolijke, positieve persoonlijkheid en motivatie. Heel veel succes!

Alberta, crazy cat lady, thanks for being such a nice and vibrant colleague. It was always nice to have you around at borrels, to annoy you with remarks about pineapple pizza and to drink a Duveltje or two. It's been a pleasure to teach you some Dutch curse words while working in the cell culture lab, I hope you'll never have to use them. I wish you all the best for the coming years, I'm sure you will write a great thesis!

Estefanía, it was really nice having you as a colleague. Everything is one big project, right? Thanks for the help with lipoprotein particles in the Influx, I hope you can figure out how to get rid of them in the near future. And thanks for helping me keep my Spanish vocabulary on point, I'm sure it will become rusty once I leave. I wish you all the best finishing your thesis, and may the flow be with you!

Xiaogang, Master of Western blots, thanks for all the help with lipoprotein-related questions. I'm always amazed how much work you get done in (what seems to me like) a short time, and I hope this efficiency will guide you towards a very good thesis. I think you're on the right track, maybe you have enough data already ;-) ? It was really nice to have you as a colleague, and I wish you the best for your final year and years to come.

Marije, bedankt voor de gezelligheid op de verschillende congressen en symposia. Ik hoop dat de parasiet-vesicles lief voor je zijn en dat je een geweldig proefschrift gaat afleveren. 
Sanne, bedankt voor de vele neutrofiel-proeven, daar is in no-time toch een mooi hoofdstuk uit voortgekomen. Ik hoop dat je ook nog iets aan mijn PCR data hebt. Ook jij bent lekker bezig in je PhD project, ik wens je heel veel succes in de komende jaren!

Willem, bedankt voor alle stimulerende discussies in de wandelgangen over de mogelijke functies van allerhande types RNA. En ook bedankt voor alle kritische vragen en opmerkingen tijdens de vrijdagochtend werkbesprekingen, het is altijd goed om in een vroeg stadium tegengas te krijgen. Ik zal mijn best doen om in de toekomst net zo'n wandelende encyclopedie te worden net als jij. Bedankt voor alles!

Ger, bedankt voor alle vele uurtjes achter de Influx, daar keek ik altijd erg naar uit. Al had ik nog zoveel gradiënten om te meten, jij draaide er je hand niet voor om. Natuurlijk was er ook altijd tijd voor gezellige gesprekken over vakanties, lekker eten en natuurlijk talloze muzikale discussies, daardoor vloog de tijd altijd voorbij. Heel erg bedankt voor alle ondersteuning!

Richard, bedankt voor de gezamenlijke stageprojecten die we hebben vormgegeven. Het was leuk om samen allerlei wilde ideeën uit te werken, want jouw enthousiasme en vindingrijkheid werken aanstekelijk. Kon ik me maar in tweeën splitsen, dan had ik handen genoeg om eindelijk die fusie events tussen EV en cellen op de gevoelige plaat vast te leggen. Met de kennis van nu denk ik dat je daar gerust een hele PhD aan kunt besteden. Ik hoop dat het je in de toekomst gaat lukken!

Aike, bedankt voor alle gezelligheid en niet-lab-gerelateerde activiteiten, dinertjes en concerten. Die avond waarop we hebben bewezen dat je overal een bitterbal van kunt maken zal ik niet snel vergeten. Het is fijn om te horen dat je een leuk lab hebt gevonden in Groningen, verder wens ik je veel succes met je verdediging zeer binnenkort!

Martijn, jij ook bedankt voor alle gezelligheid op bovenstaande niet-lab-gerelateerde activiteiten en natuurlijk alle zelfgebrouwen biertjes van jou en Aike, waarvoor ik me graag als proefkonijn wilde inzetten. Ik denk dat ik nog veel kan leren van jouw R-skills en ik zal zeker mijn best doen om jouw lipidomics tools te promoten binnen het EV-veld. Veel succes in de loop naar je promotie en natuurlijk ook bij het vervolg in Genève!

Ruud, bedanktvoor hetoplossen vanallerhandetechnische problemen, kapotgedraaide centrifugebuizen, lege stikstofvaten en opgebruikte competente E.coli's. Daarnaast ook bedankt voor het luisterende oor dat je aanbood op de zwaardere momenten en uiteraard ook voor alle gezelligheid binnen en buiten het lab om. Nu ga je de labs op de faculteit een stukje veiliger maken, heel veel succes daarbij! 
Liz, thanks a lot for the mental support you provided during the last months of my project. It was a very stressful period and you always offered a listening ear. Your overseas PhD students must be blessed with you as a supervisor. I wish you all the best during your postdoc here. And don't forget the goat!

Maya, Nafiseh, Laura, Ziying, Min, Jie, thanks for all the conversations and the good vibes during lunch at the coffee table. I wish you all the best in finishing your PhD projects.

Bernd, Dora, Bart, Martin, Chris, Jos, Bas, Nick, Celia, Jeroen, Rachid, Theo, Carla, Jillis, Petra, Louise, Esther V en Esther Z, jullie ook bedankt voor alles!

Dan mijn studenten, Matthijs, Sharnilla, Maarten en Delaram, bedankt voor jullie inzet en bedankt dat ik jullie mocht begeleiden tijdens jullie stages. Ik heb veel geleerd van het supervisen, proefopzetten bedenken, samen op ruwe data puzzelen en jullie de juiste weg op sturen. Ik hoop dat jullie hier een goede tijd hebben gehad en dat ik iets van mijn enthousiasme voor onderzoek doen op jullie heb kunnen overbrengen. Ik wens jullie heel veel succes in jullie verdere loopbaan!

Amy, thanks for hosting me in your lab in Edinburgh. In the short time I had to perform experiments, you really taught me to plan experiments efficiently and think them through in advance. In the end, things worked out really well, so thanks a lot for your help and enthousiasm. I hope we'll stay in touch and that we'll meet again at future conferences.

Franklin, thanks for supervising me in Amy's lab and helping me out with the Northern blots. It was great working with you, always cheerful and willing to help out. It was a pleasure to show you around when you visited Amsterdam. I hope I'll have the chance to visit you in Hong Kong. Good luck with your postdoc project there, we will stay in touch!

Peter-Bram, bedankt voor de hulp bij het ontwerp van de RNA-sequencing experimenten, de hulp bij en de kritische blik op de statistische analyses. Henk, bedankt voor alle bioinformatische analyses en het organiseren van de vele sequencing runs. Yavuz, bedankt voor alle technische hulp met het maken van de sequencing libraries, ik heb veel geleerd van jouw technische expertise.

Alexander, Robin, Coert, Erhard, Esther, Anna-Linda, Sanne, Boukje, Anne-Marieke, Thom, veel dank voor jullie hulp met de verschillende bloedcellen en bloedplaatjes, 
en uiteraard ook voor het delen van de klinische samples en bijbehorende data. Uit onze samenwerking is een mooi hoofdstuk voortgekomen, ik heb veel geleerd van dit project en denk hier met veel plezier aan terug.

Bernard en Pascal, ik ben blij dat we ondanks alle werkzaamheden in het buitenland en alle reisavonturen regelmatig zijn blijven afspreken. Heel erg fijn dat jullie zo hebben meegeleefd en dat we mijn eerste paper samen hebben kunnen vieren. Daarnaast was het ook fijn om het over minder wetenschappelijke dingen te hebben. Als ik straks in de US zit zijn jullie uiteraard van harte welkom!

Joris, bedankt voor alle dansjes die we op Lowlands en in 013 hebben gemaakt. Af en toe moet je gewoon het lab uit en even uit de band springen. Ik ben blij dat we dat gewoon hebben volgehouden de afgelopen jaren.

Cas, jij ook bedankt voor de gezelligheid in de afgelopen jaren, muziekjes uitwisselen, nachtjes losgaan bij Mr. Scruff en lekker skaten op de boulevard van Scheveningen.

Jery, bedankt voor de vele skatetochtjes die we samen hebben gemaakt, naar de March for Science en de tourtochtjes in omgeving Utrecht. Samen in de prijzen vallen op het I\&I PhD retreat was ook een mooi moment. Succes met het afronden van jouw PhD project!

Verder aan alle Dutch Distance Skaters, in het bijzonder Lennart, Lonneke, Rick, Stan, Patrick, Anton, Joey, Eric en Saskia: bedankt voor alle longboard events die me hebben gemotiveerd om in vorm te blijven. Met een goede conditie is het makkelijker om door te blijven ploeteren in het lab. Natuurlijk ook bedankt voor alle kletspraat en slechte grappen tijdens de lange tochten!

Paps, mams, bedankt dat jullie me altijd gestimuleerd en ondersteund hebben. Zonder jullie support was ik niet gekomen waar ik nu ben. Helaas kun je van spelen met Lego niet je beroep maken, in plaats daarvan komt spelen met plastic eppendorf buisjes toch vrij dicht in de buurt. Tijdens het doen van alle proeven is wel weer gebleken dat kleine details vaak een groot verschil kunnen maken, dus ik ben blij dat jullie een pietje-precies van me hebben gemaakt. De rest is een kwestie van doorgaan en je boerenverstand gebruiken (toch, mam?). En paps, je hebt toch gelijk gekregen dat je werkelijk overal aan kan rekenen, alleen zijn het bij mij geen moeren en bouten geworden. Bedankt dat jullie me vrij hebben gelaten om hier in het lab de volle $100 \%$ te kunnen geven, alle keren dat ik mijn frustraties en onzekerheden kon delen en alle onvoorwaardelijke support. Jullie zijn de beste! 
Nicky, lief zusje, bedankt voor de steun die ik van je heb gehad. Het was fijn dat we enkele jaren samen in het PhD-schuitje zaten, ook al lag jij iets op mij voor. Het was fijn om onze ervaringen en frustraties met elkaar te kunnen delen en er voor elkaar te kunnen zijn. Jij wist precies wat ik doormaakte en andersom. Super bedankt dat je me als paranimf wil ondersteunen tijdens mijn verdediging. Luuk, jij ook heel erg bedankt voor je steun in de afgelopen jaren.

Jannie en Chris, Linda en Sjoerd, bedankt voor alle gezelligheid in Tilburg en jullie steun de afgelopen jaren. Het was altijd fijn om bij jullie over de vloer te komen en lief dat jullie zo met mij en Ellen hebben meegeleefd.

Koos, jij mag als pluizige, miauwende scientific advisor op pootjes niet ontbreken in dit rijtje. Bedankt voor alle kopjes en alle keren dat je me 's ochtends uit bed hebt gehaald omdat je brokjes wilde, alle stress die ik bij jou heb mogen weg-aaien en voor alle keren dat je over mijn toetsenbord hebt gewandeld (ook al hebben deze toevoegingen het proefschrift niet gehaald...). Je bent het liefste poezebeest wat er is!

Lieve Ellen, wat ontzettend lief dat je er altijd voor me bent geweest. Fijn dat we samen in hetzelfde schuitje zaten en dat we dit PhD avontuur samen hebben beleefd, dat je mijn chagrijnige buien hebt doorstaan, dat je eindeloos rondjes met me hebt gewandeld en dat je me hebt gemotiveerd om te blijven sporten en spinnen. Lief dat je straks het volgende avontuur in Baltimore met me aan wil gaan. Het zal vast even wennen zijn om een nieuw leven op te bouwen, maar weet zeker dat we daar net zoveel lol gaan hebben als hier. Het is de afgelopen jaren maar weer gebleken dat we een geweldig team zijn, nu in het bijzonder als husband \& wife. Je bent de liefste van de wereld! 
